\title{
$D R-0179-1$
}

\section{UNITED STATES DEPARTMENT OF THE INTERIOR \\ GEOLOGICAL SURVEY}

\section{WYOMING}

basic data for thermal springs and wells

as recorded in GEOTHERM

Open-File Report $83-439$

\author{
By \\ James D. Bliss
}

USGS-OFR- $-83-439$

TI84 900976

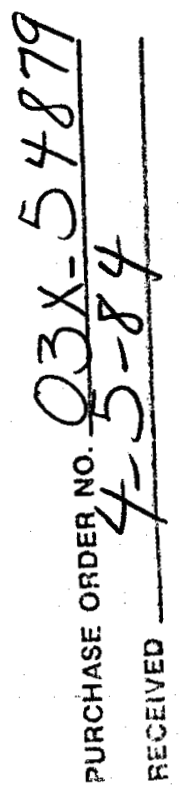

This report is preliminary and has not been reviewed for conformity with U.S. Geological Survey editorial standards and stratigraphic nomenclature. Any use of trade names is for descriptive purposes only and does not imply endorsement by the USGS.

$$
\text { Menio Park, California }
$$

May, 1983

orp

ESTREBUTON OF THS DOCUMEN IS UNLIMITED 


\section{DISCLAIMER}

This report was prepared as an account of work sponsored by an agency of the United States Government. Neither the United States Government nor any agency Thereof, nor any of their employees, makes any warranty, express or implied, or assumes any legal liability or responsibility for the accuracy, completeness, or usefulness of any information, apparatus, product, or process disclosed, or represents that its use would not infringe privately owned rights. Reference herein to any specific commercial product, process, or service by trade name, trademark, manufacturer, or otherwise does not necessarily constitute or imply its endorsement, recommendation, or favoring by the United States Government or any agency thereof. The views and opinions of authors expressed herein do not necessarily state or reflect those of the United States Government or any agency thereof. 


\section{DISCLAIMER}

Portions of this document may be illegible in electronic image products. Images are produced from the best available original document. 


\section{LEGIBILITY NOTICE}

A major purpose of the Technical Information Center is to provide the broadest possible dissemination of information contained in DOE's Research and Development Reports to business, industry, the academic community, and federal, state, and local governments. Non-DOE originated information is also disseminated by the Technical Information Center to support ongoing DOE programs.

Although large portions of this report are not reproducible, it is being made available only in paper copy form to facilitate the availability of those parts of the document which are legible. Copies may be obtained from the National Technical Information Service. Authorized recipients may obtain a copy directly from the Department of Energy's Technical Information Center. 


\section{INTRODUCTION}

GEOTHERM, a computerized information system now off-line, was used to maintain data on the geology, geochemistry and hydrology of geothermal sites primarily within the United States. The system was proposed at the First Geothermal Implementation Conference in New Zealand in 1974 (Swanson, 1977) and was active unt11 1983. The primary mission was to provide a broad informational framework for the Geothermal Research Program (Duffield and Guffanti, 1981). GEOTHERM was used to support national geothermal assessments--in 1978 (Muffler, 1979) and 1982 (Reed, 1983). It was however a public system and provided data to both public and private sectors. A detailed discussion on databases in GEOTHERM and a general scheme of how the information system operated can be found in 81iss and Rapport (1983).

This report on Wyoming is one of a series intended to preserve the data collected for GEOTHERM and make the data available to the public. States with significant geochemical data for geothemal fluids will be covered in individual reports such as this. A report will also be issued to cover miscellaneous data collected for sites in the central and eastern United States. The data found in this series is also available as a data file on the internationally-available General Electric Mark III service, a timeshare network. Those interested in accessing that system should contact the Energy Resource Center, University of OK1ahoma, Norman, OKlahoma 73070 . It is anticipated that a portion of the data will also be available on magnetic tape from the National Technical Information Service, U. S. Department of Commerce, Springfield, VA 22161 . It will not be available until after the completion of the open-file series.

\section{GEOTHERM INDEXES}

Three computer-generated indexes are found in appendices $A$, $B$, and $C$ of this report. The indexes give one line summaries of each GEOTHERM record. Each index is sorted by different variables to assist the user in locating geothermal records describing specific sites.

Appendix A (p. 188-194) is sorted by the county name and the name of the source. Also given are latitude, longitude (both in decimal minutes), township, range, section, GEOTHERM record identifier, and temperature $\left(O^{\circ}\right)$. In conducting a search of Appendix $A$, site names are quite useful for locating springs or wells for which a specific name is commonly used, but sites which do not have specific names are more difficult to locate. It is suggested that site titles which begin with words such as warm, hot, unnamed, pumped, weil, or spring be checked. Descriptive text found as part of the site name and the site coordinates should be used to assist in determining location. 
Appendix B (p. 195-201) is sorted by county, township, range, and section. Also given are name of source, GEOTHERM record identifier, and temperature $\left({ }^{\circ} \mathrm{C}\right)$. Records missing items used for sorting will be listed first.

Appendix C (p. 202-209) is first sorted into one-degree blocks by ratitude, and longitude, and then by name of source. Adjacent one-degree blocks which are published as a $1: 250,000$ map are combined under the appropriate map name. Also given are GEOTHERM record identifier, and temperature $\left({ }^{\circ} \mathrm{C}\right)$. Records missing items used for sorting will be listed first. Numbers with a blank in the same position as zero will be given first.

\section{GEOTHERM SAMPLE FILE}

GEOTHERM sample file contains 356 records for Wyoming. (Table 1). Records may be present which are duplicates. A record may contain data on location, sample description, analysis type (water, condensate, or gas), collection condition, flow rates, and the chemical and physical properties of the fiuid. Stable and radioactive isotopic data are occasionally available. Some records may contain only location and temperature. When sufficient chemical data was available, the charge balance (percentage of difference in anion- and cation-milliequivalents) was computed and added to the record. Many of the numeric fields in the sample file can be directly qualified. The qualifier code precedes the number when appropriate. The codes and their meaning are given in Table 1.

Each thermal spring or well is usually represented by several records. This may document temporal changes in the geothermal fluids. Judgement on what constituted acceptable data was extremely complicated and the primary attempt was to insure that each GEOTHERM record faithfully reproduced the pub1 ished data. On occasion, glaring inconsistencies or data clearly of poor quality were excluded. Regrettably, no database can be constructed or supported wi thout the introduction of errors. The user, therefore, is advised to check with the published literature whenever possible. Users should carefully and critically evaluate the records they use.

This compilation should contain all of the chemical data for geothermal fluids in Wyoming available as of December, 1981. However, no claim is made for completeness, and published sources have probably been missed. A substantial number of observations for geothemal features in Kellowstone National Park have been omitted. All of the records in this list contains information which was published at the time of data entry. A critically evalusted and corrected 1ist of over 2000 records for the united States was extracted from the sample file and issued as a reference document for the national low-temperature geothermal resource assessment (Reed and others, 1983). This, along with a list of geothermal springs by Berry, and others (1980) may be helpful to some users. 


\section{GEOTHERM BIBLIOGRAPHY}

A bibliography is given in Appendix $D$ (p. 210-211). The abbreviated form of the reference (called code) is identifed as the record source in the full record list and is used to sort the entries in this appendix. Codes with a leading " $*$ " identify records based on information which was unpublished at the time the record was prepared.

\section{ACKNOWLEDGMENTS}

Contributions and support to GEOTHERM have been made by many in both federal and state agencies. This includes the U.S. Department of Energy (and associated contractors), and U.S. National Oceanic and Atmospheric Administration. Data-entry forms for most sites in Wyoming were prepared by the staff of the University of Wyoming or the U.S. Geological Survey.

\section{REFERENCES CITED}

Berry, G. W., Grim, P. J., and Ikelman, J. A., 1980, Thermal springs list for the United States: National Oceanic and Amospheric Administration, Key to Geophysical Records Document No. $12,59 \mathrm{p}$.

Bliss, J. D., and Rapport, Amy, 1983, GEOTHERM: the U.S. Geological Survey geothermal information system: Computers \& Geosciences, v. 9 , no. 1, p. 35-39.

Duffield, W. A., and Guffanti, Marianne, 1981, The geothermal research program of the U.S. Geological Survey: U.S. Geological Survey Open-File Report 81-564, $108 \mathrm{p}$.

Muffler, L.J. P., ed., 1979, Assessment of geothermal resources of the United States--1978: U.S. Geological Survey Circular $790,163 \mathrm{p}$.

Reed, M. J., ed., 1983, Assessment of low-temperature geothemal resources of the United States--1982: U.S. Geological Survey Circular 892 .

Reed, M. J., Mariner, R. H., Brook, C. A., and Sorey, M. L., 1983, Selected data for low-temperature (less than $90^{\circ} \mathrm{C}$ ) geothermal systems in the United States; reference data for U.S. Geological Survex Circular 892: U.S. Geological Survey Open-File Report 83-250, 129 p.

Swanson, J. R., 1977, GEOTHERM data file: Geothermal Resources Council Transactions, v. 1, p. 285. 
TABLE 1

\begin{abstract}
State of Wyoming: computer-generated listing of records describing geothermal-fluid samples. [A few records may be for cold springs or wells--this was to provide ground-water references for some studies.]
\end{abstract}

ORGANIZATION: Records are sorted by county and then by the name of the spring or well. Order is the same in Appendix A.

UTM: The UTM Easting label was omitted. The UTM Easting figure will be given directly below the Northing 1 abel.

QUALIFICATION CODES: AII numeric attributes may be qualified. The codes and their meaning:

$$
\begin{aligned}
& L=\text { less than } \\
& G=\text { greater than } \\
& E=\text { estimated } \\
& T=\text { trace (no numeric value reported) } \\
& N=\text { not detected (not followed by number) } \\
& Q=\text { qualified (other data in qualification fierd) } \\
& R=\text { midpoint of range (actual range in qualification field) }
\end{aligned}
$$

REFERENCE: An expanded citation of the reference is found in Appendix $D$. The abbreviated form used in this table is called "CODE" if the appendix. Unpublished sources are preceded with "*". 


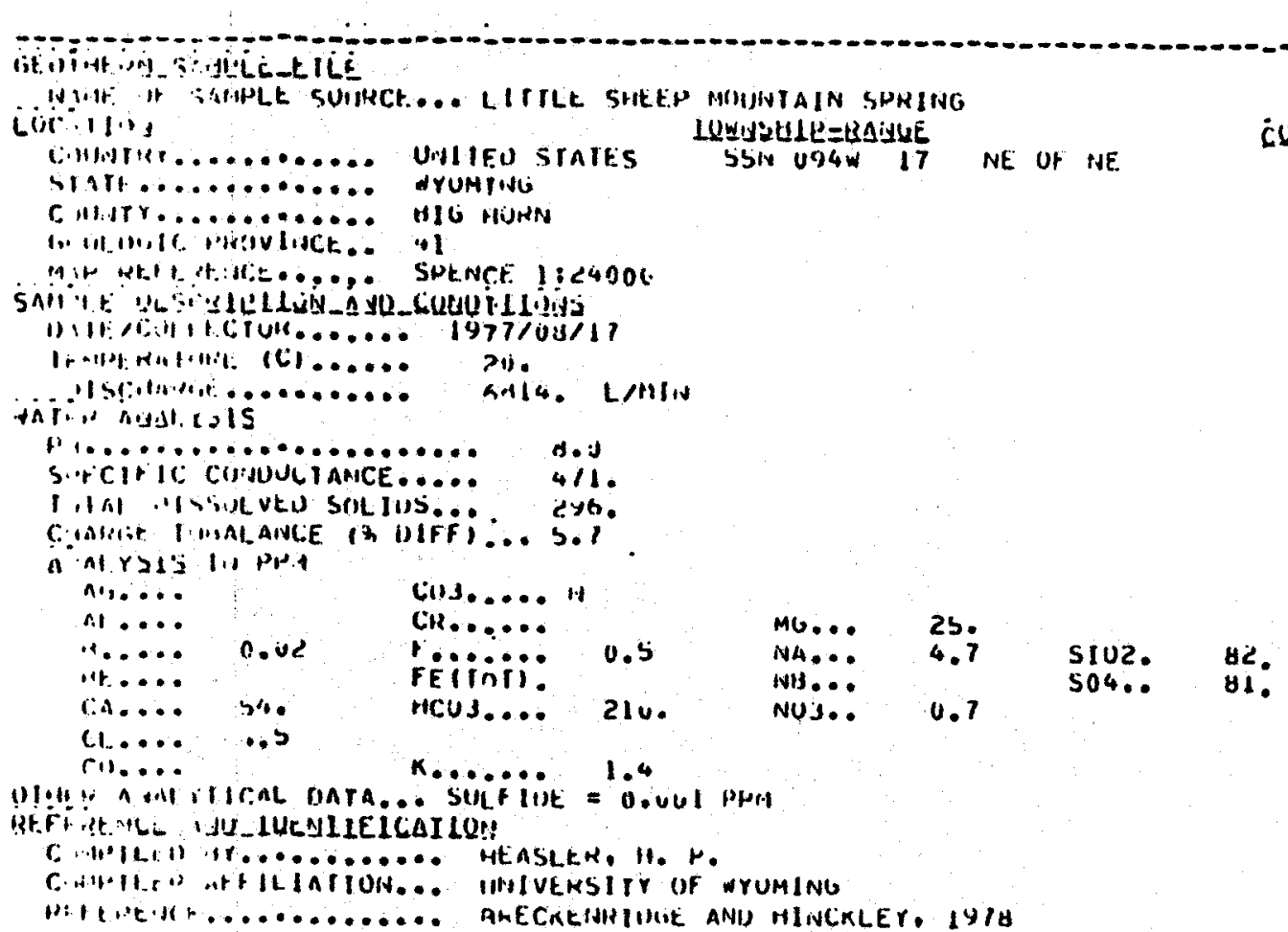

RECURD DOOOI

\section{CUQRULUaIts}

LAI/LUNG... 44-44.90 N 108-11.3S N

UIM ZUNE... 12

NURTHING... 4\$SHGCI.

$722 b 12$.

\section{ISULOEES LCLUL}

HECUNO OUOOZ

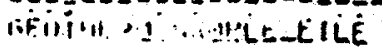

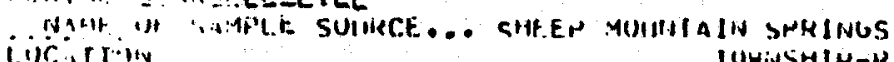

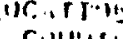

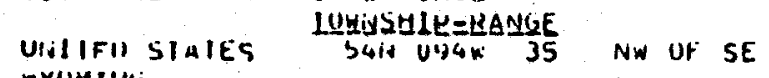

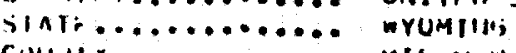

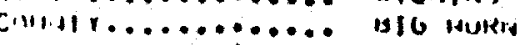

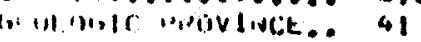

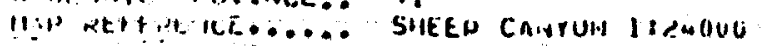

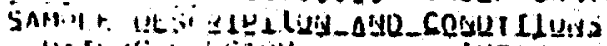

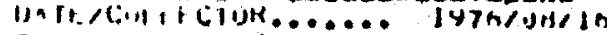

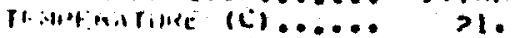

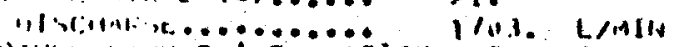

o!ll a

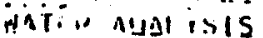

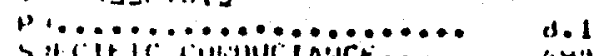

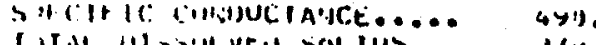

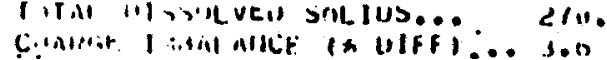

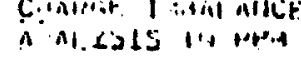

I. $1 .$.

CuUEuLuaIts:

LAT/LUNU... 44-36.45 N 10B-08.13 W

UTM ZUNE... 112

NUHIHING... 4943125.
(1) $3 . \ldots . .1$
ar.....

ISULUEES_LLOLLL 

cir.......
116...
22.3
5102.
into...

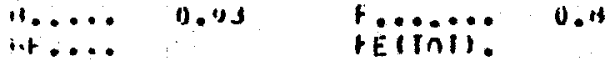
in.... si. neus... 230 .
No.s.:
n........ 1.4
r.o. $\ldots$

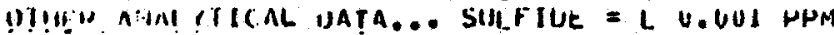
KEFFIFNC IJU_HUEIEIGAILUN
C.ANILII "T........... HEASLER, H. H.
C.
H.telet.rit.

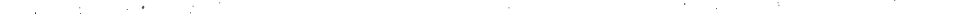

i,Enintion

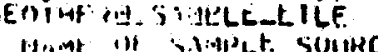

Lijeifini

SIMIF........... WYOMINI;

C.MIIT............ Ciatinn

(i.)(11.1)11: phlivlivet...4 41

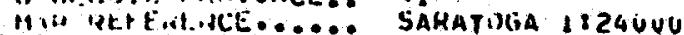

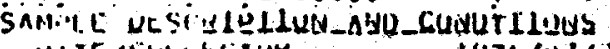

JNTF/CUI I H.CIUK........ $147 \mathrm{~b} / U 1 / 20$

IC

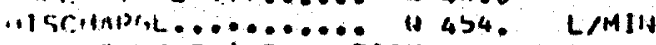

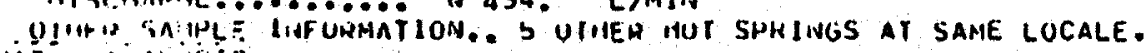

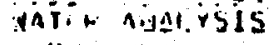

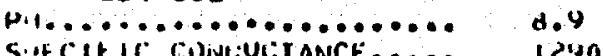

12\%000

Cinielit linne AnCE (h llff;i

$A$ ritiesti lif plit coj

A.i.....

$11 \ldots \ldots 1 . . .1$

ili.....

r.a... Jund

Coj. . 24.

24.

LI...

11. $5 . .$.

0.075

ISUIURES lec0ul

(i) .....

F. Bini:

NA...

NOS.:

450.

5102.
504.

$57 \%$.

Cin.....

nCos.... 130.

K........ 23.

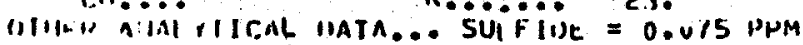

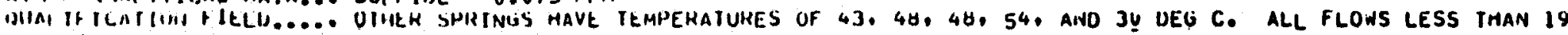

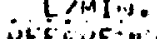

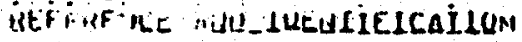

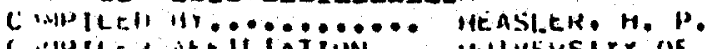

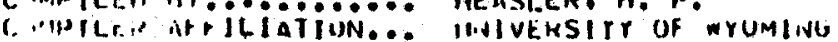

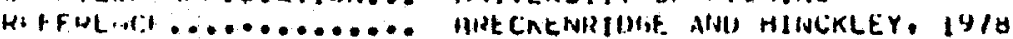

RECUKU OOOOA

cit iifition

GEUTHEKM FILE IUI VOBSUOG

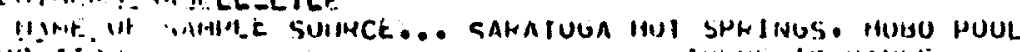

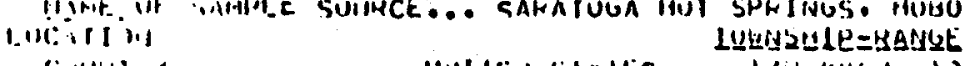

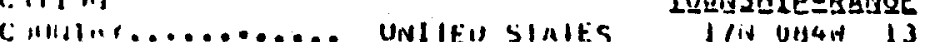

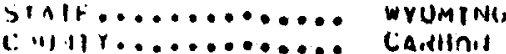

\section{CQuURLUALS}

LAI/LUING... 41-27.00 if $106-40.40 \mathrm{~m}$

UIM LUIVE... +13

NUHIHING... 4590U/O.

MECUHU OUOOS

GEUTHEHM FILE IU: UU75002

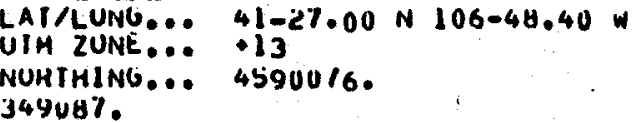

. 


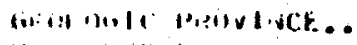

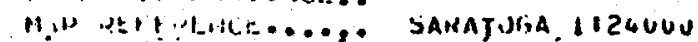

$34908 \%$

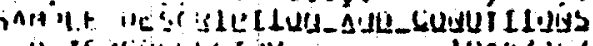

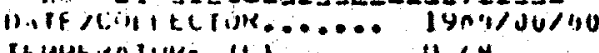

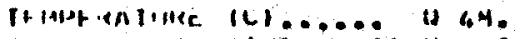

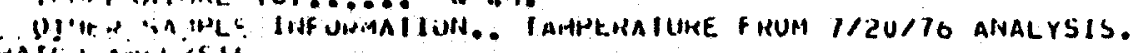

antion nighel

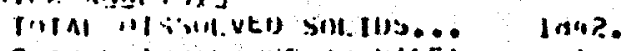

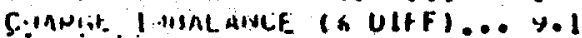

Aint risis l:o pron

Cu3.... 14

Ali.....

Cive.......

14.

LSULOEES LOCOUL

11......

ir.

1:.... I11

(i.......

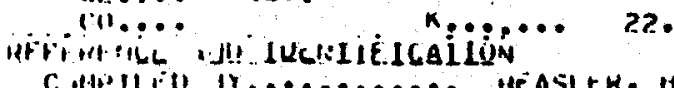

C MPILLEN H........... MEASLEK, H, P.

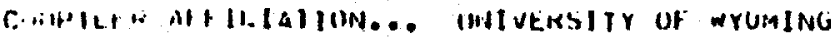

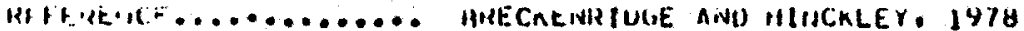

RECURO 00005

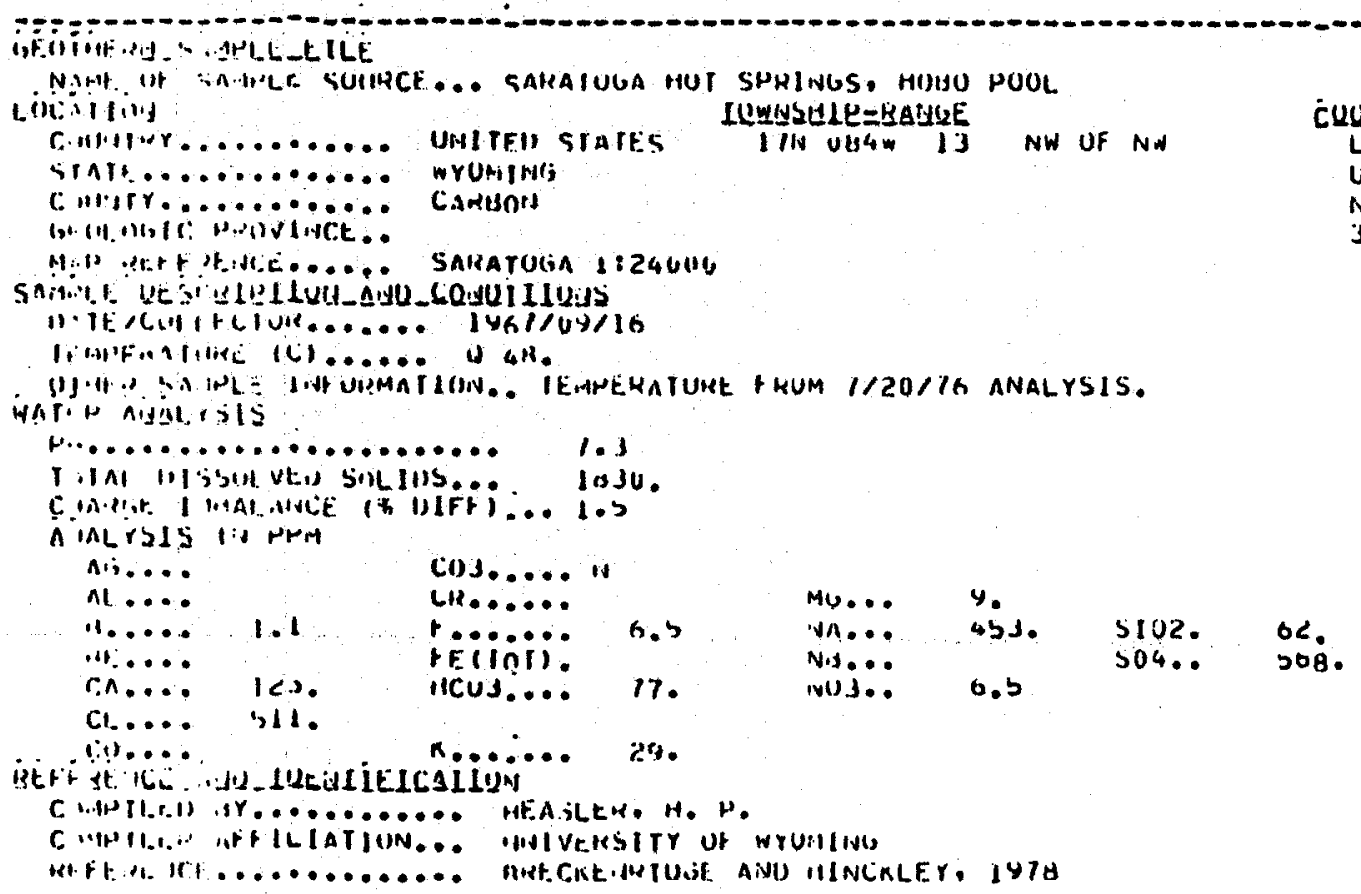

CUUGULUAIES

LAT/LUNG... 41-27.00 N 106-48.40

UIM 2UINE... +13

NUHTHING... 4590016

उ4YU⿺辶. 


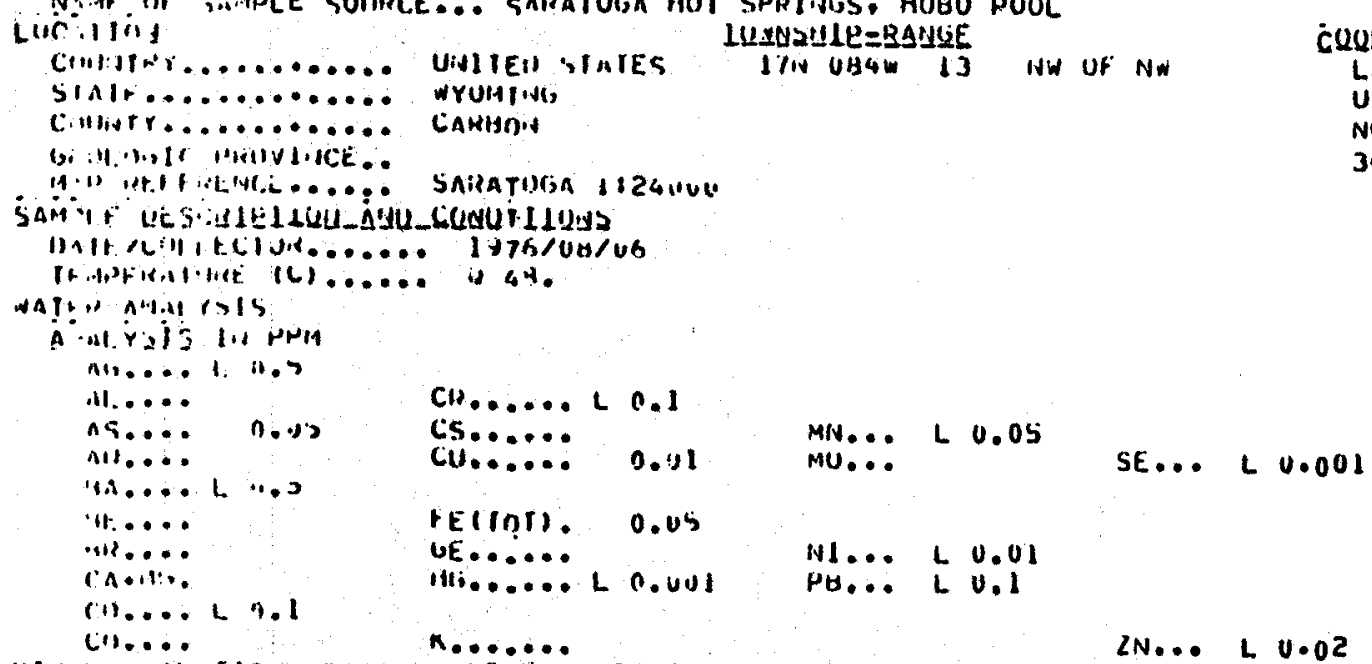

ISULOEES COLOUI

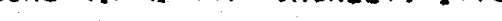

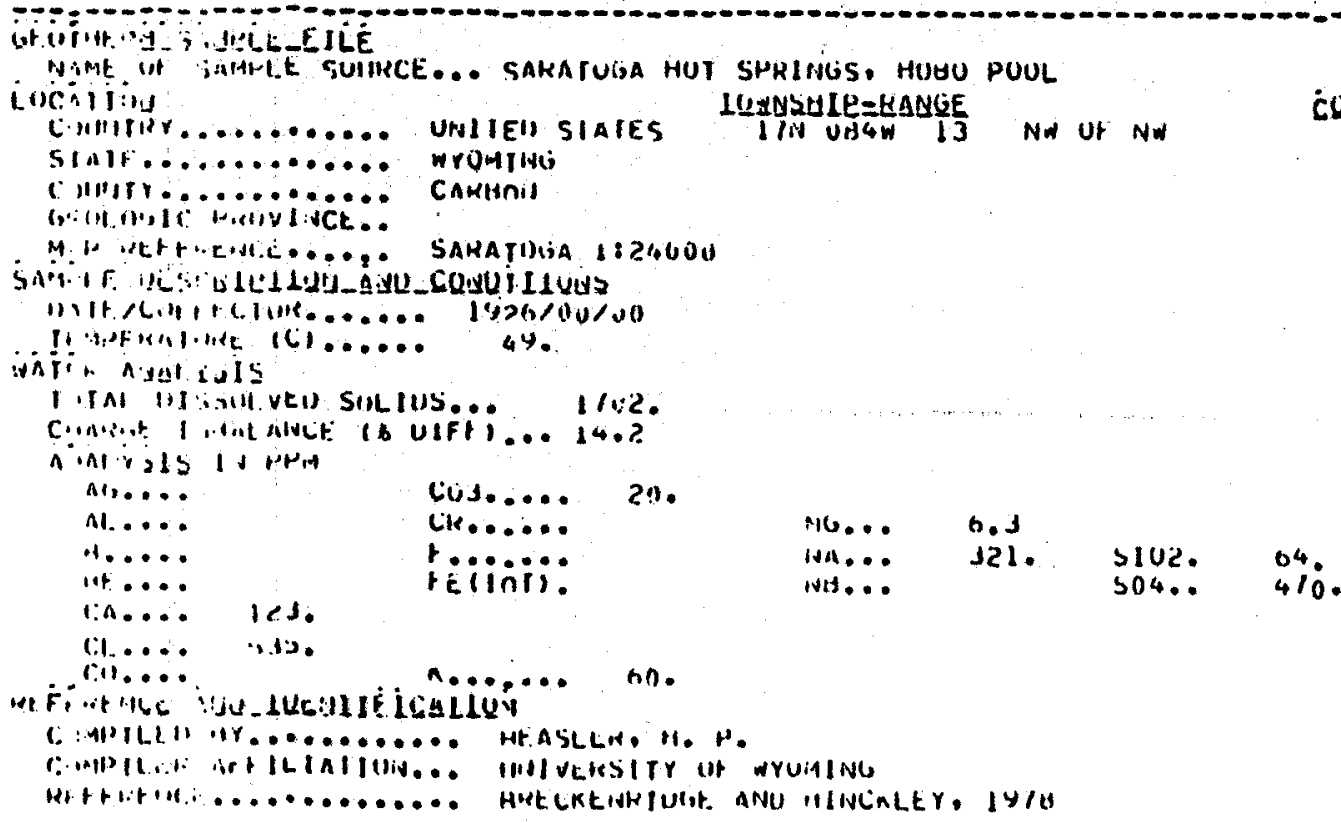

116... 15a.. J2l. S102. 04.

ISQLQEES CUCQOL 


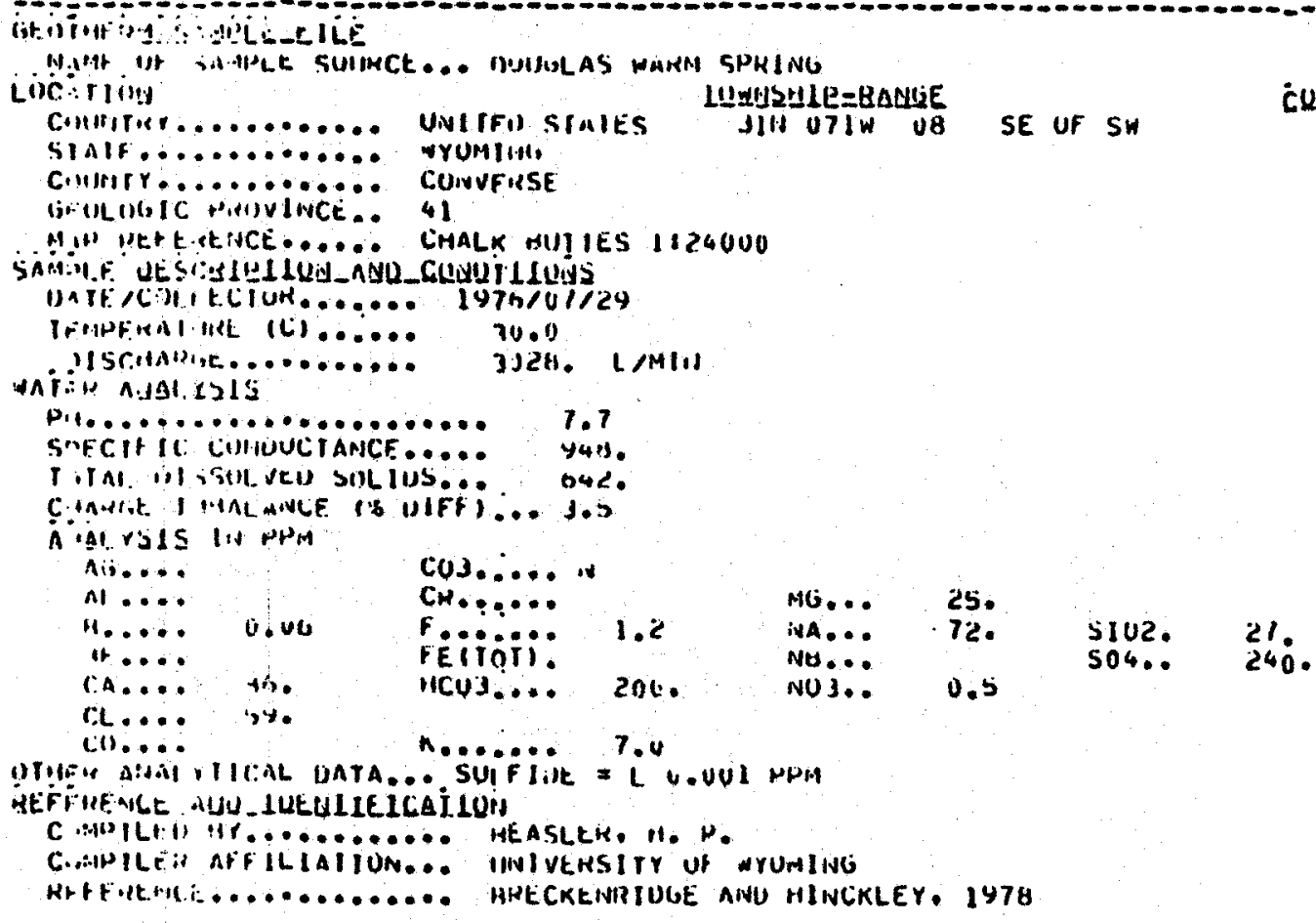

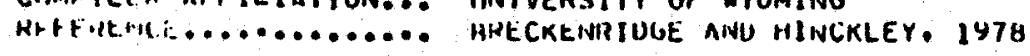

HECURU OUDUY

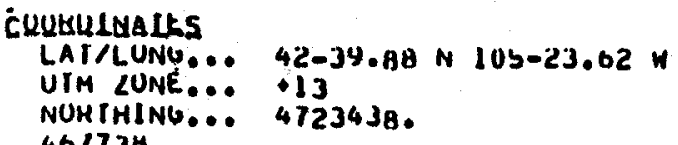

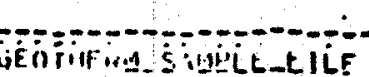

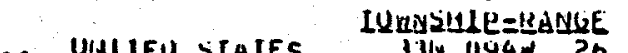

SIMIF........... WrUmllwi;

JJ14 UY4W 20 SE UF SE

Collongli PativinCE. 41

LAT/LUNG.

MaO ut 1 ut

HLUE GULCH 1:टथUVO

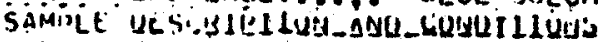

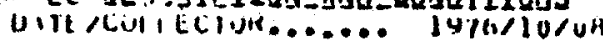

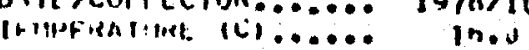

wari, aust tsis

0.1................. T.1

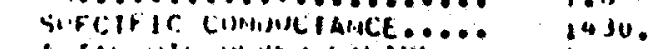

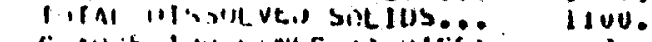

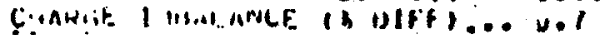

Aint Cals lit Hod
nl,.....
cos......
che.....

sy.

LWULEES IVLOUL 


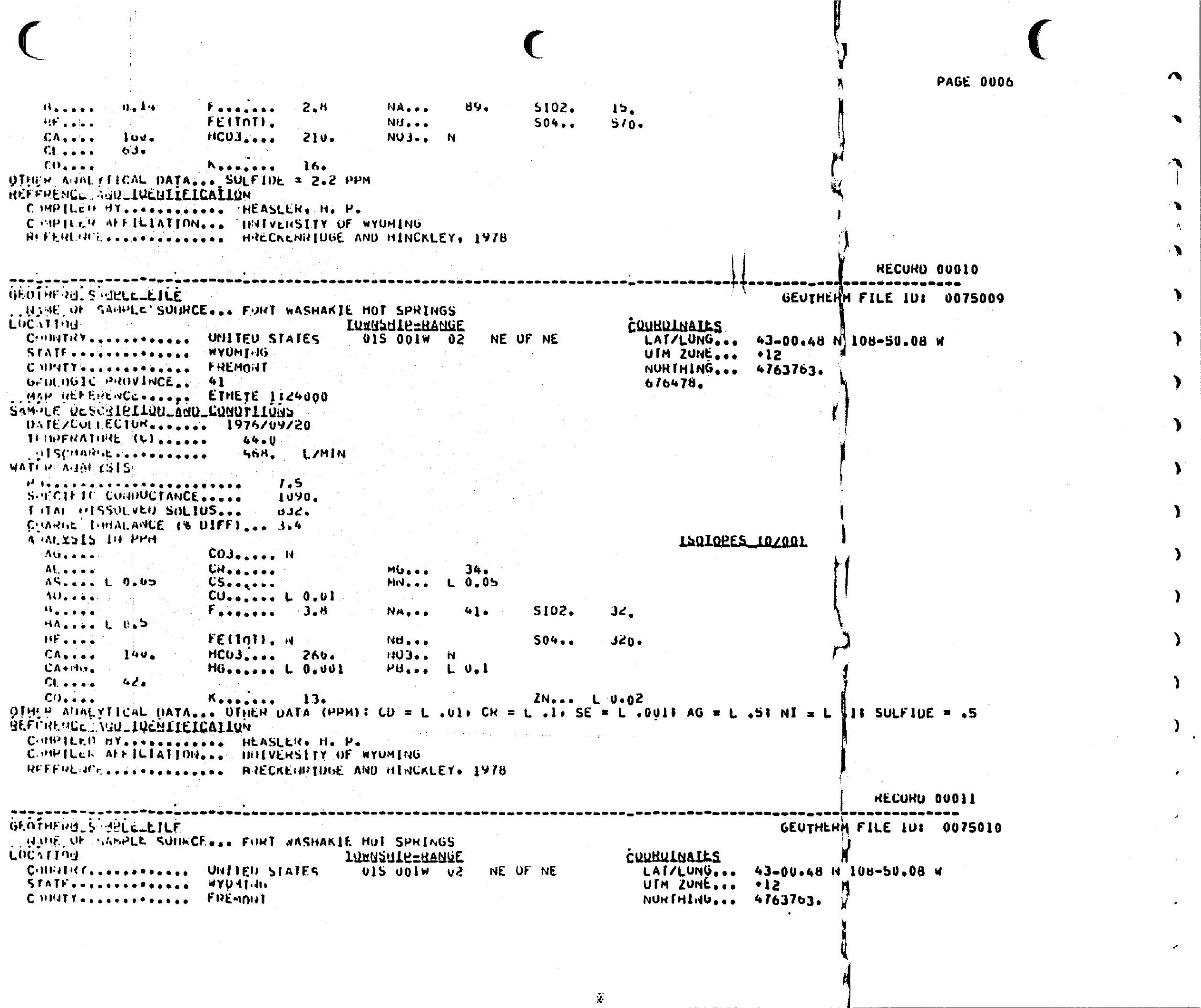




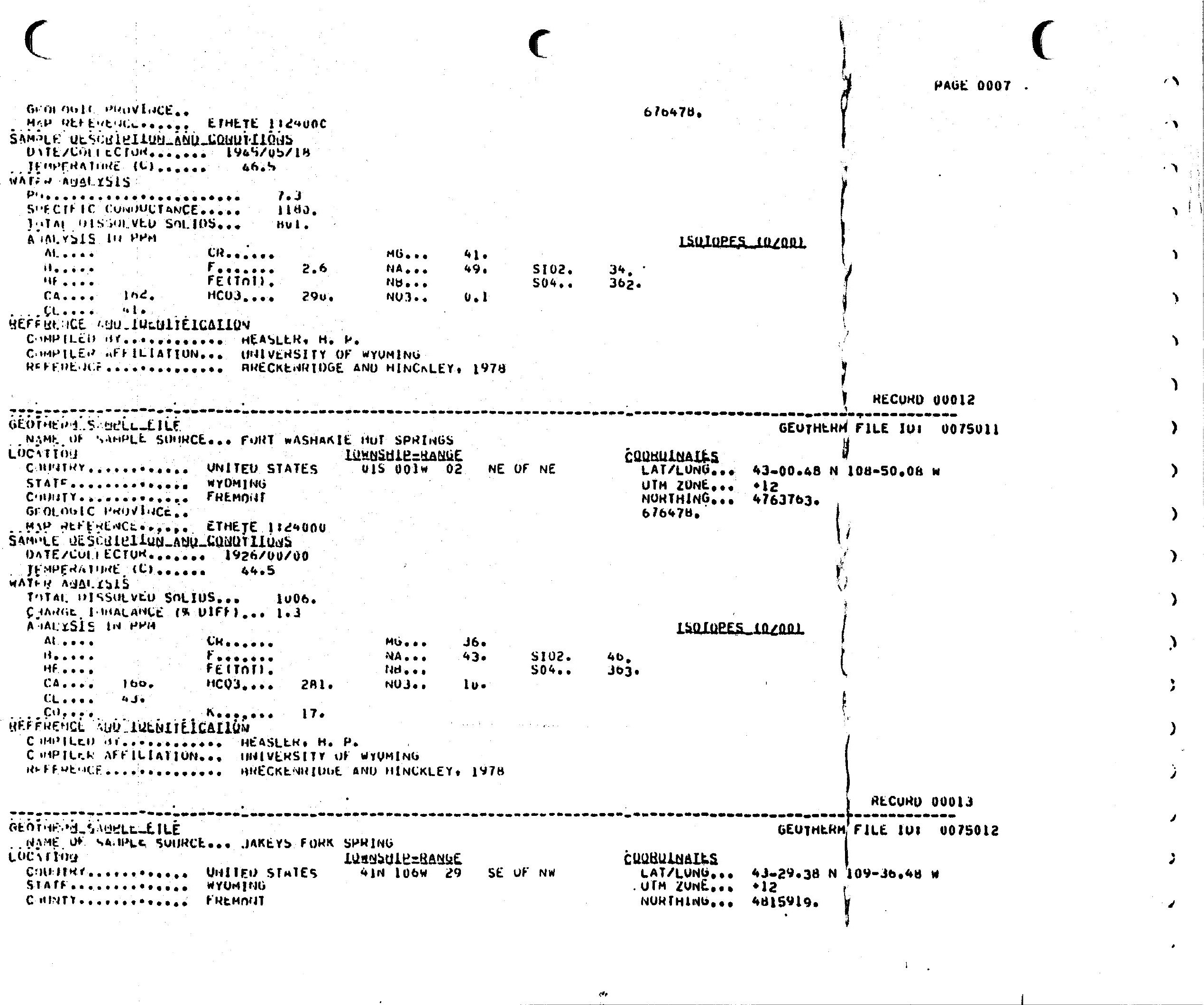




\section{C}

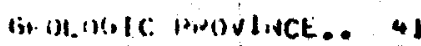

MAN REFE"EINLE..... TOHKEY LAKE 112400V

612558.

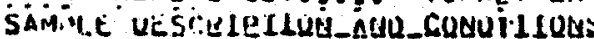

DATE,CEIIECIUK....... IYTIJOS/26

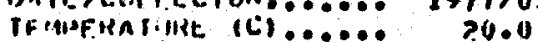

ilschaplit .......... I5. L/HIN

AnTt AUAL ISIS

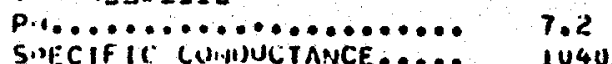

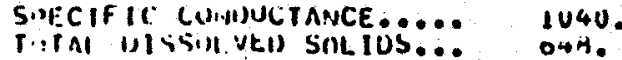

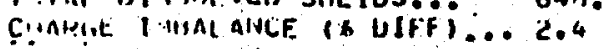

Ainlirals lin poid

Ali... cus..... is

H.....

Ch.......

1.6

EIIOIi.

1.6

$n+0,0.000$

1.... 1160

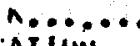

b.1

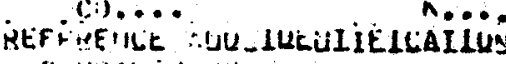

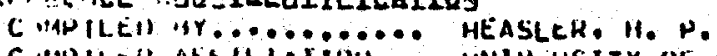

C "HPILE" AF ILIAIION... IINIVEKSITY UF WYUMING

HI FEDE WCF............. HKECKENHIDGE ANU HINCKLEY, 1978

RECUHO 00014

(iti)

. NAII: UR SAlHLE SUIRCE... I.ITTLE WAKM SPHING

iocirity

collitiler

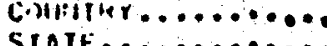

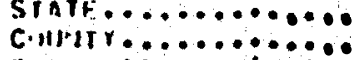

IUWYSALP $=8 A N G$

$4 \mathrm{NN}_{107 \mathrm{~W}} 14 \mathrm{NW}$ of $\mathrm{NW}$

WrOMIIST

CRULINAIES

LATRONG... 43-31.22 N 109-40.00

UTH ZUNE... 12

NURTHING... 48I9247.

b0 7760 .

Hib 2t+tutnc..... vissurs 1124000

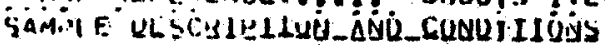

DIEACIIECIJK....... 1977/US/26

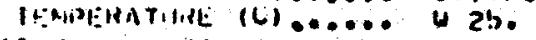

WA!t? Aivil rSIS

AN.risis

Aij..... 6 n.us

AL..... 0.0

$+11 \ldots \ldots$ L 11,5

lit......

ith.....

$\operatorname{C.A}+1,1$,

c.1).... L 0.101

Cil.....

CH. . . . L 0.61

CSE......

CS......... 0.01

Cir...... L

FEITOII. H

UE........

0.uvI

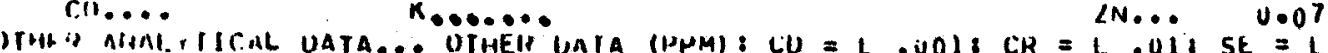

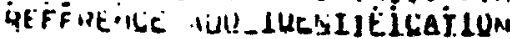

C.MHILIGH 117........... HEASLEH, H. H.

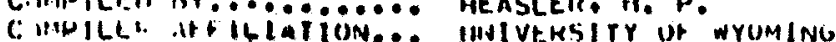

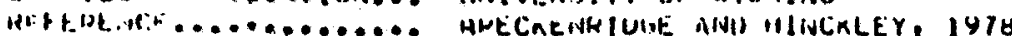

LSUIOPES 10<001

GEUTHEKM FILE IUI 0075014

miv... L 0.05

SE... L $U .001$ 
AECUKU 00015

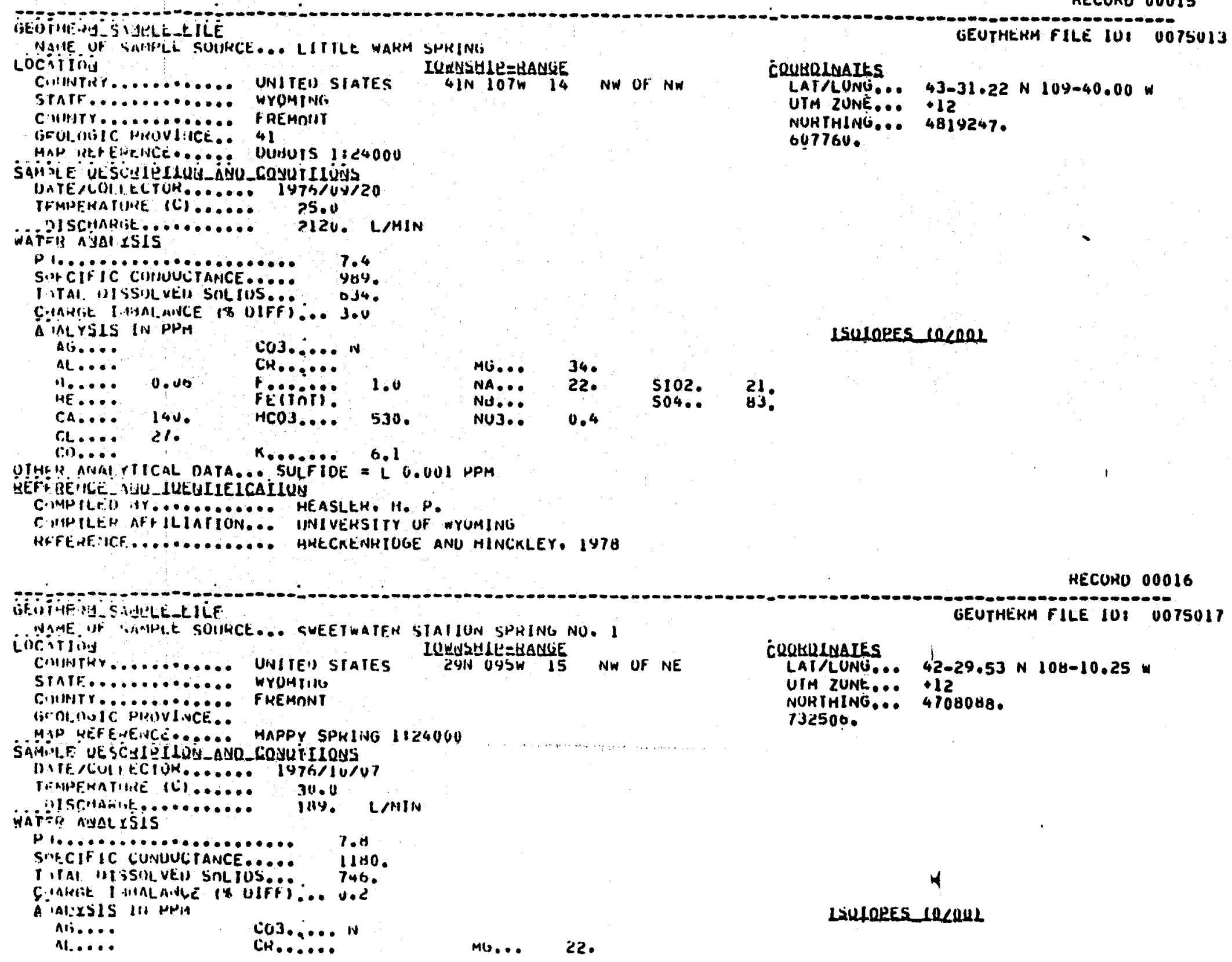




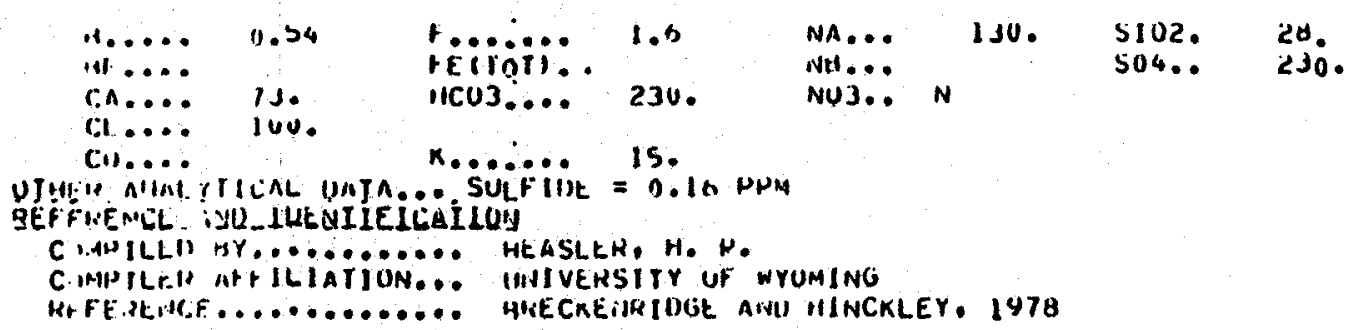

HECUKD 00017

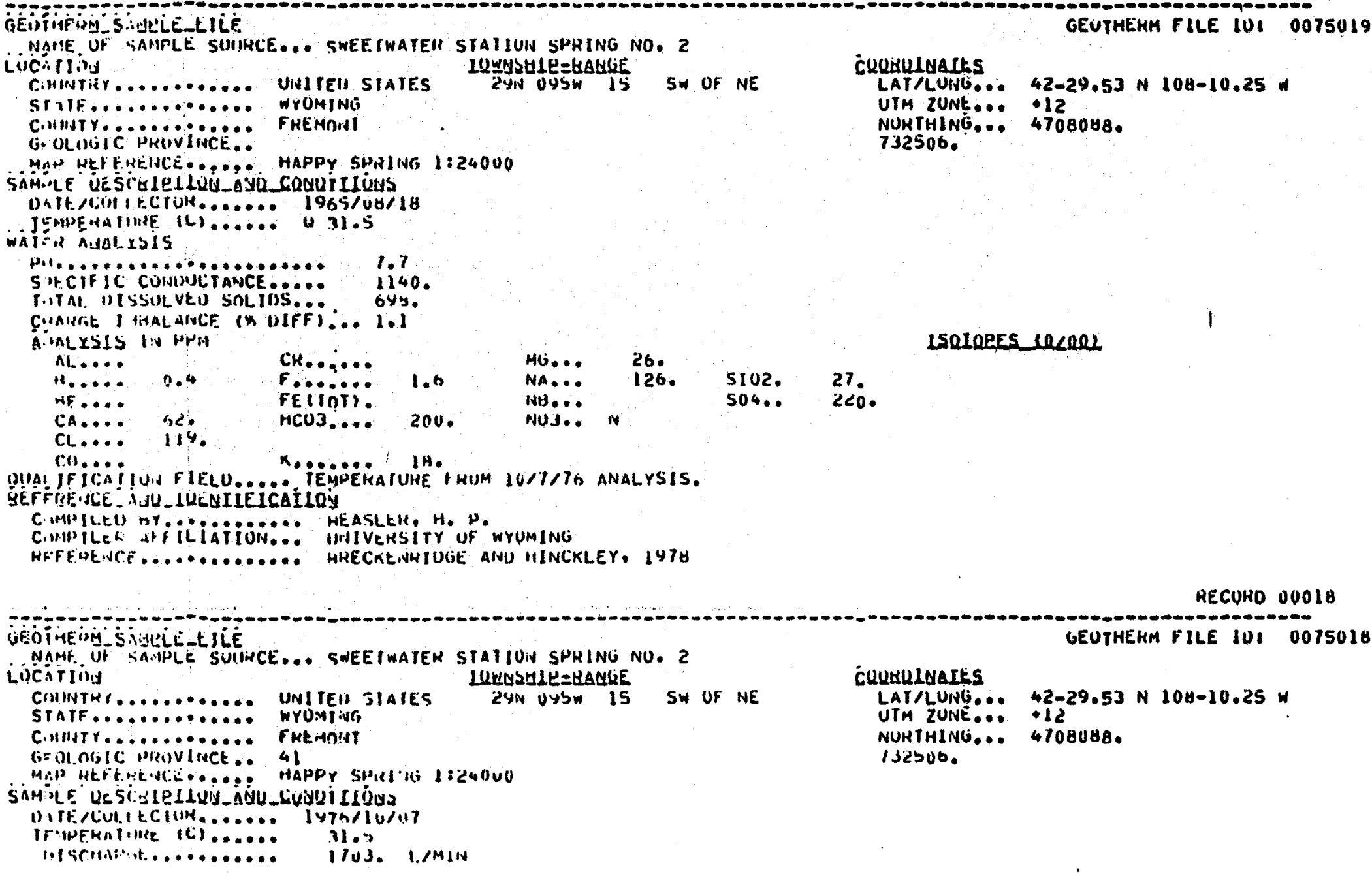

.. NAIIE. UF FAMPLE SUIHCE... SWEETWATEU STAIIUIN SPRING NO. 2 Liverilling

Strif............ WrOMIni

2YIN OYSW IS SW OF NE

G. OLOUIC PHCIVIINCE...

MAP HEF FHEINCE..... HAPPY SPAING 1:24000

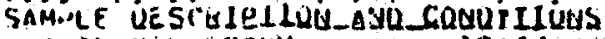

DAIt diN I ECTUK....... 1965/u8/18

WAifr nivalrols

p.1.................... 1.7

SHCIFIC CUINUTCTANCE.... 1140

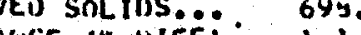

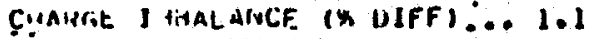

AinLYSIS IN HPM

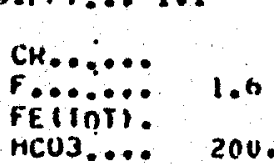

n.......

0.4

n?....

Hi... 26.

5QLORES CUCORL

GEUTHEKA FILE IUI 0075019

CQuguLAarts

LAT/LUIN6... 42-29.53 N 108-10.25 N

UTM ZUNE... 12

NUKTHING... 4708088.

732506.

CL.... 119.

(ci)....

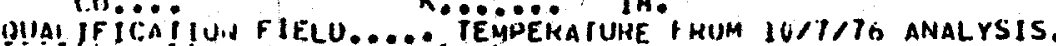

Nus.:

504.

220

GEFF!II.JCE AUU_LUGLIEICAILO

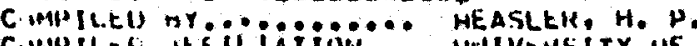

WYUMING

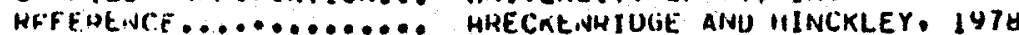

RECUHO ONO18

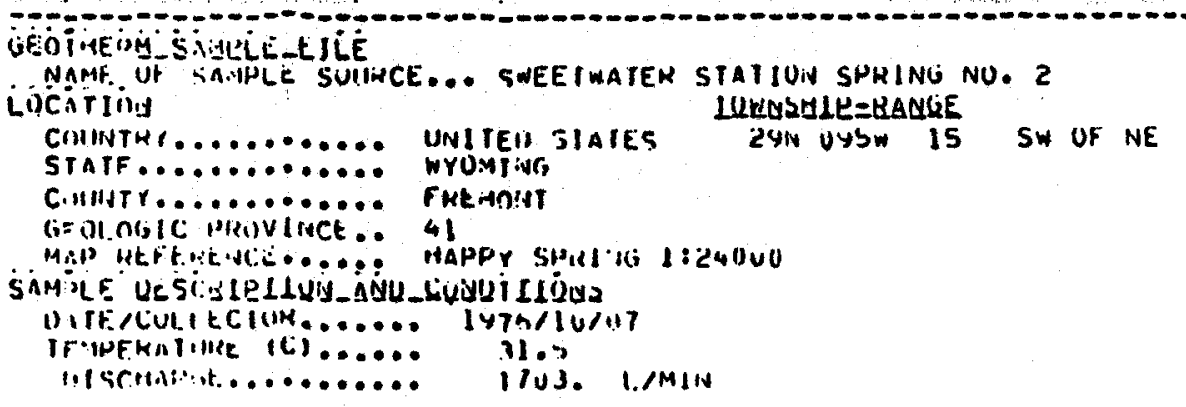

CLURLLWAIES

LAYLUUNG... 42-29.53 N 108-10.25 W

4708088. 


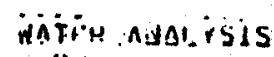

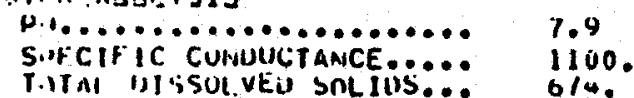

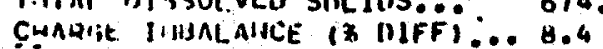

ÄIALISSIS IN PHA

LG

c03..... in

AL... CH. C.....

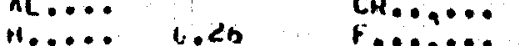

Fiinii: 1.0

MCUJ....

230 .

$\begin{array}{lll}C A . \ldots . & 130 . & \text { MCU3.... } 230 . \\ \text { CL.... 12U. } & \text { K....... } 15 .\end{array}$

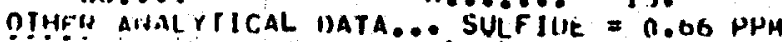

HEFFIBE KE AUU IULUIIEICAILON

C MHILEN HY......... HEASLE. H. $P$.

DERTING

RFFEHEHCE............ AIRECKEND IULEE ANO HINCKLEY, 1978

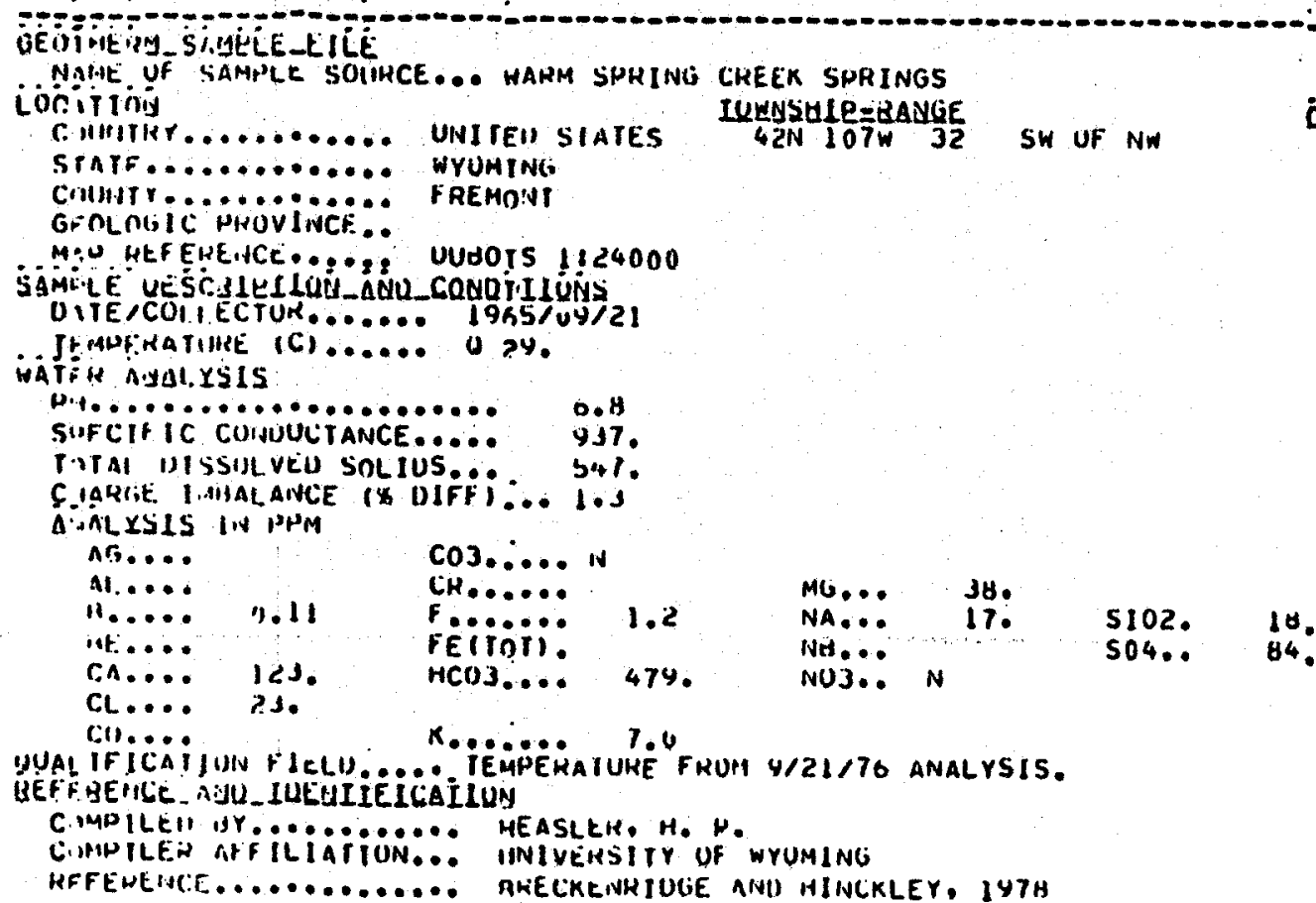

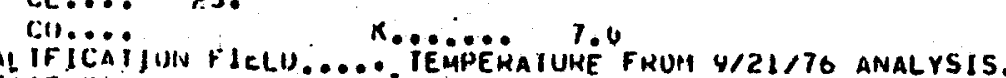
UEFFHEICE AJU_LUEUIËICAILUY

C.MPILEII UY............ HEASLEM, H. H.

CIAPILEN ATF ILIATION... IINIVEKSITY UF WYUMING

RFFEWEWLE............. AKECKLINKIULE ANU HINCKLEY, IY7H

RECORO 00019

\section{CQQHQLAAIES}

LAT/LONG... 43-33.60 N 109-43.88 W UTH ZUNE.. +12 NOKTHINC... 4823571. 0.02466.

RECUHU 00020 


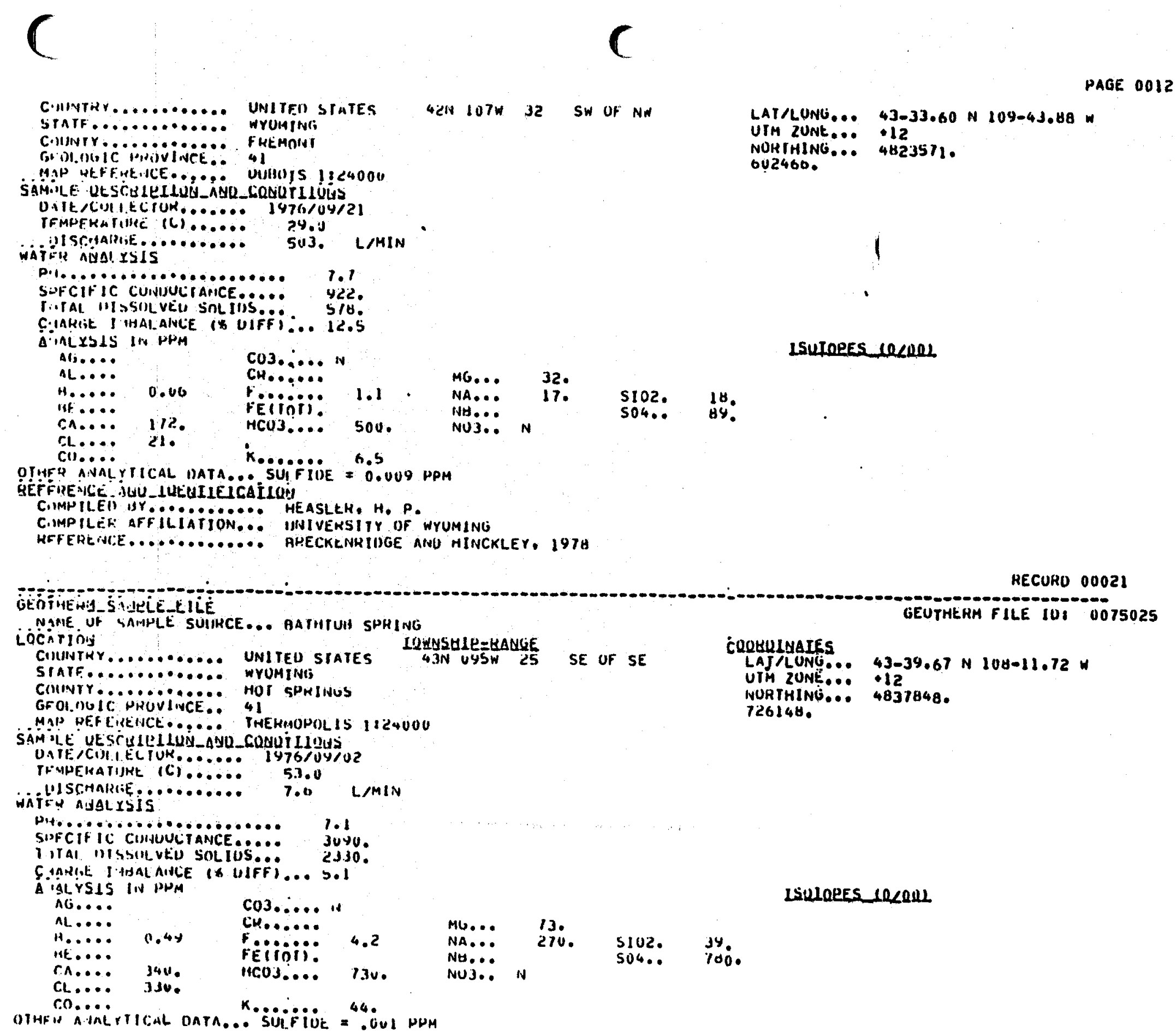




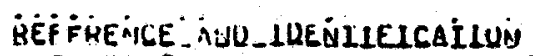

C.UAPILED UY........... HEASLER, H. $\%$.

C'IAPILER AFFILIATION... UNIVENSITY OF WYUMING

HEFE.PEHCE............. AHECKEIHIIOLE ANU HINCKLEY, 1978

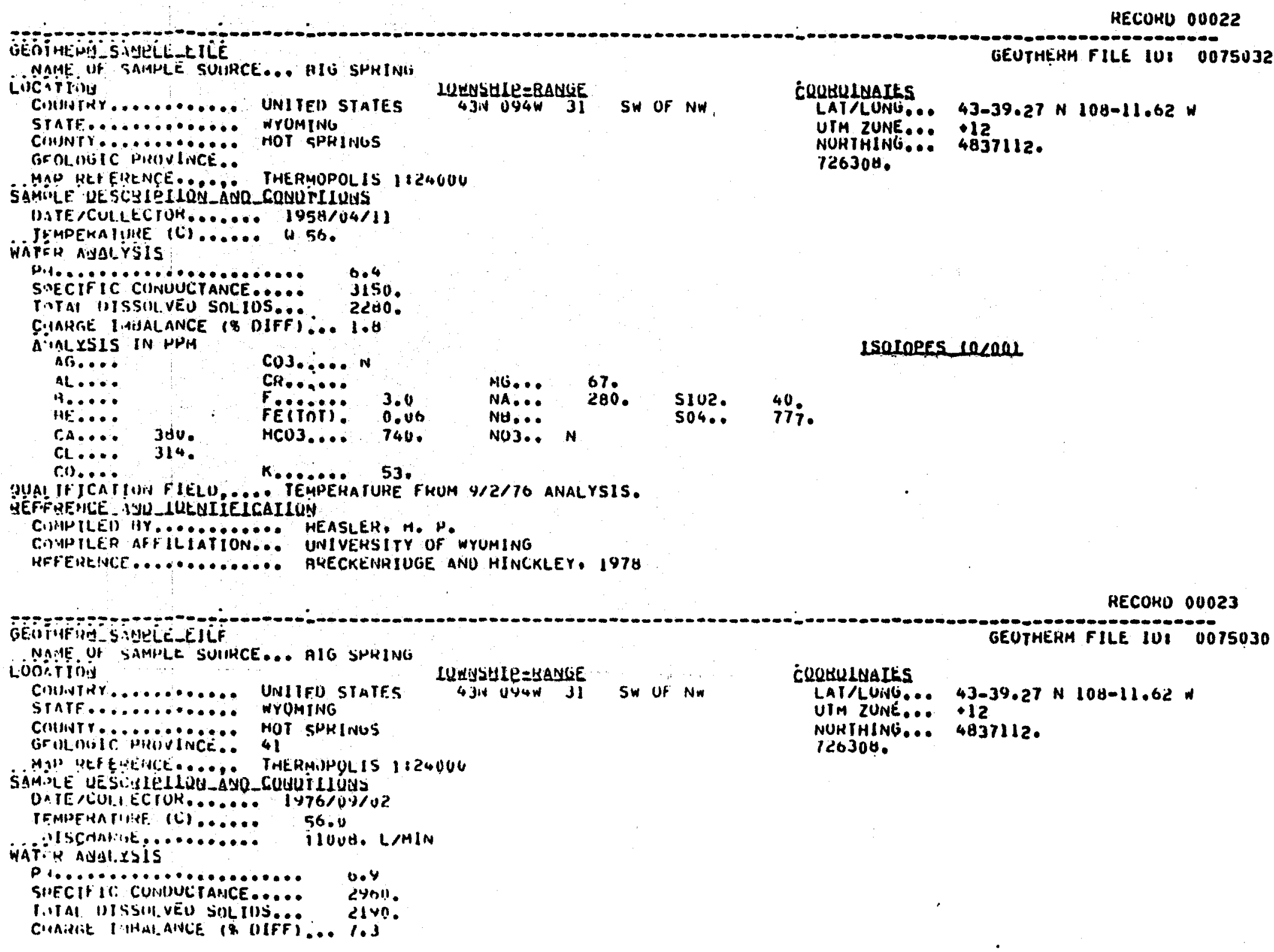


ÄIALESIS IN HAM ali.

Al. .....

AS..... 1. 0.0S

AII.

H..... n. no

HA..... 60,3

HF....

hit....

CA..... 310.

CA+Hil.

C1).... 0.0

Cl..... 30.

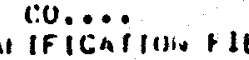

TS 57 DE: $\mathrm{C}$.

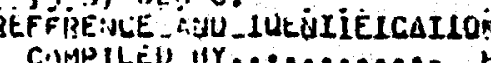

C.IMPILCU HY.......... HEASLEH, H.P.

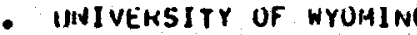

KFFEHENCE.............. AHECKENRIUGE ANII HINCKLEY, 1978

RECOKU 00024

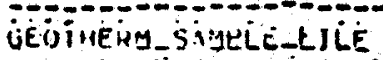

NAMR UF 'ialARLE SUIJHCE... Alt SHAINO

iocision

CIIINTHY . . ...... UNITEI STATES

STATt ............ WYUATHO

43N US4W 31 SW OF NW

Cillintr............ HOT sHKIINGS

Gentonir pinivluce.

MGP HEFETHCE...... THEKMUPOLIS $112400 \mathrm{O}$

LSULORES LOKOLL

cuublelnatis

LAT/LONG... 43-39.27 N 108-11.62

NATM $20 N E \cdot \cdots \cdot 12$

7837112

$72630 \mathrm{~g}$.

EUTHERH FILE IUI 0075035

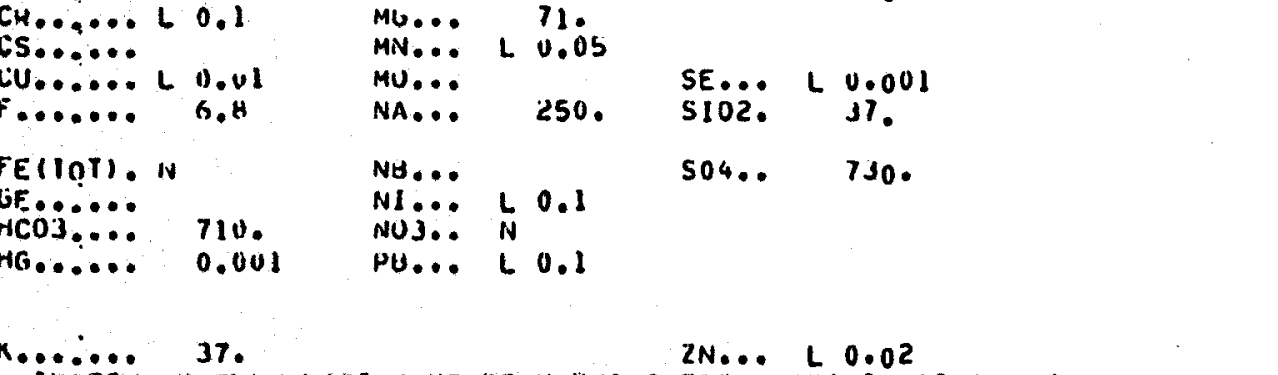

\begin{tabular}{|c|c|c|c|c|}
\hline $\begin{array}{l}\text { LI... } \\
\text { MU... } \\
\text { MN... }\end{array}$ & $\begin{array}{l}71.05 \\
0.05\end{array}$ & s.... & & 0.006 \\
\hline $\begin{array}{l}\text { MU.... } \\
\text { NA... }\end{array}$ & 250. & $\begin{array}{l}\text { SE... } \\
\text { SIOZ. }\end{array}$ & L & $\begin{array}{l}0.001 \\
37 .\end{array}$ \\
\hline NB & & SO4... & & 730. \\
\hline
\end{tabular}

NI... L 0.1

PU... ᄂ 60.1

$2 \mathrm{~N} . \mathrm{O} \quad 0.02$

TEO TEMPERATURES 56

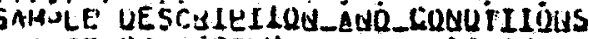

DITE,CULIECTUK....... 19n6/00/U0

TAMPEHATIMRE IC;...... O 56 .

WajEH aivalrsis

Aini YSIS IN PNM

al.....

AL.........

.

CK.

113.

FE(IOI), 2.5 Nd... S SO4..

CA. 113.

Clin.....

x. $\quad \therefore$ on

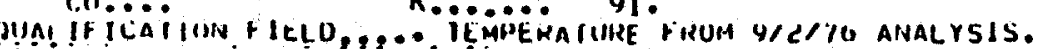

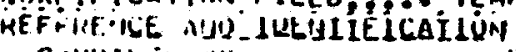

C.IMH ILEII HY .......... HEASLEH, H. P.

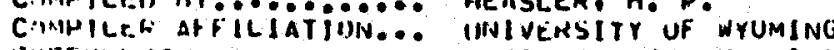

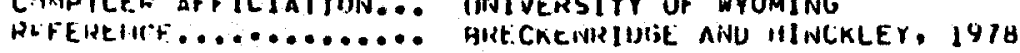

RECUHU 00025

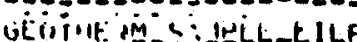

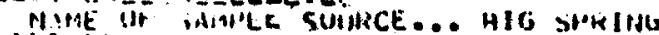
iociritid

Ciningidro

Start............. numinti

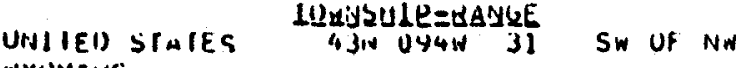

C mhir

\section{ISOLORES UECOLL}


Grotingle nizovince.

MAD REF FIENCE...... THEHMUPOL IS 1:24000

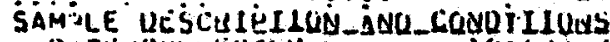

D.TE八CUI.ECTUK....... I9Ph/UO/00

TFMPERATIRE (CI....... 50 .

IIITCHalRiti

10S10. LAMIN

WATEM AVALYS is

aidirsts IN HPM

A1......

$3 . \ldots . .$.

14F......

C.A... jis.

CL.... J.3.

cil

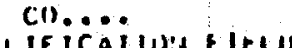

TEMPERATURE FKUM 9/2/76 ANALYSIS.

C.MMPILEI AY............ HEASLER, 11.P.

F WYUMING

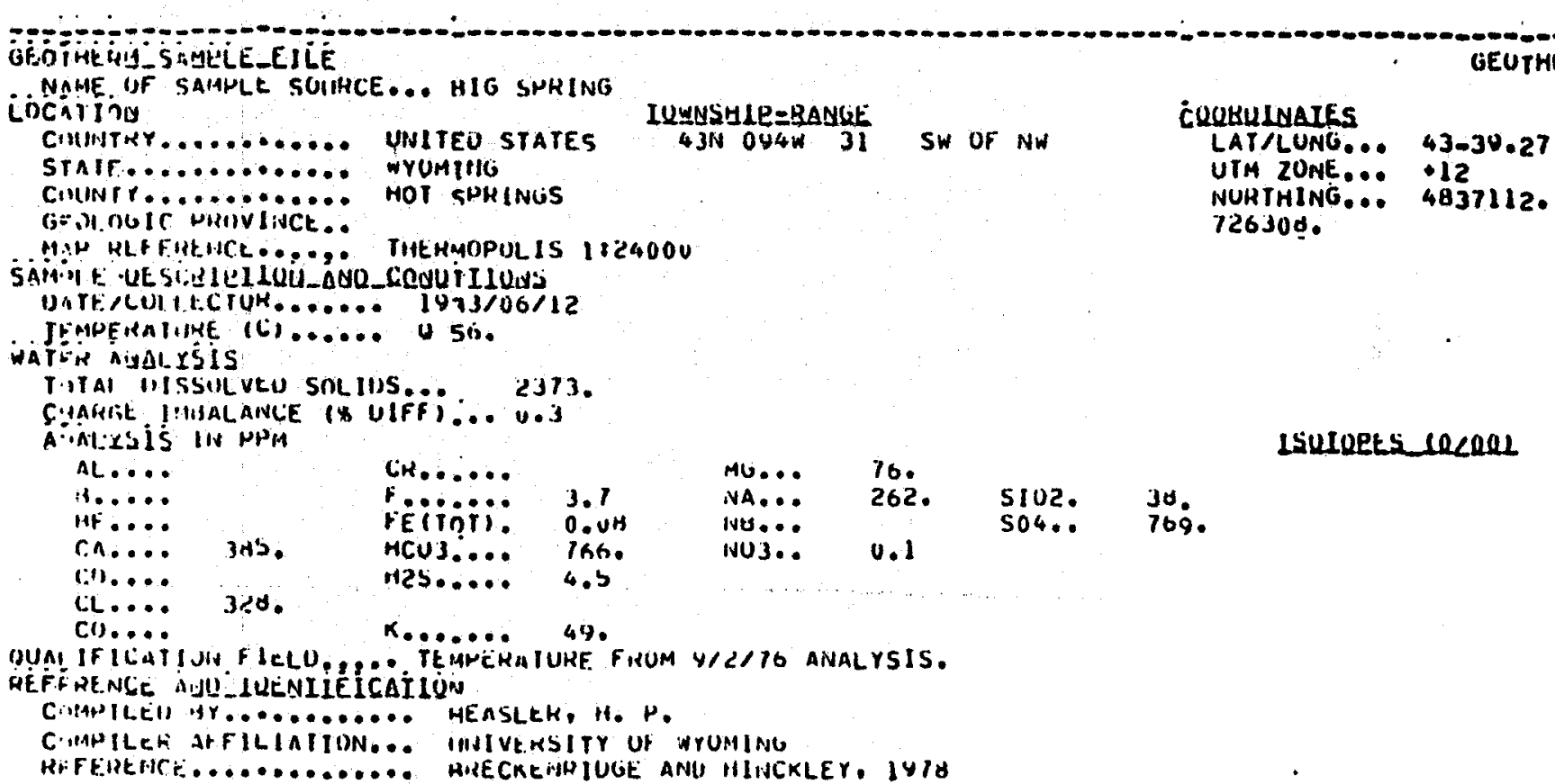

NAHE OF SAHHLE SUIHCE... HIG SHRING ideitiva

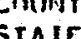

UNITEO STATES

IUEMSHLE $=$ BANGLE

$43 \mathrm{~N}$ OY4W 31 SW OF $\mathrm{NW}$

STAIT............ WYUMIlli

CIHUNTr............. MOT SPHINLS

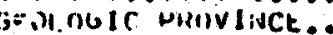

H.W RLF FULWCL.... THEHMOPUL IS 1824000

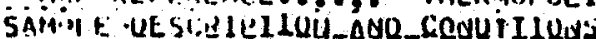

DITE,CUI T.CTUR........ 1973/06/12

JEMPERA IIINE (C) ...... 5 St.

WATHR AIIALISIS

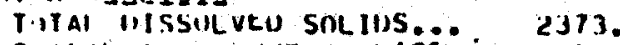

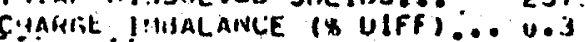

a in:zis in pPM

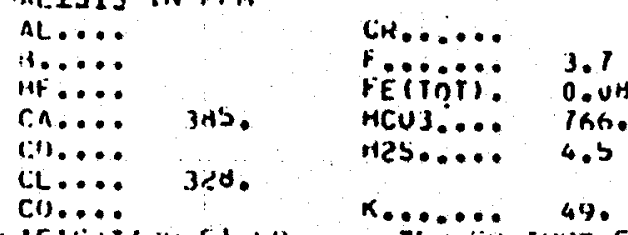

SuIOEES coe001

CQUBULAAIFS

UTM ZONE... 12

NUHIHING... 4837112.

$72630 \mathrm{~d}$.

NA... 262. SIU2. J6.

504.. 769.

0.1

gUA! IFICAT! JW FICLO, . TEMREKAIUKE. FINUM Y/2/16 ANALYSIS.

QEFFRENCE AUU LUENIEÉLCAILU'N

COPAPILEU HY............ HEASLEH, H. P.

C.NAHILEK ATFILIAIION... INIIVERSITY UF WYUMING

Hi:FEHENCH.............. HIECKE HHIUGE ANU HINCKLEY, IYIO

RECORU 00026

GEUTHEKM FILE IUI 0075033

LAT/LUNG... 43-30.27 N 108-11.62 W

HECURU 00027

Givion

NIME. UF IAMRLE SUINCE... AIS SMMIMIG Locitiving

Cining

UHIIEI SIATES

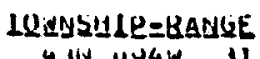

4 Jiv UY4W 31 SW UF NW

GEUTHEKM FILE IUI 0OTSO3!

COIINTYY........... UIHSIEII SIATES

LAI/LUNG... 43-39.27 N 108-11.02 * 


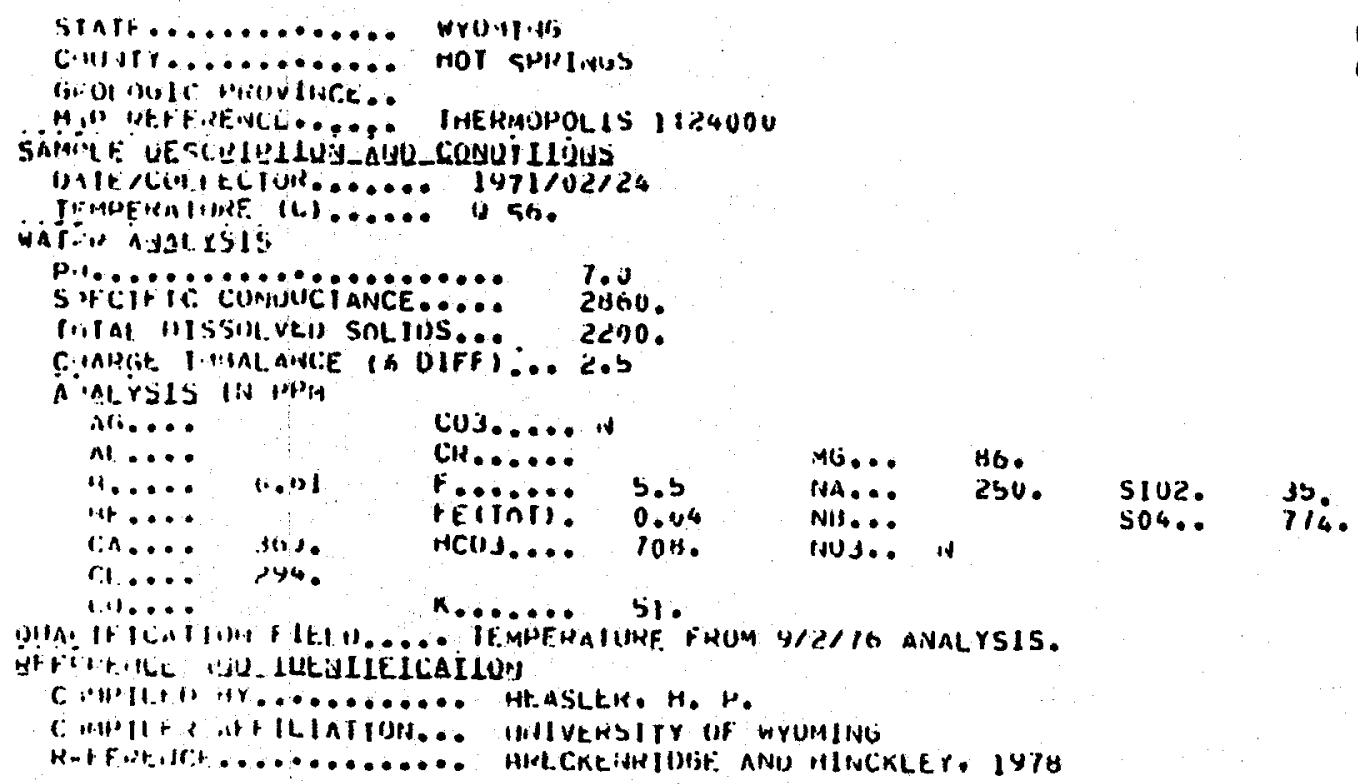

RECUHO 00028

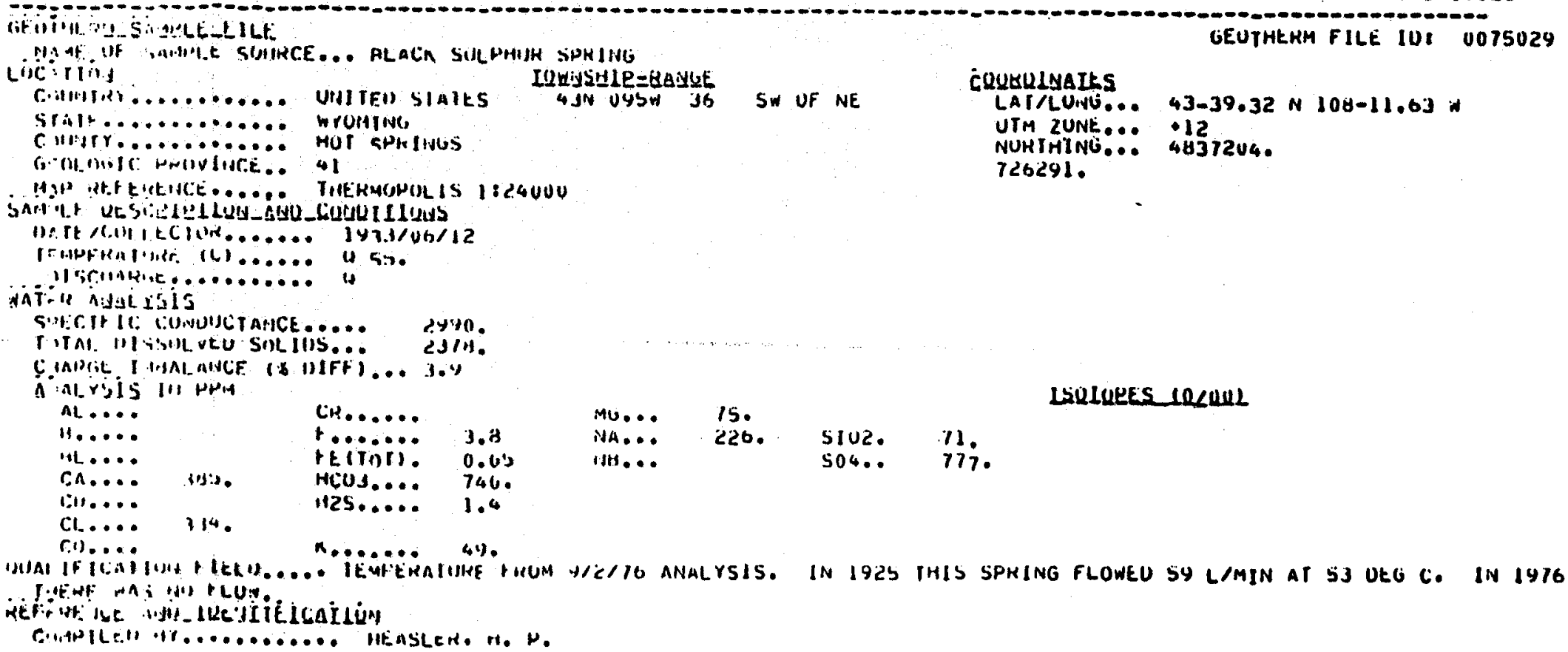


C MIILEI MFILIAIIUN... AHIVERSIIY OF WYUIING

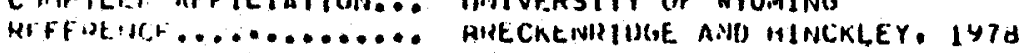

HECUHI 00029

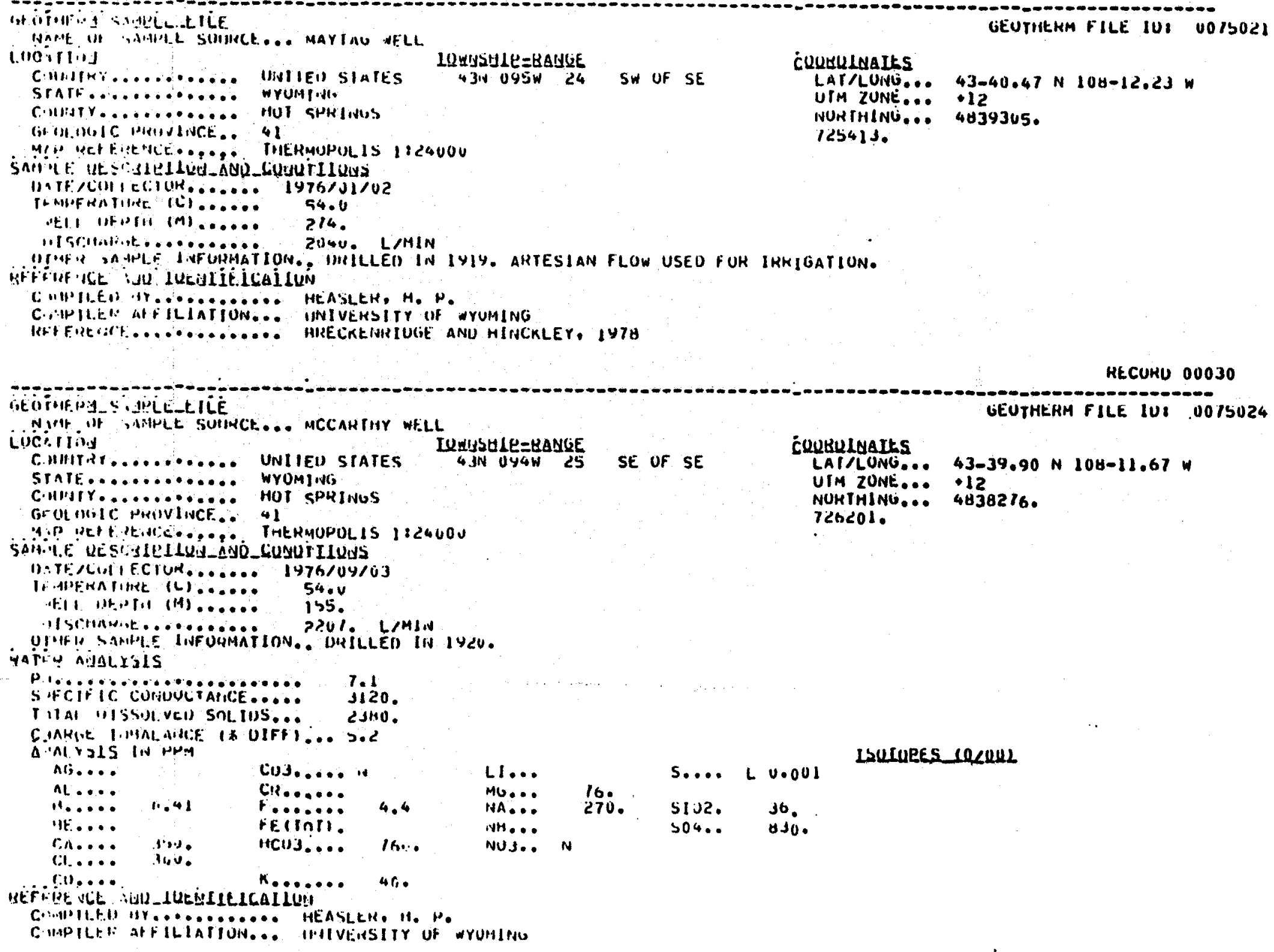

HECUKU 00030 


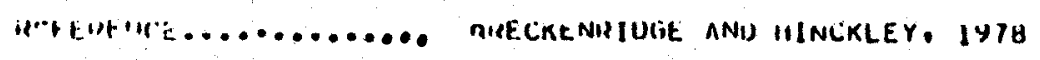

RECUHU ONO31

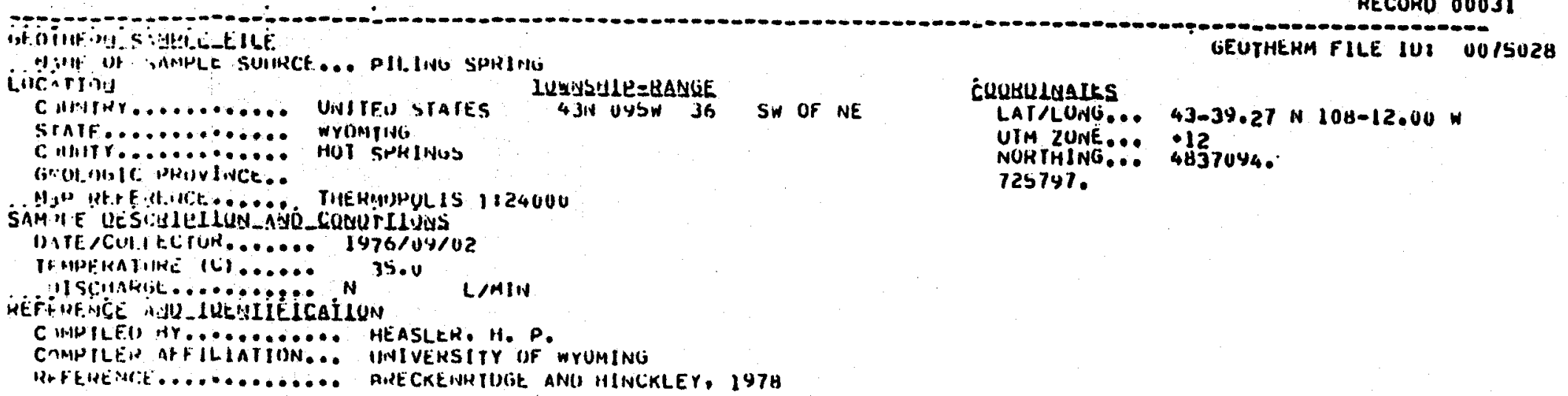

HECUHO 00032

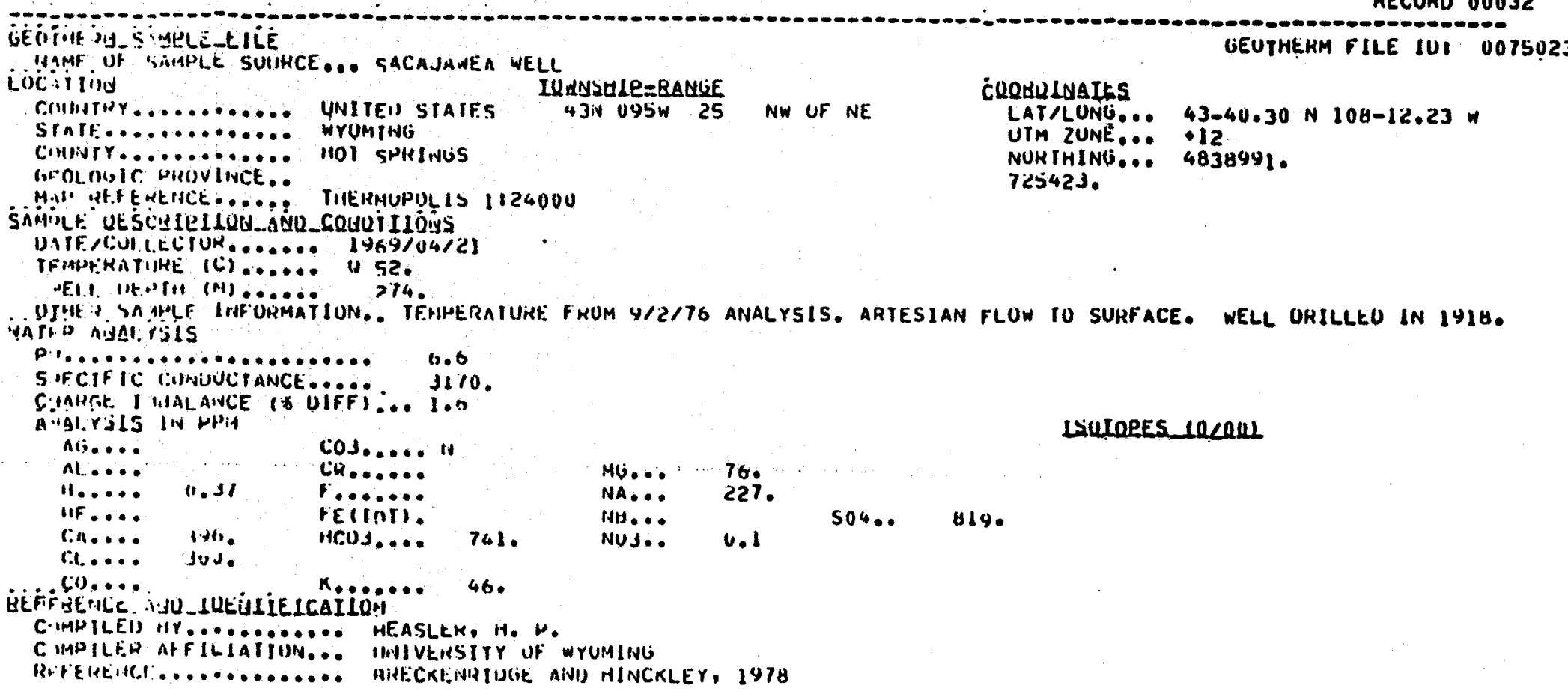

RECOKO 00033 


\section{C}

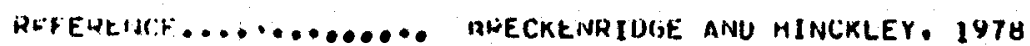

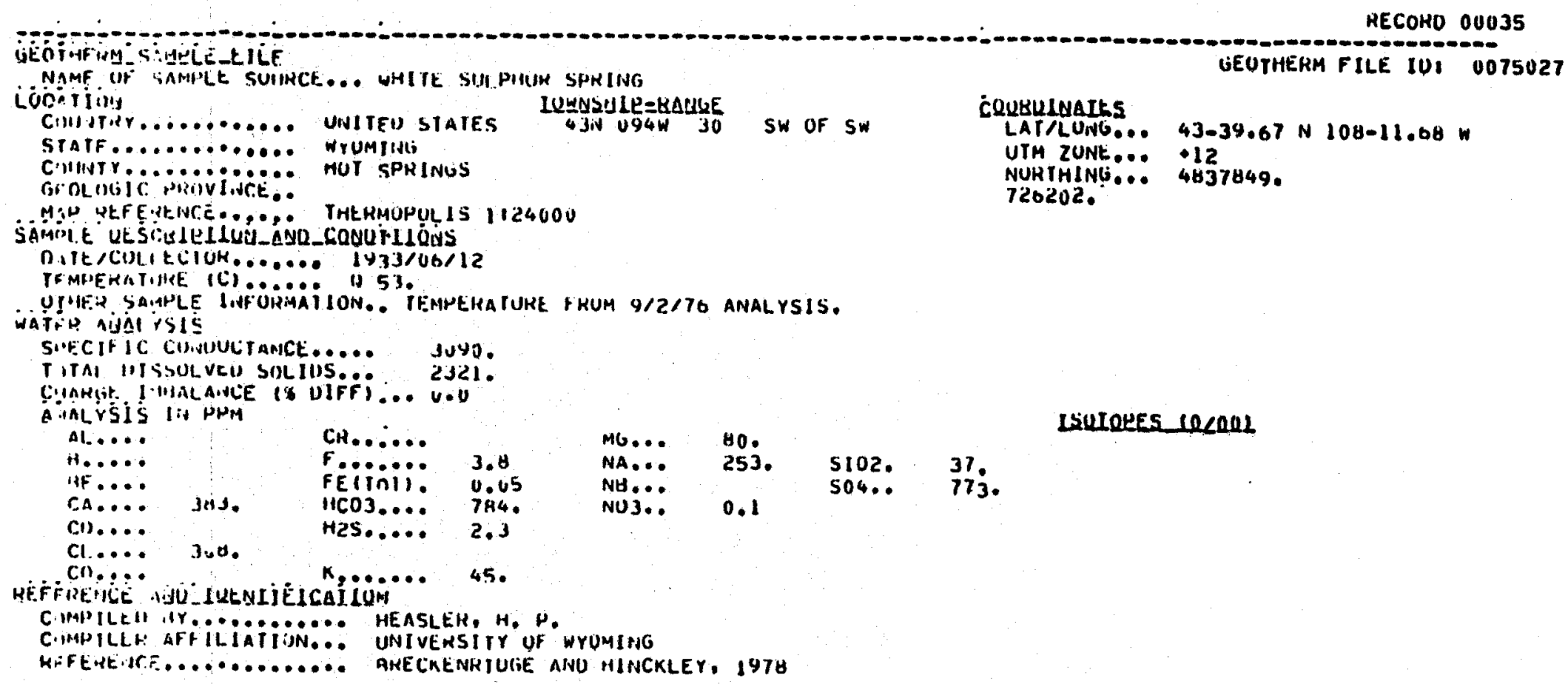

HECURU OUOJ6

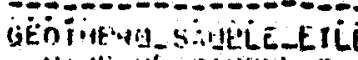

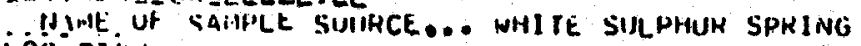

iociurios

ciniming.

Stutt

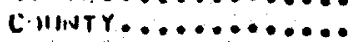

Grulnoll poruvinee.

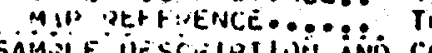

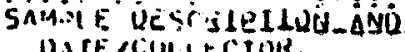

ONE COMICCTOR......

TEMIPFA I.MRE (C).......

ilschlalinte.

WAT:" AUAL XS15

UNITEU STATE

\section{WNSTIE-8}

4.

UT SPRINOS

作

JN U94W 30 SW OF SW

Sutious

प76/0\%/U2

S.د.

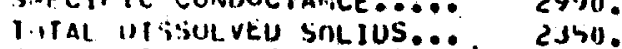

s:inwtic I lifalance ib uIfFi... b.

A'II YSIS IN HMM

di......

cos..... in

A1......

cil.......

4.45
4.1
GEUTHERM FILE IU: 0075026

\section{CUUHKLUAIES}

LAT/LUNG... 43-39.67 N $108-11.68$ W

INUNTHING...

$1 \angle 6202$. 


\section{C}

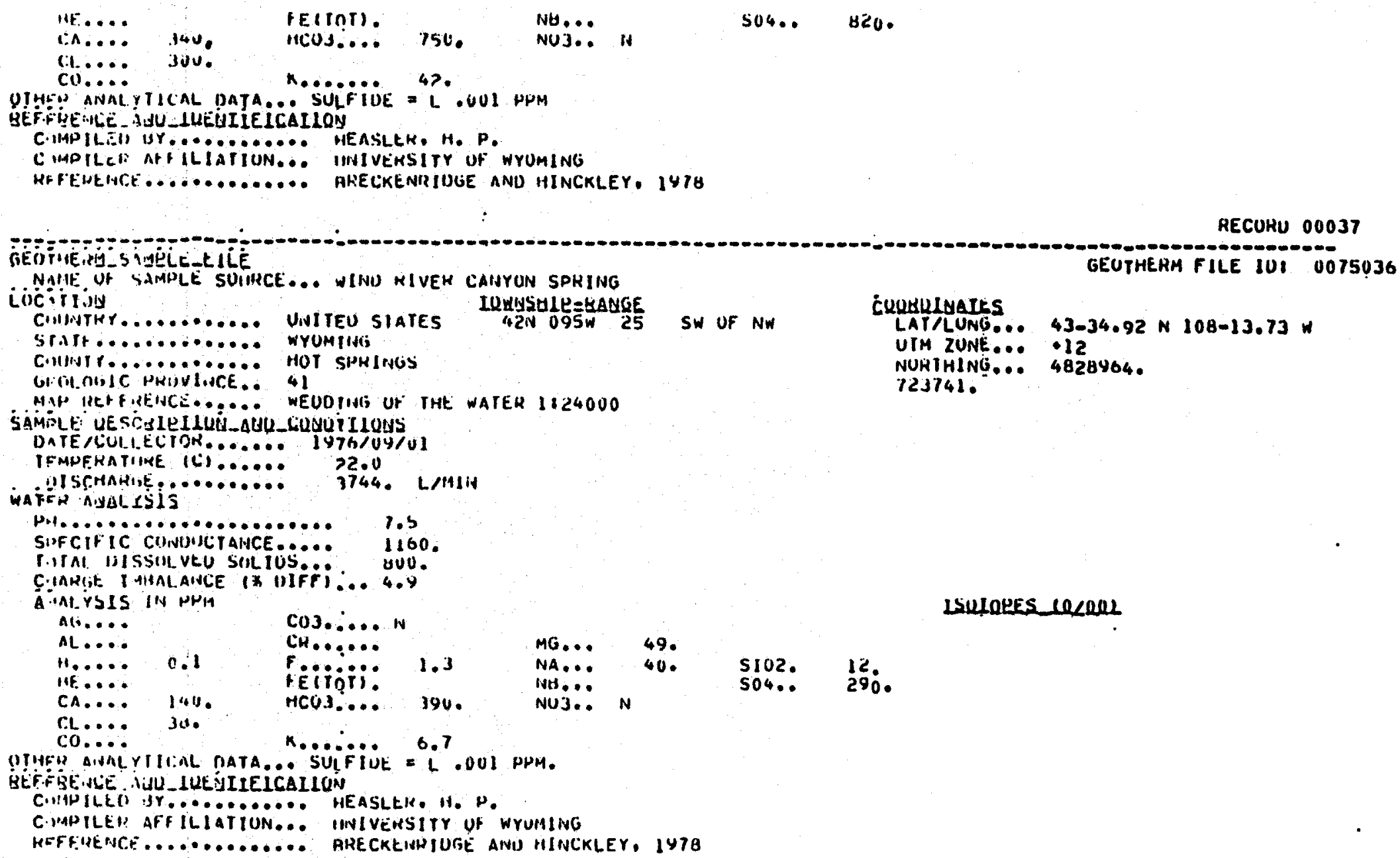
UIM ZUNE.
NUHTHING
723741.

\subsection{3}


wärez nivni.rsis 1

D.4.................. b.

Sifcit ic cumudirance..... liso.

ITTAl blisillueu Snlius... Isy.

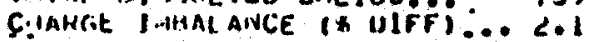

Cial isis liv nith

Ali.... CU3..... N

(1, 0.0

cive........

:IE....

0.12

FEITọii:

C.A. 146.

HCU3....

1.2
0.09

K....... 7,4

cii....

IEMPERA TUHE FRUH $9 / 1 / 16$ ANALYSIS.

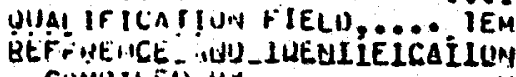

C.IMAILEN HY........... HEASLER. H. P.

C. NAPILEI: APFILIAPIUN... INIVEKSITY OF WYUMING

KEFEUtiURE............... AWECKEIVRIUGE AND HINCKLEY, 1978

$\begin{array}{ll}5102 . & 13 \\ 504 & 276 .\end{array}$

RECUHO OUOJY

GEUTHERH FILE IU: 0000779

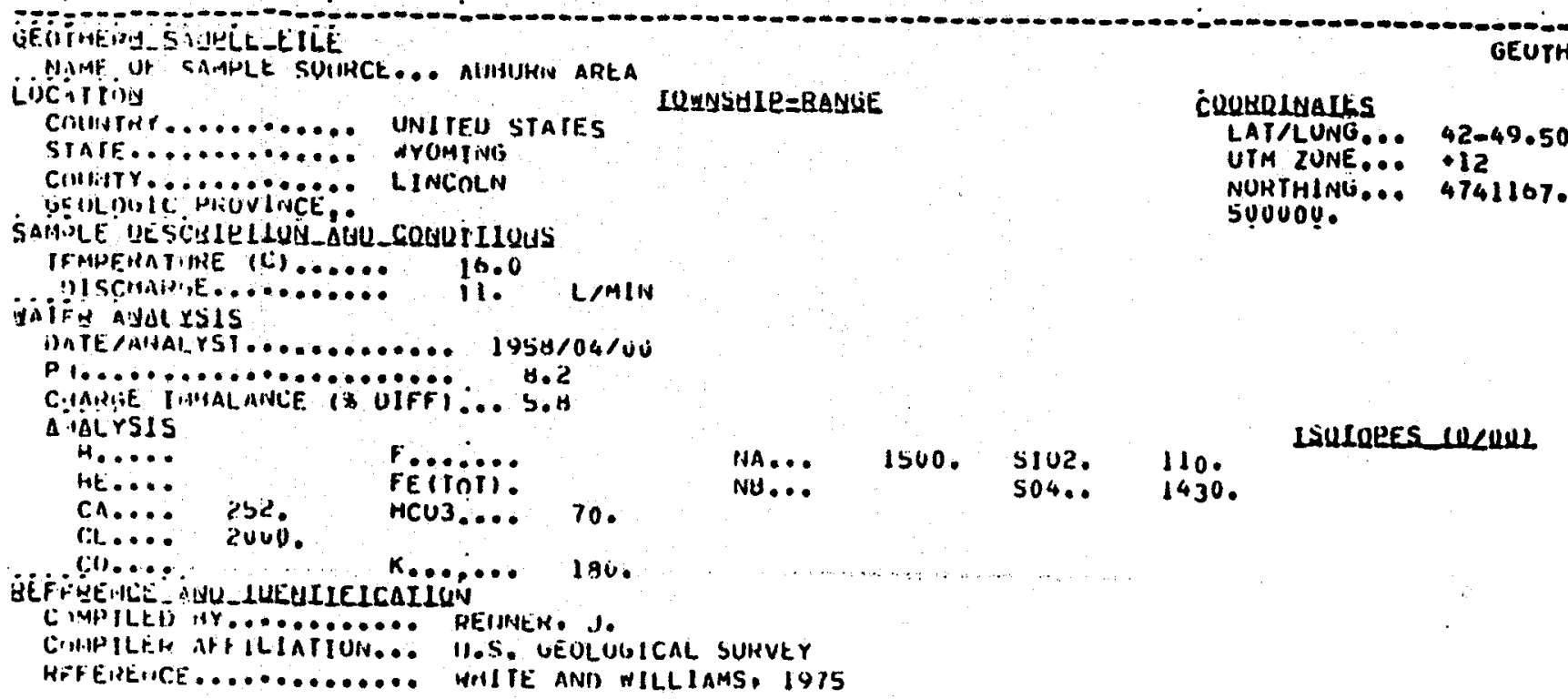
HF̈EIRE WCE.............. WHITE AND WILLIAMS, I975

LUYNSHIR=BANGE

ClQBQLAALSS

UTM ZUNE... 12

NURTHING... 4741107.

SUOUOU.

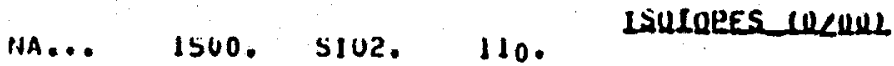

$1430^{\circ}$

HECUKO 00040 GEUTHERM FILE IUI OOT504I GE

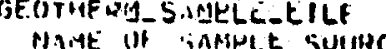

Löintint

c.nivtur ............

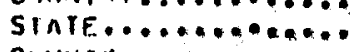

Chuntr........e.e....

(i. i)loulC PHIJVINCE.

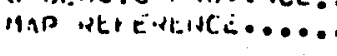

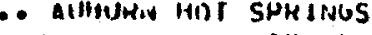

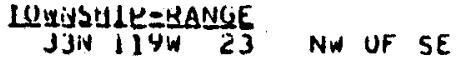

UNITRII SIATES

GrLinING

AEIITIIU I:24060
CUUKULAaLES

LATHLNG... 42-50.42 N 110-54.50 W

UIM ZUINE... +12

NUHIHING... 4/42810.

SUUGOL. 


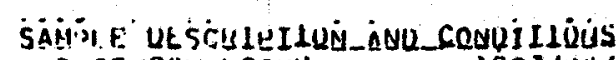

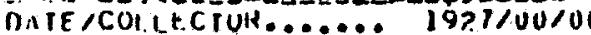

TFINFHATIME (C)

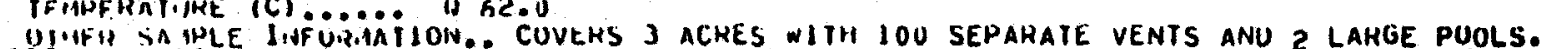
WATH AIVIT YSIS

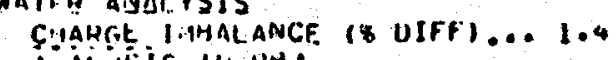

A.Ai.ris IN HW

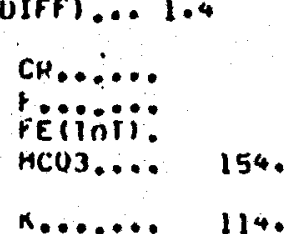

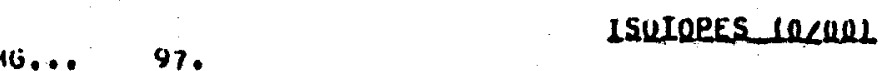

il.....

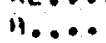

HF......

CA.... 431.

Col......

ilib.

K....... 114

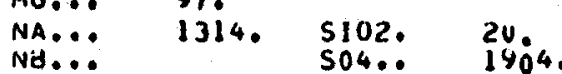

IUAI IF ICAIIIIN FILLU..... TEMPERAIUHE I HUM 9/Z2/76 ANALYSIS. GEOCHEMICAL WURK SUGGESTS SUBSURFACE TEMPEKATUHE UF I5O

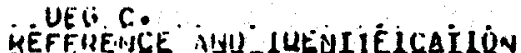

C.IMN ILEU "Y............. HEASLER, H. P.

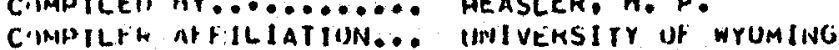

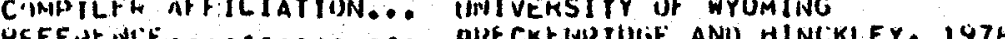

RECUHD OUO4L

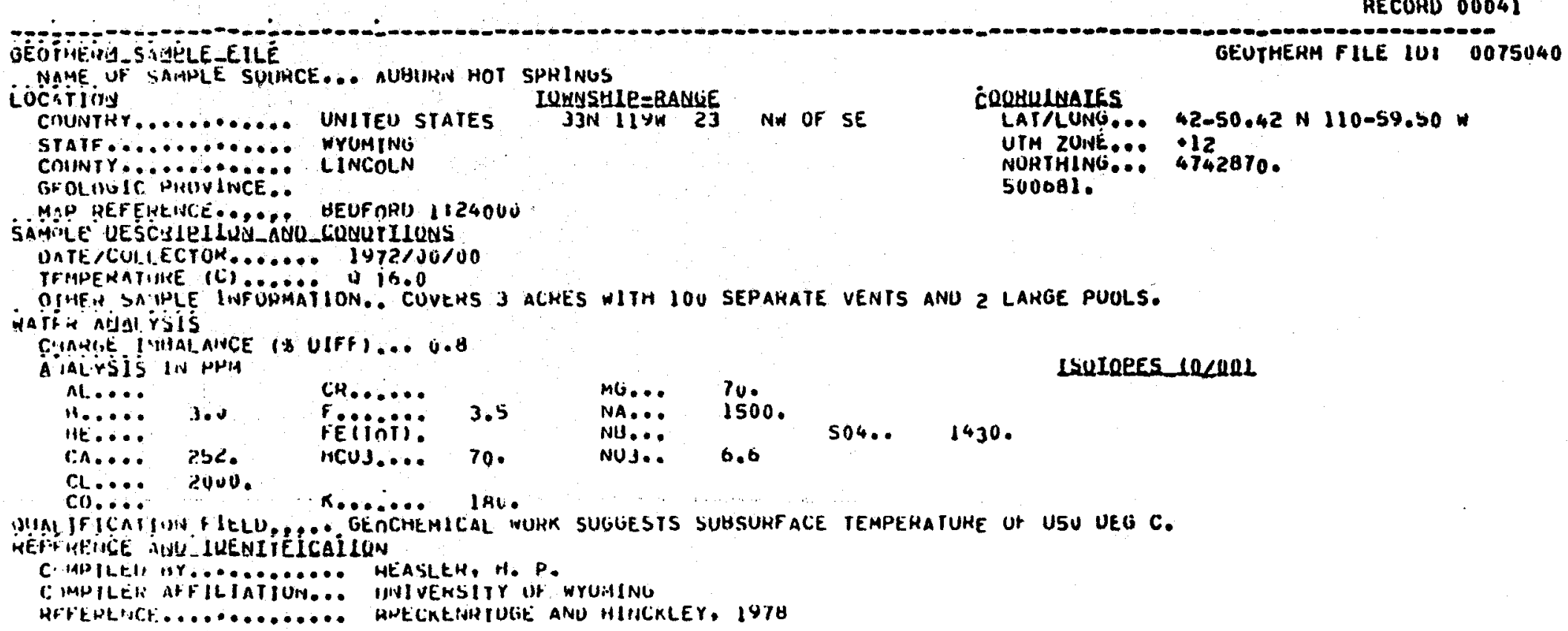

RECUKU 00042

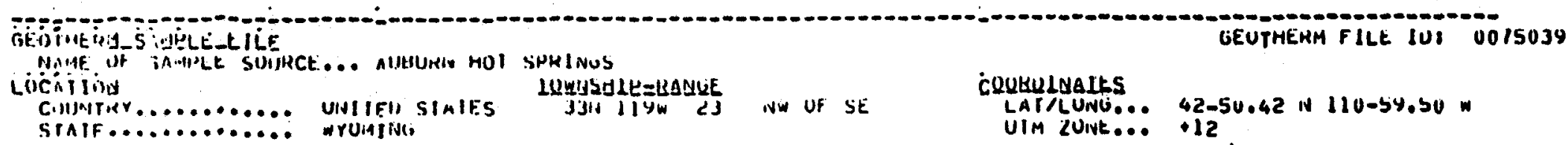


C.nlidtr........... Llivini.n

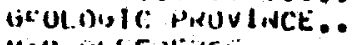

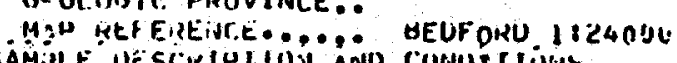

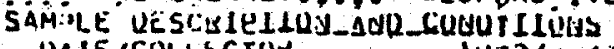

UAIE,COAIECTOR....... IY72/UU/UO

IFMPERATIJAE (C)....... W GR.

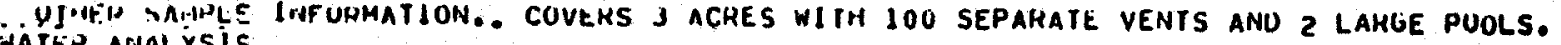
t.rsis

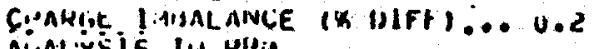

Ai.AL.YSIS lit PHiT CH......

H...... 1.3

C...... 114

Finio 3.5

CL.... IHYU.

FE(IOT).

HCO3.... 94.

MG...

740

C..... K....... 150.

JUA! IFICAT!UN FIELU PE G. GENCHEMICAL WORK SUGGESTS SUGSURFACE TEMPERATURE UF ISU UEG C.

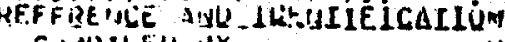

C. MPILEU IY............ HEASLEH, H. P.

C.MPILE: ATF ILIATION... INIIVERSITY UF WYUMING

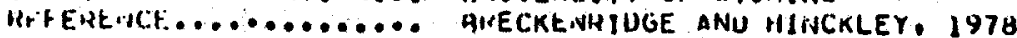

NOHIMING... 4142810

PAGE 0024

\section{ISUFURES COCROL}

WH

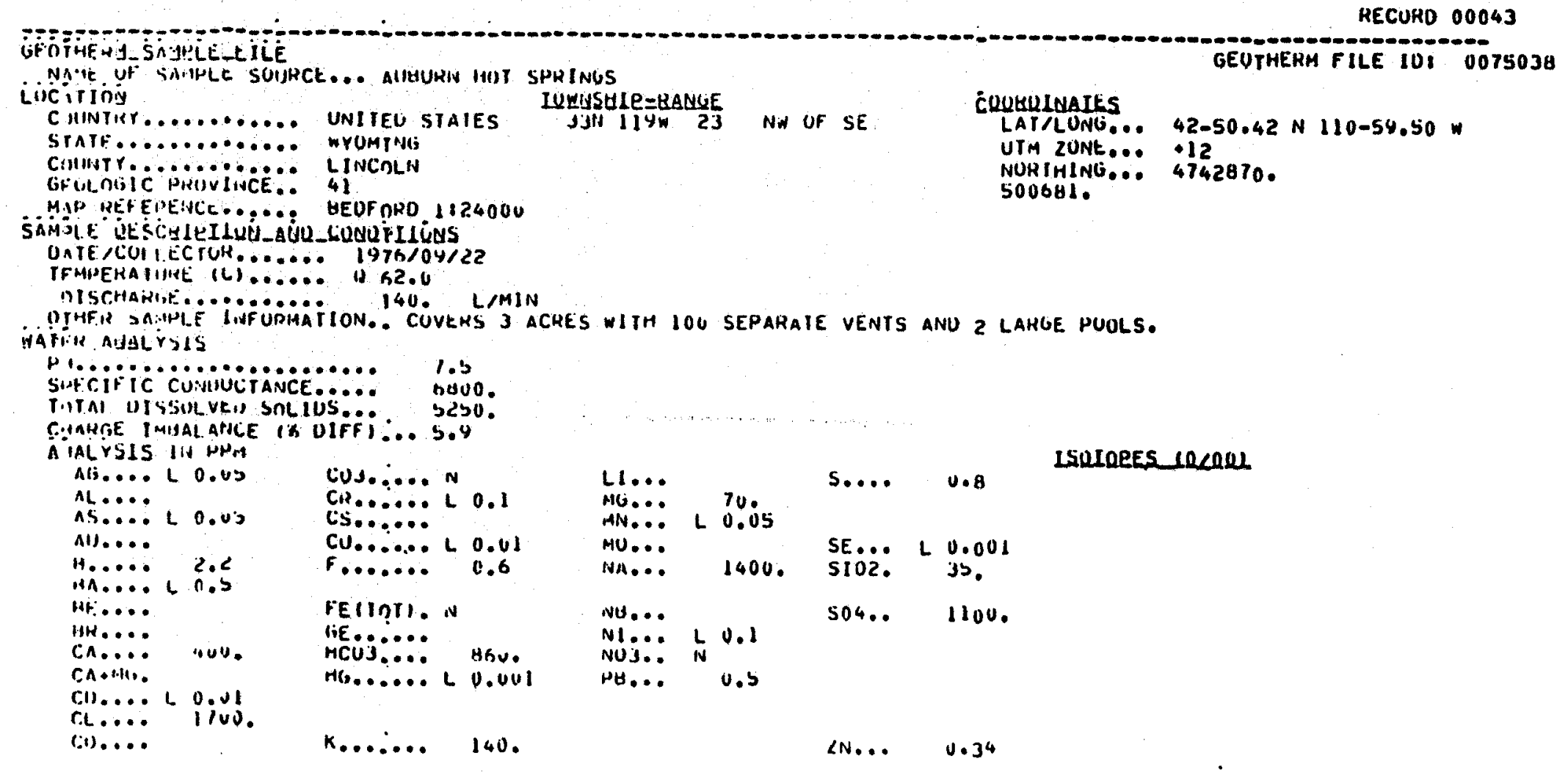


DUA IFICATIUI FIELU..... GENCHEMILAL WUHK SUGGeSIS SUGSURFaCE TEMPERATURE UF. 150 UEg C. ISEFFEENIEE AGU_LUELIEICAILON

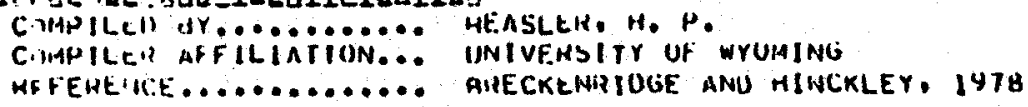

RECUKU 00044

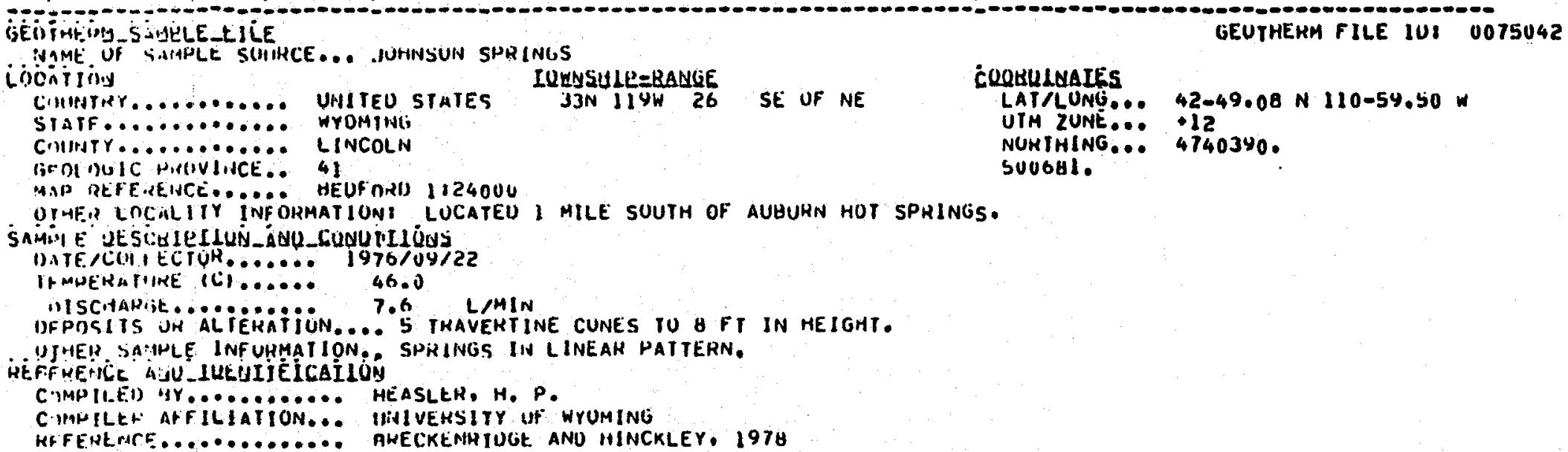

SUUGBl.

RECURO 00045

LSULOEES_RCOWL 


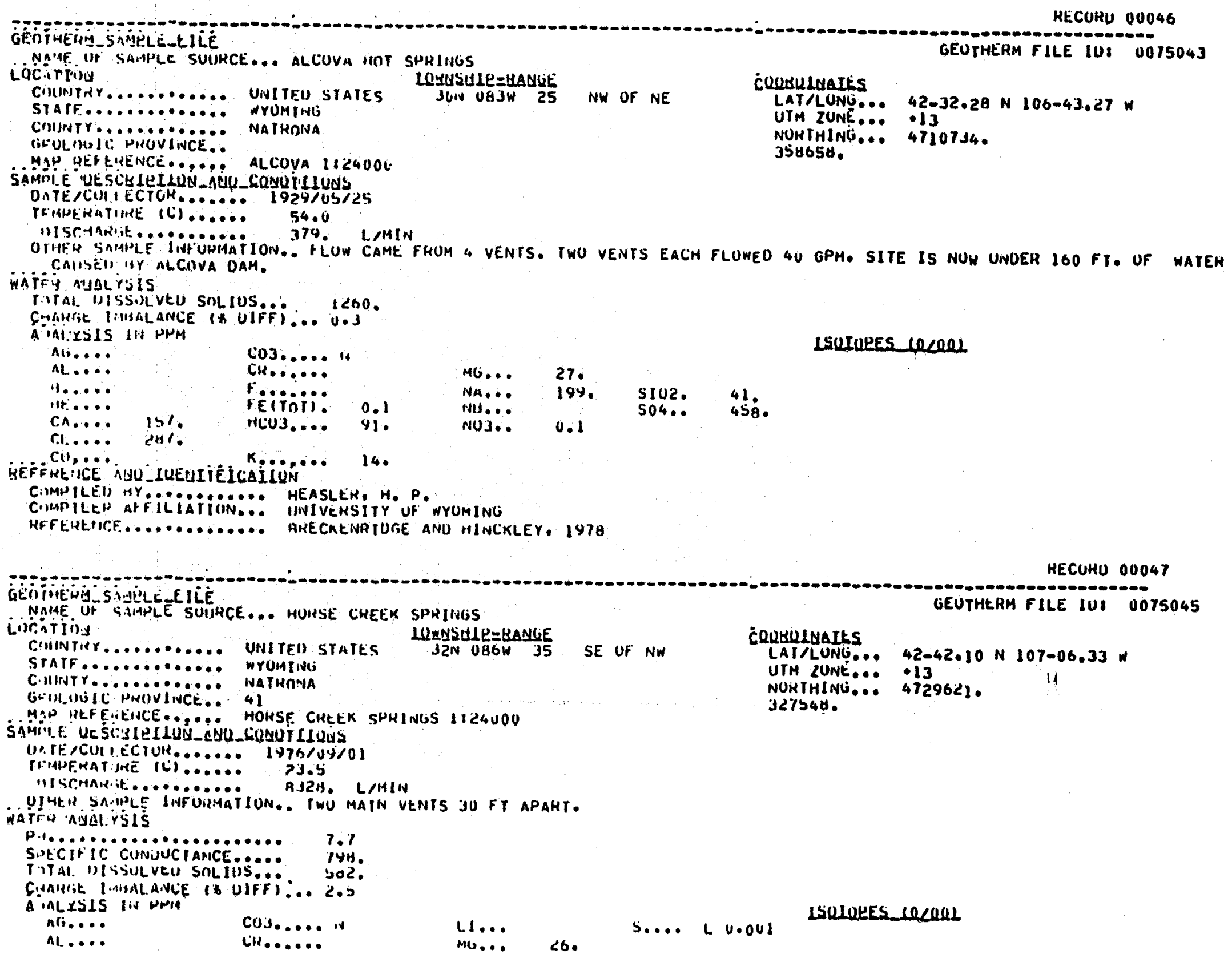

KECUHU 00047

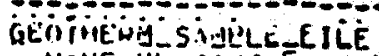

NAME UH SAHPLE SUIRCE... HUISSE CHEEK SPHIHGS

Linsitilis

LUMESU

STANRA............ UNITED STATES

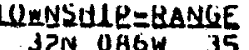

SUIURES $10 / 001$

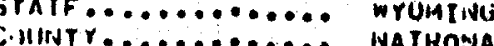

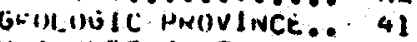

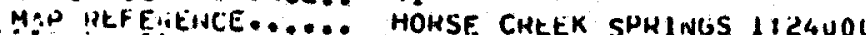

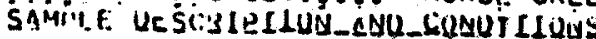

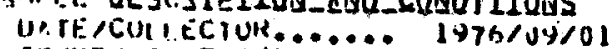

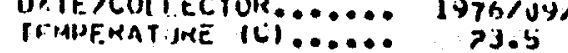

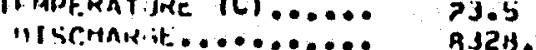

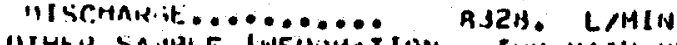

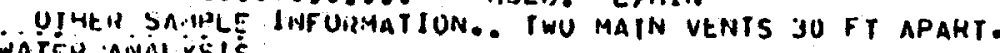
WATris nivel rists

P.1................. 7.7

S.MECIFIC CUNUUCCIANCE..... TY.

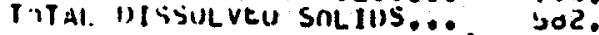

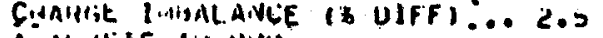

A inL LSIS liv PMit
coss..... is

nh.....

in........

L...

Mo...

«6.

\section{CRQBULAAIES}

LAI/LUNG... 42-42.10 N 107-06.33 W

UTM 2 UNE... 113

NOHTHINO... 4729621.

14 


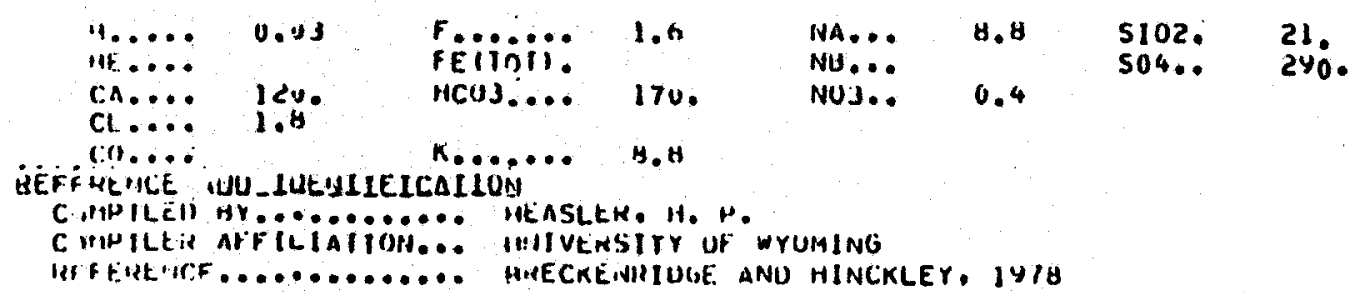

RECURU 00049

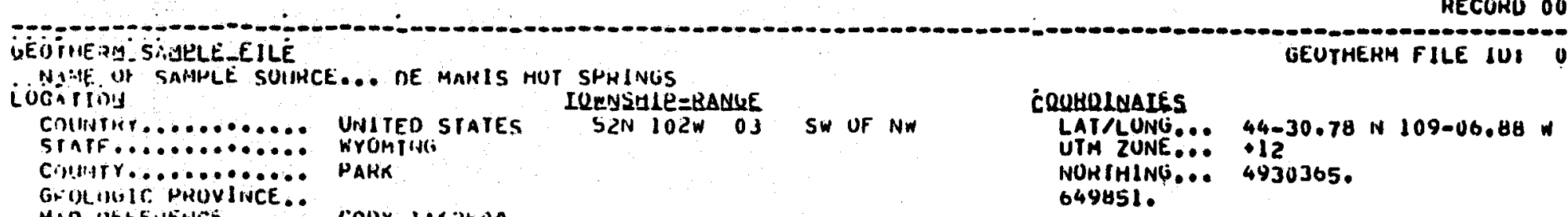

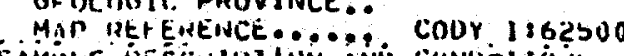

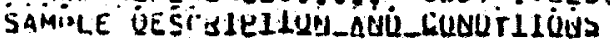

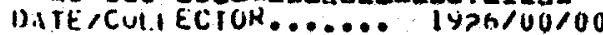

TFMPFWAIURE (6)..... U 24 .

nIST+mint

WIIER SAINLE INFUPMATION. SEVEN VEINTS. CMEMISTKY FUH UNSPECIFIEU VENT.

WAItU Airni.rSIS

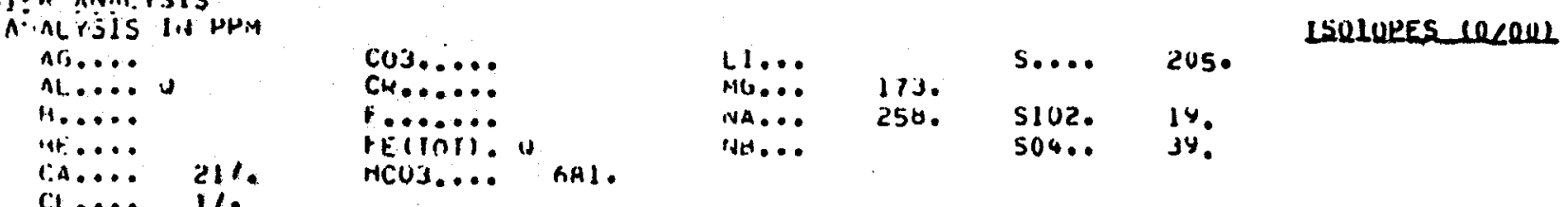

C.... 2l. NCUJ.... hAl.

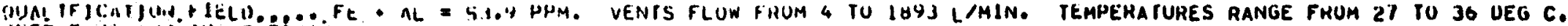

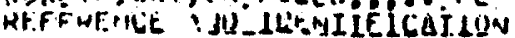

CitpILEU ir........... HEASLEK, H. 


\section{C}

CIMNILES ARFILIATION... INIIVERSITY UF WYUMING

H.FEHF.MCF.............. AIJECKENRIUGE ANIS HINCKLEY, IY78

HECUNU OUOSO

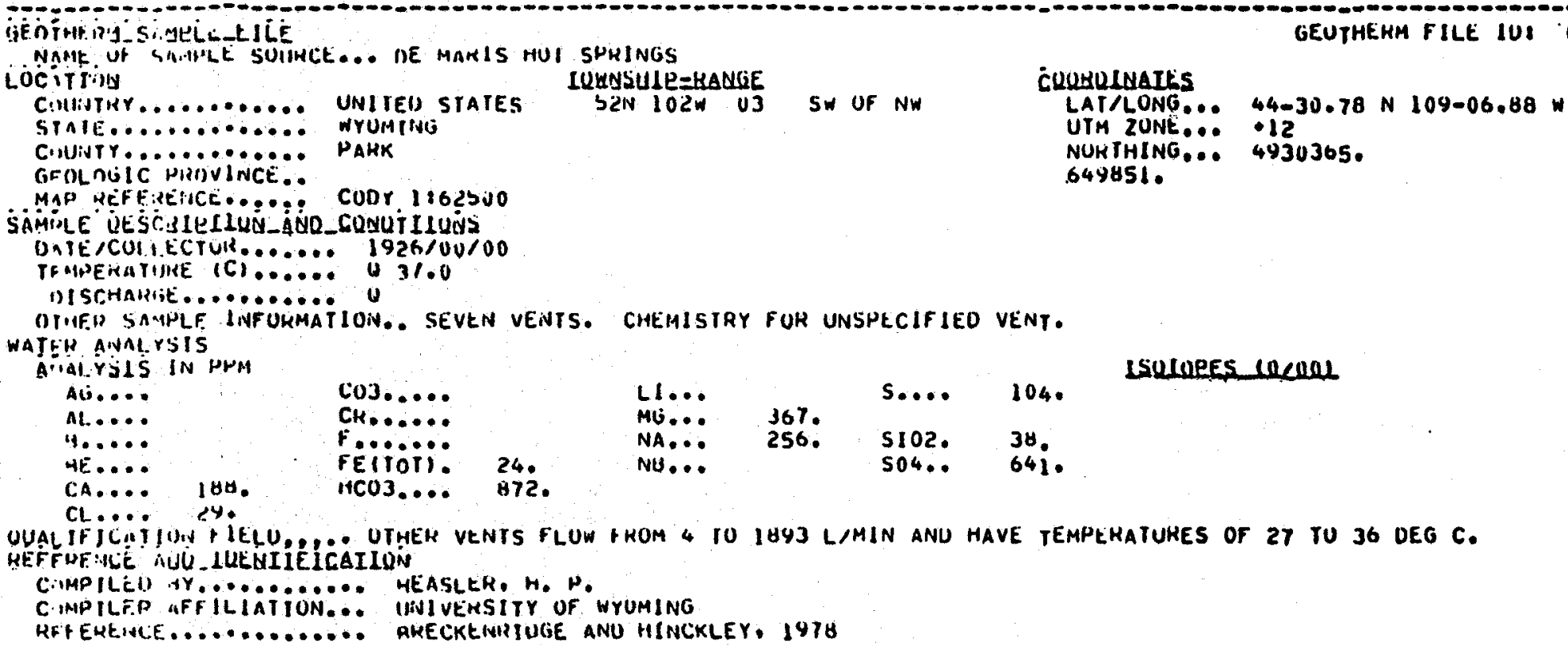

\section{CughuLNAItS}

LAT/LONG... 44-30.78 N 109-06,88

UTM ZONE... 12

NOHIHING... 4Y30365.

044051.

4430365

\section{LSULUEES LeCULL}




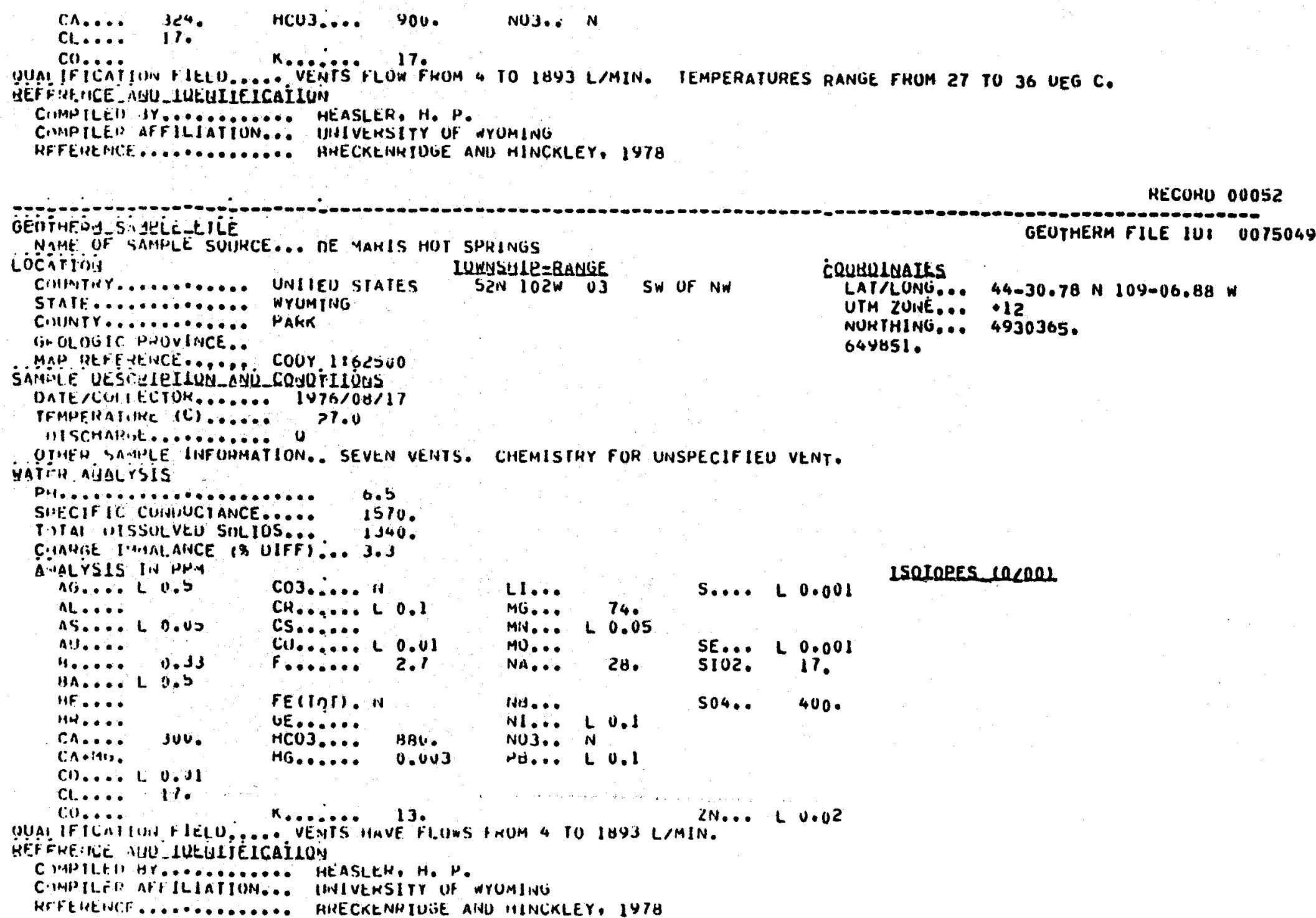

RECOHU 00053 


\section{C}

conintr............. pakk

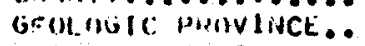

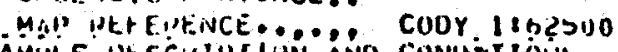

SAMLLF UESCSIEILUL_ANQ_CONUPILOUS

D.sTF, CUI.L.ECIUH....... 19RA/U2/15

TCIDEHATIIRE (C) ..... $0>1,0$

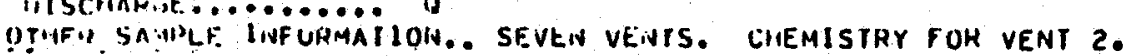

WATH AIYALYSIS

ค.1................. 1.1

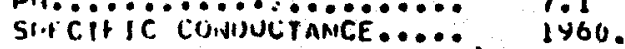

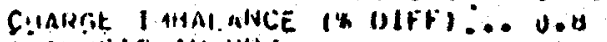

A AL YSLLS IIN HHIA

\begin{tabular}{|c|c|c|c|c|c|c|}
\hline $\begin{array}{l}\text { АL... } \\
\text { H..... } \\
\text { HE.... } \\
\text { C..... }\end{array}$ & stay. & 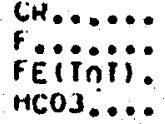 & $\begin{array}{l}2.0 \\
0.2 \\
943 .\end{array}$ & $\begin{array}{l}\text { MU... } \\
\text { NA... } \\
\text { NB... } \\
\text { NU3.. }\end{array}$ & $\begin{array}{l}03 . \\
330 \\
0.1\end{array}$ & $\begin{array}{l}5102 . \\
\text { So4.. }\end{array}$ \\
\hline
\end{tabular}

NURIHING... 4930365 .

649851 .

RAGE NOJO

LUUOEES leCARL

CL.... 2u.

C11.... K...... 16

UUA! IFICAT!ON + IELU..... VENTS FLUW FHUM 4 IU 1893 L/MIN. TEMPERATURES RANOE FRUM 27 TO 36 UEG C.

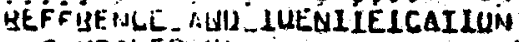

CIMPILEO HY............ HEASLEH, H. P.

CYNPILER GFI ILIATIOH... IIVIVERSITY UF WYUMING

RFFEIENC:............ AHECKENRIUGE ANU HINCKLEY, 1978

RECURU OUOS4

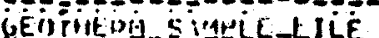

NiAt, UF TAFHPL SUIRCE... DE MAKIS HOT SPKINULS

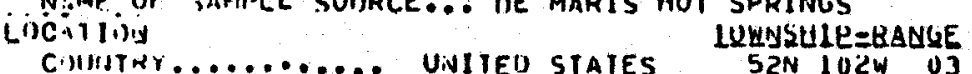

SiatF............. Wromjint

$52 N 102 W$ OJ

SW OF NW

C.IIIITY

Give Bili phivitide...

PARK

\section{¿QQRULLHAIES}

LAT/LUNG... 44-30.78 N 109-06.88

UTM ZUNE... +12

NUKIHING... 4930365.

644851 .

Mar ptefterdit...... Cour 1102500

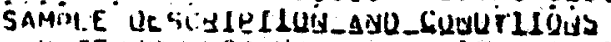

DITF, CUII ECTUH....... 197U/OH/14

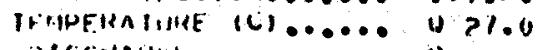

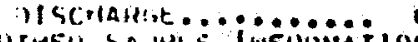

$$
27.0
$$

IMFi SA.MPL LINFURNATION. SEVEN VENTS. CHEMISIRY FOK VENI 1.

WATEN A.JULYSIS

SIPFiric Cunisuitance..... IOU

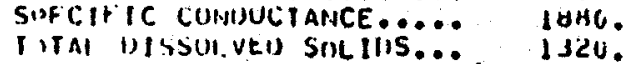

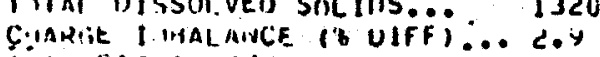

a IALYSIS I:I PMM

nI....

H..... 0. 0.

ilf.....

s<.

CH......

iini: 1.4

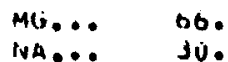

cl. 0.0 J 100

HCos... yit?

Nuj:-

c1).... K....... 1H.

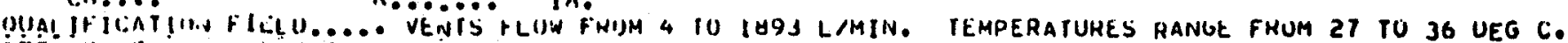

BEFHOENCE DUU _LELIIEICALLO

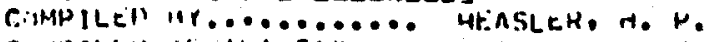

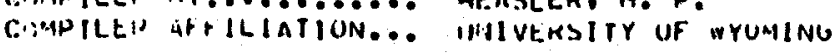


GÉ

NOMF. UI SAAHLE SUIHCE... DE MAKIS HUT SPRINGS

iacirting

CIIIITHKY. . . . . U... UNIIEH SIAIES

SIATF............. WYUMTING

CIIINTY........... HAHK

GrOLOUIC NHDVINCE.

MAP PEFE सtUCE

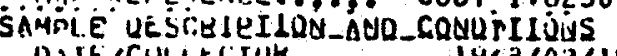

OAIE,CUI, ECIUK....... IYKA/02/15

IFMPFKATUKE (C) ...... 0 ? 28.0

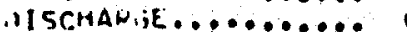

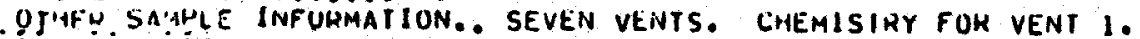
WîtFi iulat is

p.................. 0.9

T.TAL VISSULVEU SOLIUS.:P IY70.

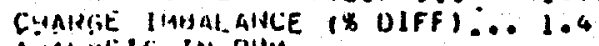

A IOLYSIS IN PHIA

Ais.....

11.....

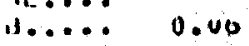

IIE......

C.4.... 354

Coj.

FEini: 2.0

Cl. . . . ?

nCo3 952

$52 N 102 W$ U

K....... Ih.

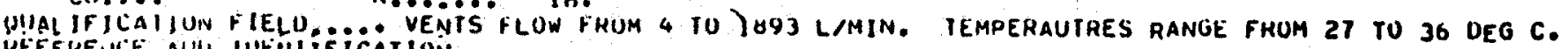

CE NUU-IULELIIEICALION

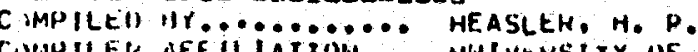

CIIPILER AFF ILIATION... MINIVEKSITY OF WYUMING

.... AKECKE HH IDCLE ANU HINCKLEY, 19/8

RECUKD 00056

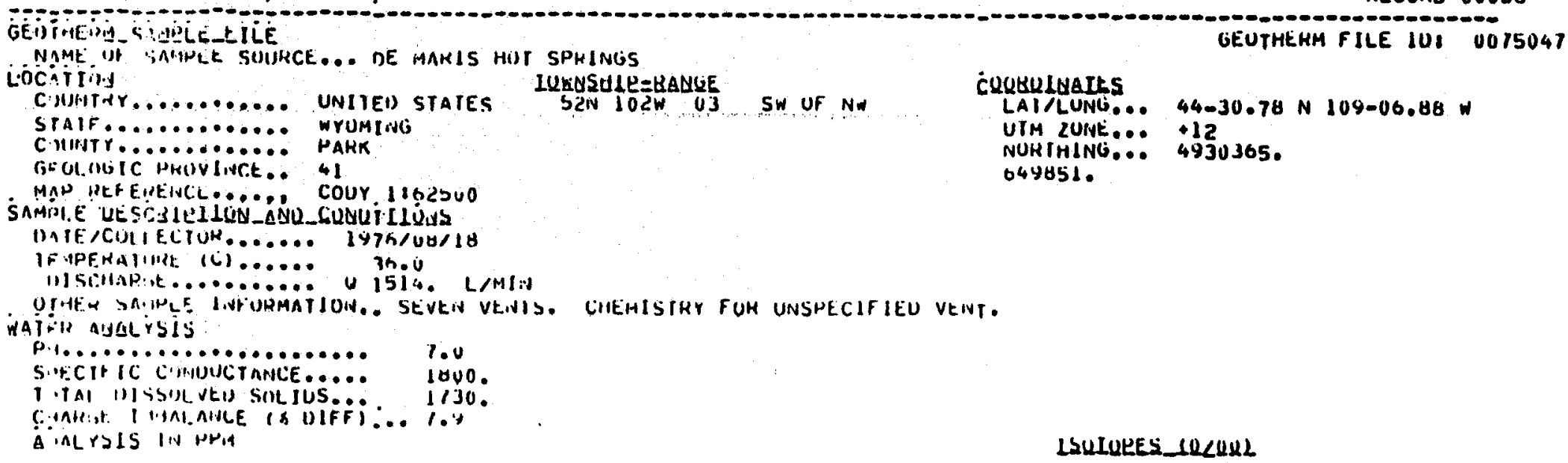

SW of $\mathrm{NW}$

LEOTHERM FILE IVI U0.75USI

CUQHULUAIES

UIM LUNE... 12

649851 .

LOIOEES leCOO

33.

NH... 33. SO4.. 422.

0.1

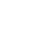

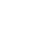

S.... 16 


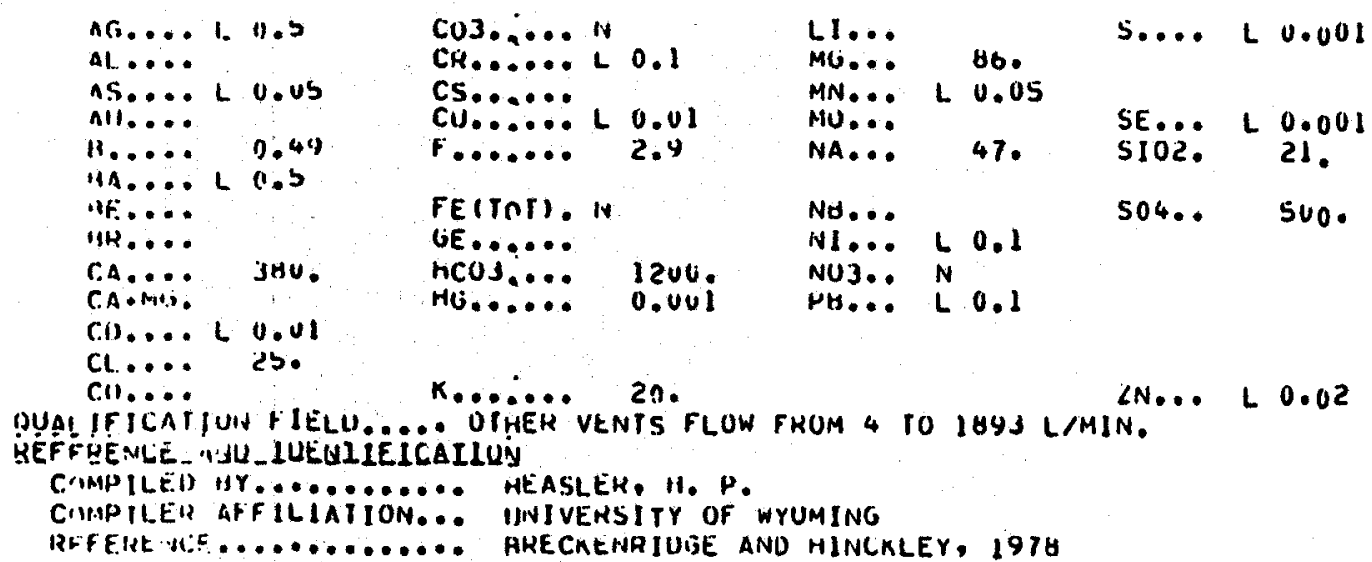

RECURO 0005

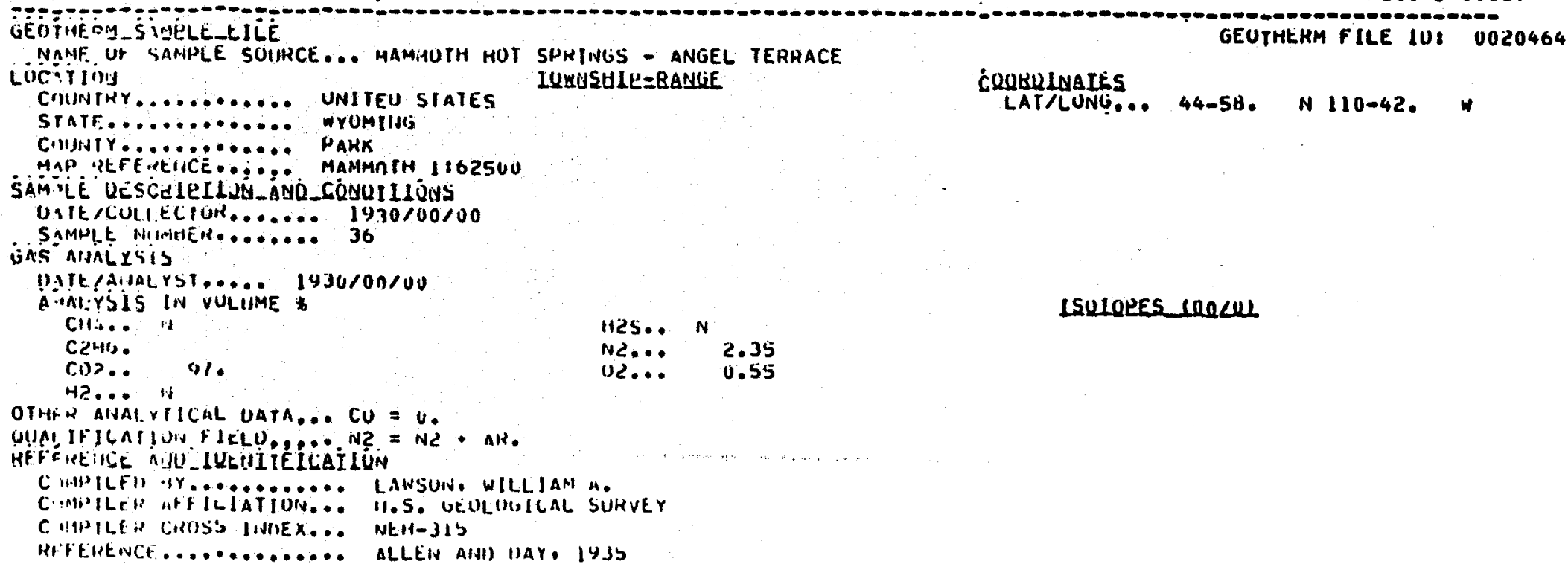

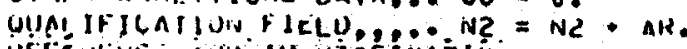

HEF

C WHILF" IY........... LANSUN, WILLIAM A.

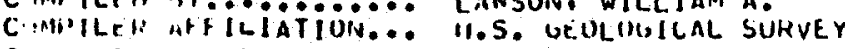

C.MPILEH CWOSS IINIEX... NEM-3IS

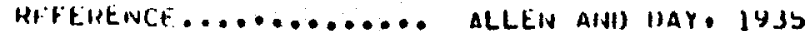

RECURU 00058

C. IIIITKY........... UHIIIFI) SIATES LUWNSILLE $=$ HAIVLE 


\section{c}

PAGE 0033

DATE八OIIECTUK....... 1965/UO/00 GUNIEK ANU MUSGHAVE SAMHLE H. MMIJEH........ IIA

JEMHEKAIIIRE (C)

GAS ANALYSIS

DATE/ANALYST..... 1965/ON/00 GUNTEH ANU MUSGKAVE

Aini-is IS IN HULE

acisis in

cirta.

Cop.: 100

1v2... 0.16

T.冂. 14

$02 \ldots 00.08$

LSULUEES leQCOS

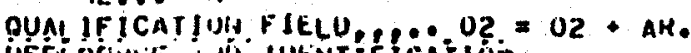

QEFF RENLE NYU IULNIIEICALLON

C.IHHILEU UY.......... L.AWSUN, WILLIAM A

CIMAPILEH ATFILIATION... H.S. GEOLOGICAL SUHVEY

C INIPILËH CKUSS INIJEX... NEH-3Z I

HECORO 00059

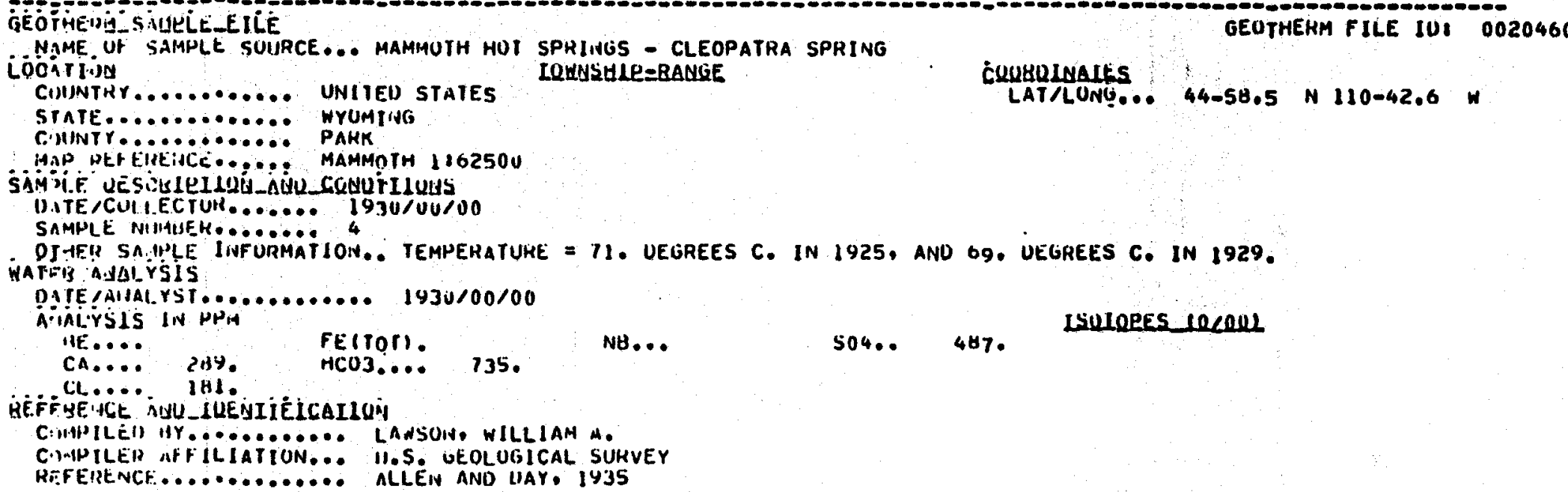

. NAME UF SAMPLE SUURCE... MAMMUTH hOT SPRIIUS - CLEOPATRA SPRING

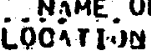

CIIINTRY ......... UNIIEI STATES LQUUSHLE=RANGE

STATE.

C.NINTY........... PAKK

HAP PEF EllEiHCE..... MAMMOIH 1862500

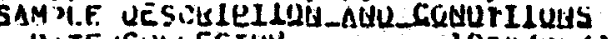

D.TTE /CULTECTUR....... 1930/0U/00

SAMPLE NIIAUEH...

OJ HE! SA IPLE INFURMATION.. TEMPEHATUKE = 71 . UEGREES C. IN 1925, aNO 69. UEGREES C. IN 1929. WATEB A VALYSIS

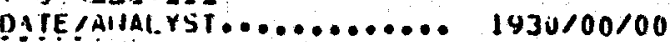

Aini.rists lis pois

ALYSIS IN HPH

FE(TOT)

NH...

$504 . . \quad 487$.

LivLORES leCNOL

Ca.

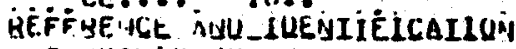

C:HIPILEU HY............ LANSOHS, WILLIAM A.

C.MIPILER AFFILIATION... H.S. UEOLUGICAL SUHVEY

RIFEILNCF............ ALLEN AND LIAY, IY3S

RECOHO 00060

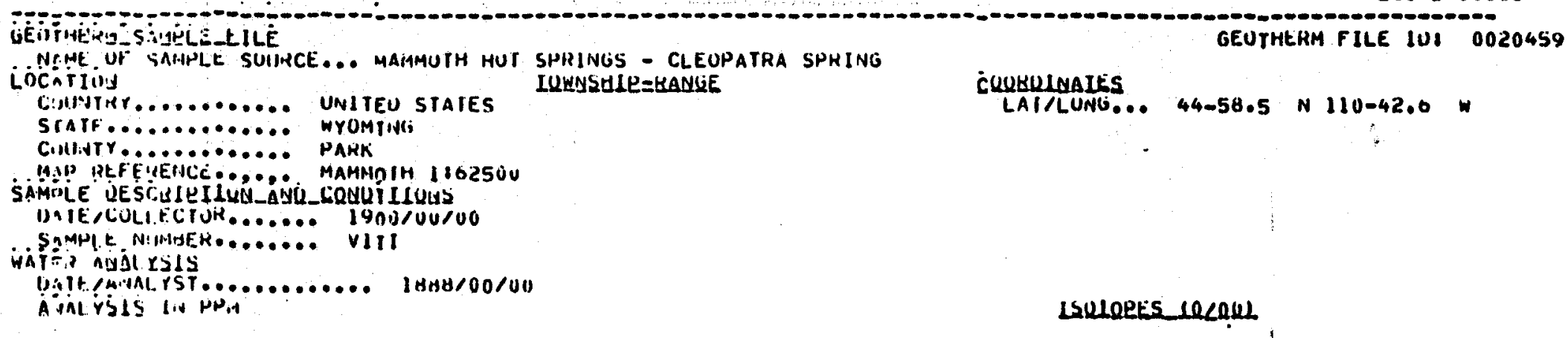

ini vis 


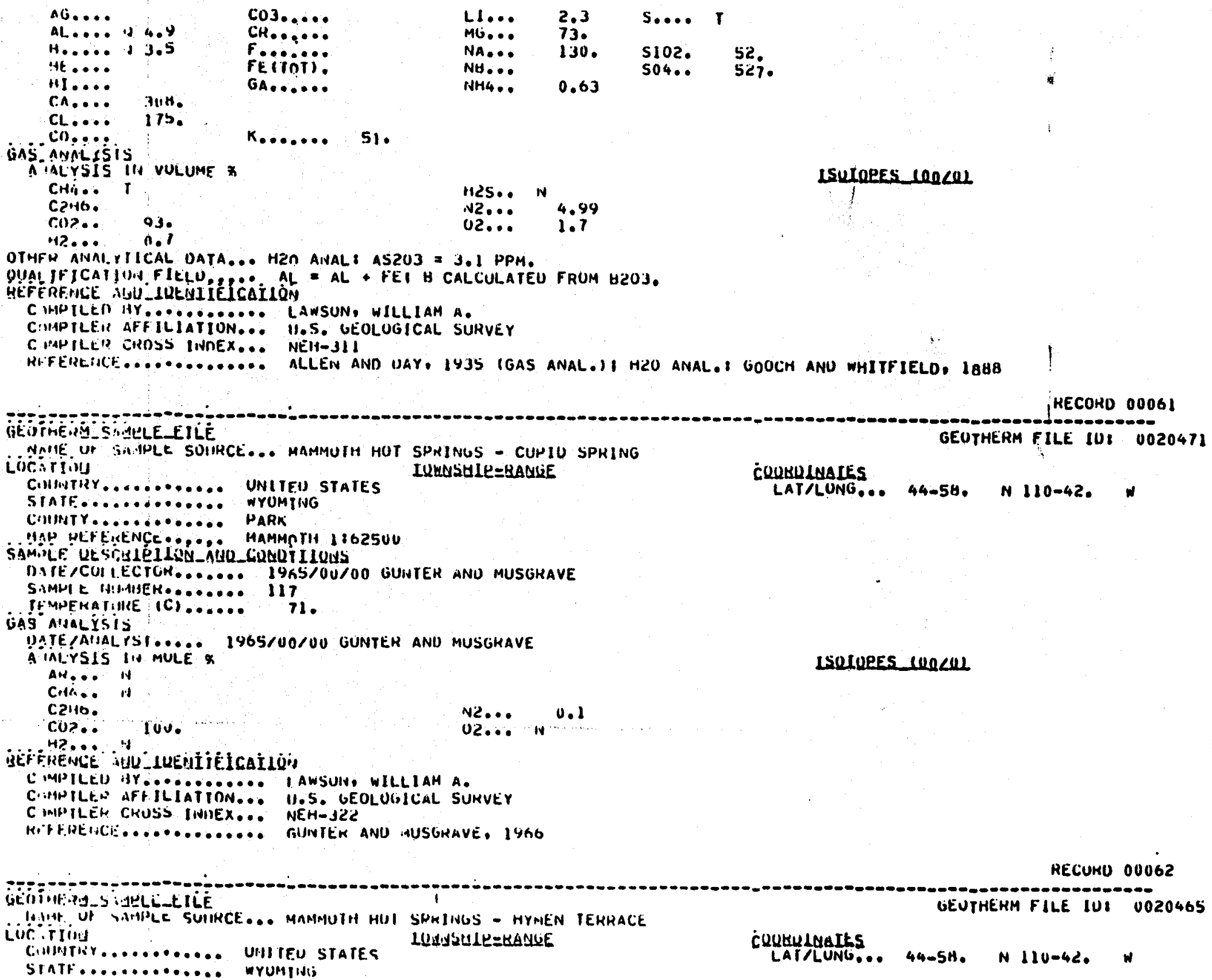


c.enr........... pank

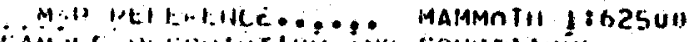

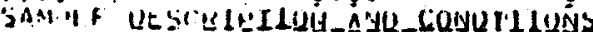

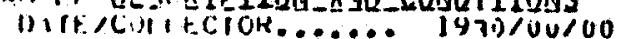

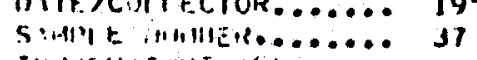

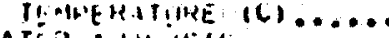

37 . 70.5

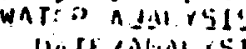

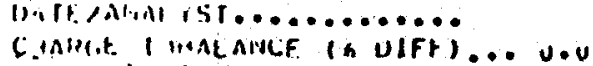

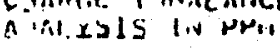

A1......

cos...... in

$11, \ldots$

an

c..... H. H.

C........

c.... H.1. HCW3... Tob.

ins

a. A1:risis lia vULIHAE 8

riti... त

(i) in:? *

(i).

4.)

ALLEN, E. I.

n...... \$7.

(4)... 11

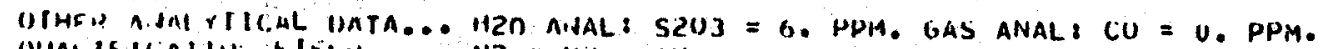

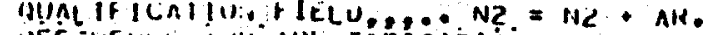

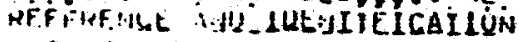

C.MPILC.11 Ir........... LAWSUIV, WILLIAM A.

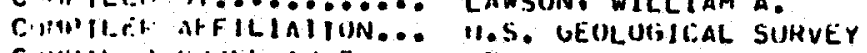

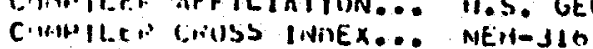

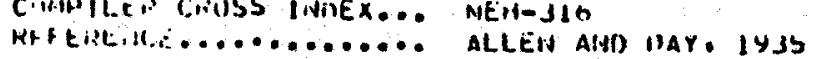

LI...

S.... N

ISULQESS_UCOQL

NA... I3j. SIOZ. 24.

NG...

24.

ISQIQRES_LROCUL

$12 \ldots 00.00$

KECUHU ONOGJ

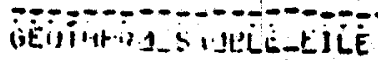

GEUTHEKM FILE IU: 002040 ?

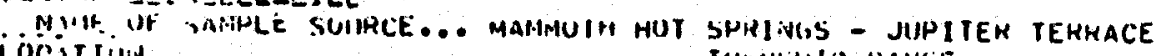

Locitring

cillitirito

wrulifins

Cihlitr............. Pakn

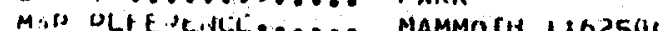

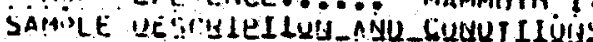

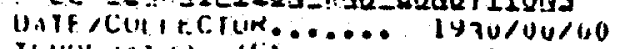

cins a.talusis

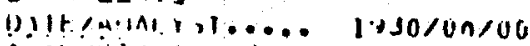

Aini risis lla Vulinie t

citim.

r.siln.

(i)... live

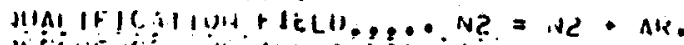

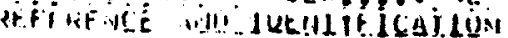

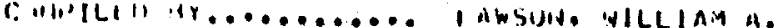

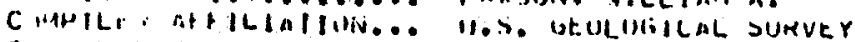

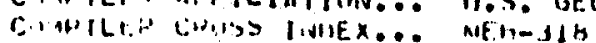

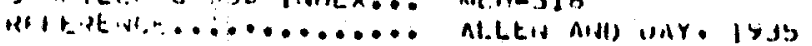

IUnNYZILLE = UANGiE

HLS.. in

$1,12 \ldots$ is 0.35

LSULUKES IONCOL

(1)... U.

\section{CUUBULAAIES}

LAT/LUNG... 44-58. N 110-42. 
RECUHO 00064

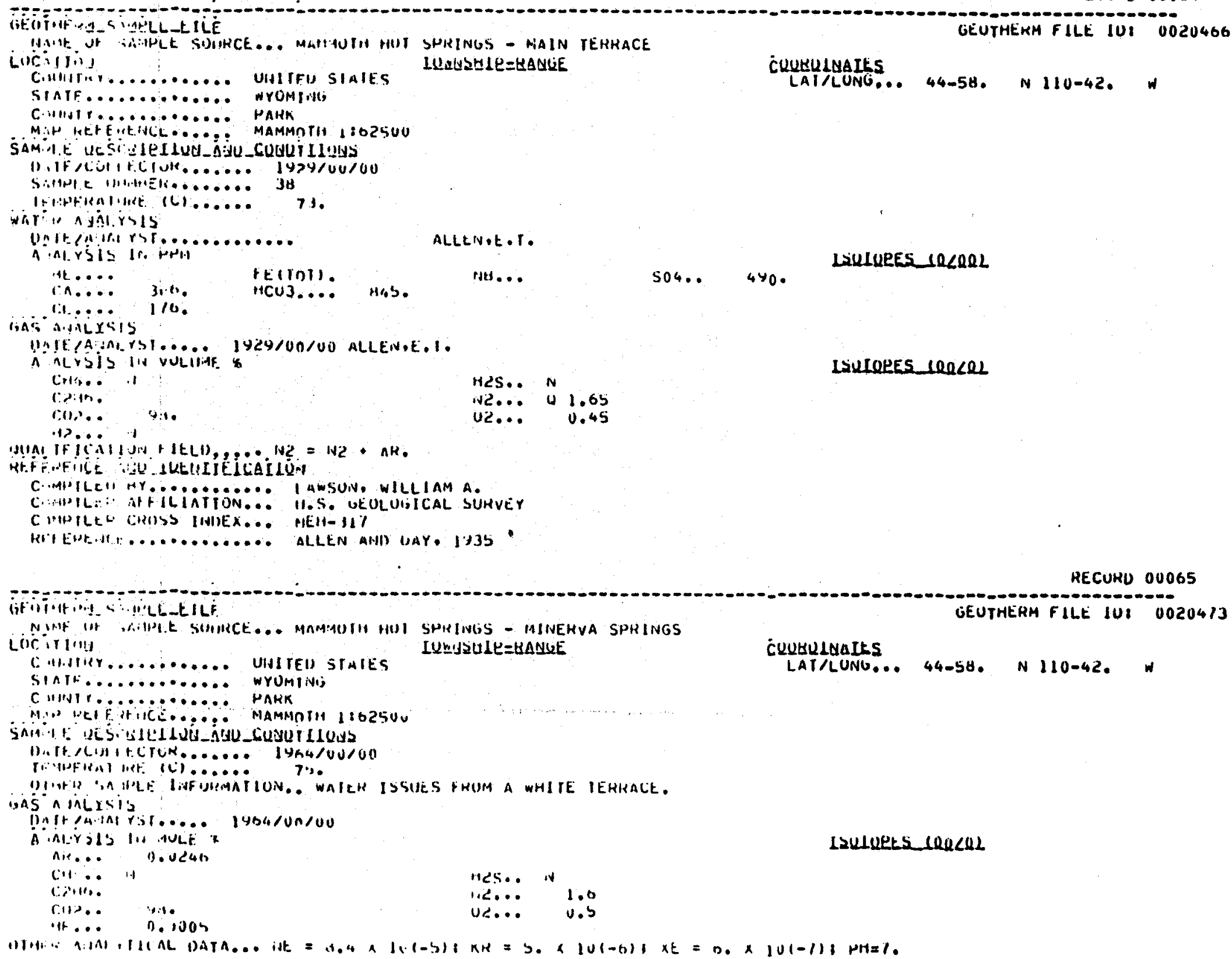

RECUKU 00065

CUUELLALITS

LAI/LUNG... 44-5H. N110-42. N

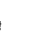

LIULURES IRLRRL

\section{LIUIOEES IROCRL}




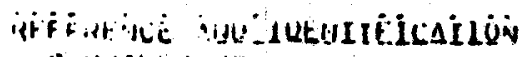

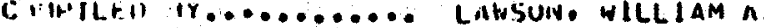

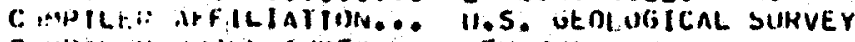

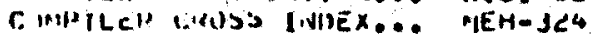

KECUHU OUO66

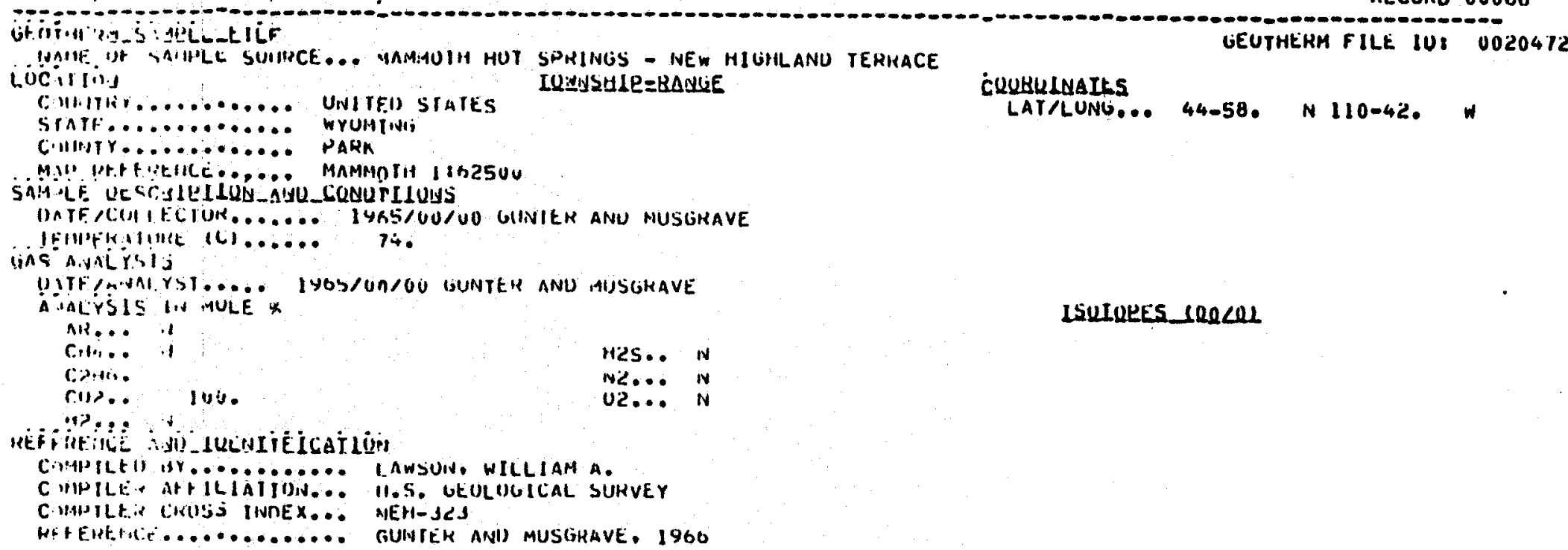

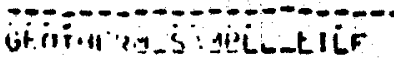

IUAME. "H SAIHLE SUIHCE... YAM:AUIH HUT SORINGS - NEW MIGHLLANU TERHACE

Liociling

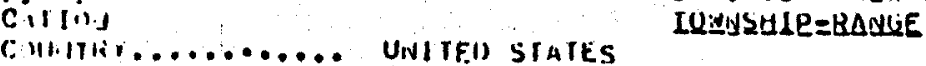

SintF........... WYUMini;

C.IIIHTY

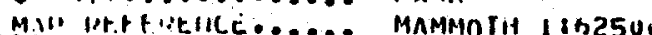

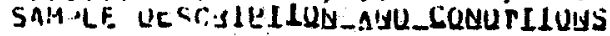

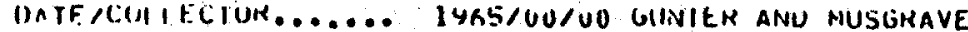

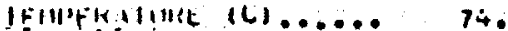

ins aivitisio

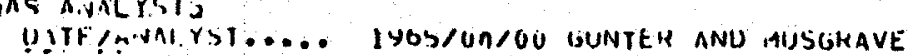

AALYSIS IN MULE *

AR... I

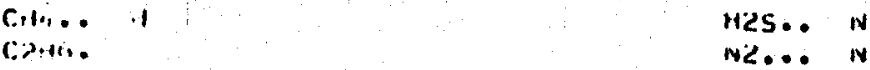

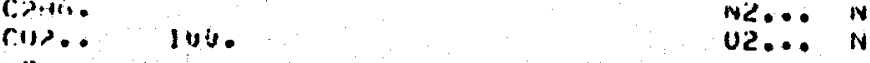

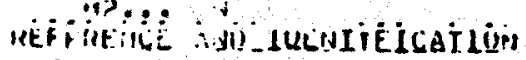

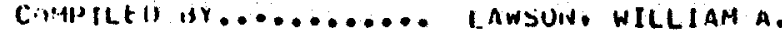

C IIPILE. ATH ILIAIJUIN... II.S. ULULUGICAL SURVEY

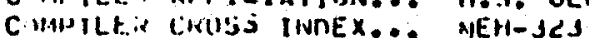

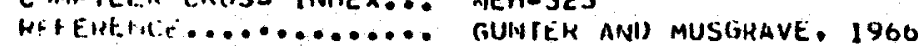

ISUIORES_ROCU

GEUTHEKM FILE IUI V020472

CQUHULNAILS

LAT/LUNG... 44-58. N 110-42. N

HECOHU 00067

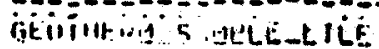

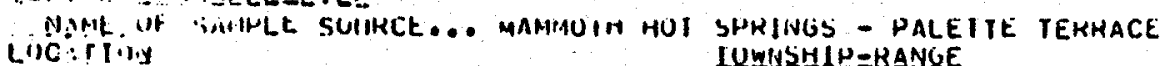

C.PIIVY

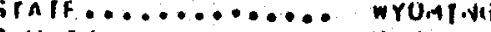

CHATH ........... PAKK

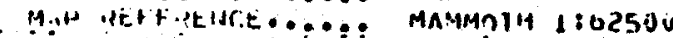

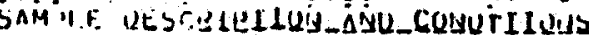

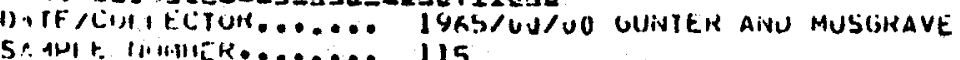

iAS ANALLYCIS

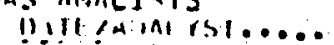

nivisis lis MULE

YOSS/CN/OU GUNTER ANIS MUSURAVE

cili..

c.entise

S.

196

ISULQEES_CQRLIL

GEUTHEKM FILE IUI 0020469

(i) $1 . .$.

4.5

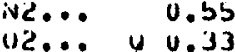

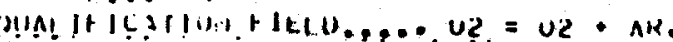

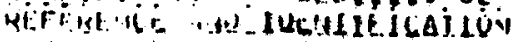

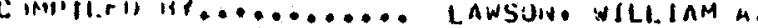

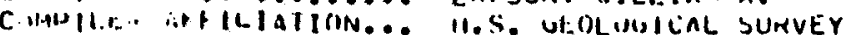

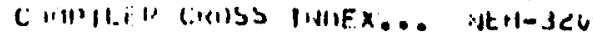

cousuluaIts

LAT/LUNG... 44-58. N 110-42. W 


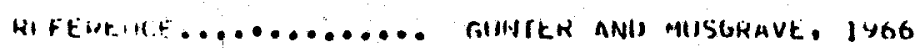

RECUND UNOOH

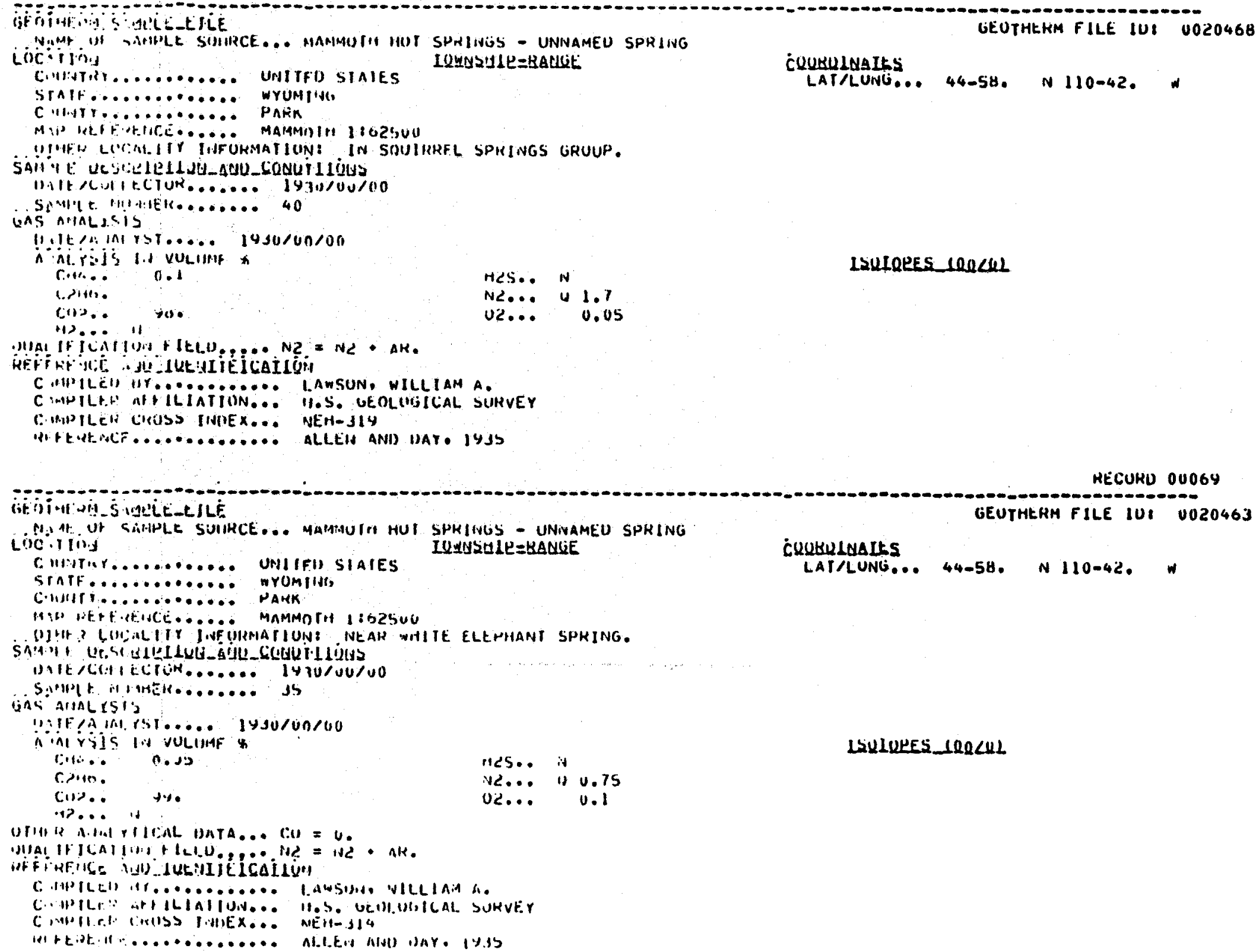

LSULOEES 100CUL

HECUKU 00069

(it) ii

M: 1F. Ut SAHHL SUIHCE... MAMAOUIO HUI SPKIFUS - UNIVAMEU SPRING Licitiong

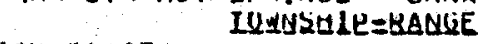

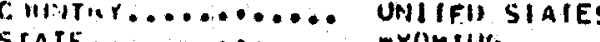

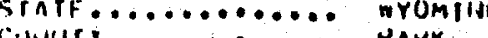

cine

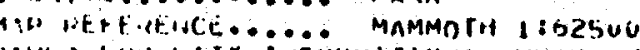

SAM

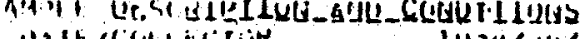

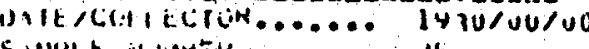

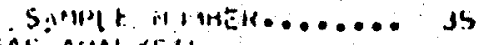

ind $\operatorname{AIIAL} 151$ is

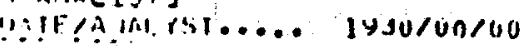

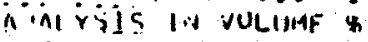

rit... 0.J

c.jon.

tיo.

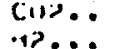

. 2 ... in 0.75

LSULUHES_LRCHL

UIII IR N.1.A PIICAL UNTA... C:U =

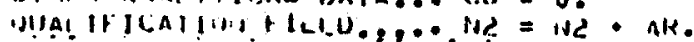

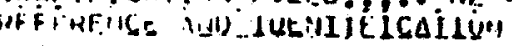

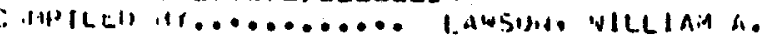

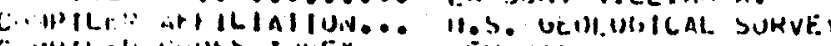

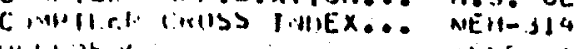

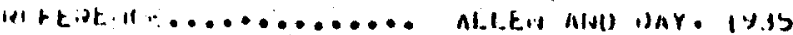


HECOND 00070

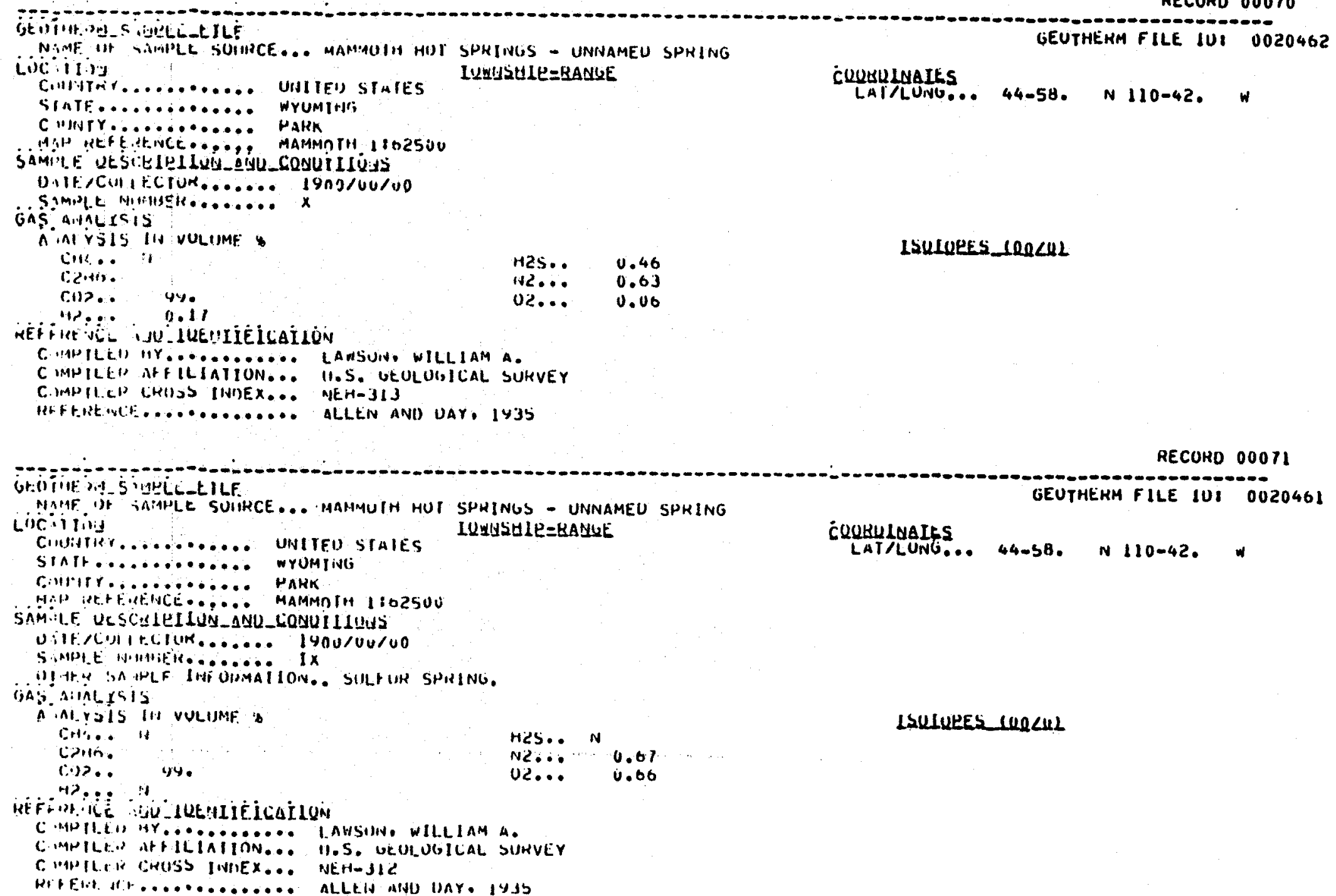

RECUKO 00071

HECUNU UUOTZ 


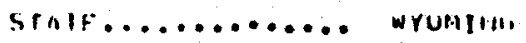

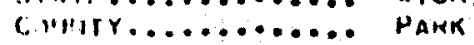

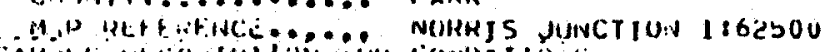

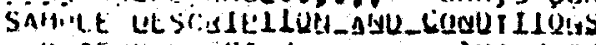

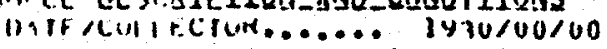

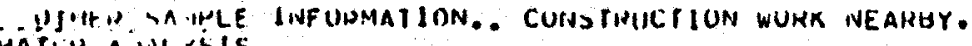

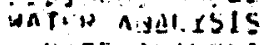

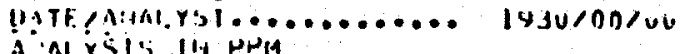

HECUHO NOO73

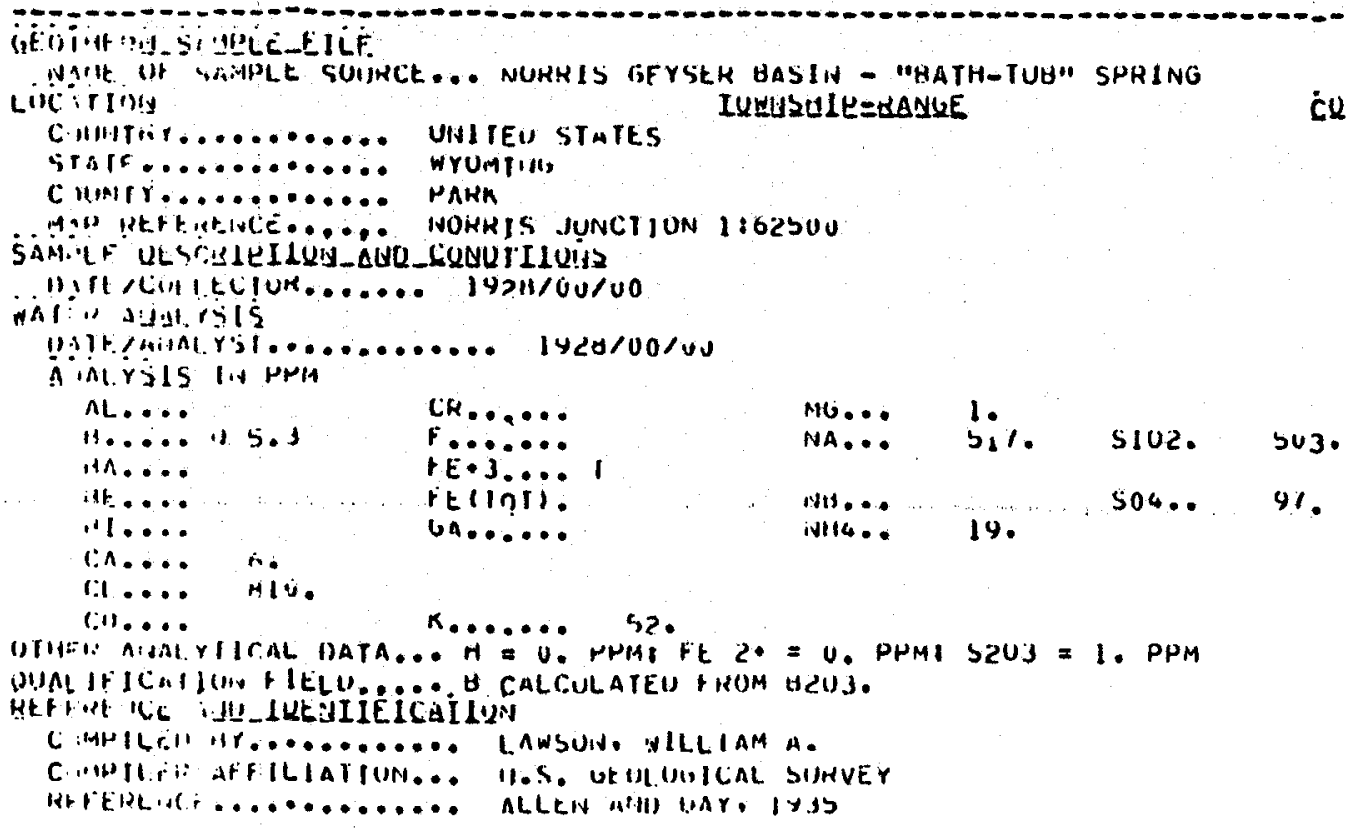




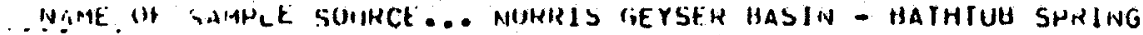
$\operatorname{loc}: 1 \mathrm{ling}$

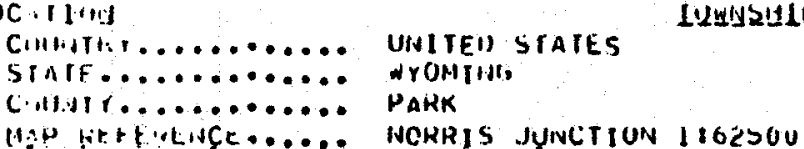

\section{CQUBulaIts}

LAT/LUNG... 44-43. N110-43.

ISULEES HUCUL

RECUKU 00075

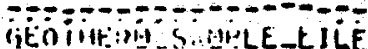

GEUTHEKM FILE IU: 0020426 incirting 10HNSULE=GANGE.

Contulir o.

Sintt.............. Wrumino

C.llNTr............ HakK

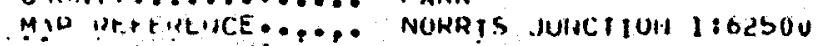

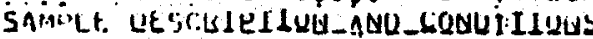

Diticiliceruk....... 19hs/vo/un

SIMPI E MHABEK....... 54

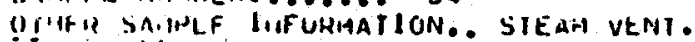

IAS MIJALYSIJ

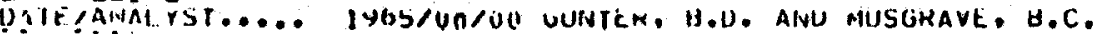

A int rsis liw WULE

C+16.. . .

cherie:

(c)

HEF FUE L 20

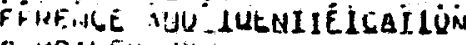

C IMRILEH HY............ IANSUIV, WILLIAM A.

C.AHILEK ATHILIAIION... I1.S. UEULUGICAL SURVEY

CIMARILtR CIASSS IIVI) X... NEH-2YI

HEFF.ME:HE............ GUIVIEK ANU MUSURAVE, 1960

RECUHU 00016

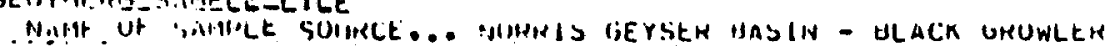

iveilin 


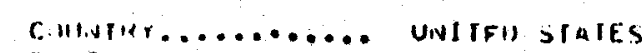
Wriminge

Sintr.......... WrUmin

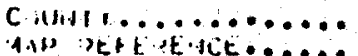

NUKAIS JUINCTIUN 1:02500

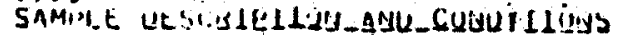

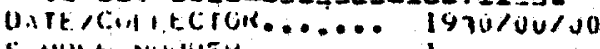

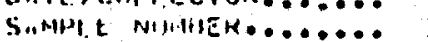

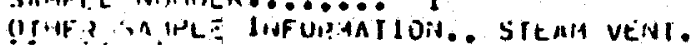

rias nnitissis

Aital ruis lit ruluate
cilce. 3.1
$+125$.
cov>.. 910
N2... iv 0.45

$$
47 \text {. } \quad 3.4
$$

LSUIUEES CaUCOL

PAGE OU42

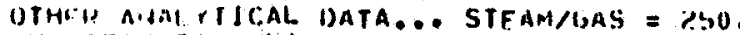

JUAI IF!LAI!UN H ItLLI)... N2 = N2 * AK.

HEFITHILL WULILENLIELICAILUN

C.HAILEW HY............ IAWSOIN. WILLIAM A.

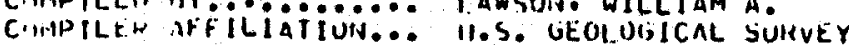

C.MAPILER CHUSS INDEX... NEH-2HO

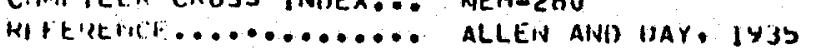

LAI/LUNG... 44-43. N 110-43.

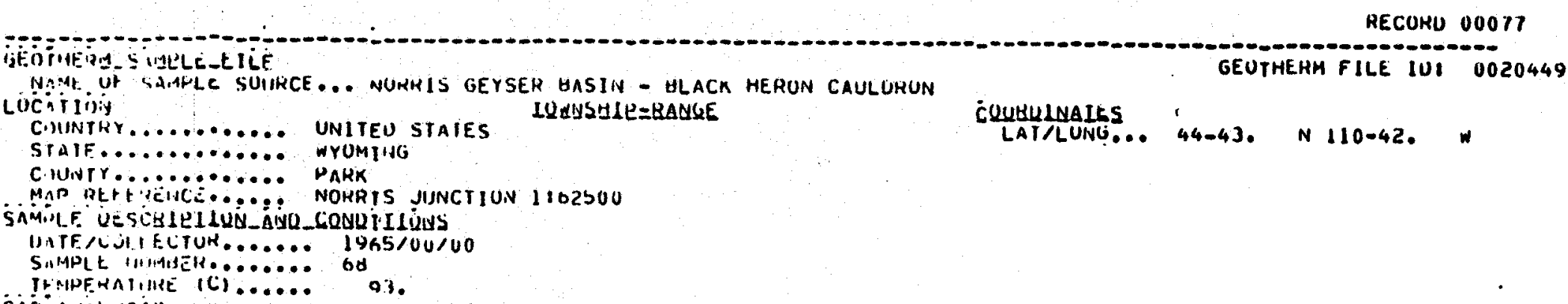

LSUIQEES INRCUI

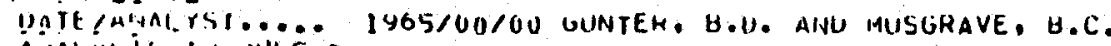

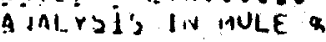

Air... U. U.

cirin... 0.18

rip.ti.

rus.. 40

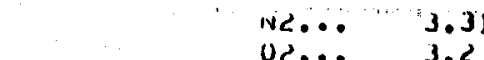

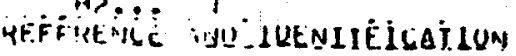

C.IIPILEI1 ilr............ LAWSUIN, WILLIAM A.

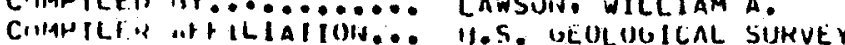

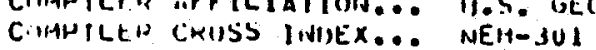

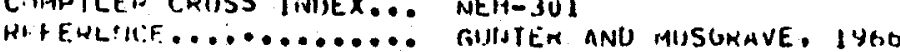

HECUKU OOUTE

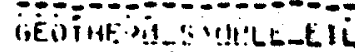

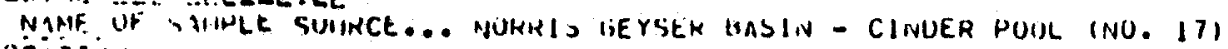

Lociting

UNAFII

GEUTHEHM FILE IU: 0020431

CUUBULNALES

LAT/LUNG... 44-44. in 110-4J. W 
C. $+1+11+\ldots \ldots \ldots \ldots \ldots$. PAKK

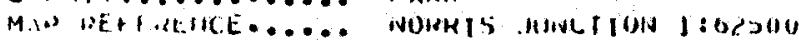

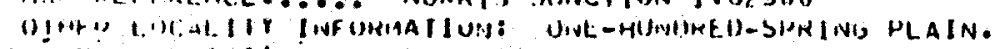

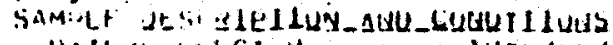

1) 1 1

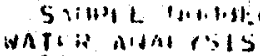

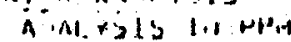

al.

$11 . \cdots, 1$.

15.... r.

11,.....

in.

i1 $\ldots$....

Ch....

Ca... H.

cil.....

ins allicrios

GA.B...

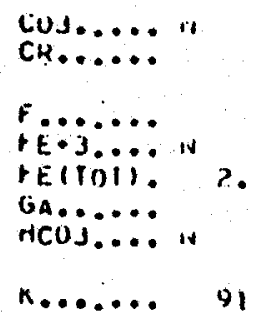

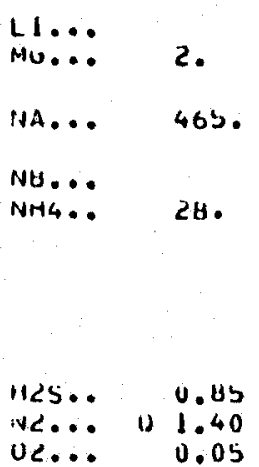

LSUIUEES IOCOUL

A M ralis lid VUlIJME

c.i... n.

ive...

1.40

(i)

itie

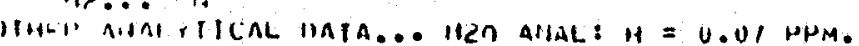

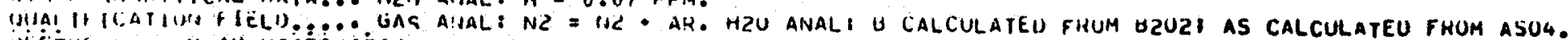

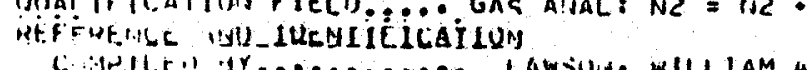

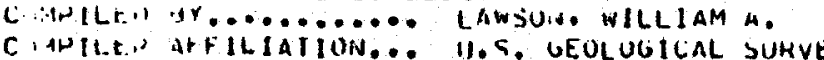

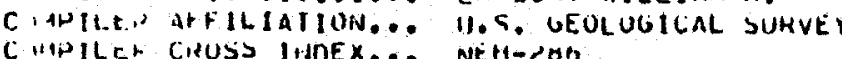

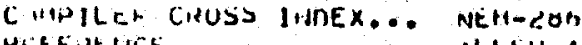

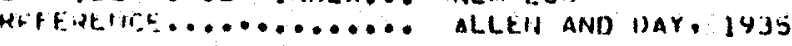

RECUKU 0U079

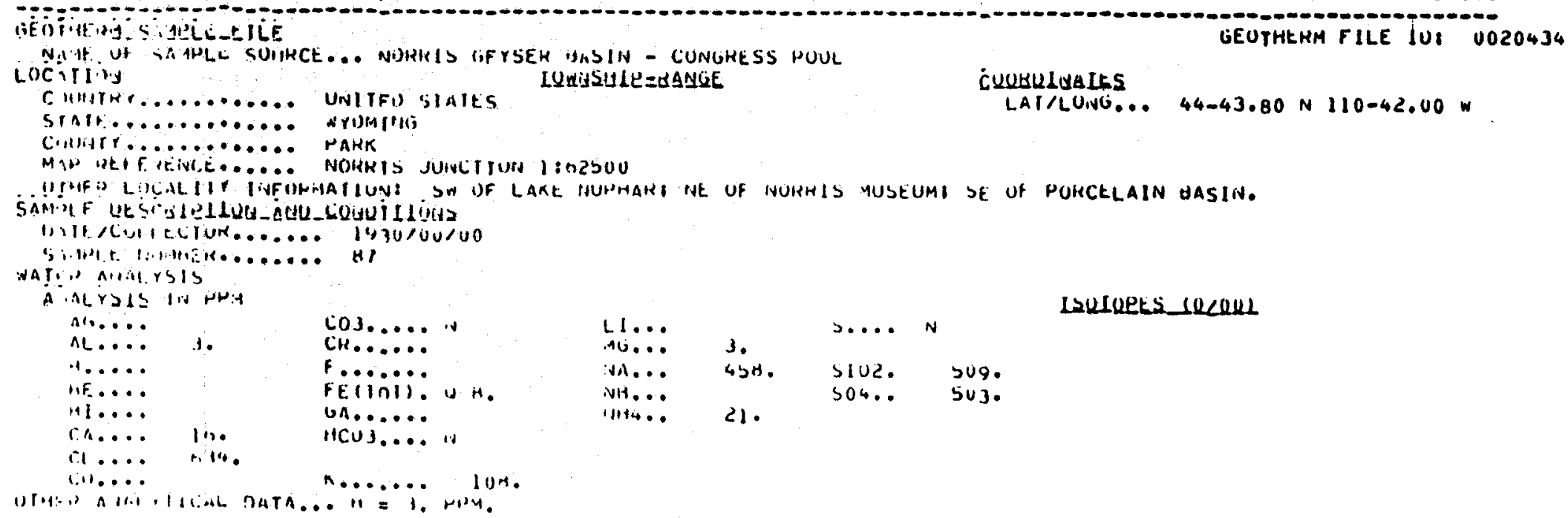




\section{C}

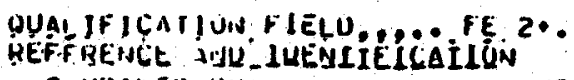

CIMPILEN HY.......... LAWSUN, WILLIAM A.

C MAPILEH AT FiliatiUn... M.S. UEOLMGICAL SUKVEY

RHFEREINCE............ ALLEN AND MAY, JYJS

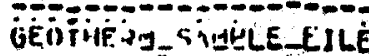

RECURL DOOBO

NAME UF SAMPLE SUIHCE... NUHKIS GEYSEH HASIN - CUHAL SPRING Lócirinis IQWNSHLP=BANLIE

C.HIITTH . .......... UNITEU STATES

STATE.......... WYUHINT

CIUNTY............ PARK

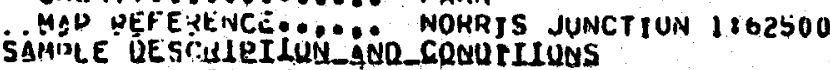

DNTE CCOL I.ECTUR....... 1900/00/00

SARIPLE N!MUEN....... VI

GAS. ANALYSIS

A JAi:YSIS IN VULUME \&

CHi. I

C.MA...

Cosi.

44

H2S.. N

N2.0 45.54

LSUIQRES CONCOL

GEUTHERM FILE IUI 0020421

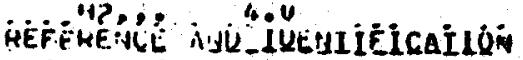

C:MPILEO WY .......... LAWSUN, WILLIAM A.

CIMPILEN AFFILIATIUN... II.S. GEOLUGICAL SURVEY

C.MMPILED CROSS INNEX... NEH-ZIH

RFFEHENCE,........... ALLEIN AND UAY, 1935

RECUHD OUOHI

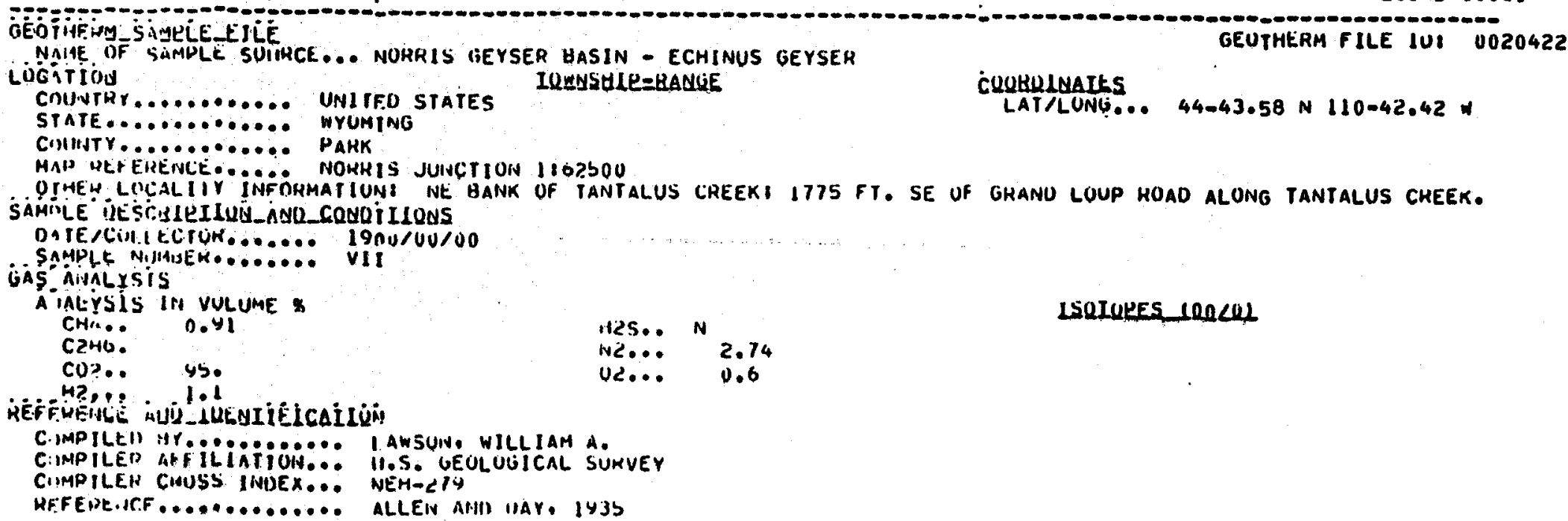

LSQLUEES CROCOL

2.74

$42 \ldots .0 .6$ 


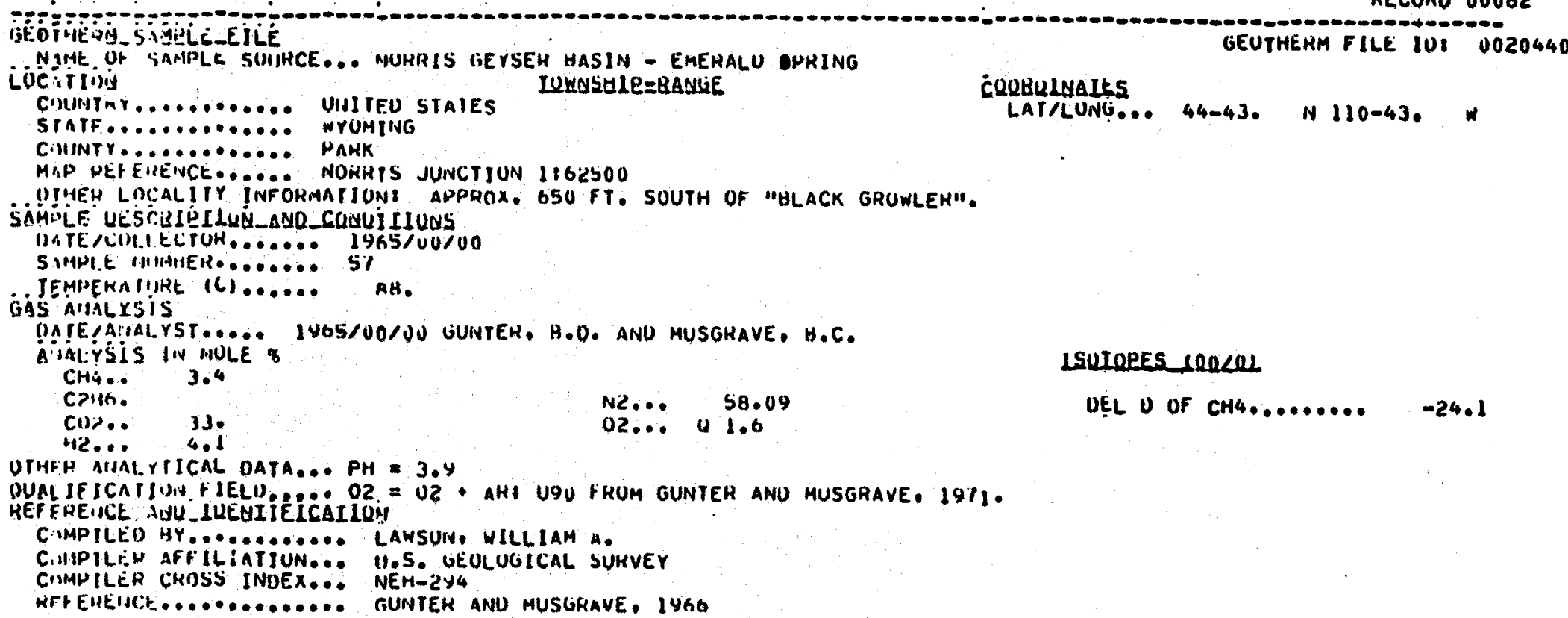

RECUKO 00083

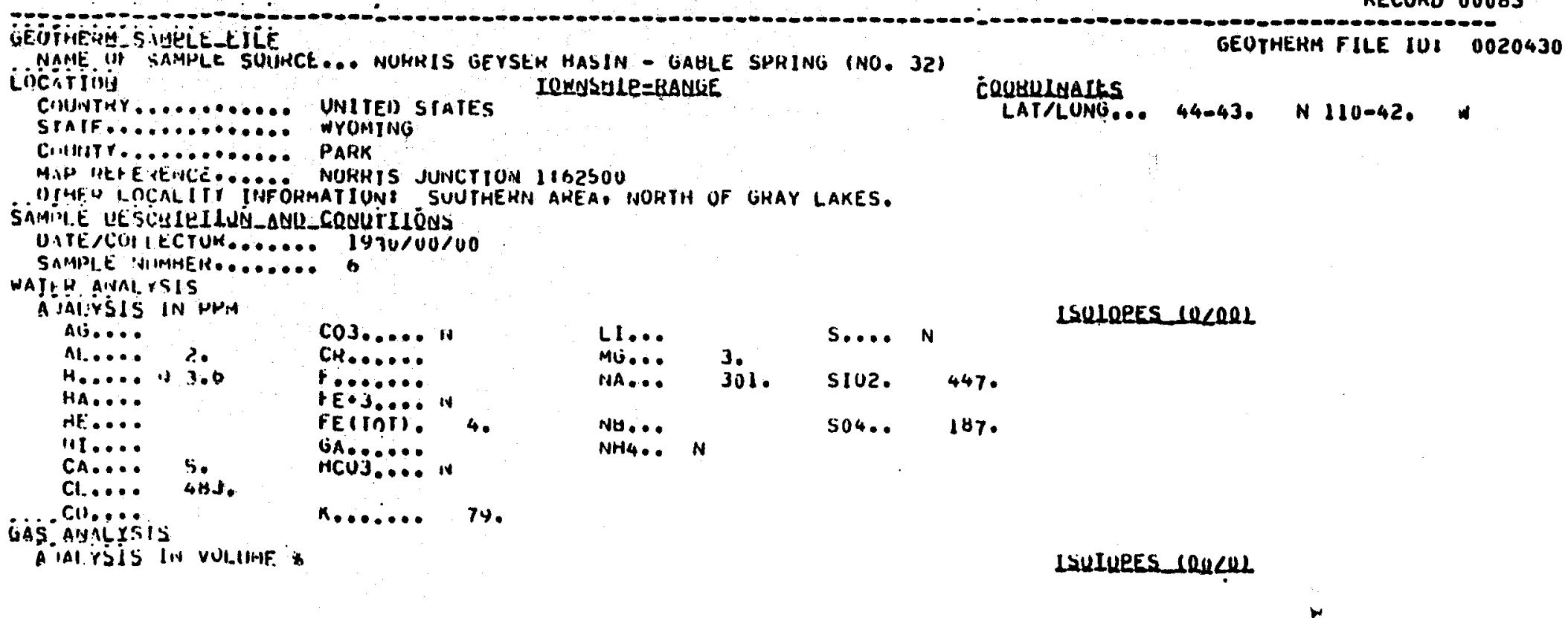

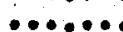

$B$ E. 3... in FE(III) 4 ACUj:-...

NG.. 301.

HE.

CA..... K....... 7y 

riti... 0.3

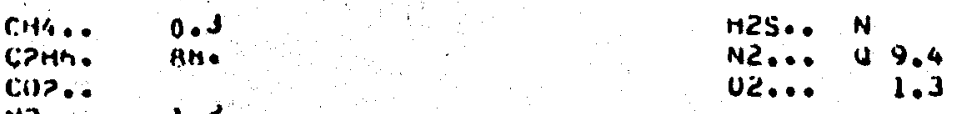
$\rightarrow 2.0 \quad 1.2$
U2.

OTHF' AUAT YTICAL DATA... MZO ANALI H $=1.4$ PPM.

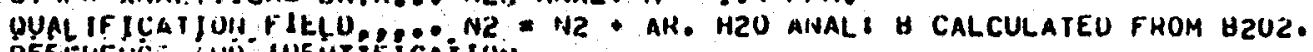

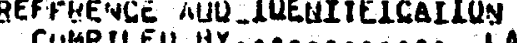

C.HAPILEU HY............ LAWSUIV. WILLIAM A.

C. MPILER AFFILIATIUN... II.S. DEOLOGICAL SUHVEY

C'MMPILFR CHISS INIDEX... NEH-ZUS

HFFEKEINCF.............. ALLEN AND UAY, JY35

RECUHO OOOB

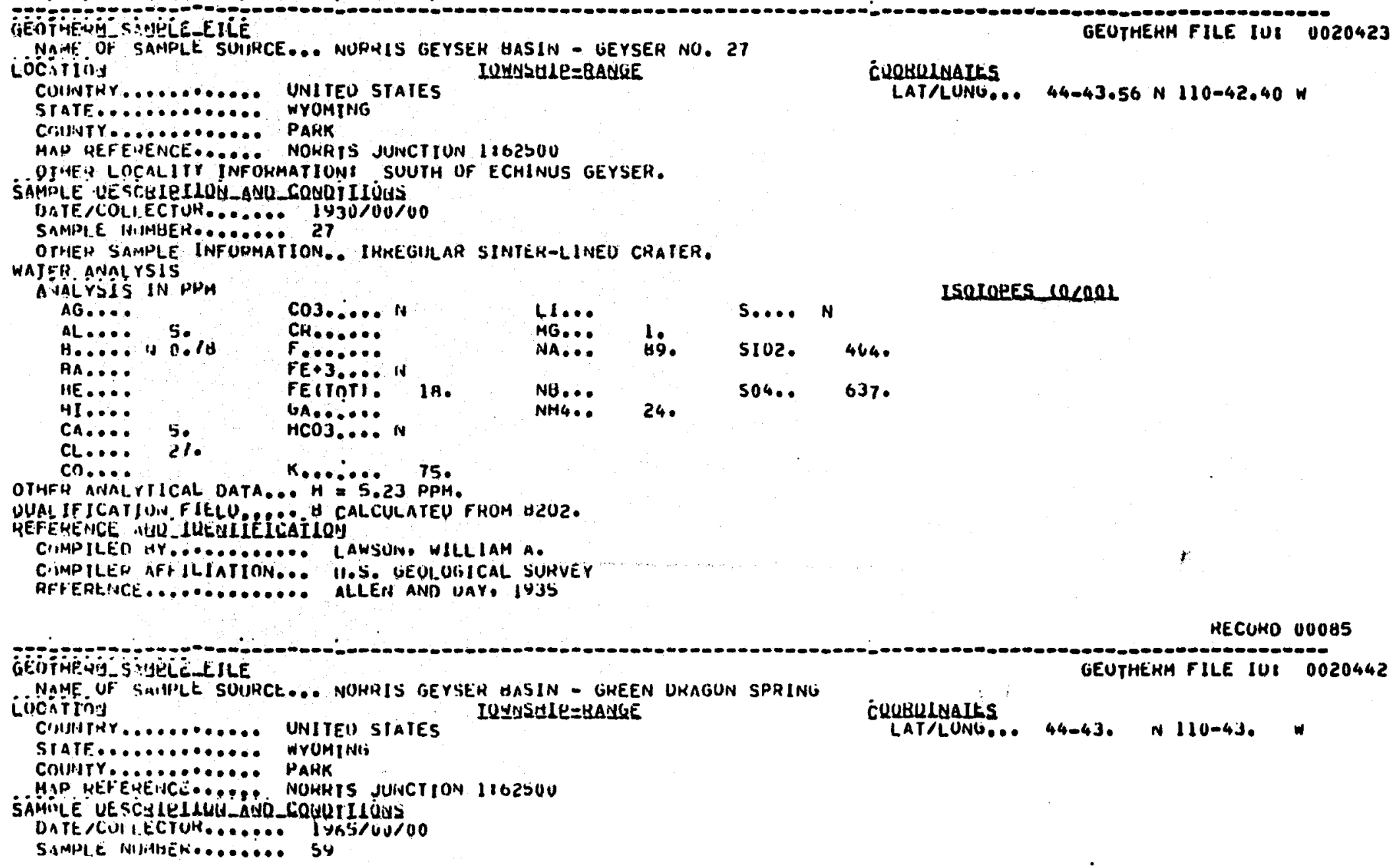


JEMPEMatume (C) ...... A4.

GïS AMinLSis

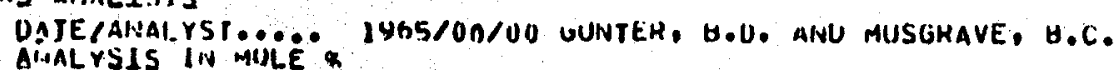

CHA.. IN MOA

C2H6.

44.

N2... 4.88

LSUIORES LeRCUI.

c12... I

OTHEH AIVAT.YTICAL UATA... PH $=3$.

gUA IF ICATIUU, FIELU

BEFFRENGE NULIUEQIEIEICAILUN

C.MMPILEU) UY ........ LAHSON, WILLIAM A.

C.MPPILEN ARF ILIATION... 11.5 . UEOLUGICAL SURVEY

CNAPPILER CHOSS INDEX... NEH-2Y6

RFFEHENCF.............. GLINTER ANI HUSGKAVE, 1966

.

HECUKD 00086

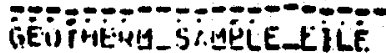

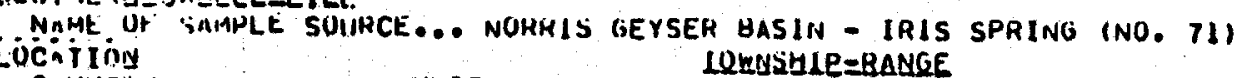

CIIINITEY . . ...... UNIIEU SIAIES LUESSHLE=RANGE

STATF............. WrUMIING

Conintr............. PARK

MAP REF EIEENCE...... NORRTS JUINCTION 1162300

OP!EE! LOCALIII jiNFURMAT ION? PURCELAIN UASIN.

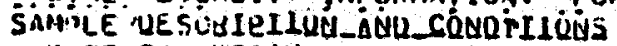

D.TE /CULIECTOK....... 1930/UU/00

WAJER ANALYSIS

A IAL YSSIS IN HPM

AG....

$\cos \ldots . . . \mathrm{N}$

AL..... $3,4.0$

CH......

HA....

HF....

chi....

FE.......

FETIOTI: iN 2

iAA.......

HCOj..... N

CL..... 56H.

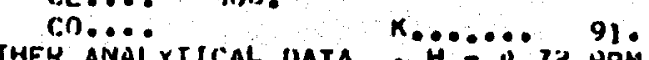

UTHER ANALYTICAL DATA... H = O.T2 PPM.

DUA IF ICATIUIN + IELU..... G CALCULATEU FRUM BZU.3.

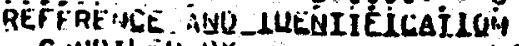

C MMP ILËI HY........... LAWSUN. HILLIAM a.

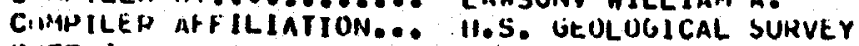

HFFEMEINEF............... ALLEN ANO WAY. lY3S

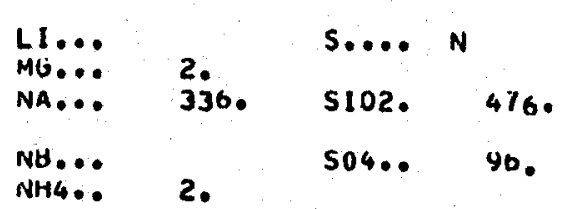

KECUHO 00087

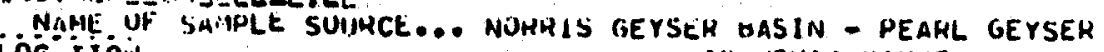
Lócirining

c.llinrer............ UNITEU SIATES IUENSHLE=HANLE

Stath

CININTY............ PAKK

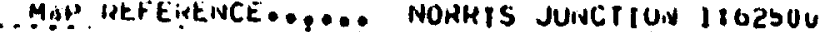

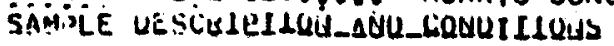


DATE/COI ECTCH.

SAMPLE NIHALER....... 5B

JEMPEKAT!IHE (C) ...... AS.

QÁs diyaítsis

OATE!ANALYST,...? 1965/00/00 GUNTEH, H.D. ANU MUSGHAVE, H.C.

CHit.. 0.00

CHin.

cor?.

Hi.

N2... $\quad 14.37$

LSOLORES CRaCWL

$12 . \cdots$

$02 \ldots 0.4 .4$

OTHEH AIVALYIICAL DATA... PH $=6.5$.

gUAL JF ICA IJUN FlELU P OZ OZ 02 - AR.

REF FHENCE AUL_IKENIIÉCCAILO'N

CIMP ILED UY......... LAWSUN, WILLIAM A.

CIMPILER AFFILIAIION... II.S. GEULUGICAL SUKVEY

C.IMPILEH CHOSS INDEX... NEH-295

RECOKO 00088

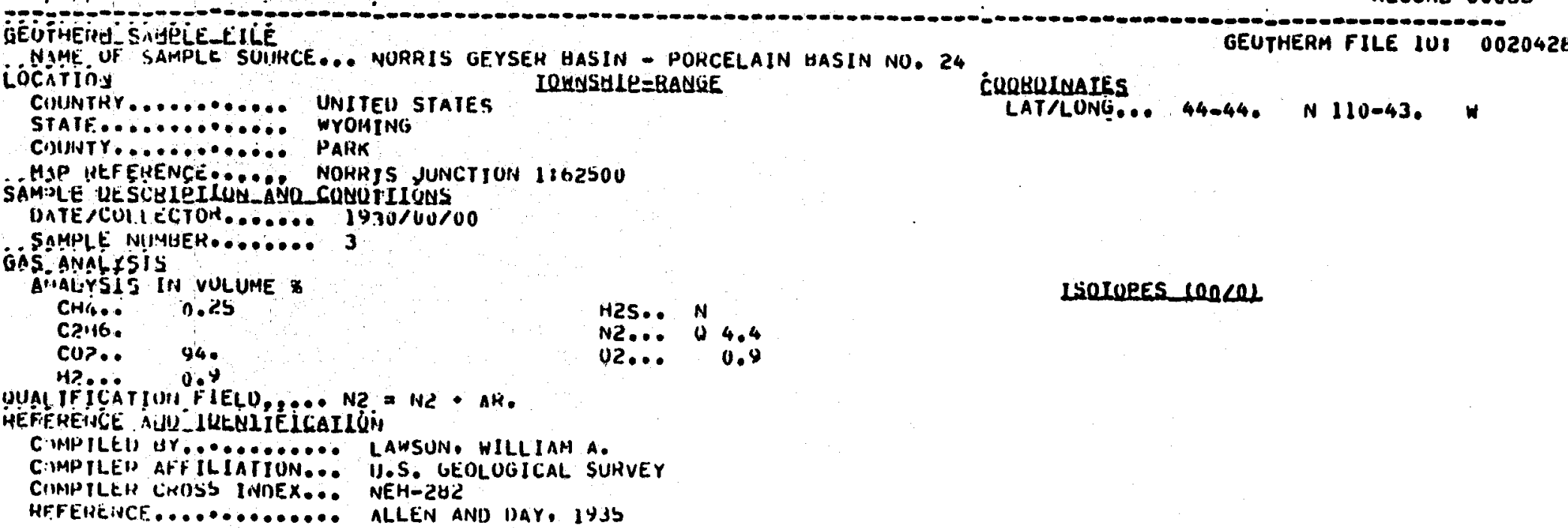
WOMEE UF SAMPLE SUIIKCE... NORRIS GEYSEH HASIN - PORCELAIN BASIN NO. 24
IOWNSHLE=RANGE

STATR............ WYOAING

CIIINTY............ PARK

MSP HEFEHENCE. HO... NOHKJS JUNCTION 1862500

SAM:JE UESCBIEILUA-ANO_COUUTILN

DNTE/CUILCTOH....... 19.30/U0/00

SAMPLE NUMUER_..... 3

GAS.ANALFSIS

AiALYSIS IN VULUME $\$$

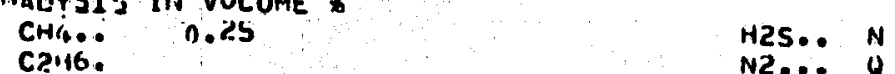

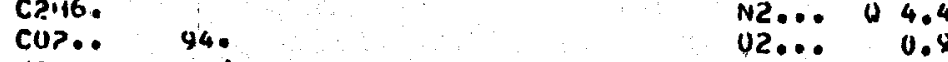

LOLIURES $100 \angle 0)$

GEUTHERM FILE IU: 0020428

CRQBLLNATES

LAT/LONG... 44-44. N 110-43. N

JUAL IFICATIUH FIELD,

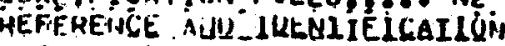

C MMPILEU UY ........... LAWSUN, WILLIAH A.

C:MMPILE" AFFILIATIUN... W.S. GEOLOGICAL SURVEY

CINAPILEH CHOSS INNEX... NEH-ZYZ

HFFEIHEINCF........... ALLEN AND IJAY, 1YJS

RECUKO OOOHO

GEUTHEKM FILE IU: 0020427

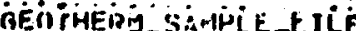

N.WHE IIF SMNIPLE SOINCE... NURKIS GEYSER HASIIS - POHCELAIN HASIN NU. 70

Lócirions

LUWASHLE=HAQGE

Conintar........... UnITE.) STATF

STATF ............ WrUMtNe

CIIINTY

MORE JUNCTIUN 186250u

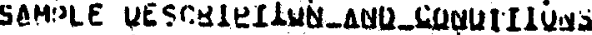

UATE CULI ECTUR....... ICTO/UU/JO

WATris AIHAI rís 
CHARGE I:AHALAINCE (q OIFF):.. U. ini.XSIS IN $\mathrm{MPM}$

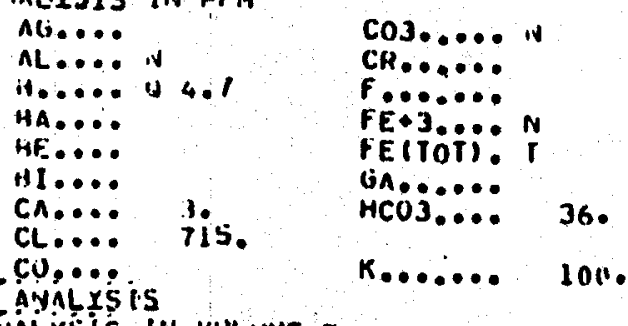

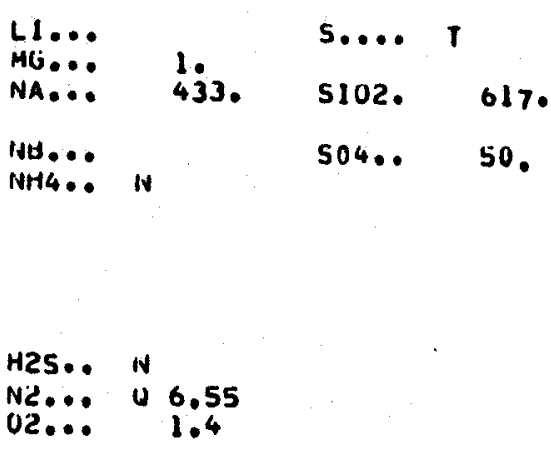

LSULOES COCURL

Aivai ÝS IN

CHA VULUME $\%$

C2Hi.

CO?.

BY.
2.3

N2... 6.55

-

UTHF. ANAL YTICAL DATA 0 CO

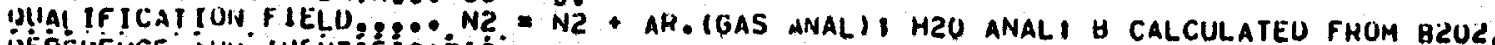

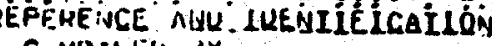

C.MWILEIS AY ......... LAWSUN, WILLIAM A.

CiMPILEH ATFILIATION... H.S. GEOLOGICAL SUHVEY

CIMPILEK CHOSS IHUEX... NEH-28

HFFEREINCE $\ldots \ldots \ldots \ldots$ ALEN AND UAY, IYJS

RECURD 00090

GEOTHERMSTHELEALE

LSULOES LOCOLL

NAME UF SAMPLE SUIIHCE... NORHIS GEYSEH HASIN - SPRING NU. 1

iocitiving

C'NINTHY

SIATF 00000000000 UYUMTUE STARES

C.UIVTY

MAN HET E: ENCE.

OPHEH LICALITY INFURMATIONI EASTEKN EUGE OF HASIN IN SOUTH SECTION.

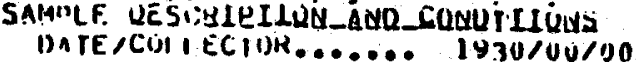

OTHE.L SAIAPLE INF ORMATION.. LAKUE MUIS SPRIING.

WAJER. ANAL RSIS

Aialiysis IN ppit

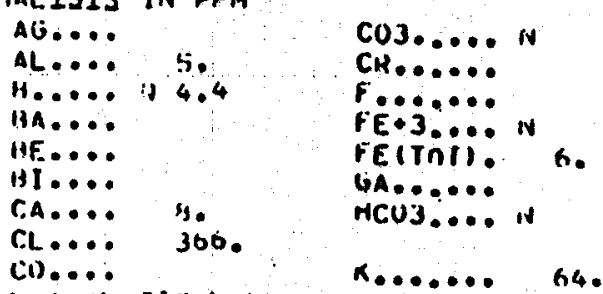

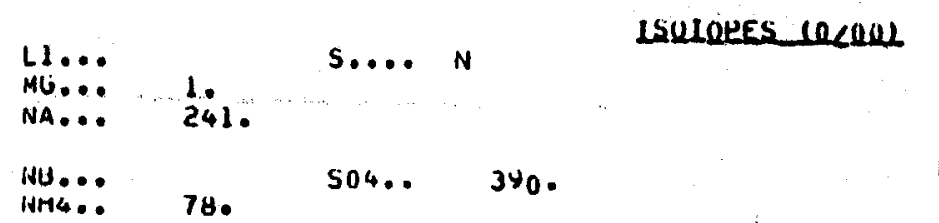

GEUTHEKM FILE IO: 0020403

CRoHULALIES

LAT/LUNG... 44-43. N 110-42, *

OTHW ANGALPTICAL IMATA... $11=0.04$ HPM.

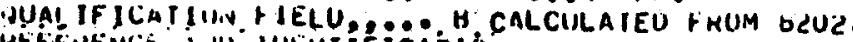

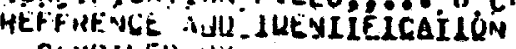

C'MHPILEU ISY............ l.AMSUIV, WILLIAM A.

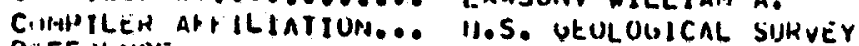

RFFERENCE.............. MLLEIN ANI IIAY, IYJS 


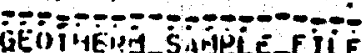

ZUOAAIE UF SAMPLE SOIIKCE... AIURRIS GEYSER BASIN - SPHING NU. IS Cocisions

C.HINTRY............ UNITEOS STATES LULUSHIR=RANGE

STATE............ WYUMTING

CINUNTY.......... PAHK

MAN REF ERENCE..... NORHIS JUNCTION 1862500

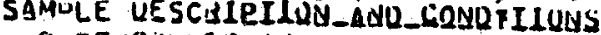

OITE/CUL.LECTOK....... 193.J/00/UO

WAIER ANAI.YSIS

A InL YSIS LIN PPM

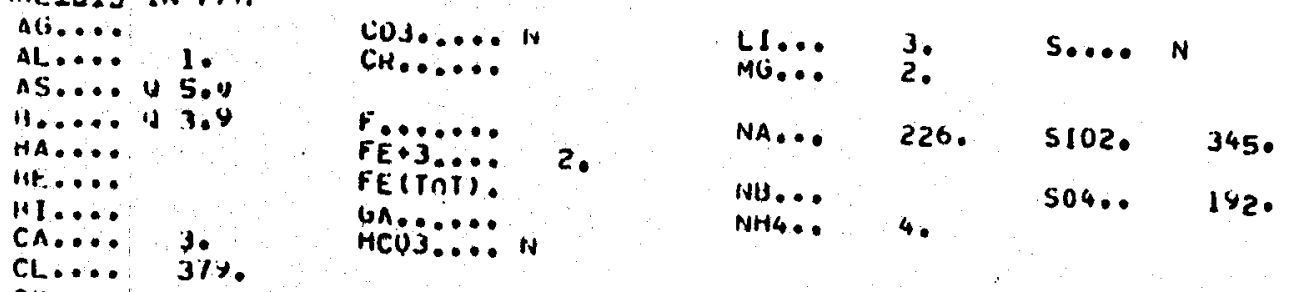

CL.... 3\%. 3\%.

THER MiNCi Y YICAL DATA K...... 77

PPAI FE 20 = 2. PPM.

BEFFEENCL

COMPILEO UY.

CIMP

RFFEREINCF............... ALLEN ANIO DAY, IYJS

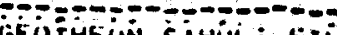

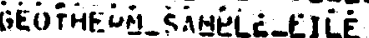

OOCAME UH SAMPLE SOIHCE

Locarting

CIUINTKY..............

STATE................

CinUivtr..............

SAMIDE UE SENCE...... NOHRIS JUNCTION 1862300

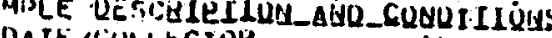

HA IEV AVAL YSIS

A IAL XSiSS IN PNA

UTHE

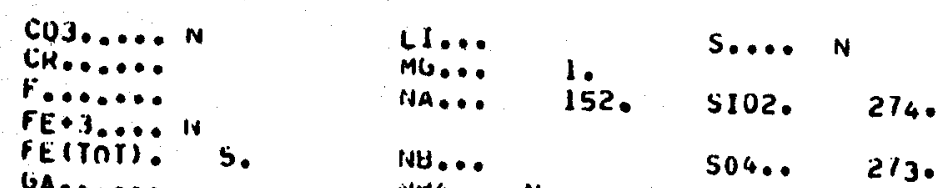

V.JS MPM. ASUZ $=0$.

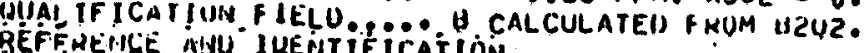

RECUKD 00091

GEUTHERM FILE IU: 0020409

\section{CQuBuivaIts}

LATLUNGE... 44-43. N 110-42, N

LSULOPÉS_ULCQR

RECUHD 00092

GEUTHEHM FILE IU: 0020410

\section{¿UquULMaIts}

LATLONG... 44-43. N $110-42$, W

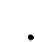

\section{ISULORES COCOOL}


CIMPILE⿰ HY........... LAWSUN, WILLIAM A.

CIMPILE: AFFILIAIIUN... U.S. UEOLUGICAL SUIRVEY

HFFERERSE............. ALLEIN AND DAY, 1935

RECUHD 00093

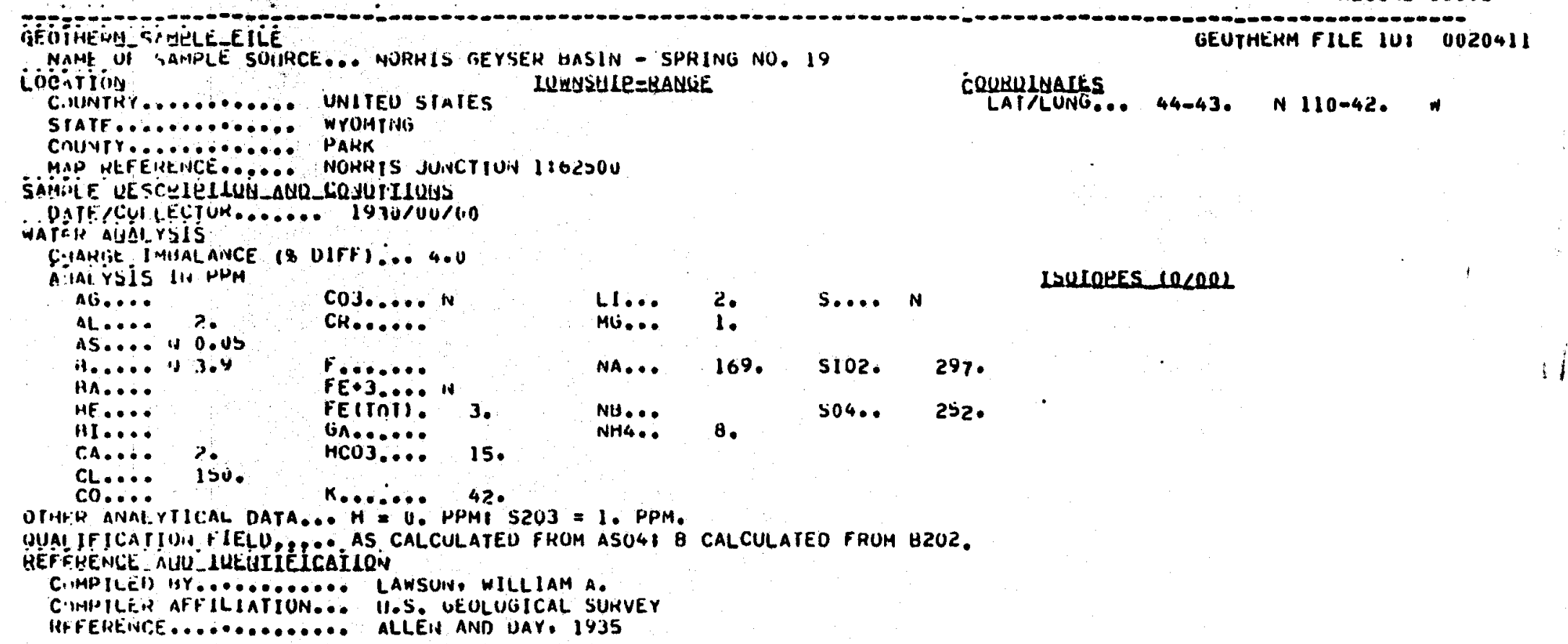

RECOHU 00094

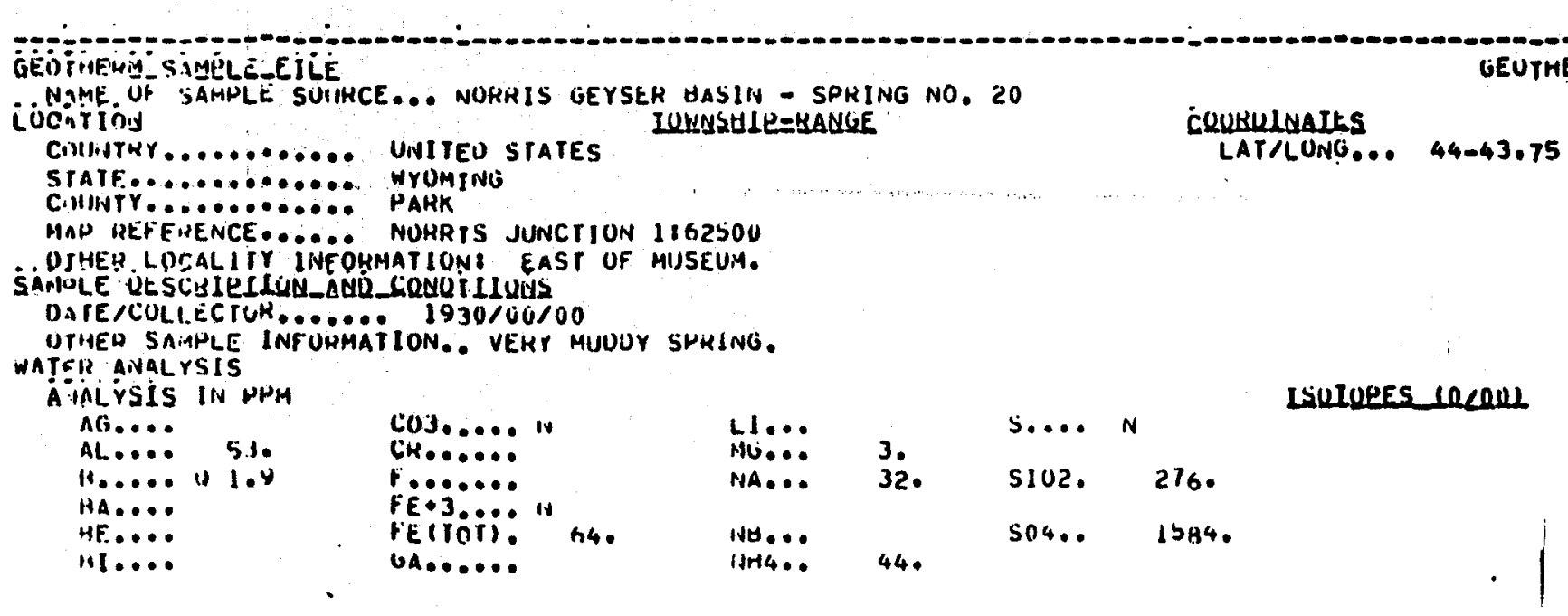




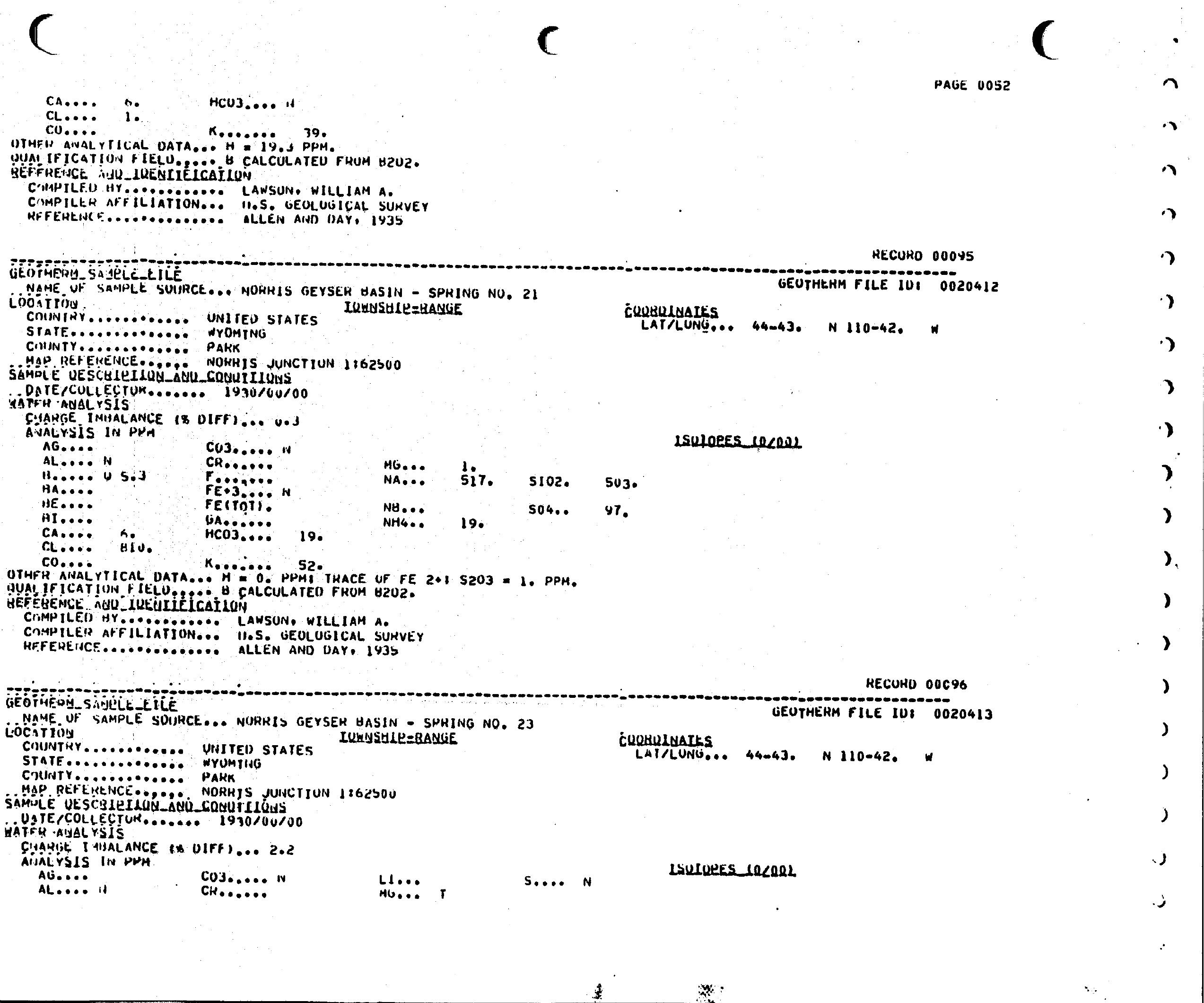




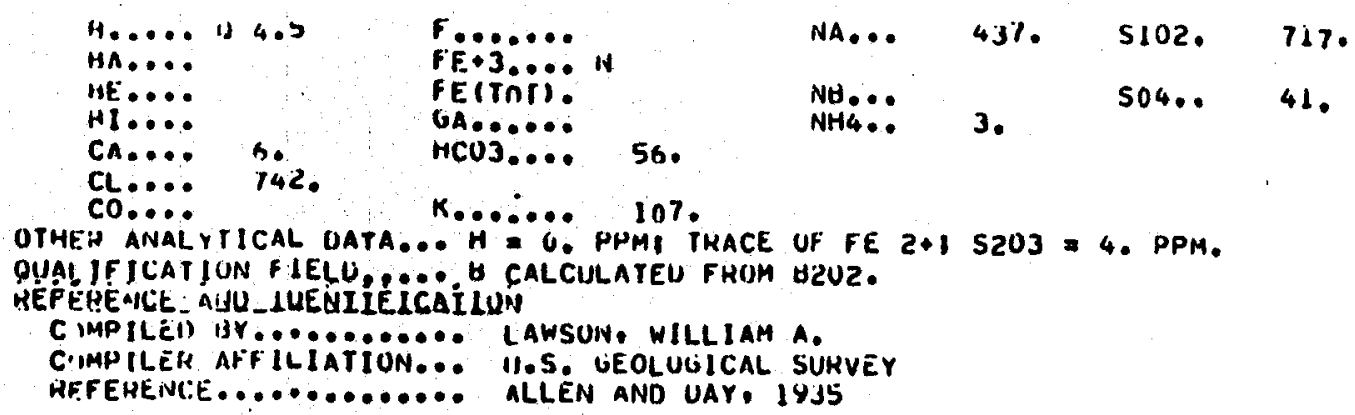

RECUKU 00097

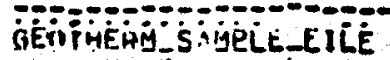

NAME UF SAMPLE SOURCE... NURRIS GEYSER HASIN - SPKING NO. 25 Locitivis

IUHUSHLE=BALLE

STATE............ HYMTNG

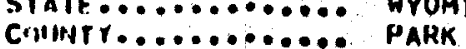

MAP HEFEMENCE.?. PARK

OPHEM LOCALITY INFORMATION: PURCELAIN BASIN NEAL CONGRESS POOL.

SAMISLE HESCQLPILU_ÄUD_CQUUTIIUUS

UATE,CUILECTUH........ IY.3U/UÜ

SAMPIE NIITUEK.........

WAIER AIHAL YSIS

A IAL YSIS IN PNM
AG.....
AL.... N
$11 . \ldots .$.
CA.
CL...... Y Y Y
A UALYYSis IN VULIIME $*$
CHA.. O.

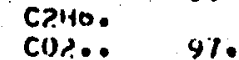
Hz....

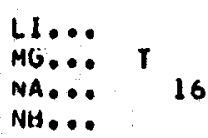

GÄS ANALÝSTIS

ILE IU

GEUTHERM FILE IU: 0020436

GQUBULNAILS

LAT/LUNG... 44-43.81 N 110-42.03 *

OTHFK AIVAL YTICAL DATA ... MZO ANAL: H $=20$ HPH.

gUAL IFICAT!UIN F IELU, ,.. N2 = N2 * AH.

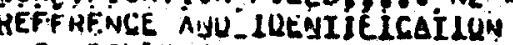

C MMP ILEI) BY.......... LAWSUIV, WILLIAM A.

CIMPILER AFFILIATION... M.S. GEOLUGICAL SURVEY

CIIMPILER CHUSS INDEX... NEH-ZO

HFFEREIHCE............... ALLEN AND UAY, IYJS

RECURO 00098

GËTOTFCASS SMELE_EILE

NAME UF SMADLE SOIIHCË... VUHRIS GEYSE.H BASIN - SPKING NU. 30 consititis.

UNITEU STAIES
IUUUSHLE= HAHLE:

GEUTHEHM FILE JUI 0020414

CUUGULAaIts

LATRUNU.... 44-43. N 110-42. 
STATE.

C.UINTTY Y........... PARK

MAP REFEUEINCE..... NORHIS JUNCTION 1162500

OIHË LOCALIIP IMFOHHATION EAST EOGE OF BASIN, SOUTH OF COAAL SPHING.

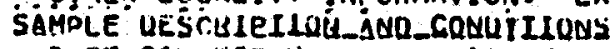

OATE/COI.IECTUK.... 1970/00/00

ORHE'H SAIIPLE INFORMATION.. LARGE DEEP POUL.

WATEA ANALYSIS

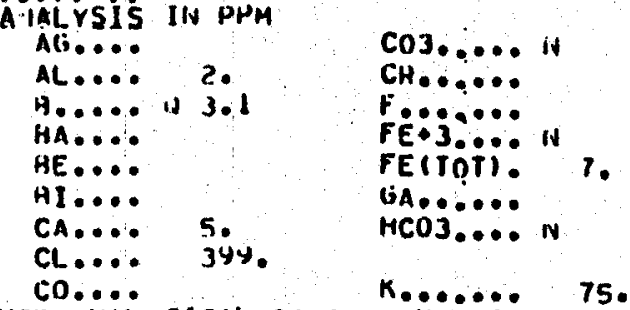

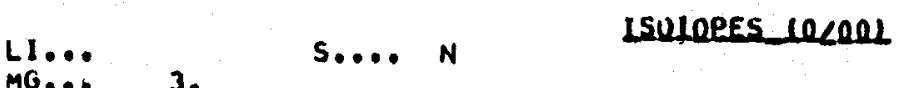

LI... S...

NA... 270. SI02. 406.

So4.. 375.

NH...

NH4.:

9.

CO

JUAL IF ICAT!UN FJELU, ... B CALCULATEU FHUM HZUZ. BEPE HENCE AUU_IUENIIELLILION

CNMH ILEO HY.......... LAWSUN, WILLIAM A.

CIMPILER AFF ILIATION... II.S. GEOLOGICAL SUKVEY

HFFERENCE............. MLLEIY AND UAY, IYJS

RECORO 00099

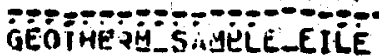

GEOTHEKM FILE IU: 0020415

Lócíitio

STATE. CoUTr.

- SPRING NO. 33

STATE............ WYOMING

CIJUNT Y............ PAKK

. MAE REFEPEIVCE...... NORRIS JUINCTIUN 1862500

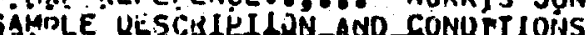

DATE./COL.LCTUH...... 1930/00/00

WATEH AIVALYSIS

AINAL YSIŚ IN HPH

$\cos 30.000$

LI... $\quad$ 2. S... N N

CquQuluarts

LAT/LONG... 44-43, N 110-42, N

A..... \& 0.40

Ch.......

H...... I1 1.2

HA.....

fon.jo.

48. SIU2. 303

LSUIOEES ceCal

\section{N 2100420}

IIE. $\cdots$.

CA.

FE+3Tïi: H

CA. … 4, 4.

IA

NG...

S04.. 1105.

CL.....

$\mathrm{HCO}_{3} . . \mathrm{N}$

NH4..

342.

CTHFH ANAL

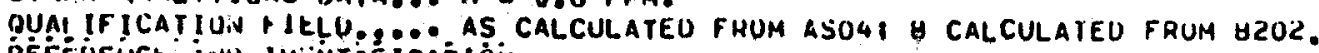

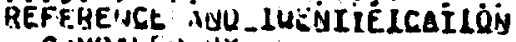

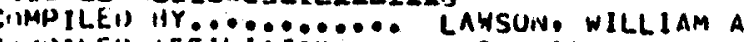

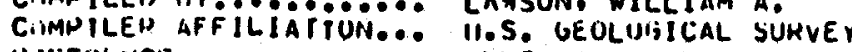

HFHEHENCE............... ALLE AN AN JAY, IYSS 


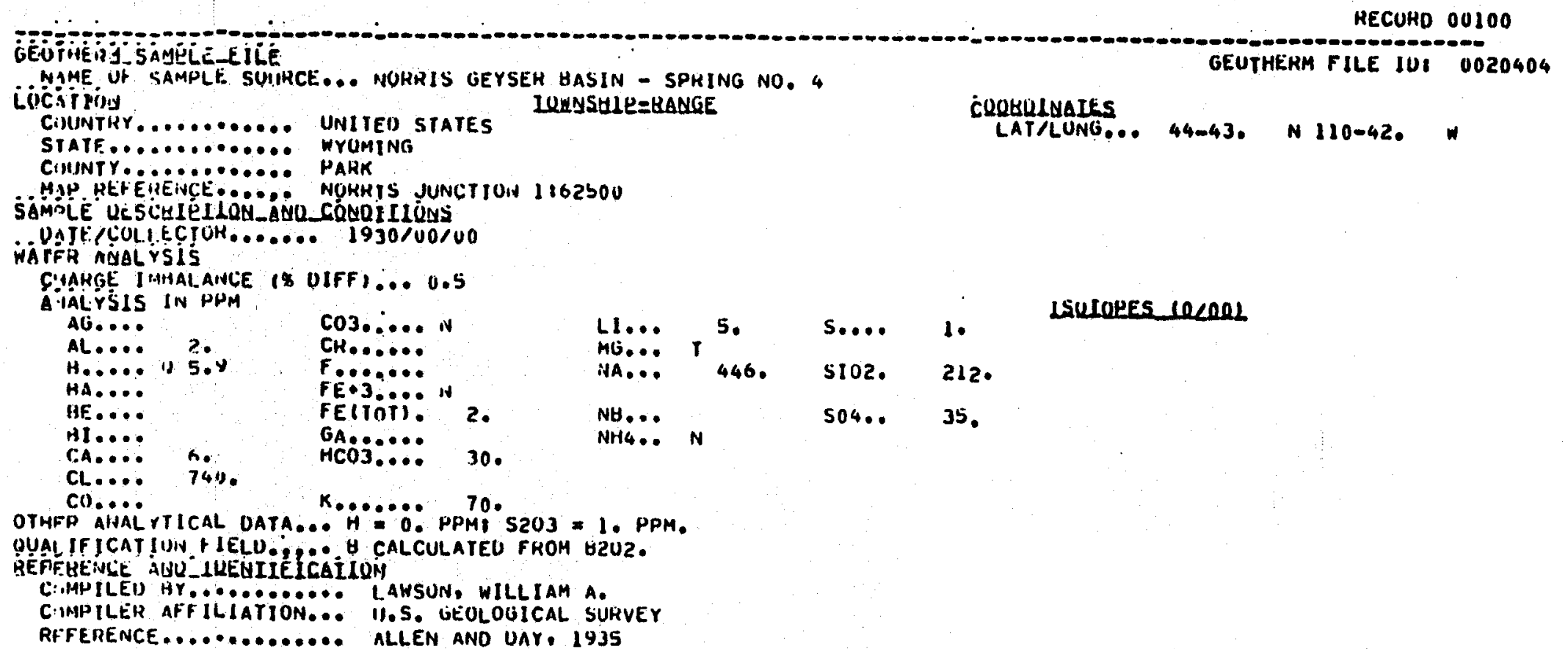

RECURO 00101

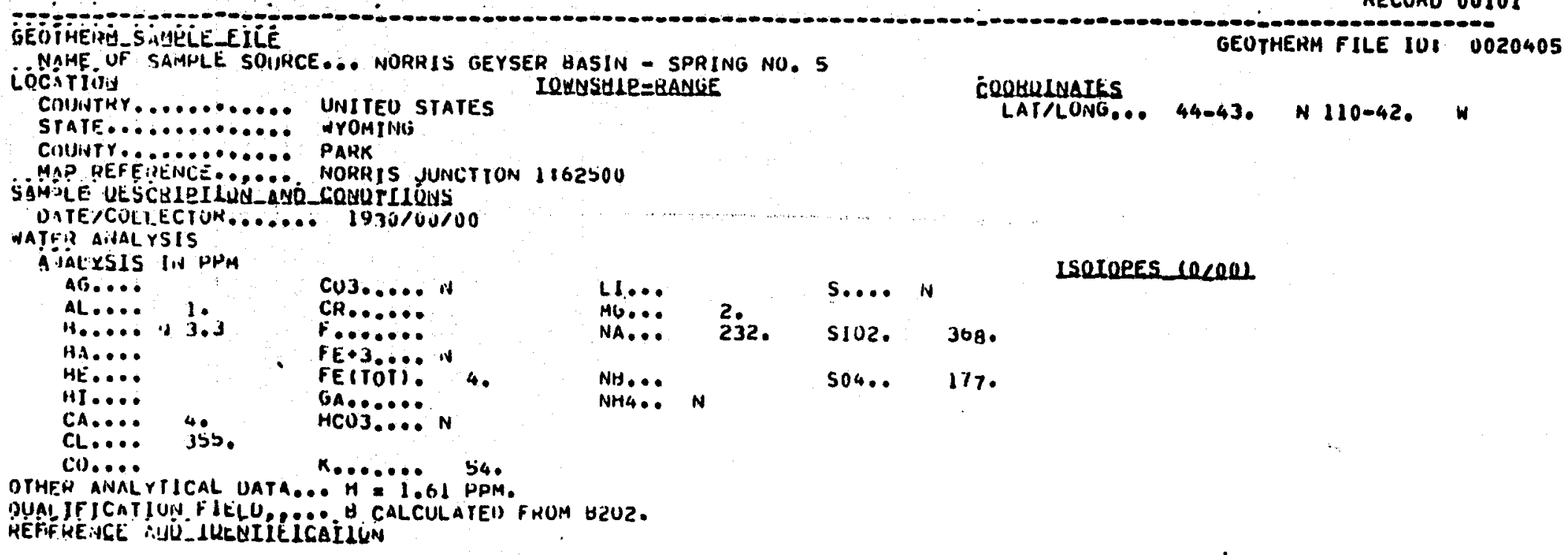


C.IMHILEII HY.......... LAWSON. WILLIAM A.

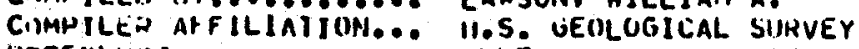

RFFEREINCE.............. ALLEN AND UAY, IY3S

RECUHU 00102

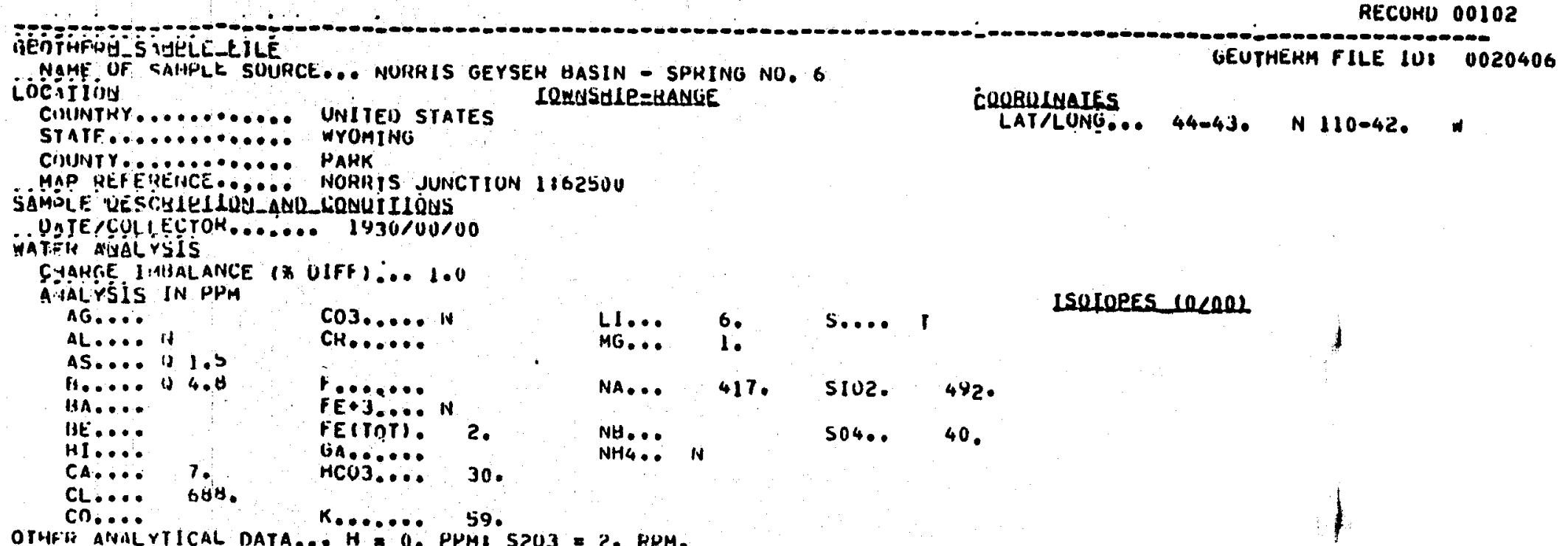

RECOKO 00103

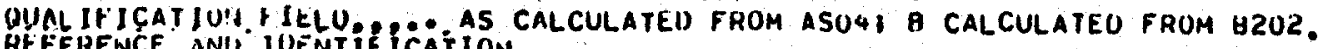
Comp ILE HY

BY........... LAWSUN, WILLIAM A.

CIIAPILEL AFFILIATION... 11.5. GEOLOGICAL SURVEY

RFFEIRENCE........... ALLEN AND DAY, 1935

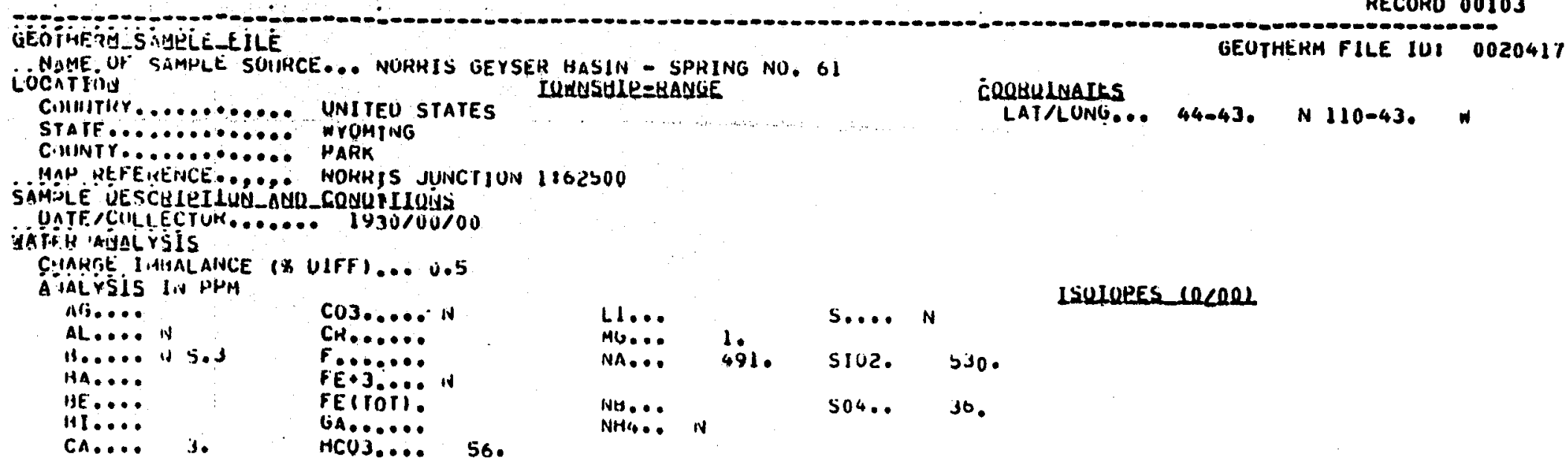

lakre liatialaluce (क UIfF)... u.5

NHA, N

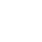


CL... Ho Ho

Co.... K..... 101.

OTHFL AINALYIICAL DATA... H $=0$. PPHI IHACE UF FE $2015203=4$. PPM.

UIUI IF ICAT! UN FIELLU... \& CALCULATEO FRUM BZOZ.

HEFFRENCE AUU_LLEIIELCAILÚN

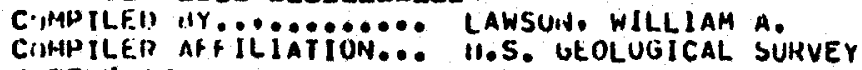

MFFEREINCF............... ALEN ANO UAY, 1935

RECUHD 00104

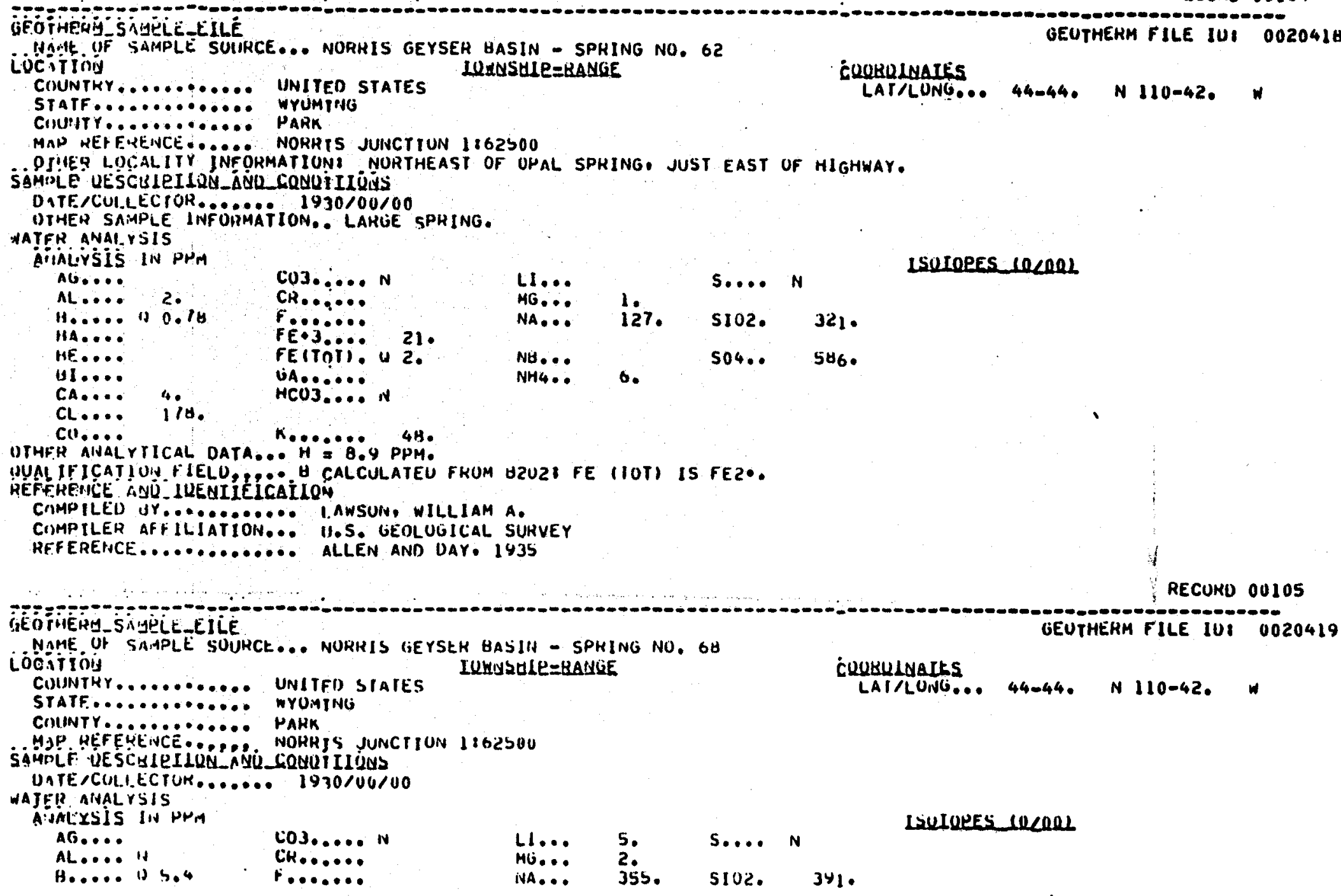

JUA! IFICATIUN FIELU, H CALCULATEU FKUM GZUZI FE IOTI IS FEZ•.

CIMPILED UY......... I. AWSUIN, WILLIAM A.

CIMPILER AFFILIATION... H.S. GEOLUGICAL SUHVEY

HFFEREHCE............ ALLEN AND DAY, 1935

IVA... 35S. SI02. 341. 


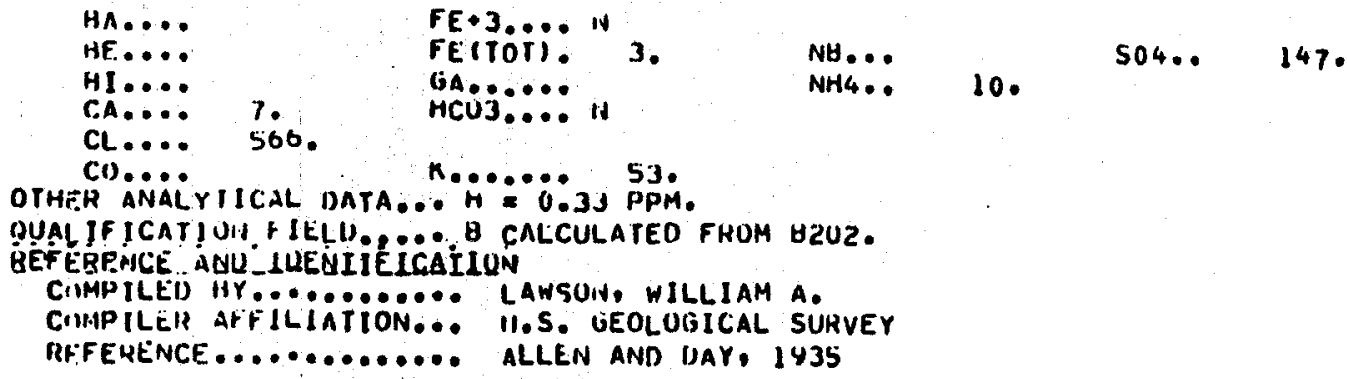

$\boldsymbol{\omega}$

RFFEREINCE............. ALLEN AND UAY, IYJS

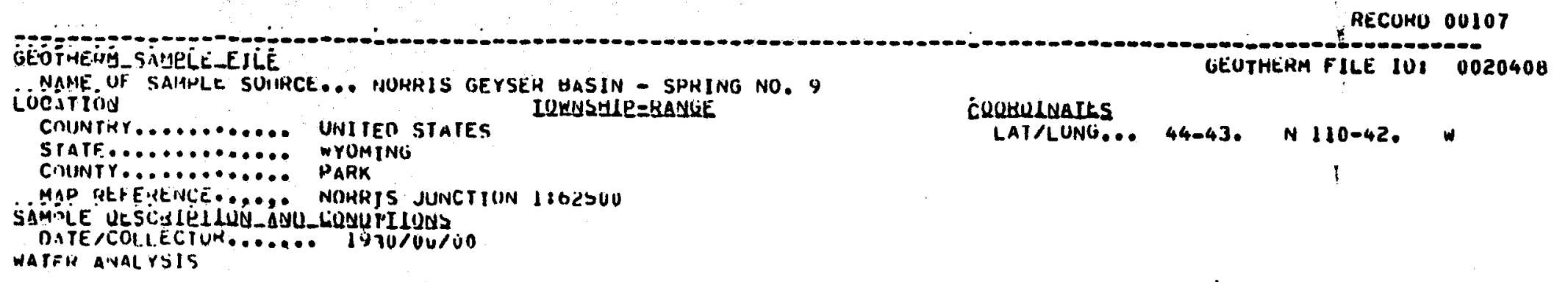


MaP REFERENCE..... NOHRIS JUNCTION 1862300

OSHEH LOCALITY INFORMATION? $2 M$ NOHTH OF VERMILLION POOL.

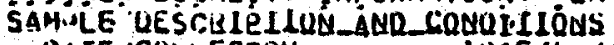

OATE/COLI.ECTOH....... 1965/60/00

SIMPLE WIIHAERO...... 48

JEHPEHATUHE (C)..... 79.

GAS ANALYSIS

DATE,GNALYST..... 1965/00/00 GUNTER, B.U. ANU MUSGRAVE. B.C.

a TALTSIS IN MULE

CHi 0.12

COH6. 93.

U2... U 0.85

OUAR ANALYIICAL UATA... PH $=2.3$.

MUAL IF ICAI JUP FIELU, , $02=02$ - AR.

C.NENCE, ANU LLEUIREICAILON

CIMPILEN $\triangle Y . . \ldots \ldots \ldots$ LAWSUN, WILLIAM A....

C.MPILER AFFILIATION... HES GEOLOGICAL SURVEY
CIMMPILER CHOSS INDEX... NEH-2UB

RH:FEHENCE............. GUNTER ANU MUSGRAVE, 1966

ISOTOPES COREOL

KECUHD OOI1O

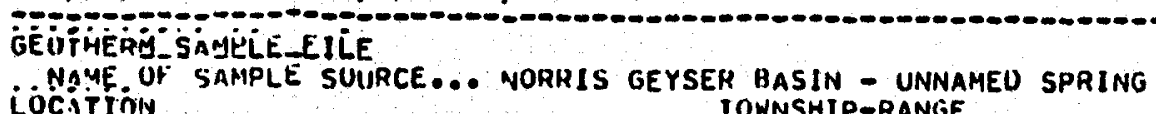

UNITFO STATES LUWWHIR=BANGE

STATE

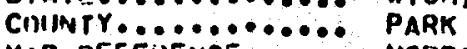

MAP REFERENCE..... NORRTS JUNCTION 1162500

O IHEQ LOCALITY INFORMATIONI IUM SOUIH OF HATHTUY SPRING

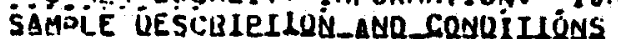

DA TE, COLLECTUK...... 1965/00/00

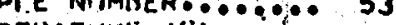

00.6

GAS ANAĹYSIS

DITE ANALYST.... 1965/00/00 UUNTER, B.U. ANO MUSGHAVE, B.C.

ANALYSIS IN MULE 9

CH4.. 0.05

C.?H6.

N2... 4.46

CO5.: 940

GEOTHËRM FILE IUI 0020439

\section{CQQRQLUATES}

LAT/LUNG... 44-43. N 110-43. W

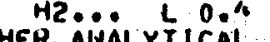

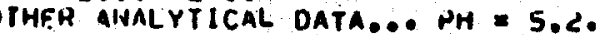

OUAL IF ICAT I UIN F IELU, OO OZ = OZ * AH.

REFFAENCE INUL_LLELIÉLCAILON

CIMPILEU HY.......... LAWSUIN, WILLIAM A.

C.IMPILER AFFILIATION... N.S. WEULUGICAL SURVEY

CIMPILER CHOSS IINISX... NEH-2У2

RFFERENCE............. GINTER ANI) MUSGHAVE, 1966

ISUIORES COOCOI

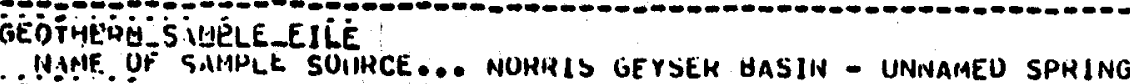

RECUHO .00111 Liocitiviog UNI TEI STATES

IUUNSULE=BANGE

GEUTHEKM FILE IU: 0020438

CQRQRULNAIES

LAT/LUNG... 44-43. N 110-43. N 
STATF

COINTY MAPE HEFENCE... PAKK

NORHIS JUINCTION 1862SOU.

OIHE!! LOÇALITY IWFOHMATIONI SM NORTHWEST OF HATHTUB SPRING.

SAMMLE UESOHLILQU_AQU_CQUUTILOUS

DNTE/COLIECTUH....... 1965/00/00

SAMPLE W: IMHEH...... 52

TFMPERATURE (C)..... 92.

Gís ANALYS I.S

DATEAANALYST M... I9G5/ON/OO GUNTER. B.D. ANO MUSGRAVE, B.C. CH4.: 0.06

Cil?.: 96.

H2... L 0.4

OTHFH ANAL YTICAL DATA... PH $=6$.

DUAL IF ICATIUIV FIELO, \&O $02=02 \bullet A H$.

REFEMENGE TUL LUEUIIEILAILOON

CIMPILEO OY............ LAWSUN, WILLIAH A.

CMMPILER AFFïIATiON... II.S. GEOLOGICAL SUHVEY

CMIPILER CHUSS INNEX... NEH-2YI

$\begin{array}{lll}N 2 & 3.18 \\ 02 \ldots 0 & 0.31\end{array}$

LSOTOEES CONCOL

HFFE QEINCE............. GUNTEK ANO MUSGRAVE, 1966

RECURO 00112

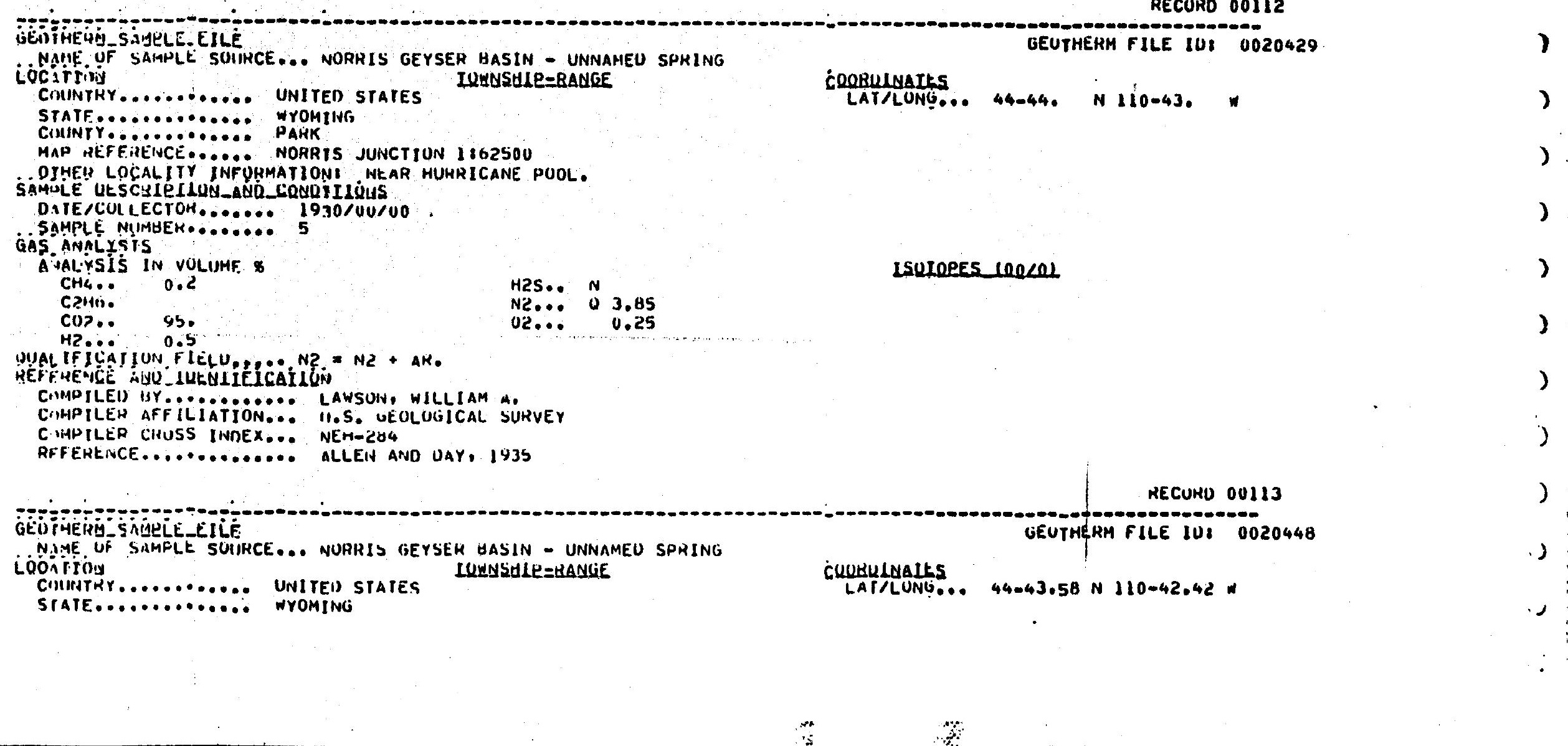


C')UINTY

PAKK

MAP REFEIREINCE... NORKIS JUIVCTION 1862500

OIHEQ LOGALITY INFORMATION OUM SOUTH UF FUOTBRIDGE ON WEST SIUE OF TRAIL PAST ECHINUS GEYSEH.

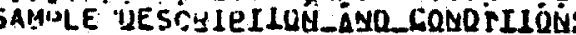

IIATE,COLI.tCTOK....... $1965 / 00100$

SAMPLE NMHAER

UIHE!? SAMIPE INFORMATION. COLLUIDAL SULFUR POOL.

Lias ANALYSIS

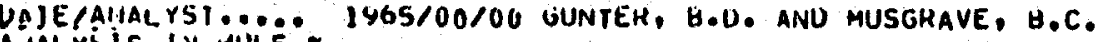

A IALYSIS IN IULE

AN $2.0 \quad 0.03$

CHili.: N

Crilo. O.

co?

98.

N2... 2.04

ISULORES IOOCOL.

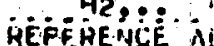

UUL_LENIIÉLCAILÕN

C MPILEU HY............ LAWSUN, WILLIAM A.

C IMPILED AFFILIATION... H.S. GEULUGICAL SURVEY

CIMPILEH CHOSS IHDEX... NEH-JUO

REFEHENCE ............ GUNTEK ANO MUSGRAVE, 1966

2.04

RECOHO OO114

GEOFHE ST SAMPLE SUUHCE... NORRIS GEYSEK HASIN - UNINAMEU SPRING

Gón

IUYASHIE $=$ BANGE

COINHTR............ UNITEU STATES

43.58 N $110-42.42$ N

STATE............. WrUIATNG

CIIINTY............. PARK

MAP HEFERENCE...... YELLOWSTUNE NATIUNAL PARK $1: 125000$

PJHË LOCALIIT INF ORMATION ZUM EAST OF ECHINUS GEYSER.

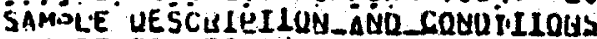

DATE,CULI. ETUK....... 1965/U0100

SAMPLE NIMAUEH....... 66

TFMPEKATIJKE (C)

OTHEI SAMPLE INFORMATION YELLUW POOL.

GAS ANALYSIS

DAJE/A'VAI, YST..... 1965/00/00 GUNTER, H.O. ANU MUSGKAVE, H.C.

A:IAT:YSIS IN MULE

AH... T

0.06

C?H6.

C1)?. 94.

H) 20.4

CQRULNAIES

ATILONG.

$44-43 \cdot 58$

$110-42.42$

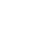

IYIEK AIVALYTICAL OATA... PII $=2.4$.

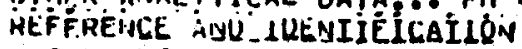

C.MMPILED OY ......... I.AWSUN, WILLIAM A.

CIMPP ILER AFFiliäion...

CIMPP ILER CRUSS INDEX... NËH-ZYY

HFFEREHCE............. GIUNTEK ANU MUSGHAVE, 1966

$$
\text { N2... } 0.85
$$

LSUIUPES CROCOL 


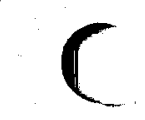

ióöititivy

COUNTHY........... UNITEU STATES

IRESSHLE $=$ RANGE

STATE........... WYOMTNG

CIUNTY........... PARK

MAP MEFEHENCE..... NORRIS JUNCTIUN 1862500

OTHFY LUCALITY INFORMATION REALGAIL CREEK.

SAMOLE UESCHIELLUU_AUD COUUTIIONS

DATE/COLI ECTUH....... 1955/00/00

SAMPIE MUMAER........ 85

GAS ANALYSIS

OATE GANALYST_.... 1965/00/00 GUNTEK AND MUSGRAVE

AVALYSIS IN MULE \&

AL. 0.03

CH4.: 0.U

C2H6. 46.

CO?.:

$4 ?: \cdots N$

$\begin{array}{rr}N 2 \ldots & 3.47 \\ 02 \ldots & 0.09\end{array}$

CQRHuLAatis

LAT/LUNG... 44-43.8 N 110-42.5 N

PAGE V063

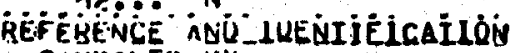

CIMPILED HY.......... LAWSON, WILLIAM A.

CIMPILER AFFILIATION... N.5. UEOLOGICAL SURVEY

CIMPILER CHOSS INDEX... NEH-JU6

RFFEHEIICE............. GUNTER ANU MUSGRAVE, 1966

AECURU 00116

GËTHE SAMELE EJLE

NAME. UF SAMPLE SUIRCE... NUKHIS GEYSER BASIN - UNNAMEU SPHINO

LOOOSTISO

CUUNTHY ...... UNITEU STATES IQUUSUIR=RAMGE

STATE ............ WYOMING

COINTY Y.:. PARK

MAP HEFEHEINCE...... NORKIS JUIVCTIUN 1862500

OJHEG LOCAL ITY INFORMATIONI HEALGAR CHEEK

SAMOLE UESCUIPILUA AND CONO MIIÓNS

DATE COLLECTOH...... 1965/6U/00

SAMPLE NUMHER........ B4

Gins ANAĹYS IS

DATE/AHALYST.... 1965/0n/00 GUNTER AND MUSGRAVE

A iníxsis liviule

AK. IS IN TAULE

LSOROPES LOCOL

GEUTHEHM FILE IOI 0020456

COQRULHAIES

LAT/LUNG ... 44-43.8 N 110-42.5 W

$\mathrm{CH}_{4} \ldots 0.21$

C2H6.
COT.

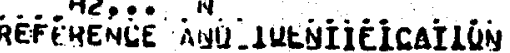

C.MPILED HY.......... LAWSUIV, WILLIAM A.

C:MHPILER AF FiLIATION... M.S. GEOLUGICAL SURVEY

CIAIPILER CROSS INOEX... NEH-3US

HFFERE INCE........... GIJNIEK ANII MUSUHAVE, 1966

RECUHU OQ117

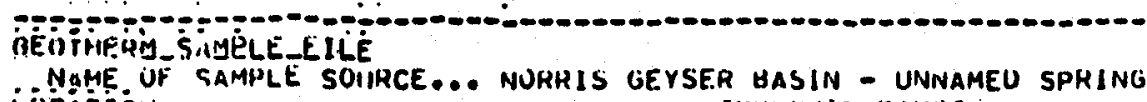

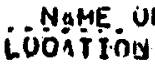

LUYNSULE=BANGE

GEUTHERM FILE IU: 0020455

CQUULLLAIES

LATLLUNG... 44-43.7 N 110-42.6 N 


\section{COIIITY \\ M.IH HEFE. ZE NCE...... NOKRIS JUNCTION 1862500}

OJHË LOCALITY INFOHMATION AUUUT $2 S M$ NORTHEAST OF NO. 71.

SAMULE UESCUIEI IQU_ANQ CQUUTLLONS

OATE COLLECTUK. . ..... 1971100/0O GUNTER, H.D.

SAHPIE NIJABEK...... T3

TFIHFERATIHE (C)

OJHE? SAPARLE INFORMATION.. MURKY DAHK SPRING WITH SHALL OVERFLOW GAS ANALISIS

DAIE /AHALYST.... 1971/00/00 GUNTER, B.D.

Aini YSIS IN MULE

DH. THSIS IN MULE

CHA

$$
\text { O. St }
$$

ISOLORES IONCOL

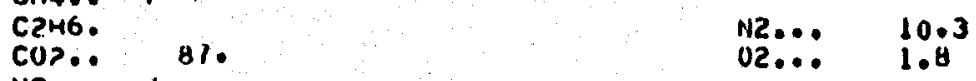

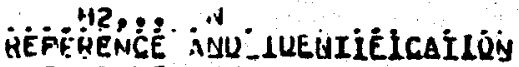

CIMPILEU WY........... I.AWSUN. WILLIAM A.

C.IMPILER AFFILIATION... H,S. UEOLULICAL SUFVEY

CIMPILER CROSS ININEX... NEH-3U9

HFFERENCE . . . GUNTEH, 1973

RECOHD 00118

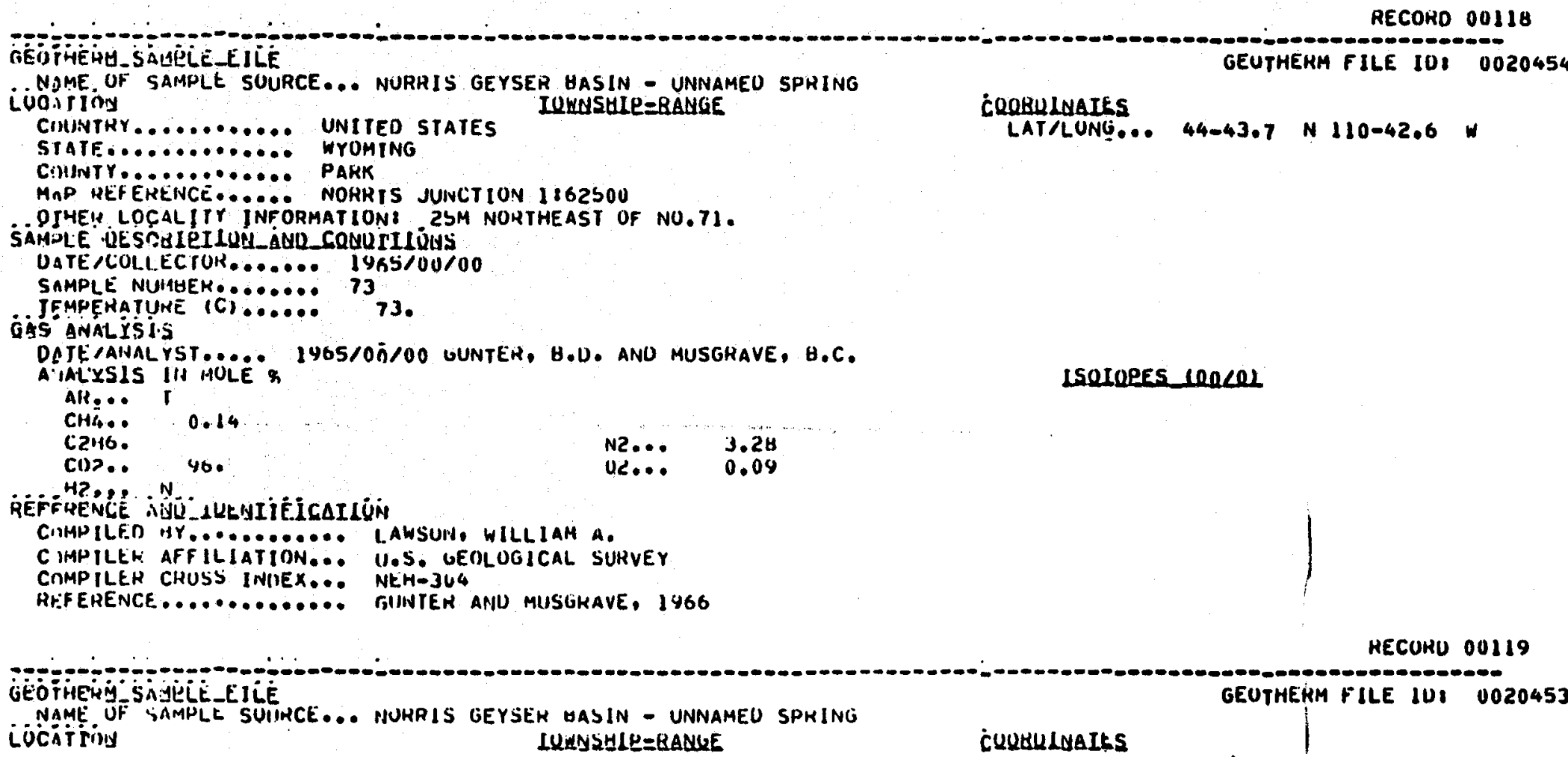


COUNTKY.

STATE............ WYOMTING

C.NINTY......... PAKK

MIP REFENENCE...... NORRIS JUNCTION 1162500

OTUE? LOCALITY INFURMATIONI ABUUT SM NUKIH OF NO.T1.

SAMPLE UESCULILIUYS ANU CONUIIIOUS

DATE CULIELIUR....... IGTI/UU/UO GUNIER, $B . D$.

SAMPLE NIMUER.

TFMPENATINAE (C)

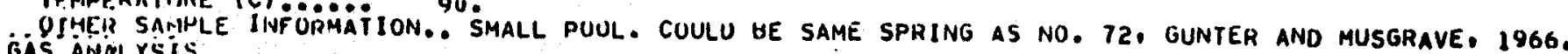

TAS ENRLYSIS

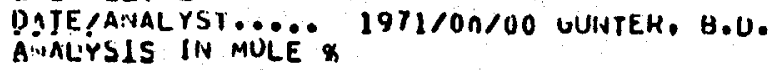

Al.

CHin.

CuP.: 22.

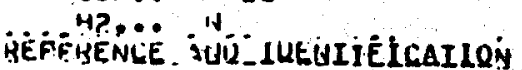

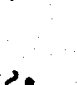

N2... 65.1

LSUIOEES LROCO

CINPILEN BY............ LAWSUIV. WILLIAM A

CWIPILER AFFILIATION... W.S. GEOLUGICAL SURVEY

CIMPILER CHUSS INDEX... NEH-3UE

R.FEHEICE............. GUHTER, 1973

KECUKD 00120

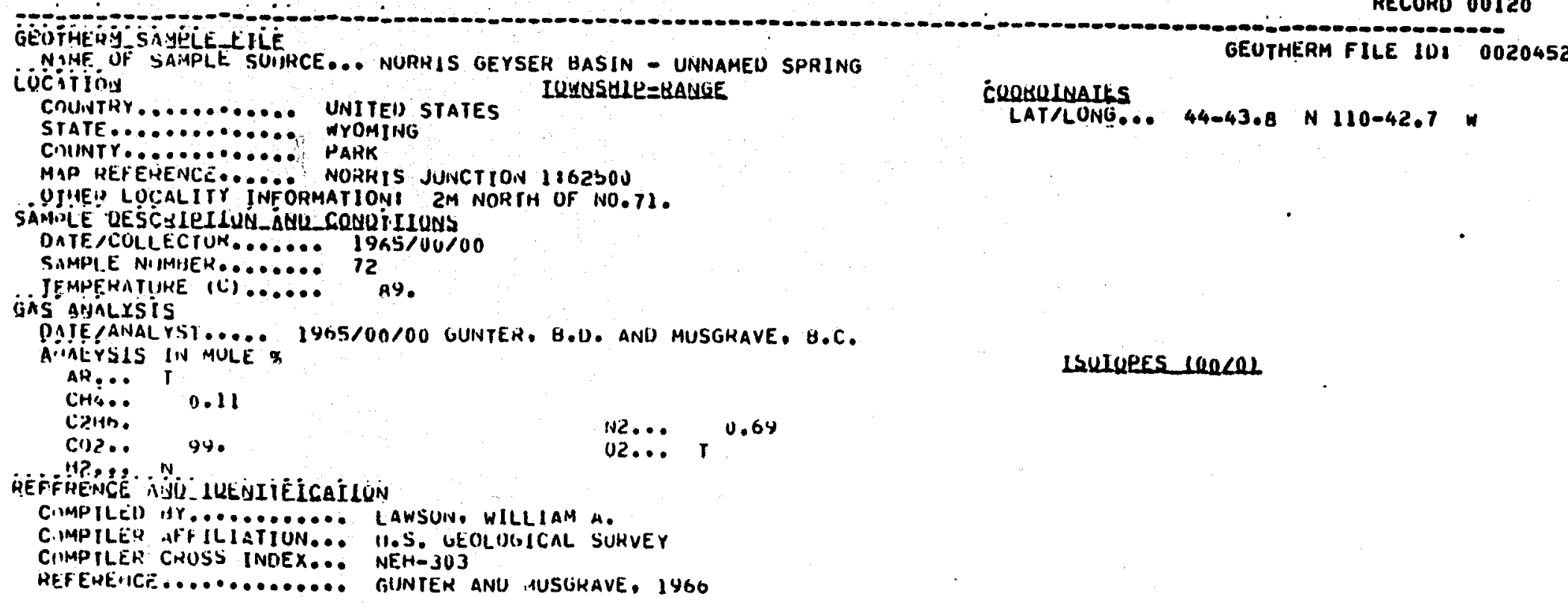

AECUHO 00121 
iuciritioj

C Vuntir............ UNIIEU SIAIES

STATE:

IQWUSAIE $=$ KANLE

Srate.............. Wrumillo

MaY UEF 9 .

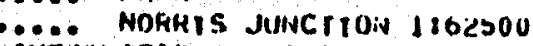

SAH:D UESCIOL

DATECCUI IECTUK....... 1971/UU/UO BVAIER, B.U.

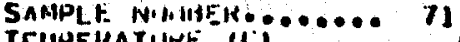

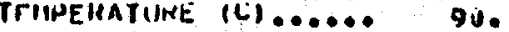

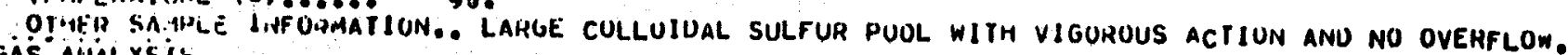
GAS Arinixs is

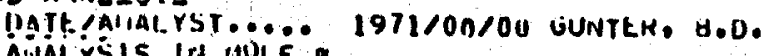

A"IAL YSIS INI HULE

all... n.. 1

C114..

cints.

C1)

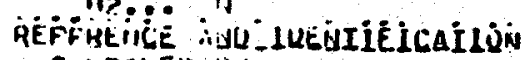

CIMPILEO $118 . . . . . . . .$. LAWSUN, WILLIAM A.

CIMPILEH AFFILIAIION... M.S. GE.OLOLICAL SURVE

C.MAILILEA C:ROSS IINIEX... NEH-JUT

HFFF,HEWCF............. GIWIEK, IYI3

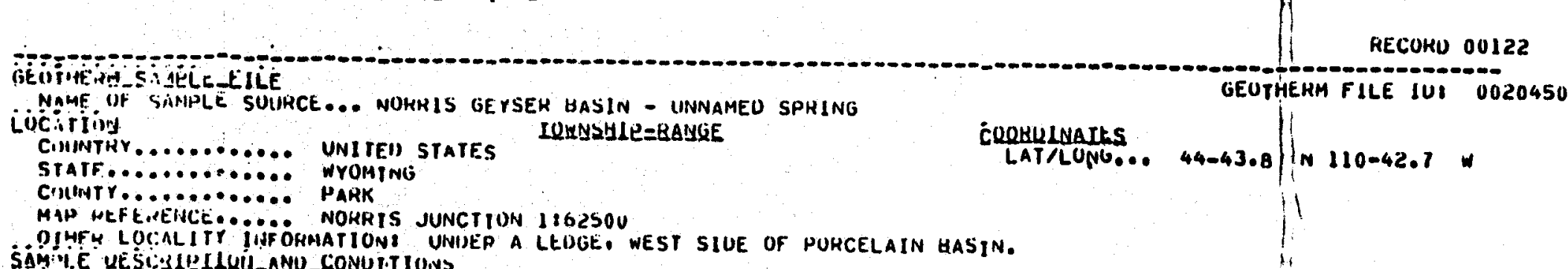


RECUHO OU123

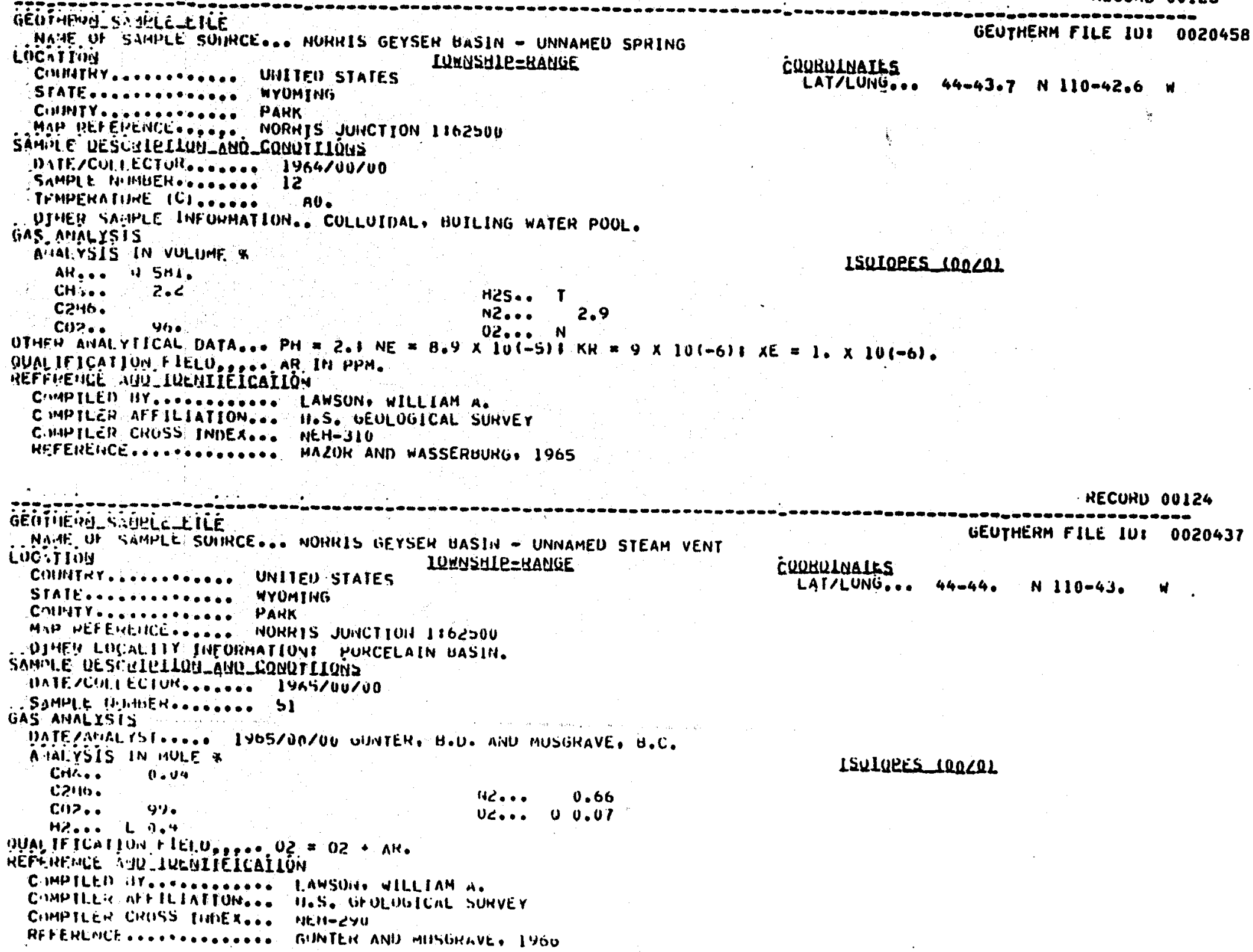




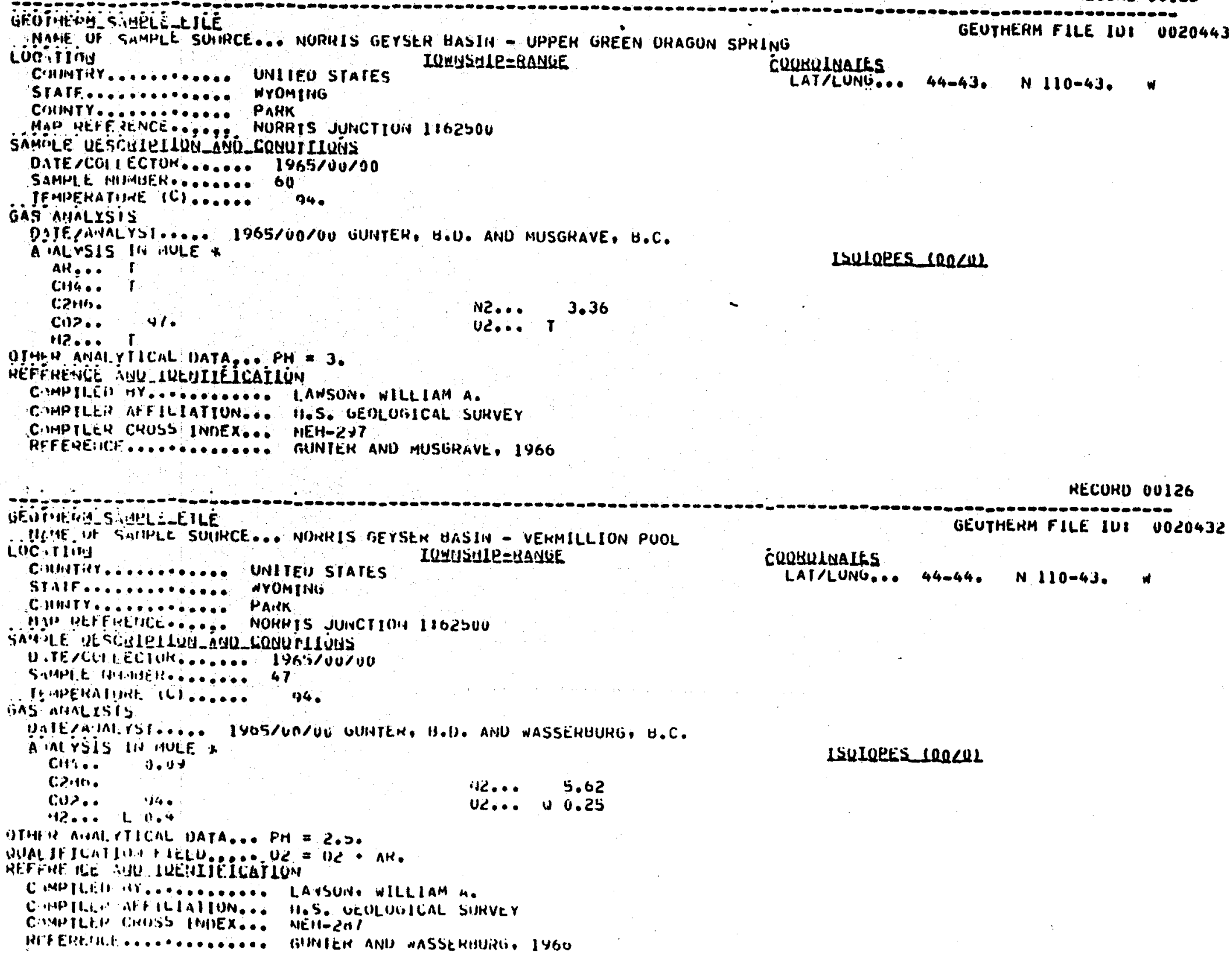

RECOKO OOI26 


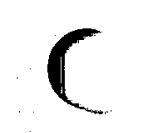

PAGE 006Y

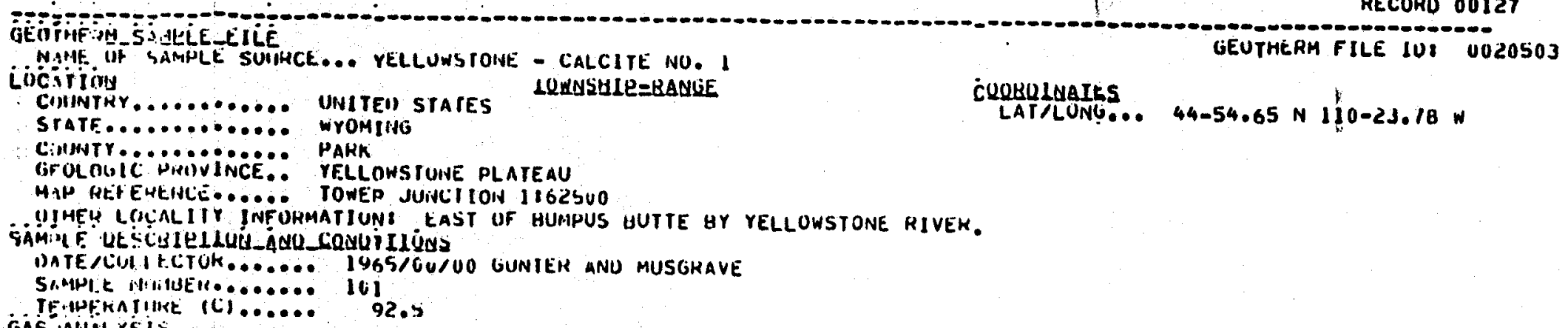

\section{CUORULUAItS \\ LATRLOW... 44-54.65 N 110-23.18W}

C.गilh

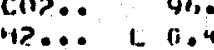

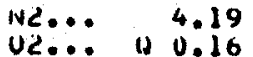

UTHEK AIIN YIICAL DATA... PH $=7 . Y$.

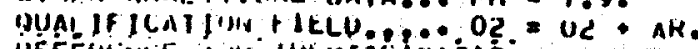

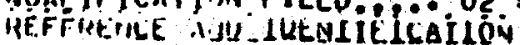

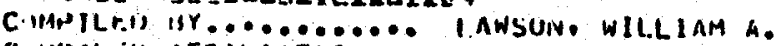

CIMBILFIS MFFILIATION... II.S. GEOLOGICAL SUHVEY

C.MHILER CHOSS INNEX... NEH-JSZ

HFFEPCHCF............. GUNTEK ANU MUSGRAVE, 1966

EQTiAt: गIS_S ABLLEILE

RECURO OO12B

NAML: UF TAPIPLE SUIHCE... VELLUWSTONE - CALCITE NO. 2

ILE IU: 0020505 
CWHII EU AY........... LaWSUN, WILLIAM A.

CHIPILC'S GAFILIAIION... M.S. ULOLUGICAL SURVEY

C. WATILEF CHUSS IWNEX... MEH-J J3

MFFF.HE:JIE.............. GUUIEH ANIS MUSGKAVE, 1966

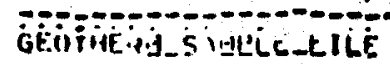

NANE. UI IAIHLL SHIHCE... YELLUWSTOHE - CALCITE SPKINGS

Livesillivis

C.. YELLUWSTUNE - CALCITE SPKINGS

CHIIVTIRY.......... UNITEU STATES

QWNSHLE $=8 B N$ LEE

STATF............ WYUMINO

CIHIIITY............ PAHK

GrollinilC PirUVi.VCE. TELLOWSTUINE PLATEAU

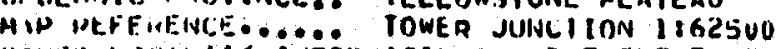

U!'HF! LOCALII INFORMAIIUNE AI EXTREME NUKTH ENU OF AREA.

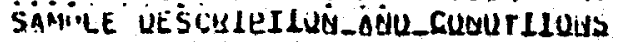

DATF /COLECIUH...... 1930/00/00

AAT:H AidOL YSIS

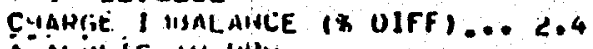

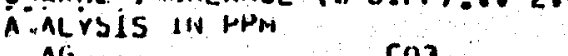

Coj.

al.

cho.....

IIE. . . .

41....

retinit?

CA... I, I,

lin.......

CL.... 21\%

HCO.3... 174 .

Co......

K........

OIIUE AlHAL YTICAL UATA $\ldots . \$ 5201=21$. PPA.

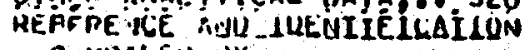

C IMPILEU, HY........... LAWSOIN, WILLIAM A.

COSAPILEH ATR ILIATION... MIS. VEOLURIICAL SUKVEY

RIFEHE.HCF ............... ALLE AND DAY IYJ

GiviNG S

IJ.S. VEOLUCIICAL SUKVEY

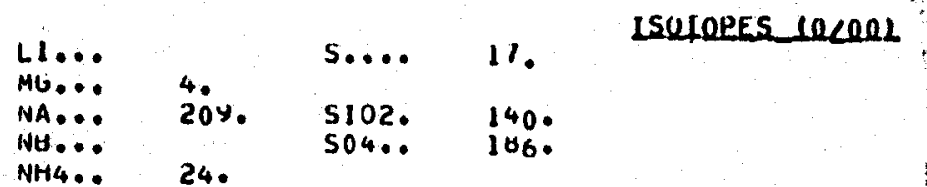

86.

HECUHU OU129

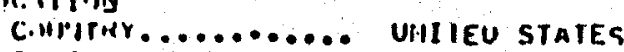
UTIIEU

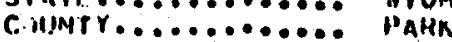

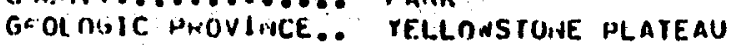

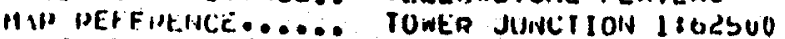

!!!HEY LOCALITY INF IHHATIONI SUUTH ENO OF TALUS SLUPE. IS FT FRUM HIVLR.

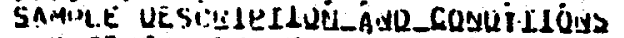

UATE/CINIECIUR....... 1970/6U/00

SIMPLE HIIMUER.

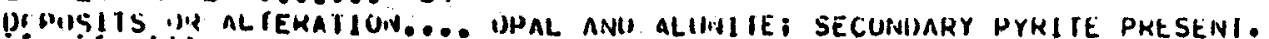
vatio nivbl isis

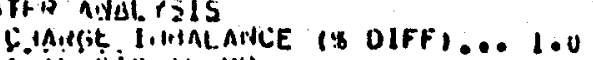

inirisis lin Ho:

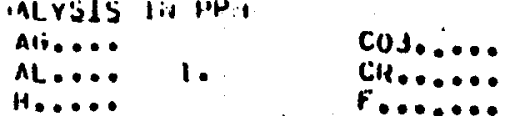

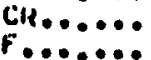

L1...

S.... I

LZQLOEES 10COLL:

H.....

NA...

2us.

SIJ2.

$245 \cdot$ 


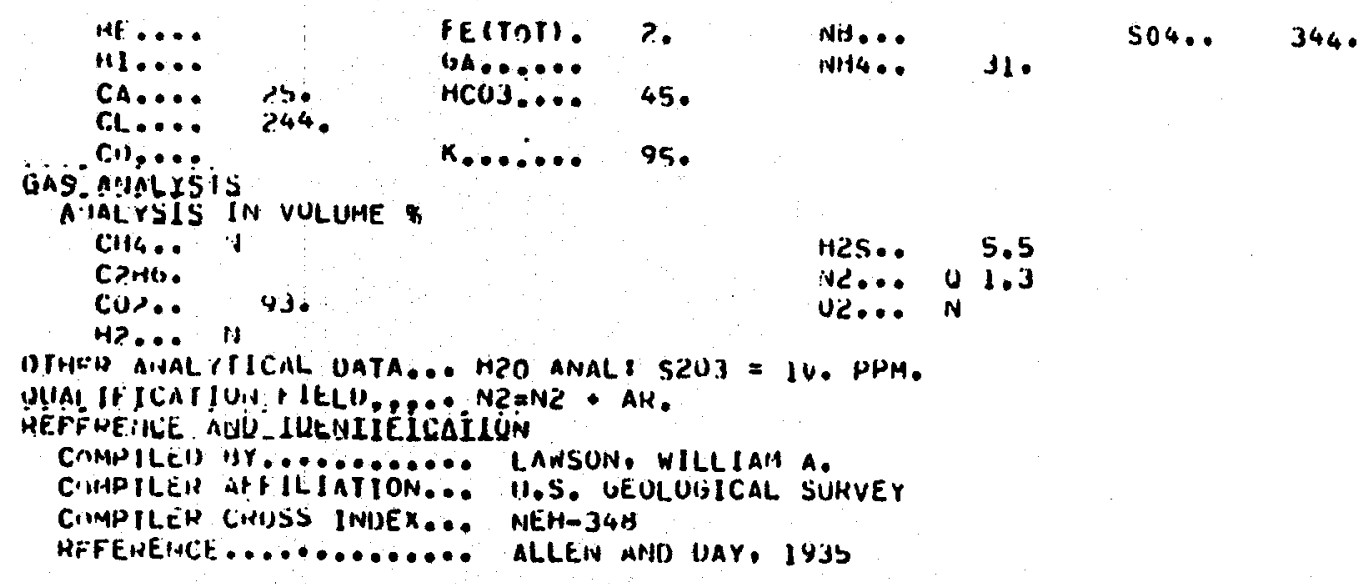

\section{LSQLQEES $100 \angle O L$}

page voll

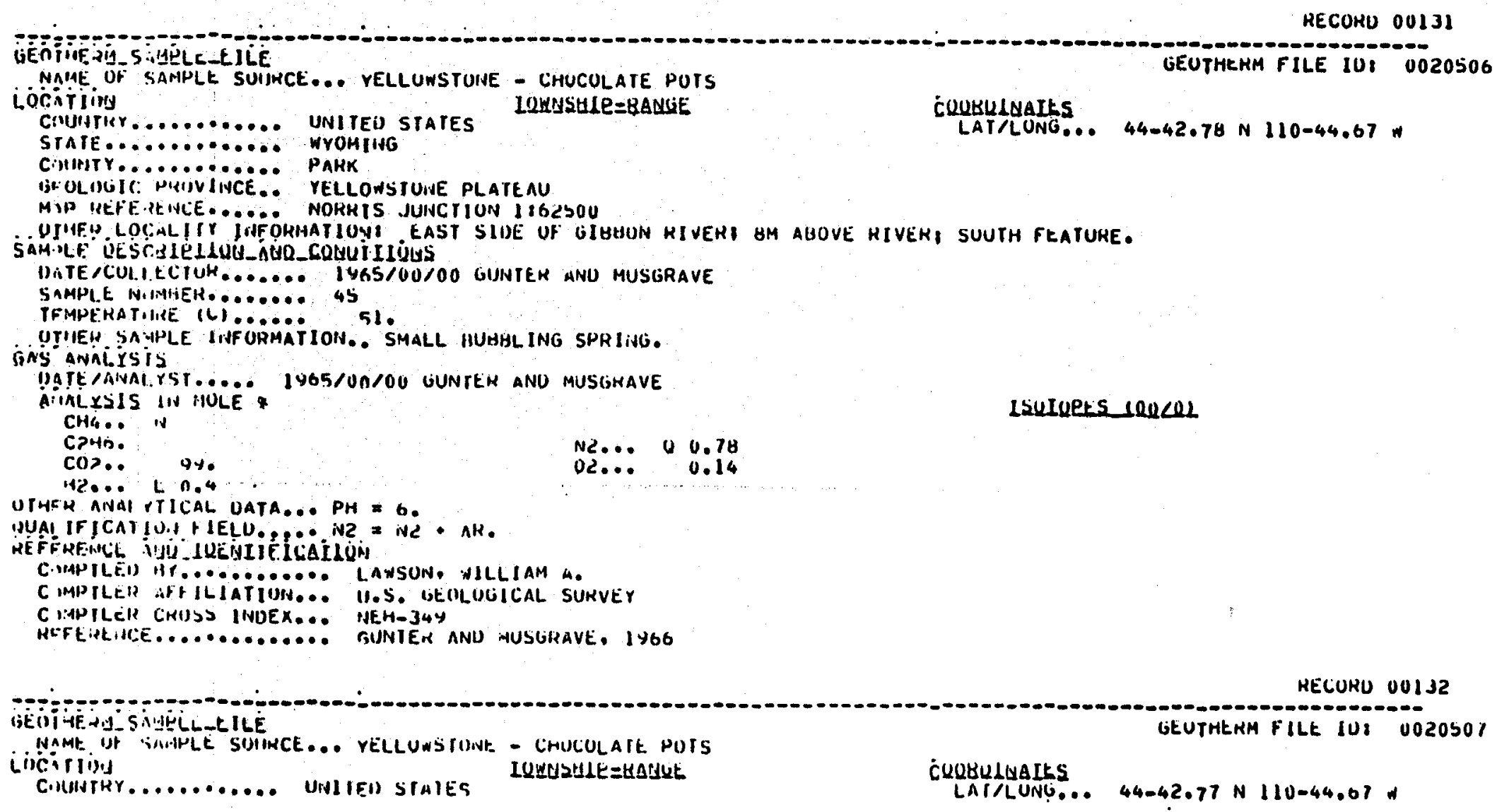




\section{STAIF............ WYOAlIN \\ CIIIHTY............ PAKK

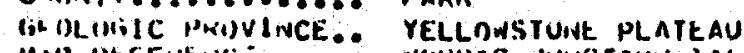

MAlP IJEFE REINTE...... NUHKIS JUIVCTIUN 1:62b00

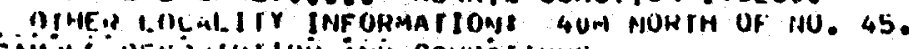

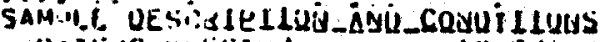

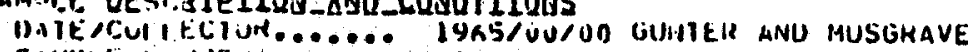

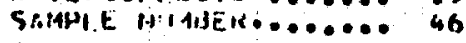

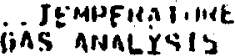

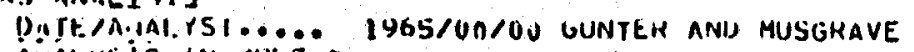

A IMLYSIS IN HULE

chl1..

LSULORES LURCOL

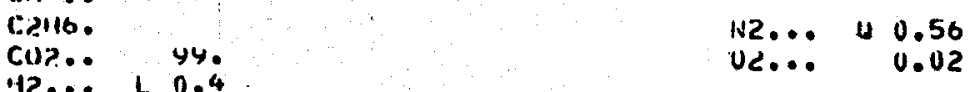

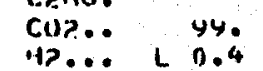

OTHEW ANAI.YIICAL WATA... PH $=6$.

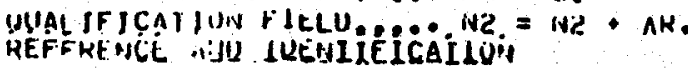

C MPILE) HY........... LaWsuiv, WILLIAM a.

C MAPII.E" afF ILIATION... U.S. GLOLUGICAL SIJKVEY

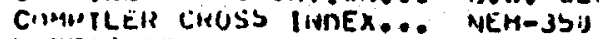

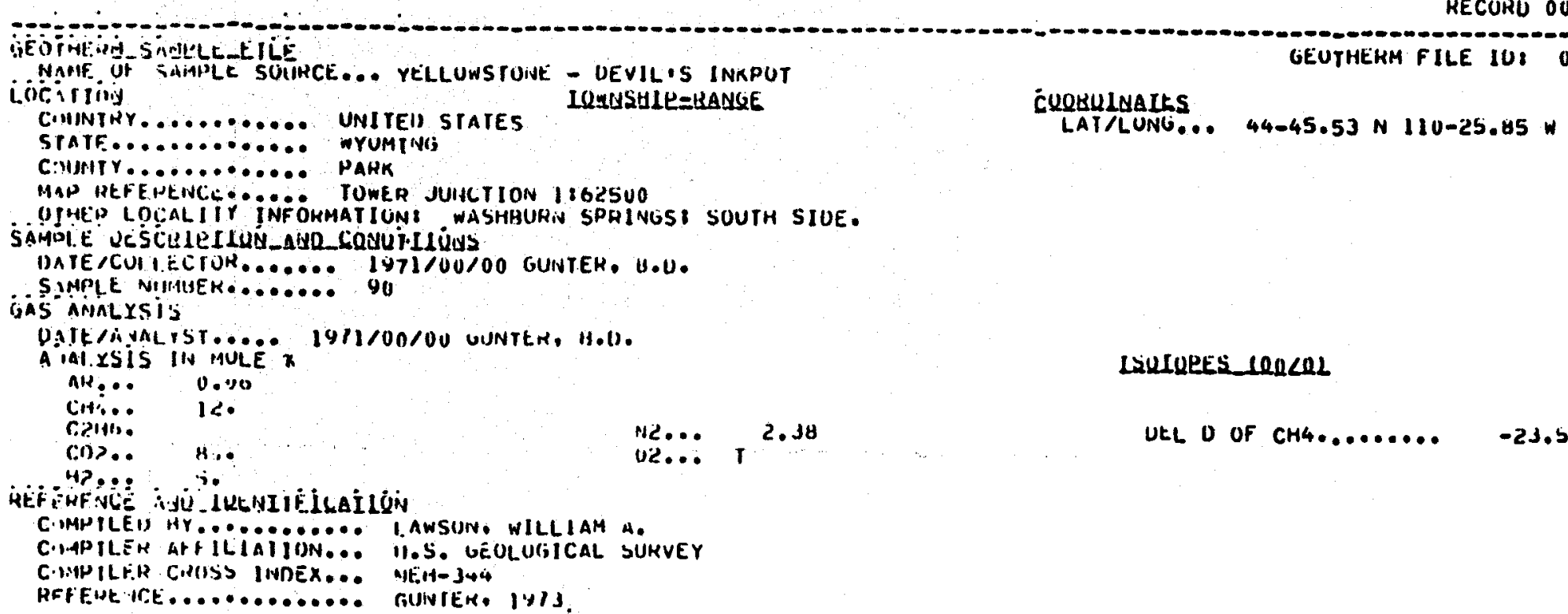

NANIF UF SAIAPLE SUINCE... YELLUWSTUIVE - DEVIL'S INKPUT

LOCiriniv

CUININY. . . . . . UNITEL STATES

STATF............ WYMMTIV;

C:VINIY........... PAHK

MAP HEFENENCE..... TOWER JUIKCTION 18625UO

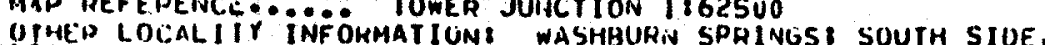

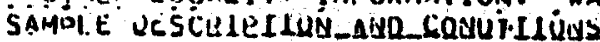

DATE/CUIIECTUK....... $1971 / 00 / 00$ GUNTER. U.U.

SIMPLE NIIIUSER.......90

GAS MiNLYSTI'

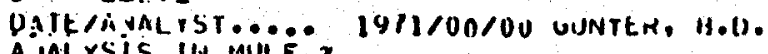

nie... 0.00

citio.. 160

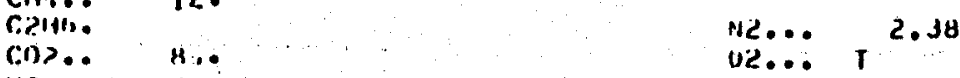

H?

IVtNIIFILAILOON

REFENF VCE CJU IKLNIIELCAILON

C.MPILEN AR IILIAIIION... II.S. GEOLUI;ICAL SUHVEY

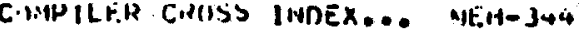

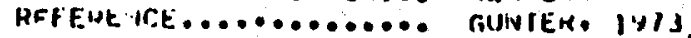

LSULUEES $100<02$

UtL O OF CH4....... -2J,5

\section{CUQBULUAILS}

LAT/LUNG... 44-45.53 N 110-25.85 W 


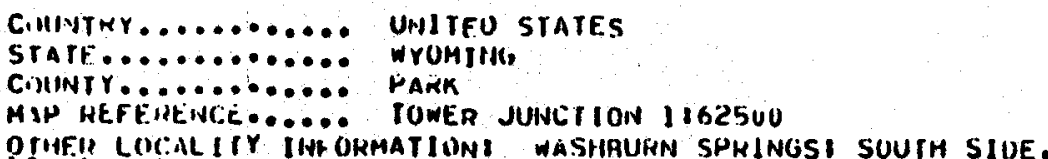

JHFA AINAL YIICAL DATA... AS2O3 $=0$. PPM CU2 $=64.7$ PPMI H $=0.2$ PPM

UUAL IFICATIUI FIELU, ... D CALCULATEII FKUM UZU3.

REFFRFHCE AUU LUEUIIÉICAILUN

C MMPILEI HY........... LANSUN, WILLIAM A.

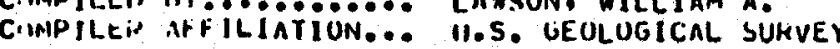

WH:FEHEHC.H............. ALLEN ANO DAY, IY3S

RECUKU 00135

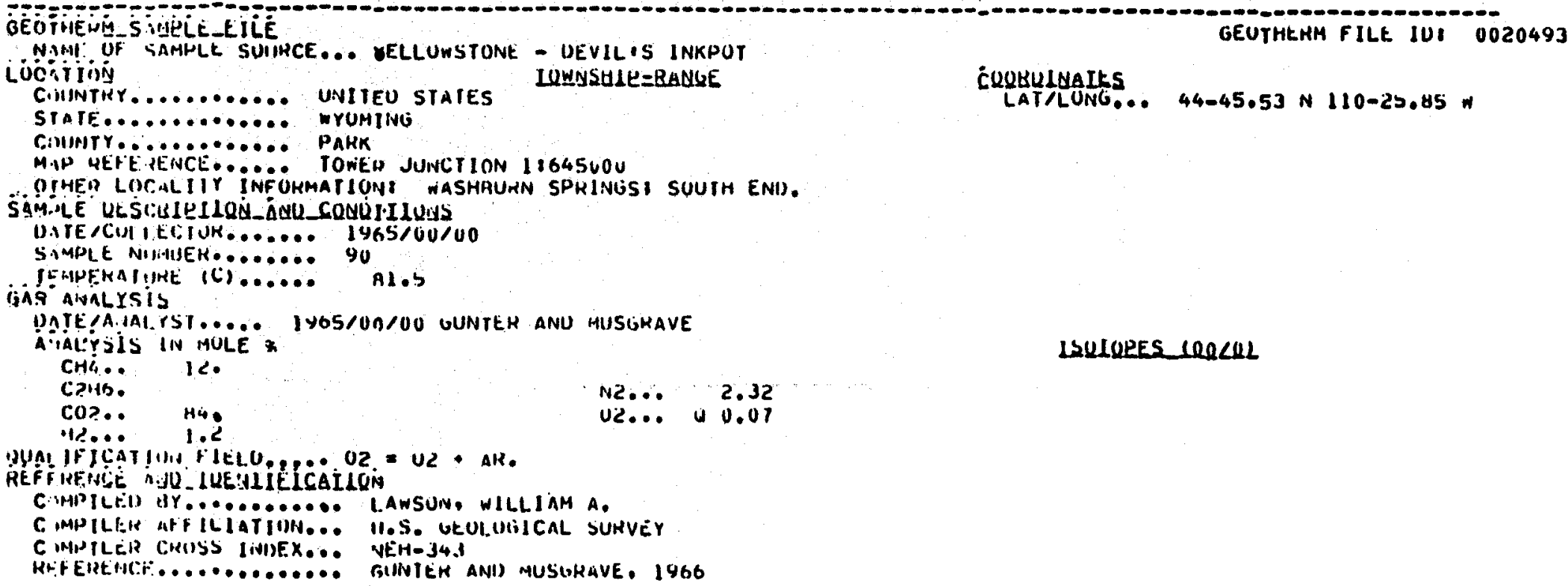


Ali.....

iti......

Cl. $\cdots$

Co3..... is

HCUj N8... SO N 1272.

LSOIULES LCOUII

CQ $P$ C.

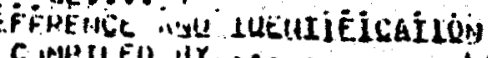

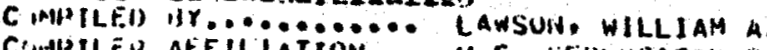

C',HPILER AFFILIATION... 11.5. UEULUGICAL SUKVEY

RFFEHENCF.............. ALLEN AIND UAY, IY3S

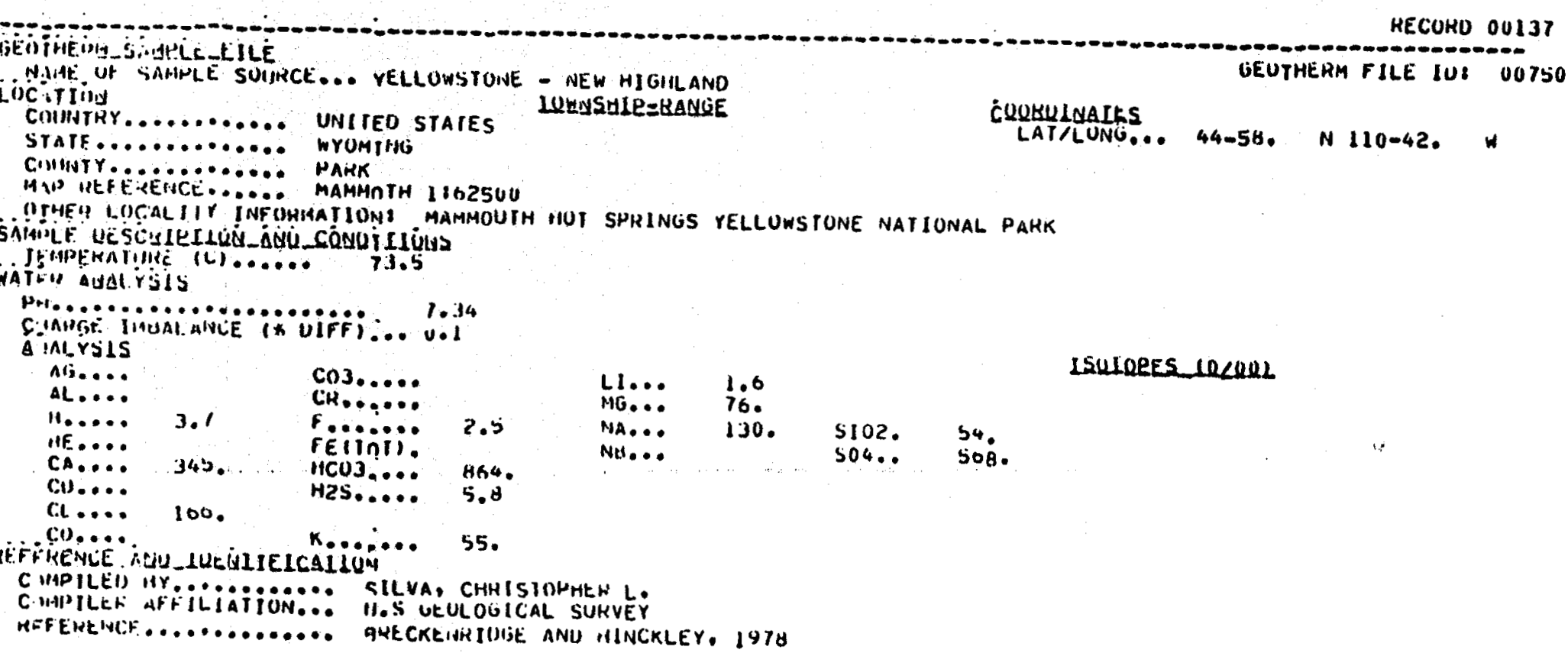

CUUHULNAIKS

LAT/LUNG... 44-37.28 N 110-25.58

HECUKU OU136 


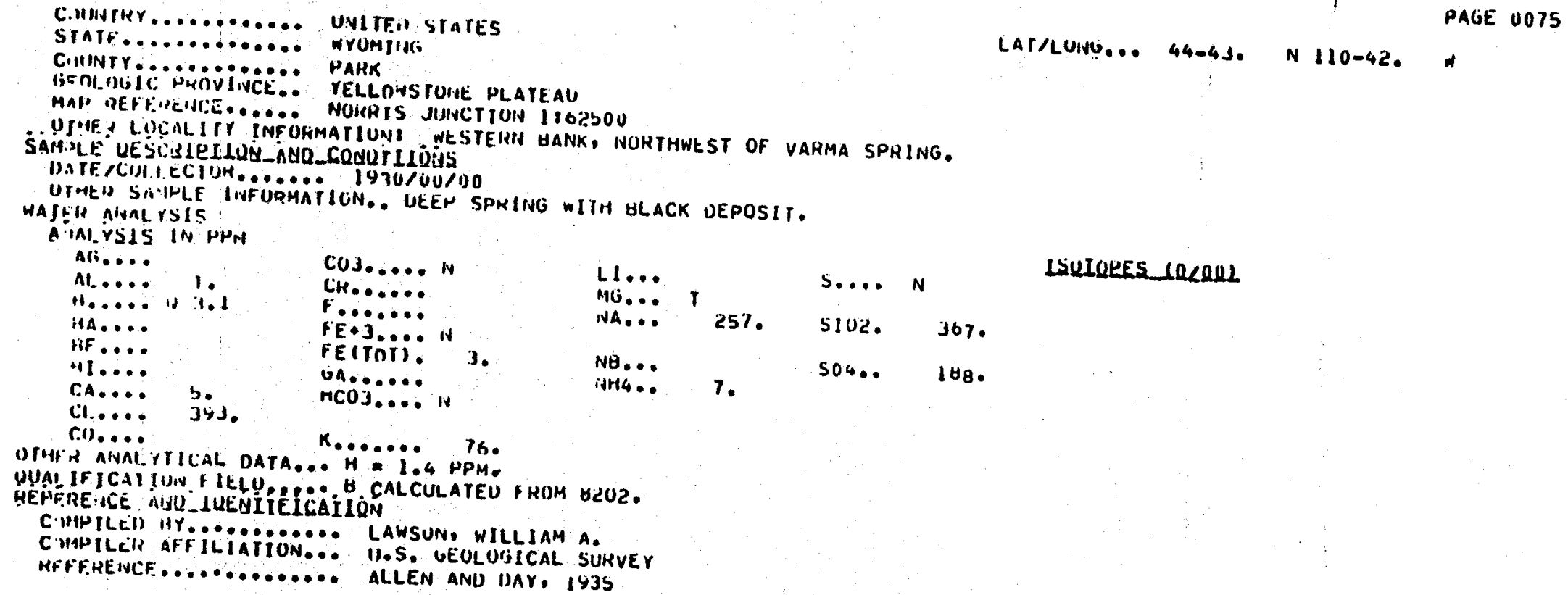


SIATF............. WYOMING

Cinintir............ PaRK

MAT PET EHEIVCE..... CANYON VILLATE 1362500

IIJHFY LOGALIIT INFOKHAIIUNI UY MUU VULCANO.

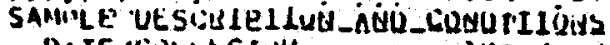

ONTE八CIII.ECTUK....... 19.30/U0/U0

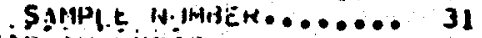

Dins ANALYSIS

$$
\text { ANIALYSIS IN VULUME o }
$$

CH.

0.1

Hiss..

(⿻)丨

0.1

LSULQRES IORLOI

CQRGULNALES

LAT/LUNG... 44-37.28 N 110-25.SH W

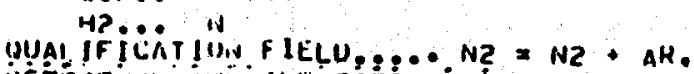

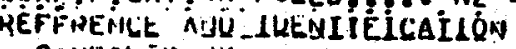

C MMP ILËI IY........... LAWSUN, WILLIAM A.

CIMPILEP AFFILIATIOON... M.5. GEULUGICAL SUHVEY

CIHIN ILER CKOSS INDEX... NEH-34I

HFFEHENCF.............. ALLEN AND UAY, 1935

RECUHO 00141

GEUTHEKM FILE IVI UU20S08

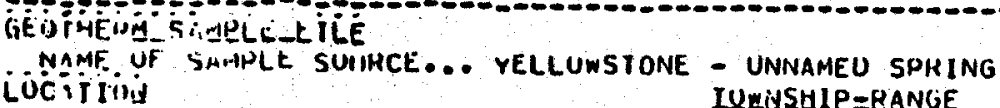

CIMNTKY.......... UHITEU SIATES

IUEXSHIE $=$ BANGE

STATE............ WrOMIING

CIHIJTY............ PARK

GHOLOSIC PMUUVINCE.. YELLOWSTUINE PLATEAU

O! HE: L.OCAL!IY INFOHMATIUN: NEAR GIUGON RIVEK.

SAMPLE UESCOSLEILQU_AQU_COQUUTILDUS

UAIE COLLIECTUN....... 1965\%00\%00 GUUNTER ANU MUSGHAVE

S.MMPI.E NIUULER....... H6

DI:AER SA IPLE INFORMATION.. STEAMING SULFIIK AREA.

GAS A.JALYSIS

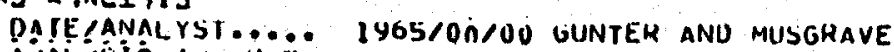

A JALYSIŚ IN MULE

AH... 0.03

CHC.. 0.2

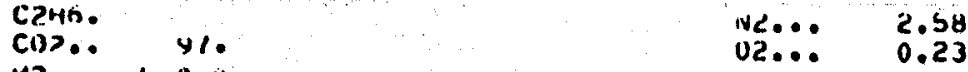

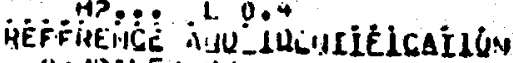

C MPILES MP............ LAWSUN, WILLIAM A.

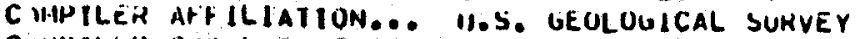

CIMPILILER CIXIISS [NIIEX... NEH-Jb]

RFFEHEIVCE............: REUTIEH ANI MUSGRAVE, 1960

LSULOEES CURCOL

cqueLLNAIES

RECOHU 00142

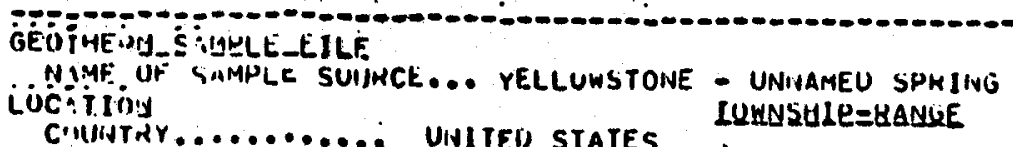

NaMF UF SAMPLE SUIMCE... YELLUUSTONE - UNIVAMEU SPKIIVG

cilintrir. VINITED STATES

GEUTHEKM FILE IU: 0020499 


\section{C}

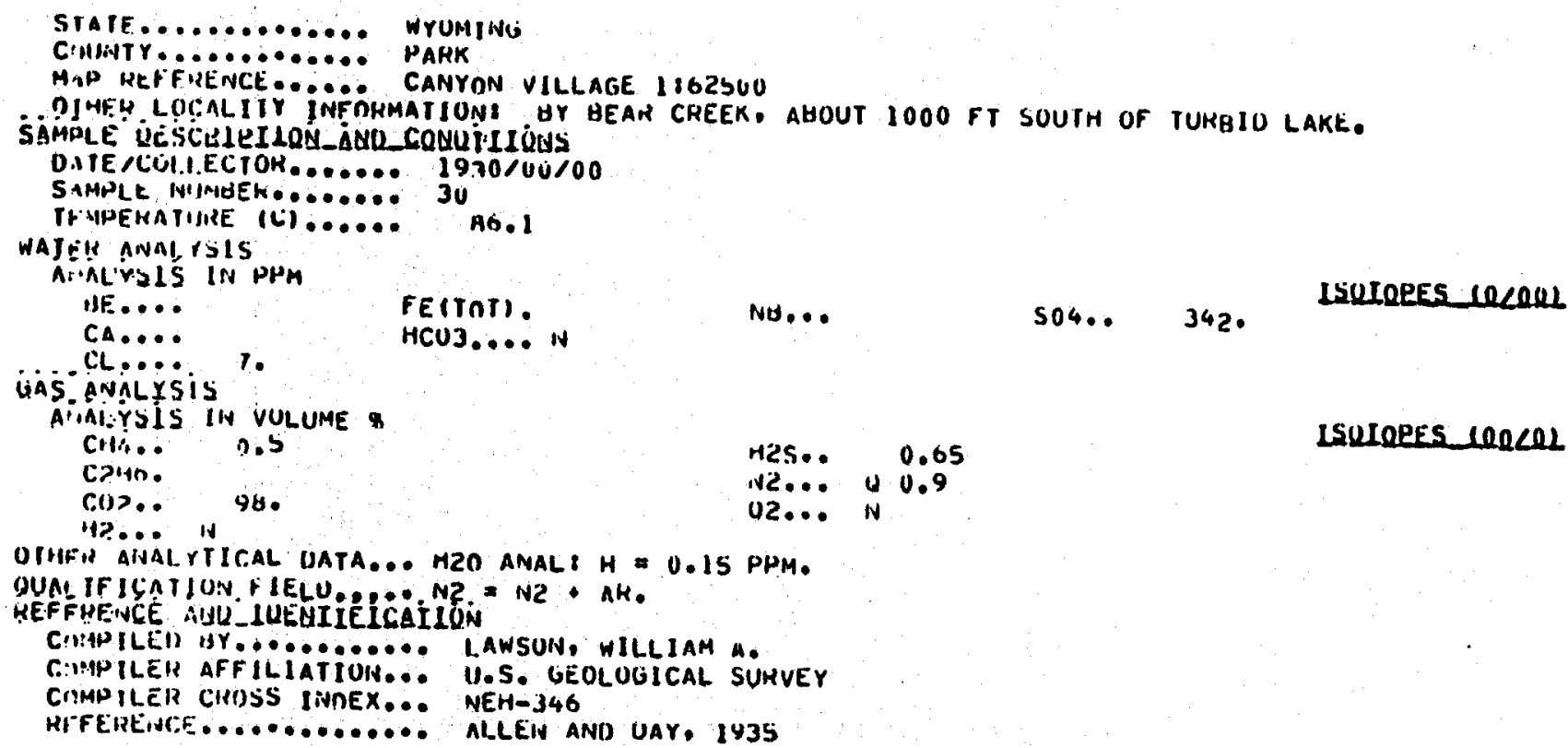

AECOKO 00143

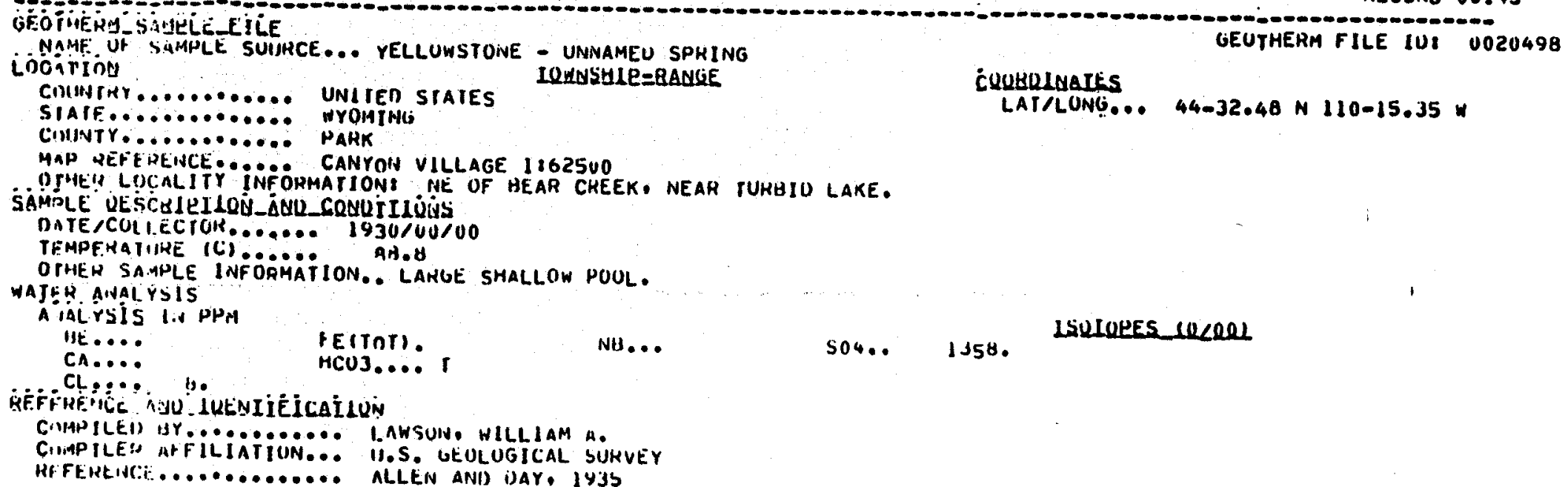


CIMUThr.

C.MUNir

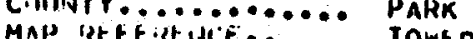

Mar

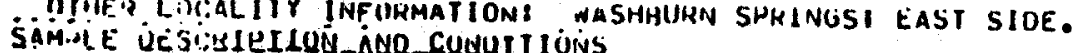

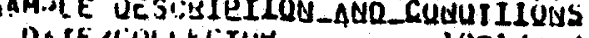

OATE COH LCTUH....... $1971 / 00 / 00$

SAMHIE RHIMHEH

TFIHFEATIHE (C) ...... AB.

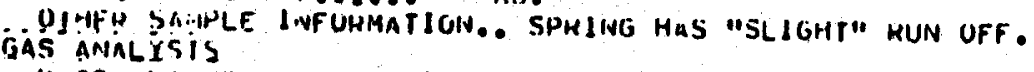

DAIE,ALJALYST..... IYII/UO/OO BUNTEK. H.O.

A IALYSIS IH MULE

AH... 0.10

CHit.. 14.

Caits.

14.

1.2

U2... I

2.98

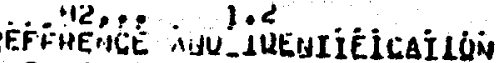

C MPILEN AY........... I.AWSUN, WILLIAM A.

C IMPILE! AFFILIATION... I1.5. GEOLUGILAL SURVEY

CIMPILER CWOSS IINHEX... NEH-345

H.FE:RLICE............. GIJNTER, 1973

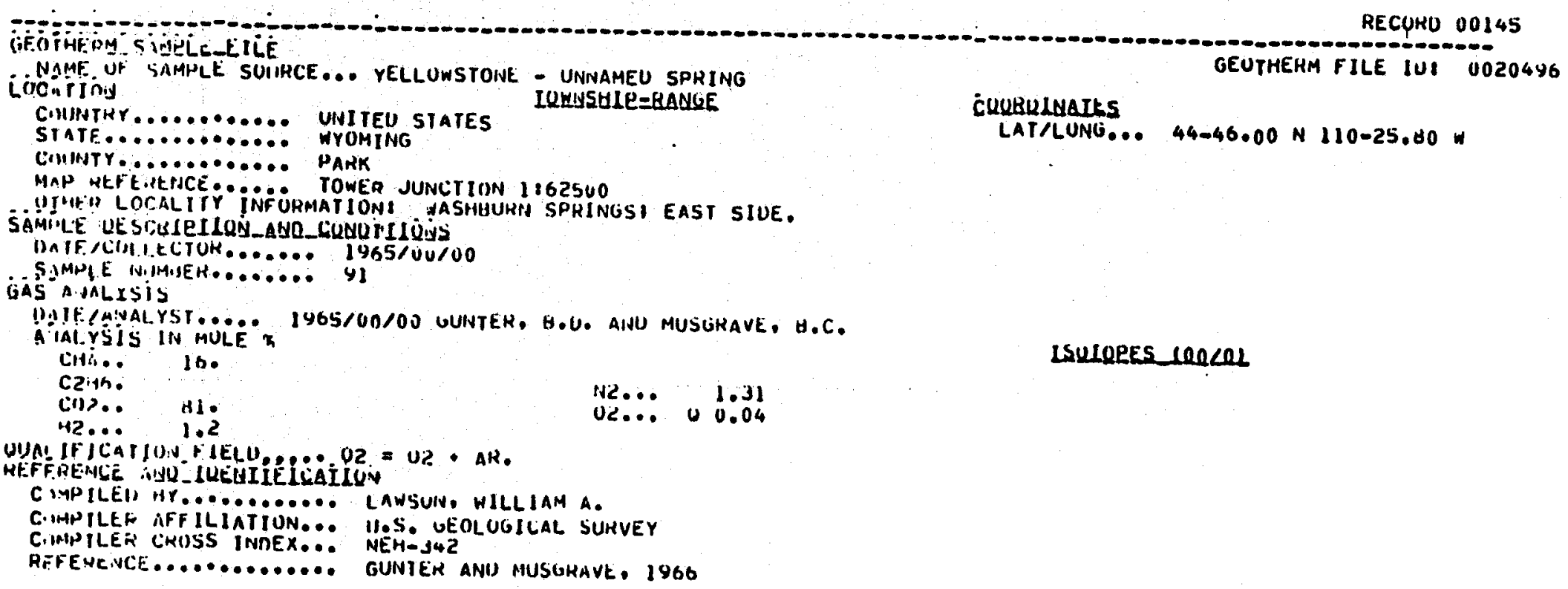

\section{LSOLOEES LURCOL}

UțL $O$ of $C H 4, \ldots \ldots \ldots . . .26 .6$

RECOHU 00146

MAME UF SAMPLE SUIHACE... YELLUWSTONE - UINAMAEU SHKING

GEUTHEKH FILE IU: 0020495

Liocirivin IUEMSAIN = HAIVGE

cugruinaIEs 


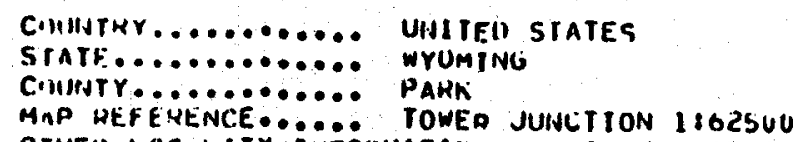

HFF FILEIVCF............. ALLEN AHD UAY, 1935

RECUHO 00147

GE THFitis

iUCANE UF SAMPLE SOIBRCE... YELLUWSTONE - UNNAMEO SPHING

Comivith LOUHSULE=BANGE

STATE

COUNTY

MAP REF EHENCE..... CANYON VILLAGE 1162500

HATE HILLSI EAST SIUE.

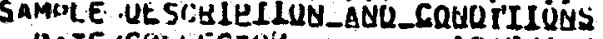

INTE/COLLETUK....... 1965/00/00 GUNIER ANU MUSLGAVE

SAMPLE NTHEH

Trapenatiuke (C)......

OJHEP SAHPLE IINFORMATION.. SULFUR POOL.

GAS ANALXSIS

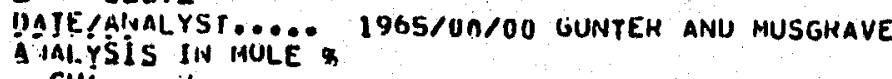

CHit.. iv

C.246.

COP. $\quad 99$.

OTHFR AHALYTICAL DATA... PM $=3.4$

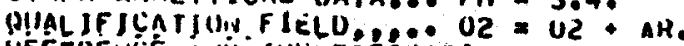

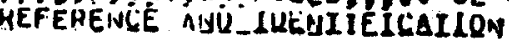

C:IMPILEU WY........... LAWSUN, WILLIAM A.

CIMHILEH AFFILIATION... H.S. WEOLUGICAL SURVEY

C:IMPILEK CKOSS. INDEX... NEN-J4O

HFFEREINCE............ GUNTEK AND MUSISHAVE, 1966

ISUIORES IONOOL

\section{CUQHLLWAIES}

LAT/LUNG.... 44-39.53 N 110-28.15 W

GEUTHEKM FILE IUI 0020491

N2.. 0.79

$02 \ldots 0.02$

RECUHD 00148

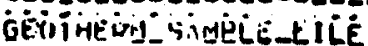

NAME UF SAMPLE SUIHCE... YELLUWSTONE - UNNAHED SHHINO

ionoitrivis
GEUTHEKM FILE IUI 0020490 


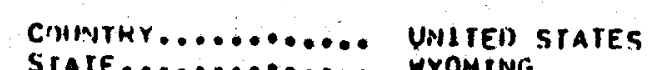

STATE............. WYOHING

Comb Tr........... PALK

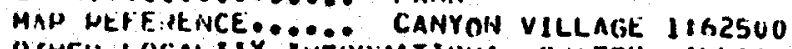

SÄMTI E UESCBYIETUQ

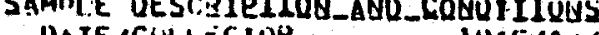

DATE CCULLCCIOH...... 1965/00/00 GUNIER ANU MUSGRAVE

SAMPLE NIMUER.........

TFMPERATIJKE (C) .......

70.6

OI'HA SA MPLE INFUHMATION.. SHALL SULFEN POOL.

Gins anfiLYSis

D.ITE AINALYST..... 1965/Un/00 GUINTER ANU MUSGRAVE

A MALSIS IN HULE \&

Cita. "

N2... 40.36

C1)?.. 49.

H?... N

OUA! IFICATIU: FIELL, $10.02=02+A R$.

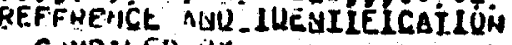

CIMPJLED AY........... LAWSUN, WILLIAM A.

C MPILER AFFiLIATION...: 11.5 . UEOLUGICAL SURVEY

CIMPILER CHOSS INDEX... MEH-339

RLFEHE NCi:.............. GUNTEk ano musgrave, 1966

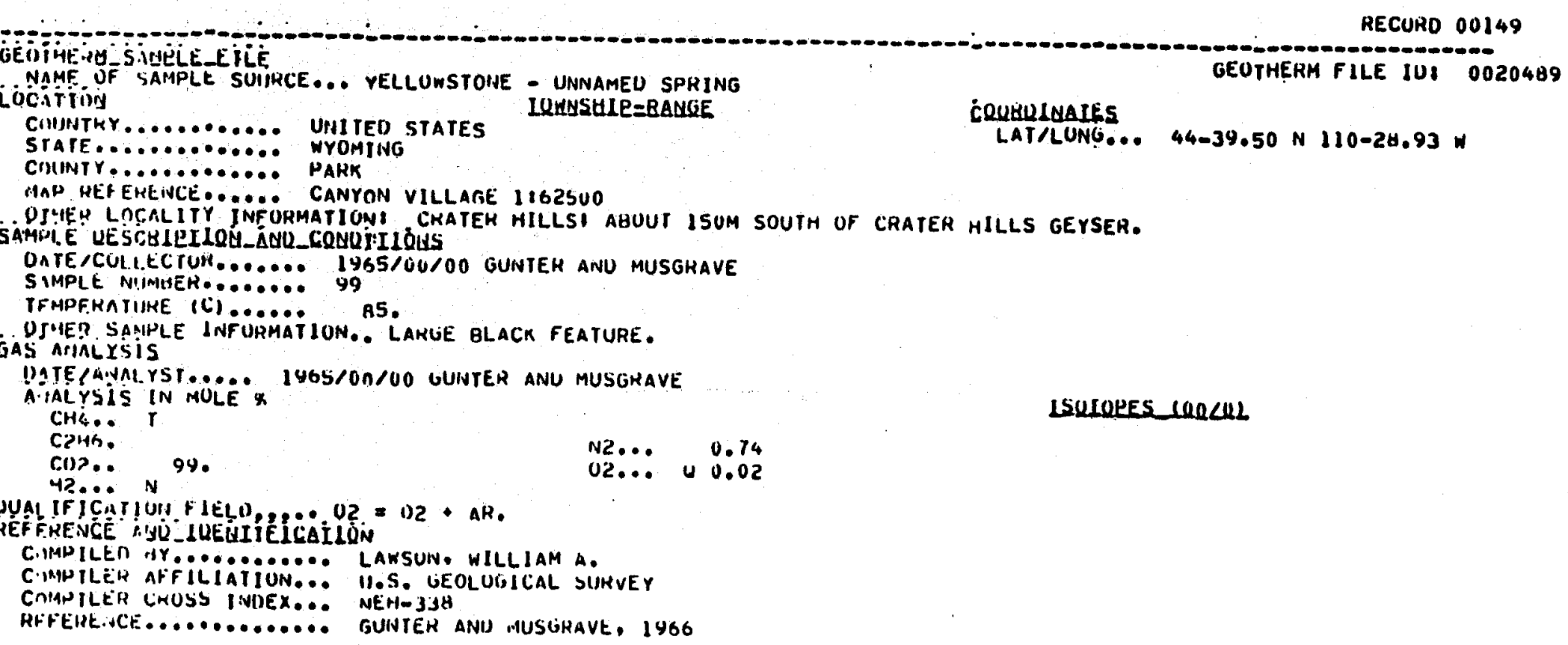




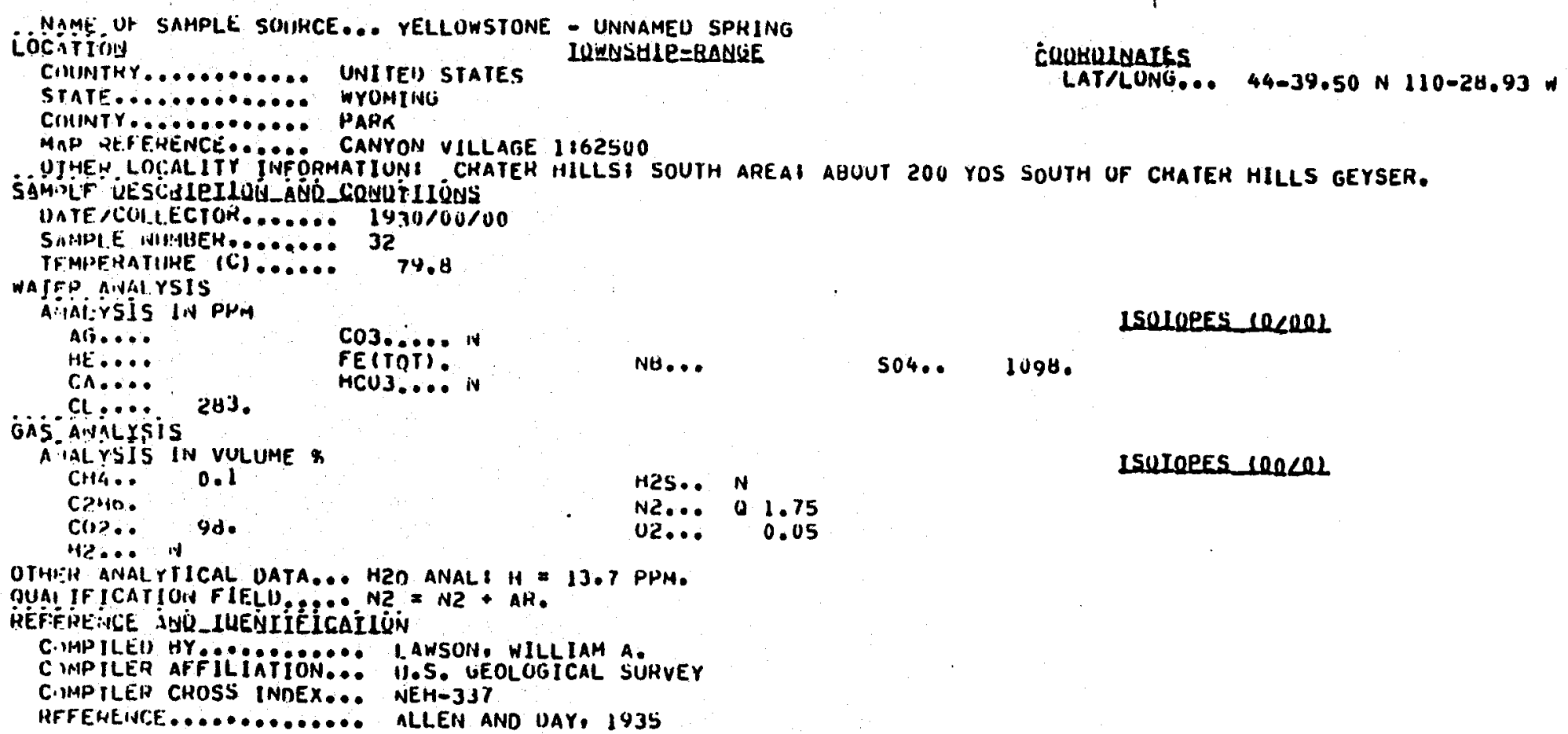

RFFEHEINCE............ ALLEN AND UAY, 1935

\section{ClluHULUATES}

LAT/LONG... 44-39.50 N 110-24.93 N

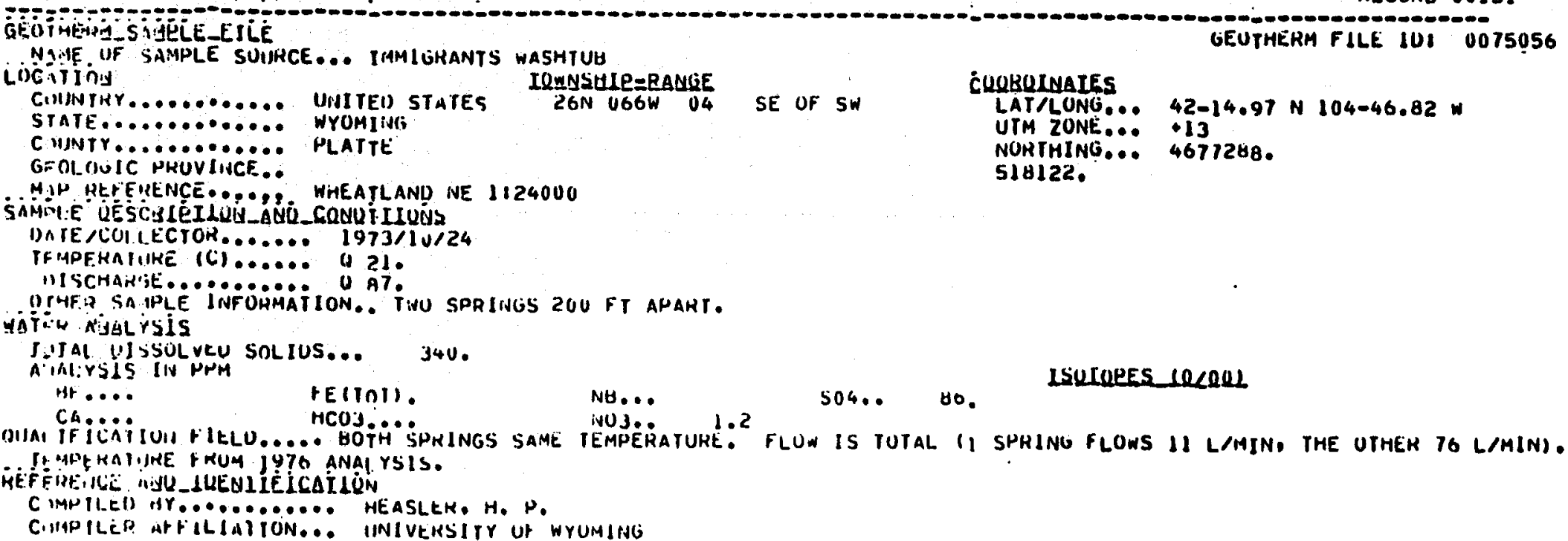




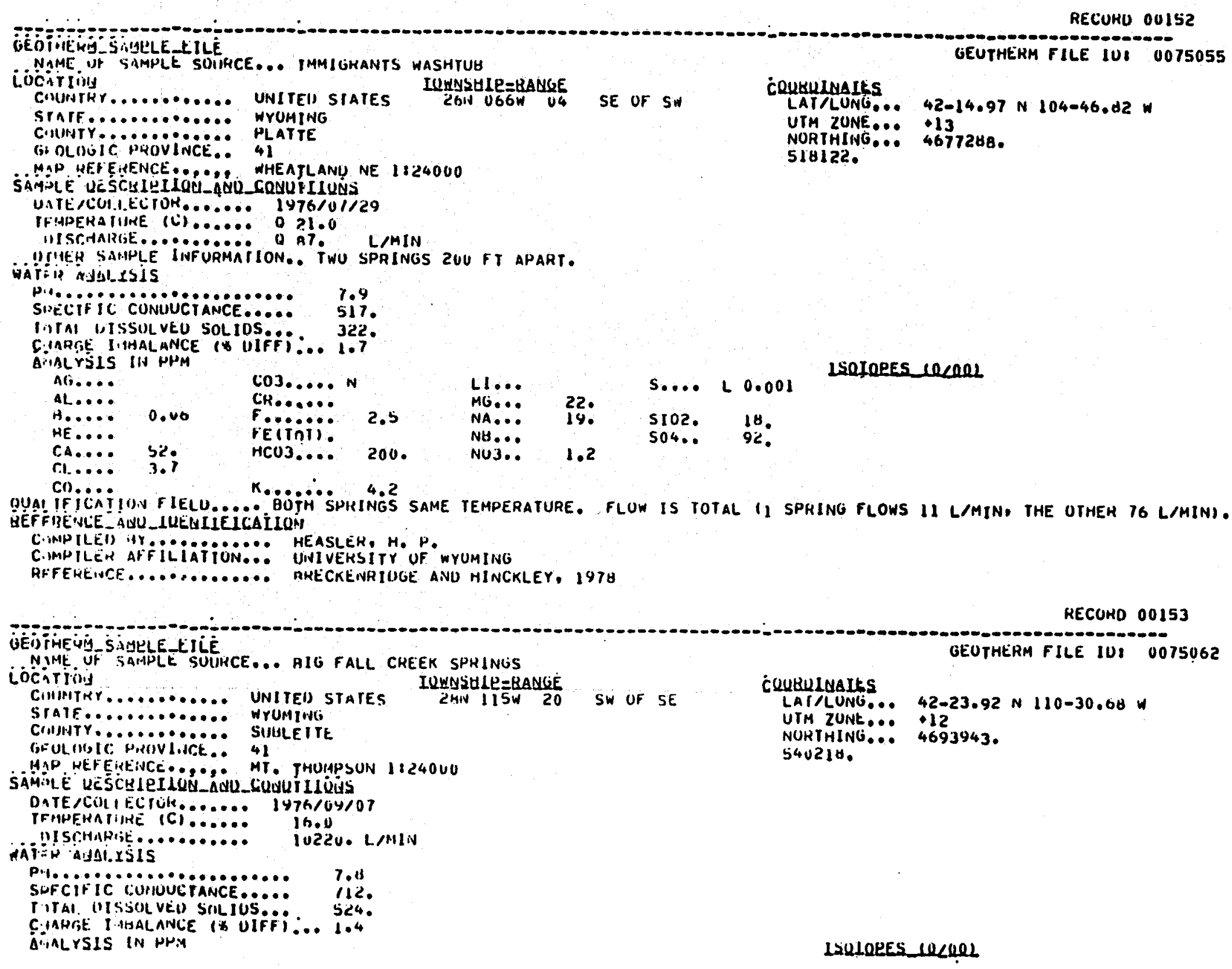




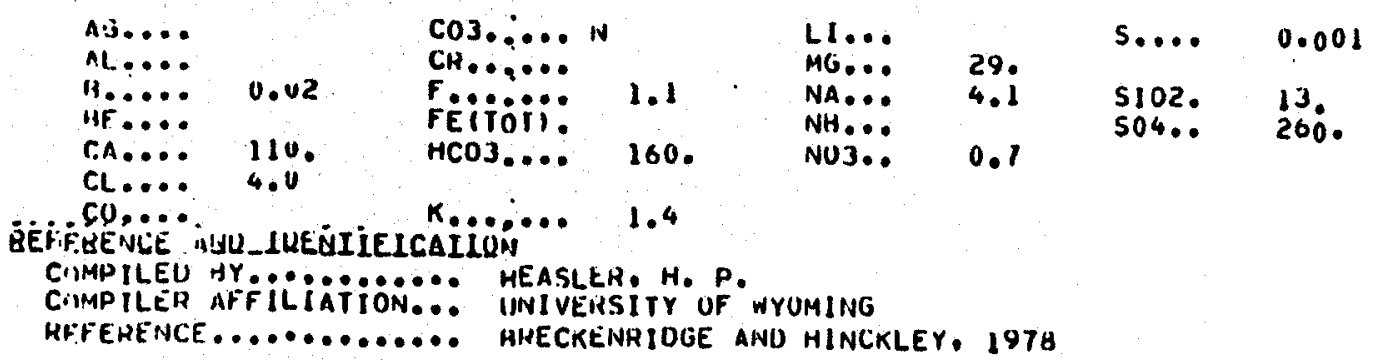

HECUHO 00154

\section{NAYIF UF SAHPLE SOIHCE... KENUALL WARM SPKINGS} Locitinit

COUNTIT............. UNITEO STARES 3BN IJOW 02

STATE.............. WYOMTNO

C:MinTr

GLULOHIC PRUVINCE.

MPP REFEYEHCE.... KLONDIKE HILL 1:24000

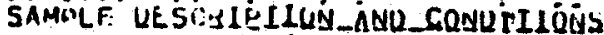

D. IE./COI. LECTUR...... 1976/U4/24

TFIHFFAIIIKE IC) ...... 29.5

IISCHARISE.......... IJ627 L/MIN

OJHER 5 AMPLE LINFOPMATLON. 6 MAIN SPHINGS WATrí AVAL TSIS

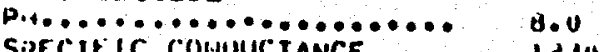

TITAL UISSULVEU SOL IISTO

Tirai OISSULVEU SOLIDS. 1060

gyingGE I Misalaivie (\% UIFF)... S.3

AIALYSIS IN $\mathrm{NHM}$

ali....

coj..... N

H..... 0. Us

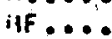

c1...

Ch.......

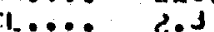

F.:.? 0.4

FEITOTI.

MCO3.... 130.

K. 0.0

QBEFFEF

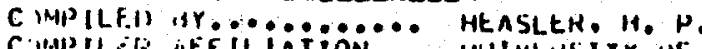

C'MIILIS AFF ILIATION.. HUIVtKSITY UF WYUAlIVII

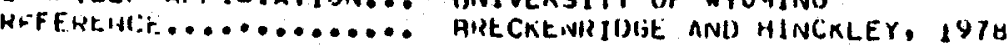

COA

\section{COUBULUALISS}

UTH ZONE... 12

579609.

\section{GEUTHËH FILE IUI 0075057}

UNIUUE SPECIES OF FISH IN AHEA.

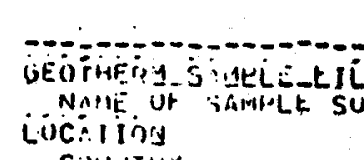

Cimitith

.............

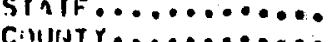

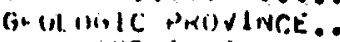

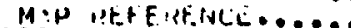

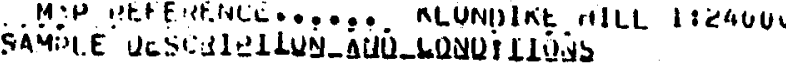

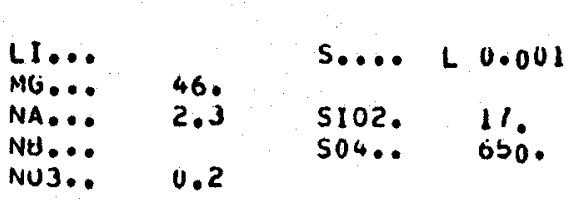

RECUHU OUIS5

CUUEULUELS

43-16.93 i 110-01.13 W

UIM ZUNH... 1 II

INURIHIIVG... 4/924U2. 


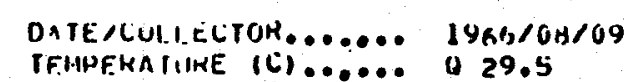

OI'AE SAIALE INFORMATION.. O MAIN SPRINGS. UNIUUE SPECIES OF FISH IN AREA. WATHO AOALLYSIS

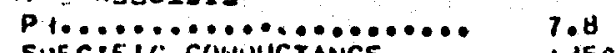

SHECIF IC CONUUCTANCE.... 1250.

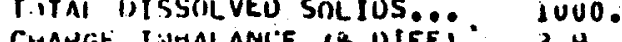

CHAM(it I HALANCE IS DIFF):.. 2.9

a.alisis In phid

Ch......

AL.....

H......

HE....

HE.... 2IS. HE(IñT).

CL.... $\quad 3.2$

2.1

Mti...

52.

LSOLOEES leCONL

PAGE 0084

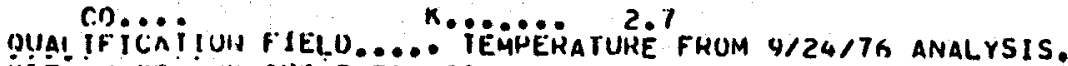

UEEFBE ICE hUU_LUELIEICALLN

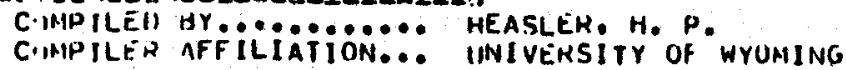

RFFE.ALISCE............ HHECKE.NHIUSE ANU HINCKLEY, IYTO

RECURO 00156

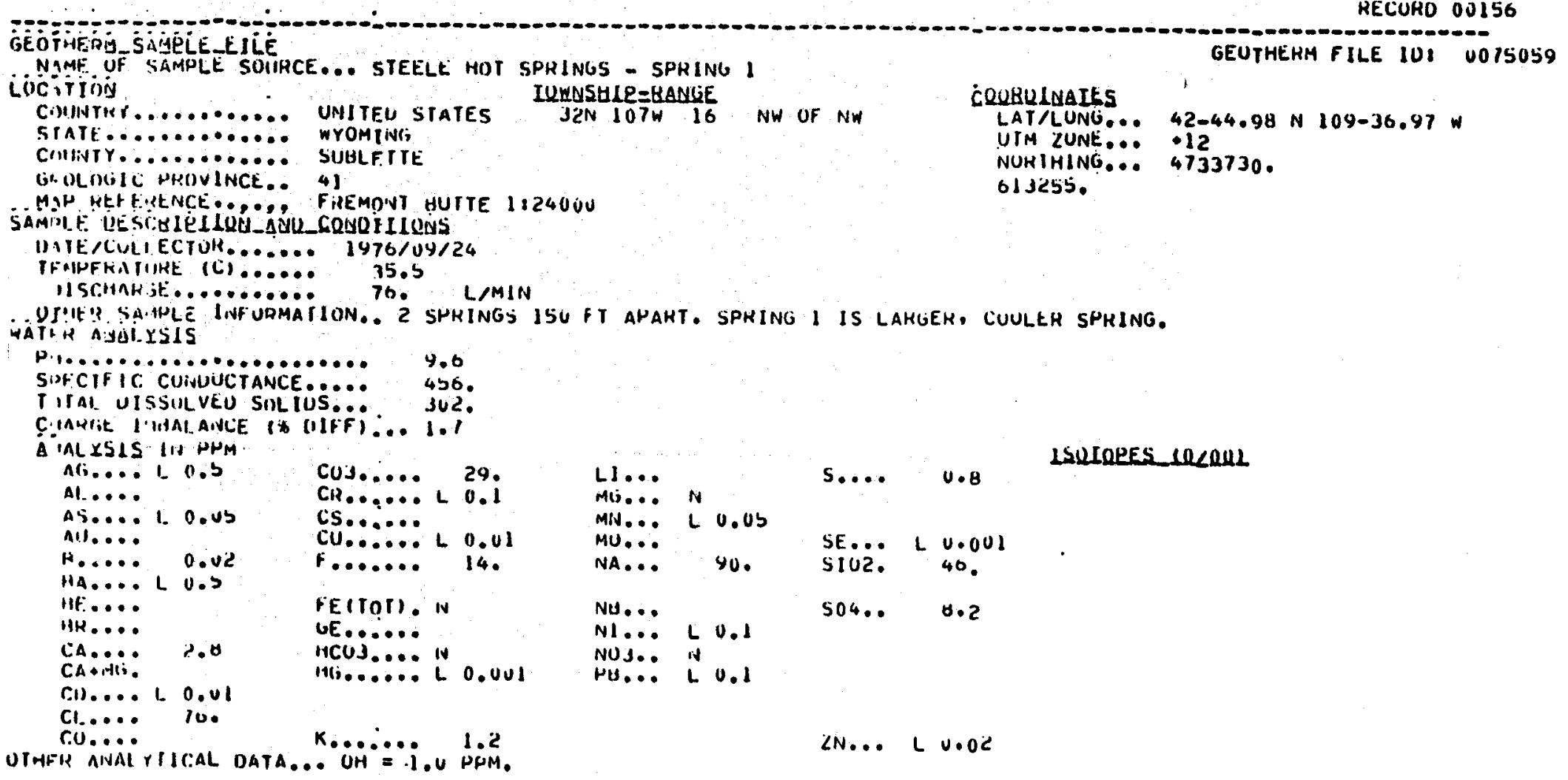

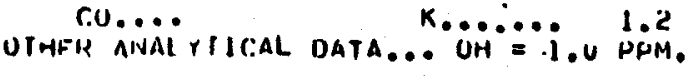




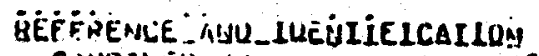

C.MPILEN Ar........... HEASLEH, H. H.

CIMPILER ARFILIATIOH... INIVERSITY OF WYUMING

HFFEAENCF............ ARECKENAIUGE ANU HINCKLEY, 1978

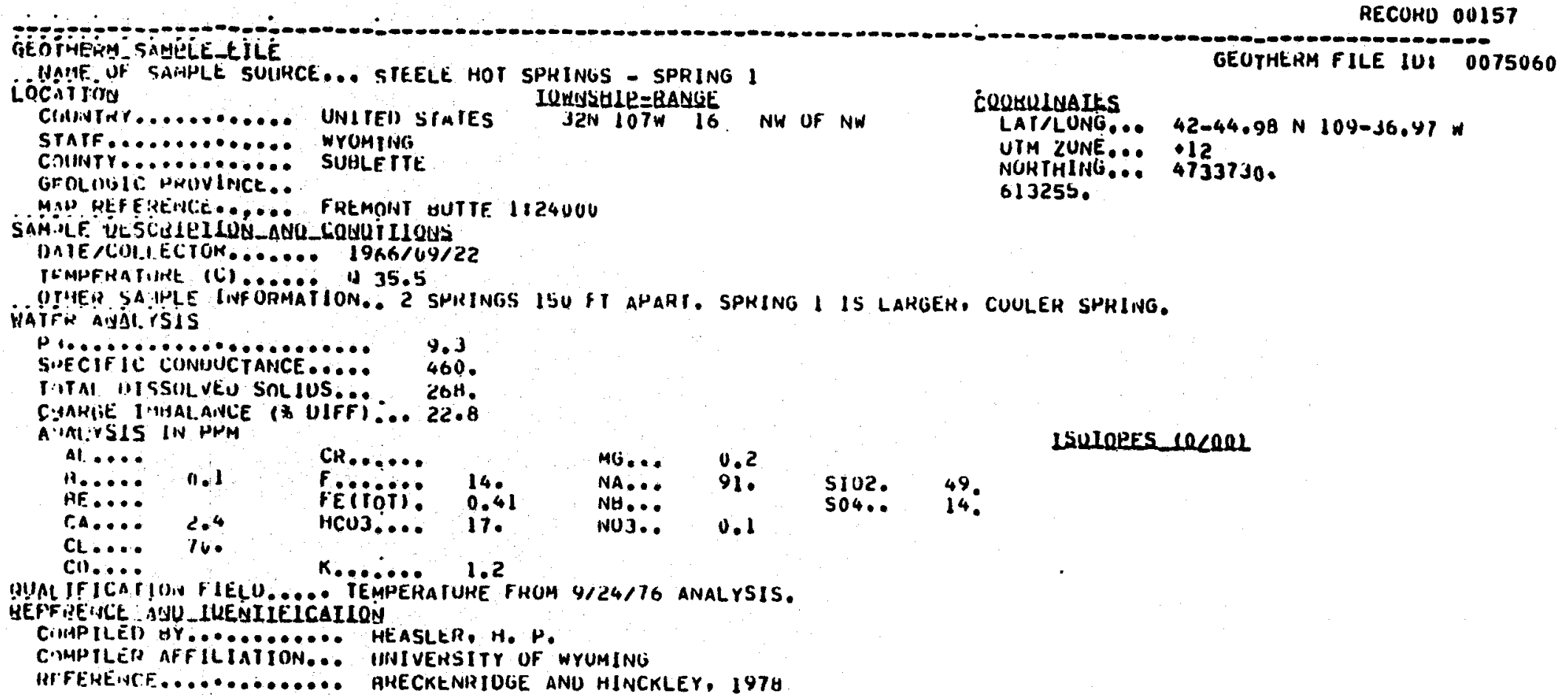

RECUHO 00157

RECURD OU15B

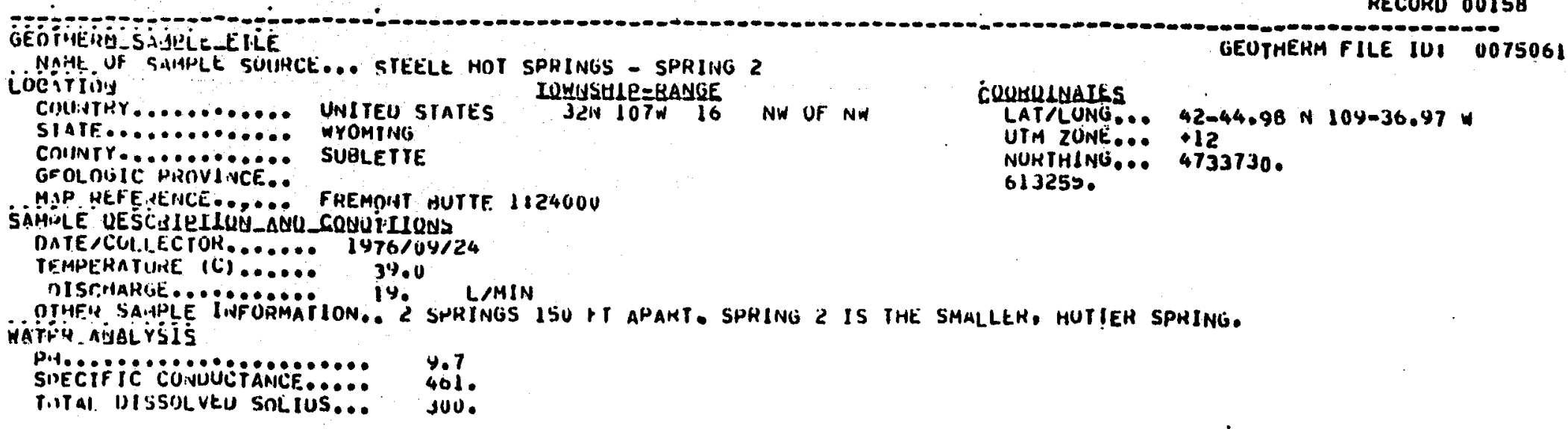

SINECIF IC CUNUUCTANICE...... 401 


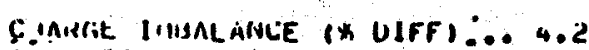

A,Mi.XSTS IN PP:A

Aï.0.0 L 0 o.

IL....

coj.... 30;

LI...

AS.... L L a.us

CH...... L 0.1

CS......

MN.... $L$

All.....

H...... $0.0 \mathrm{~J}$

HA.... L

Ht.....

$411 . . .2$

cA.... 2.1

F.000000 0.U

MO....

....... 14.

Na...

92.

HE(TOT). IN

C.4.iti:

GE. . : . .

HCU3.... N

No...

N1... L 0.1

$1: 0 . \ldots 1.0 .01$

H(i....... L 0.001

NO3.0

PH... L U.I

$$
\text { Cl...... }
$$

K....... 0.9

UEE F̈UE

C. WAH ILEI HY ........... HEASLEH. H.

CWHILER AFFILIATIUN... INIIVERSITY UF WYUMING

REF E.IPENCE............. PHECKENAIUGE ANU HINCKLEY; 1978

KECOHO 00159

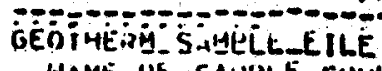

WAME UF SAHPLE SUIACE... AHEHCHOMAIE WARM SPRINGS icienting cintiky.

\section{TULASULE =BAMLE}

$41 \mathrm{~W} 116 \mathrm{~W} 02$ NW UF $5 \mathrm{~W}$

STATE............ WYOMINS

CIIINTY.......... TETON

GFOLIIUIC PAIOVINCE.. 41

MAP HEFE.ATHCE.... GROS VENIKE JUNCTIOH 1824000

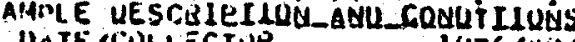

UNTE/CII,I.CCTUR...... 1976/U9/23

TFMPEKATIJHE (C)...... 27.0

.'ISCHARIit........... 946. L/MIN

WATF A AYULLSIS

P.HE IFic CONIUCTARCE....

T.ITAL lIISSUL VEU SOLIUS.

C:IAYGiE I M!IALAIYCE IS UIFF):.. Z.H

AinLXSIS IN PHM

GGOSIS IN PHM

AG.... L 0.5

C03..... in

AL.......L G.US

CH...... L 0.1

A11....

$11 \ldots \ldots 0.1$

CS......

C11...... 0.61

HA..... L 0.3

HE.....

HIR ....

LEITITI. N

CA.... J5.

r.a. ants.

MCOj.... 19, 14.

Ci)... L 0.01

H6...... L $0.0 \mathrm{~V}$

cri....

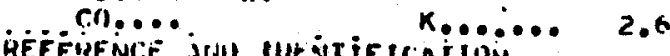

C. MMH ILED UYY

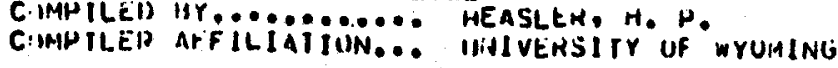

\begin{tabular}{|c|c|c|c|}
\hline $\begin{array}{l}\text { L.... } \\
M G . \ldots \\
\text { HN.... } \\
\text { MU... } \\
\text { HA... }\end{array}$ & $\begin{array}{l}17.05 \\
0.0\end{array}$ & $\begin{array}{l}\text { SE... } \\
\text { SIOZ. }\end{array}$ & $\begin{array}{l}0.002 \\
14 .\end{array}$ \\
\hline $\begin{array}{l}\text { NH... } \\
\text { N1.... } \\
\text { NU3... } \\
P G . . .\end{array}$ & $\begin{array}{ll}L & 0.1 \\
1.9 \\
L & 0.1\end{array}$ & $504 \ldots$ & 20. \\
\hline
\end{tabular}

ISULUEES LQCUUL

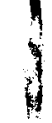

$S E \ldots L 0.001$

SO4.. I1.

2N... L U.02

GEOTHEHM FILE IOI 0075063

EROBDLALITS

LAT/LUNG... 43-32.75 N 110-44.37 N

UTA ZUNE... \$12

NUHTHING... 4821249.

521045 .

\section{1.}

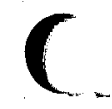


RFFEHLHCF............. ARECAEINHIOGE ARU HINCKLEY, 1978

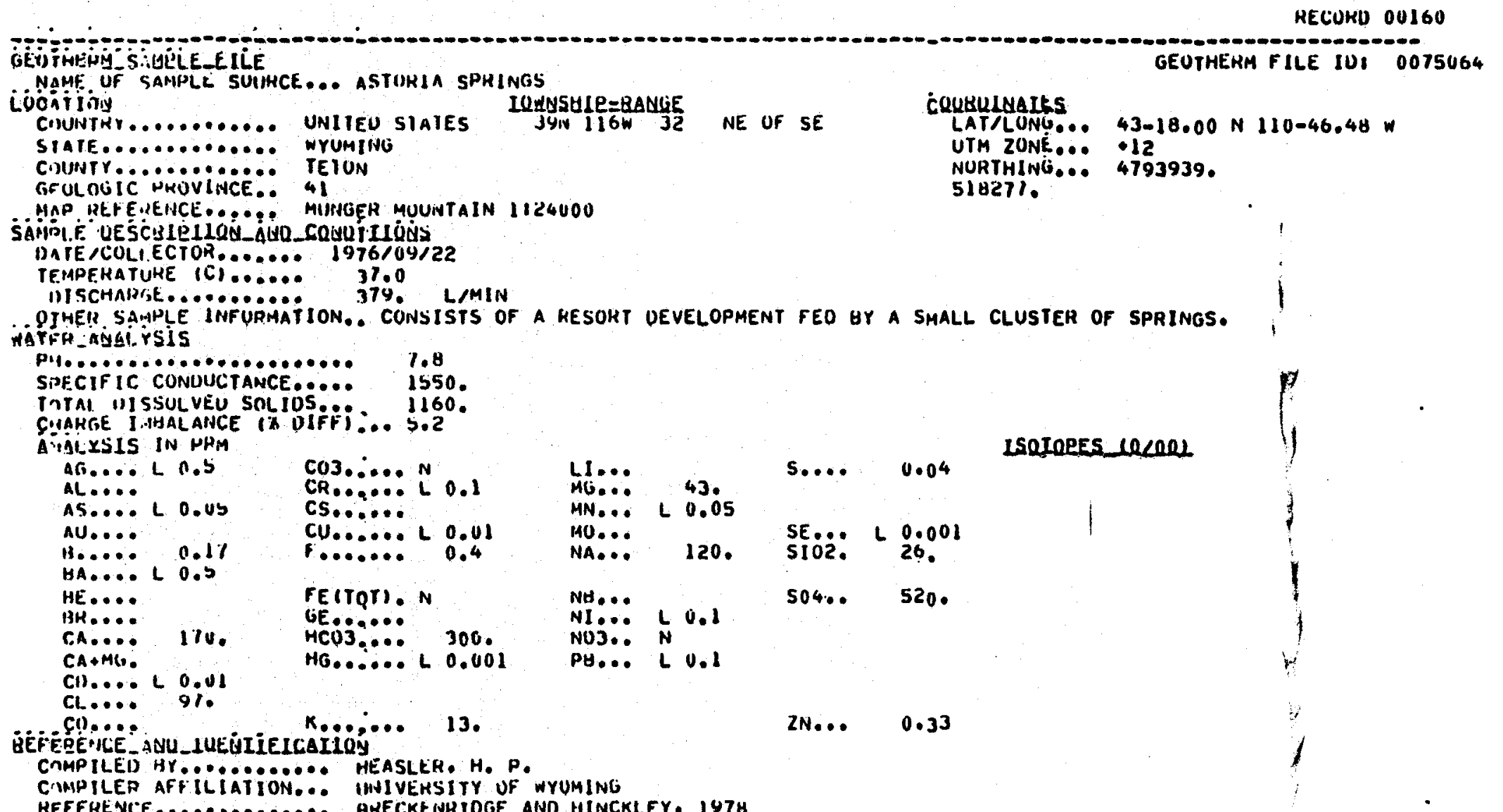

RECUKO 00161

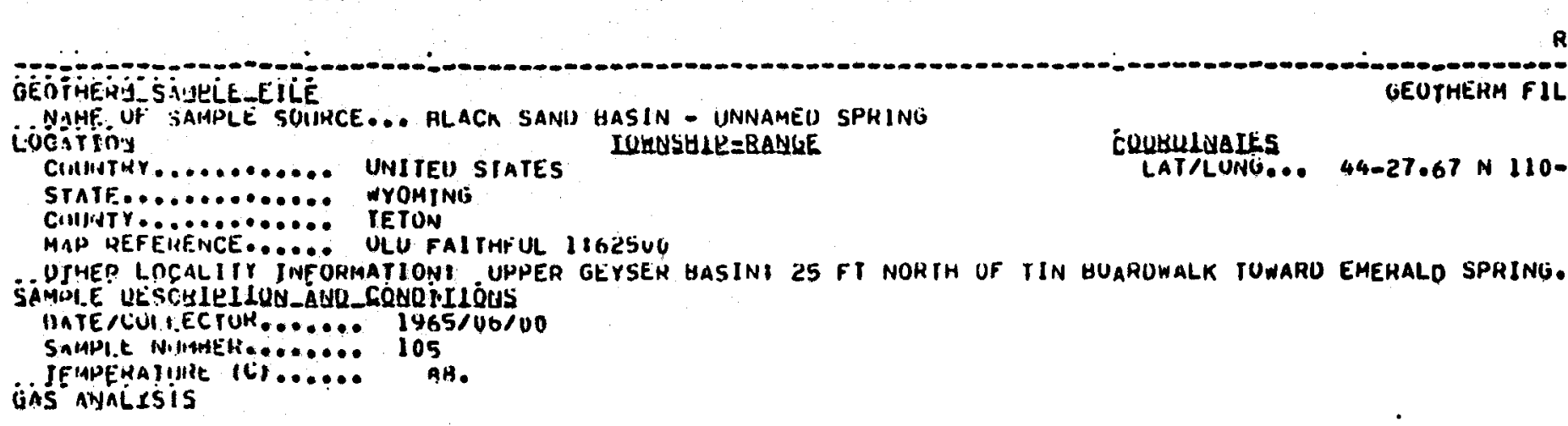

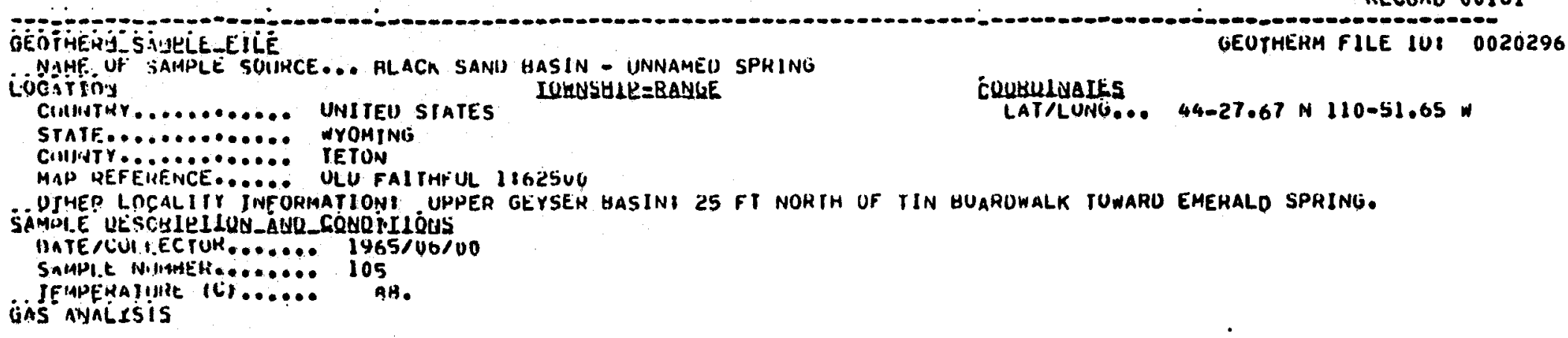


DIIE/AIALYST..... 1965/06/UU GUNTER AND MUSGRAVE

A AILYSIS IN MULE \&

CH4.. 1.1

CPith.

chis..

Ha.

0.4

N2... 10.7

OTHFH ANML YIICAL DATA... PH (EST. WITH PH PAPER) =

OUAL IFICA IION.FIELU, O.. OZ = 02 . AH.

HEFFeE

CIBAPILEU IY........... LAWSON, WILLJAM A.

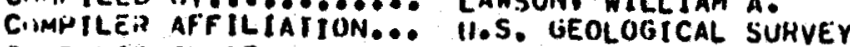

CIMPILËH CHUSS INNEX... NEH-2UT

RFFEREIICE............... GUNTEH ANU MUSGRAVE, 1966

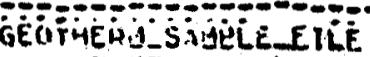

NAPE UF SAMPLE SUUHCE... HLACK SAND BASIN - UNNAMEU SPRING

Luoitiols

CIINTHY........ UNITEO STATES LOUUSHLE=RANGE

STATF............ WYOMINL

CINUNTY............ TETOM

MAP NEREREINCE...... ULU FALTHFUL 1862500

SIIYEY LOCALIIY INFQRMATIUN: NUHTH OF SPOUTER SPRING, UPPER GEYSER HASIN.

DLE UESCHIPIION

UATE,COLLECTOR...... 1930/00/00

SAMPL E NIMALEH....... 14

GAS. ANALYSIS

ANALYSIS IN VULIIME

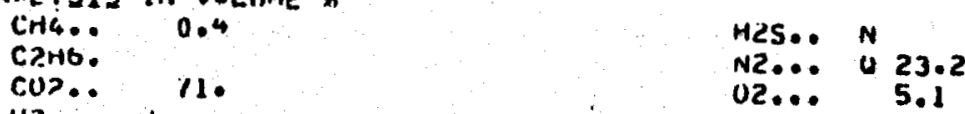

H2 ... in

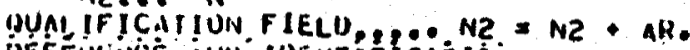

REFFHE VIE ALUL_LUEIIELCAILÓN

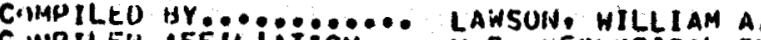

C.MPILEN AFFILIATION... U.S. WEULUGICAL SURVEY

C MANILL.R CHUSS INOEX... NEH-176

REFEREHCF............. ALLEN AND DAY, IYJS.

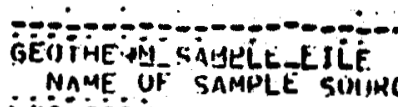

NAME UF SAMPLE SUIIRCE... AOYLES HILL SPRINGS

\section{Lociningy}

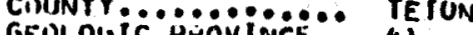

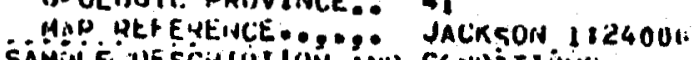

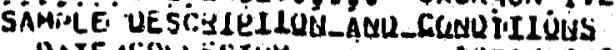

DATE COU I ECTUK.

IFAPERAIIHE (C)...... 311.0

IISCHAHTiE ......... IdY. LAMIN

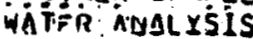

RECOHO 00162

ISUIORES 100402

GEUTHEAM FILE IU: 0020245

CQUBULUAIES

LAT/LUNĞ... 44-27.7 N 110-51.3 W

RECUKD 00163

LSOIOHES toncol

LE IOI 0075065

GEUTHERM FILE IUI 0075065

CQQBULYAIFS

LAI/LUNG... 4J-28.30 N 110-50.08 N

UTM ZUINt... +12

NUKIHING... 4812992.

513373. 


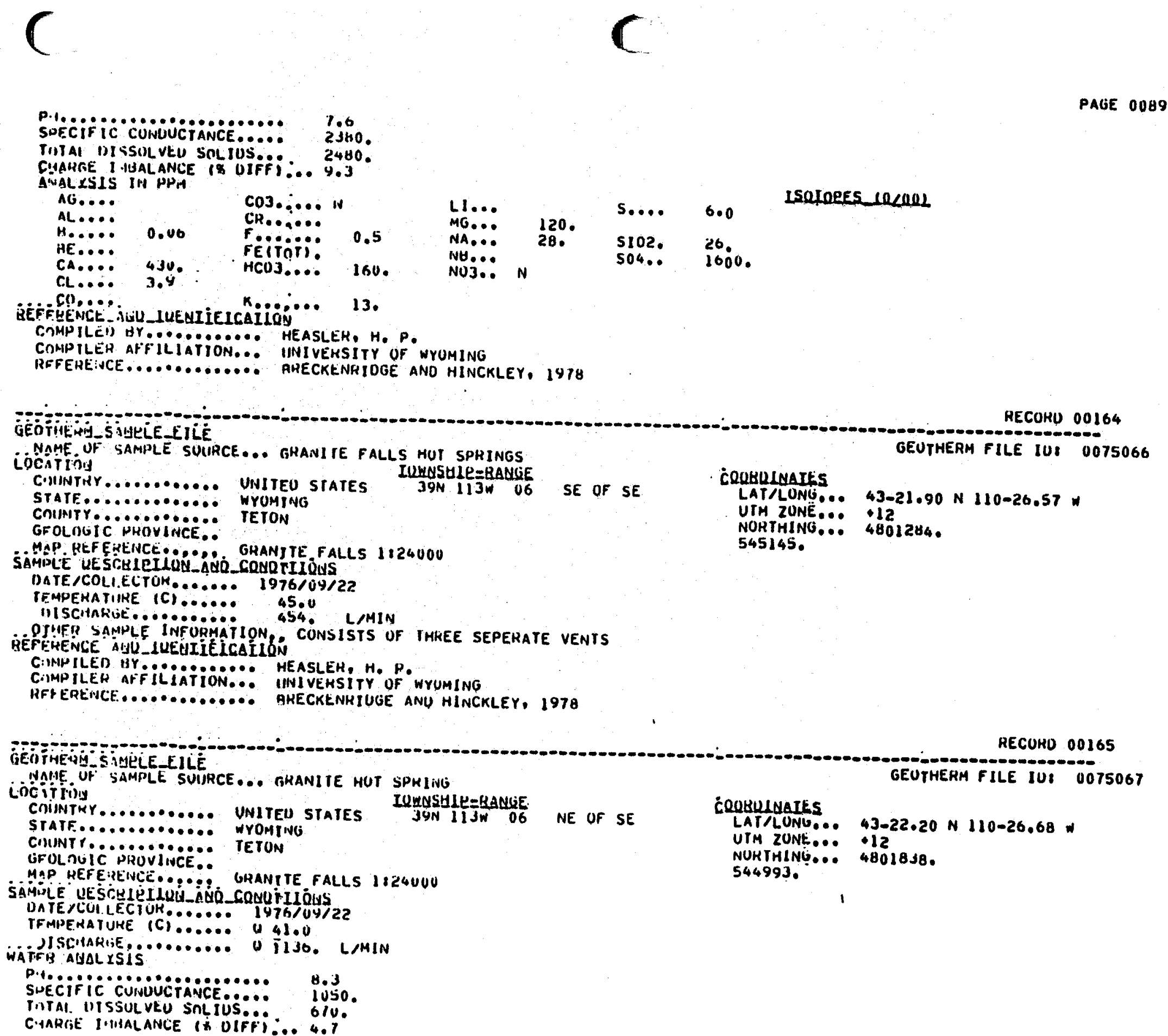$$
\text { co,.... }
$$

\section{CQRBLLAaILS}

LAT/LONGS... 43-21.90 N 110-26.57

UTH ZUNE... 112

NORTHING... 4803284.

545145.

NAME UF SAMPLE SUIIRCE ... GRANITE HUT SPKLIVG

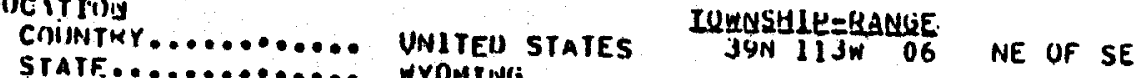

STATF............. HYMTING

CIIUNT T............. TETON

GFOLNUIC PRUVINCE.

MAP REFE:JE'NCË..... GRANTTE FALLS $18 Z 4 U U O$

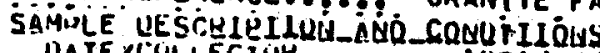

DATE YCULLECTUK...... 1976/U4/22

TFMPERATURE (CI...... U 4 41.0

WATISTCIARTEE........ U TIJO. LAMIN

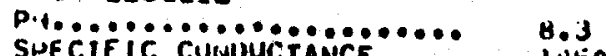

SHECIF IC CUINUUCTANCE..... lUSO.

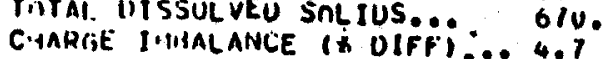


A OnLXS1S IN PPM AG.... L R.S AL.....

AS..... L 0.45

AU.....

H..... 0.61

HA..... 20.5

IE....

म4....

$C A+M G$.

C.1).... L 0.01

CL.... 140.

UYAL IF ICAI JWIN FIELO CHENCE-AUU_LEUIEICAIION

CIIMPILEI) HY CIMP ILEN AFF

CrMAPILEN AFF ILIATION... UNIVERSITY OF WYUMING

RFFEREINCE....

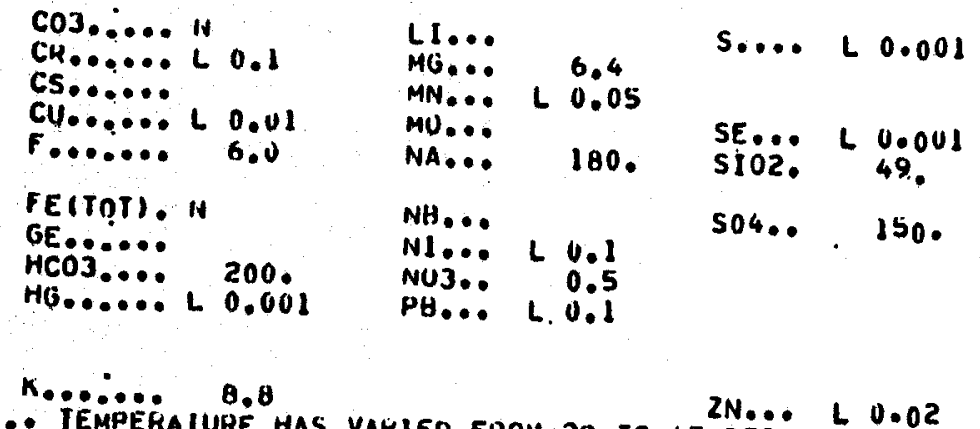

PAGE 0090

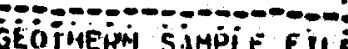

AECURO 00166

iOCAME OF SAMPLE SPIHCE... GRANITE HOT SPHING

GEOTHERM FILE 1010075068

Cociriva

CIINTHY............ UNITEU STATES LUWWSHLP=BANGE

STATE........... WYOMING

39N $113 W$ W 06 NE OF SE

GFOLOOEC HKOVINCE. TETON

GIST HROVINCE.. 4

SOMP TEFEREINCE. GRANITE FALLS 1824000

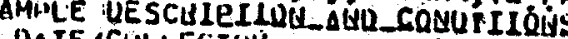

OATE/CULIECTUH....... 1973/10/02

IEMPERAIULE (C) ..... 39.0

WATER AYVALÝSIS

P.4................... 8.0

THTAI IISSULVEU SOL

CHAHTE IHALAIYCE SNLTUS.0. 547.

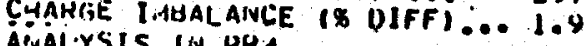

VALYYSIS IN PP:A

\begin{tabular}{|c|c|c|c|c|c|c|}
\hline $\begin{array}{l}{ }_{H} \ldots \cdots \\
H H_{\ldots} \ldots \\
C A \ldots \ldots \\
C L \ldots\end{array}$ & $\begin{array}{l}0.53 \\
32 . \\
1.40\end{array}$ & 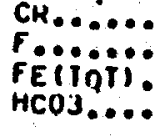 & $\begin{array}{l}5.3 \\
182 .\end{array}$ & $\begin{array}{l}\text { MG... } \\
\text { NA... } \\
\text { NH... } \\
\text { NU3.. }\end{array}$ & $\begin{array}{l}5.8 \\
160 . \\
0.2\end{array}$ & $\begin{array}{l}\mathrm{SlOL}_{1} \\
\mathrm{SO}_{4} \ldots\end{array}$ \\
\hline
\end{tabular}

ISUIORES Lecan

ISilapeschenal

NU3.: 0.2

ISOIDEES 10.002

\section{CROBOLNAIES}

LAT/LUNG... 43-22.20 N $110-26.68 \mathrm{~W}$

UTM ZUNE... 12

NOHTHING... 4801838.

544993 .

\section{8}

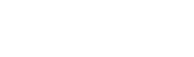

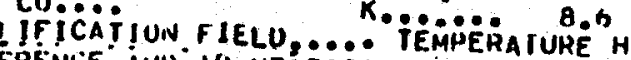

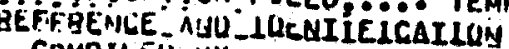

CIMPILEU UY

MYUMING

RFFEHENCE............. ARECKENRIUGE ANU HIINCKLEY, 1978

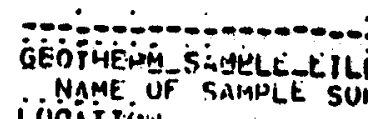




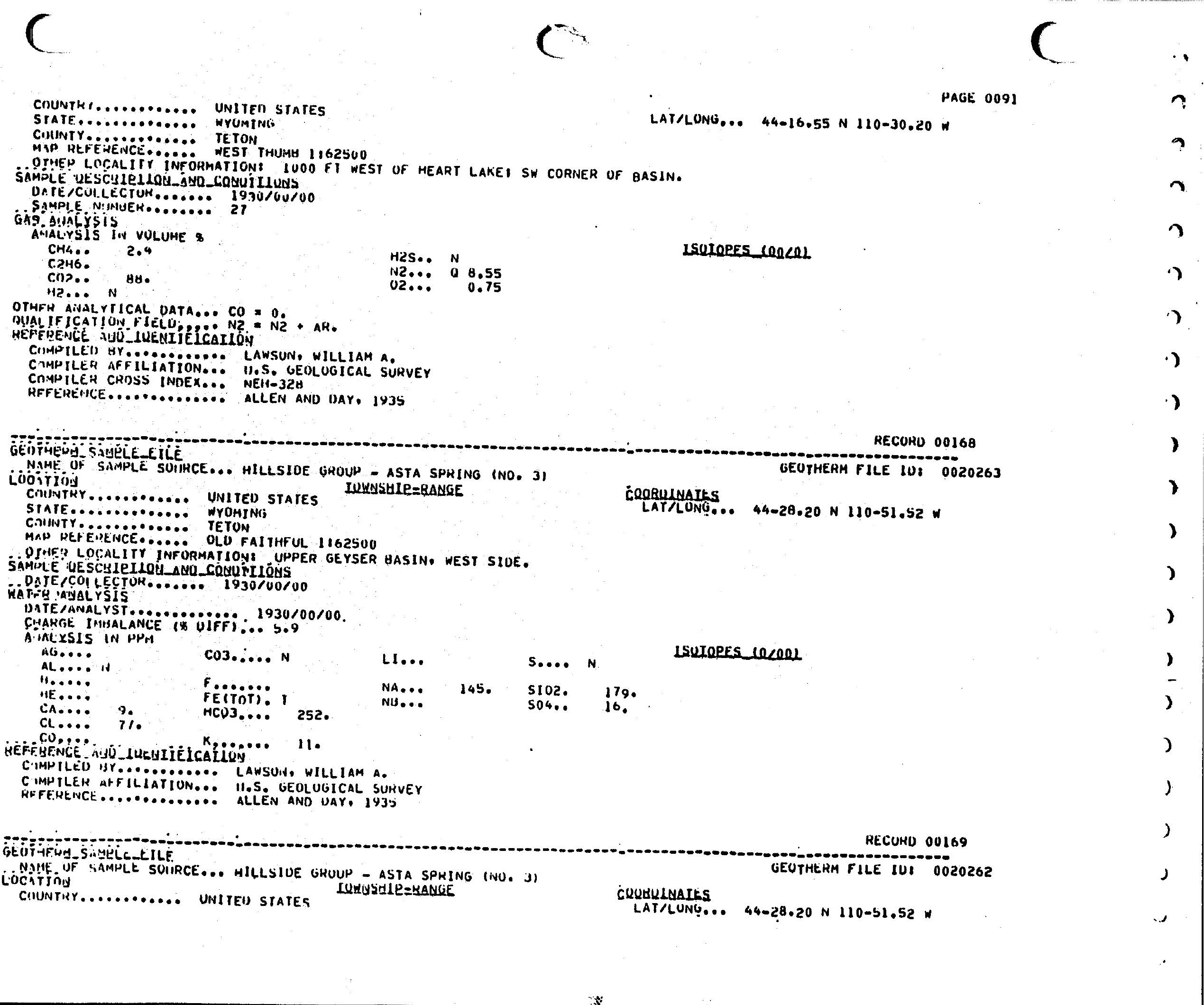




\section{STATE............. WrOMTNG} CIUNTY........... TETON

page 0092

MAP NEFERENCE...... OLD FAIIIT UL 1362500

SAMTIEY LOCNLITY INFOHMATIONI UHPER GEYSER BASIN, WEST SIOE.

法

12ATE CCHIECTOH....... 1906/00/00

GÁS ANALYS IS

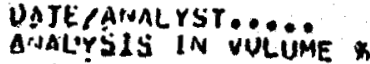

CHit.: 3,3

3..

CP? 2110

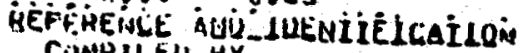

CIMPILED HY.........

C.MPILER AFFILIAIION... M.S. GEOLUGICAL SUHVEY

HFFEUETCE.

NHILLIPS, $F, C$.

Has.. $N$

N2... 62.34

6.3

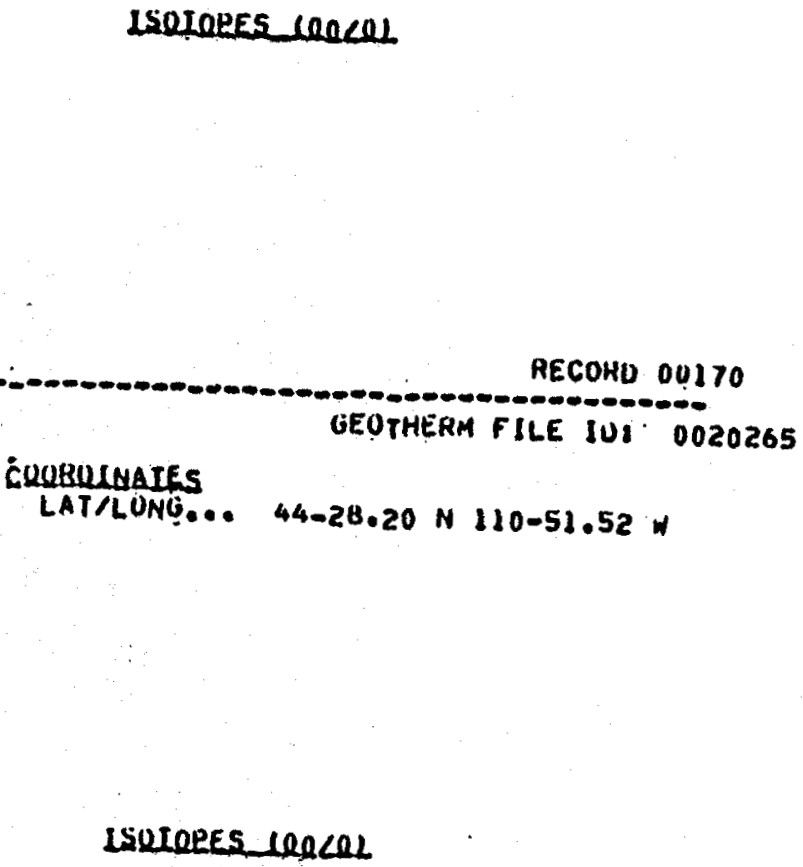

LOTORES_lOCLLL

.

IHE AWAL PTICAL MATAP PH (EST. WITH PH PAPERI $=6.4$.

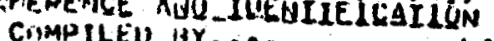

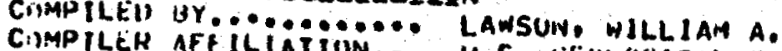

CIMMO ILER CHUSS INDEA.." II.S. WEULOGICAL SURVEY

RUNTER ANIS , AUSGKAVE, 1966 


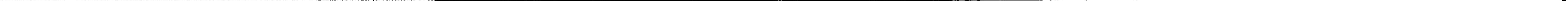



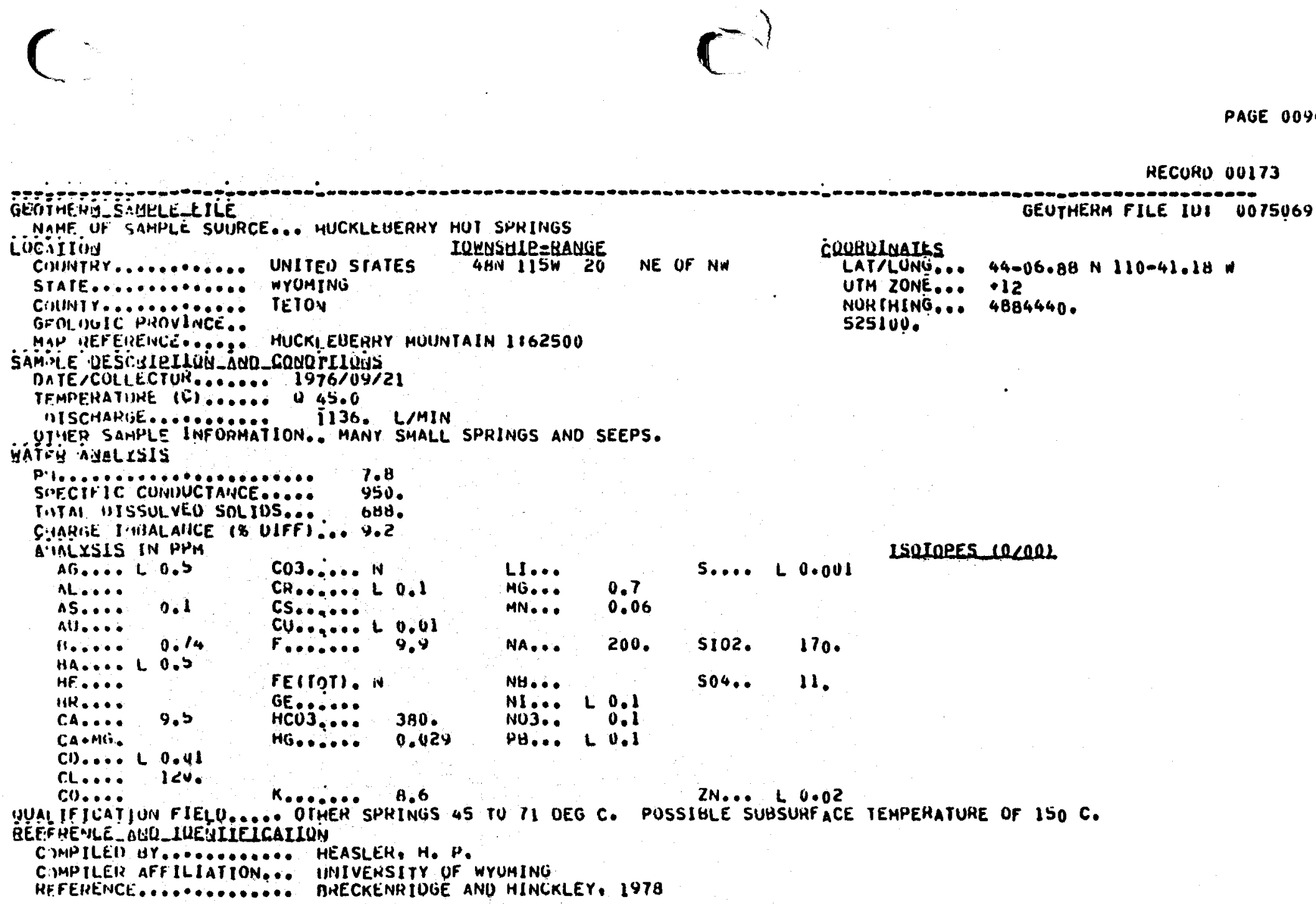
TITAL DISSULVEU SOLIOS... AJT.

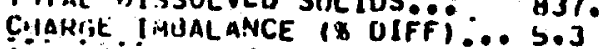

AiALYSTS IN PPM

AG.... COB.... N

CH.......

HE....

He....

FËiöii:

140

chon

HCO3....

K....... 14

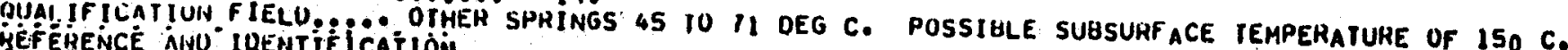

HEF EQRENCE AUU ILEUITÉLCAILOON

COMP ILEEI UY

CIMPILEP AFFILIATION..: UIIVEHSITY OF WYUMINO

HFFERENCE.............. HHECKENGIUGE AND MINCKLEY, 1978

PAGE 0095

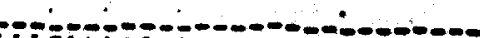

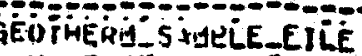

NAME UF SAMPLE SOIJRCE.

cooijionis

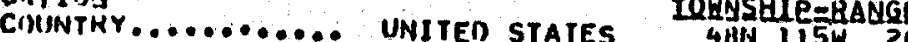

STATF............ WYOMING

4ON 115W 20 NE OF NW

GFOLUGIO. PRÖPINCE.".

MAP REFERENCE.... HUCKL EGERKY MUUNTAIN 1862500

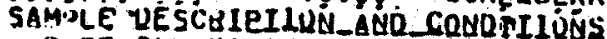

DATE/COLIECTUK....... 1973/10/U2

TFMPEHATURE (C) ..... O 61.0

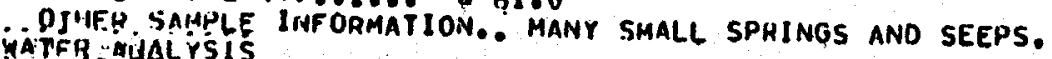

PAT:AUALSIS

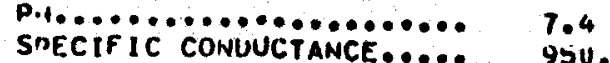

TiTTAI. "ISSOLLVO SOLIOS... G13.

C:AAHEE I MHALANCE 18 UIFFi:.. 3.1

Q IAL:YSIS IN PPH

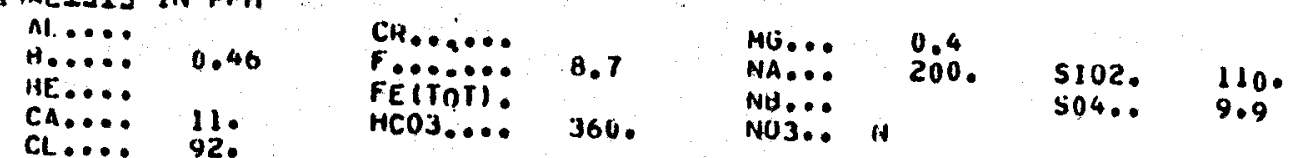

LSOJORES WeEQU

RECOHO 00175

LSULOEES COPOOL

Cn.... K........ B.t

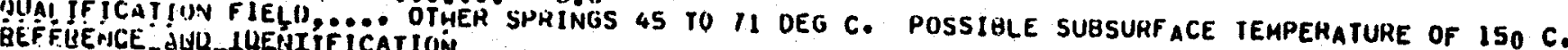

COMPILEO dUL

COMPILED dY

CMMPILEP AFFILIATION... INIVEKSITY UH WYUMINO

RFFERENCE.............. AKECKENRTULIE ANO HINCKLEY, 1978

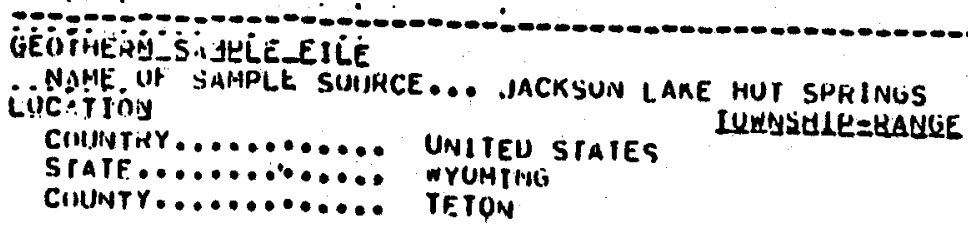

RECUKU 00176

GEUTHEKM FILE IUI 0075073

CUUGULMALES

LA1/LUNG... 43-57.50 N 110-41.75 N

UTM ZUNE... +12

NUKTHING̈... 4867074 . 


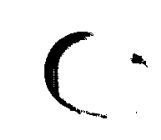

GEOLOBII: PHOVINCE.. 41

MAP REFEHENCE..... COLTFH HAY 1124000

PAGE 0096

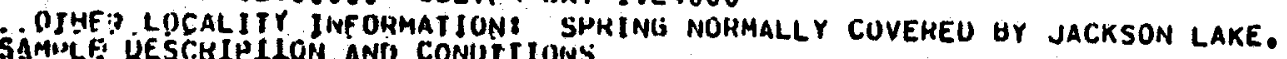

DATE/COU I ECTUH

TFMPERATINE (CI....... O 72.0

IISCHARTE........... U U

OTIER SAMPLE INFURMATION. LOW LAKE LEVELS HAVE EXPOSEO A MUMBER OF SPRINGS.

DUALIFICA I IUI F IELU

C:IMP ILEII HY CIMPILER AFFILIATIONOO. UTI

CIMPILCH HY........... HEASLER, H. P.

CIMPILE! AFFILIATION... IJNIVERSITY OF WYUMING

HFFEREINCE............ ARECKENAIOGE ANU HINCKLEY, 1978

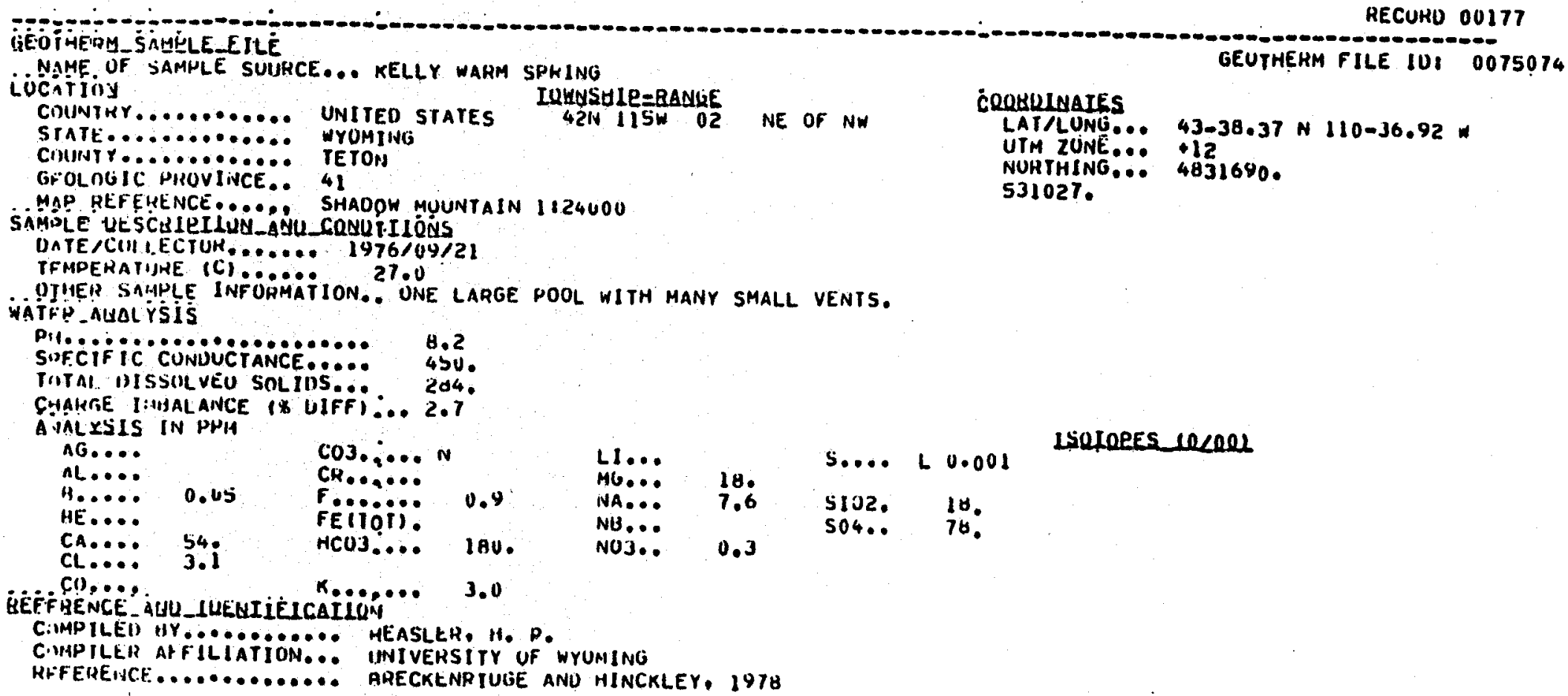

HECORU 00178

NAME UF SAIAPLE SUIJHCK... I.UNEK GEYSEK HASIN - IMHERIAL GEYSEK

ióäiliby

CINUTHKY.......... UNITEU STATES LUWUSULP=EANUE

STATE............ WYUMTHE

C.IINTYY.

MIN MË EHEINCE...... MADISUN JUIVCTIUN 1862 OUO

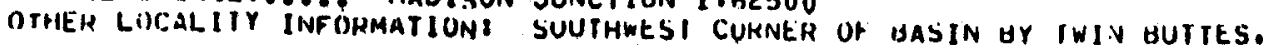




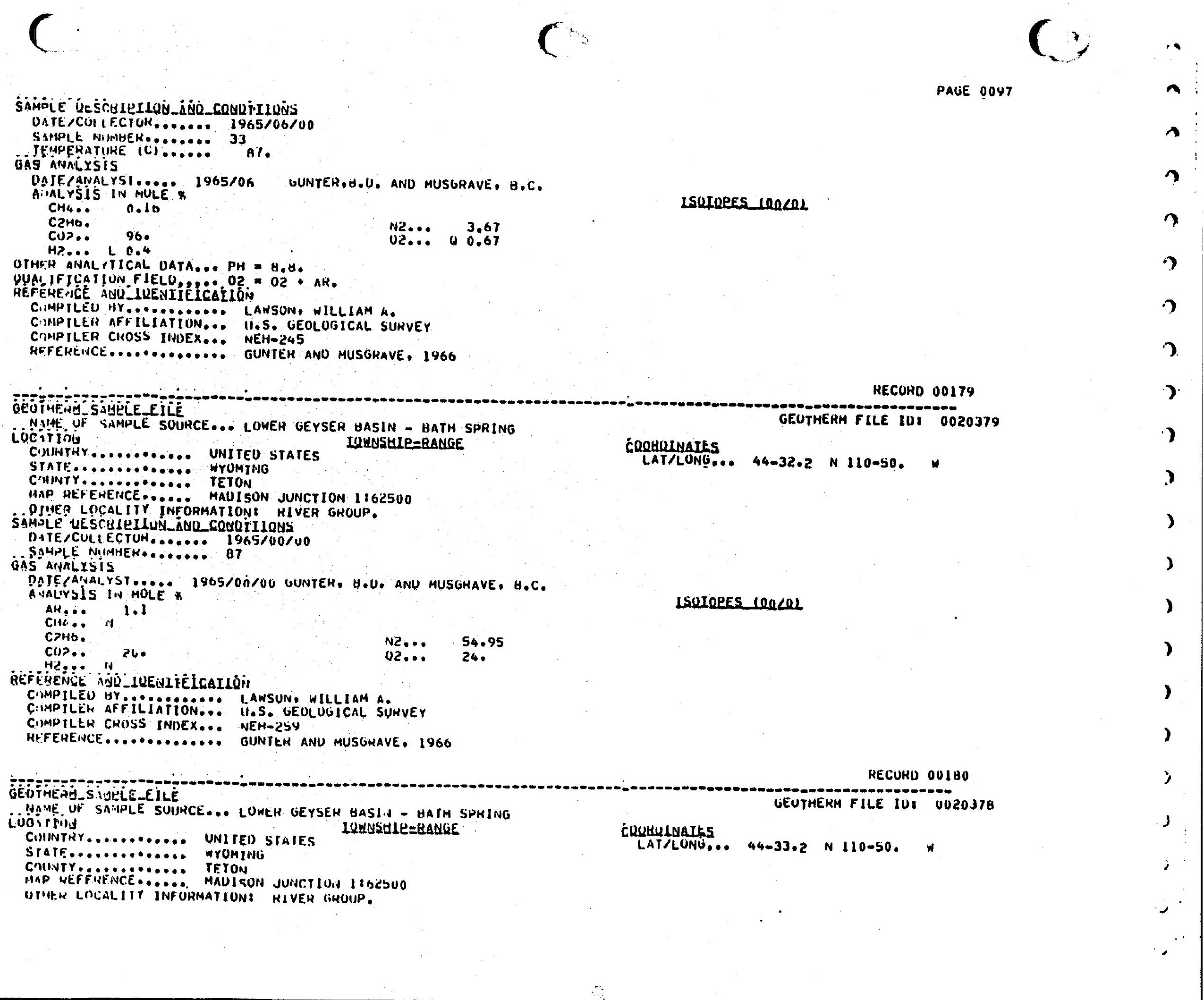




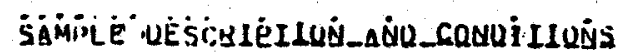

ONTE/COLIECTUK,...... 1930/UU/UO

S.MPLE NIMUEH....... 22

GAS ANALYSIS

A.jALYYSIS IN VULUME $*$

CH4.: 0.35

C1)2.

0.5

IF jCATIUN FIELO

N2 $=\mathrm{NZ}+\mathrm{AH}_{0}$

C.MENCE AUU_JUEUIIEICAIIUN

CIMPILEN HY........... LAWSUN, WILLIAM A.

CIMPILER AFFILIAIIUN... H.S. LEOLGGICAL SURVEY

CIIMPILER CHOSS INIIEX... NEH-236

LSUIUPES LReCL

N2, . O 12.85

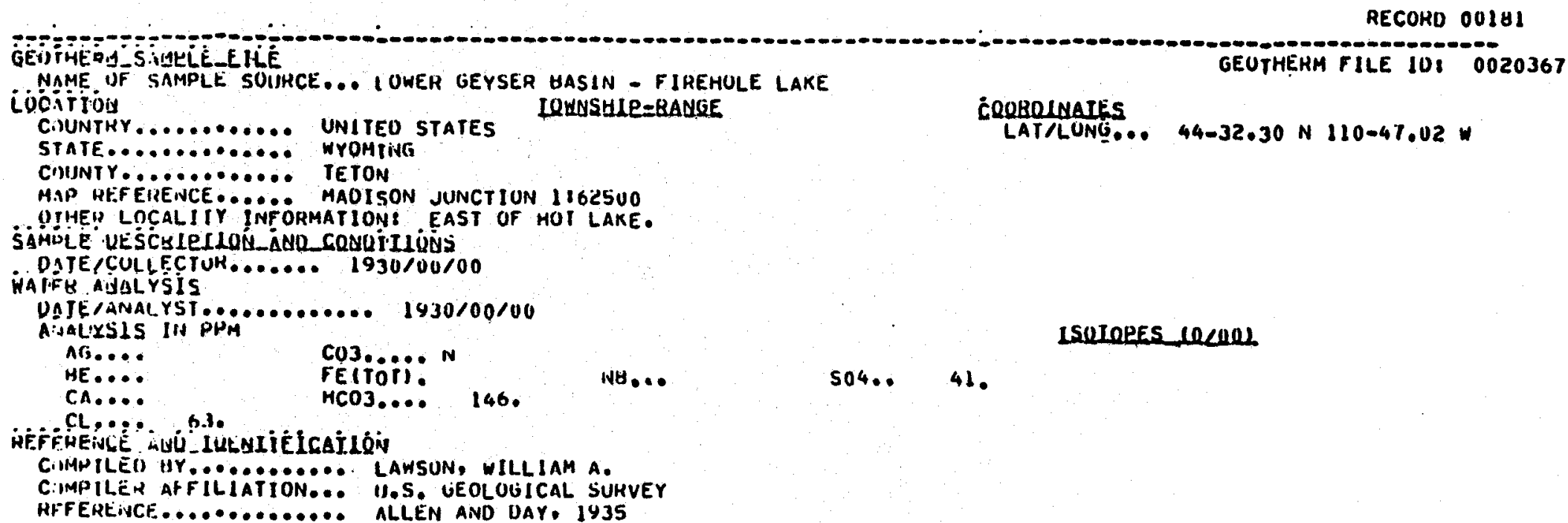

RECURO 00182

STAIF............ WrOMIHL

CMINTY............ TETUN

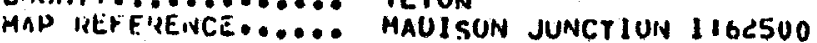

У]HE! LDCALITY IINF URMATIUN! EAST OF FIKEHOLE LAKE.

SAMIILE UESCEIRILUA_AND_CRMUTILOUS

DITE /LULIECTUK....... IYG5/06/UO ISUNIEH ANU MUSGRAVE

SIMPLE ININHEH....... 28

SEMPERATIINE (C)

90.5

GAS AMALYSis

DITE/AWALYST..... 1965/06/UU GUNTER. H.U. ANU MUSGRAVE, H.C.

\section{CUQRULAALS}

LATLUNG... 44-32.30 N $110-46.98$ N 


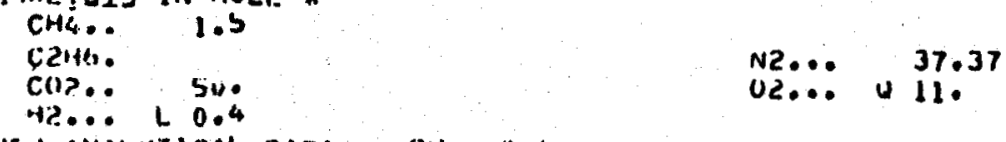

UTHE. ANAL PTICAL DATA... PH $=8.4$.

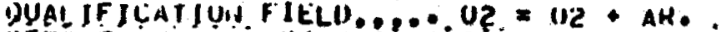

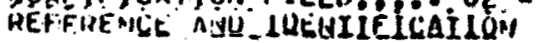

C IMH ILEII GY........... LAWSUIN. WILLIAM A.

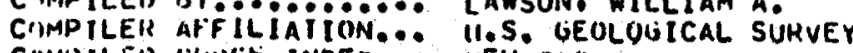

CIHHILER CHUSS INDEX... NEH-24O

H.FENTNCE.............. GUNTEH ANU MUSGRAVE. 1966

GQTHE

RECOKO 00183

GEUTHERM FILE IU: 0020360

NAME UF SAHPLE SUIJKCE... LOWEH GEYSER HASIN - FIREHOLE POUL

Licioifing

CIINTKY
UNITED STATES IUWUSHLERANGE

STATF.

CIINTTY......... TETON

MAP REFENENCE...... MADISON JUNCTION 1802500

OIHE! LOCALIIY INFORMATIONI EAST OF FIREHOLE LAKE.

SAMFLE UESCHLPLLUN_ANU_COQUKILUWS

DUTE, CUI,I ECTOK....... 1930/00/00

SIMPLE NIMUER.......... 25

Gas. Givaizs is

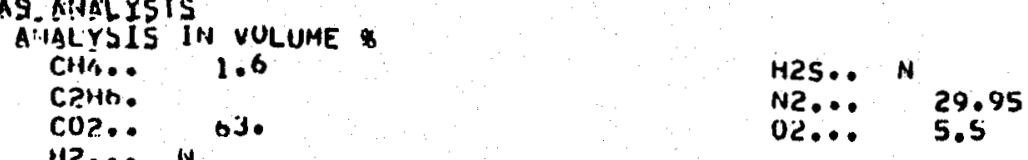

ISOLOEES LenCOL

$\mathrm{CO} 2 \ldots$ b30

$02 \ldots .5 \quad 5.5$

MUAL IF JCAIJUNA FIELU.,...NZ ₹ N2 • AR.

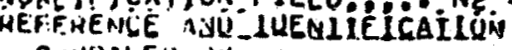

C.MPILE.U BY........... LAWSUN. WILLIAM A.

COMPILFE AFFILIATION... 11.5. GEOLOGICAL SURVEY

CIMPILEH CRUSS INIJEX... NEH-2J9

RECORO 00184

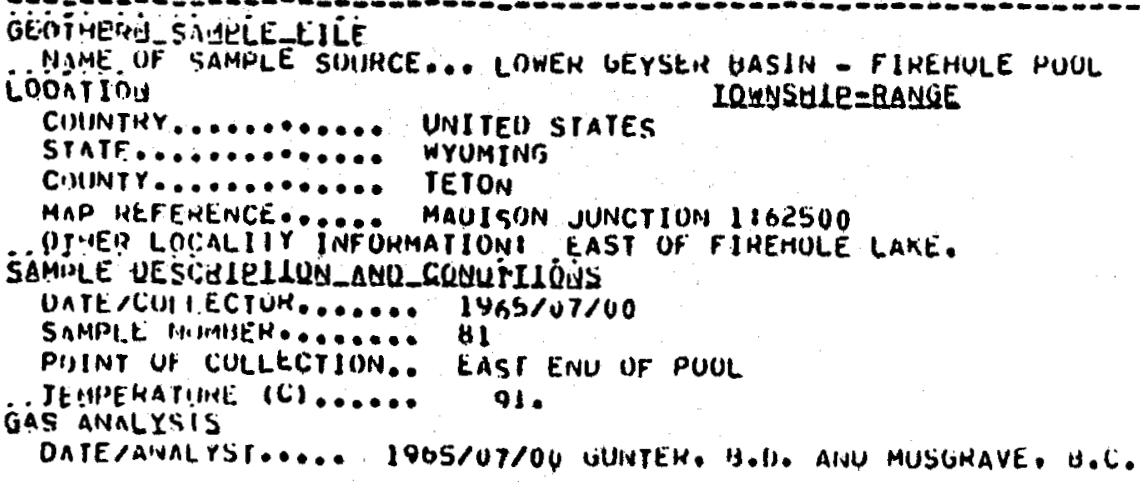

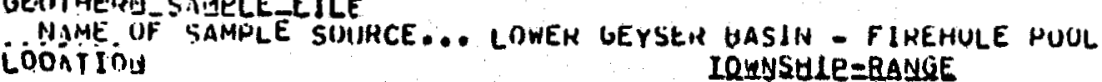

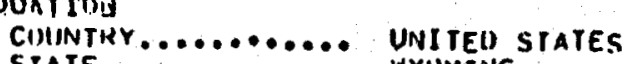
IOWNSULE = BANGE

STATE,

CIIINTY............ TETON

MAP KEFERENCE...... MAUISON JUNCTIUN 1162500

OIME! LOCALIIY INFUHMATIONI EAST OF FIREMULE LAKE.

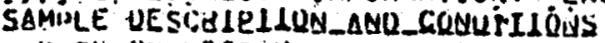

UATE CUI T.ECTUH....... 1465/U7/00

SAMPI.E MHATHER....... BI

pisint uf cullection.. East Enu uf pUUL

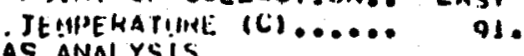

GAS ANALYSIS

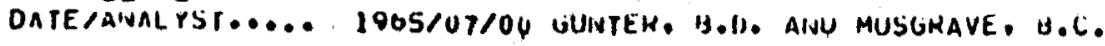




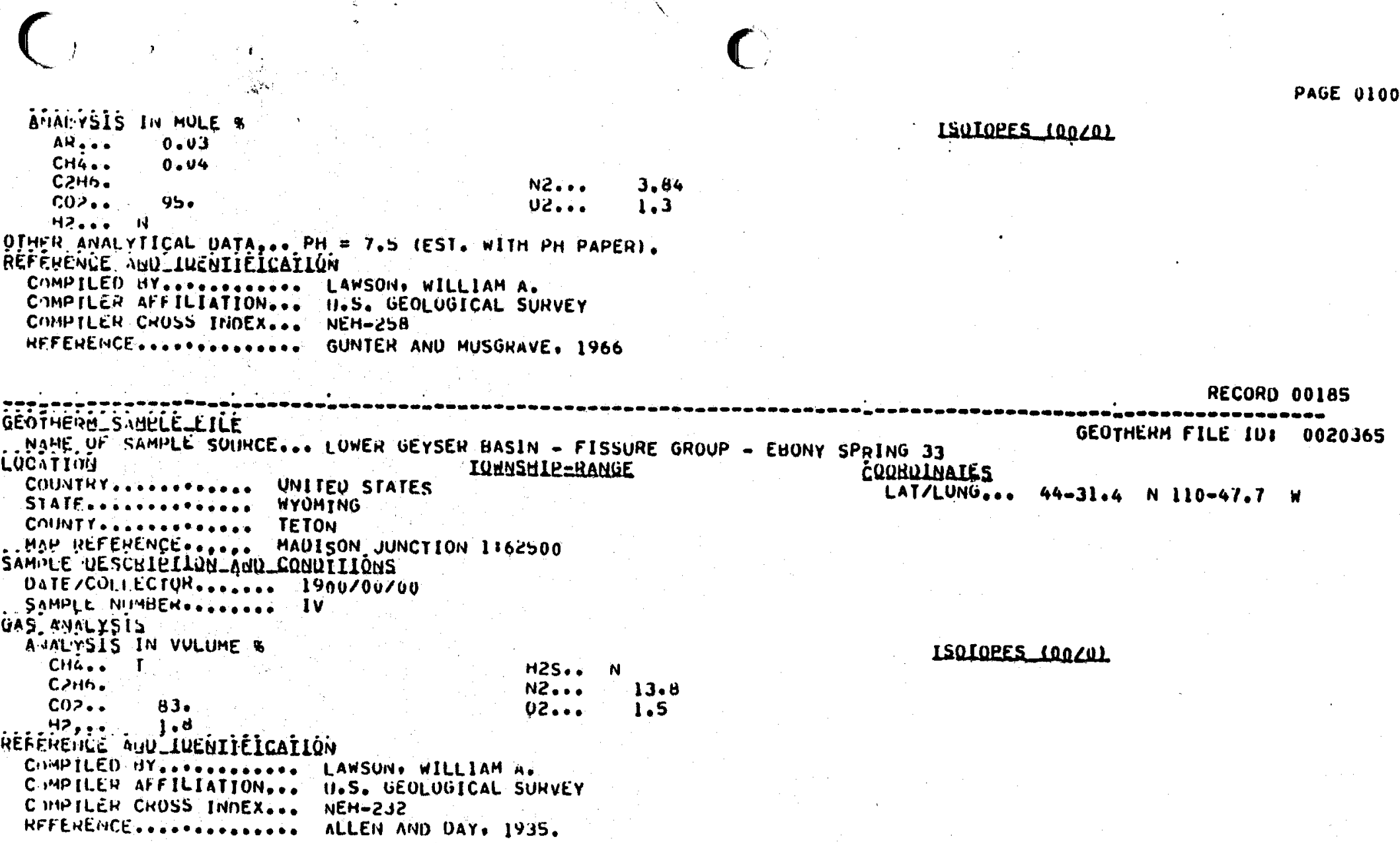

RECURD 00186

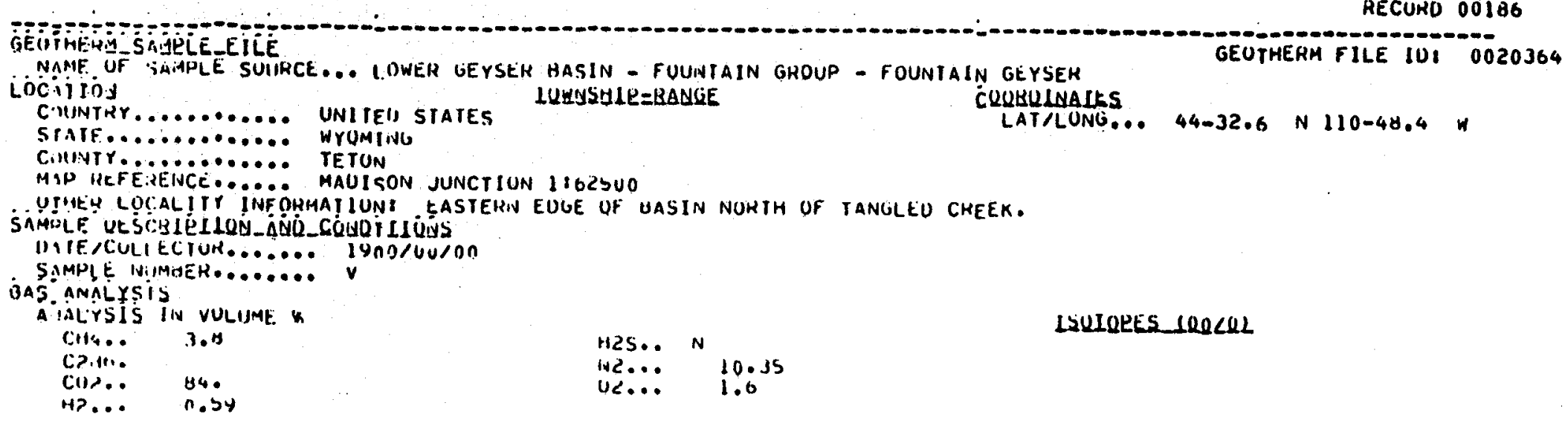

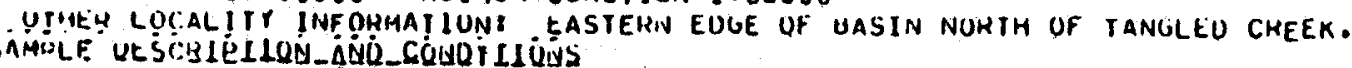

SIMP

AS AMALYSIS
chis.. 3.5
n.

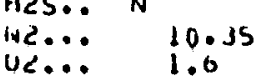




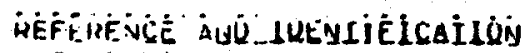

C MAPILEU IY........... LAWSUN, WILLIAM A.

C.MPILE iR ATFILIATION... M.S. GEOLUGICAL SUHVEY

C'MIPILER CHOSS INDEX... MEE-ZJJ

RECURU 0.0187

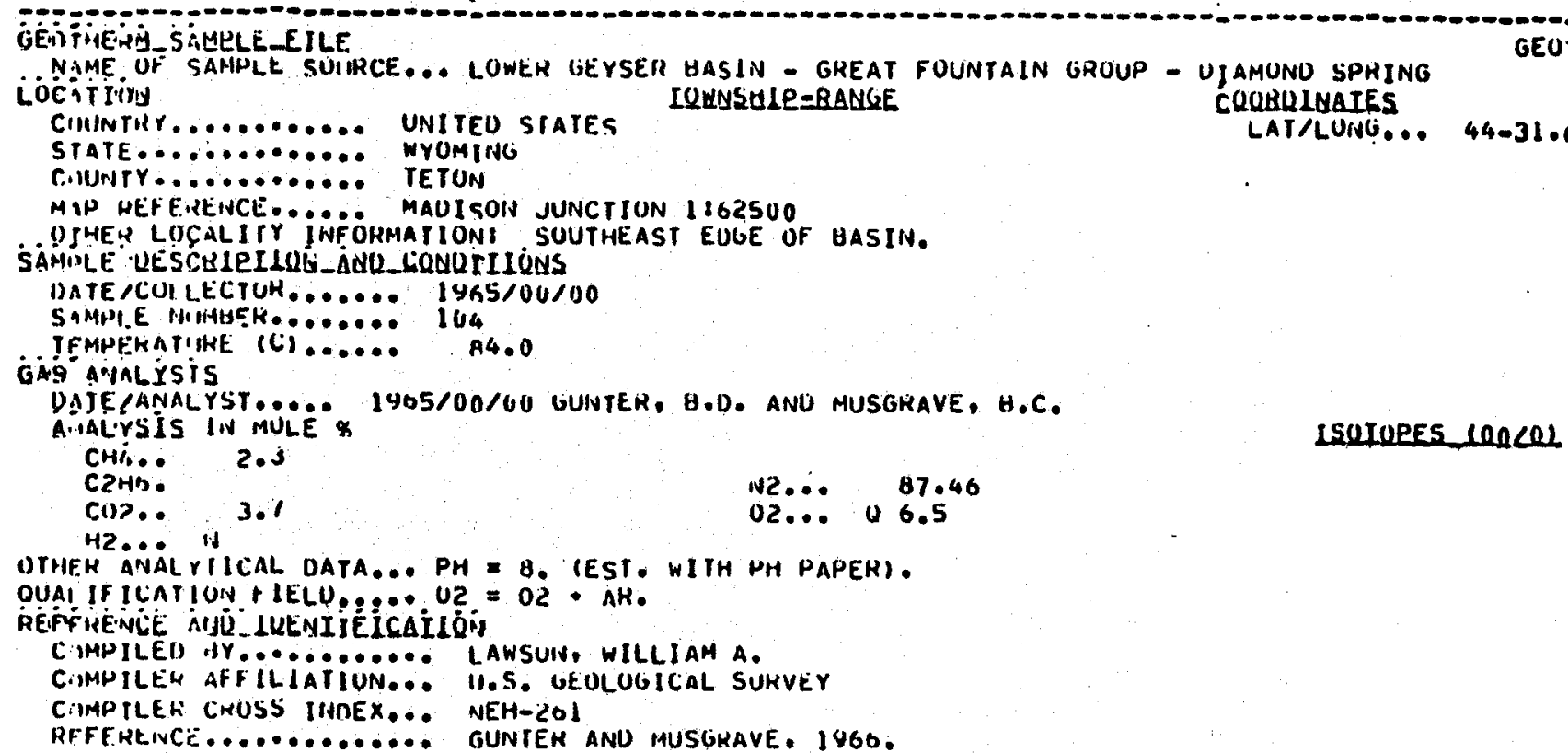

GEMTFE UF SAMLLEE SUIRCE.

iocitivity

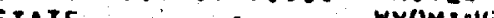

C. MUTH

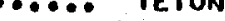

MIP HEF E.REINCE...... MAUISON JUNCTIUN 1862500

DJHEY LOCGALITY JWFOKMATIONI SUUTHEAST EUGE OF HASIN.

SAMINLE UESCBIEILQL_AUL_LQULTIIUNS

DATE/CUILECTUR....... 1965/00/00

SAMPI.E MHIHESK....... 164

TFMPEKATIIKE (C) ....... A4.0

GAO ANALÝSIS

DAJERANALYST..... 1905/00/UO GUINTER, H.D. ANU MUSGRAVE, H.C.

AuAirsis IN MULE

CHi

CHin...

2.3

3.1

N 30

H2...

$\begin{array}{ll}N 2 \ldots & 87.46 \\ 02 \cdots & 6.5\end{array}$

ISUIURES LenCOL

UTHE. ANAL YIICAL DATA... PH = O. (EST. WIIH HH PAPERI

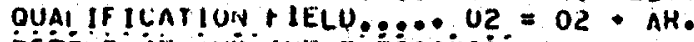

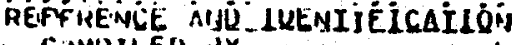

CIMPILED AY........... LAWSUIN, HILLIAM A.

CIMPILEH AFFILIATIUN... 11.5. GEULUGICAL SUKVEY

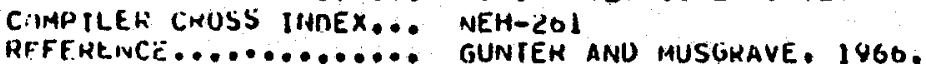

HECUKU $0018 B$

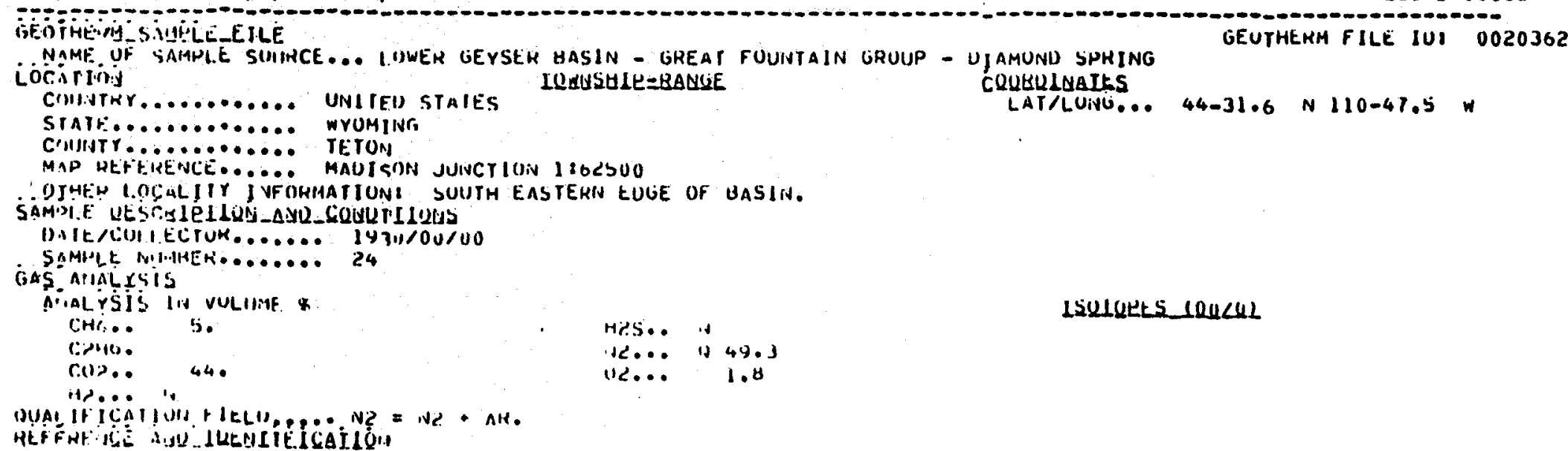


SAILPLE MUIHISH._.... 32 TFMPEHATIJKE (C)...... BY. WAJEH ANAL TSIS

A intoxsis

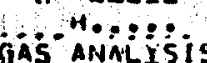

F........

NA...

5102.

0.27

LSUIOPES IRCORL

LSULORES LONCUL

DAYE GANALYST..... 1965/06/00 GIJNTEK, H.D. ANO MUSGRAVE, B.C.

VAL-YSIS IIU MULE क

C240.

N2. . $\quad 0.97$

H2. L 10.4

SUAL IF ICA I IUH FIELO, O U2 = U2

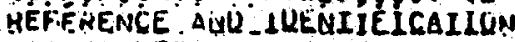

CIIMPILEN AY.......... LAWSUNA HILLIAM A

CIMPILER AFFILIATION... N.S. GEOLOGIGAL SURVEY

C. MHPTLEP CKOSS INIIEX... NEHT-244

RECUHO OUIYI

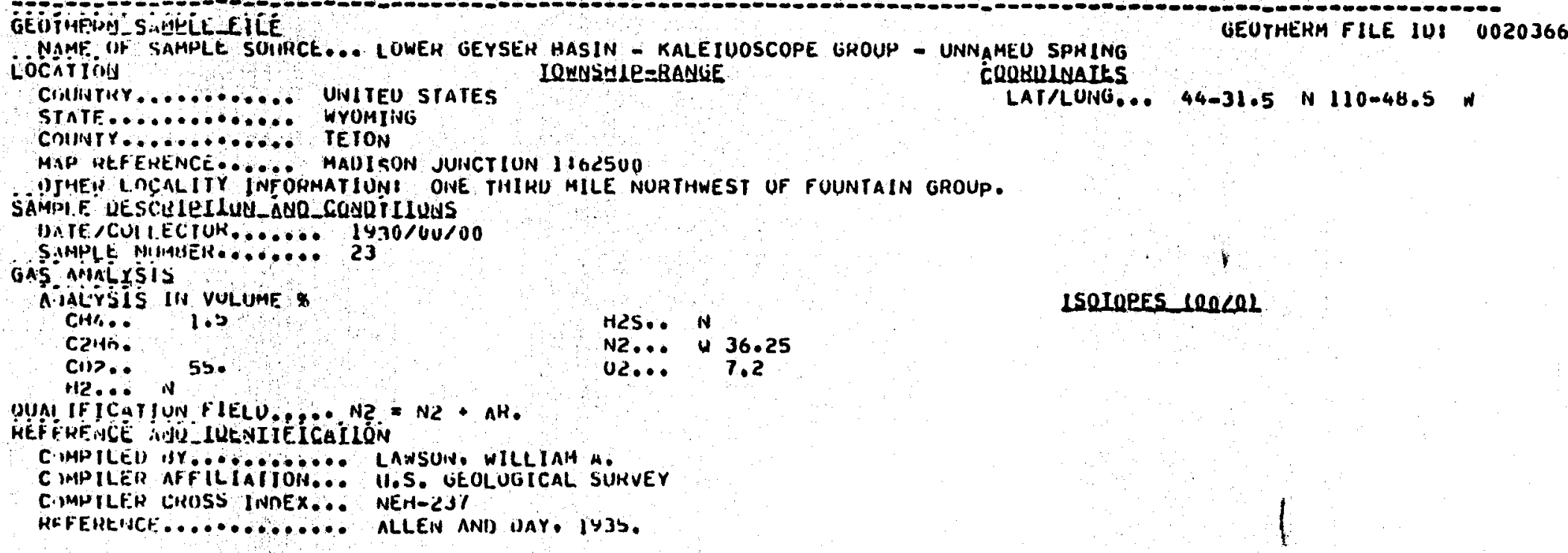

GEOTAFTi S ATELE ETLE

NAMF o

NAME UF SAMPLE SUIHCE... LOWEH GEYSEH

CITHTIUY........... UNITEU STATES

STATE............. WYOMING

MTNRY............ TEION

MAP REFERENCE...... MADISON JUINCTIUN 1162500

IIHEI LOCALITY INFOGHATIUNE OINE THIHU HILE NURTHWEST UF FOUNTAIN GROUP.

SAMIIE UESCUIEILUU_DNO_CQND ILLUS

WITE / CUITECIUK........ 1930/60/00

SIMPLE MinitSER.

Gas ninitysis

A JALYYSIS IN VULUME $x$

$\mathrm{CH}_{4} \ldots \mathrm{CH}$

cintr.

Ciil?.:

His... in

UYA! IF ICATIUN FIELU, \&. N2 = N2 • AH

HEF FRF MCE WUU_LLENIEIGAILÓN

C.MPILED IY............. LANSUIN WILLIAH H.

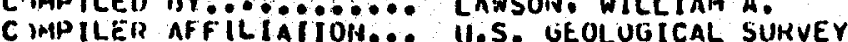

C.MMHILFR CHOSS INNEX... NEH-23l

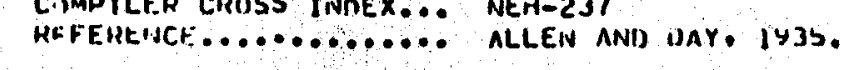

HLS. N

$N 2 \ldots-36.25$

LSOIQRES IROCAL

CROUI)INATE

LAT/LUING... 44-31.5 N $110-48.5 \mathrm{~W}$$$
\text { (1) }
$$

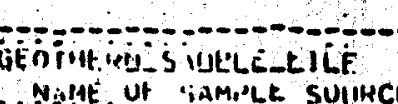

Locition CaIITy

:

RECUKU 00192

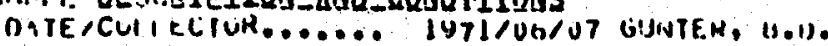


SIMII! NIMIUER........ 14

GAS ANALYSIS

DIIE!A.ALYST..... 1971/UR/UO GUNTEK, H.U.

ATAi YSIS IN MULE $\%$

AR... 0.12

ISUTOEES LOCOL

CHin:. 0.52

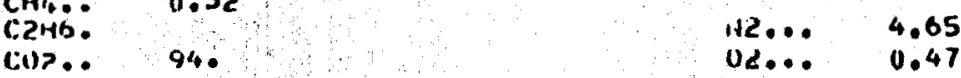

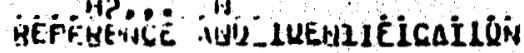

C.MPILEU ISY........... I.AWSUN, WILLLAM A.

CMIIILEK AHFILIAIION... II.S. GEOLOGICAL SUHVEY

CiMPILEP CKOSS INDEX... NEH-20Y

RECUKO 00193

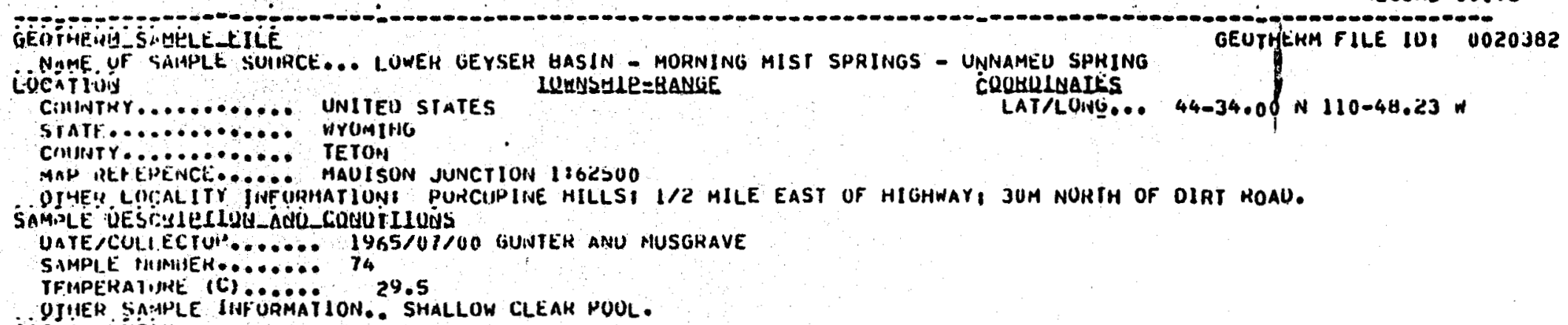

LSUIOEES IORCLL

DAS ANALYSIS

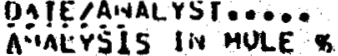

AR.. 0.04

CH4.. 0.45

C2110. N2... 2.6

U2.. 0.9

H?...L 19.4

OTHEIR AMALYTICAL DAIA O. PHבS

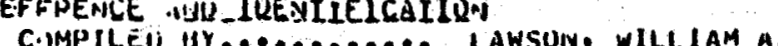

C.MMPILEN AFFILIATIOA.... U. U.S. UEULULICAL SUKVEY

CIMMPILER CHISS INIEX... MEIT-ZSI

CFFENEINCE.............. SIUNTER ANU MUSGKAVE, 1966

HECORO 00194

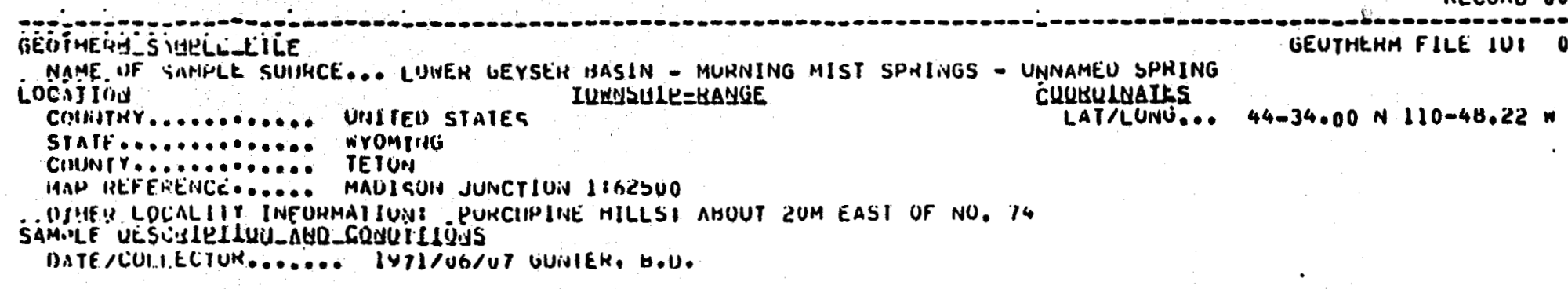


SAMPI.E IIIIISEKK...... T

TFUPEITATIIHE (C)

75.

DISCIIARIIE.

GIMEY SAMPLE IIFFOBMATION.. SHALLOW MUUUY POUL. FLUW HATE OF 4.1 L/HIN ON $1 / 10 / 72$.

TAS ANALYSTS

ANAEYSIS IN MULE

AH. 0.5

CHA. I.S

CUP. 60

CU?.

60

971/06/UO UUNTER, H.O.

HP.

3

$\begin{array}{lll}N 2 \ldots & 27.7 \\ & 02 \ldots & 2.1\end{array}$

ISQIOEES IONCUL

EFTERIE huJ IutHIIELCAILON

CHAPILEII UY........... LAWSUIN, WILLIAM A

CIMPILE' AFF ILIATION... M.S. GEOLUGICAL SUAVEY

C.MPILEH CHUSS INDEX... NEH-21O

HFFE'ULNCE.............. GUNTEK, 1973

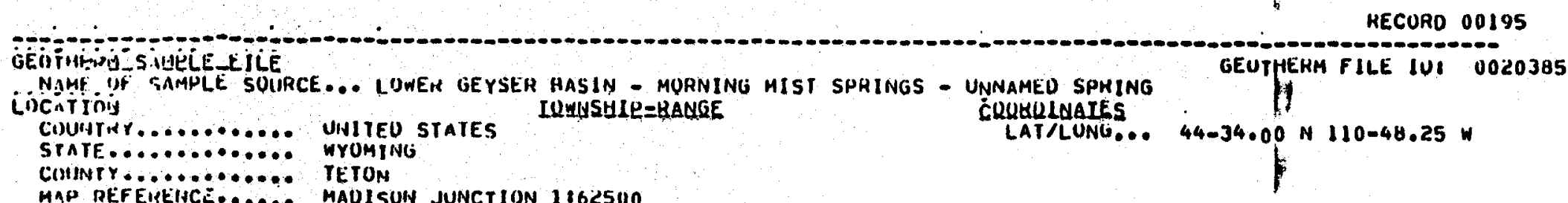

OTHE. LOCALIYY JNFOHMATIUN: FURCUPINE HILLSI $30 M$ WEST OF NO. 74

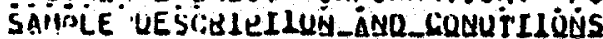

UATF.CULLECTOH....... 1965/U7/00 GUINTH ANU MUSGHAVE

SIMPIE NIMUSER....... 76

IEAPEKATINE

GAS ANALYYSIS

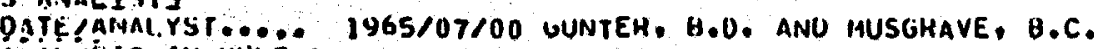

AiRi:YSIS IN MULE

Ale... 1.14

ISUIORES conCOL

Crillo. Hu

$45 \ldots$ L 0.4

N2... 16.42

UTHFH ANALYTICAL UATA... PH $=7.6$.

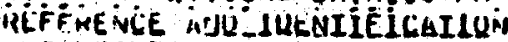

C.IMPILEI HY ........ LAWSUN WILLIAM A.

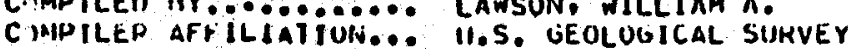

COMPILEP CKOSS INIIEX:-.. NEH-2S3

MFFEIAL,NE............ GUNTEH ANU MUSUHAVE, 1966

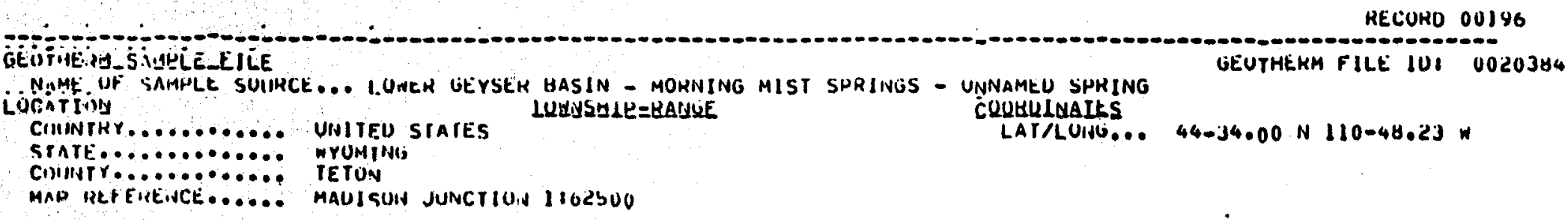

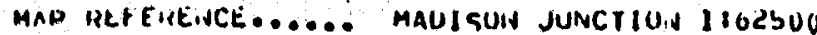




\section{c}

PIHEI LOC:ALIIY INFOKMATIUNI UINE METEH SOUIH OF NU. 741 PORCUPINE HILLS.

SAM NE UESCBLELLU_AUD_LQUUI ILUSS

DA RE/CUI ECTUK...... 1965/OT\%OO GHINTEK AND MUSGRAVE

SAMPI.E MIIMUEIIO

GAS JPHEHATHIIE

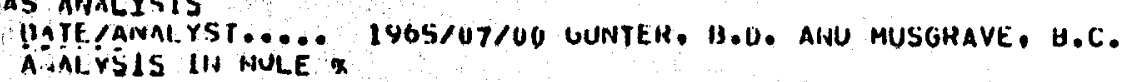

AR, 0.01

Chitio n.s

C.112. 46.

96.

ISUIORES CUNCQL )

UTYHY ANAL YI ICAL UATA OPH $=5.2$.

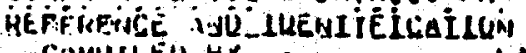

CMMILEU] HY.......... LAWSUIV, WILLIAM A.

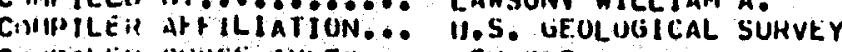

CIMMIILEH CUUSS INHEX

HECORO OU197

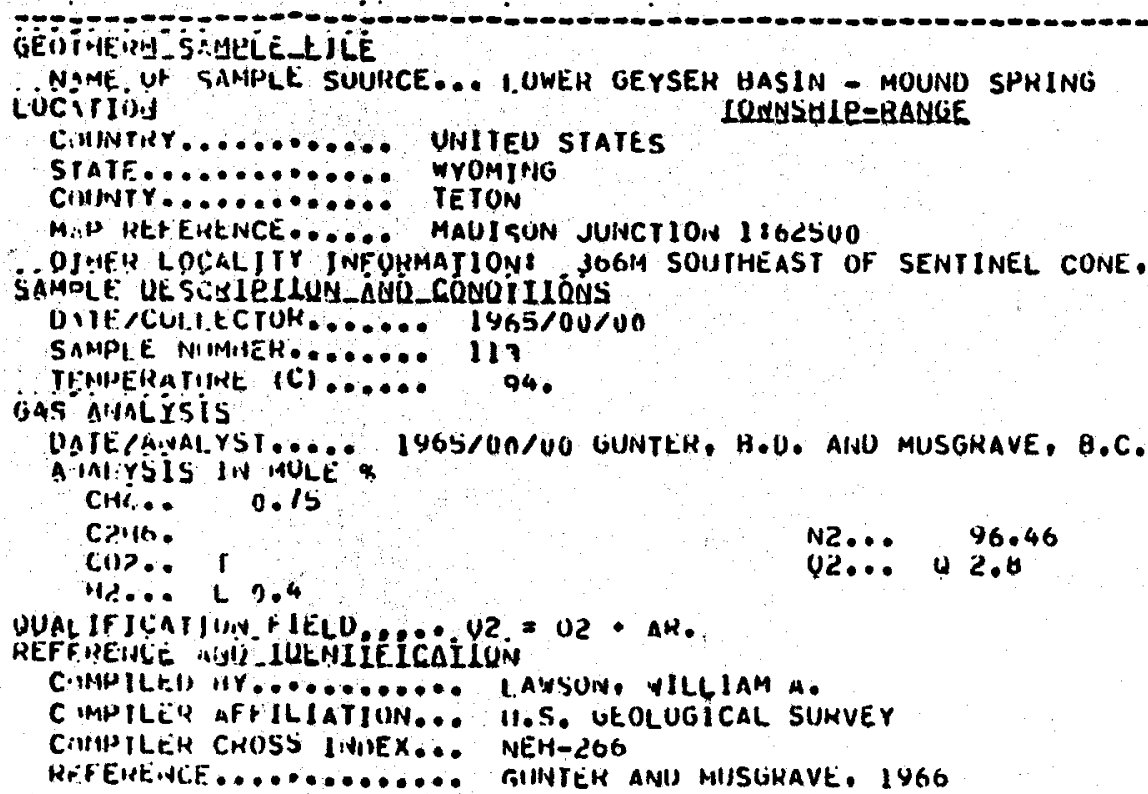

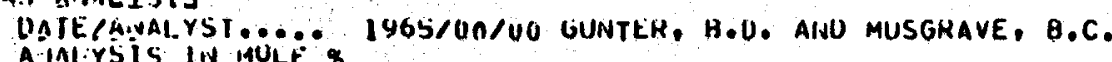

CHi:Yis IN 140 .15

N2. $\quad 96.46$

Ci) 1

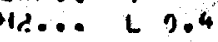

Q2... प 2.8

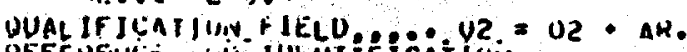

REFF RENGE NITi ILEMIIÉfCAILUN

C.MH ILFI) "Y........... LAWSUN, WILLIAM A.

C IMPILËL AFFILIATIUN.... II,S. UEOLUGICAL SUMVEY

CIIHIILEH CKOSS INIEX... NEH-2O6

R.FENE.HCE............ GIINTEK ANIS MIISLIKAVE, 1966

0.36

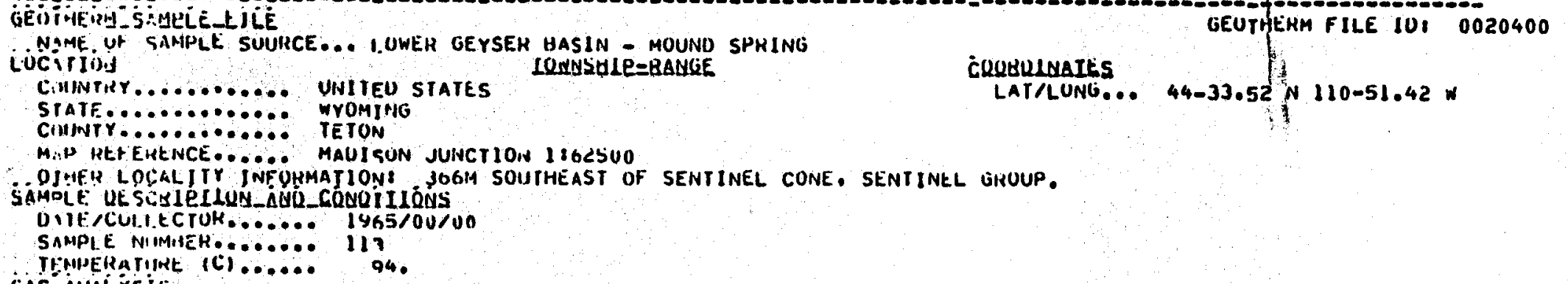

ISUIORES IRQCOL

HECORO OU19B

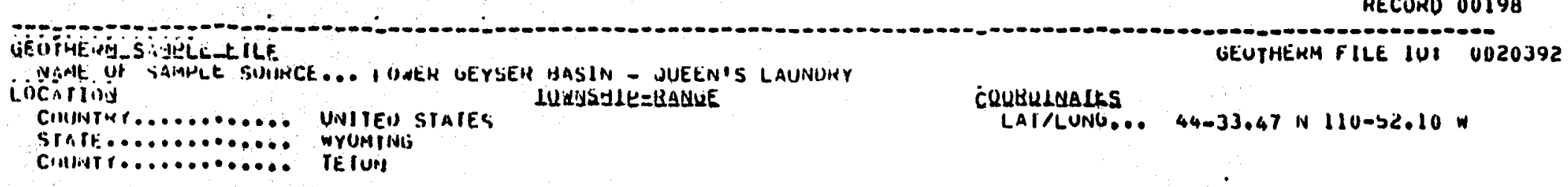




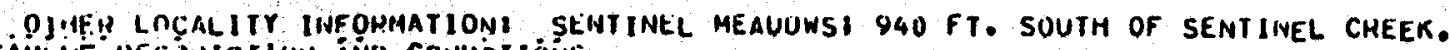

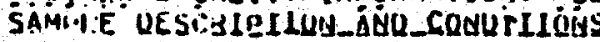

DIIE/CULLECTOH....... $1930 / 00 / 00$

WATFIS AUALYSIS

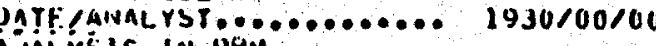

A IALYSIS IN PPM

ar.....

Coj..... 21.

FE(TIOI) $\quad$ NH...

HCU3.... 368

CA... 115.

KEFFHE ILE UU LUELIÉLCAILUN

CIMHILEU HY......... LAWSON, HILLIAM A.

C.MPILEH AFF ILIATION... M.S. UEOLUGICAL SURVEY

RJFERENCF............... ALLEN AINO DAY, IYJS

ISULORES IRCONL

RECURU DUI9Y

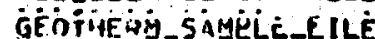

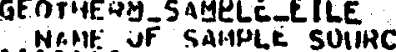

iocition

Coullivis

GEUTHERH FILE IU: 002038

STATE . . . . .

C.MINTY ........... TETON

MAP MEFFHENCE..... MAOT SON JUNCTIUIN 1862500

(1JHE!" I.ICAL II INF OHMAIIONI JUH S-SW OF SKELETUN POOL.

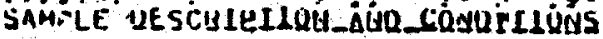

DIVICUI LECTUK........ 1971/06/OI LUNIEN, H.D.

SAMII.E WIHHEWH......

TFMPENATHUE ICI...... 63.

MISCHAHCIE........... A 3.5 LAMIII

OJUFH SA:H'LE IIHOMMATION.. LAKUE GREEN PUUL WITH NO RUNOFF. GiAs ÂnALYSIS

DATE/ANALYST..... 1971/UR/UO GUNTER, H.U.

A.AL:YSIS IN HULE

A... 1.2

ISUTOEES concol

Ciliz:. 4.5

Cos.. Iy

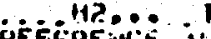

$\begin{aligned} N 2 \ldots & 68.9 \\ N 2 \ldots & 6.3\end{aligned}$

\section{CRQBULUATES}

LAT/LUNG... 44-33.

N 110-50.

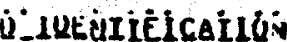

CIMHILEUI SY............ AWSUIY, WILLIAM A.

CIIMPILER ARFILIATIOH... M.S. GEOLUGICAL SIJKVEY

CIMPILEN CKUSS INIEEX... NEHEZTI

RFFEHENCE........... GIJTIE, 1973

RELUND 00200

GETFIER SAMULCE IL

iónitions

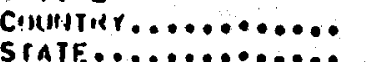

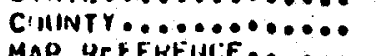

OFHË LOCALIIY IIFFUHATIONE JUM S-SW OF SKELETUN POOL.

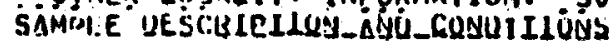

mads suly junctluin 18 besuo

IASIN - HIVER GROUP - UNNAMEU SPHING

\section{CUUBULUALES}

LAT/LUNG... 4 44-33.

GEUTHEKM FILE IUI OUZOS80

NIIEI) SIATES 


\section{C}

DITE/CUI.I ECTUN........ 1965/OI/UO

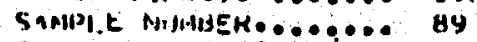

GEMPEEATHUE (C)....... A4.

GAS ANALISIS

DATE /AHALST..... 1965/07/NO UUNTEH, H.U. ANO NUSGRAVE, H.C.

Arini:rsis IN HULE

Nie... 1.1

CHi.. 1.3

C:146.

$12.0 \quad 0 . y_{3}$

OIHEF ALIAL YIICAL UATA, .. PH $=7, H$

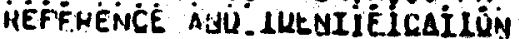

C MMPILEN OY............ I.AWSUN. WILLIAH A.

C.MMPILEF AFFILIATION.... U.5. UEOLUGICAL SURVEY

CINHPILER CHUSS

HFFEIREHCE.............. GIINTER ANU NUSGRAVE, 1966.

ISUIORES IROLO

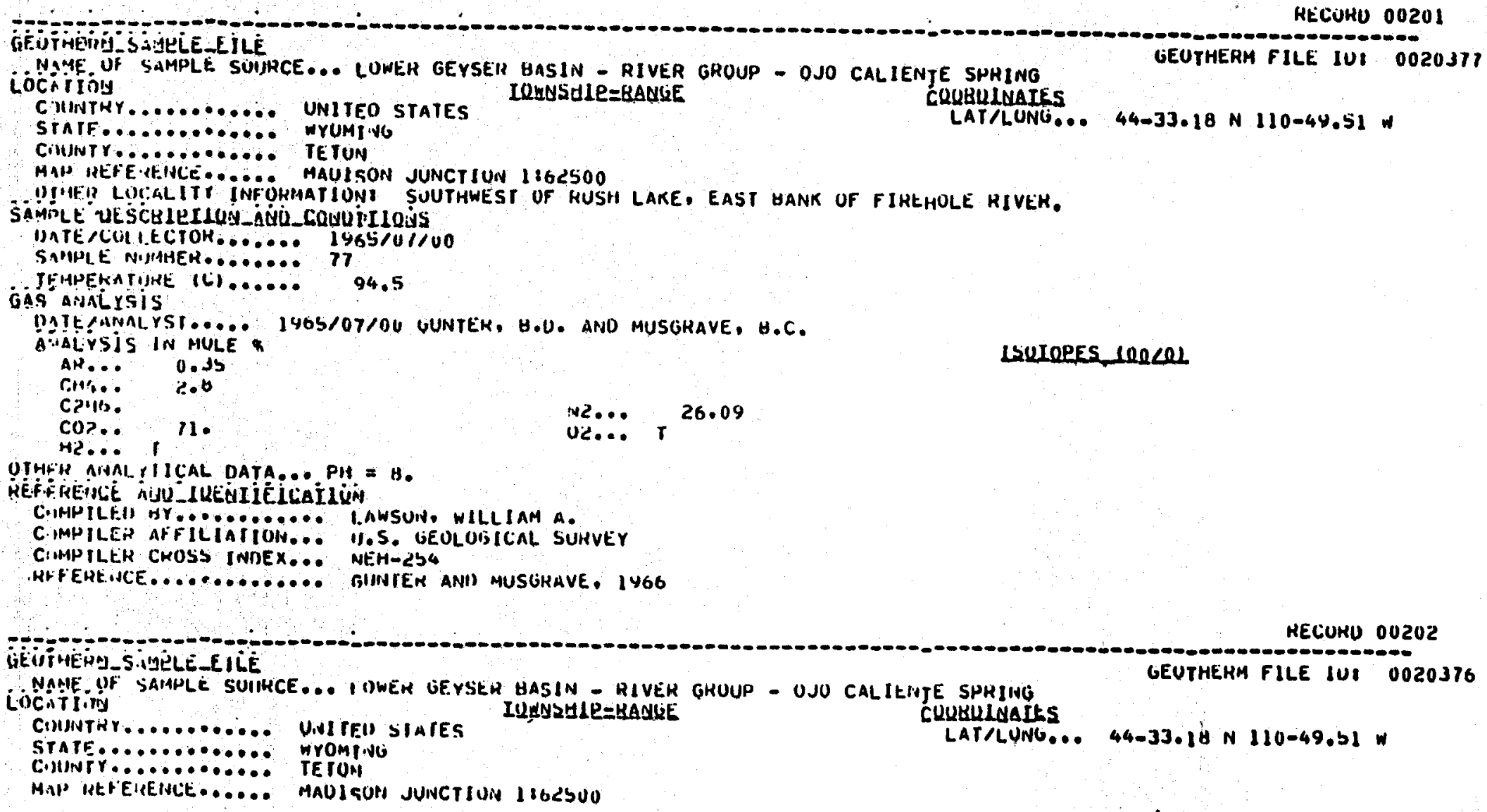


SIIIFY LOCGLITY INFOKMATION: SUUTHMEST OF NUSH LAKE, EAST GANK OF FIHEHOLE RIVEM.

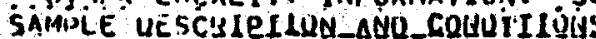

BATE, CULIECTUH....... 19.30/00/00

SAMPI.E NIIIHEK........ 5

UFWIISI IS UK AL TEHATION..... INCKUSTATION OF SULFUA ON A SINTER BURUER.

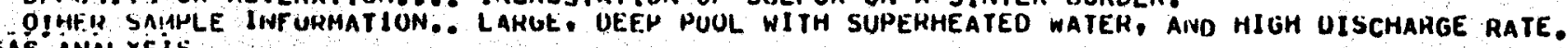

GAS ANALYSIS

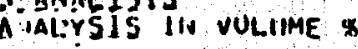

crit.. n.o

Canto.

Cis?.. of un.

Hes.. T

N2... O 19.15

QUA! IF ICAHIONY FIELL, , C COZ = CU2 + HESI N2 = N2 + AK,

U2... N

LSULOEES LOUCOL

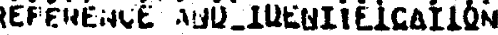

CIMMPILEUI UY.......... LAWSON, WILLIAM A.

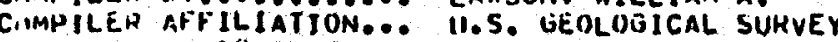

C.MMPILER CRUSS INIEX X... NEM-ZSS

RFFELEINCE.............. ALLEN AND UAY, 1935

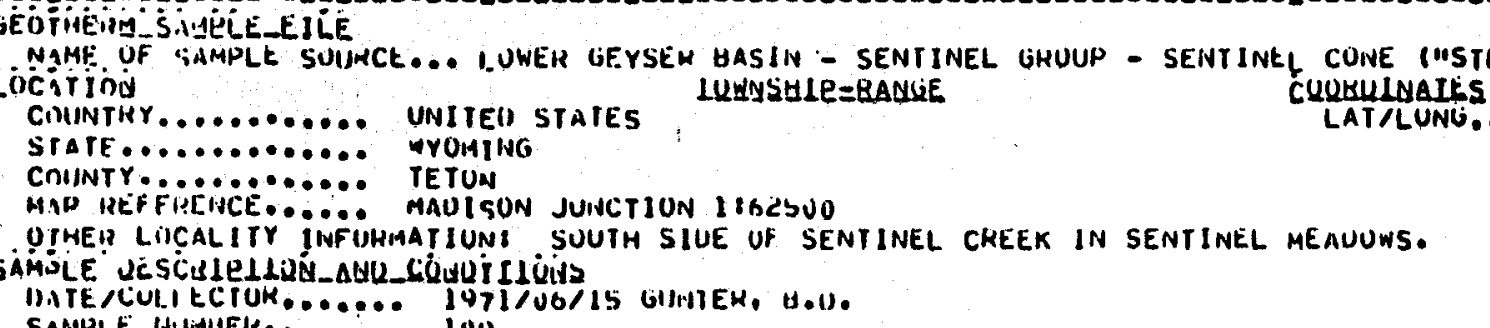

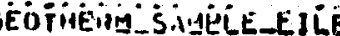




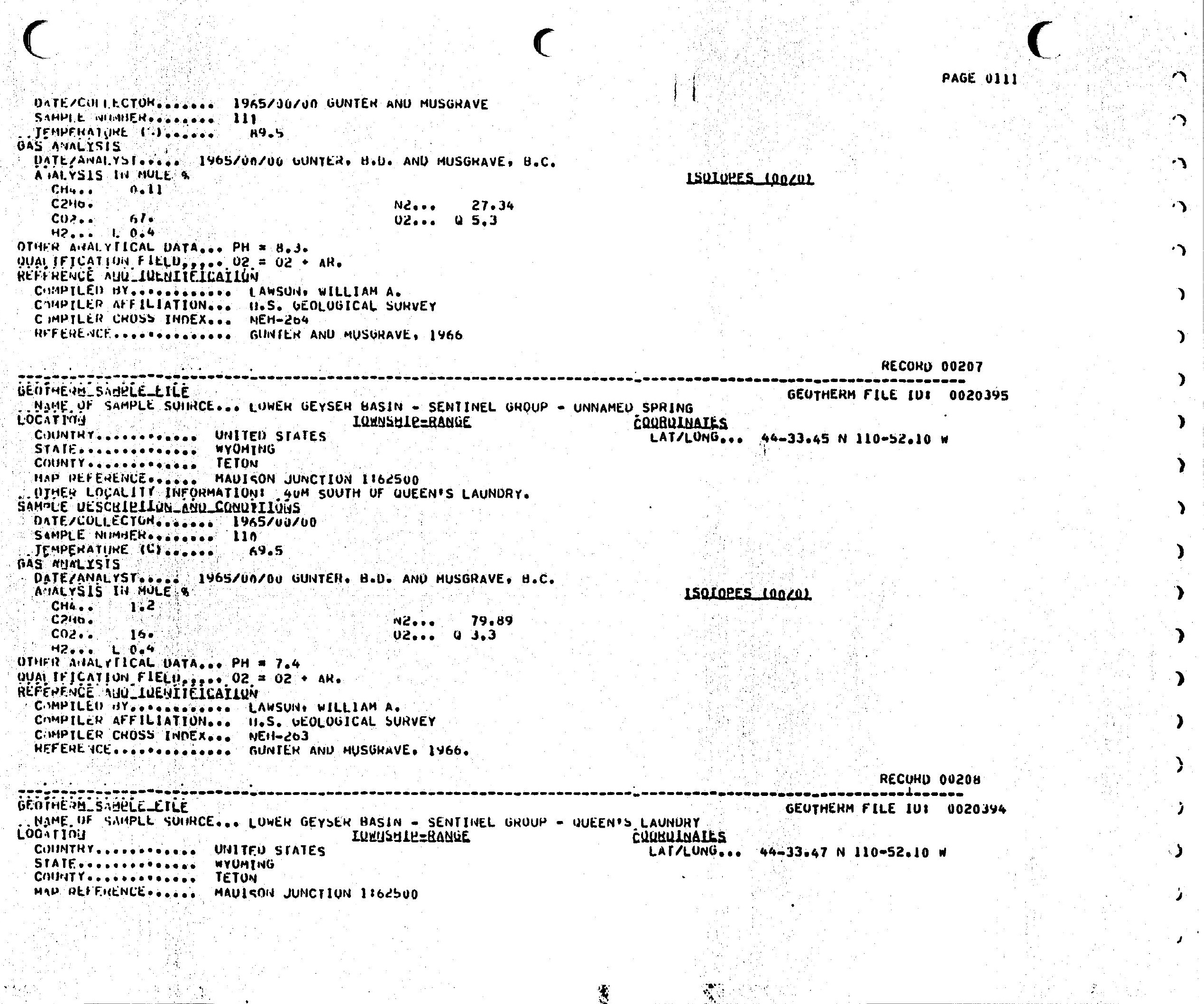




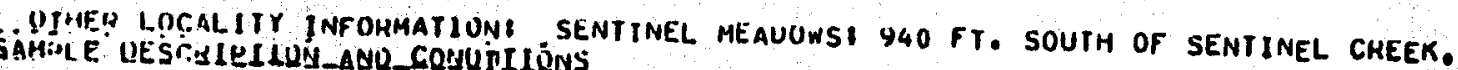

DATE CULIECTOM...... 1971/06/16 GUNIEH, H.D.

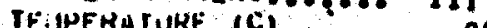

IISCrAARISE

GASHEH SAMPLE INFORMATION.. FLUW RATE OF 42S. L/MIN ON T/11/72.

DATE ALIALYST MUL $1971 / 06 / 00$ GUNTER, H.U.

ARLYSIS IN MUL

1971/06/00 GUNTER, H.U.

Crip 1.5

ISULOEES cenCOL

CदHG.

Co?.. 160

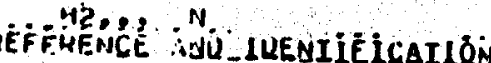

C.MAPILEI) HY........... (AWSUIY. WILLIAM A.

C.IMPILEH AFFILIATION... N.S. GEOLUGICAL SUKVEY

C.MMPILEH CHOSS INDEX... NEH-273

HFFEMENCE............ GUNTEH, 1473

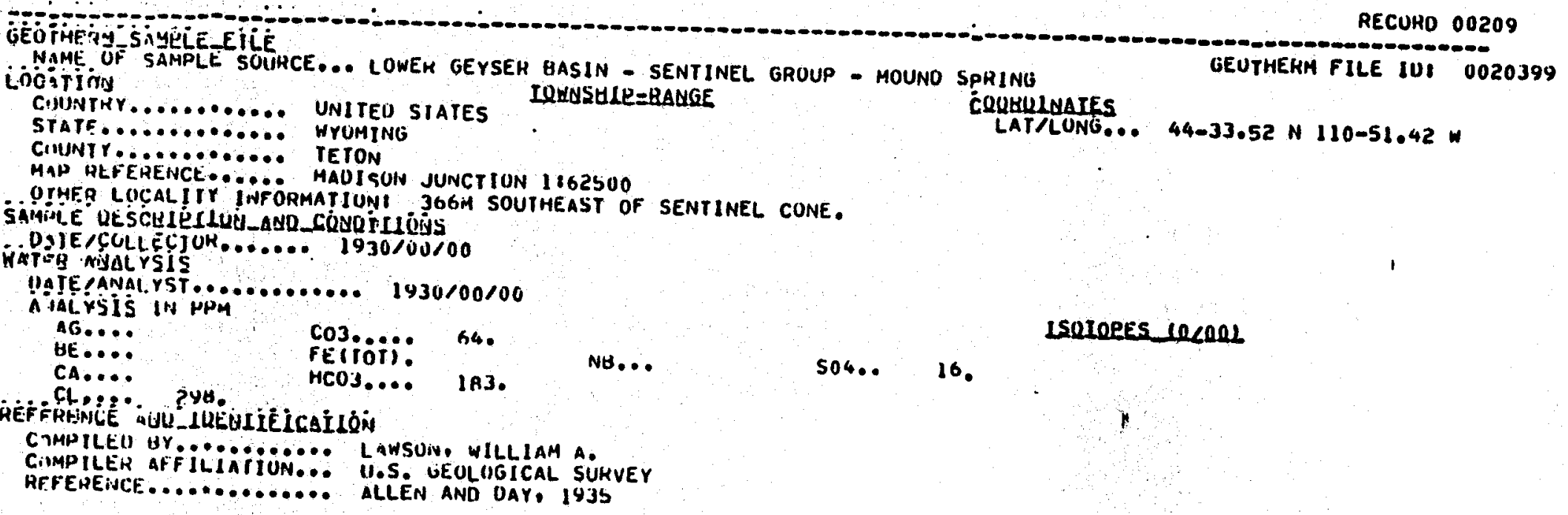

RECURU 00210

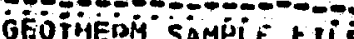

N2... 75.6

MAME UF SAMCLETILE

Loñing

Cillunthr.

UNIIEO STAIES

Crate............ mrOMING

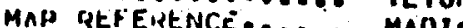

UIHEII IOCAL TY

SAMIDE UESCHIOIDU 


\section{C}

D.TENLII I ECIOR

S.IHIPI NiliHEH...... 1465/00/30

SIHIPI.E NIIAHEH....... II?

Gäs nionitrsis

OATE MANALYST.... 1965/Un/OO UINTEK, H.U. ANU MUSGHAVE, H.C.

CH4 0.15

CPHD.

$$
\text { is. }
$$

$42 \ldots 1$ L

DTHI AINAI, YTICAL UATA... PM

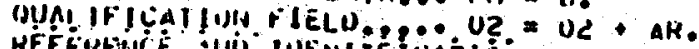

C UII JCE TUU - LUENIIELLAILǗN

C VIIILEN HY.

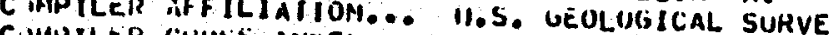

C.VMPILER CRUSS INI)EX... NEM-2OS

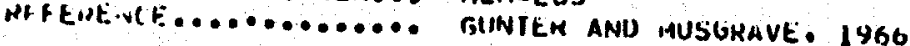

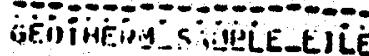

RECUHU OU211

$\begin{array}{ll}N 2 \ldots & 5.53 \\ \text { U2... } & 0.35\end{array}$

ISUIOEES LenCUL ionitility

CUIMTKR

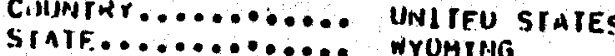

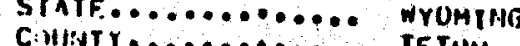

MnH HE TUN

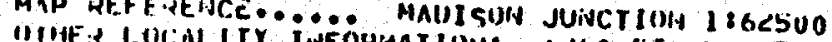

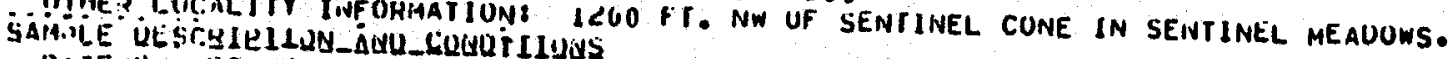

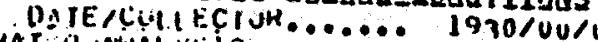

WAT- AYYAI YSIS

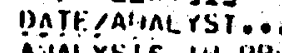

ainitisis lin pep

Ali.....

$1930 / 00 / 60$

IHE.....

Cojo.

15.

No...

LSULORES lecule

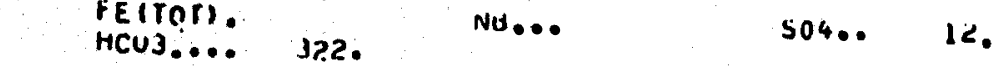

HEFGRENE

GEUTHEKH FILE IUI 0020397

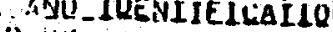

CIMP ILCI! iIY .

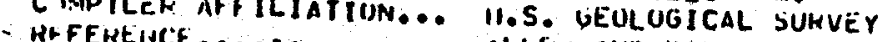

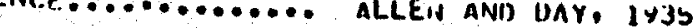

HECUKU 00212

GEOTHF

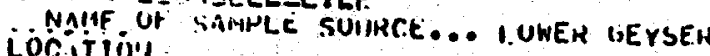
Locitiony

C.Mllitur........... UNI TEU STAIES

STATE........... UNTRE IUHNSULE= MANGE

GEUTHEKM FILE IUI 0020396

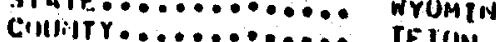

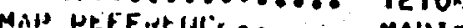

(I) IFF

SAMMILF UESTRIULLU:

vate

va To

ivitemalis

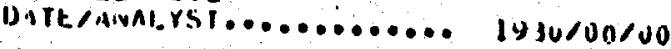


Ainl:xisls lif NMn Alj.....

cus...... Hetrini,

BF.....

43.

$3 \cdot$

273.

Na....

LSUIUES UKRQL

PAGE 0114

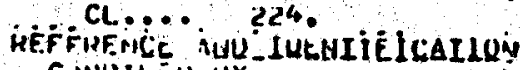

C MNILEDS HY......... LAWSUN, WILLIAM A.

C.MPILE AT ILIATION... H.S. UEOLOGICAL SUHVEY

RFFEHEINC. ............. MLLEN AND IIAY. IY3S

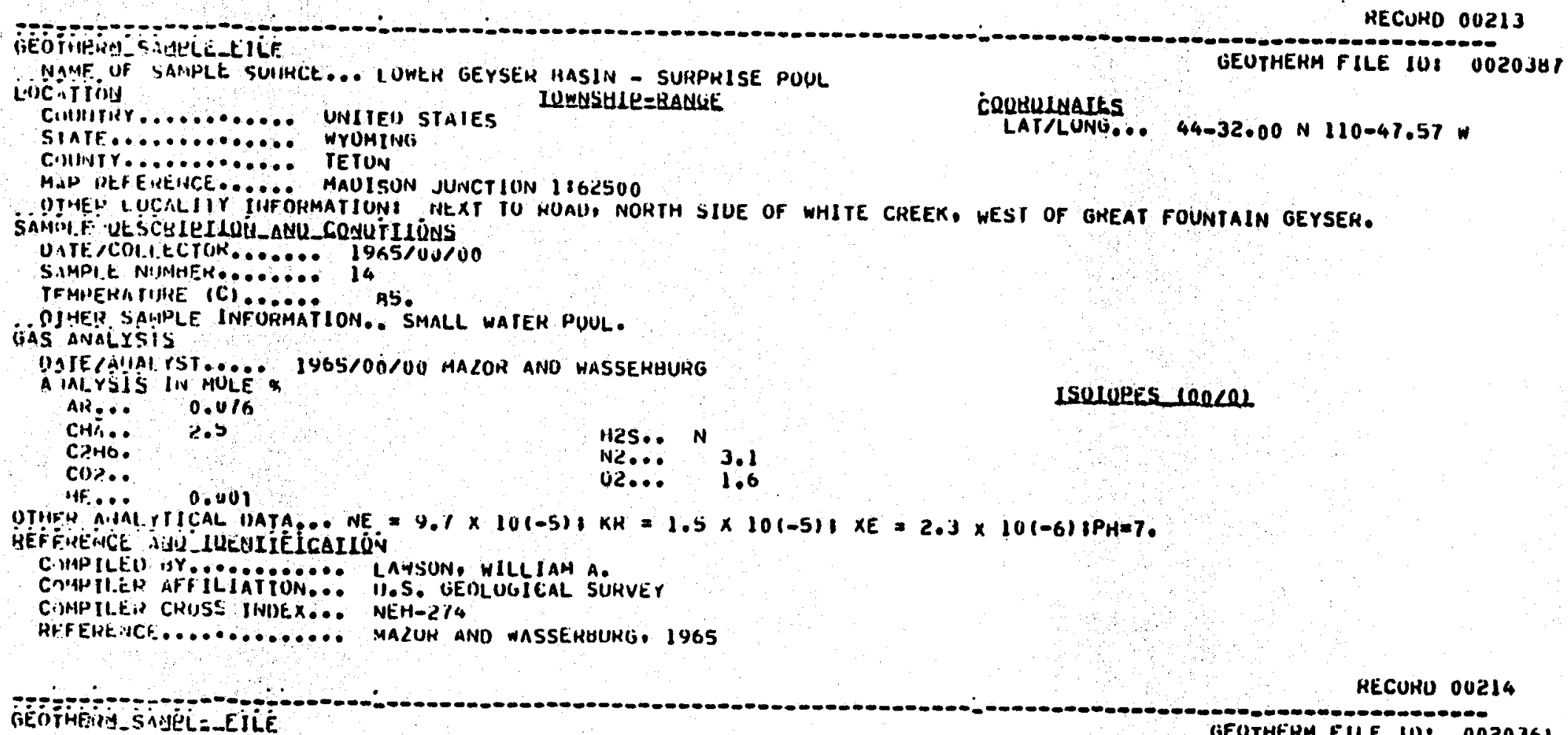

LSOLURES CoOCO

RECUHO 00213 


\section{C}

Ainitysis IN MUL

Clip... $\wedge .44$

cille.

n.44

Cor. H4.

OTHF MIVAL YTICAL

OUAL ANAL YTICAL WATA... PH $=3.3$.

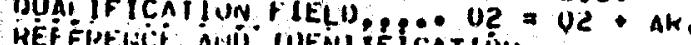

HEF FIEINCE AUU_LLEULIE.LCAILÚN

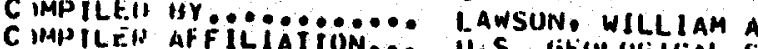

C MAPILEN AFFILIATION... JOS. GEULUGICAL SUHVEY

NEH-246

H.FERE.WCF............... GUNTEH ANII MUSGRAVE, 1966

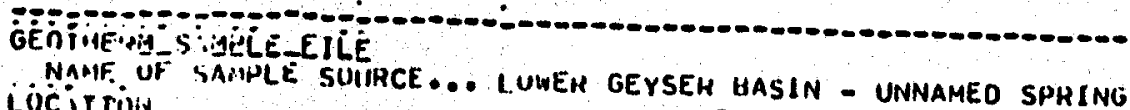

iocirring

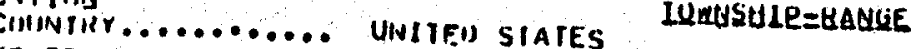

STATF............ WrUNTHC

S. JINTY Y

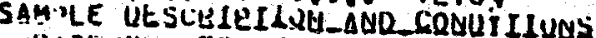

DITE CULIECTUK

SAMPIE NIJHEEK....... IO

TFAHEKN YUITE (C) .....

id.

GA' JIE! BAHALE IINF ORHATION.. SMALL BUILING WATER PUOL.

AN ALYSIS

YMIE JAIJAL YST..... 1965/UN/OO MALOR ANU WASSERUURG

Auirsis III AULE

Akt... $\quad 5.162$

$112 \ldots 0.11,43$

LULUUES IOOCOL

page OIIS

C2Hh.

Co?.. H. H.

itt.... 0.6074

N2. $\quad 6.6$

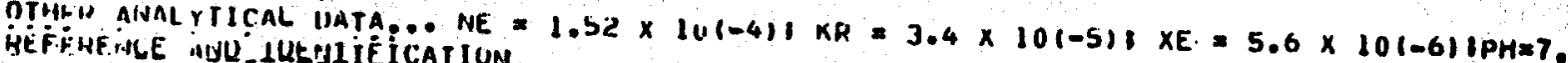

C.MH'ILCII dY.......... LAWSON, HILLIAM A.

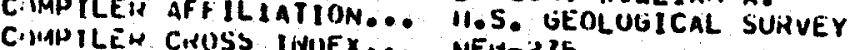

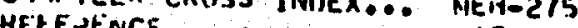

HFFEAENCE............ MAZUR ANO WASSERBUKG, 1965

RECUHO 00216

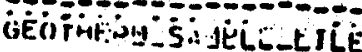

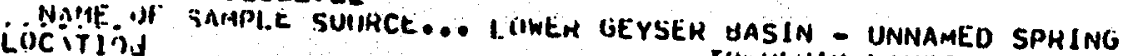

Locition

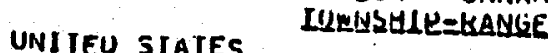

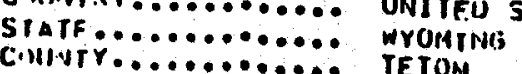

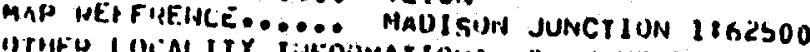

LSOLOEES IROCOL

MECUNU OU215

SAMTLE UESCETEILUO

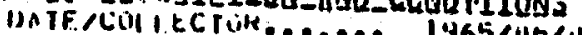

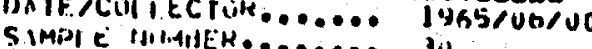

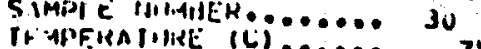

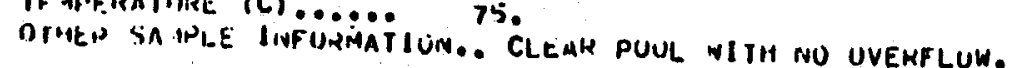


Gäs ANALISIS

UATF.ANHALYST..... 1965/G6/00 GINHEH, H.J. ANU MUSGRAVE, H.C. D MLYYSIS IN AULE $\mathrm{B}$

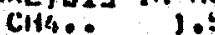
1.5

N2... 44.79

$112 \ldots<0.4$

$02 . .0 \% 7.6$

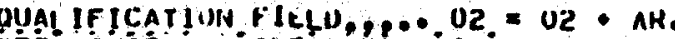

REFEHENCE NUL IUEUIIEICAILUN

CIMP ILEU HY.......... LAWSON. WILLLAM A.

COMPILEA AFFiLIAATION... M.S. GEOLUGICAL SUHVEY

COIPILEA CIOSSS INDEX... NEN-242

IBFFEREIUCE.............. GUNTEH AND MUSGRAVE, 1966

LSOTOUES 1010201

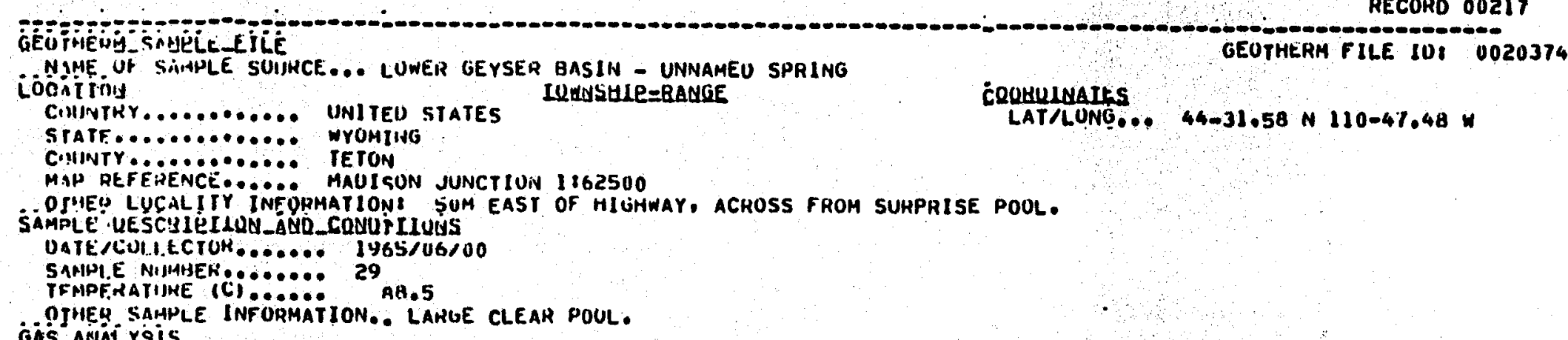

a

LSWIOEES IOQCOL

92.19
$02 . . . \quad$ o 7.6
Aini YSSIS IN MULE

CHi.. 0.23

cu2.:

$12 \ldots$ L 0.4

OTHFR ANMI.YTICAL INATA... PH $=9$

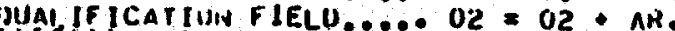

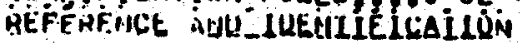

COMPPILEI) HY........... LAWSUR. WILLIAM A.

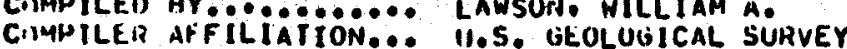

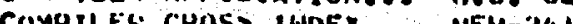

MEFE ME!UCE............ GUNTER ANU MUSGKAVE, 1966

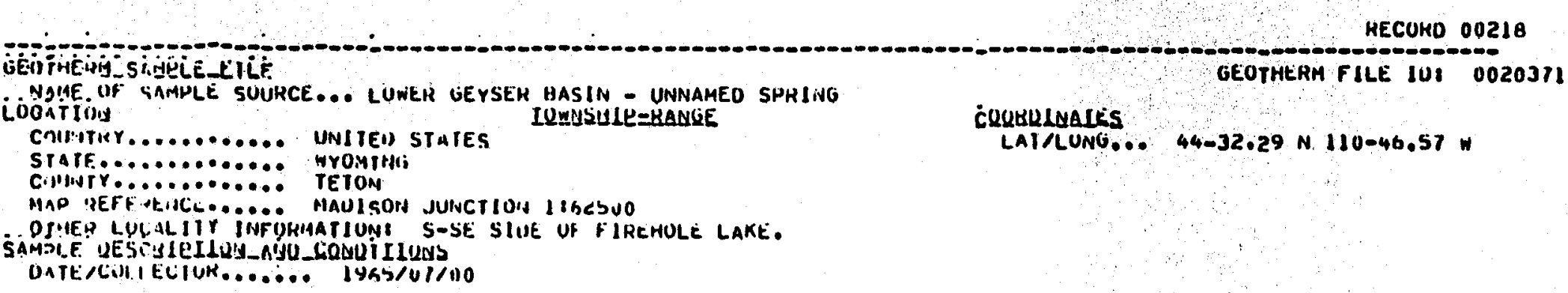


SIMHIE N. HUHER

TFMHEHAT.ME ICI,

UJHE SA IPLE INFUUMATION.: SMALL WHITE SPHING.

SAS ANALXS IS

DATEKANALYST.... 1905/OT/00 GUNTER, B.U. AND MUSGRAVE, H.C.

AH. 0.44

(स्H13.

CIIP. - L 030

$\begin{array}{ll}N 2 \ldots & 31.6 \\ 42 \ldots & 150\end{array}$

OJHEH ANALYTICAL DATA .. PH 7.5 (EST. WITM PH PAPERI

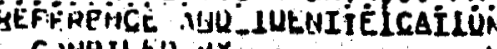

C WPILEI] AY .......... LAWSUN, WILLIAM A.

CIMPILEII AFFILIATION...: H.5. GEOLUGICAL SUHVEY

CillPILER CHUSS INDEX... NEH-ZSS

RFFEMEIUE. ............. GIJMTEN ANU MUSGRAVE, 1966.

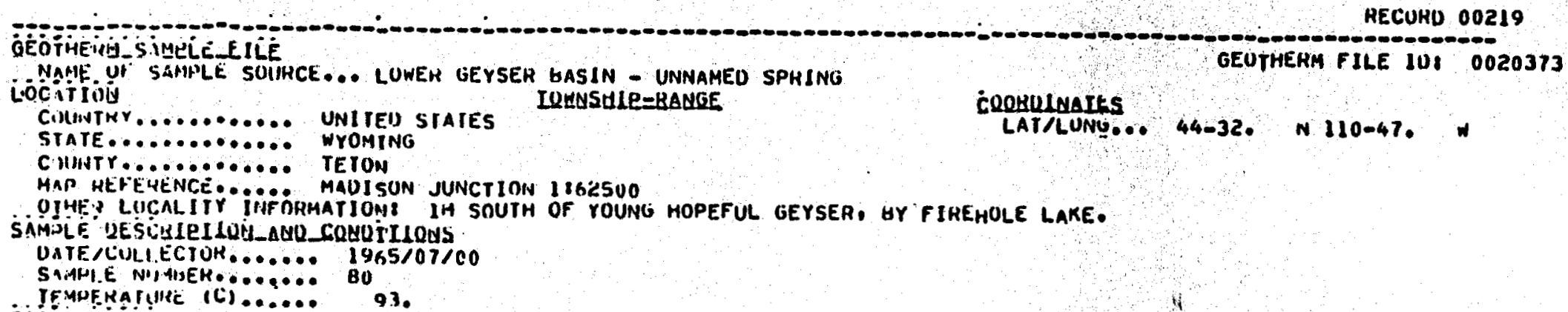
CHR:- O.JO

C(1) 1 . 96

H?...

(EST. WIIH PH PAPEH)

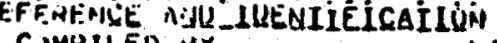

C MPPILED HY.......... LAWSUIV. WILLIAM A.

C MOLILE AFFiliation..: T.S. UEULUGICAL SUHVEY

CIHPILER CKUSS IHNEX... NEH-ZSI

KFFEREHCE............ GLWTEH ANU MUSGKAVE, 1966

\begin{tabular}{|c|c|c|}
\hline 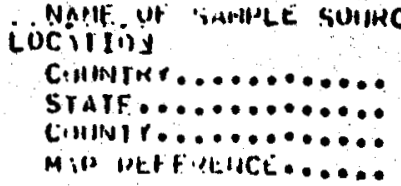 & 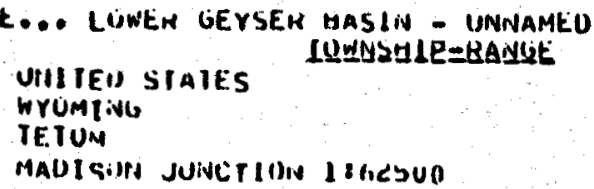 & SPHING \\
\hline
\end{tabular}

KECUKU 00220

GEUTHEHM FILE IUI N020S72

cuUELLUALS

LAT/LUNG... 44-32. N I10-41. W 
OPMF: LOCALITY INFURMATIUN OM WEST OF YUUNG HOPEFUL GEYSEK, OY FIREHOLE LAKE.

DATE/CULI.ECTOH

IFMIPEHATIUE IC)

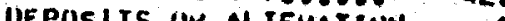

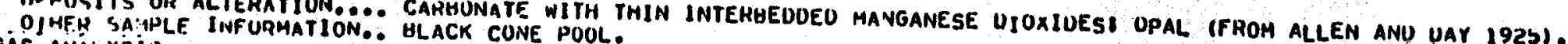
BAS MilaLYSIS

DATF !AIIAL YST ME IOE $1965 / 07 / 00$ GUNTEK, H.1). ANU MUSGRAVE, B.C.

AK. 0.06

CHí... O.UY

C.116.

42.0 .200$.

U2... 1.6

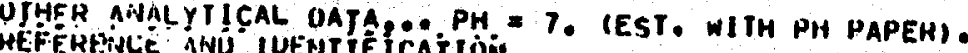

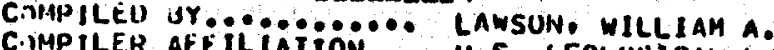

C.MMPILER AFFILIATION... M.S. GEOLUGICAL SURVEY
C.MPILER CHOSS INDEX N... MEH-ZSH

HFFEIAEACE ............. GUNTEH ANU MUSGKAVE, 1966

LSUIORES concoi

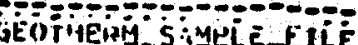

RECOHO OU22I

NAIF UF SARPLE SUIHCE... IOHEN GEYSER GASIN - UNNAMEO STEAM VENT Comininy.

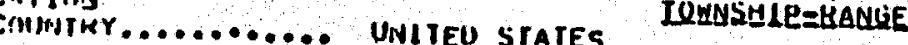

STATF ............ WYUMTING

CHINTY M........... TETUN

MIN KLFERENCE...... MADISON JUNCTION 1862540

SAMOF UESTHE

OATE,COLECTUH....... $1965 / 00 / \mathrm{JO}$

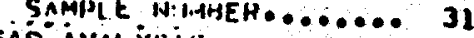

GAS ANALYSIS

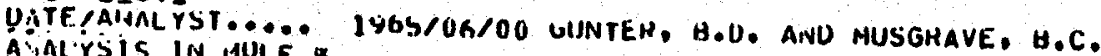

A ALYSIS IN MULE

Cil... nolo

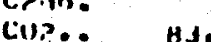

Co... HJ

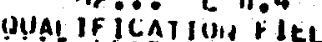

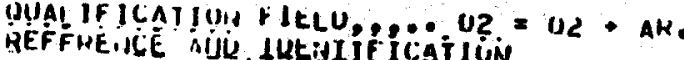

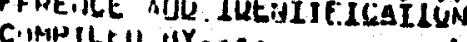

C.MINILEI) BY .

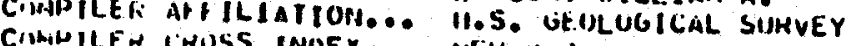

$42 \ldots \quad 14.08$

LSULOPES LONCII

CIMHIDILFH CNOSS INISEX... NEH-24J

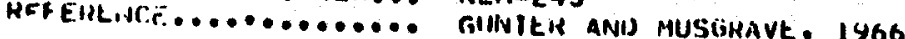

RECUNU OU2Z2

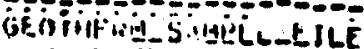

Naif Ii SAMHLE SUINCE... MIUWAR GEYSEH UASIN - ERCELSIUR GEYSER

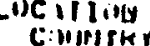

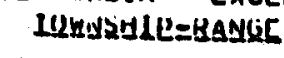

stalt........... UNIIEU SIAIE

UEUTHEKM FILE IUE 4020343 


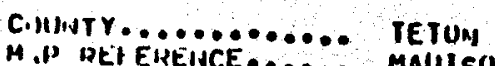

PAGE OLIS

M.D DE EREINCE...... MAUISON JUNCTIUH 1862500

SAM.PE UESCLIEILOUF IOAMATIONE JUST SE UF VABM-MIUWAY (7251. FTI.

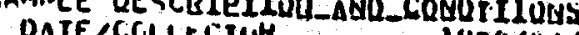

OATE/CCLCLCTUH....... 1930/00/OD

TFMPFAATIINE (C) ..... 91 .

WAYT OSF SA HLLE INFURMATION.. HIUIT RATE UF UISCHARGE.

WAYIL nigal 1 SIS

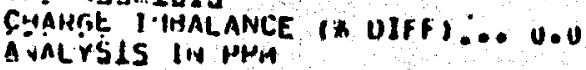
Ali.....
$\cos \ldots \ldots$ W
AL.... 1.
H...... U 2 .
CA.... 3 il.
Fon... 20.
FE(Tọr) 1.
HCo3... 562.
LI...
MU... N $N 10$
S.... 00.67
LSOIOEES LOLOOL
Co.... K....... 140
NH.
5102.

UYAL IF JCAT JUU, F IELU OO H CALC

REF TPE.HCE $\because J U$-IUtNIIEICAILON

C.HIP ILCD WY........... LAWSUIV, WILLIAM A

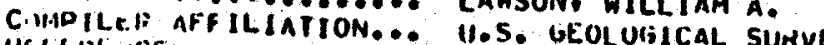

P.F.tEL ivCE.

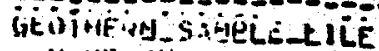

RECUND 00223

No:Al or SAlapte SUIHCE. iocition

alleit ann vay, igjs, for 1976 vata see htcuru nu. vot5U89.

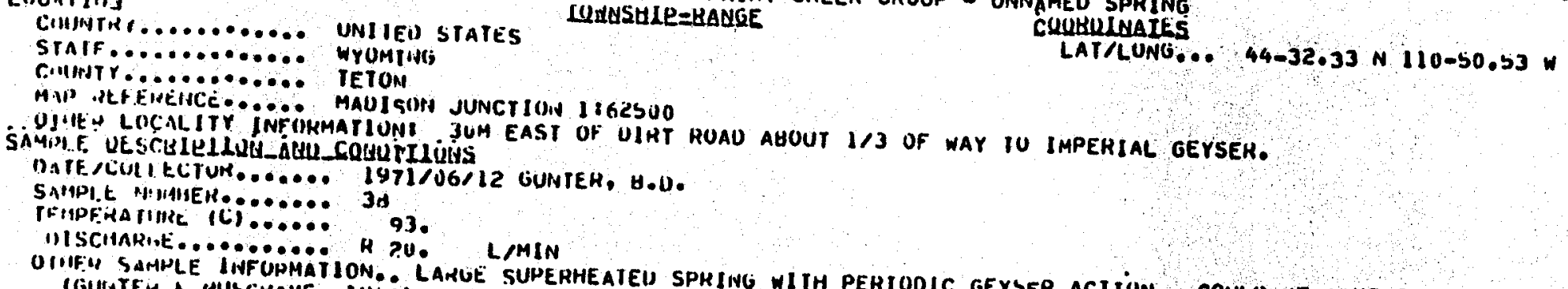


RECURO OU224

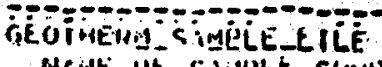

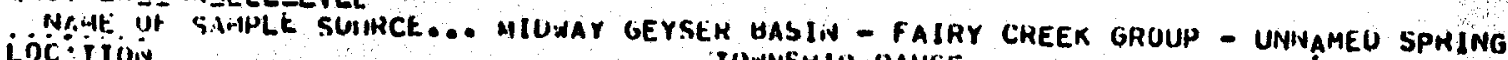
Lic iring

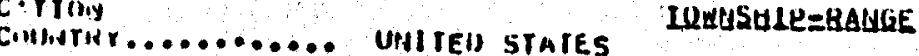

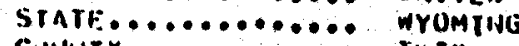

CHINTY........... TETON

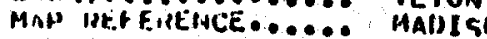

JUNCTIUIN 1162500

作

U.I

SIMPLE NIMHER....... IS

TEIAPF.RAIIITE (L) ..... 74.

GSHEY SA:APLE INFORMATION. WHITE SPHING WITH ULACK OEPOSIT.

liAs añLYSIS

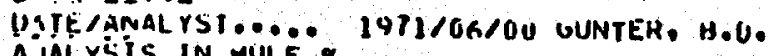

A IALYSIS IN MULE

AK... n.5

LSUIUEES (0020)

CHin 1.3

C.)

47

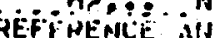

IUU_LLELIIÉICAIIÚ

CM.PILEH AY........... LAWSUN WLLIAM A.

CiHPILER AFF ILIATION... H.S. GEOLUGICAL SURVEY

GUNTEN, 1973

KECOHO 00225

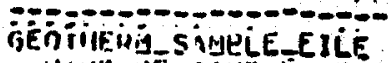

GO HAME UF SAMPLE SUIRCE... MIUWAT GEYSER HASIN - FAIRY CKEEK GROUP - UNNAMEO SPRING

GEUTHERM FILE IU: 0020355

CUUHUINATES

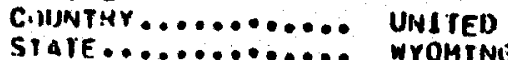

LUMNSULR=HANGE

LAT/LUNG... 44-32.33 i 110-50.53

CHINTY

MIP WEFERENCE...... MAUISON JUNCTION 1862500

DJEEE LOCALITY INFORMATIONI I/2 MILE NOGIH OF NO, 37, 30M EAST OF OIRT ROAD,

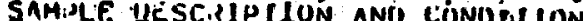

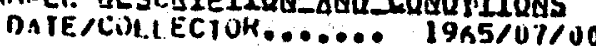

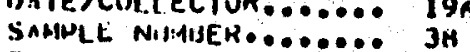

TEHPERA TIIRT (L)

IIFFORMATION.. SUPERHEATEO PUUL.

DAS AnLYSIS

DATE/ANALYST..... 1965/07/00 GUNTER, B.U. ANU MUSGHAVE, B.C. Cith.. 5.0

CPH6.

Cor.. 440

N2... 47.95

LSUIORES COOCOI

GEUTHEKM FILE IU: 0020352

HFA AHAL YTICAL OL

PH $=\mathrm{H.4}$.

El.U.. .0 U2 $=02$.

NIIELICALLÓN

lawsul, wILliam a. 
C.MPILE" AHF ILIATIUN.. II.S. UEOLUUICAL SURVEY

C.MP ILEW CROSS IMNEX

HFFELE VRE. ............. NINTEK ANI MUSGKAVE, 1966

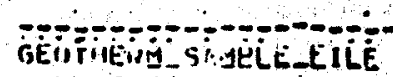

RECORO DO226

NAMF UF SAMPLE SUIRCE... MIUNAT GEYSEh BASIN - FAIKY CHEEK GROUP - UNNAMEd SPHINO

GEUTHEKM FILE IVI 0020354

Liếcition

UNITEO STATES

LUWASULE $=$ BAALIE

CQRHQLNAIKS

STATE............ WYUMING

LATRONB... 44-32.15 N110-51.10 N

C IINTY Y

MAP REFERENCE...... MAULSON JUNCTION 1862500

ORHF. LOCALIIY INFOHMATION: JUOM EAST OF ROAD.

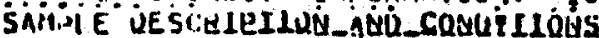

UNTE/CULIECTUR....... 19R5/U7/U0

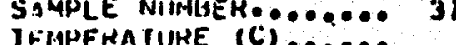

THMPERA TIRE TCI...... A2.0

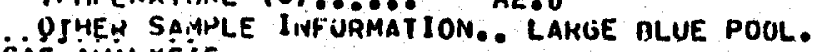

GAS AIVALYS IS

OAIE GANALYST BOE I965/07/0O GUNTER, B.U. ANO MUSGRAVE, B.C.
A.IALYSIS IN MULE

CH4.

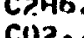

C1)?. 740

${ }_{02}^{N 2} 19,61$

LUTORES ION $20 L$

OTHER AIUALYTICAL DATA... PH = B.5.

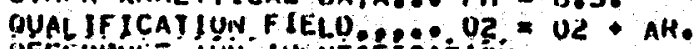

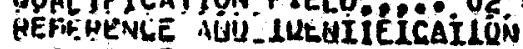

CIIMP ILEI) HY ........... LAWSON, WILLIAM A.

C.IMPILER AFF ILIATION... I, U, UEOLUGICAL SUHVEY

CIMPILER CHOSS INIEX... NEH-Z49

RFFEREHCE............ GUNTER ANU MUSGRAVE, 1966

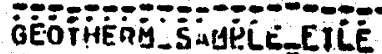

NAMY UF SAMPLE SOMJRCE... MIUWAY GEYSER HASIN - FAIHY CREEK GRUUP - UNNAMED SPRINO

ióition

CIIINTHY ............. UNITEO STATES

IUWYSHIP=BANGE

STATE............ WYOMING

COUNTY............ TETON

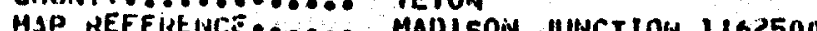

DI'tË? LOCALITY INFORMATIONE JUUM WEST UF NO. IS.

SAMIDE UESCESLEILUN ANQ COQUI IIONS

DATE/CULIÉCTUR....... 1965/UT/UO

SIMPI.E NIMUER ...... 36

TEMPF. HA TIVE (C)

UTHE!? SAMALL LINF URMATION.. VERY SMALL PUOL.

GÁ Aivilisis

VATE AAISAL.YST..... 1965/UT/OO UUNTEH, H.U. ANU MULRAVE, B.U.

at.rís IN quLE

CHe.0 0,40

Chr.:

N2... $\quad 20.64$

HECOND 00227

co?

710

LSOLOPES HOLZLL

\section{CQUHOLAAIES}

GeUTHERM FILE IUI 0020353

LAT/LUNG... 44-32.18 N 110-51.27W 
OTHFH ANAL YIICAL OATA... PH = H.S.

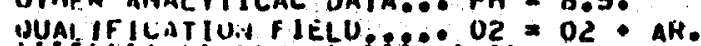

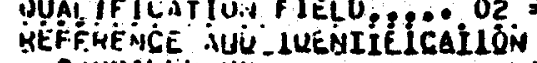

C IMPILEU HY............. l.AWSUN, WILLIAM A.

C.MMPILEH AFFILIATIUN... W. W. GEOLUGICAL SUHVEY

CIIIPILER CKUSS IMNEX... NEH-24H

HEFERENCF............. GUNIER AND MUSGKAVE, 1966

RECUHD 00228

QjEOTHEOU_SAUELE_LILE
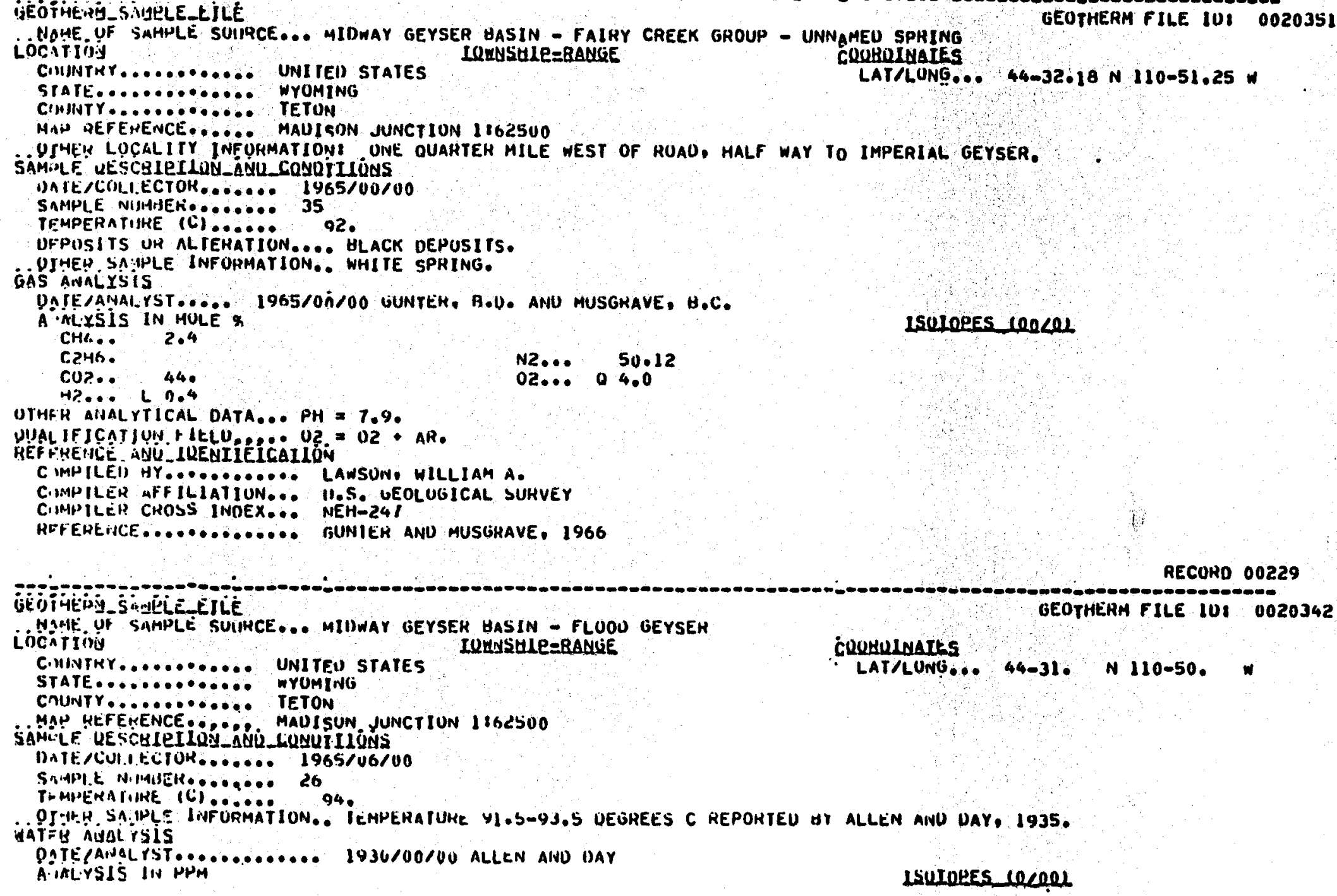


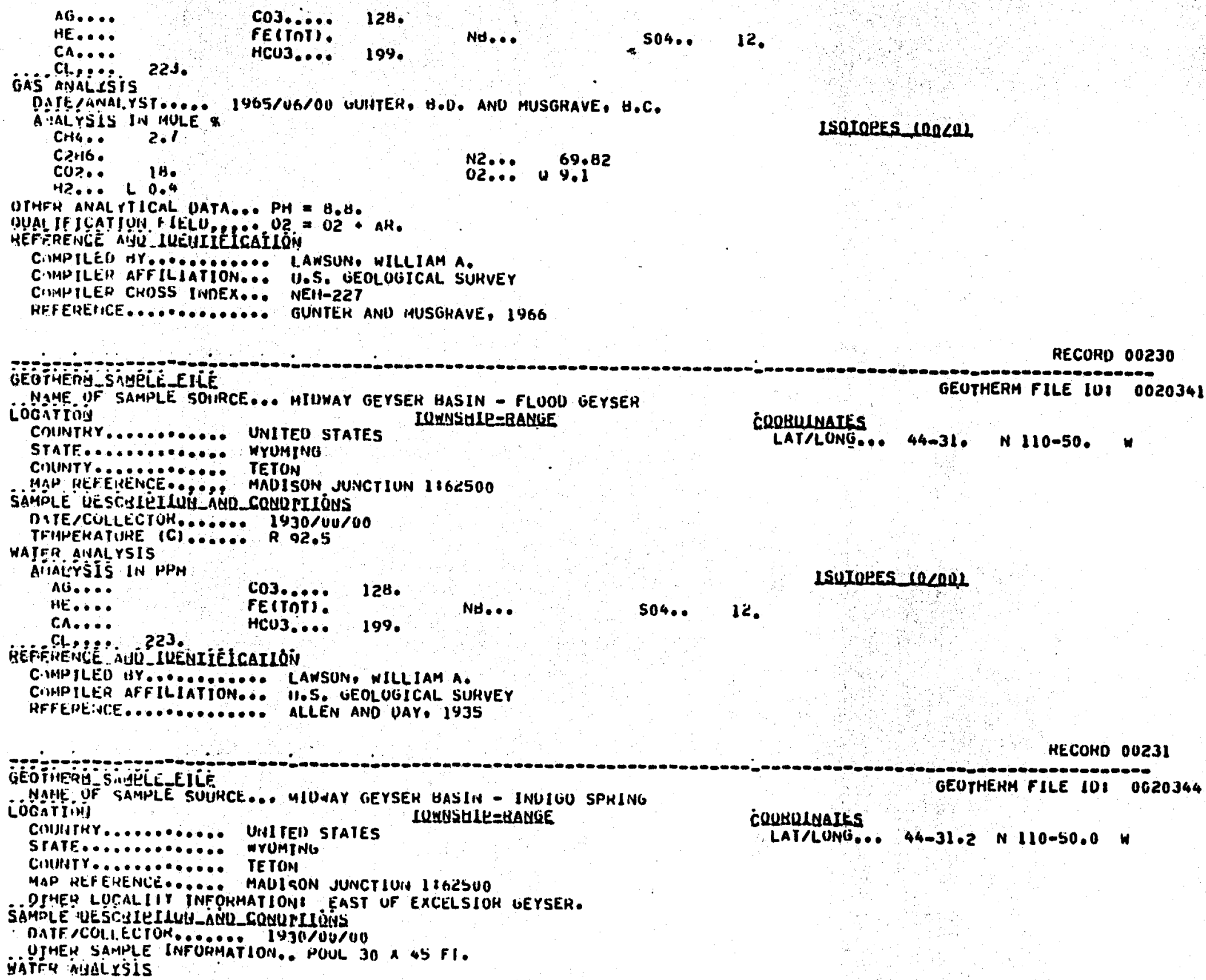


C:IAHTYE J JAIALANCE (\$ UIFF) ¿. 6.6

Aini YSIS liv HPH
AG....
Co3.... 3A
LI.:
AL . . 3
ISË....
CA,
CA... 3.
CH.?.?.
FElTnT) $\int_{469}$
Co...:
MG...
NA.०
K....... 13
NH. $\bullet$

\begin{tabular}{|c|c|c|}
\hline & s.... 0 & 0.67 \\
\hline $\begin{array}{l}4 \\
\therefore\end{array}$ & $\begin{array}{l}\text { S102. } \\
\text { s04... }\end{array}$ & $\begin{array}{l}272 \\
16 .\end{array}$ \\
\hline
\end{tabular}
YAL IFICATION FIELO, . S CALCULATEO FHOM SZOZ.

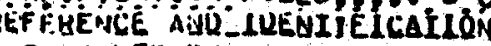
C:MM ILEO HY.*...... LAWSUN, WILLIAM A
CIMPILEN AFF ILIATION... N.S. GEOLUGICAL SUKVEY
HFFENEHCC̈_........... ALLEN AND DAY,1935

LSOTORES COENOL

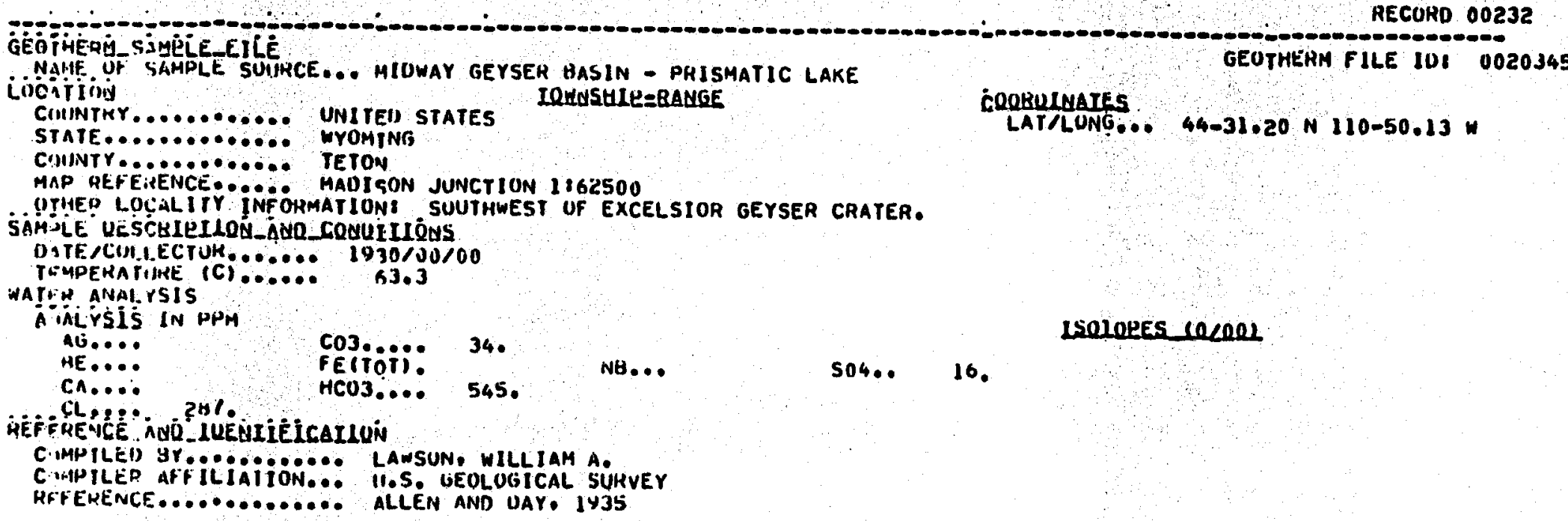

RECUHO 00233

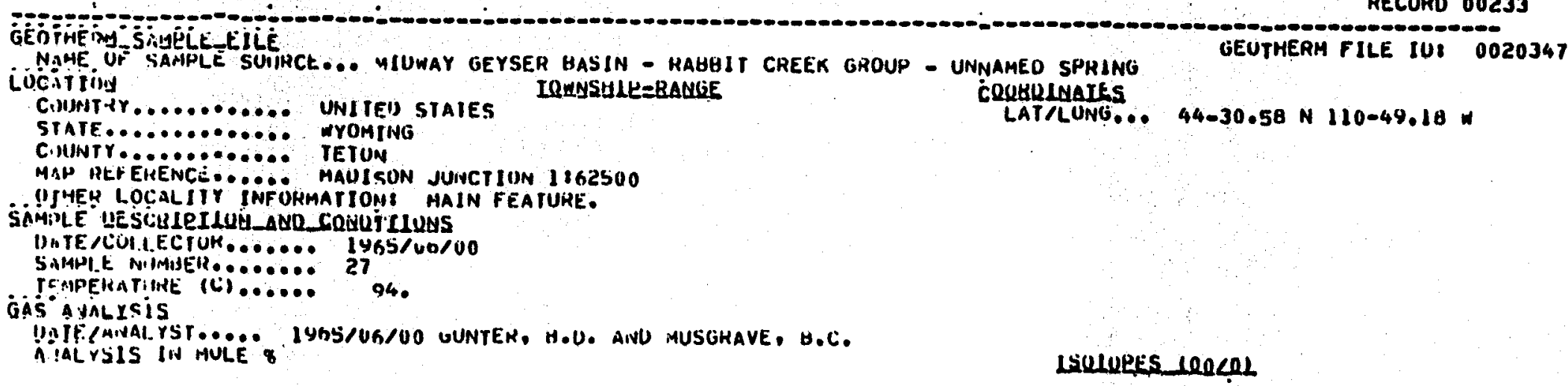


CHi... 2.3

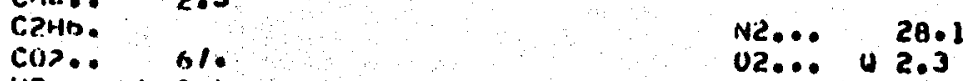

$$
\text { COS.: का }
$$

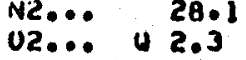

UTHFH AVAL, YTICAL DATA... PH $\approx$ B.J.

JUAL IFICATJUN_t IELO, . $02=02$. AK.

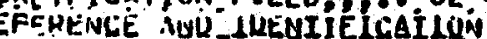

CIMP ILËI) HY............ LAHSUN, WILLIAM A.

CIMPILEN AFF ILIATION... No. W. GEOLUGICAL SUHVEY

C.MMNILER CIROSS INDEX... NEM-ZZH

HEFERENCE........... TUNTEK ANU MUSGHAVE, 1966

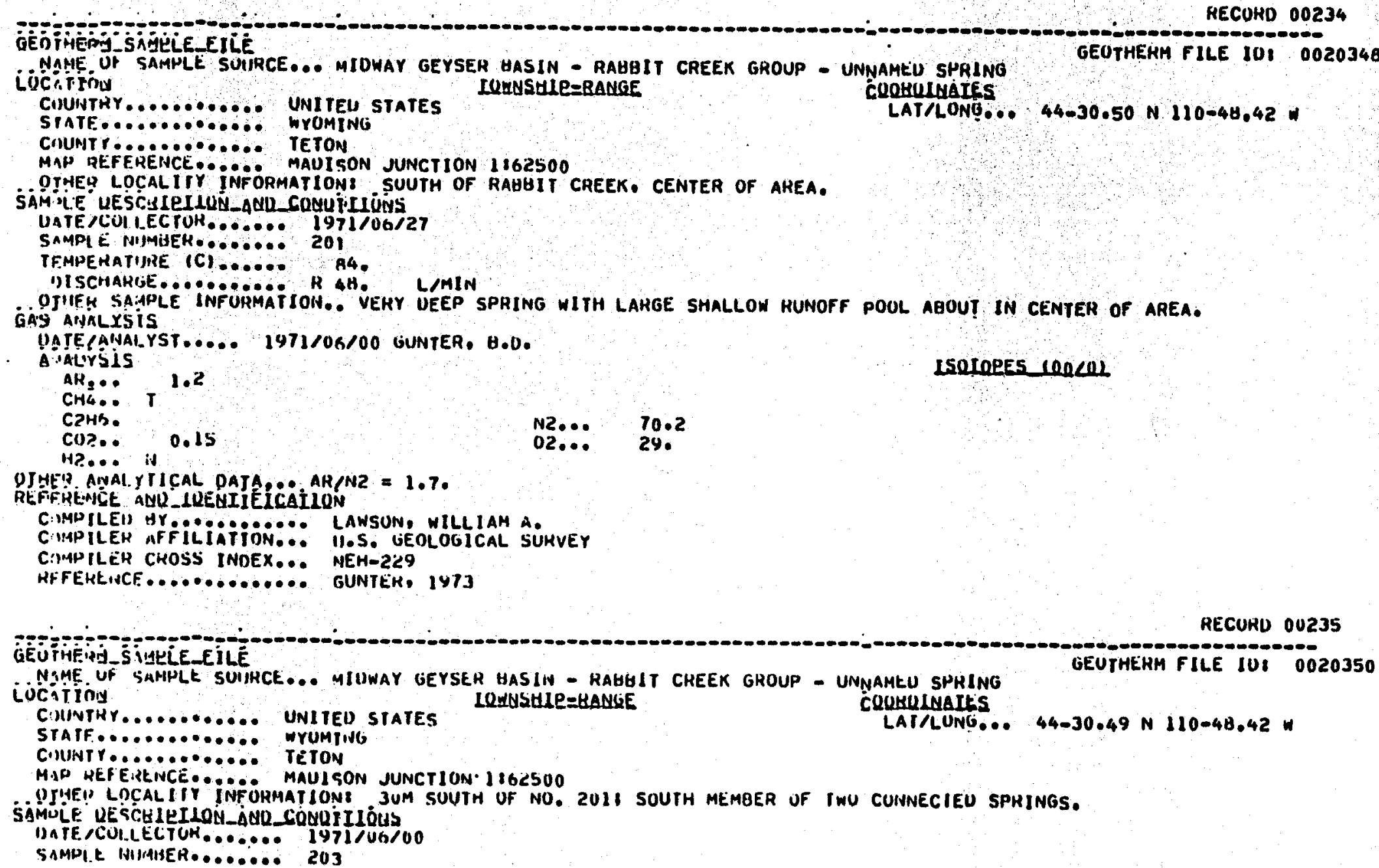

LSOTORES $10 R \angle I I$ 
TF.MPEHATIIRE (C)..... Q Q0.

IISCHIAHITE........... H 67. L/MLN

OIMFO SAHHL INFUMMATION. LARGE CLEAH SPHING WITH CAVED IN EDGE. GÁS MUALÝSTS

DATE PANALYST MÜ. $1971 / 66 / 00$ GUNTEH, H.U.

CHe 1.3

LSOTORES Cancel

CHA.?

C2:16.

Cu2..

H? $\ldots$ is in

1. $\quad \begin{array}{ll}\mathrm{N} 2 \ldots & 85.8 \\ 02 \ldots & 12.0\end{array}$

OJHEP ANALYTICAL DATA OARINZ = 1.76.

UEFERËWCE,ALU_ILENIIEICAIION

CIIMPILED HY.......... LAWSUN, WILLLAM A.

CIMPILER AFFILIATION... U.S. GEOLOGICAL SUHVEY

CIMPILER CKOSS INIJEX... NEH-2JI

RFFE HEIYCE............. FUNTEK, 1973

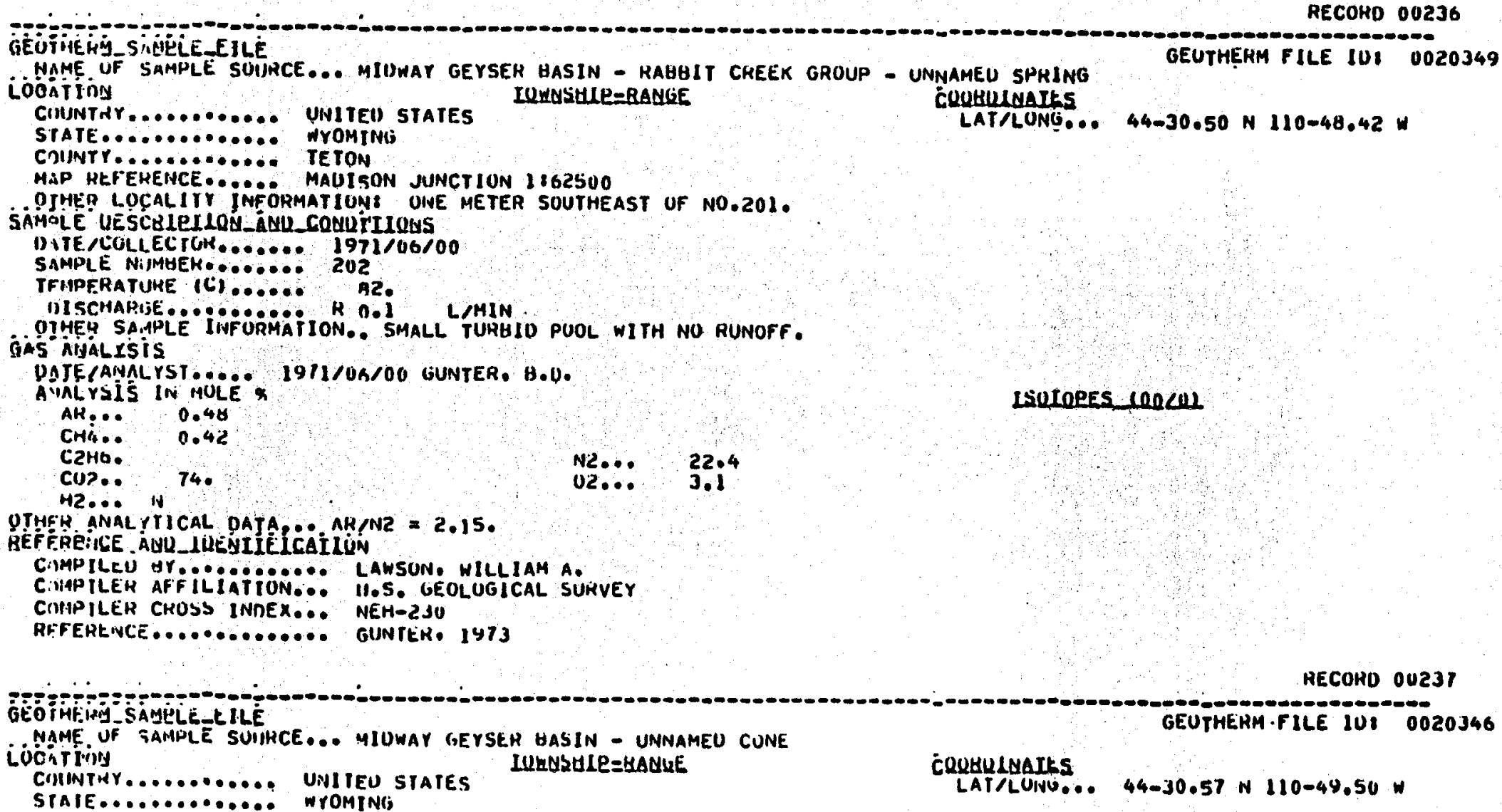

RECOHD 00236

STAIE.......... WHOMING; 
C.MINTY

MAP ZEFEAKINCE...... MALISON JUNCTION 1162500

USHEY LOCALIIY IMEORMATIONE HIÖHT HANK OF FIREHOLE RIVER, 150 YOS, HELOW UPPER BHIOGE.

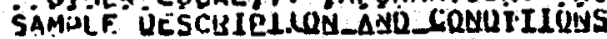

IIATE/CULIECTOR....... 19.30/00/00

TFMHEKATIKE (C)....... 92.6

WATER ANALYSIS

AIC

IALYSIS. IN HOH

Co3 15

CA....

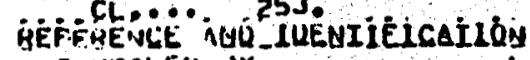

COHPILEU HY............ LAWSUN, WILLIAM A.

C.MMPILER AFFILIATION... II.S. ULULOHICAL SUKVEY

HFIEHEINCE............... ALLEN AND UAY, 1935

RECURD 00238

GEOTHENM SIMPLELEILE

GEUTHERM FILE IOI 0020339

NAME UH SAIPLE SOIRCE... MIOWAY GEYSER UASIN - UNNAMEU SPRING

inciling

IOWNSHIP=HANGE

COIINTHY............. UINLTEO STATES

IOWNSHIP-HANGE igohulNaIES

LATILUNG 44-31, N 110-50. N

STATF.

COUNTY........... TETON

MAP UE FENENCË. .... MAUISON JUNCTION 1862500

OJ'HEY LOCALITY INF OHMAIIUN? EAST BANK OF FIREHOLE RIVER.

SAMLE UESCHIEILQU_AUD_COUDTILUS

DATEACULIECTCK....... 1930100/00 SAMPLE NIIAHEH........ 19

GAS ANALYSIS

a uni ís Is liN vUluHE

CH4.. $n .2$

HeS.: n

C) 20.91

12... 4.95

ISOTOEES $10 \angle Q O L$

che.

91

U2...

0.3

DUA IF iCAllUU FIELD, O N2 = HZ - AHO

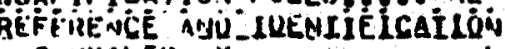

CIHPILEU IYY........... LAWSUN, WILLIAM A.

CIMPILCH AfFiliaTiON... 11.S. UEOLUGICAL SUHVEY

CIMAILEK CRUSS INDEX... NEH-225

RFFERENCE.............. ALLEN AND OAY, 1935.

RECUHO 00239

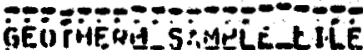
Nalle UF

Lócirition

UIIKCE... NORIA HUFFALU FUKK SPAINGS - GROUP 1

STATF............. MYATIVG

45 iv III 32 NE UF $S E$

CInINIY

GFOLIJGIC ORCVIATCE..

TETON

MAP REF F.REIYCE...... JOY PEAK 1824000

OJHEL LOCILITY INFOHMATIUPI GKUUP UN EASI SIOE OF BUFFALO FORK AT JUNCTIUN WITH SOUA FORK.

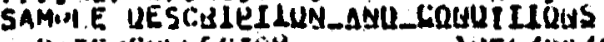

DATEACULI.ECTOH....... IY5h/UO/00 
TMPERATIHE IC HOS THE GROUP FOR TMIS ANALYSIS IS OESCHIBEU AS VIOLENTLY BUBGLING, NISCHARGE. INFUHMATION. IWU GROUPS

UTHEH SAMPLE TMF WNO A SULFUH SMELL. SOMALS TASTE ANO A SULF

BËFrGENCE AUU-LLEIIEICAIL HEASLEH. H. P.

CIMPILEU HY

CIMPILER AFFILIATION... INIVERSITY OF WYUMING HINCKLEY, 1978

RFFEHEHCE..........

RECOKD 00240

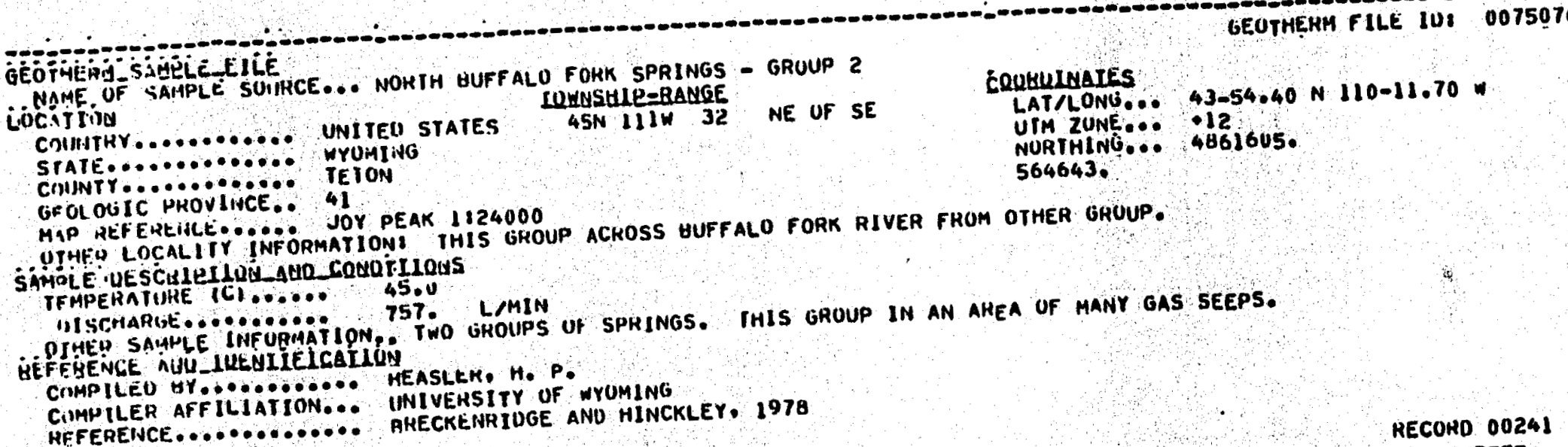

GEOTHERM FILE IUI 0020287

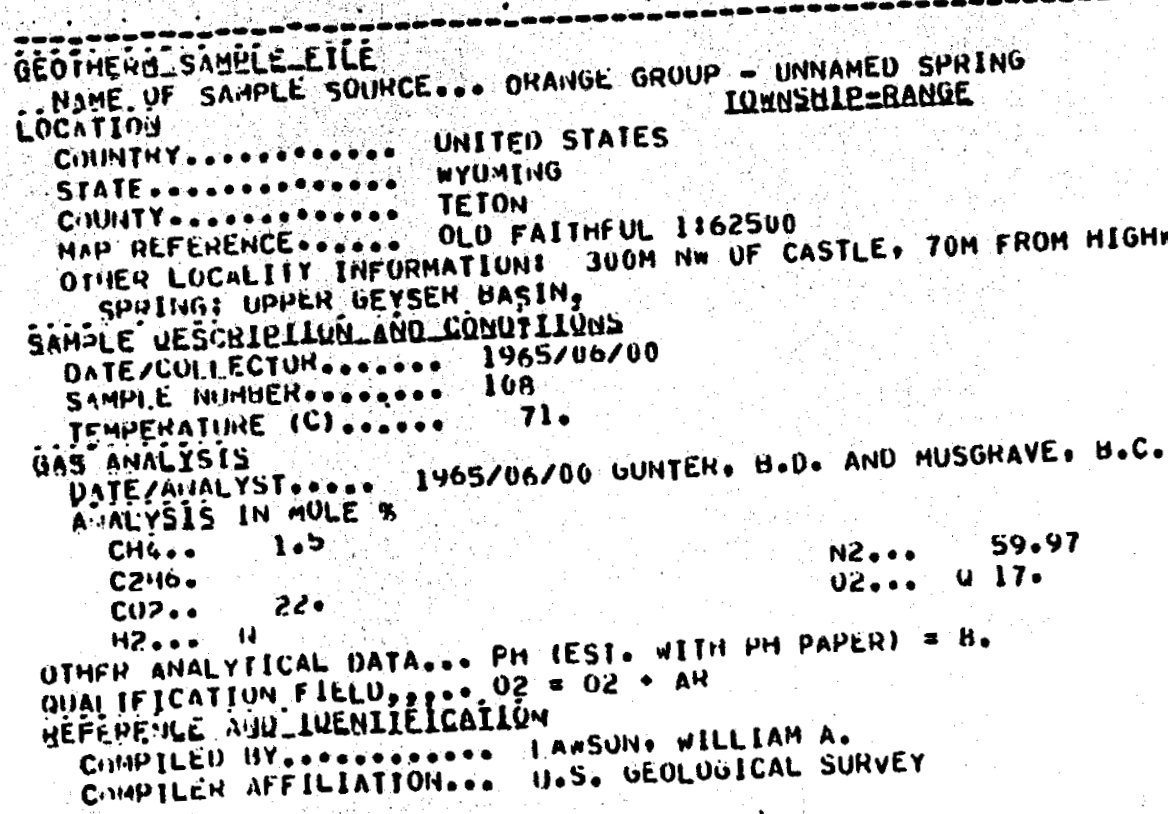

NAME UF SATPLE SOUHCE... OKANGE GRUUP TOUSELE=RANGE
COCATINIY

STATE

D TIEQ LUCALIYH GEYSEK GASIN

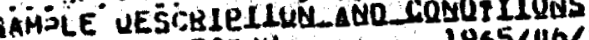

OATE COLLECTUR...... IOS

Samperto 710

As Anvilisis

OTE KAUALYST IN MULE

CH4.. 1.5

C2416.

0... 417.

HP.... I I

OTHFN ANALYPICAL DATA,O PH EST WITH

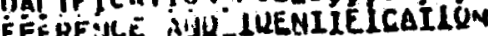

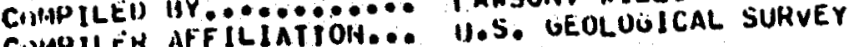

\section{EOOHULUAISS}

LAT/LON6... 44-27.77 N 110-50.75

LSULOPES HOCLOL 
C.MPILEH CHUSS INNEX... NEH-210

HFFEHENCE............. GUNTEK ANI MUSGRAVE, 1966

RECOHD 00242

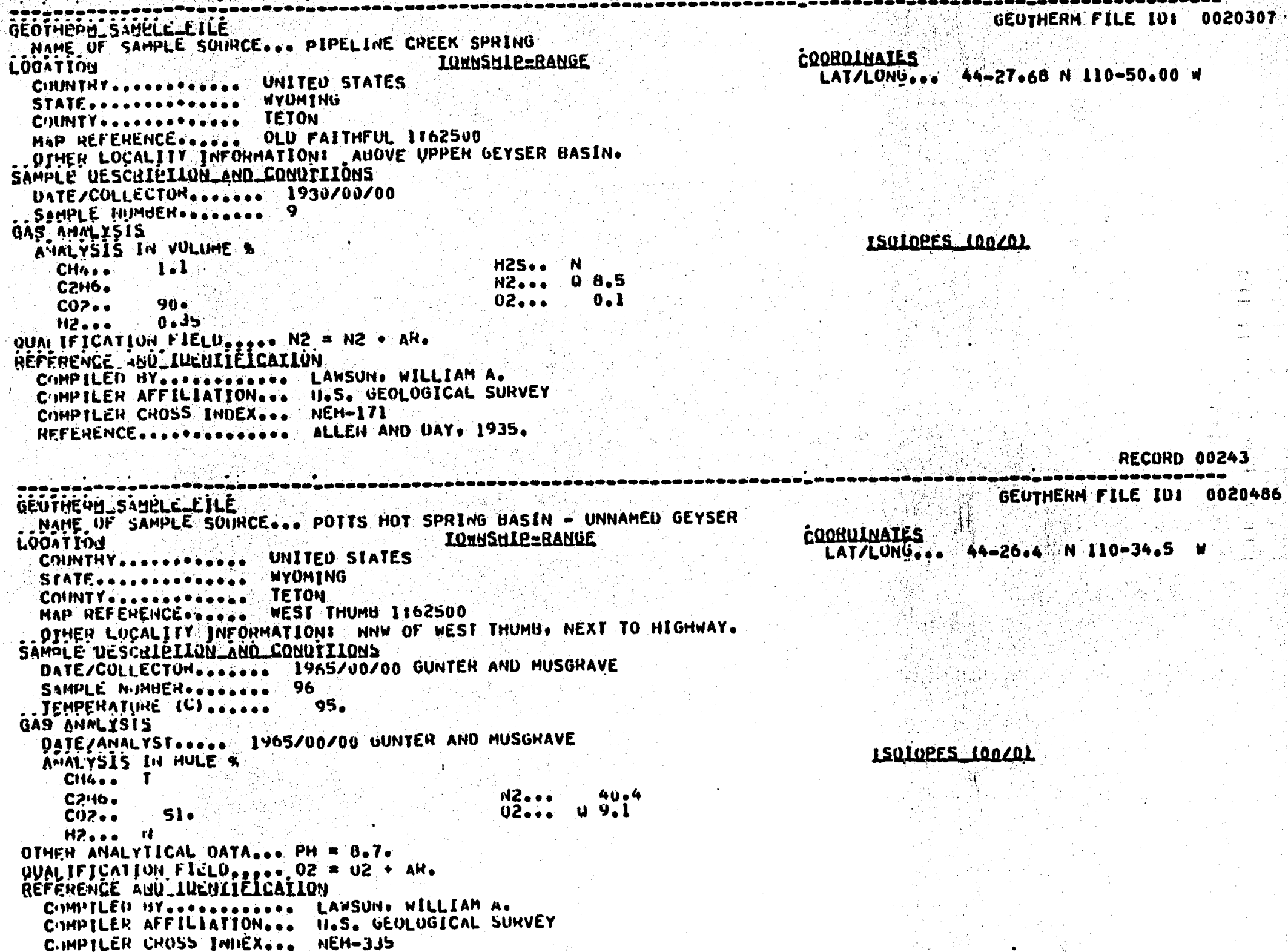

RFFERENCE ............. ALLEN AND UAY. 1935

ISULEES $100 / 0 \mathrm{C}$

RECORO 00243 


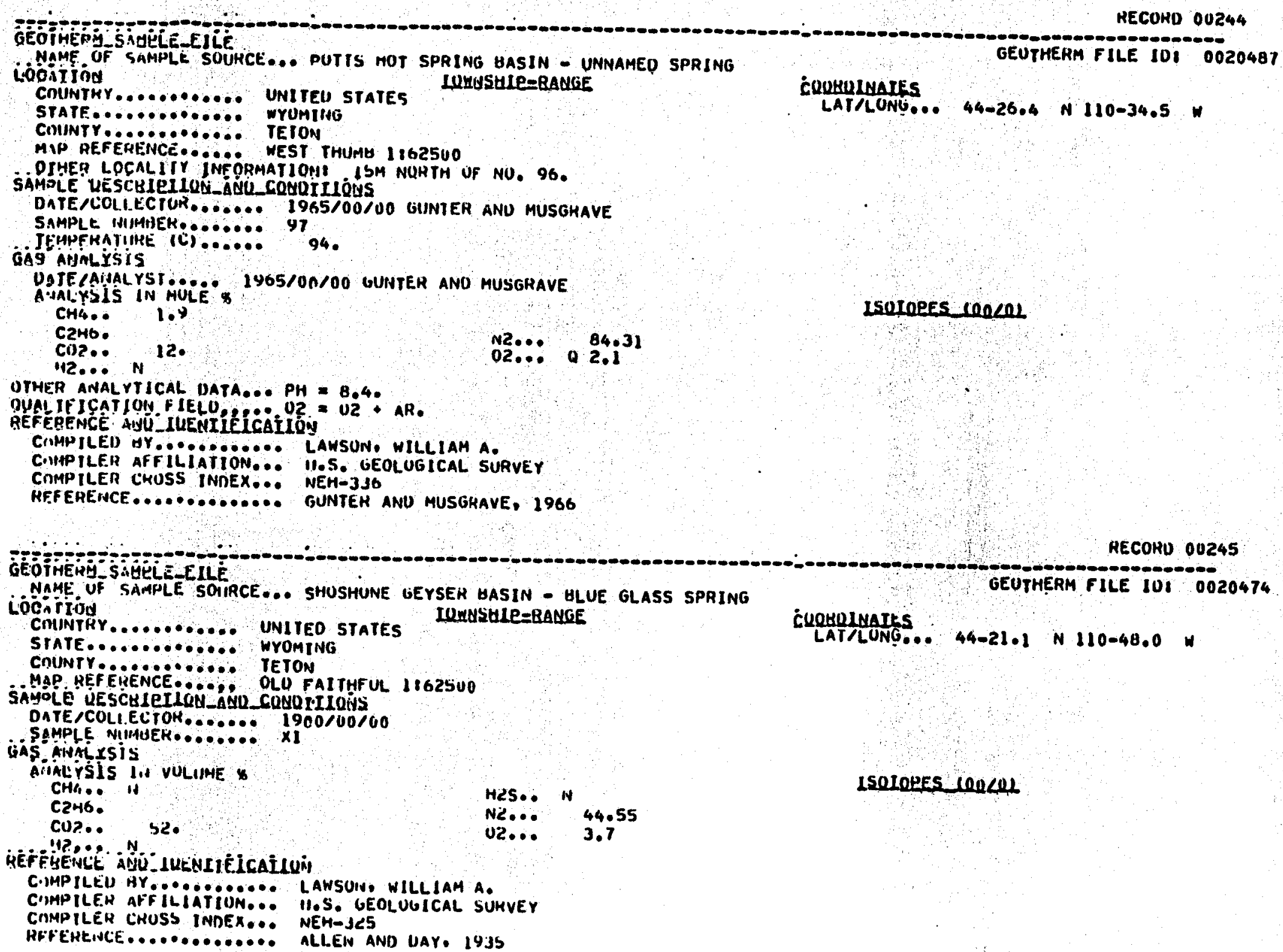

GEOTHETH SAULEELILE

NAHE UF SAMPLE SOIRCE... SHUSHUNE GEYSER UASIN - HLUE GLASS SPRING

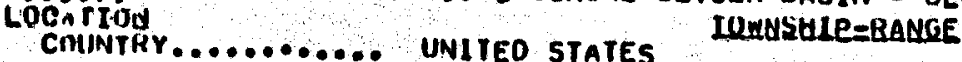

STATE............... WYOMING

CNUNTY............. TETON

MAP HEFERENCE.... OLU FAITHFUL 116250

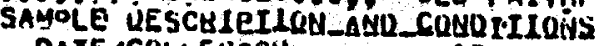

DATE/COLIECTOH....... $1900 / 00 / 60$

SAMPLE NIHAUER०..... XI

Gas AivMirsis

AiinLYYSIS I.A VULLAME

CHA...

C246.

Cur.. S2.

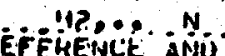

C.PENCE ANÜ-IUENIEEICAIUUA

C.MMPILEUI AY............ LAWSUIN, WILLIAM A.

CIMPILE.H AFFILIATIUN... N.S. GEOLUGICAL SUHVEY

COMPILEA CKUSS INDEX... NEH-JLS

RFFEHEINCE............. ALLEIV AND VAY. 1935

RECOHD 00244

GEUTHERM FILE I0! 0020487

Counuimarts

LAT/LONE... 44-26.4 N $110-34.5$

LSOIOEES CONCOL

RECOHO OUZ45

GEUTHERM FILE IOI 0020474

\section{CUOHOLNATIS}

LAT/LONG .. 44-21.1 N $110-48.0$ N

LSOLOLES (00LUL

N2S... N $\quad 44.55$

U2... $\quad 3.7$ 


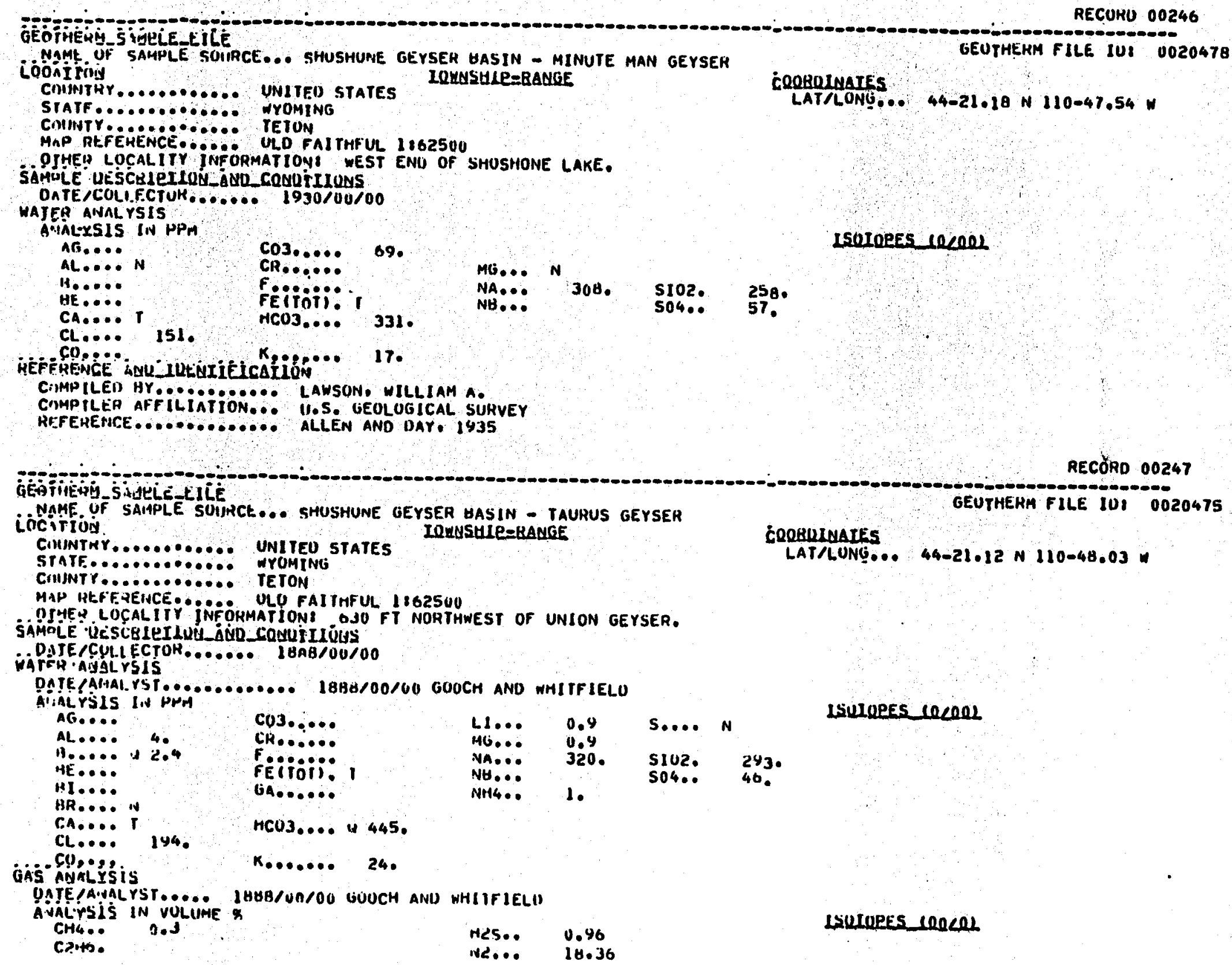


con? 780

U2... 0.23

UTHFH ANALVTICAL UATA... HZO ANALI ASZOS - U. 7 HPM.

GUAL IF ICAIJUI, IELU, IE HCO3 IS A CALCULATEO VALUE, B CALCULATEO FROH HZO3,

CIMPILEU HY.......... LAWSUN, WILLIAM A.

C.MPILEH AFFILIATION... M.S. GEOLUGICAL SURVEY

CAMPILEK CHUSS IWNEX... NEH- 326

REFEHENCE............ ALLEN AND UAY, 1935

GEOTHE SAULLELE

MAIE. UF SAMPLE SOINCE... SHOSHUNE GEYSER HASIN - UNION GEYSER

Liogifives

CIUNTHY

STATF.

COUNTY

MAP QEFEKENCE

SIHEH LOCALITY INFOHMATIONB WEST SIUE OF SHOSHUNE LAKEI APPROX. 2700 FT WEST OF. LAKE.

SAMRLE QESCAIEILU_AQLCOAURLUUS

DATFR AUALYSIS

CYAPGE IMUALANCE (\$ UIFFI... I2.1

AiAtrsís IN PHM

AG... IN PPM

AL.... I

B......

HE.

CA... 20. 235

Co3.... 77 .

LI...

S.... N

ISOIOEES IOCOUI

Feitio

NA...

364.

5102.

332.

CU....

MCO3... 310

NH....

so4..

45.

RECOHO 00248

GEUTHERM FILE IU: 0020477

cookunNarts

LAT/LUNG .. $44-20.48$ N $110-47.55$ W

KHE.... 130

OJHER ANALYTICAL DATA. $0.5203=1.5 \mathrm{PPH}$

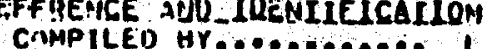

COMPILED HY..@..... LAWSUN, WILLIAM A.

CIMPILER AFFILIATION... 1.5. GEOLUGICAL SUHVEY

RFFERENCE................ ALLEN AND DAY, 1 Y35

HECURD 00249

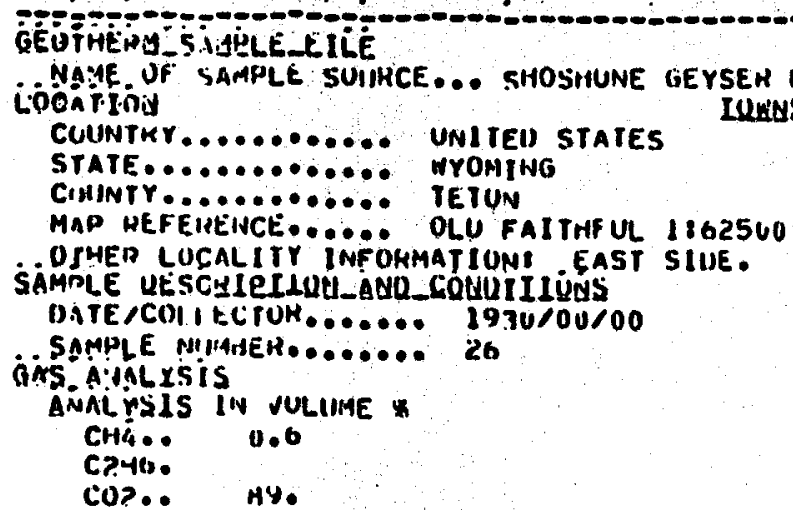

HY.

1152

N20.. 4.7

ISULOEES COOCOL

क.


H2... n.05

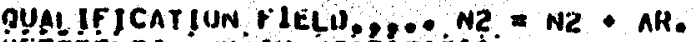

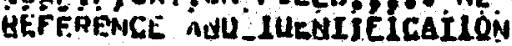

CTMPILEN HY......... LAWSUN, WILLIAM A.

CIMPILEH AFF ILIATION.- H.S. GEOLOGICAL SURVEY

COHAPILEP CHOSS TWREX... NEH-JZT

RFFEREHICE............. ALLEN AND DAY, 1935

GÉOTHÉM̆ SAMULE EILE

iochílion

CIUNTKY........... UNITEU STATES LQ... ULALEQAHCE

STATF.

CIIUNTY ........... TETON

MAP JEFEREINCE...... MAUISON JUIVCTION 1162500

OPIAES LOCALITY INFPRMATIONE NEXT TO ROAD, NORIH SIDE, APPROX. 3345 FT. NOHTHEAST OF MADISUN JUNCTION.

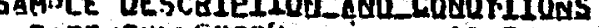

DATE/CULLECTOK........ $1965 / 00 / 00$

SAMPLE NIMUER

TEMPERATIRE (C)

GAS ANALÝYis

DOJE YANALYST BÖE 1905/0O/00 GUNTEK, H.D. ANU MUSGRAVE, H.C.

CHá 0.4

LSULORES concul

C2HO. 0.4

H2... L 0.4

OTHTH AINAL YTICAL DATA... PH $=6.0$

DUA! IFICATIUN FIELO, $02=02+A K$

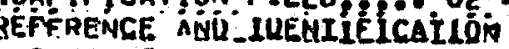

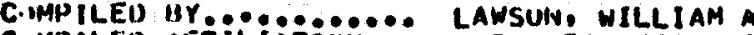

C.MPPILER AFFILIATIUN... 11.5. GEOLUGICAL SUHVE

C.MHP ILEH CHUSS IWDEX... NEM-ZII

HEFERENCE............. GUNTEK ANU MUSGKAVE, 1966

RECUKD 00251

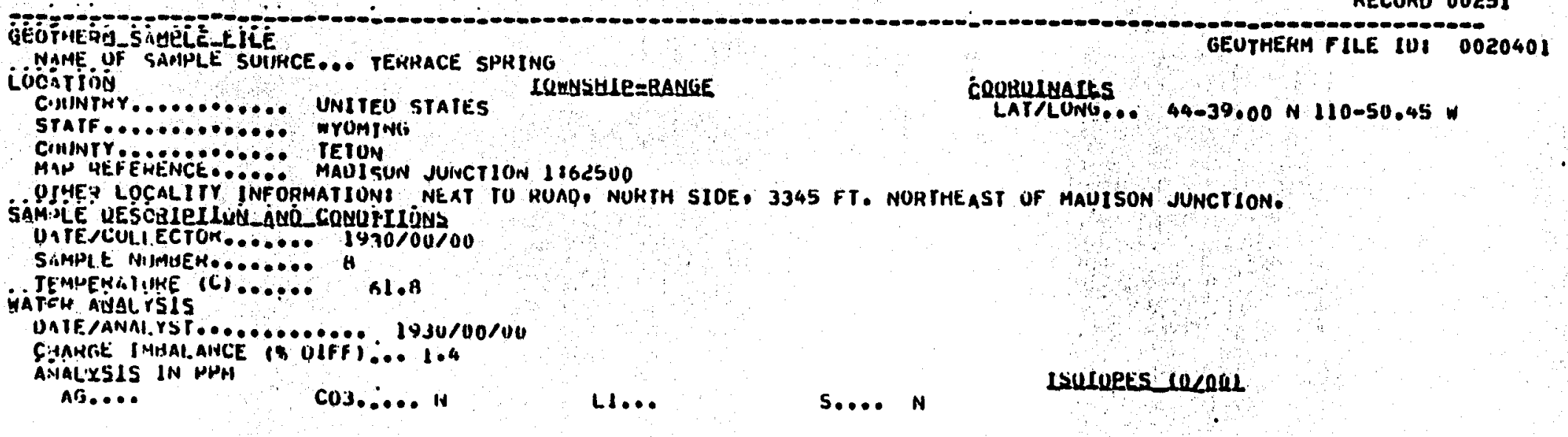

RECOKD 00250

GEUTHERM FILE 1010020402

COUKOLNATES

LAT/LONU.. 44-39.00 N110-50.45 W

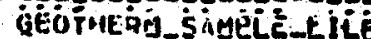

GEUTHEKM FILE IU! 002040

OOANE UF

Caring

UNITEO STAIES

QRuLuarts

AM:LE UESCALILIVA-4MÓ CQNUEIIUNS

UTEACULI.ECTOK..... $1930 / 00 / 00$

ATEH AUALCISIS

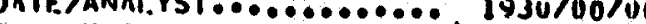

cos...... N

L...

S.... $N$ 


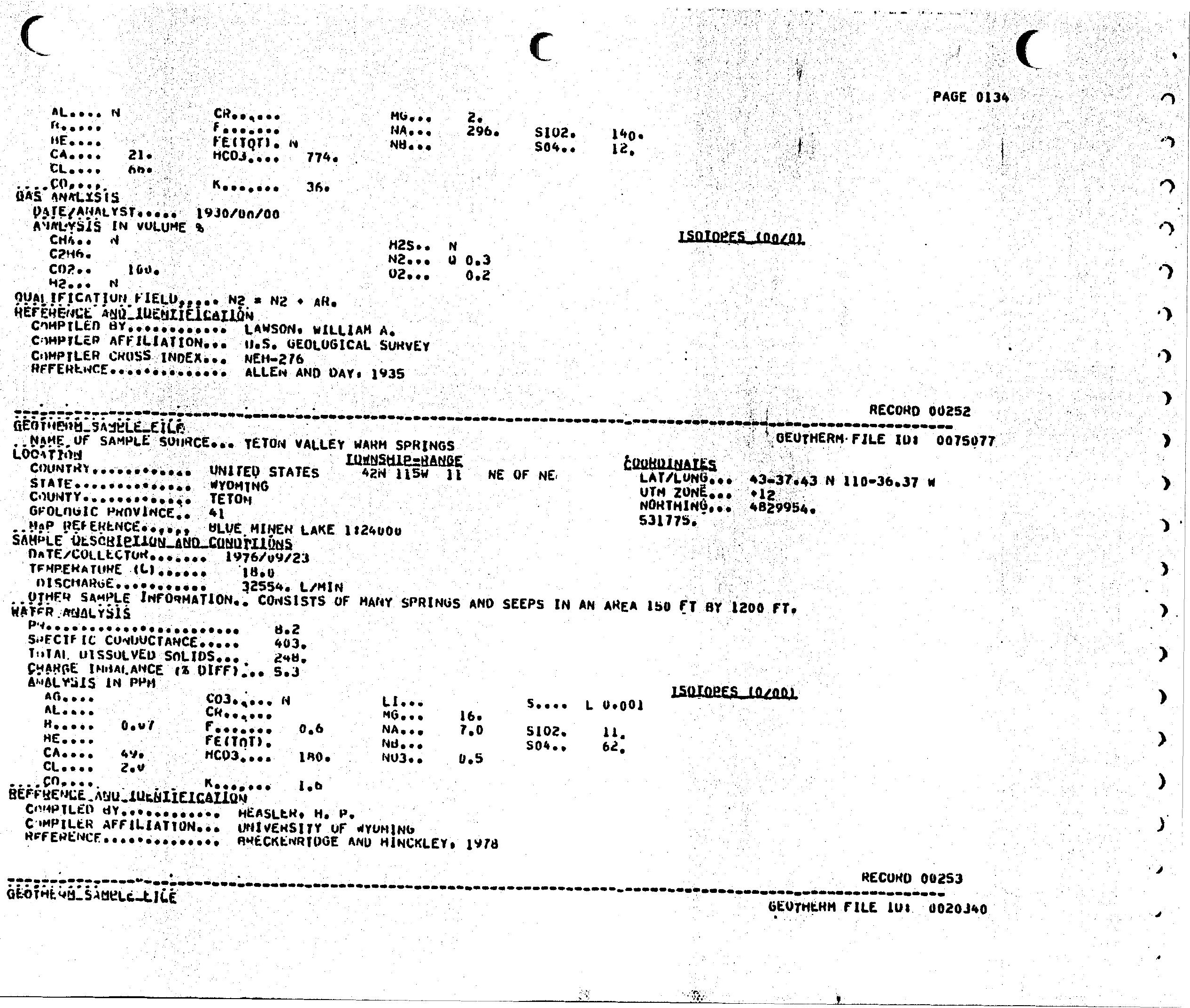


NAME UF SAMPLE SOINCE... IINNAMED SPHING Locitioy

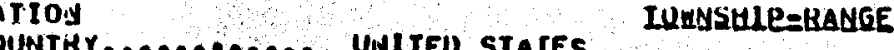

STATE............ WROMINR

COATIY.... W

MAP AEFEKENCE - MAOISON JUNCTION 1162500

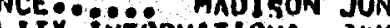

S

Ja

DATE/COLIECTOH...... 1965/06/00

SAMPLE NIMUEH...... 25

IEMPEKATUNE (C) ...... 94.

GAS DNALYS IS

DATE /ANALYST..... 1965/06/UO GUNTER, H.D. ANO MUSGHAVE, B.C.

A VACYSIS IN MULE

CH4.: 0.41

C246.

H2... L 0.4

UTHEH ANALYTICAL DATA... PH $=8.7$.

QUAL IF ICAT IUN FIELU,

REFERETCE ANU - LULUIEICAILO

CIMPILEO HY........... LAWSUN, WILLIAM A.

COMPILER AFFILIATION... H.S. GEOLOGICAL SUKVEY

CIMPILER CHUSS INDEX... NEH-226

RFFERENCE............. GINTEK AND MUSGRAVE, 1966

\section{couHulNaIts}

LAT/LONG, 44-31, N 110-50, N

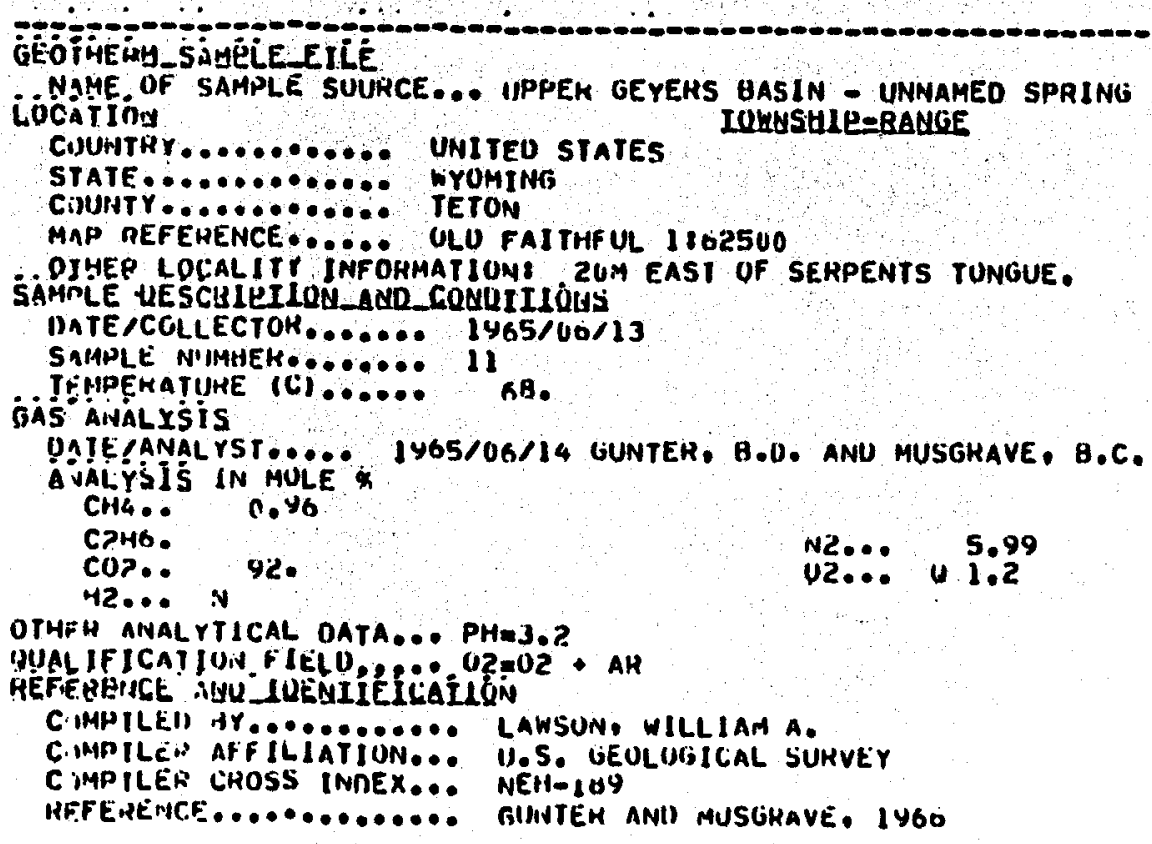

\section{RECUKD 0025}

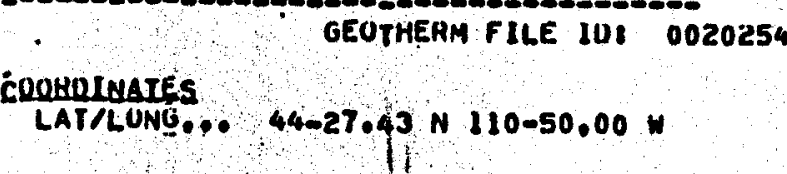




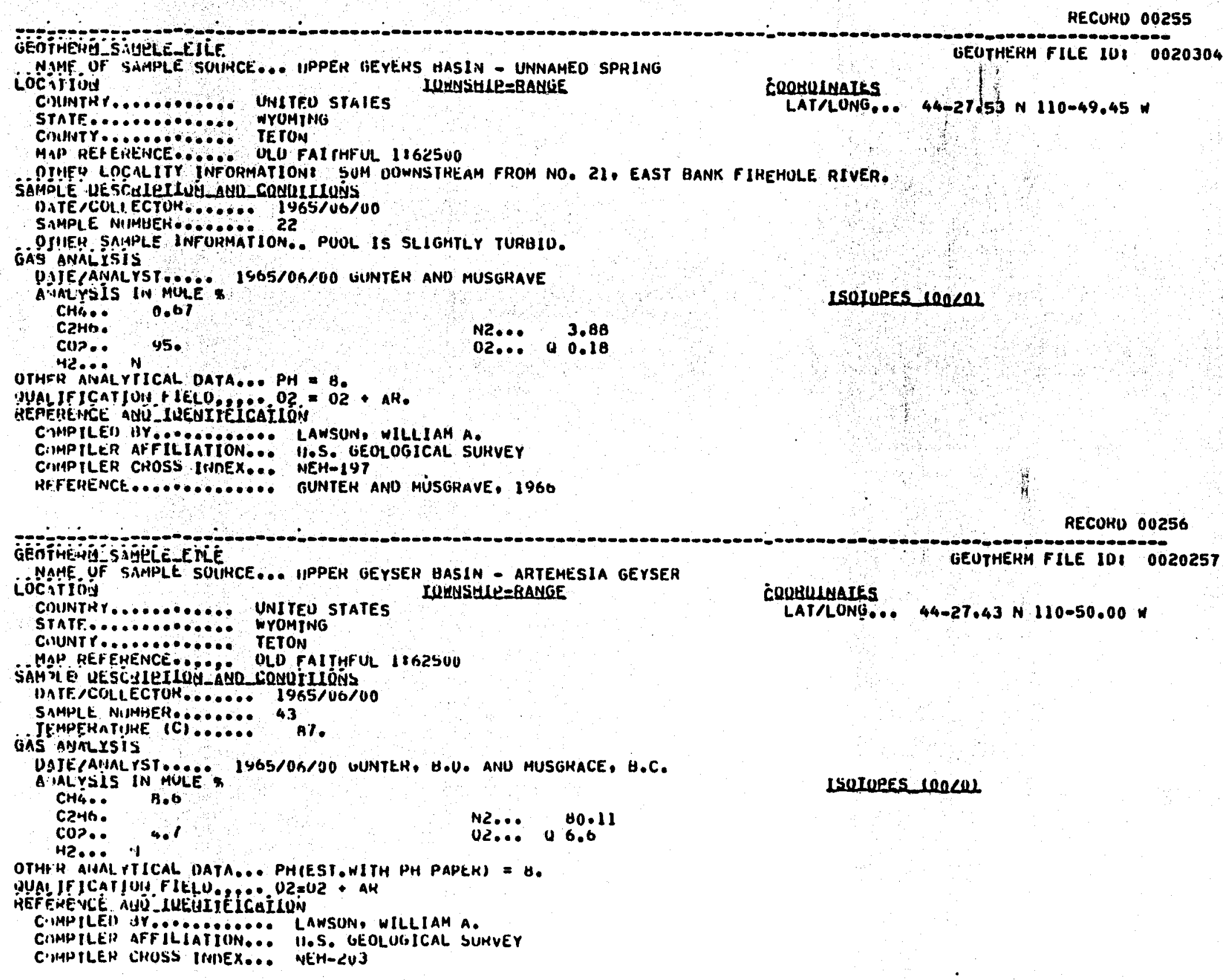

DOJE/AHALYST..... IY65/0K/00 UUNTER, H.V. ANU MUSGKACE, H.C

CHLSLS IN MULE$$
\text { N2... } \quad 80.1
$$

H2 $\ldots$. I

OTHF AIJAL FTICAL DATA... PHIESI.WITH PH PAPEK) $={ }_{0}$

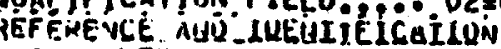

CIMPILER AFFILIATIÖN.... II, S. GEOLUGICAL SURVEY

CIMHILER EHOSS IMINEX... NEH-LUJ 
CUINTHY.......... UNITEU STATES

STATE

CINUNTY........... VETUN

MAP QEF EIRENCE,, 0, OLO FAITHFUL 182500

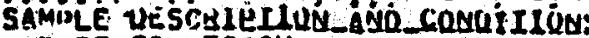

DATE COL.ECTOK

SAMPLE NIMHFHE...... 16

OTHEH SAIHLE INFURMATION.. TEMHERATURE VAKIES FROM 83.7 TO 91.5 WAJEH AWALYSIS

Aival.rSIS IN HPH

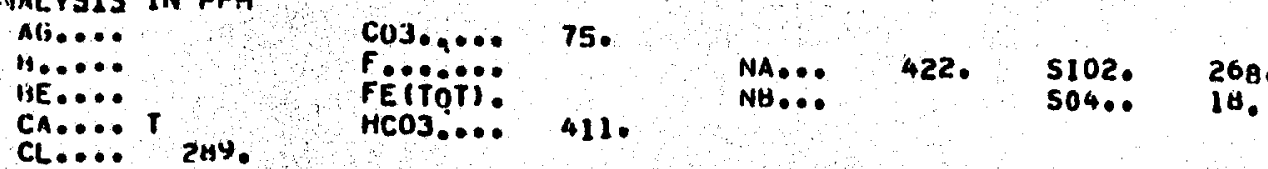

Co, K..... 16.

AAS ANALYSIS

CH4.. 11 .

CPHS.

11.

C.:

UTHFH ANALYTICAL DATA . O $8203=10$. PPM

WUAL IF ICAT IUN FIELO OE NE NZWN2 - AH

CMMPILED HY OOP... LAWSUN, WILLIAM A.

CINAPILER AFFILIATION... H.5. LEOLOGICAL SURVEY

CINAPILER CHOSS INDEX... MEH-I 18

HFFEKËHCE ............ ALLEN AND UAY, 1935.

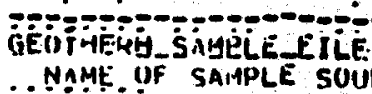

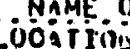

CiUNTHY

STATE . . . .... UNITEU STATES

CIUHT........... WYOMING

CIIINT T............ TETU

AIItUUL 186540

UMTE

ONTE CULLECTOK....... 1900/00/00

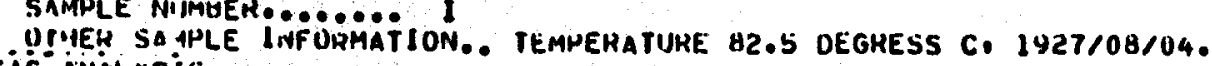

GAS ANALIS IS

D.TE/AINAL PST......

Gialoysis in vulume

corlace. in

C246.

cur.:

L.d
NHILLIHS, B.C.

$\begin{array}{ll}H 25 \cdots N & N \\ \text { N2... } & 94.08 \\ 02 \ldots N & 3.5\end{array}$

AECOHD 00258

ĊUHULUATES

LAT/LUNG... 44-27.43 N 110-50.00
CQUBULUATES

LATRUNG... 44-27.43 N110-50.00

ISOTORES inenOL

ISOIORES $100 / 01$

ISUIOPES loneOL 


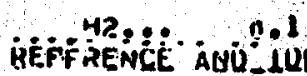

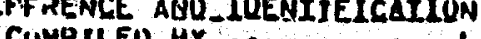

CIMP ILEI UY HO

CIMPILER AFFILIATTON... H.S. WEOLOGICAL SURVEY

CIMPILER CHOSS INDEX... NEH-16O

HFFEREIJCE ............. ALLEN AND WAY 1935

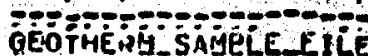

RECUHD 00259

NAME UF SAMPLE SOIJKCE... IPPPEH GEYSER BASIN - BEEHIVE GEYSEH iociritió

IOUNSHLE=RAULCE

STATE.............. WY UYMING

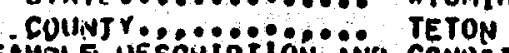

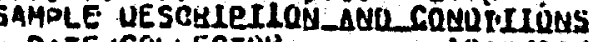

DATE/COLLECTOH

TFMPEHATIME

WAIFH ANAL YSIS
AVIAL

AG.....

co3..... 29.

L1...

S.... a 1.

LOIOEES WeCOQL

H...... 128.4

HE.....

F.........

NA....

322.

s102.

CA..... I

FElTOTI. N

NB...

$504 .$.

294 .

CL.... 378.

HCO3.... 102 .

K....... 18.

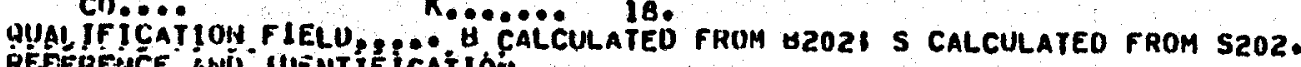
REFERETCE AQUL JUEUIIEICAIIÓN

COMP ILEO HY........ LAWSON, WILLIAM A.

COMPTLEH AFFiLiAïiön:.: IISS. GEOLOGICAL SUHUEY

HFFEHEINCE.............. ALLEN AND DAY, 1935

GEO

RECOHO 00260

iöcivitiols

CONUNTHY............. UNITEU STATES

LUENSHIR-RAMGE

STATE............ WYOHTNG

CHATE 00000000000 MYOMINC

SBMDLE UESCHIPIO

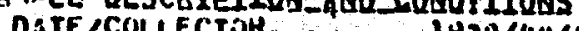

DSTE/COLI.ECTOK........ 19.30/00/00

TFMPEHATIIHE ICI...... A. A.?

WAIEH ANAL YSIS

Auaie is Ís IN PPM

AG....

AL.... N

H..... 1 11.81

HE....

HI....

CA.... 1.

Cli.....

1..

Co3..... N

ISOIOPES IOCARL

GEUTHERM FILE IOI 002032

CoOHULAAItS

EUTHEHM FILE IDI U020325

Cootuluates

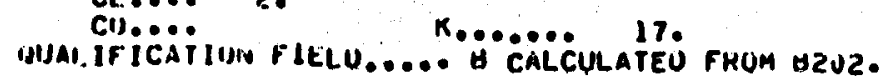




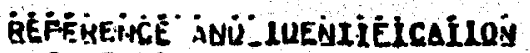

CIMPPILED GY........... LAHSON, WILLIAM A.

CIMPPILE. AFFILIATION... M.S. WEOLOLIICAL SURVEY

RFFERENCE............ ALLEN AND UAY, 1 I3S

RECUHD OU261

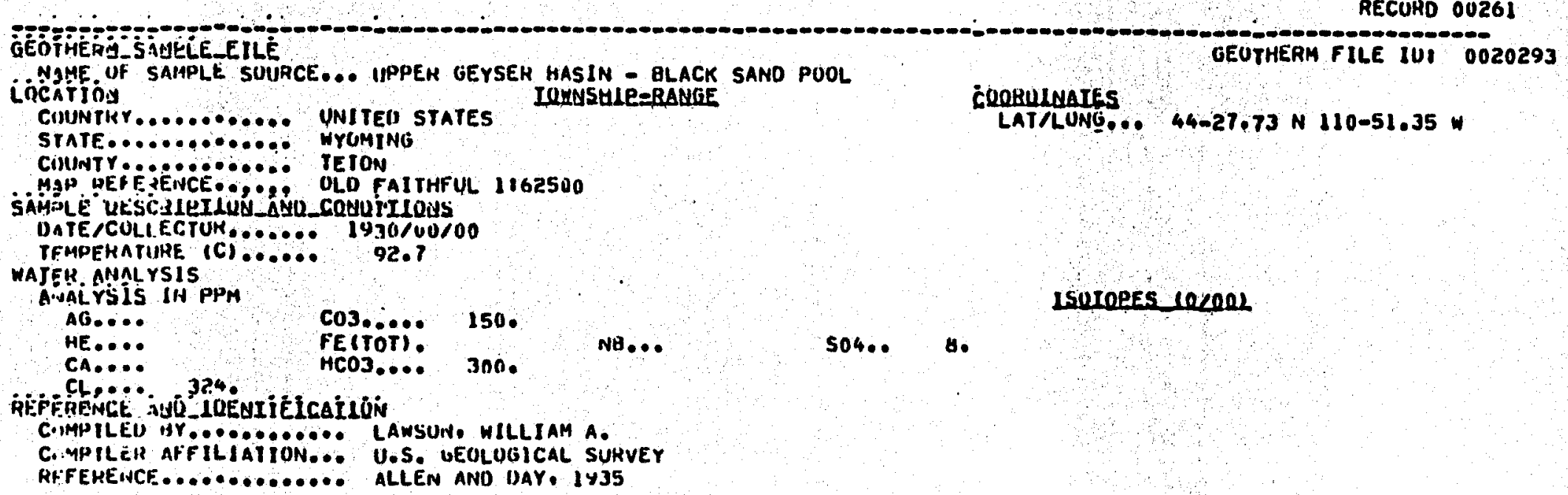

RECUHO OU262

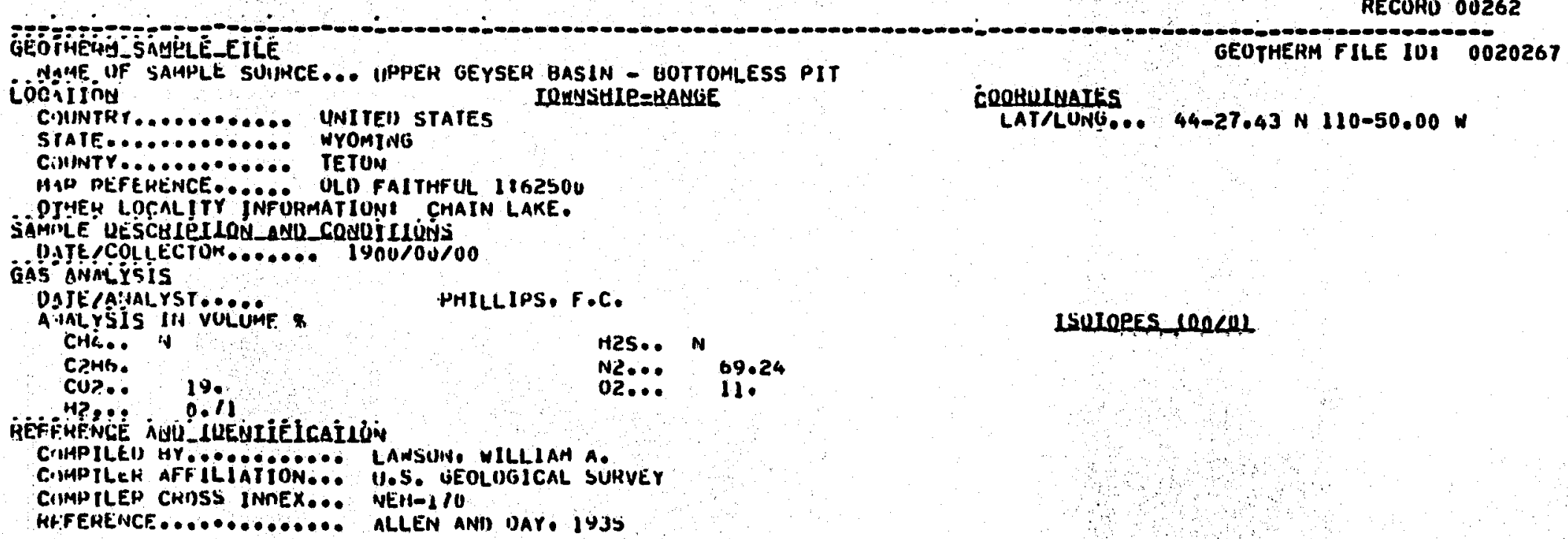

RECUHD 00263 
INAME, UF SAMPLE SUIHCE... IIPPER GEYSEH HASIN - CALTHOS SPRING Locitioü

COUNTHY U....... UNITED STATES LUHSHIE-BANGE

IUHWSHIE-BANGE CUQHULNATES

STATE

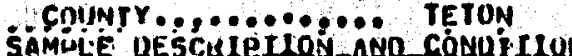

DATE CCOLLETOH....... $19.30 \% 00 / 00$

TFBPF,KA TIIRE ICI...... 94.5

WAJEK ANALYSIS

Avilirsis IN HHM

AG...

Co3.... 42.

FE(TOI):

HE....

CA. … 280.

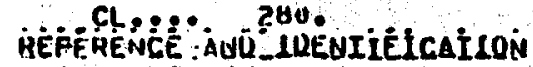

CTHPILEU BY......... LAWSON, WILLIAM A.

CAMPILER AFFILIATION... U.S. GEOLUGICAL SURVEY

HFFERENCE............. ALLEN AND UAY, 1935

ISOTORES COCOOL

$(\cdots$,

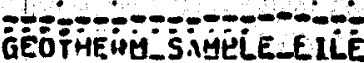

NIME OF SAMPLE SOURCE... ITPPER GEYSER BASIN - CASTLE GEYSEK

Coontrivis

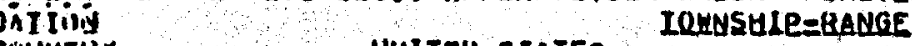

COUNTKY........... UNITEU STATES

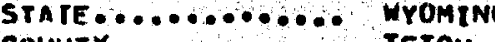

COUNTY........... TETON

MAP QEFEHENCE

SAMOL UESCHIEILOA AŃL CÓNOLIIOUS

OATE/COLLECTUH....... $1930 / 00 / 00$

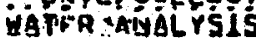

CHARGE IAHALANCE (\$ DIFF) ... 5.2

ANÄLiVSis IIN PHM

AG....

Co3. 42

LI...

AL.... is

H.... is 2 .

Ch.......

FËPöi

HE.....

DI $\cdots \cdots$

FE(TOT).

CA... 1 Jy

GA.....

t

PAGE OI4O

co.

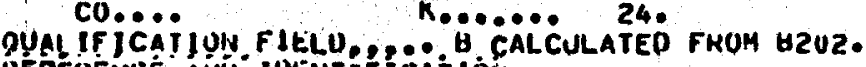

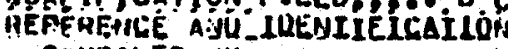

CMHPILED AY.......... LAWSUN, WILLIAM A.

CMHPILEP AFFILIATION... ll.s. GEOLUGICAL SUHVEY

REFEHEINCE........... ALLEN AND UAY, IYJS

AECUNO 00265

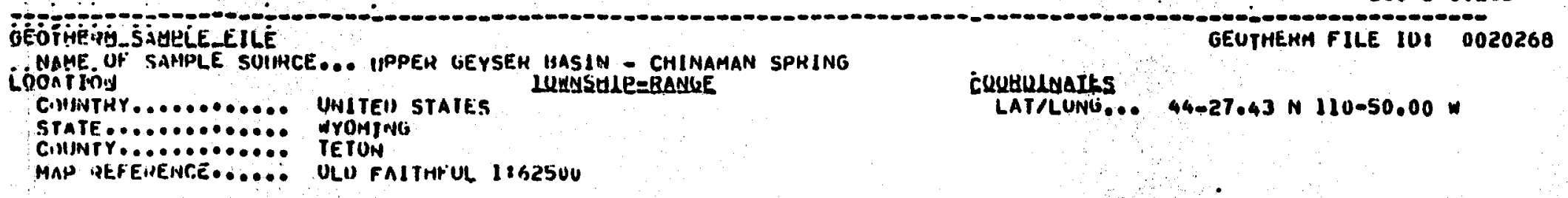

RECURO 00264

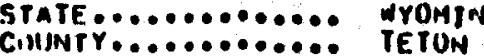

MAP REFEUENCEE...... ULU FAITHFUL I:G25UU 


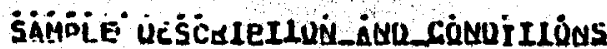

$$
\text { DATE/CÜLLCTOH....... 1930/00/00 }
$$

TFMPEKATIJHE (C) ...... 93.5

MATER ANALYSIS

- iAi Ýsis IIN PNM

AG.....

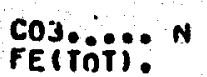

CA....

FE(ToT):

HCO3.... 174

NH...

LSOIOEES $10 \mathrm{COOL}$

GASG CLABAZÝSTS

A UALUSSIS IN VOLUME

CTHA: 0.15

C?HO.

प्र2.

COP.:

0.45

OUN IF ICATIOW FIELU ,O NZ = N2 - AK.

REFFHENCE MÜLLEUIÉlCAILÚ

C.MMPILEU UY

C.MMPILER AFFILIATION... H.S. GEOLOGICAL SURVEY

CIMPILER CHOSS INDEX... NEH-172

KEFERENCE............ ALLEN AND DAY, 1935.

RECOKO 00266

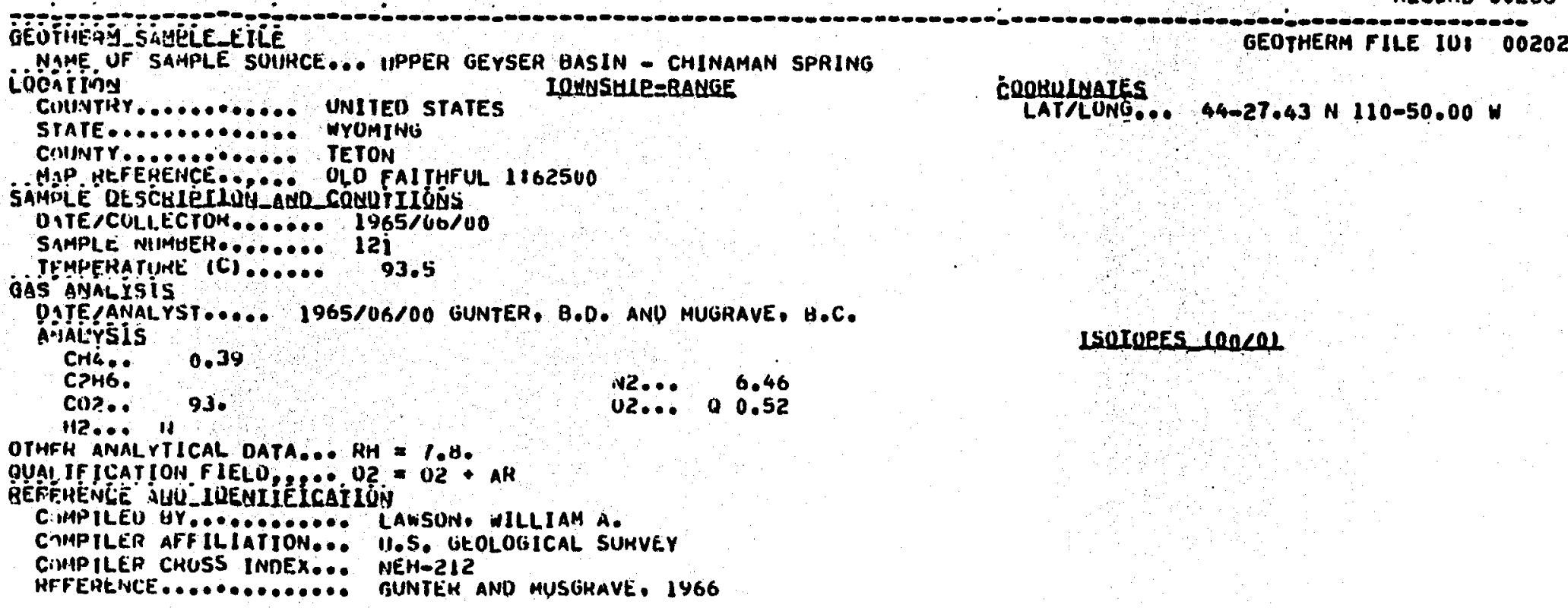

LSOIREES CORCOL

RECUKO OU267 


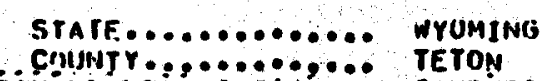

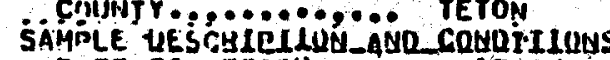

D.TE /COLI.ECTOH........ 1930/NO/UO

TFMPEHATIJKE (C) ...... \$9.6

WAJER AINAL YSIS

Q.vAlOSSIS IN PHM

AG....

(2)

UE....

co3.... 10.

FE(TIT) NH...

CL. 300.

590.

A

CFUENCE ANU LUEUIIÉlCAILON

COMPILEO BY .......... LAWSON, WILLIAM A.

COMPILËP AFFILIATION.:- W.S. GEULOGICAL SUHVEY

HFFERENCE .............. ALLEN ANO YAY. 1935

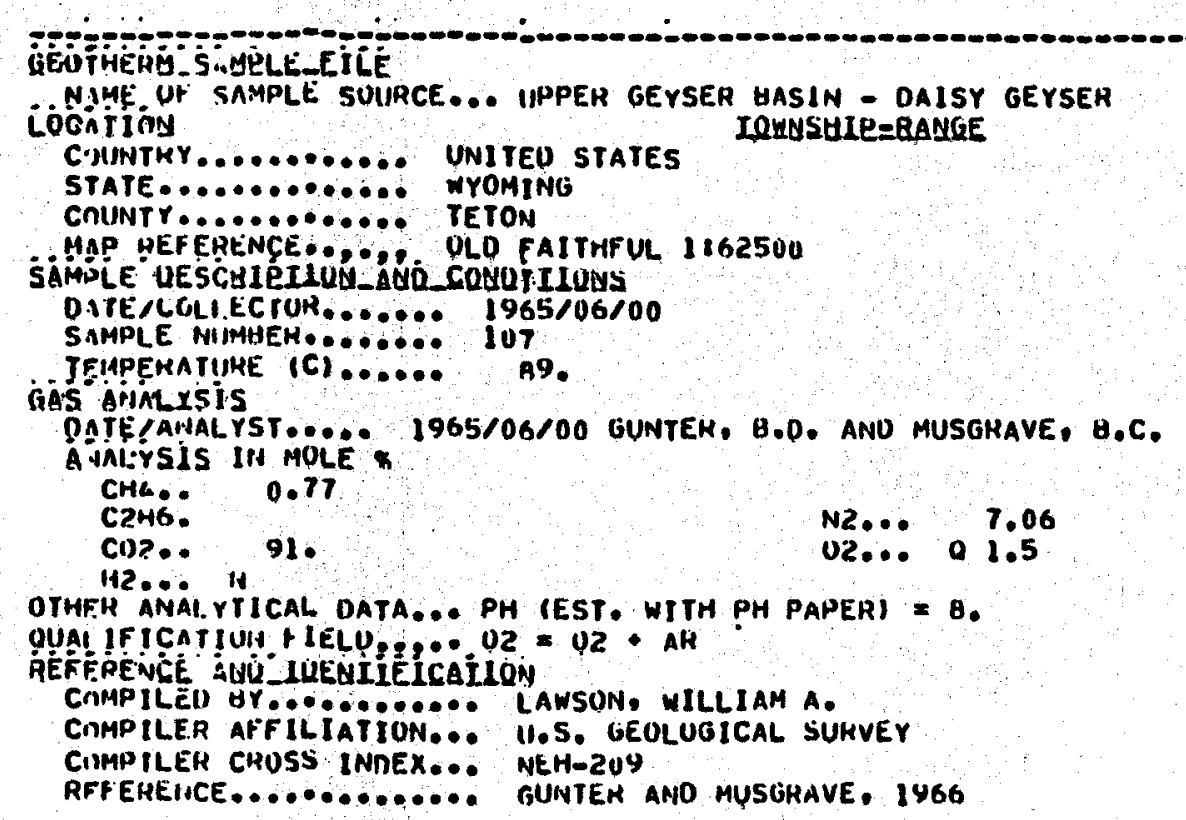

KECOHO 00268

GEOTHERM FILE IU: 0020277

LSUIOPES loenOL

25 


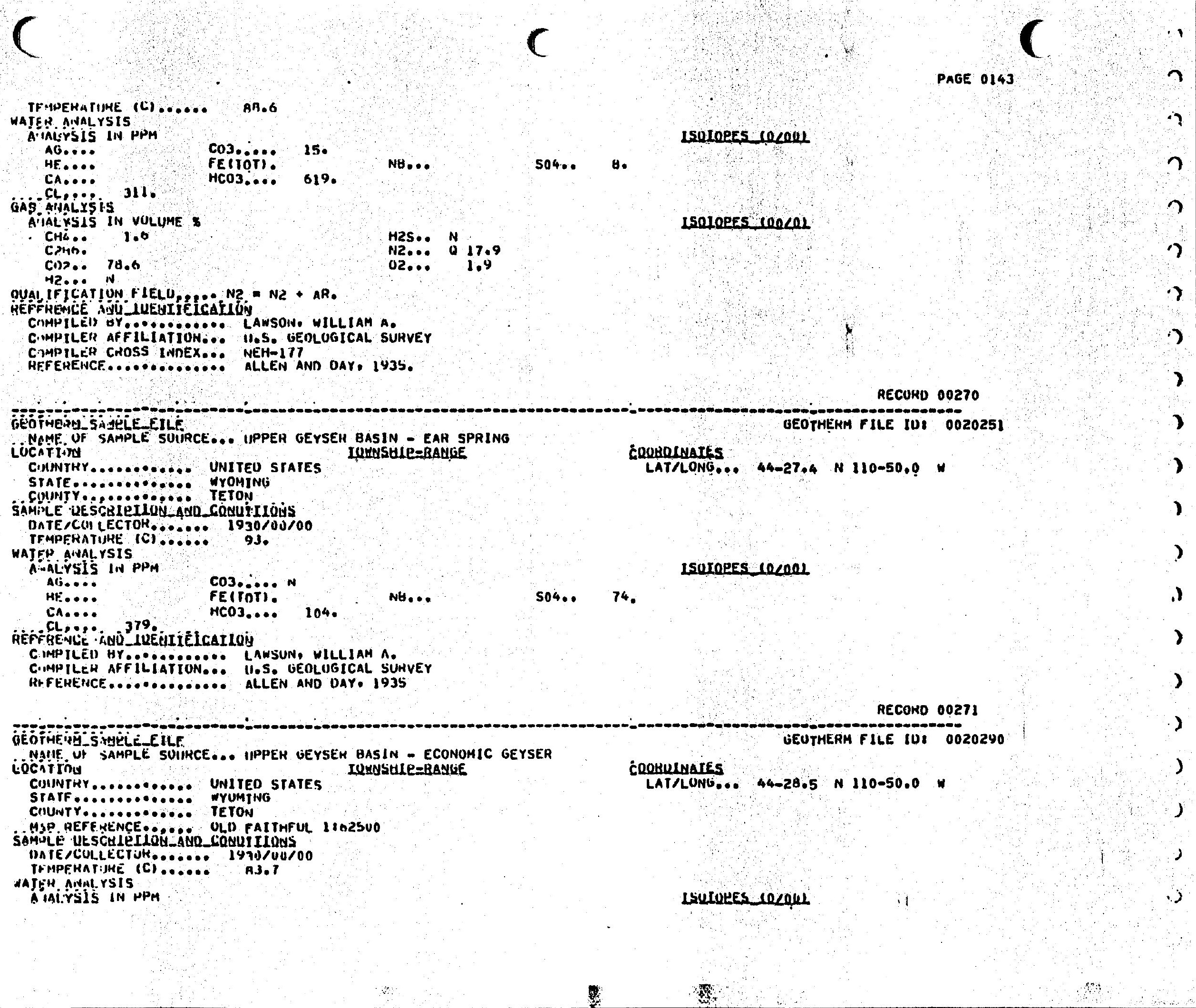


CA... J TOT. HCO3... 362.

PAGE 0145

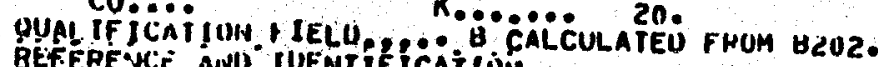

BEFEPE HC AUL IUENIIELCAILON

C:IMPILED HY H....... LAWSUN, WILLIAM A.

COMPILER AFFILIATION... H.S. LEOLOGICAL SUHVEY

RFFERENCF............. ALLEN AND WAY, 1935

GEOTHEHY SAMELE TIL

RECUNU 00274

LOCAME UF SANPLE SUIRCE

-. IPPER GEYSER BASIN - GIANT BEYSER

STATE.....

OHASHIL=RANGE

TrUMING

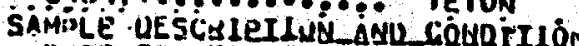

DATE,COLLECTUR..... $1930 \% 0 \%$

TFMPEHATUHE (Ci

ATEMPKATURE 94.3

AJE ANAL YSIS

HALSIS IN PPH

A6....

$603 \ldots \ldots 52$

L. .

$M \ldots \ldots$ in 2.6

$M E$. . . .

CA..... I

CL.... 247.

Co....

FËTÖT:

HCO3... W 487

NA...

NH...

5102.

0.0 .33

ISOIORES COCOOL

GlukDinaIES

GEUTHERM FILE IOS 0020324

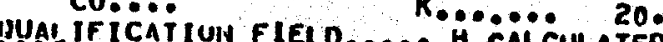

REFERFMCE ANU IUENITEICAIO

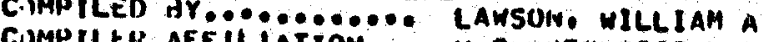

RFFERENCE

HFFERENCE............. ALLENA AND UAY, 19Jj

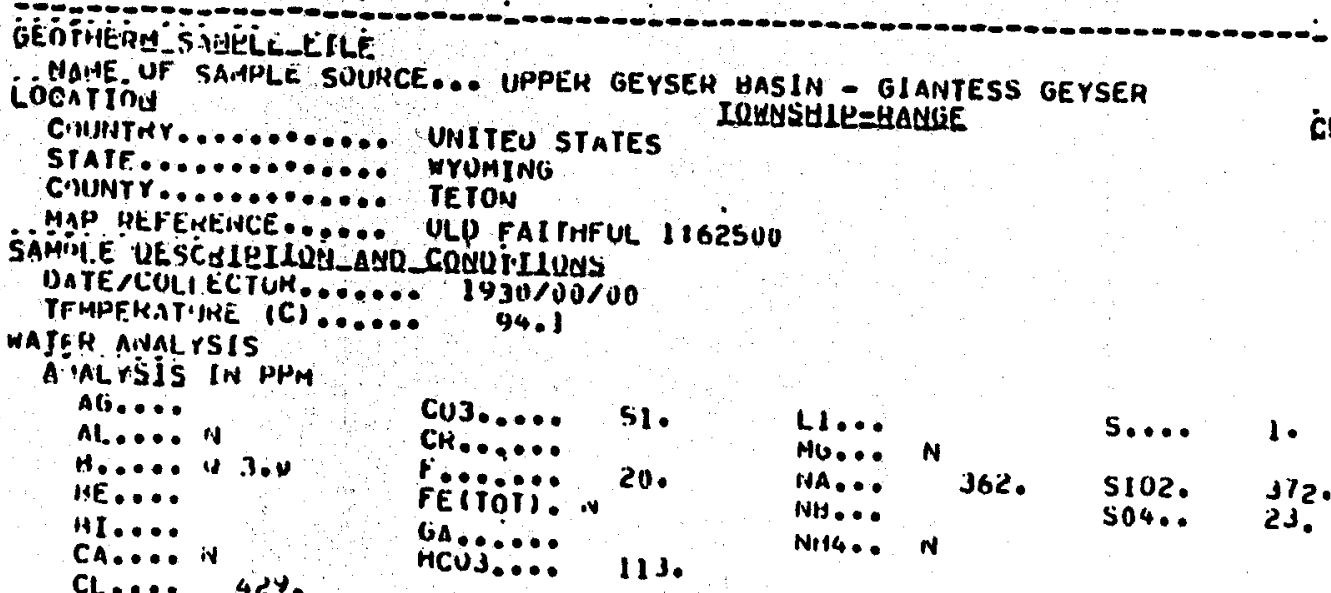

RECUHU 00275

GEUTHERM FILE IUI 0020249

QUHDLNATES

LAT/LUNG... 44-27.7 N110-49,5 W

)

-)

$\rightarrow$

)

)

)

)

)

)

)

)

) 
Co.....

K....... 20

QUAL IF ICAIIUIN FIELO, . U CALCULATED FHOM HZUZ.

REFERENCE AUULLLEUIELCAILÓY

CIIAPILED UY.

CIMPILEH AFFILIATION... H.S. GEULUGICAL SUHVEY

HFFEREINCE.............. ALLEA AND DAY, 1935

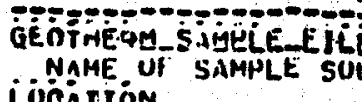

NAME UF SAMPLE SUIRCE... UPPEH GEYSER UASIN - GKAND GEYSER Loninisos

IOHNSULPaBadlie

STATE............ WYMTING

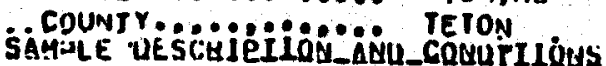

DATE,COLIECTOH......... 1970/00/00

IFMHEKA TIIKE (C)...... 79.

WAIER AIVAL YSIS

Ainivisis lin pap

AG....

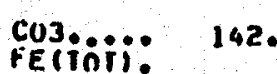

Cosionii:

142. NB.

IUIORES $10<00$

CA.....

MCO3.... in

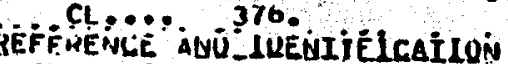

CIMPILEN HY........... LAWSOIV. WILLIAM A.

C MPILEN AFFILIATION... W.S. GEULOGICAL SURVEY

RFFEREITE.............. ALEN AND DAY I935

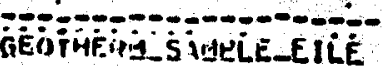

HECONO 00277

NIME OF SAMPLE SUIARCE... IPPEK GEYSEK HASIN - HILLSIUE NO. Licition

CIINTMY ........... UIVITEO STAIES IUUNSHLE=BANGE

STAT

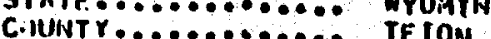

MAI" REFEIEINCE...... ULO FAITHFUL $1: 62500$

OJWE COCALITY INFGGMATIUN WEST SIUE.

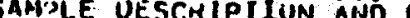

D IE COU I CTUH

Närir nivsl, isis

CHAYIjE IMHALANCE (\$ UIFF)... 3.6

ial rsis lo phia

Co3......

Al. . . in iv

H...... 1.0

HE. $\cdots$

CH.......

HI....

foron.

CA.... Y.

Perroris.

Cl..... S' So

MCUj

$\mathrm{con}$

cquHuLaIts

GEUTHERM FILE IVI 0020332

UAA IF ICAT Jud + ItIL

?.- Y CALCIRATEU fHUM dzOz.

IELCAILU

C.MHJLED AY............ LAWSUIV. HILLIAM A.

CIMDILER AH ILIATIUN... H.S. VEOLULICAL SUIKUEY

S... N LLLLOES LOCOUL

MG.... I

NA... 124.

GEUTHERM FILE IUI 0020264

CQuguLaIKS

LAT/LUNG... 44-28.20 N 110-51.52 W

SIU2. 170.

so4.. I. I.
)

)

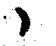

)

)

)

)

)

1

)

$$
\text { , }
$$

)

$$
\text { ) }
$$

\section{,}




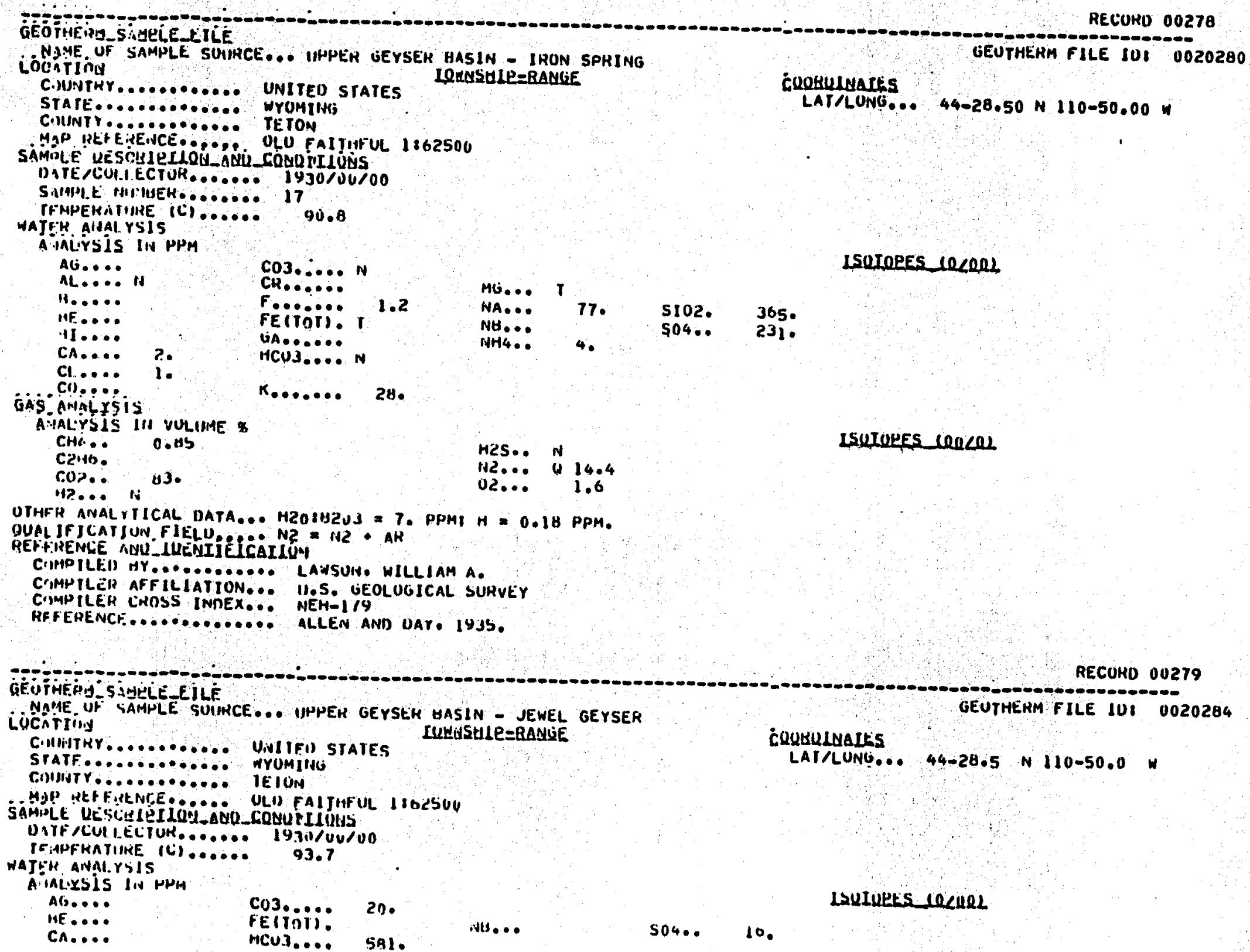

Cn.....

5月1. Nts... 


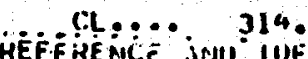

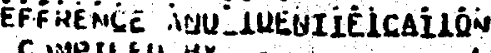

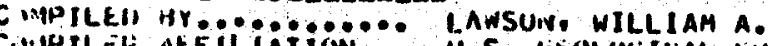

C. H.S. GEOLUGILAL SURVEY

HHFEDECF.............. ALLEN AHD UAY. 1935

RECOHO OU2BO

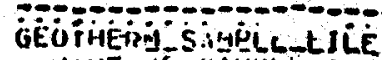

WAHE UF SÄILLE SUUHCE... IIPPEH TEEYSEH HASIN - JEWEL GEYSER

ioeitjiog

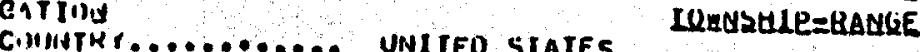

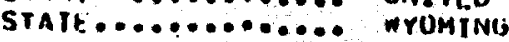

C.llINTY............. TETON

HAP MEL FUEHCE

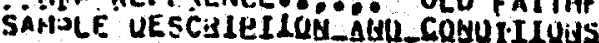

DIEACUI ECTOK....... 1965/00/00

SAMNI. NIIHUEH....... 40

TEMISEHA TIIIE (C)..... AH.

Dajerasals

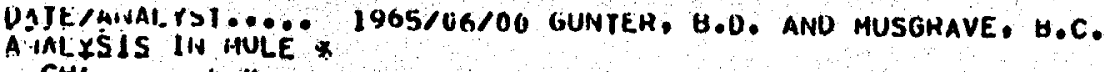

CH4.. 4.0

C?40.

cos?.

IV $^{4 \mathrm{H}}$

U2.

E 1080020285

GEUTHERH FILE IUI 0C20285

CQRQRLUATES

LAT/LUNG... 44-28.5 N 110.50 .0 W

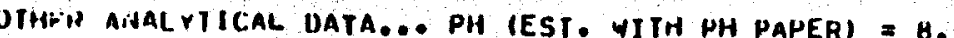

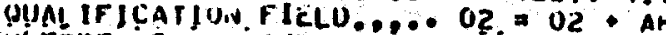

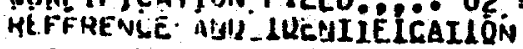

C.MILED "Y.

C.MPILrid AFFILIAIIIN...: II.S. VEOLOGICAL SUMVEY

CIMPPILEN CKUSS IMHEX... NEMTZUU

HIFEKE TIEE.............. GUNILK ANU MUSGRAVE, 1066

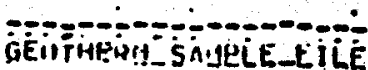

\section{í.}

RECONO OO281

IJ: ME WF SA.IPLE SUIJCE... IUPPER UEYSER HASIN - LIUNESS GEYSER

iocitivins

U....... UNITEU SIATES

LUWNSULL= BANLE

GEUTHEKM FILE IUI 0020J28

STATE ............ WYUMING

G CUIINT Y.......... TETON

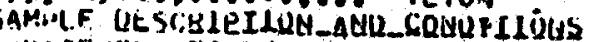

UAIF/CCLI.ECIUR....... 1930/00/00

THMDEYATIRE (C) ....... 94.

LSOIOEES (ROCO)

naini risis IN PHM

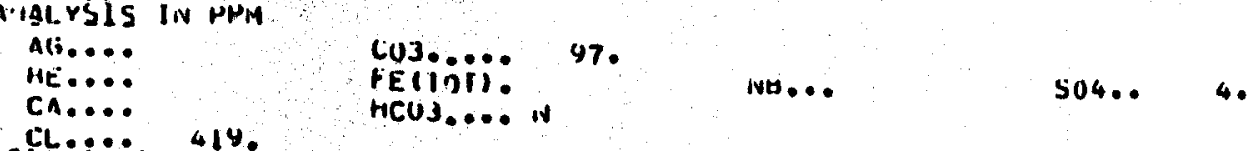

ISULOEES IR OOOL

coukulualfs

CA. 419

CFEP

C.MMP ILE. IY........... LAWSUIN, WILLIAM A.

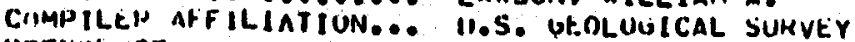

REFE.HE.NCF.............. ALLEN AND DAYY 1 . 


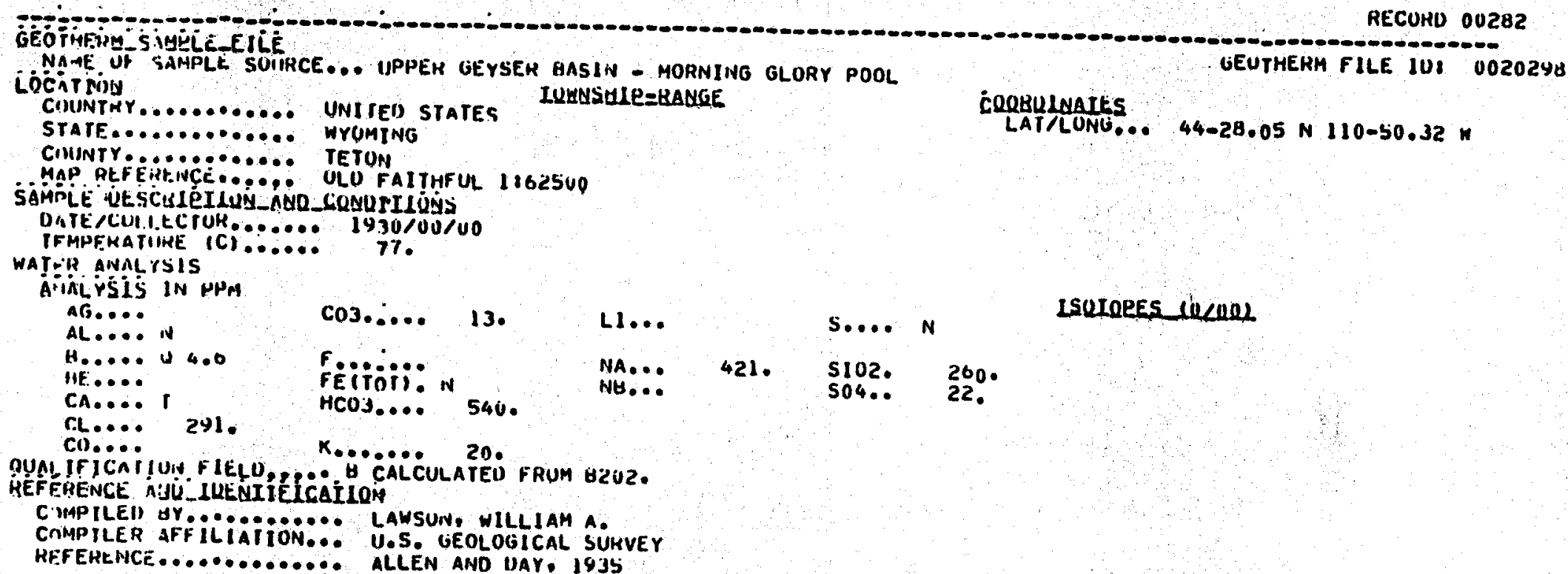

CQQBULNAItS

LAT/LONG... 44-28.05 N 110-50.32 N

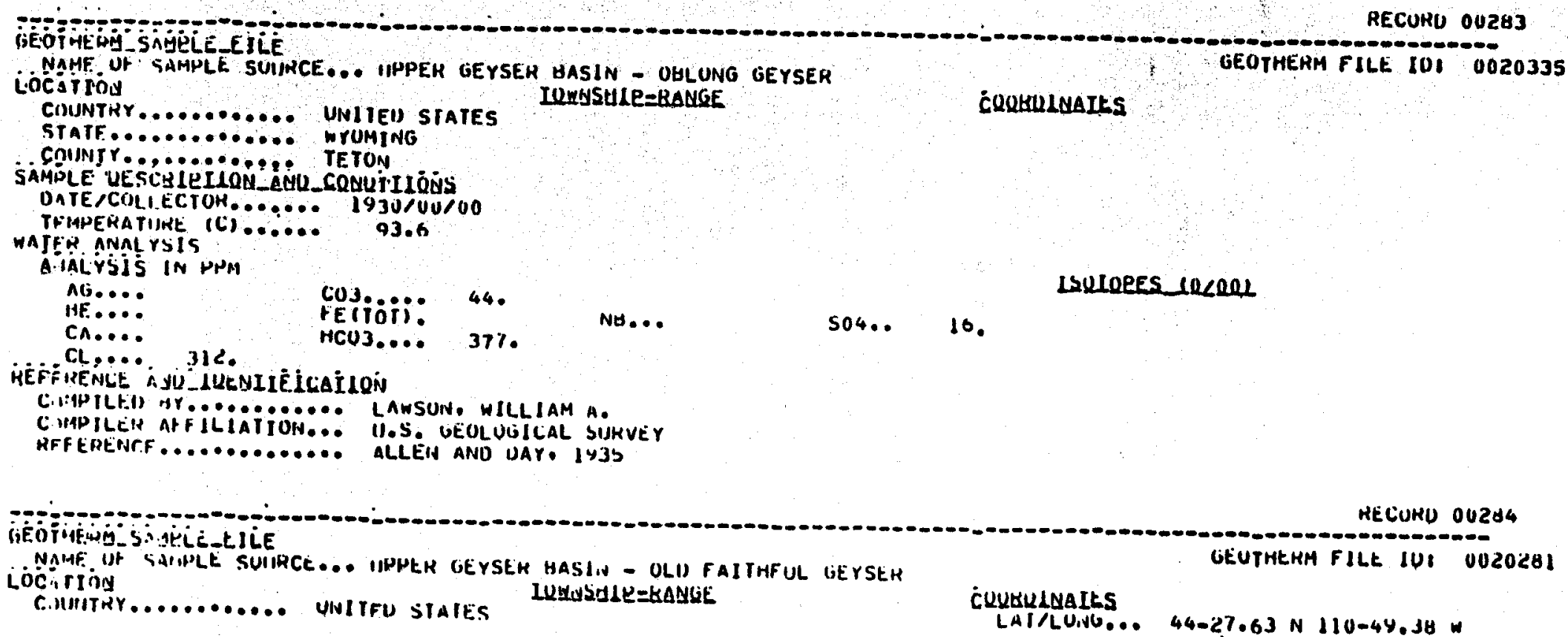

\section{CQQHULNAItS}

SO4.. 10.




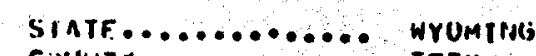

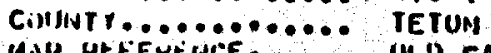

(4.)11 HEFEMEILE...... ULU FAITHGUL 1202500

OPHFY LUCALIIY INFORMATION: NURTHEAST OF QLO FAITHFUL INN.

SAMRLE ULSCEIEILUL_AQD_COUQRIIUUYS

VOTE CULLECJOK....... 19.30/00/00

CiARGE IMISALANCE (\$ UIFF)... U. U

A iAi. ÝS ÍS IN HMH

nlo....

coj..... 10y.

L...

S... T

ISUIORES coCOUL

AL.... II

H.... 3,6

HE.

Feifiti, 22 .

NA...

372.

5102.

357.

CL... 4

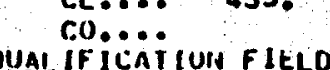

UUA IF ICA IUU FILLO, B GALCULATEU FROM UZOZ. REFHENGE "UU LULUIIÉLCAILÓN

C.MAPILED AY........... LAWSUN, WILLIAM A.

CIMPILER AFFILIATIUN... M.5. GEULUGICAL SUHVEY

HFF ERE INCE............ ALLEN AND DAY 1935

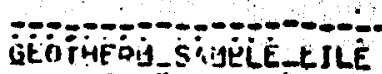

chen ano bar. jyss

RECUKD OU285

NARE UF S

Lositinis

C.MINTHY . . . U. UNITEO STAIES

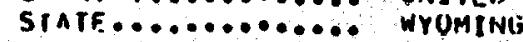

CAIJNTY............. TETON

MAP METEHENCE.... OLO FAI THT UL 1262500

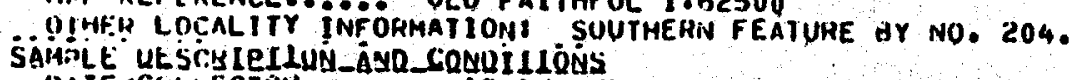

DATE,CULI ECTOH....... 1971/UG/29

SnMHI.t NHIHHEH....... 205

THMERATIJKE (C) .......

II SCHARTt.

IHEO SA.AMLE INFURMAIION OO SUMK

Gis hivalysis

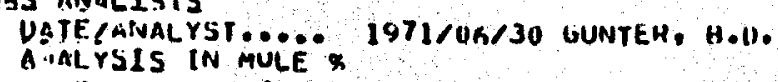

nh. 1.2

C.14.10 $\quad 0.49$

C.10.10. 3so

4R... N

(12.

OIHEN ANAL YI ICAL DATA, .. AH/N2 $=2,17$.

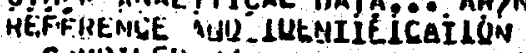

C.HPILEU IT ........... LAWSUIV, HILLIAM A.

C MMPILE AF ILIATIUN... II.S. UEOLUUICAL SUKVEY

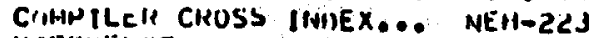

HFFEUENCE............ GUIVIEK, $197 \mathrm{~J}$ 


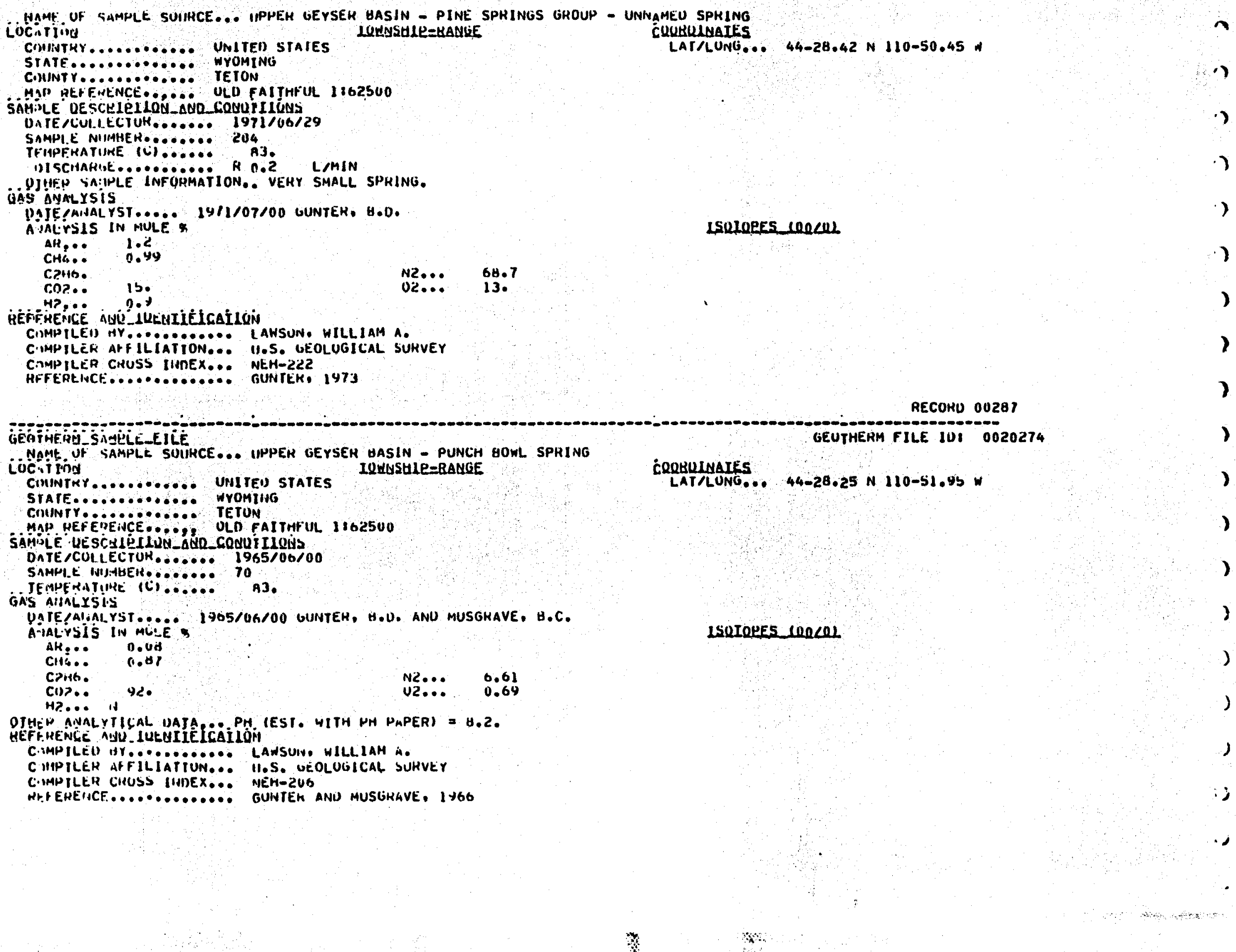


RECOHO 0U2HU

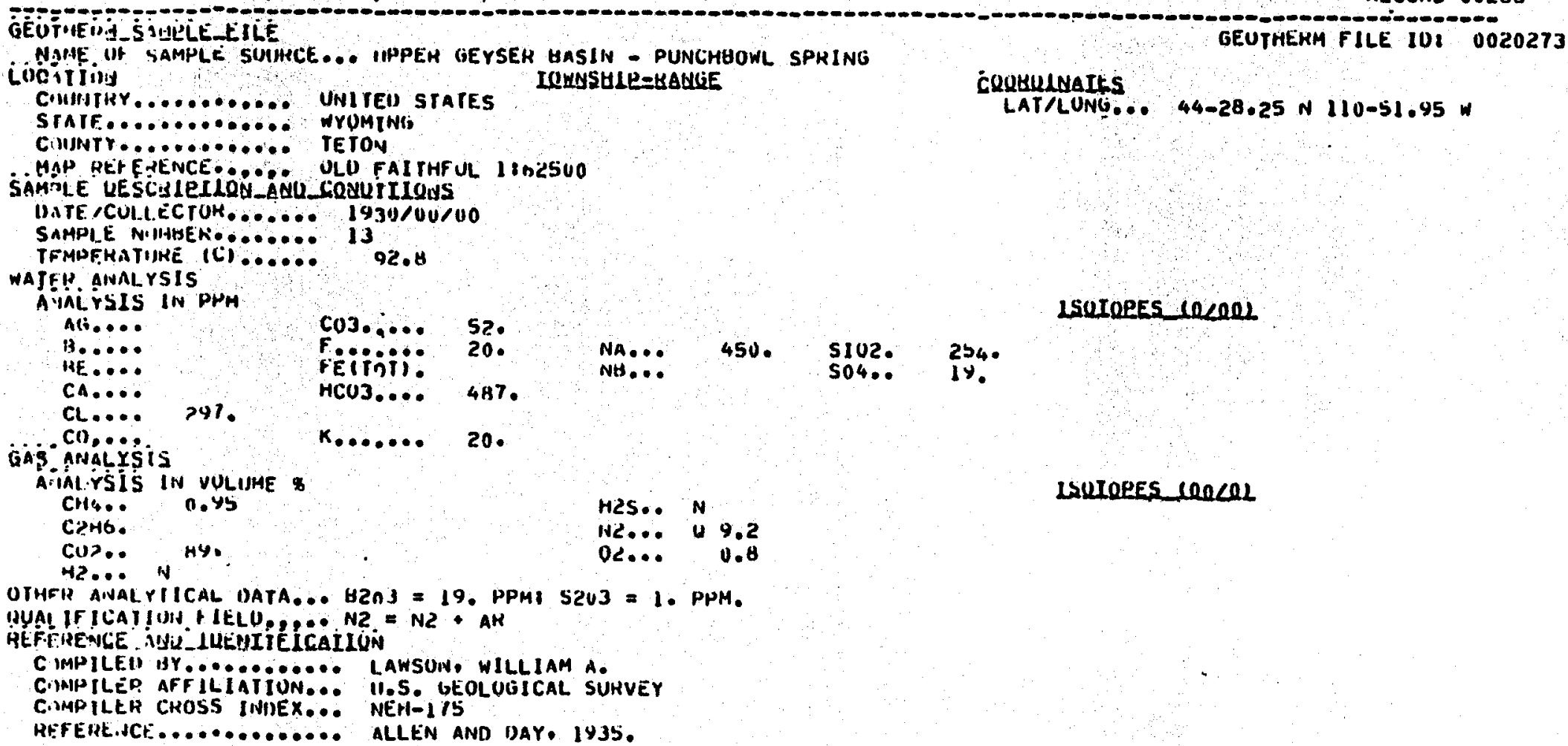

RECUKO 00289

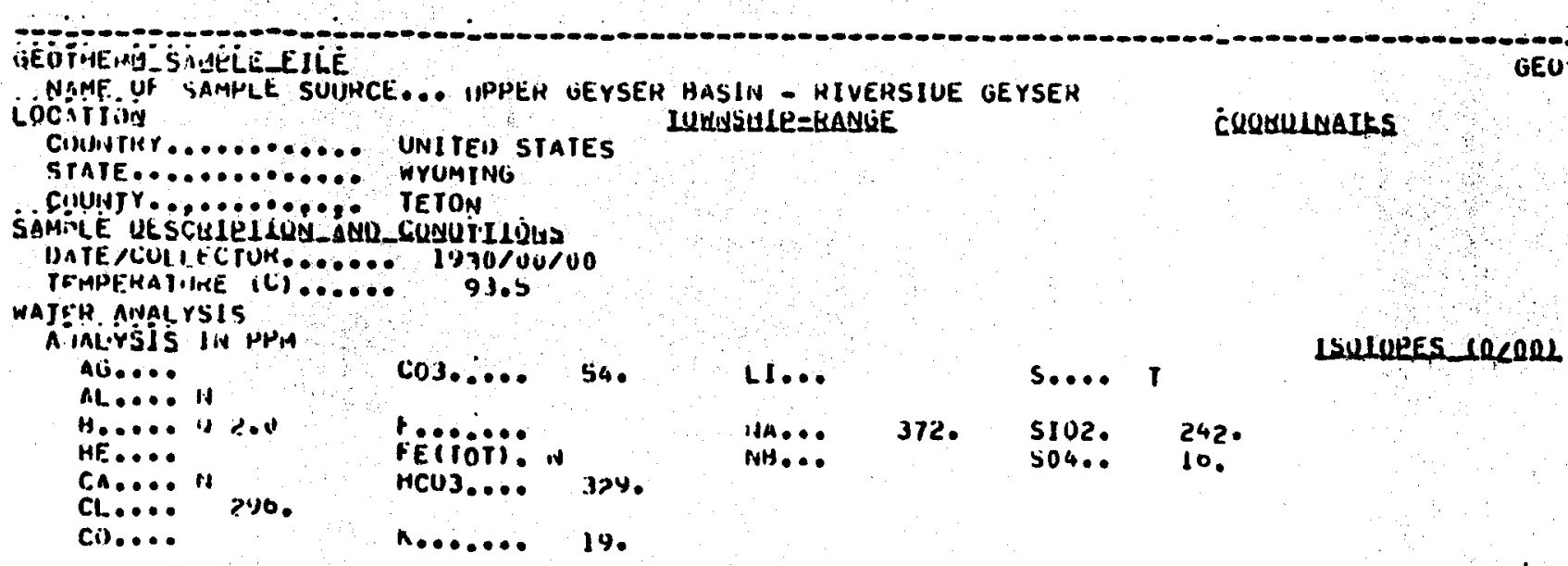


OTHFK AIVMI. ITICAL IIATA... SZIZ = J. PHM.

MUA! IFJCA IIU! FIELU, B. H CALCULATEU FHUM GZUZ.

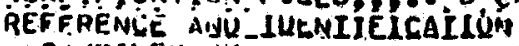

CriAPILEU BY............ LAWSUN, WILLIAM A.

CIMIPILE"I AFFILIATION... II.S. LEOLULIICAL SUHVEY

KFFEHEINCE ............... ALLEN AND UAY, 1935

HECURU OOZYO

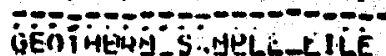

NMMË GH SAMPLE SUIMCE... IIPPEH GEYSEK HASIN - SAPPHIRE SPRING Locition

LUHASULE =8ANLIE

CINHTKY........... UNITEO STATES

STATE............ WYOMING

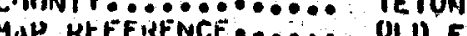

MAD KLFETENCE.... OLQ FAI THFUL 1162500

OJHEY LOCALI IY INFOHMATIONE NUKTH ENU UF HISOUIT BASIN.

SAMDLE UESCHIELLOE_ANO_CQUUIILOÜS

DATE/CULLECTUR........ 1930/00/00

JEMPENATIHE (C)...... 94.5

WATFH AYULYSIS

$$
94.5
$$

CHAHCEE IHHALANCE (\$ VIFF) ... I.O

Anaírsis lit PHM

Ali.....

$4 \ldots \ldots$ I 2.4

Co3....66 66

F.......

Fitio 22

HE. . .

GA.......

HCU3... 466

CA.... 4.

CL.... 3ul.

GEUTHEHM FILE IUI 002025

\section{Cquanlatas}

LAT/LUNG.... 44-28.48 N 110-51.15 N

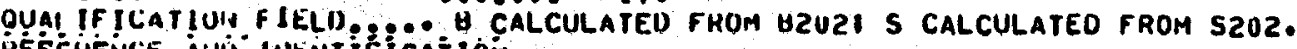

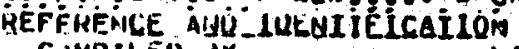

C MMP ILEDI HY......... LAWSUIN, WILLIAM A.

C.MPILEH AFFILIATIUN... W.S. GEOLUGICAL SURVEY

RFFERtNCF............ ALLEN AND DAY, 1935

RECURO 00291

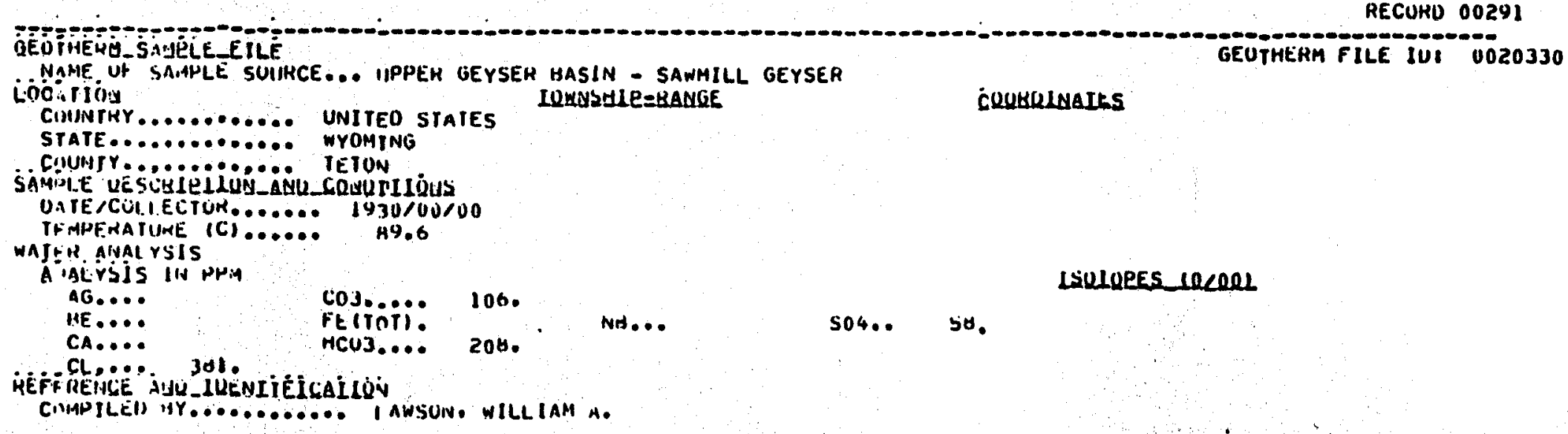

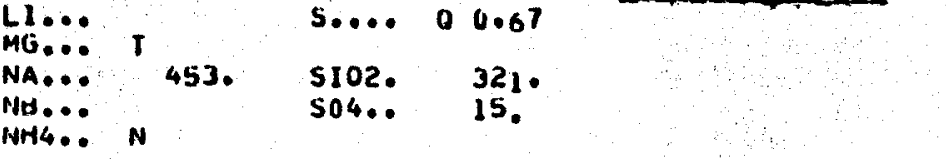

,




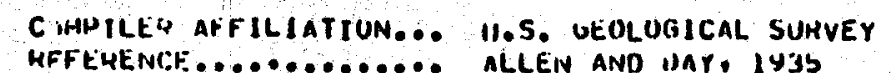

STATE

LUWUSALE=BANGE

CQRHULNAIFS

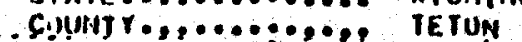

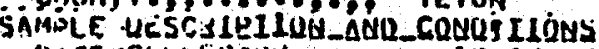

UATE/CGLLETUN........ 1930/UU/UO

TFMPF RATIHE ICI

WAJEQ ANAI YSIS

AHL TSIS IN PHN

AG....

Cu3..... 90.

SUIOPES $10<002$

CA. . .

FE(IQT). NH..

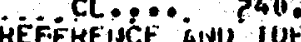

504.. 12.

LEIIÉlcaíló

C MAPILED AY ............ LANSON, WILLIAM A.

CMMPILER AHFILIATION... H.S. VEOLOSILAL SUHVEY

HIF EHENRE ................. ALLEN AND DAY, IYJS

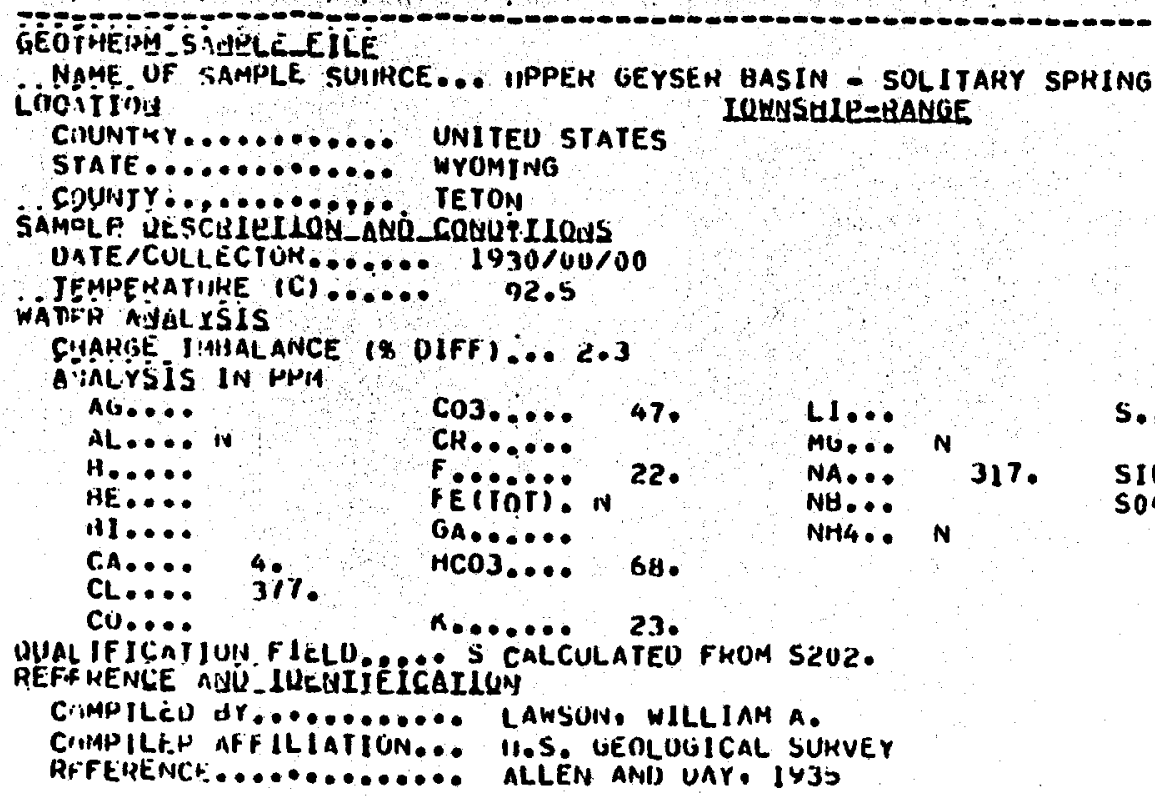

HECUHU 00294 
N YME UF SAMPLE SUIHCE... IMPPEH GEYSEM HASIN - SPASMUDIC GEYSEH Leciation

CITINTHE.

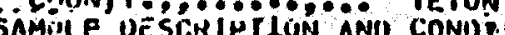

UITE $/ C O L .1$ ECTUH,

TFMPEHATIJRE (C)..... 93.5

WATEM AiVAL YSIS

AVAEYSIS IN PPM

$A G$.

co3.....

IOWNSHIP=BANGE

UIE....

FE(TII).

NH...

Cl. $36 \mathrm{H}$

IUENIIÉICAILUA

CYM IY......... LAWSUN, HILLIAM A.

CMPILEN AFFILIATION... H.S GEOL UGICAL SUHVE

HEFEREMCE.............. ALLEN AND VAY, 1935

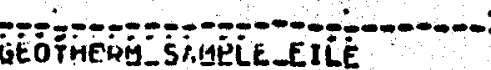

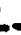

iocition

Cuintk

CIMINTKY.......... UNITEU STATES

STATE............ WYOMIHS

CyNNIY

(O)

NTE/COI.IECIOK....... 1930/00/00

TFMPEFATLHE $(C) \ldots \ldots . . . .93 .5$

ATEK ANALYSIS

AnAitisis IN PHA

HE....

CA....

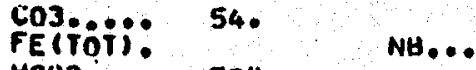

NH...

538.

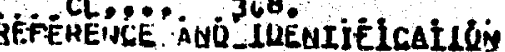

C.MPILEU AY........... LAWSUIN, WILLIAM A.

CMIIPILER AFFILIATION... II.S. GEOLOGICAL SUHVEY

HFFEREIYCE............ II.S GEOLOGICAL SUH

GËTHE SOM STMLE_LILE

NGIAE UF SATALE SUURCE ... IIPPER GEYSER GASIN - SPONGE GEYSER

iogostion

COUNTHY.......... UNITED STATES

IULUSHLE-BANGE

STATE

COUNJY ............ TETUN

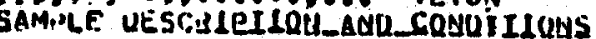

UATEACGLLCTOK....... 197U/UU/00

TRIAPERATURE (CI....... 93.5

WATEH ANAL YSIS

iaíríls In PPM

afi....

COJ.
FEIIITI:

105.

No...

LUIOEES HOCHUL
SUIORES IOLOOL

CuonuLatEs

HECURO 00295

GEOTHEAM FILE IU: 0020336

\section{COQBOIMAIES}

ISOIOEES IOCOOL

$504 \ldots 25$

RECUKU 00296

GEUTHEHM FILE IUI 0020327

GUQGULAAIES

GEUTHEHM FILE IVI 0020327 
Ging AnMigisis $42 \%$

Aing

A InL

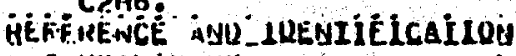

C.MMPILTIU UY........ LAWSON. WILLIAM A.

C.MMPILEK AFFiLIATION... W.S. GEOLUGICAL SUHVEY

RFFERENCE.............. ALLEN AND UAY. 1 Y35

LSUROPES CORLWL

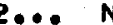

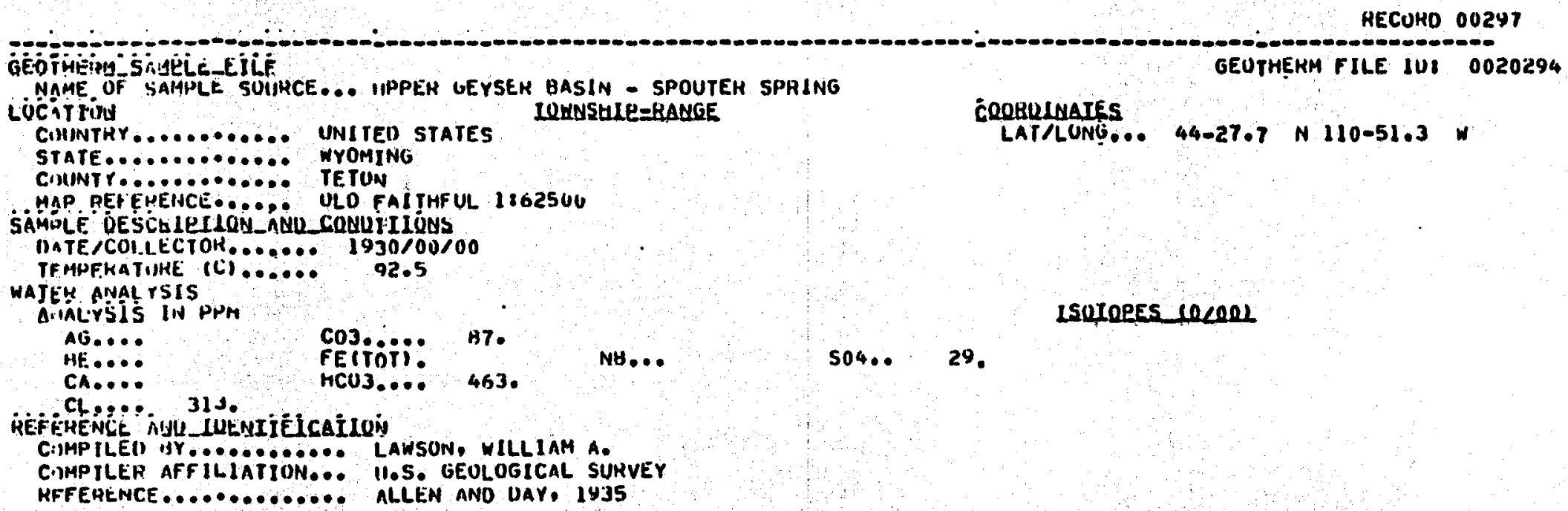

HECOKD 00298

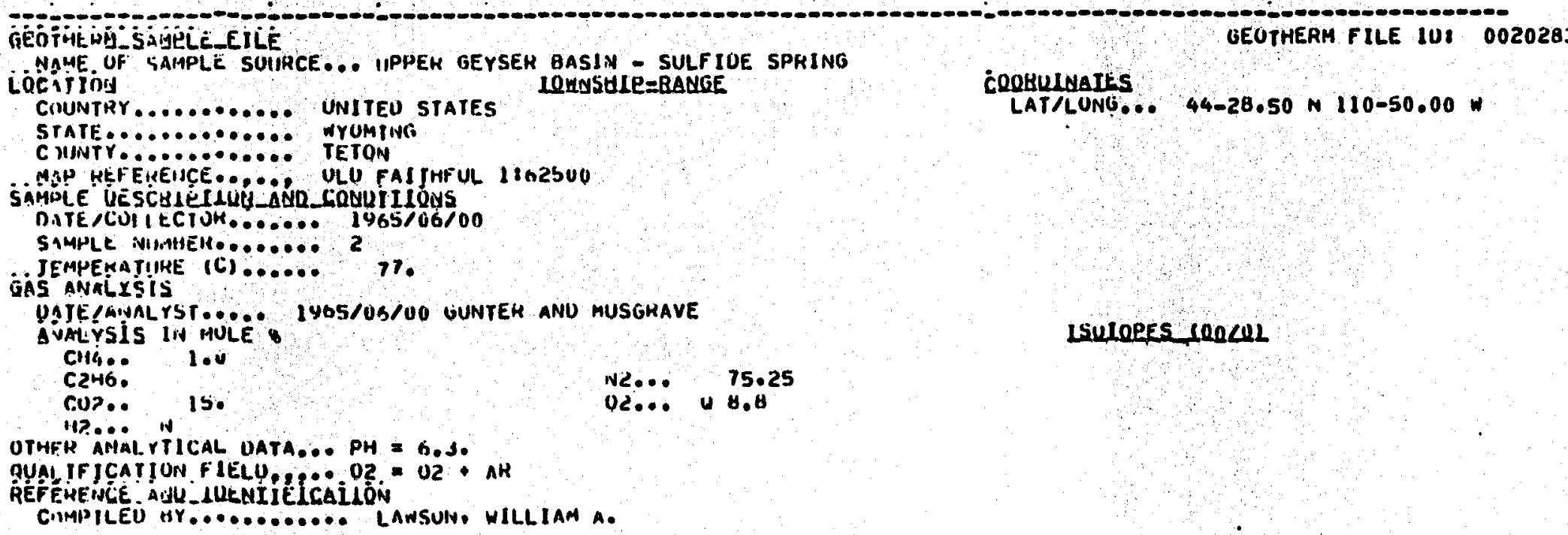

$02 . . .48 .8$

15.

LSUIORES COQCUL

OTHER ANIAL YTICAL DATA... PH $=6.50$

QUA IF JCATIUN FIELU ... O2 = O2: AH

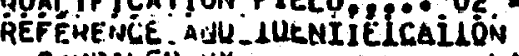

CIMIILEU HY........... LANSUIN, WILLIAM A. 
CIMPILER AFFILIATION... 11.5. GEOLUGICAL SUHVEY

COMPILER CROSS JINIEX... NEH-IUL

MFFENENCE............. GUNTEK ANU MLSGKAVE, 1966

RECURD 00299

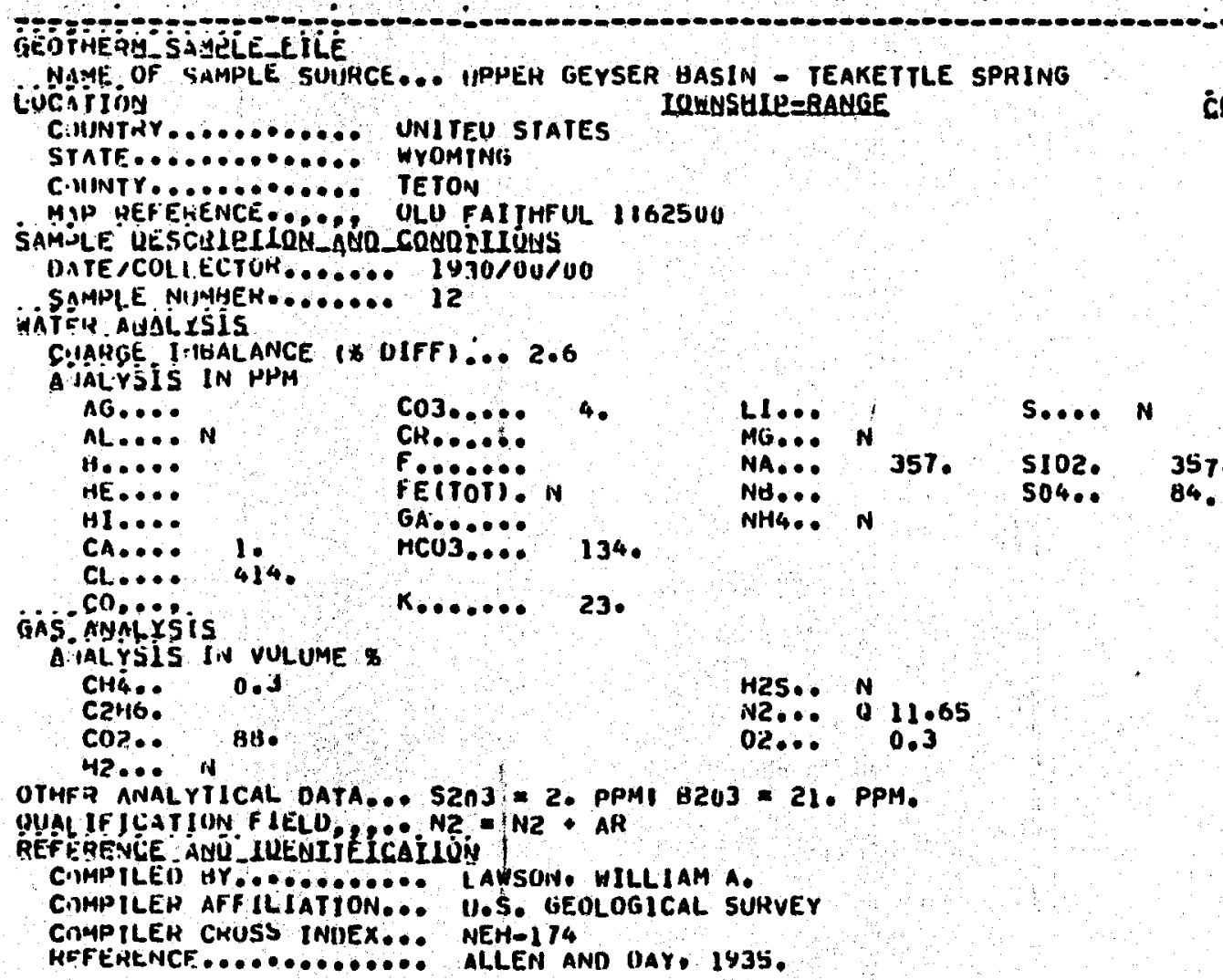

RECOHO 00300

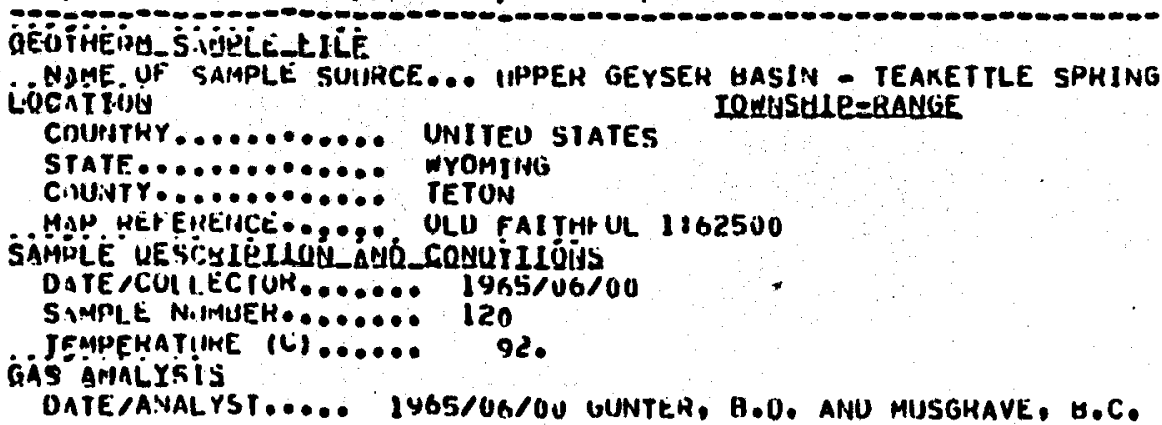

OATE/AVALYST..... IY65/OG/OU GUNTER, B.0. ANU MUSGHAVE, B.C. 
Ajaírisis IN MULE a

CHi... O.UB

C? ?h+

0.08

C2... w

OTHFH ANAL RIICAL DATA... PH = S.3.

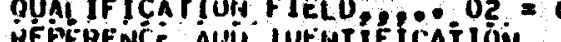

REFFRENCE ALU IUEGIIÉCACAILÓN

C. IMPILEO HY........... LAWSUN, WILLIAM A.

C.MPPILEK AFF ILIBTiGîn... U.S. UEOLUGICAL SUKVEY

C.)MPILEK CHOSS INIIEX... NEH-ZII

H+rEHENCE............. GUNIEK ANU MUSGGRAVE, 1966
LSOLORES $100 \angle 01$

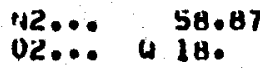

RECOHO 0030

GEUTHERA FILE IN: 0020278

NIME. UF SAMPLE SOIARCE... UNPER GEYSEH HASIN - THREE SISTERS SPRING iocitriour

CINUWTKY

STATE.............. UY UYMING

CIIUNTY............. TETON

HAP WEFEHENCE...... ULU FALTHE UL 1262500

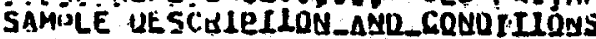

DITE COLIECIOH....... 1930/00/00

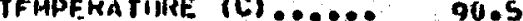

WATH ANAL RSIS

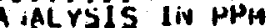

AG... COB.... 117

BE....

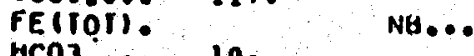

CO3.... 10 .

Cl.

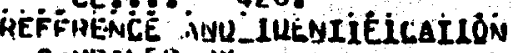

C. IAP ILEN WY........... LAWSUN, WILLIAM A.

CINAPILEK AFFILIATION... 11.5 . GEOLUGICAL SURVEY

REFENEINCE............. ALLEN AND DAY, 1935

RECOHO 00302

GEOTHEQM STMELE EILE

A.

iociring

STATF.0.0.000.0.

Cnilintr. . . . . . .

MD LEFEIXENCE

UNIIEU STATES

LOMASHLP=RAMLE

WYOMTNGS

TEIuin

velu fal tartul ligzsula

LAT/LONG̣... 44-28.5 N 110-50.0

LSOTUPES COCOOL

SO4.. 74.

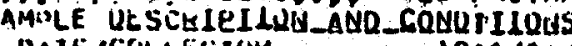

DIIE/COL LECIUK....... 1965/00/00

.S\$MPLE NUNHEK........

ins aifalisis

Da IE IA VALYST..... 1465/0h/00 GUINTER aNU mUSGKAVE

A AALYSIS IIA MULE

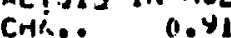

ISULOHES IOMCOL

GEUTHERM FILE IUI U020279

cootulnarts

LAT/LONG... 44-28.5 N 110-50.0 W

C2HK.

N2... $\quad 87.73$

Cils.:

2.06

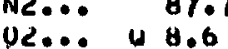

ITHFH AUNI.YTICAL IJATA... PH $=8 . Y$. 


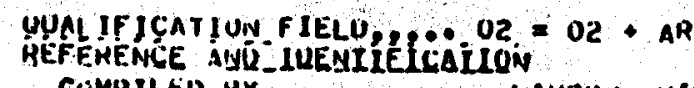

C.MPPILED) HY.

C. MHPILEF AFFILIATiON... 11.5 . GEOLOG ICAL SUHVEY

CIMPILER CHOSS INNEX... NEH-IHI

REFEPENCE............... GUHTEK ANU MUSGHAVE, 1966

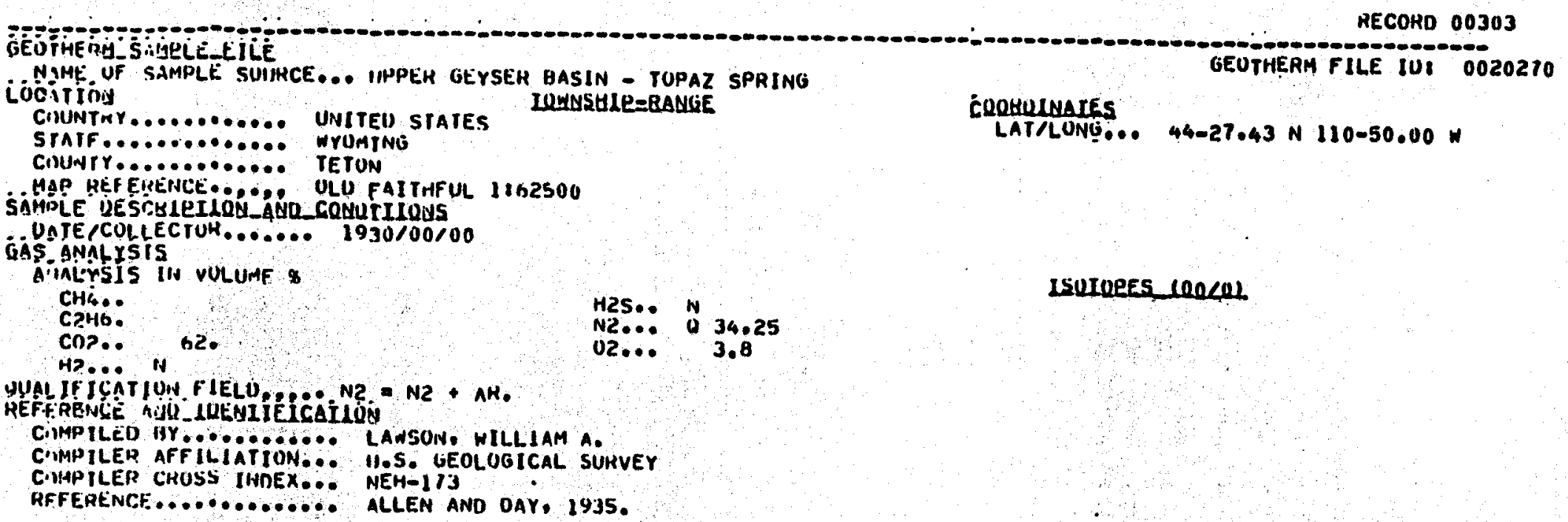

RECORU 00304

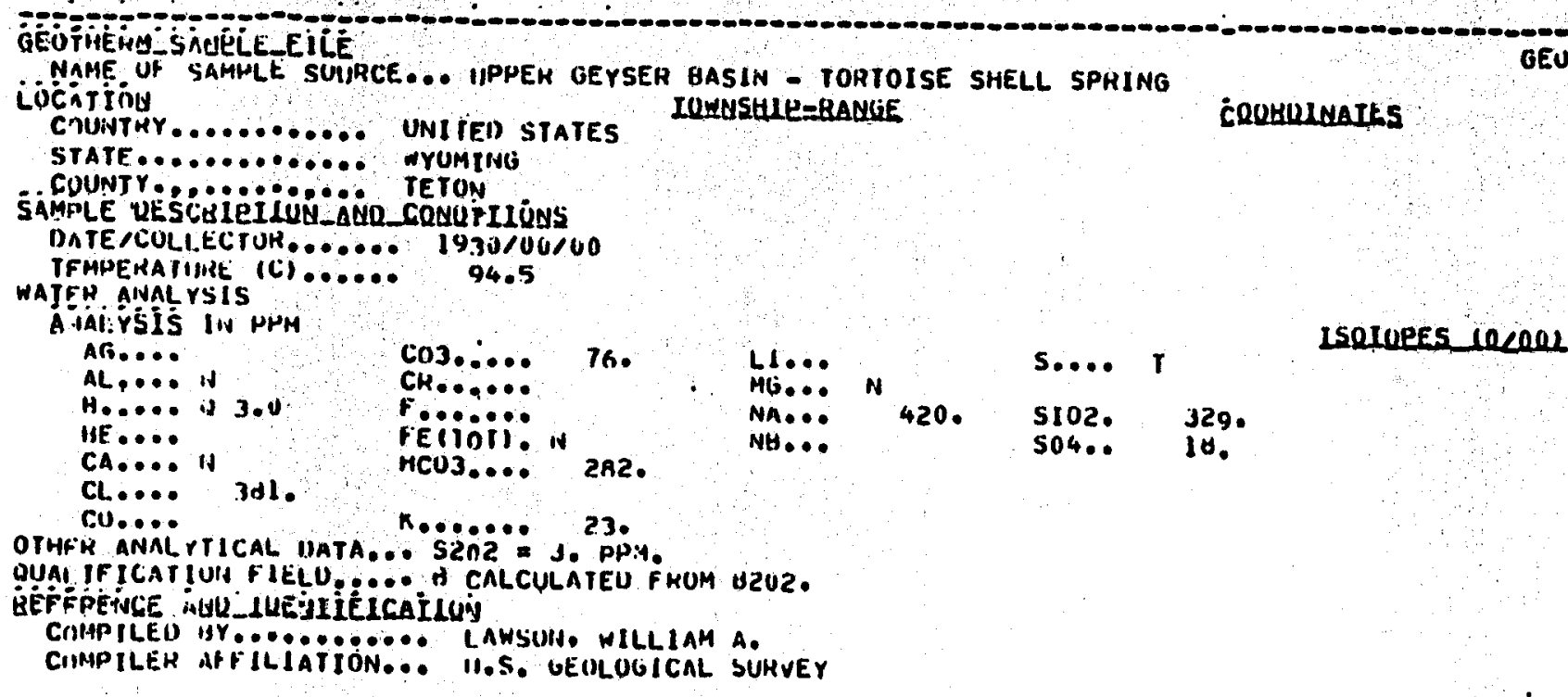

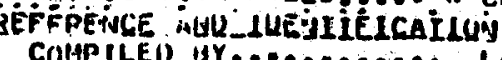

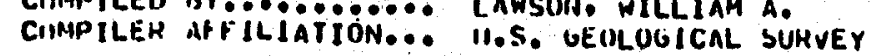




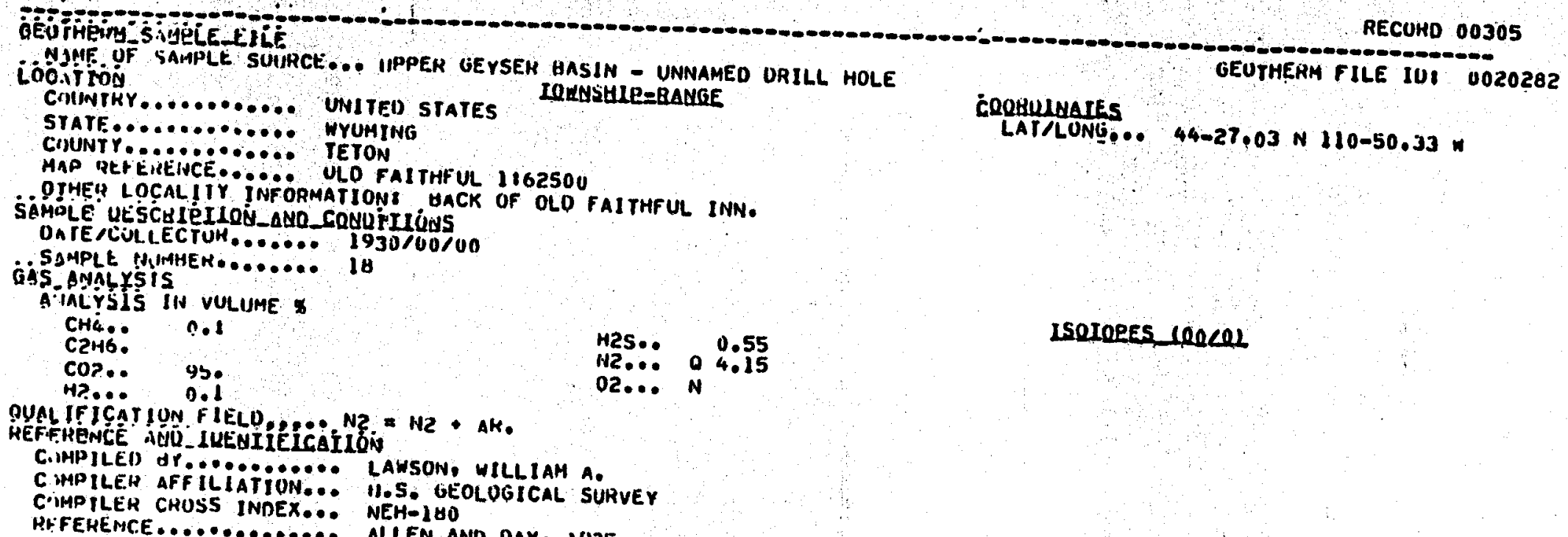

H2S.. 0.55 ISOIORES IOCLOL

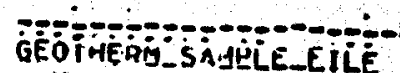
RECUHD 00306

G NAME UF SAMPLE SUUACE... UPPPEH GEYSER BASIN - UNMAMED SPRING CoIINTKY

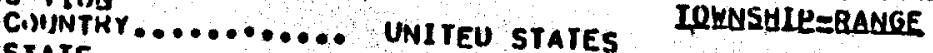

STATE............. WYOMTNG

C.)UNTY.......... TEIUN

OIMER LOCAL

O PMEQ LOCALIIY INFORMATIUNE GUM SOUTH OF MORMING OLURY PODL

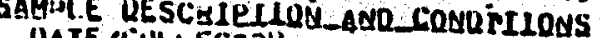

DATE/CUI IECTOK....... 1965/06/00

SAMPLE NIIMHEK....... 10

JEMPEKA TIME (C)...... 55

Gis AnMixsis

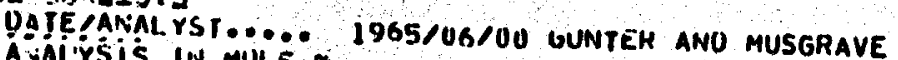

A ALírSis IN MULE

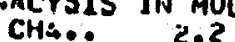

Canb.

$$
2.2
$$

CO2.. $N^{H .1}$

N2... 15,19

LSULUES $100 \angle 1)$

GEUTHERM FILE IUI 0020J00

Cugunuats

LAT/LONG... 44-27.43 N $110-50.00$,

OTHER ANALYTICAL DATA... PH $=6.5$

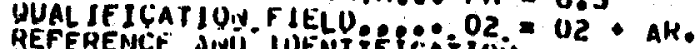

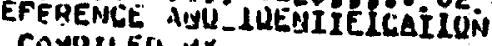

COMPILED Ar..̈....... LAWSON. WILLIAM A.

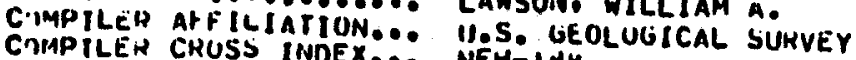

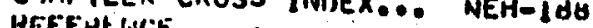

runter aND husGrave, 1966

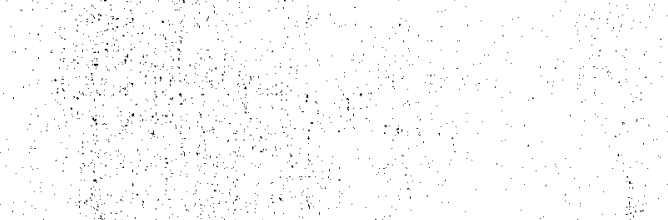




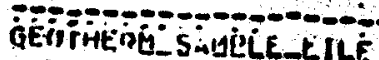

HECUHU 00307

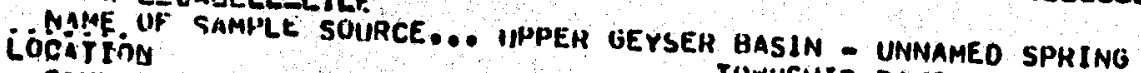

IUEUSHIP =RANGE

\section{STMTE.......... UNITEU STATES}

STMRE............ WYUMING

CAPS

AnP HEF ETNCE.... OLO FAITHFUL 1162500

SAMMAL OES

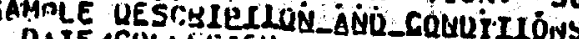

OATE/COL.ECTOK...... 1965/06/00

SAMPI.E NIPHHENO......

JEMPERATIHE (C)

ANMLYSIS

PAIE GANALYST MUE $1905 / 06 / 00$ GUNTER ANU MUSGRAVE

ATIYSIS IN MULE

CHa...

$1 \cdot 3$

CदHh.

$1 \cdot 3$

OTHFH

N2. $\quad \varphi_{0}$

LSUIORES COOCOL

GEOTHERM FILE IU: 002029

\section{CoOGULAares}

LAT/LUNG... 44-27.43 N $110-50.00$ W

UUAI IFICAT IOIC FIELI

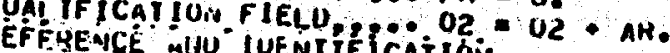

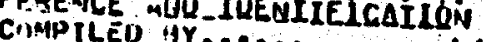

COHPILCH AFF ï ï.̈..... LAWSON. WILLIAM A.

COMPTLER GHUSS INDEX... H.5. GEULOGICAL SURVEY

HFFERE.NCF.

BUNTEH ANU MUSGRAVE, 1960

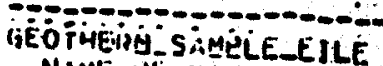

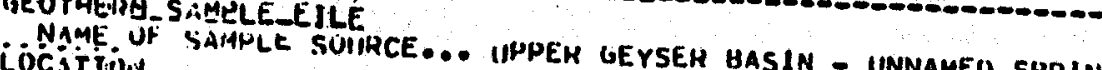

HECOHD OU3OB

cocitivin

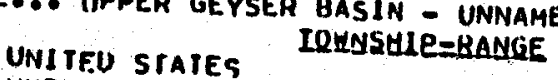

SIATF................ UnITEU SIATES

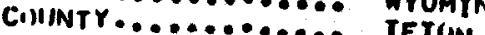

MAP HER E'EEPACE.... OLO FAI

OIHEQ LOCAL IIY IIHFOKHAT FAI THFUL 1262500

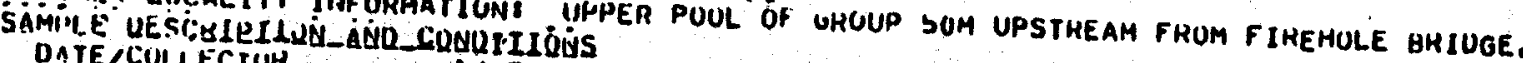

SNMPI E NOPMHEH ........ 1965/06/00

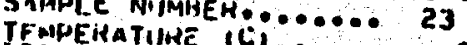

CAS BNALESTIS

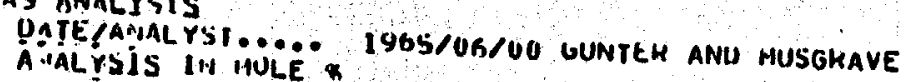

Citi... 0.64

LSULUESS LOOCOL

$$
\begin{aligned}
& \text { r.12... : } \\
& \text { 46. }
\end{aligned}
$$

OTHLH ANAL YTICAL DATA... PH (EST. WYTH PH PAPER) = S.

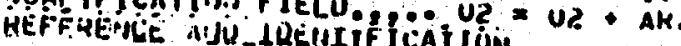

C MNILEI) ar P.OP

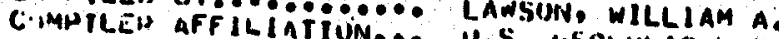




\section{C}

C.MMPILER CHOSS INDEX... NEH-IYY

PAGE 0162

RFFERE ITCF, .............

GUNTEK ANII MUSGRAVE, 1966

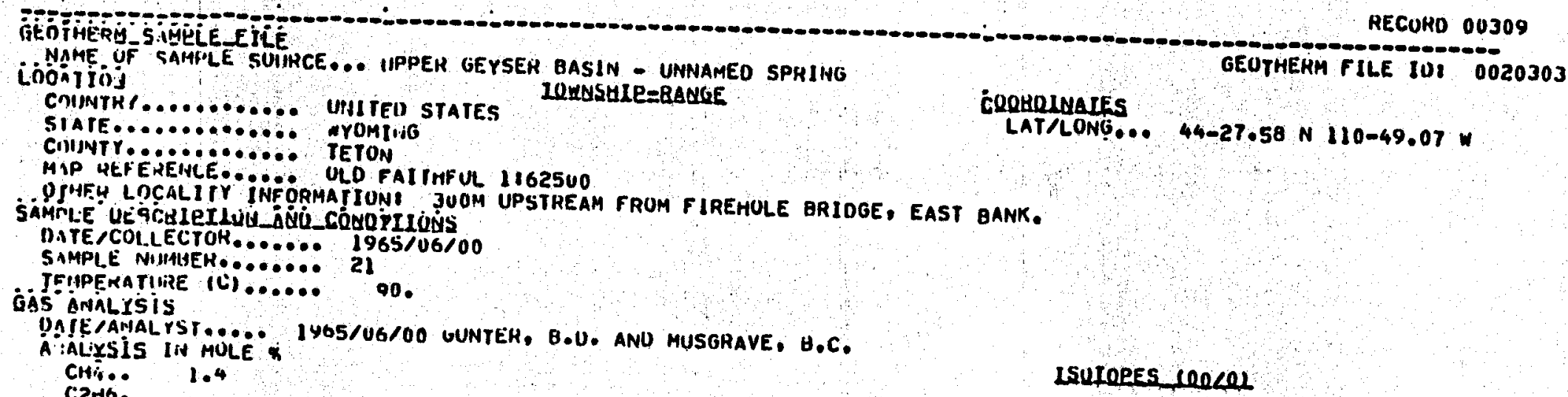




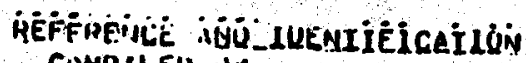

CIMPILEI) Hr.......... LAWSON, WILLIAH A.

C.MPILCP AFFILIATION.... N.5. GEULUGICAL SURVEY

RFIEHEINCE ............. SIINTEH ANU MUSGRAVE, 1966

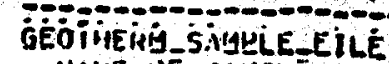

RECOKU 00311 LÜOATI UF SAMPLE SUIITCE... UPPEH GEYSER GASIN - UNNAMED SPRING

COUNTRY

STATF.

CIUUTY

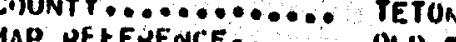

MAP REF ENENCE.... OLO FATIHFUL 1862500

SAMU UE SCLIT IMFORMATIONI ZM ABOVE PIPELINE CHEEK.

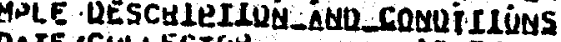

SAMPIE NIJAGER..... 1965/06/00

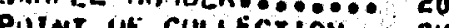

PIINI OF CULLECTION. SMALL CUNE

GAS ANALYSIS

QATELAHALYST.... 1965/UT/OO GUNTER ANU MUSGKAVE

A GaL ÝSLS IN MULE $\alpha$

chin. 0.4

Cri?.

93.

1965/U7100 GUNTER ANU MUSGKAVE

$\begin{array}{lll}N 2 \ldots & 4.88 \\ 02 \ldots & 1.3\end{array}$

ISUTOEES loneOL

GEUTHEHA FILE IU: 0020312

$42 \ldots 10.4$

JUMI IFICAIJUN FIELO, . O2 = U2 $A R$.

HEFEHENCE AIVU_LLUIIELCÁILON

C. HAPILEN IY........... LAWSON, WILLIAM A.

CiMPILEC AF FILIATION... II.S. GEOLUGICAL SURVE

CIAHPILER CHOSS INDEX.D. NEH-IYS

HEFERE,UCF............... GUNTEH ANO MUSGRAVE, 1966

COOBWLLATES

LAT/LONG... 44-28.0 N110-50.0

ETHFHA STMRLETIL'

RECORO OUJ12

Natie UF SAMLELE

Nonke UF

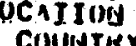

COMINTKY.

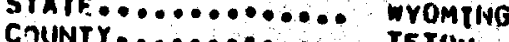

MAD REFERE UCE OO TETON

OIHE LOCALIT

SAMSIE UESCAL

DATE, COLLECTUH A ASTCOUUTILUAD

SATHPI. E NIIMUEH........ I4

JEIPENATUHE (C).....

QR ANALYSIS

OAJE ANVALYST CH4.: 0.62

1965/OG/00 GUNTEK ANU MUSGKAVE

C2Hs.

ISUIOPES leOCOL

h5... in

N2... 31.43

CQRguinares

LAT/LUNG... 44-27.35 N 110-49.37

THFH ANAL.YTICAL INATA... PH IEST. WITH HH PAPERI $=7$. 
YUAL IF ICAI IOW FIELO, , \& OZ \& O2 \& AK.

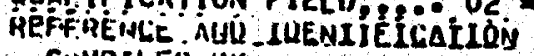

CIMP ILEO HY ........ LAASUIN, WILLIAM A.

C:DAPILEH AFFiLIAIIOH... W.S. GEOLOGICAL SURVEY

CIMPILER CHOSS INIEX,

AEFEQENCE............. GUNTEH ANO MUSGHAVE, 1966

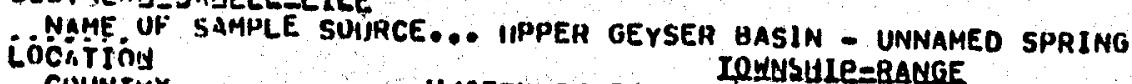

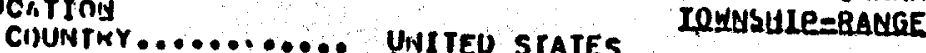

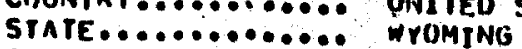

CHIHTY

MAP WER EKEENCË...... OLD FATTHFUL 116250

SAMIRE? LnCALITY INFORMATION SUUGH OF PIPELINE CREEK.

OATE/COLIECTUR....... 1965/06/23 GUNTER ANU MUSGRAVE

SAMPLE NOIMESER...००... 18

TFMPEHATIHE TCI..... 71

OIHFE SA ARLE liNFORMATION. LARGE CLEAK ULUE SPRING.

GAS AMALYSIS

DAJE GANAL YST MÜE $1965 / 07 / 00$ GUNTER ANU MUSGHAVE

AP... 0.12

CHAं. 1,1

C2H6.

cos...

$8 \%$

N2... 11.22

$02 \ldots 0.31$

LSUIDPES leOCOL

OTHEH ANALYTIÇAL OATA, PH $=6.4$

COMP IE ËU -LEAIIEICAILON

CIMPILED HY........ LAWSUN, WILLIAM A.

CAMPILER AFFILIATION... U.S. GEOLUGICAL SURVEY

CIMPILER CHOSS IHOEX... NEH-1Y4

REFEHENCE............ GUNTEH AND MUSGRAVE, 1966

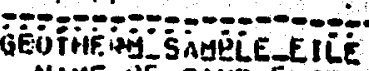

RECUKD 00314

NAME UF SAMPLE SUIHCE... IPPEH GEYSER BASIN - UNNAMEO SPHING ócitisiog

C.JUNTHY . . . . W..... UNITED STATES

IOUSSULE-RANGE

SIATF............. WYOMING

CANTY

SOPIEA COCALITY INFORMATIONI JUST HELOW FAN GEYSEH.

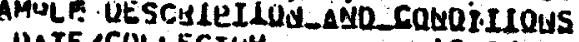

OATE/COILECTUK...... 1930/00/UO

TEMPEKATIJE ICI...... O2.5

WATE:L ANALYSIS

AvAL 'YSIS II HRH

Ati....

co3.

CA.....

121.

FElTOR: NH...

LSULOEES IOLOOL

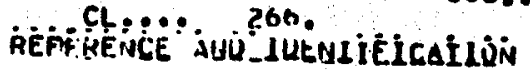

S04..

21. 
CIMPILCH AFFILIATION... II.S. GEULUGICAL SUHVEY
CIMPILER CHOSS INDEX... NEH-IYZ
HFFEHEFCE...

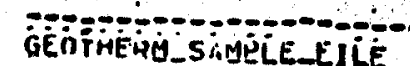

ZÖAME UF SAMPLE SUUHCE... UPPER GEYSEK UASIN - UNNAMED SPRING

RECUKO 00317

Lósiriny.

CIIINTHY.......... UNITEO STATES IOUNSHLE-RANGE

STATE

COIJNTY MAHER HOMTNG

MAH HEFËRENCË.? TETON

OIHE LOCAL ITY

SAMTLE UE SCIEI ILOMF OHMATION: NUKTH OF PIPELINE CREEK.

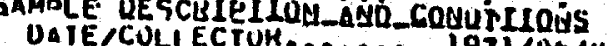

DAIE/CULLECTUH...... 1971/06/U2

SAMPLE NIHBER.

TFMPEKATIIKE (C)

DISCHAFGE

GA THEQ SAMPLE INFGRMATION. LAKLE BKOWN SPKING WITH PERIODIC OVERFLOW. DATE/ANAL YST

AK.

CHi. 0.10

C246.

$1 \cdot 9$

Cor.

$T$

S.

N2...

12.5

LE IUI 0020309

OTHEY ANALYTICAL DATA_.. AR/N2 = 1.48

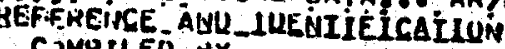

CMPILEO AY

C.MPILER AFFILIATIUN... U.S. GEOLOGICAL SURVEY

CIMPILER CHOSS INDEX... NEH-Z15 HFFEHEIUCE............. GUNTEK, 1973

HECUHD 00318

GEOHE:AU_STMṔLE ETIĹ

COCITITE UF SAMPLE SUURCE... IIPPEK GEYSER BASIN - UNNAMEO SPAING

CONINTKY . ........... UNITEU STATES LOWHSHL=BANGE

SIATE.

CIINTYY.......... TETON

MaP RE ELETYCË..... OLU FAITHFUL 1862500

SAMDLE UESCGIQILUQ_GQU_CQUUTLIUUS

STMPLE NIMLIER......... $1971 / 06 / 03$ GUNTEH, 3.0.

GAS ANALYSIS

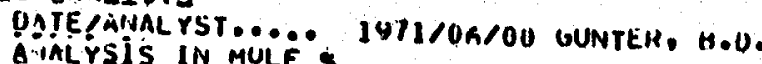

AH. IN MULE

AH... $\quad 0.47$

C?:16.

ISUIURES inecul

Cur.. 3s.

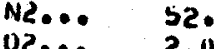




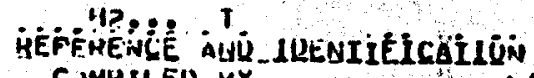

CIMHILEI HY......... LAWSUN, WILLIAM A.

C.MPILEN AFFILIATION. H. H.S. UEULUGICAL SUHVEY

C.MMPILER CHUSS IIUDEX... NEH-2I4

HFFEREWCE............. GUNTEK, 1973

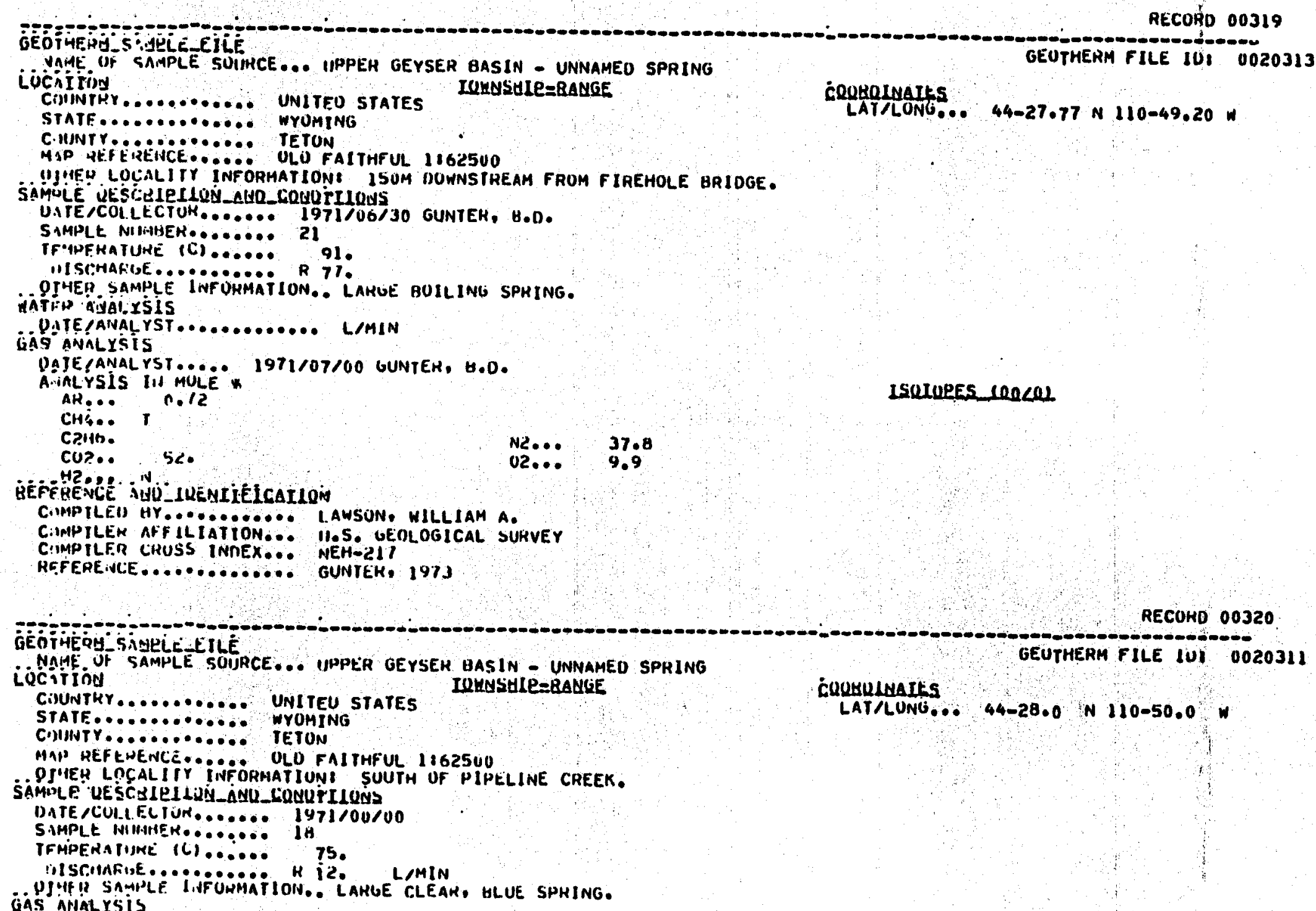

RECOŔD 00319

LSULURES_anCOL

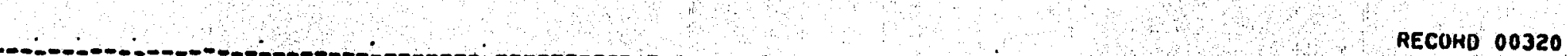
GQC NAME UF SAMPLE SOIJRCE... UPPER GEYSEK BASIN _ UNMAMEO SPRING

CiUUNTHY............ UNITEU STATES IOHOSHIR =RAMGE

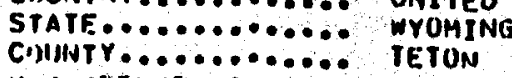

MAP REFEHENCE....... OLO FAI THFUL 1862500

PI'AEY LOCALIYY INFFORMATIUN! SUUTH UF PIPELINE CREEK.

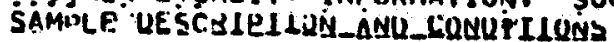

DATE CUIAEC IUN.

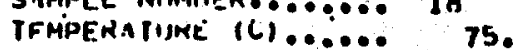

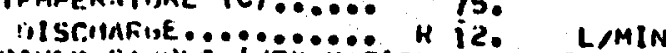

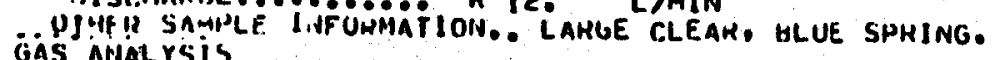


DATE ANAA RST..... $1971 / 0 O / O O$ OUNTEH. H.U. IAI.VSIS IN HULE

AK, D. O.J6

ISULOEES IONCOL

C.H... I.S

CPHE. His

hP... I

OTHEN ANAI Y TICAL UATA AKINZ = 2.29

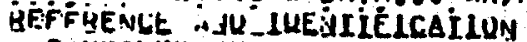

C.MPILEIS HY............ LAWSUIV WILLIAM

CIMMPILEF AF FILIATION... II.S. GEOLUGICAL SURVEY

C. MAPIt.El CHOSS INDEX... NEH-LIO

RFFEREISCE............. GUHTEH, 1973

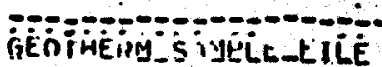

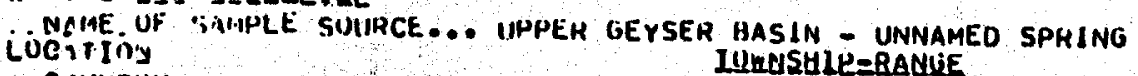

COUHTRY......... UNITEU STAIES LUWUSHLE=BANLE

HYOMINIS

C.NINT P............ TETON

MAP HEFEHEINCE...... ULU FAITHFUL 1862500

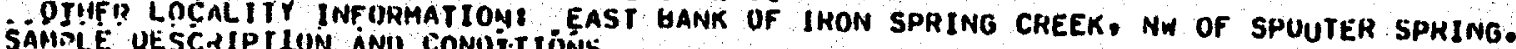

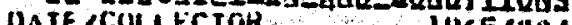

SIMPLE

S.MPLE NIIAEH

Gis nivatisis

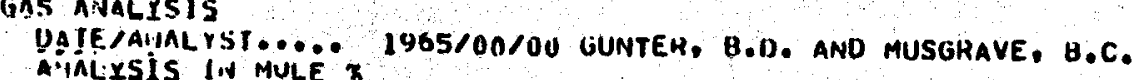

iAlissis lin mule

CHe... 1.5

c:7+46.

cit?...

OTHFA aival YTICAL

UUIA IF I LAI I UIY I I I LATA... PH (EST. WIIH PH PAPER) = 8

REFFEE UC AU

C MPPILEL UY

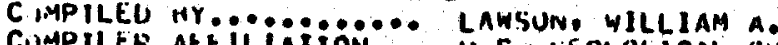

CIMP TLE. AFF ILIAIION... 11.5. LEOLOGICAL SURVEY

C. 1,APILER LHOSS INDEX... NE NH-ZUB

RIFEHE:AF............ hUNTEK ANU HUSGKAVE, 1966

LSULOUES inOCOL

CQUHULMATES

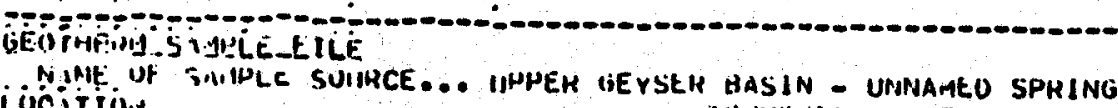

RECURO 00322

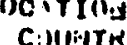

LUWSILL = WANLEF

CTATF

nromitist

CININTY........... TEION

MAP METE.NE.HCE...... ULL FALTHAIL I InCSUO

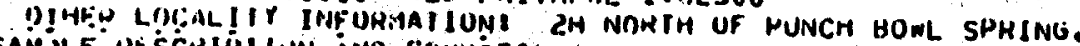

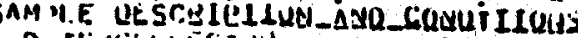

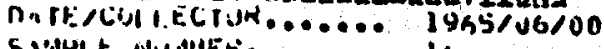

S N.IPI.t. ivi.111ER......... 14

RECUKD 0032

GEUTHEKM FILE IOI 0020289

LAT/LUNG... 44-28.50 N 110-50.00 W 


\section{C}

JE MPEKITIUE (C) ...... A4.

GAS ANMLYSIS

UATE/AIUALYSI_.... 1965/US/OG GUNTER, B.D. ARU MUSGRAVE, H.C.

Oini rsis dit tuie

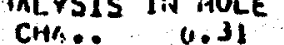

caitio

$$
\text { (.31 }
$$

cos?

$4 \cdot 0$

N2.. 62.87

H... is

טL... 12 .

UTHFH ANAL ITICAL DATA... PH $=9,2$

OUAL If JCAT IUIN FIELU, O. UZ $=02$ AR

HEFFPFITE HULLUEIIEICAILUN

CMPILEII OY.......... IAWSUN, HILLIAM

CHOILER AFFILIATION..: M.5. GEOLUGICAL SURVEY

C SMH ILEH CRUSS INDEX... NEH-IYO

HFFFHERCE............. TIUNIEK AND MUSGHAVE, 1966

LULCLEES cenCQL

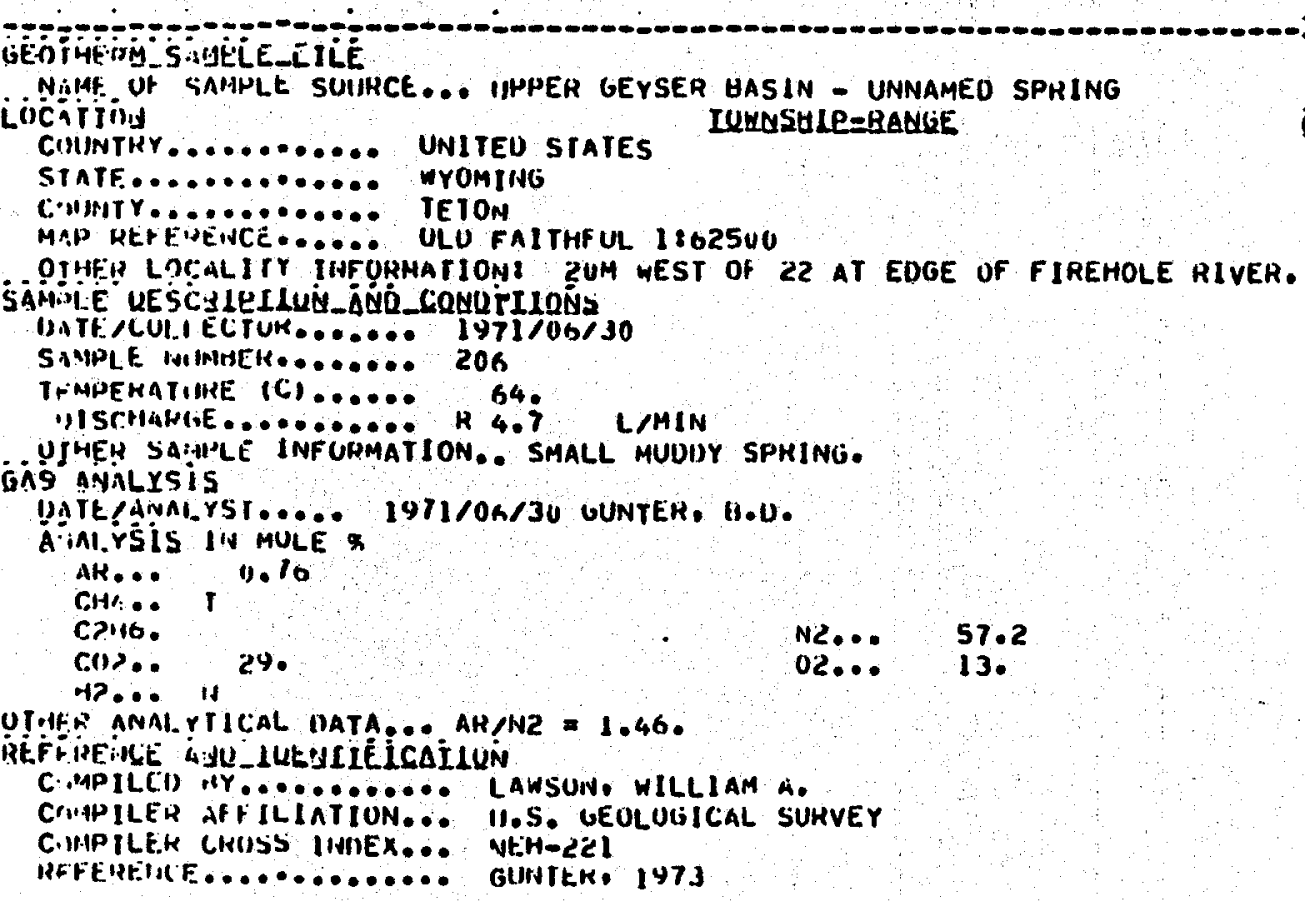

KECOHD 00323

GEUTHEKM FILE IOI 0020317

CQQHULCAIES

AT/LONG... 44-27.53 N $110-49.46 \mathrm{~W}$

SUIOPES InOCOI 
QTHEË LOÇALIIY INF ORMATIOAI EAST HANK OF FIREHOLE RIVERI JUST NORIH UF NEW GRIUGE.

SAMILE UESCALELLU_AUL_CONOTHÓNS

DITE/CULIECTON

SAMPLE NIIIAEEKO....... 200

TFMHEHATHIRE (C) $\ldots \ldots . . .$. A9.

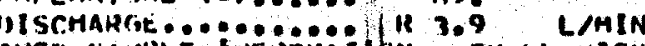

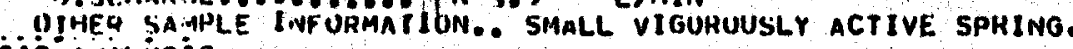

GÁs anALÝSTIS

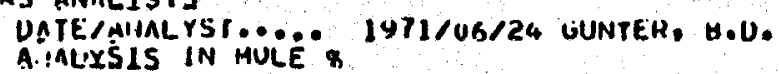

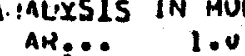

ISUIQLES LON $\angle 01$

CHin.. 1.1

CP.

H?... In

N2... 80.9

02

THËH ANAL YIICAL PAJA .. AH/NZ $=1.320$

REFHETICE HUL_LCLIIÉICAILLUN

CIMPILED HY H. OA... LANSUN, WILLIAM A

C IPPILEA AYFILIATION... NOS. GEOLOGICAL SUHVEY

CIIPILER CHOSS INDEX... NEH-2ZO

REFEREIICF.............. GUNTEK, 1973

RECUKO 00325

GEO THE G SMPLE LILE

LOCAMTIOY SAMPLE SOIJRCE... IIPPEH GEYSEA HASIN - UNNAMEO
LQLUSHLE=BAMGE.

Cntunth

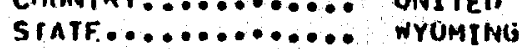

CIIINT

MAS QEFE

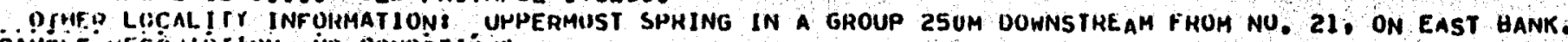

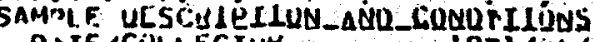

DITE/CUI.LECTUK.

SIMPLE HIIUEK....... 23

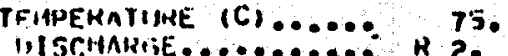

GAŚ Aivalysís

DATE!AIALLYST..... 1971/0R/24 GUNTER, H.U.

A TALYSIS IN MULE

AR... 0.20

Bist...

C)Mti.

C.11D..

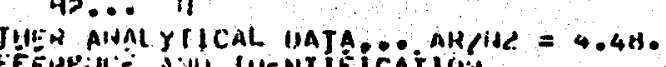

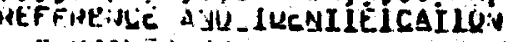

C IMPILEU HY............ LAWSUN. WILLIAM A.

CIMPIIE iR AFFILIAIIION... 11.5. vËULUGICAL SIIKVEY

C.IMIDILEH EHUSS IIVIIEX... NEH-ZIY

HIFEHE.ICF............. FIIISTE, $19 / 3$ 


\section{iocitiós}

CUUNTKY............ UNIIED SIATES

STATF............. WYOMTNG

COUNTY.......... TETUN

MIP REP EDETICE..... OLII FAITHFUL 1862560

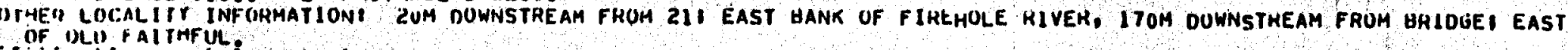

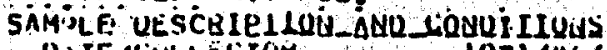

D.T TF. CUI.LECTOK..... 1971/06/30

SIMPIE NIJMUEH....... 22

TFHARFUMTINE (L)

2

IIISCitairit.

MLE INFUMATION.

SPRING IS SLIGHILY TUFHIO.

\section{AS ANRL XS IS}

USTE /ANALYST _... $1911 / 0$ / $/ 30$ UUNTEH, H.D.

IALYYSIS IN HULE

AR.... 1.0

CPit6.

cur..

16.

\author{
ISULORES IONCOL
}

CogBuLNAIts
LAT/LONG... 44-27.53 N 110-49.45

N2... 65.9

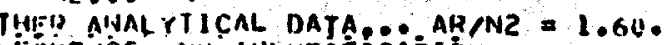

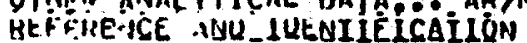

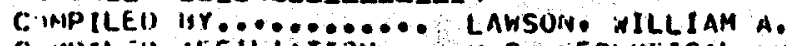

C IAPILER AFFILIATION... N.S. WEOLUGICAL SUHVEY

C. HNILER CRISSS INNEX... NEH-2IB

WHFEL.UCR............... nUNTEK, 1913

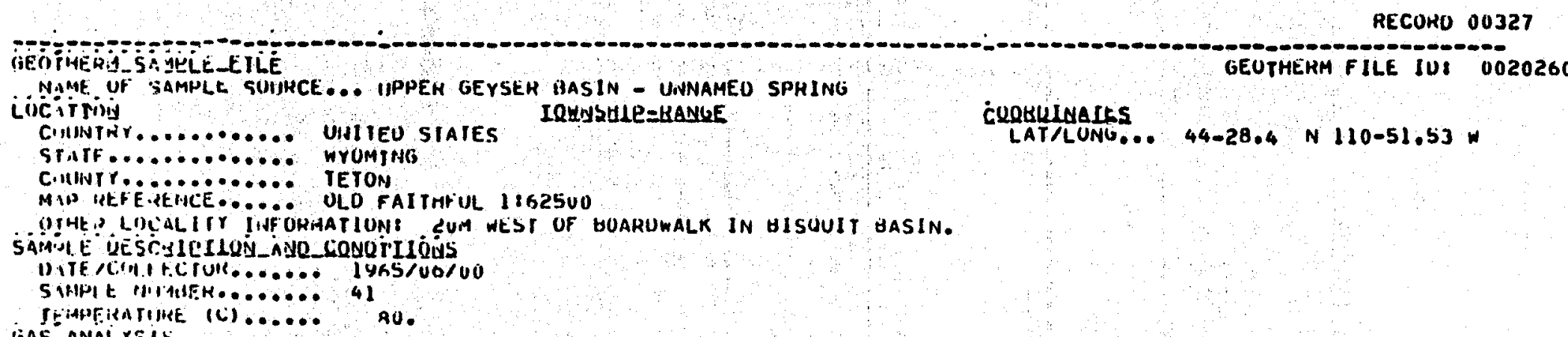

LULUEES COOCUL

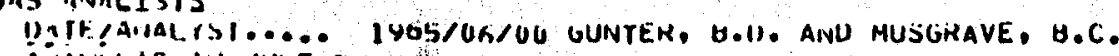

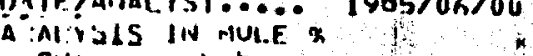

cili... bob

.

$$
\text { N2.. } 44.36
$$

ciis.. ase a

"ITHI "ANAI PTICAL WATA... PHIEST. WITH PH PAPEK) = 7.S.

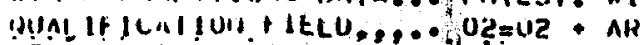

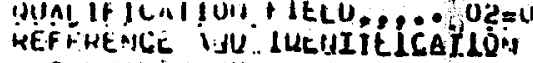

C. Wisll.i.11 ir............. LANSUIr, WILLIAM A.

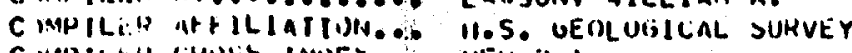

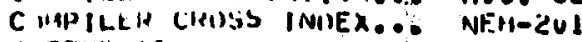

RifFHE IICF.............. GUNIEH ANU MUSGRAVE. I9Go 


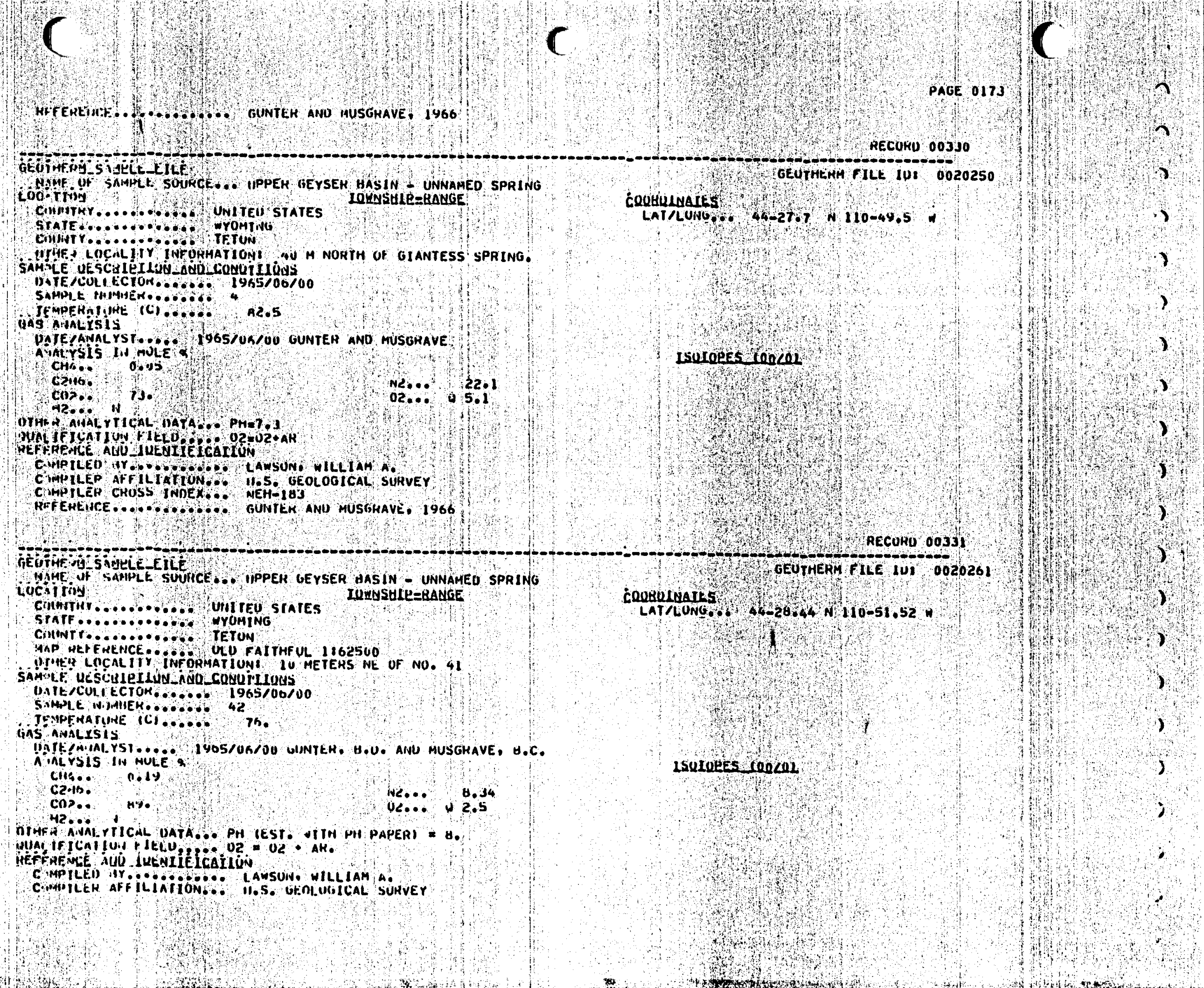


C.MPILER CKUSS INDEX... NEH-ZUZ

HFFEME YCE.............. GUNTEK ANU MUSGHAVE, 1966

RECORO 00332

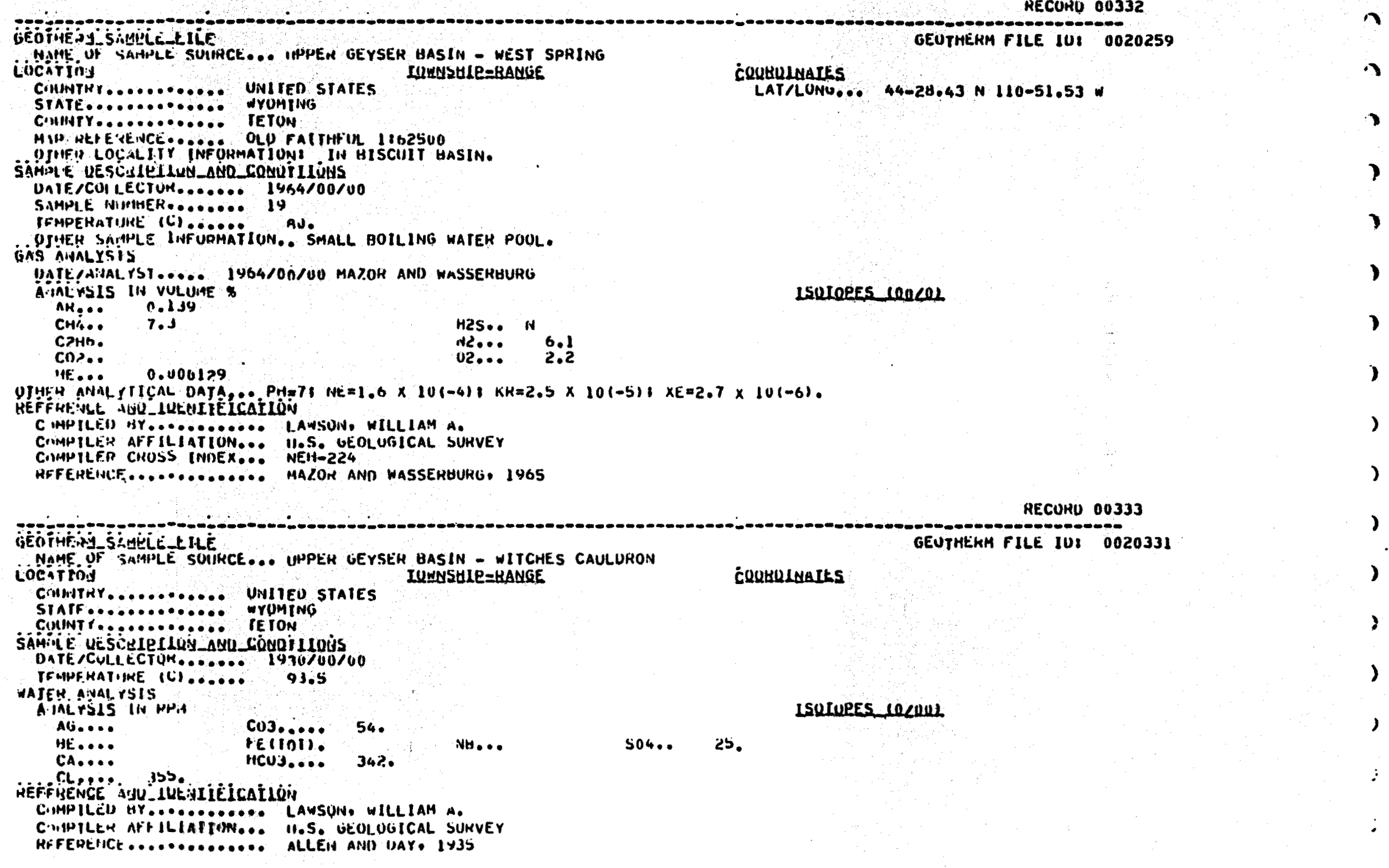




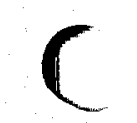

PAGE 0175

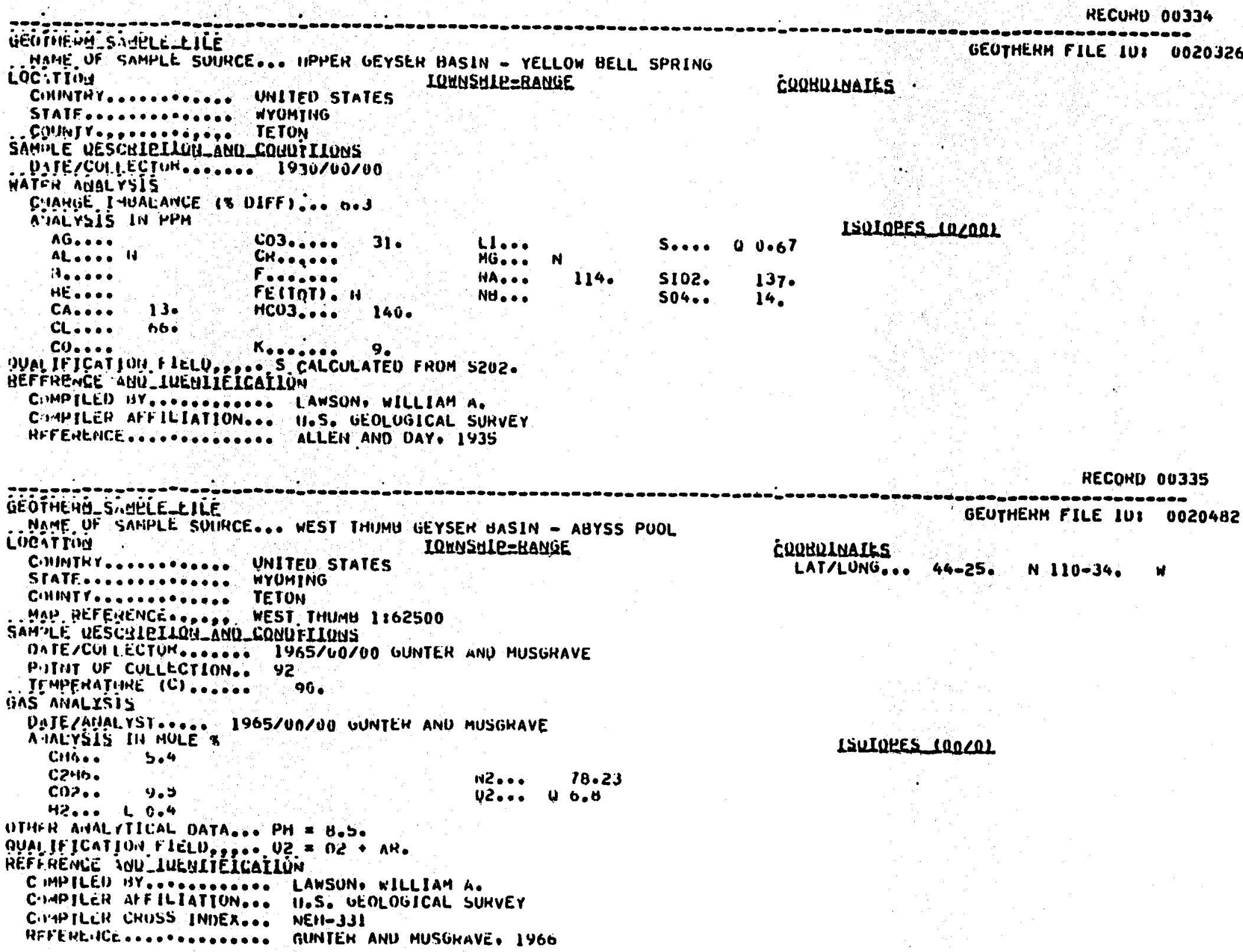

RECOKL OO335 NAME UF SARPLE SUIIKCE... WEST IHIJMU LEYSER OASIN - ABYSS PUOL Lieñirivi IQWUSULP=HANGE CIMTIKr........... UNITEO STATES Cratr. ............ HYOMING ChINTr.0.0.0.0.0. TETOM MAL HEFEAENCE...... WEST THUMH 1862500

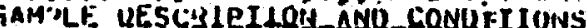

OATE/CUI LECTUK. OA. I965/L0/00 GUNTEH ANU MUSGHAVE D.jint uf cullection.. yz Ir Mptr.hat!inte (C) GAS ANALYSIS

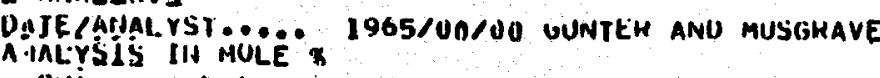

cirio.. 3.4

5

Coj.. 9.3

THEK AIHLPTICAL DATA... PH $=8.3$.

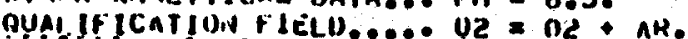

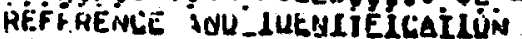

C MMPILEI) HY.......... LAWSUN. WILLIAM A.

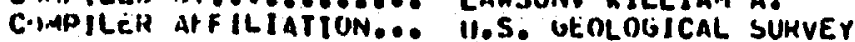

CIMPILLIR CHISSS INIDEX... NEIIOJJI

HFFEHE.HCE............. GIWIEK AND MUSGKAVE. 1960 
HECUKU 00336

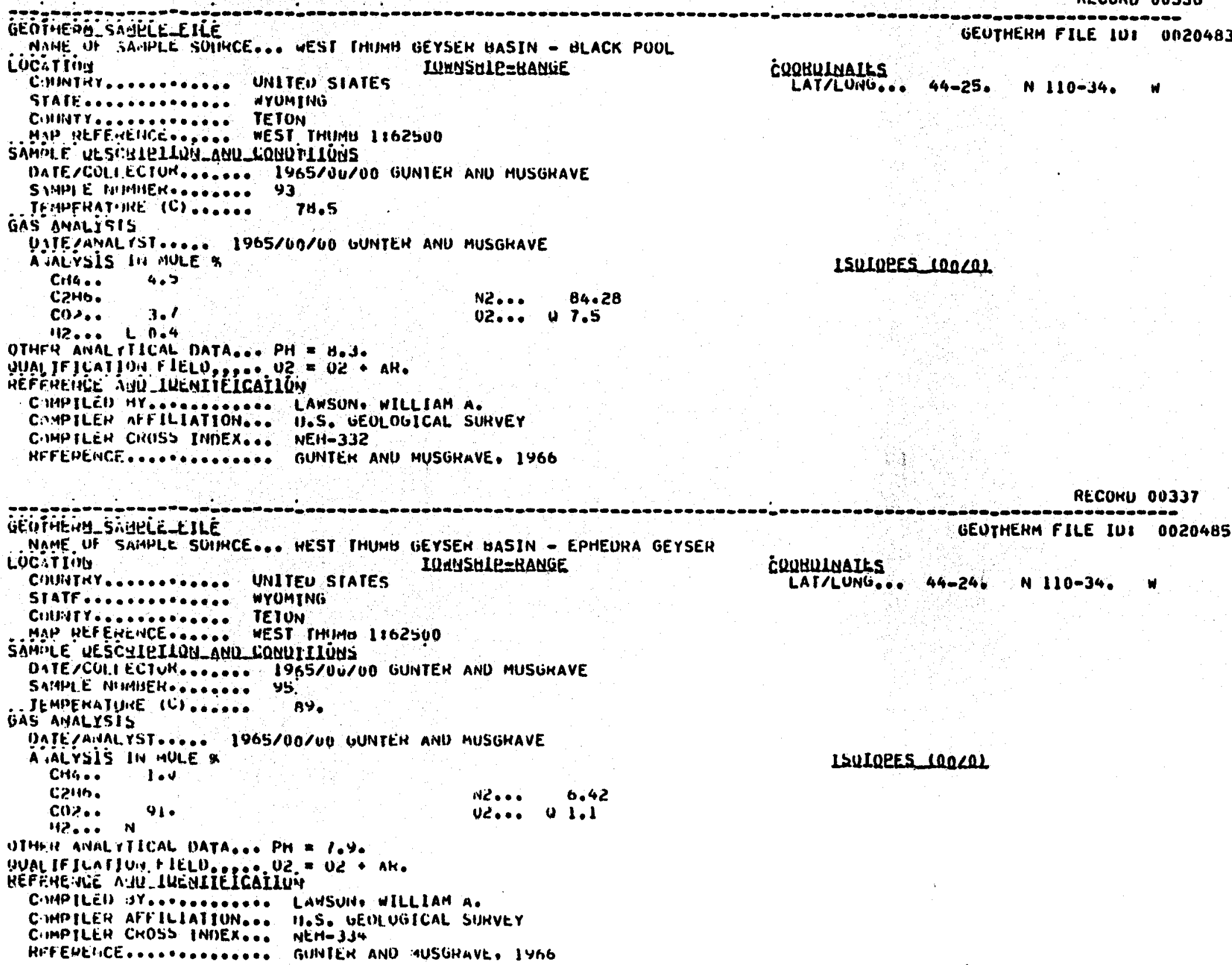

RECOKU 00337




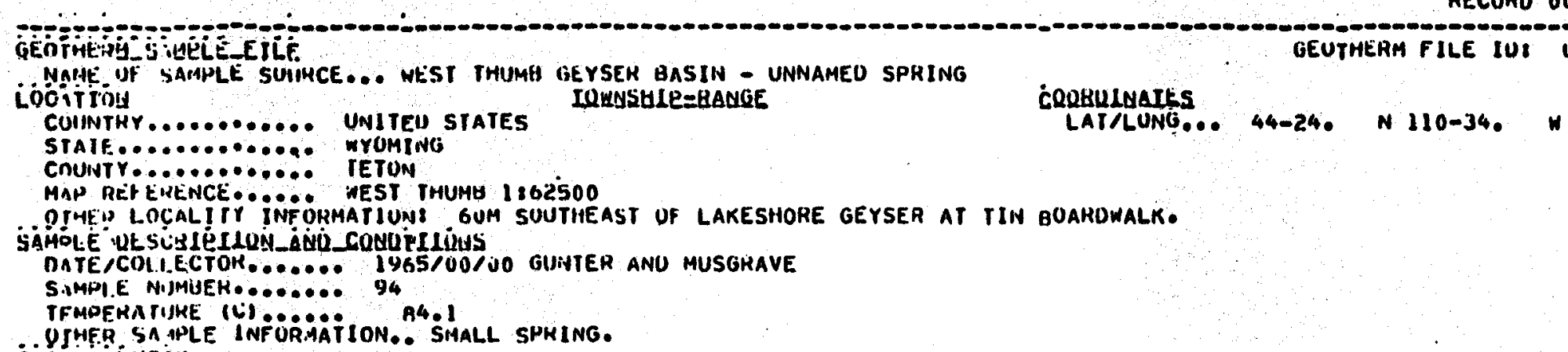

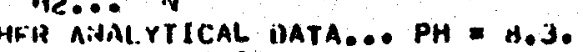

OUAL IF ICATJUIU FIELU.... UZ = UZ AR.

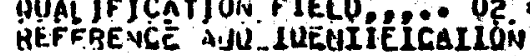

CIMPILEW AY............ L IAWSON, WILLIAM A.

C IMPILECH AFFILIATION... W.S. GEOLOGICAL SURVEY

CIMP ILEH CKOSS INDEX... NEH-333

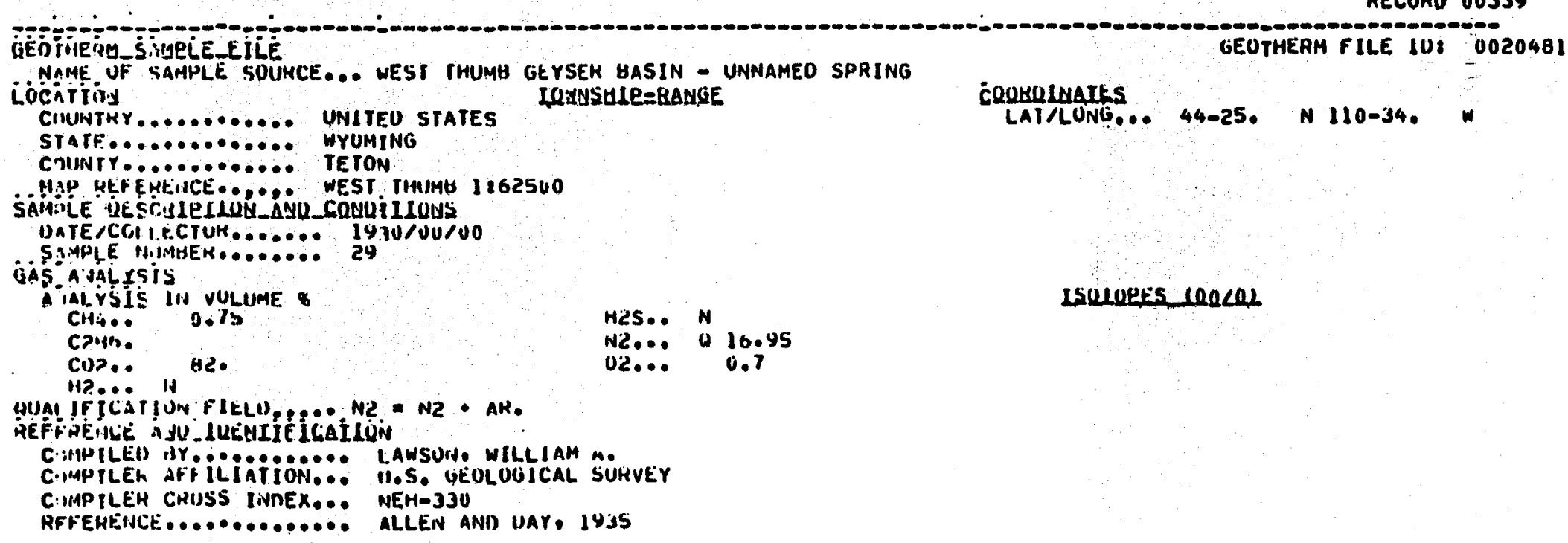

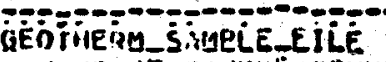

-

NAME UF SAMPLE SOUHCE... WESI THUMB GEYSEK BASIN - UNNAMED SPRING iocition

CoIUSHLE=BANGE

STATF............... WYUMING

CDINTHY............ TETON

MAP HEF EMEIUCE...... WEST THUMU 1862500

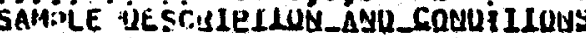

DATE/CCA I.E.CTUK....... 19.30/N0/00

ST.MPLE MIMMSER........ 29

GAS. A JAL TSIS

Cial Ýsís lin vUlume o

caith.

(a)

HES.:

corpo

820

0.0 .95

LSLLUEAS lenCOL

HUAL IF ICATIUN FIELL, .... N2 = N2 • AH.

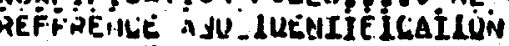

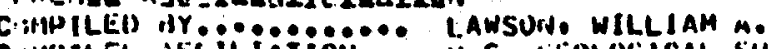

NeS. gEOLLGical SUHVEY

C:MPPILEE CRUSS INUEX.... NEH-330

RFFEHEENCE.............. ALLEN ANI UAY, 1YJS

LAT/LONG.... 44-25. N 110-34, N

AFERERENCE.............. GUNTEK AND hUSGRAVE, 1966$$
\text { gunter ano musGrave, } 1966
$$

NECOHD OU33H

LSUIOPES 100COL

RECOHO 00339 
RECUHD OU340

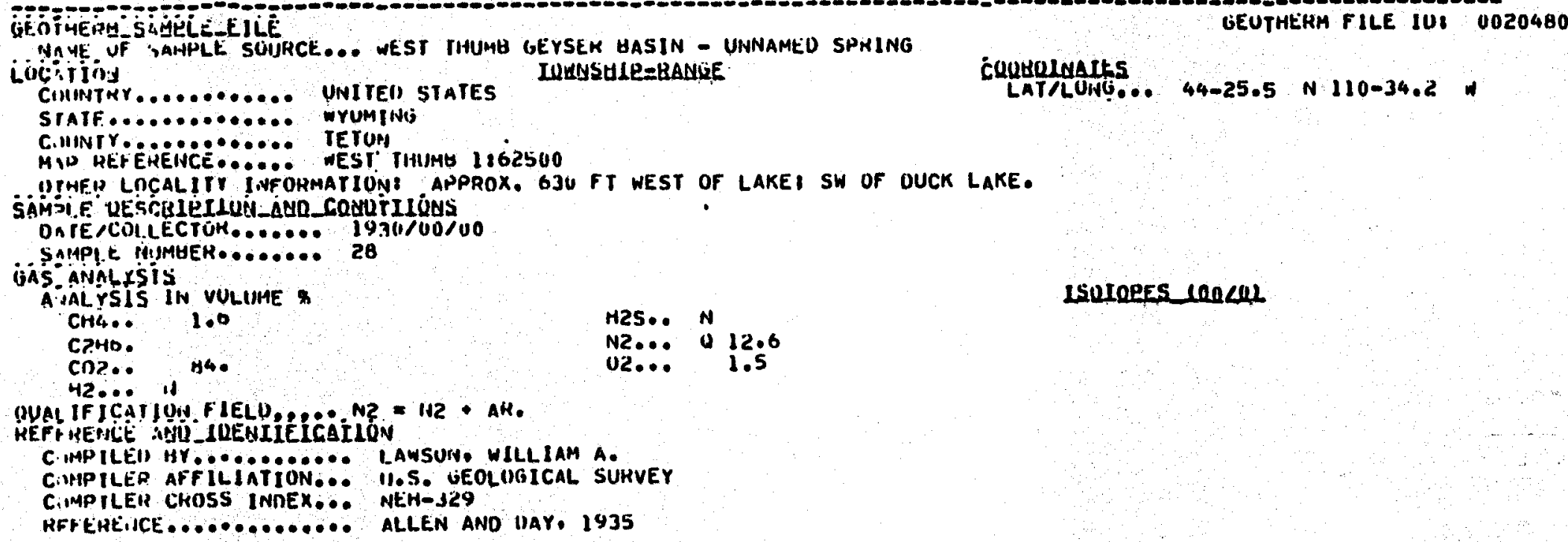

HECOHO 00341 


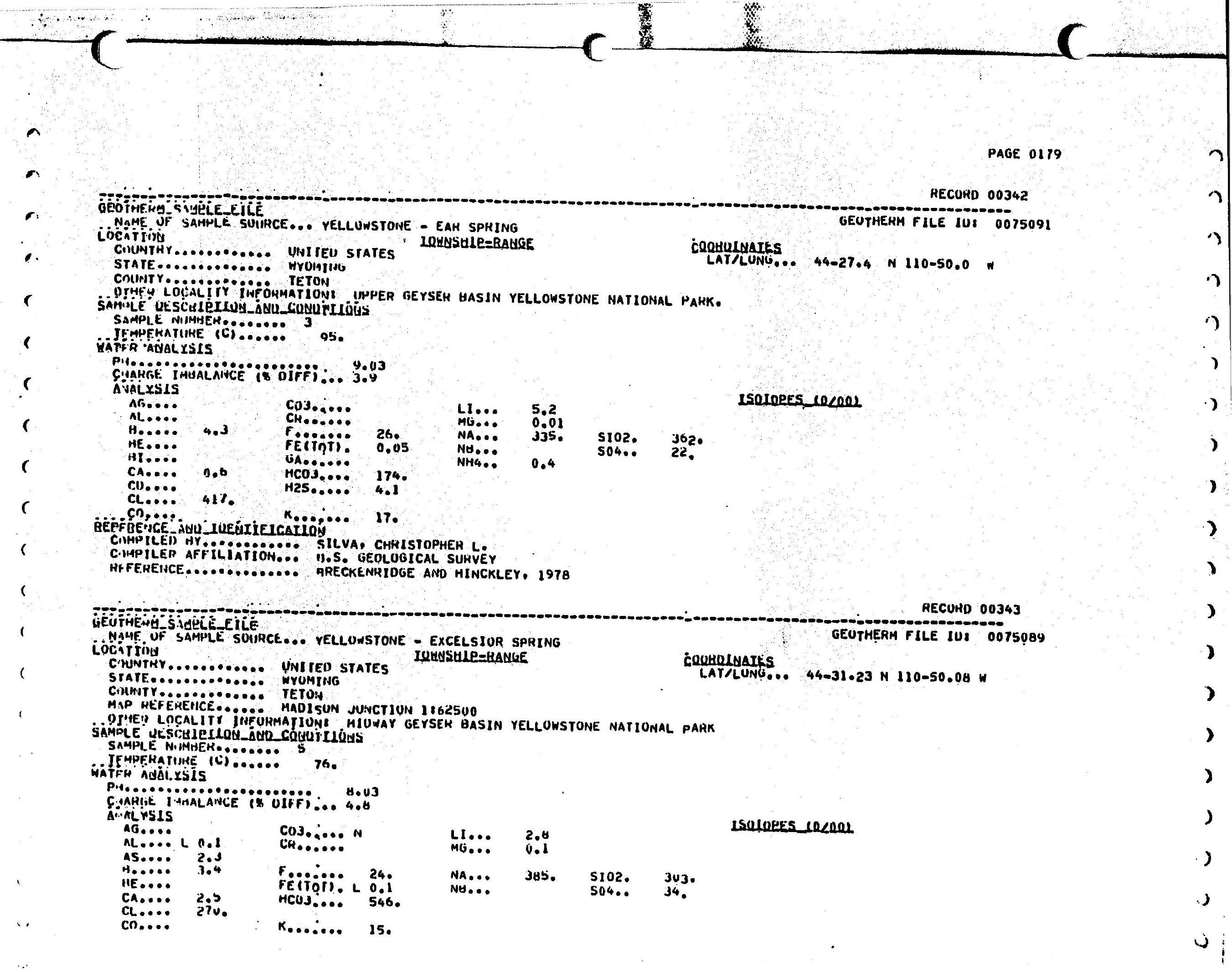


GéFFAF ICE TUQ LUÉLIELCALLON

C. 2HWILEN dY............ SILVA, CHHISTOPHEH L.

C.1HILE⿰R GFFILIATION... H.S. GEOLUGICAL SUHVEY

HI EDENCE............. ARECKEINHIUTEE AHU IHINCKLEY, 1970

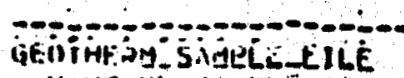

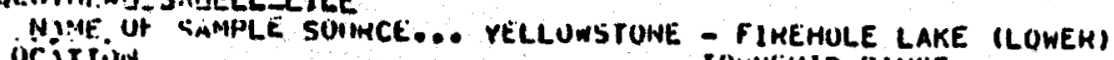

Lucitring

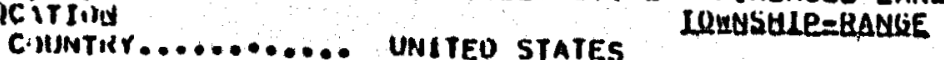

STAF

CONATR

MAP REF EHETELE...... MAUISUN JUNCTIUN 1862500

SAOSHEY LOGALITY INFORMATIONZ LUWER GEYSER GASINI EAST OF FIREHOLE LAKE.

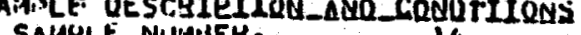

SMAPI E NIHABEH....... 14

TFIHERAIUNE (C)

IUN.... OILUIE TRAVERTINE VEPUSTIS

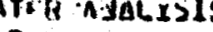

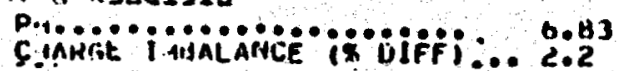

A.IALISIS

AG.....

AL.....

H.....

HA.....

AF.....

HI

C.A... is

coj.....

CU....

CL....

iaś foursi

Chone?

F.o.t. 10.

LI...

0.4

SOIUEES LOCOOL

RECUNO 00344

A ULLYSIS

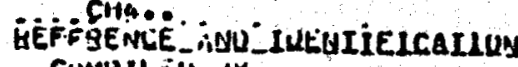

$10 . \quad$ NA... B5.

85. S102. 213.

NH...

SN...

NH4..

0.1

213

OEL C113) of CO2 ... - -4.4

GEUTHERM FILE IUI 007508

CUQHQLAAIES

LAT/LONOO... 44-32.30 N $110-47.02 N$

PAGE U 180

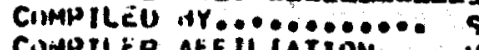

CIMAPILER AFFILIATION...

165

27.

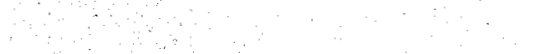

LOLORES IONCOL

SEL C (113) OF CH4..... -26.1

AECOHD 00345

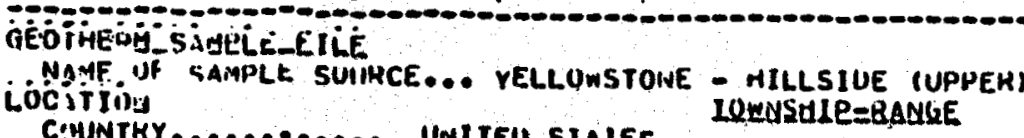

C:HINTHY............. UNITEU SIAIES

STATE............ wrominto

C.JUTIY......... TETON

C.

OJPE. LOCALITY INFORMATIUNE LUAER GEYSEK HASIN. WESI SIUE.

SAMDLE UE SCYLELLUU_ANU CCQUU

TrMPERATIKE (C)...... 94.5

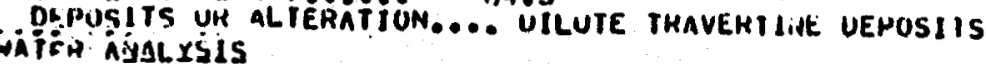


P..................... 6.

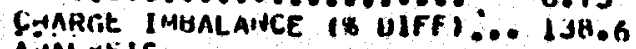

A.NALYSIS

AGSIS CO3.

C.18

$4, \ldots$

1.1

CR......

6.
2.

L....

MG...

0.8

HI.....

CA... 1,9

FEITOTi.

NQ...

149.

5102.
504.

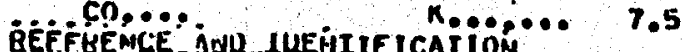

C MNNILEU ANY IUEUIIELCALIO

C.MPILEH AFFIIAT... SILVA, CHRISTOPHË L.

il. G. Gelogical surVey

truesuale anu fouknieh, 1976

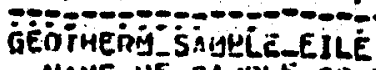

RECORD 00346

NAME UF SAMPLE SUIACE... YELLOWSTONE - LITTLE WHIRLIGIG GEYSER

LOOA IIOA

CiNINITH

STATE $0.0 .0 . . .$. UNITEO STATES

GEUTHERM FILE IU: 0075092

STATE.0.0....... WYUMING

CNUNTY $.00 \cdots 0.00$ TETON

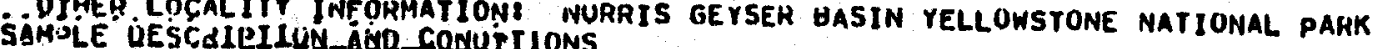

SAMPIE NIMHEH

JEMPERA THUE

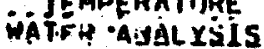

PH.

J.24

AG.. CO3

AL... $2.1 \quad C_{0} \quad C_{0.10}$

H..... 9.2

HE....

CA....

CL.....

K._.... B3.

NA... 349.

NH...

S102. 420.

ISUIOPES IOCOOL

C.IMPILED AY

CIMMILER AFFiliation....

SILVA, CHRISTUPHER L.

U.5. GEOLUGICAL SUKVEY

HFFERETHCF............... AHECKENRTUGE ANU HINCKLEY, 1978

RECUHD 00347

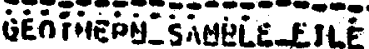

NARE UF SA.MPLE SUIRCE... YELLUWSTONE - NEAH CLEPSEDRA GEYSEH

Lócitiou

CMINTHY....... UNITEU STATES LQUHSHLE-BANGE

STATE............. WYOMING

COHINTY........... TETON

OP TEE. LOCALI ITY INFOHMATIONB LUWER GEYSER HASIN VELLOWSTUNE NATIONAL PARK

SAMPLE N.JAHEK. .

IEMPERATINE (C)

HAFFH MINALIYSIS

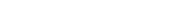


B.1.

CYAHF, О.............. 9.03

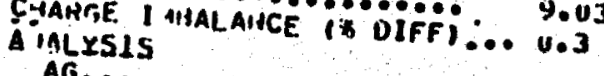

AG....

Cro.........

HE.....

Ml....

CA.....

3.0

Fiini:.:

38.
0.05

$\mathrm{CL}=\cdots$

0.5

GA.......

NA.P.

400

MCoj.... 159.

NH4.:

0.1

414.

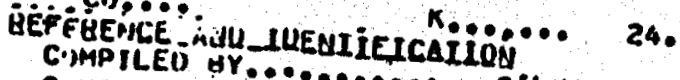

CIAHPILER AFFILIATIO - SILVA. CHAISTUPMEH

RFFEAEIYCE O.0000

A... AHECKENRIUGE AND HINCKLEY, 1978

ARECKENRIUGE AND HINCKLEY, 1978

GEOTHÉg SAMELE ETIE

CONAME YF SAMPLE SOURCE。

\section{Couritir.}

cinviry

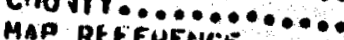

UNITEO sTATES

mromins

TETON

OTHEH LOCALITY INFO MAUISUN

HIYEH

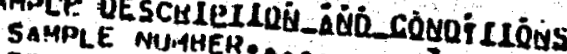

JEMPEHAJUKE (C) ......? 7

WATFE MÜALXSIS

9.5.

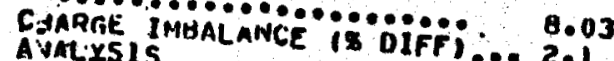

Avintris

AG....

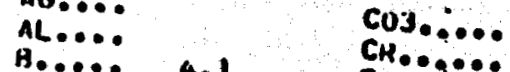

H.... 4.1

ME:

HI....:

Ca.... 0.9

C1).....

Co.....

Giśtouns is

331.

rLYSIS

FË30. 31.

FEITiTi:

GA

$M C 039.0$

M25. 225

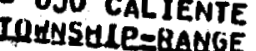

SPRING

ING

IRIVER GHUYP)

\section{CAT/LONG.}

ATAONG... 44-33,18 N $110-49.51$

RECOHO 00348

GEUTHERM FILE IUI 00750

1.3

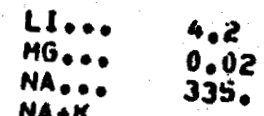

NG.:

NH4.:

K....... 9.5

5102. 230

$504 .$.

24.

LSOTORES loenol

PAGE 0182

ËFrit.

C.JMPILEO GOLLLELIEICAILUY

CAMPILEH AFFILIATIUN... S

AFFERENCE. AFILIATIUN... U.S. GEOLUGICAL SUHV.

ARECKENRIOLEAL SURVEY

SW OF RUSH LAKE, EAST UANK OF FIREHOLE

Lutoes locan

$\therefore$

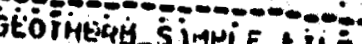

NAME UF SAMULE

RECOKO 00349

GEUTHEKM FILE IOS 0075085

ISOIOEES (ROCOL
SEL C(I3) of CH4.....

$-20.6$ 
Löoiriois

COUNTHY......... UNITEU STATES

STATE. CHUTY

CTWES LOCAL ITY JNEORMATUN

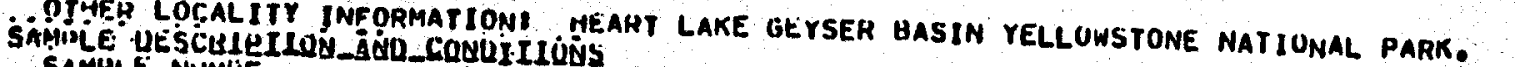

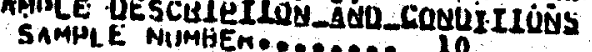

TEMPEKATIJHE (C) O.

HATFe NUSLYSLS

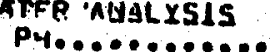

91.

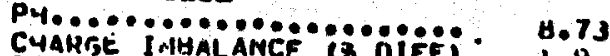

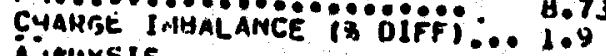

A JALYSIS

A6.....

AL.... CO3.

AL...

HI.....

CA.... 1.2

FEitio:

BEFFEO KOE KO 16.

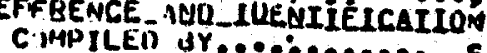

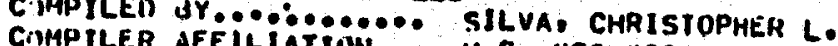

CIMAPILER AFFILIATION... H.S. GEOLUGICAL SUHVEY

RFFEAENCE .......... RHECKENRIOGE ANO HINCKLEY, 1978

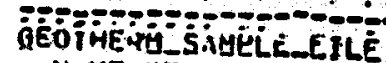

\section{cRQRULUATES}

PAGE 0183

MAME UF SAMPLE SOUACE Cöonitió

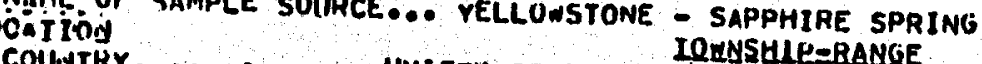

STATE

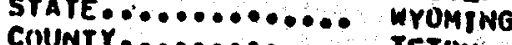

CIUNTY

MAP REFERENCE.-... OLO FAITHFUL 1162500

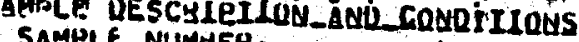

SAMPLE NUMHER०........

. IEMPENATINE (C)

45.

Pro ANIAL YSIS

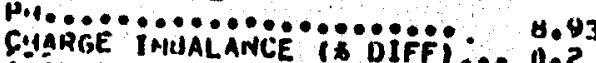

ONALYSIS

A6.....

AL

AE $\cdots \cdots$

HI.

CA.....

cis.....

3.2

Co3......

CL.

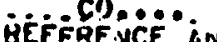

FËPọTi.

0.3.

HCO3.

LI...

MG... 0.05

NA...:

405 .

0.1

ISUIORES cenes

PAOE OI83

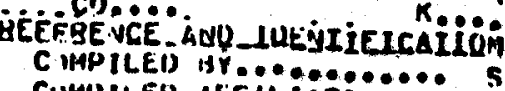

C.IHPILER AFFIL

RFFEREMCE..... U.S. GEOLOGICAL SURVEY

RECOHO 00350

GEUTHENA FILE I0: 0075090

Contuluares

LAT/LONO... 44-28.48 N 110-51.15 N

$\therefore$

NURTH END OF BISOUIT BASIN.

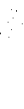




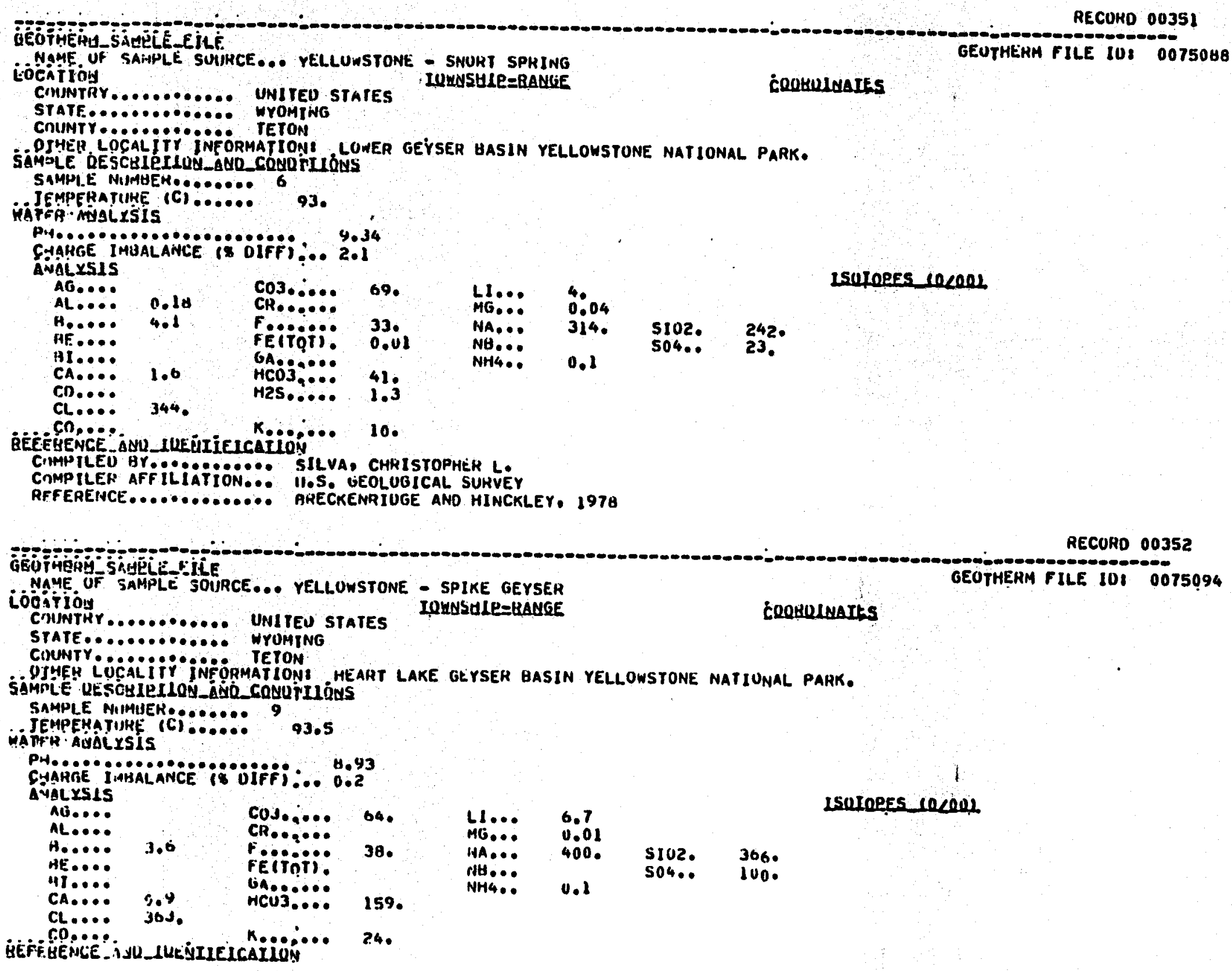


CIMP ILED BY

C:IMPILEH AFFILIATION... H.5. LEOLUGICAL SUKVEY

REFEIRENCE............ TRUESUALE AND FOURNIER, 1976

AECOHO 00353

GËTHETM SAMULE LIIE

NAME OH SAMPLE SUUACE... YELLONSTONE - SPKING 192

GEUTHEAH FILE IU: 007508

CoUhth

COUHTY........ WYOMING

OJHEY LOCALIT INFOHMATIONE SHUSHONE GEYSEN GASIN YELLOWSTONE NATIONAL PARK

Le

JEMPERATUKE (C)

11.99

THE NUÁLYSIS

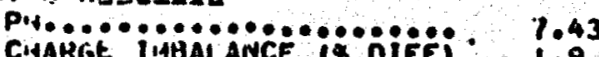

CIAAKGE IHBALANCE (\$ OIFFI:.. I.9

WALESIS

AGESIS CO3.....

Co3.....

H..... 3.

AE......

HI....

CA..... C. 3

F..... 25 .

MGO $\quad 0.5$

NA... 350 .

CL... 323.

FE(InT).

NH4:

$$
0.1
$$

350.

LSOIORES (NeOOS

K.

230

EFFUENCE AUU_LUEIIEICAIIOO

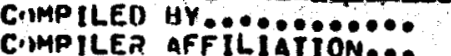

CIMPILEA AFFILIATION

TRUE GUOLOGICAL SURVEY

RECUHD 00354

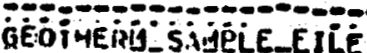

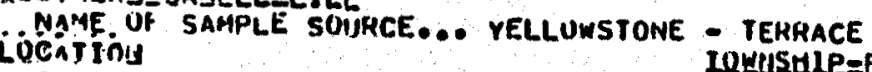

united states LQLUSUle=Bange

SthtF............. Wromtho

CUNTY........ TETUN

MAP HEF EAENCE..... MADISON

TETON

E IV: 0075080

HOAU. sämöie

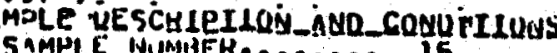

SAMPLE INIAHBEK....... 15

ab.

DEPOS!JS UR ALIEKATION.... UILUTE THAVEHTINE DEPUSITS

Phr AnAI XSIS

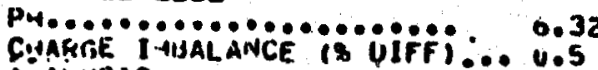

YALYSLS

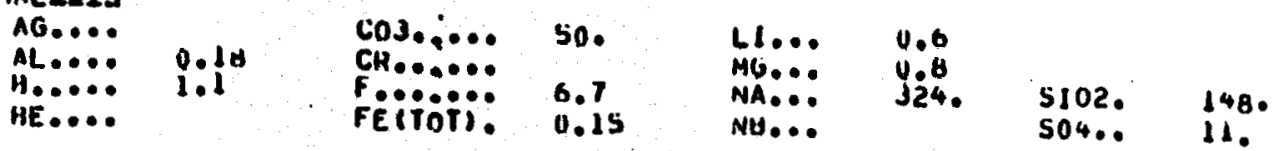

LSUIORES CRCOQ4

HE...

Feirioi:

NU...

SO4..

11. 


\section{C}

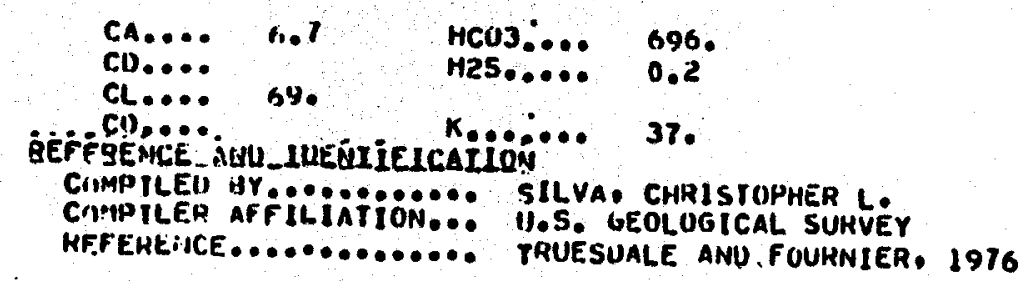

PAGE 0186

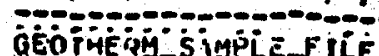

RECOHO 00355

\section{NAME OF SAMPLE SOINKCE}

iócitious

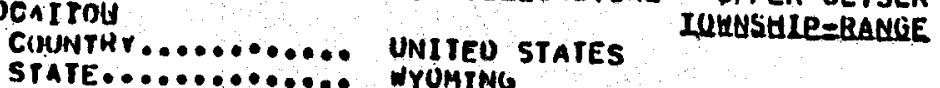

MAP REFEHENCE

FaITHFUL 1862500

GEUTHẸR FILE IO: 0020308

LAT/LONGE. $44-28.5$ N $110-50.0$ N

SAM

QESCIELLU_AOR_CQNORIIOAS

D.TE COL.I ECTOK....... 19R5/06/60

SAMPLE N!IHAEH

GJ'HER SAMPLE INFURMATION. LARGE BHOWN POUL IN STREAM.

IIATELYSIS

WAIE LANAL YST MOE. 1965/00/00 GUNTER ANU MUSGHAVE

WALYSIS IN MOLE

AR... 0.05

CHA.: 1.0

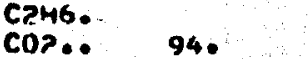

H2 — N N N$$
\text { N }
$$

U2 $\ldots \ldots, 5.4$

ISPIOEES conen

PTHEH ANALYTICAL OATA $\ldots$ PH $=6.1$

REPERE:UCE AUU_LUEUIÉLCAILÓN

CIMPILED WY .......... LAWSUN, WILLIAM A.

C.MMPILEH AFFILIATION..: NoS. GEOLOGICAL SUHVEY

COMMILER CKUSS INDEX... NEH-143

RFFEREIVCE............. RUWTEK ANU MUSGRAVE, 1966

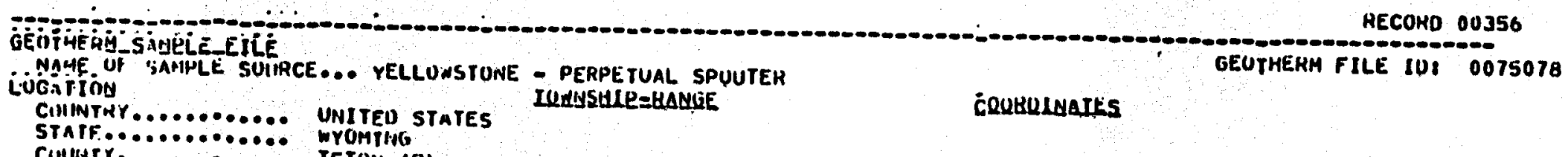

AECOHD 00356

Cinjutr.

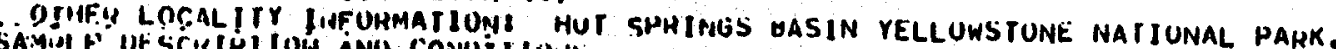

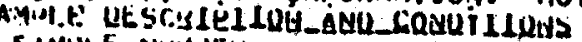

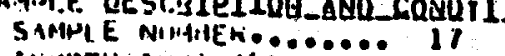

JEMPEFADUNE (C)...... A9.

WAT+U aIJAL YSIS

B.ilisis

Ai.....

$\cos . . .$.

$1 \ldots 0.04$

LULIOEES HenW 


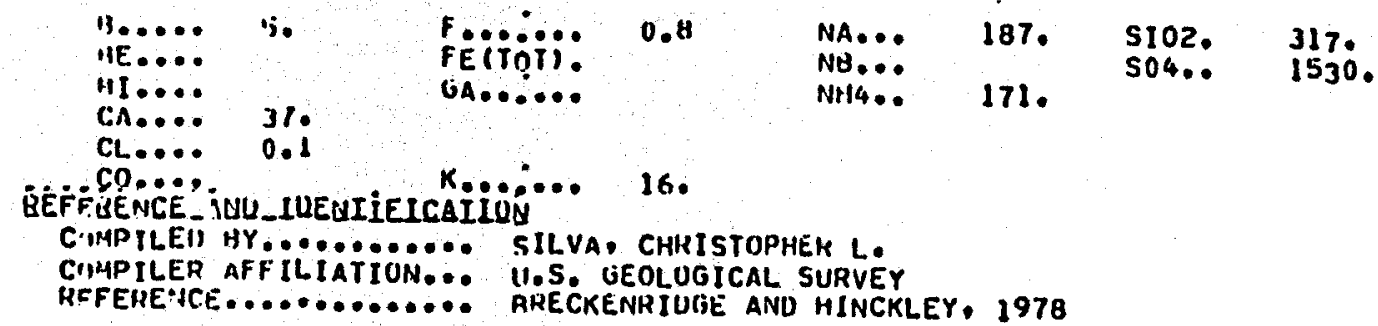


APPENDIX A

Index to GEOTHERA's sample file for the state of Hyoning. This computer generated appendix contains some trucated fields. The index is sorted by county and name of the source. rus township, RNG range, sect. - section, 1.0. - GEOTHERM record Identifier, Temp; - temperature oc

County

BIG HORH

BIG HORH

CARBON

CARBOH

CAnBon

CARBon

CONYERSE

FREMONT

FREYONT

FREMONT

FREMONT

FREMONT

FREMONT

FREMONT

FREMONT

FREHONT

FREMONT

FREMONT

FREMONT

HOT SPRINES

HOT SPRINGS

HOT SPRINGS

HOT SPRIHGS

HOT SPRINGS

HOT SPRINGS

HOT SPRINGS

HOT SPRIMGS

HOT SPRINCS

Hor SPRINGS

HOT SPRIMES

HOT SPRINGS

HOT SPRINGS

HOT SPRINGS

HOT SPRINGS

HOT SPRINGS

HOT SPRINGS

HOT SPRINGS

LINCOLA

IIACOLM

LIMCOLN

LINCOLN

LINCOLN

LINCOLA

MATROMA

MATRONA
Hame of Source

LITILE SHEEP MOUNTAIN SPRING

SIIEEP MOUNTAIN SPRINGS

SARATOGA HOT SPRINGS, HOBO POOL

SARATOGA HOT SPRINGS, HOBO POOL

SARATOGA HOT SPRINGS, HOBO POOL

SARATOGA HOT SPRINGS, HOBO POOL

SARATOGA HOT SPRINGS, HOBO POOL

COUGA CRAT SPRING

FORT MASHAKIE HOT SPRIMES

FORT WASHIKIE HOT SPRINGS

FORT WASHAKIE HOT SPRINGS

JAKEYS FORK SPRING

LITITE WAPH SPRING

SUEETMATER STATIOH SPRIMG NO. I

SWEETUATER STATION SPRING NO.

SWEETMATER STATION SPRING NO. 2

HARH SPRING CREEK SPRINGS

UAPU SPRIMG CREEK SPRINGS

BATHTUR SPRING

BIG SPRING

BIG SPRING

BIG SPRIKG

BIG SPRING

BLACK SULPHUR SPRING

MAYTAG MELL.

MCCARTHY MELL

PILING SPRING

SACANAWEA MELL

SACAJAMEA MELL

YAY MORMAM MELL

MHITE SULPHUR SPRING

WHITE SULPHUR SPRING

WINO RIVER CANYON SPRIMG

WINO RIVER CAHYON SPRING

AUBURH AREA

AUBURA HOT SPRINGS

AUBURA HOT SPRINGS

AUBURN HOT SPRINGS

AUBURA HOT SPRINGS

JOHNSOH SPRINGS

ALCOYA HOT SPRINGS

ALCOYA HOT SPRIMGS
Latitude Longitude INS RMG Sect. 1.D. Temp.

44-44.90 H 108-11.35 H 55M 094Y 17007500020 44-36.45 N 108-08.13 W 54M 0944 35007500121. 11-27.00 N 106-48.40 W 17H OB4H 13 0075002 0 48.0 41-27.00 N 106-48.40 W 17W 0844 130075006 O 48. 41-27.00 N 106-48.40 N $17 \mathrm{M} \mathrm{084M} 130075004$ Q 48. 41-27.00 N 106-48.40 N 17N 084N 130075003 Q 48. 41-27.00 N 106-48.40 N 17H 084K 13007500549. 42-39.88 N 105-23.62 * 311 $071 \%$ O8 007500730.0 42-48.02 N 108-03.03 K 33H 094N 26 0075008 16.0 43-00.48 N 108-50.08 * 015 0014 02 0075009 44.0 43-00.48 N 108-50.08 N 015 001W 02007501046.5 43-00.48 M 108-50.08 Y 015 001Y 02007501144.5 43-29.38 N 109-36.48 * 41K 106429007501220.0

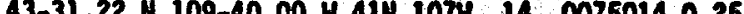
43-31. 22 "I 109-40.00 N 4II 1074 43-31.53 H $100-10.00 \mathrm{~N}$. 22-20 $422-29.50 \%$ is 0075019 Q 31.5 $42-29.53 N$ N 0075018 31.5 43-33.60 N 109-43. 43-33.60 N 109-43.88 W 42स 107 32 007501529.0 43-39.67 N 100-11.72 N $43 N$ 095H 25 0075025 53.0 43-39.27 N 100-11.62 N 43N 094N 310075032 Q 56. 43-39.27 N 108-11.62 N 43N 094W 31 0075030 56.0 43-39.27 N 108-11.62 W 43H 094X 31 0075035 Q 56. 43-39.27 N 108-11.62 N 43N 094K 310075034056. 43-39.27 N 108-11.62 W 43N 094N 31 0075033 O 56. 43-39.27 N 108-11.62 * 43N 094N 31 0075031 O 56. 43-39.32 N 108-11.63 Y 434 095\% 36.0075029 055. 43-10.47 II 108-12.23 Y 4340954 24 007502154 43-39.90 " $100-11.67$ " 431 0941 (35 43-39.27 N 430.00250

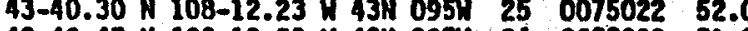

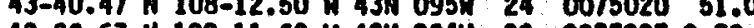
43-39.67 H 108-11.68 $\mathrm{N}$ 43k 094 300075027 Q 53. $43-39.67 \mathrm{~N}$ 108-11.66 W 43H 094W 30.007502653 .0 43-34.92 N 108-13.73 N 42N 095W 25007503622.0 43-34.92 N 108-13.73 พ 42N 095N 25.0075037 Q 22. 42-49.50 N 111-00.00 (1194 230075041 a 62.0 (25 230075040 \& 16.0 42-50.42 N 110-59.50 Y 33H 1104 33 00750380.62 .0 $42-49.08$ N $110-59.50 \times 334119425007504246.0$

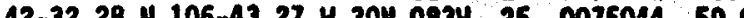
$42-32.28$ N 106-43.27 Y $30 \mathrm{~N} 083 \mathrm{M}$ 25 $0075043 \quad 54.0$ 


\begin{tabular}{l} 
YAYROKA \\
PARK \\
PARK \\
PARK \\
PARK \\
PARK \\
PARK \\
PARK \\
PARK \\
PARK \\
PAAK \\
PARK \\
PAPK \\
PARK \\
PARK \\
PARK \\
PARK \\
PARK \\
PARK \\
PARK \\
PARK \\
PARK \\
PARK \\
PARK \\
PARK \\
PARK \\
PARK \\
PARK \\
PARK \\
PARK \\
PARK \\
PARK \\
PARK \\
PARK \\
PARK \\
PARK \\
PARK \\
PARK \\
PARK \\
PARK \\
PARK \\
PARK \\
PARKK \\
PARK \\
PARK \\
PARK \\
PARK \\
PARK \\
PARK \\
PARK \\
PARK \\
\hline
\end{tabular}

HHORSE CREEK SPRIHGS

BUFFRLO BILL RESERVOIR SPRINGS

DE MARIS HOT SPRINGS

DE RARIS HOT SPRINGS

DE MARIS HOT SPRINGS

DE MARIS HOT SPRINGS

DE MARIS HOT SPRINGS

DE MARIS HOT SPRI

DE MARIS HOT SPRIHGS

DE MARIS HOT SPRIMGS

MAMNOTH HOT SPRINGS - ANGEL TERRACE

MAWOTH HOT SPRIMGS - CAYERM TERRACE

MANOTH HOT SPRIHES - CLEOPATRA SPRING

MAMOTH HOT SPRINGS - CLEOPATRA SPRING

MANOTH HOT SPRINGS - CUPID SPRING

MUMOTH HOT SPRIMGS - HYMEM TERRACE

MAMPOTH HOT SPRINGS - JUPITER TERRACE

MuMmont HOT SPRINGS - MAIH TERRACE

MANHOTH HOT SPRINGS - MINERYA SPRINGS

MANOWOTH HOT SPRINGS - MEK MIGHLAND TERRACE

PAMPOTH HOT SPRINGS - PALETTE TERRACE

MUMOTH HOT SPRINGS - UNHANEO SPRIMG

MAPMOTH HOT SPRIMGS - EMMAMED SPRING

MUNWOTH HOT SPRINGS - UMMAMED SPRIMG

MAMAOTH HOT SPRINGS - UMRAMED SPRING

MORRIS GEYSER BASIH - "BATH-TUB" SPRING

MORRIS GEYSER BASIH - "BATH-TUB" SPRING

NORRIS GEYSER BASIN - BATHTUB SPRING

HORRIS GEYSER BASIN - BLACK GROMER

MORRIS GEYSER BASIH - BLACK GROMER

MORRIS GEYSER BASIH - BLACK MEROH CAULDRON

MORRIS GEYSER BASTH - CINDER POOL (NO. I7)

WORRIS GEYSER BASIN - CONGRESS POOL

NORRIS GEYSER BASIN - CORAL SPRING

NORRIS GEYSER BASIH - ECHINUS GEYSER

MORRIS GEYSER BASIN - EMERALD SPRING

MORRIS GEYSER BASIM - GABLE SPRING (NO. 32)

MORRIS GEYSER BASIN - GEYSER NO. 27

NORRIS GEYSER BASTM - GREEN DRAGON SPRING

NORRIS GEYSER BASIN - IRIS SPRING (NO. 71)

NORRIS GEYSER BASIH - PEARI GEYSER

NORRIS GEYSER BASIH - PORCELAIM BASIN NO. 24

NORRIS GEYSER BASIN - PORCELAIN BASIN NO. 70

NORRIS GEYSER BASIN - SPRING NO. 1

MORRIS GEYSER BASIH - SPRING NO. 15

MORPIS GEYSER BASIN - SPPING NO. 18

UDPRIS GEYSER BASTH - SPRING HO. 19

WOPRIS GEYSER BASTH - SPRIG NO. 20

MORIS GEYSER BASIH - SPRING NO. 20

MORRIS GEYSER BASIN - SPRING NO. 21

HORRTS GEYSER BASIN - SPRING NO. 23

NORRIS GEYSER BASIN - SPRING NO. 25

NORRIS GEYSER BASIN - SPRING NO. 30

NORRIS GEYSER BASIN - SPRING NO. A

MORRIS GEYSER BASIN - SPRIMG MO 5
$02-42.10$ N 107-06.33 Y 32N $086 \% 350075045 \quad 23.5$ $44-28.50 \mathrm{~N} \mathrm{109-12.25}$ W $52 \mathrm{H} 103 \mathrm{Y}$ 12, 007504

44-30.78 N 109-06.88 y 52H.1024 03 ' 0075050 o 24. $44-30.78$ N 109-06.88 Y $52 N 1024030075048$ o 37.0 $44-30.78$ * 109-06.88 * 52H 1024030075054 Q 27.5 $44-30.78 \times 109-06.88 \times 52 \mathrm{~N} 102 \mathrm{~N} \quad 03007504927.0$ 44-30.78 N 109-06.88 N 52K 102N 03 0075053 Q 27.0 $44-30.78$ N. 109-06.88 N $52 \mathrm{H} 102 \mathrm{~W} 030075052 \mathrm{O} 27.0$ $44-30.78$ N 109-06.88 N 52N 102N 030075051 Q 28.0 $44-30.78$ N 109-06.88 W 52N $102 \mathrm{~N} 030075047 \quad 36.0$ 44-58. N 110-42. N 0020464 44-58. N 110-42. $44-58.5$ H $110-42.6$ $44-58.5$ H $110-42.6$ 44-58. $44-58$.

44-58. N 110-42.

44-58. N 110-42.

44-58. H 110-42.

44-58. $110-42$.

44-58. $1110-42$.

44-58. H $110-42$.

44-58. H 110-42.

44-58. I 110-42.

44-58. N 110-42.

44-43. N $110-43$.

44-43. $110-43$

44-43. N $110-43$.

44-43. H 110-43.

44-43. N 110-42.

44-44. N 110-43.

$44-43.80 \times 110-42.00$ N

44-43. N 110-42.

$44-43.58$ N $110-42.42$ W

44-43. N 110-42.

44-43.56 W 110-42.40 K

44-43. N 110-43. N

44-44. IN 110-42.

44-43. N 110-43.

44-44. $1110-43$.

44-44. N 110-43.

44-43. N 110-42.

44-43. N $110-42$.

44-43. N $110-42$.

44-43. $N$ 110-42.

44-43. N 110-42.

44-43. N 110-42.

44-43.81 N 110-42.03 W

44-43. N 110-42. N

44-43. N110-42. H

44-43. N 110-42. H
66.4

0020460

0020159

00204717.

002046570.5

0020467 71.

0020467 71.

002047375 .

002047274 .

0020469

0020468

0020463

0020462

0020461

0020445

002044693.

0020426

0020426

0020449 93.

0020431

0020434

0020421

00204228.

0020430

0020423

002044284.

0020420

85.

0020428

0020427

0020403

0020409

0020410

0020411

0020424

0020412

0020413

0020436

0020414

0020415

0020404

0020405
002044185. 

STEEIE HOT SPRIMGS - SPRING 1 STEELE HOT SPRINGS - SPRIMG 2 ABERCROMIIE UARH SPRIMGS ASTORIA SPRINGS

BLACX SAMB BASIN - UWMANED SPRIMG

BLACX SAND BASIM - UNWAMED SPRING

BOYLES HTL SPRINGS

GRAMITE FALLS HOT SPRIMGS

crenite mat sparma

GRAMITE HOT SPRIMG

HEART LAXE GEYSER BASIM - RUSTIC GROUP - UNMANE

IIILLSIDE GROUP - ASTA SPRING (NO. 3)

MILLSIDE GROUP - ASTA SPRING (NO. 3)

HILSIDE GROUP - UWHAMED SPRIMG

HILLSIDE GROUP - UWHAMED SPRING

HUCKLEBERRY HOT SPRIMGS

HUCKLEBERRY HOT SPRINGS

HUCKLEBERRY HOT SPRINGS

HUCKLEBERRY HOT SPRINGS

JACXSON LAKE HOT SPRINGS

KELLY MAPA SPRING

LOWER GEYSER BASIN - IMPERIAL GEYSER

LOWER GEYSER BASIN - BATH SPRING

LOWER GEYSER BASIN - BATH SPRING

LOMER GEYSER BASIN - FIREHOLE LAKE

LOWER GEYSER BASIH - FIREHOLE POOL

LOWER GEYSER BRSIN - FIREHOLE POOL

44-32.30 N 110-46.98 K

LOMER GEYSER BASIN - FISSURE GROL - EDOYY SPRI 44-31.4 N 110-47.7 N

CNER

LOOER GEYSER BASIH - GREAT FOUNTAIN GROUP - DIA 44-31.6 N 110-47.5 H

LONER GEYSER BASIH - GREAT FOUNTAIH GROUP - DIA 44-31.6 N 110-47.5

$44-31.47$ N $110-52.32$

COYER GEYSER BUSIY - KALEDDOSCOPE GPOUP - WMYM 44-31.5 M 110-48.5

LOVER GEYSER BASIH - MOPYING HIST SPRINGS - UNH 44-34.01 N 110-48.23 W

LOUER GEYSER BASIN - MORHING HIST SPRINGS - UNH 44-34.00 N 110-48.23 N

LONER GEYSER BRSIN - MORNING MIST SPRINGS - UNN 44-34.00 N 110-48.22 N

LOMER GEYSER BRSIN - MORNING MIST SPRINGS - UNN 44-34.00 N 110-48.25 N

LOWER GEYSER BASIN - MORNING MIST SPRINGS - UNN 44-34.00 N 110-48.23 N

LOWER GEYSER BASIN - MOUWO SPRING 44-33.52 N 110-51.42 N

LONER GEYSER BASIH - QUEEH'S LAUNDRY 44-33.47 N 110-52.10

LONER GEYSER BASIN - RIVER GROUP - UNMAMEO SPRI 44-33. N 110-50. N

LONER GEYSER BASIN - RIVER GROUP - UNHAMED SPRI 44-33. N 110-50. N

LOWER GEYSER BRSIN - RIVER GROUP - OJO CALIENTE 44-33.18 N 110-49.51 H

LOUER GEYSER BASIN - RIYER GROUP - OJO CALIENTE 44-33.18 N 110-49.51 H

LONER GEYSER BASIN - SEHTIMEL CONE - 44-33.43 N 110-51.45 H

LOWER GEYSER BASIH - SEMTINEL GROUP - SENTINEL 44-33.43 N 110-51.45 H

LOWER GEYSER BASIN - SEMTINEL GROUP - SENTINEL 44-33.43 N 110-51.45 H

LONER GEYSER BASIN - SEMTHEL GROUP - GUEEM'S L 44-33.47 N 110-52.10 H

LOUER GEYSER BASIH - SEHTIE GROUP - UUEMED S 4-33.45 M10-52.10

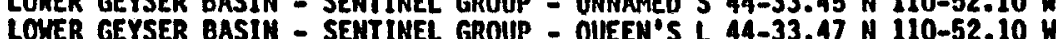

LOVER GEY RASIN - SEATEL GROP - QUEEN S 44-33.47 N 110-52.10

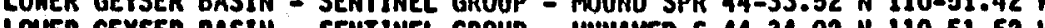

LONER GEYSER BASIN - SENTINEL GROUP - UNWAMEO S 44-34.02 N $110-51.52 \mathrm{~K}$
160075060 Q 35.5

16007506139.0

02007506327.0

007506437.0

0020296

$0075065 \quad 30.0$

007506530.0

of 0075067 a 41.0

007506839.0

0020479

0020263

0020262

002026584

002026665

o 0075072 Q 71.0

0075069045.0

0075071 Q 61.0

0075070 o 61.0

0075073 Q 72.0

007507427.0

002036087.

0020379

0020378

0020367

002036990.5

0020368

002037091 .

0020365

0020364

002036384.0

0020362

0020358

002035989.

0020366

0020363

002038229.5

002038675 .

002038575.

002038429

002040094 .

0020392.

002038163.

0020380 64.

0020376

0020389

002039193.

002039093.5

0020393389.5

$\begin{array}{ll}0020395 & 69.5 \\ 0020394 & 86 .\end{array}$

0020399

002039892.

0020397 . 
LONER GEYSER BASIN - SENTINEL GROUP - ROSETTE G 44-34.08 N 110-51.53 H LOWER GEYSER BASIN - SURPRISE POOL

LOUER GEYSER BASIN - UMNAMED MUD POT

LOWER GEYSER BASTM - SURPRISE POOL - UNAANED NUD POT

LONER GEYSER BASIM - UMNUED SPRING

LOWER GEYSER BASIM - UNNANED SPRING

LOWER GEYSER BASIN - UNAANED SPRING

LOWER GEYSER BASIN - UNAAMEO SPRING

LOWER GEYSER BASIN - UWNAMED SPRING

LOWER GEYSER BASIN - UNWAMED SPRING

LONER GEYSER BASIN - UWHAMED STEAH VENT

MIOWAY GEYSER BASIH - EXCELSIOR GEYSER

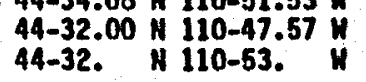

44-32.2 N 110-47.30 W

44-31.58 N 110-47.48 $\mathrm{H}$

44-32. 29 N 110-46.57

44-32. N 110-47.

44-32. N 110-42.

44-31. I 110-52.

MIDWAY GEYSER BASIH - FRIRY CREEK GROUP - UNMAM 44-32.33 N 110-50.53

MIOMAY GEYSER BASIH - FAIRY CREEK GROUP - UNNAM 44-32.18 N 110-51.25

MIDYAY GEYSER BASIH - FAIRY CREEK GROUP - UMHAM 44-32.33 N 110-50.53

MIOUAY GEYSER BASIH - FAIRY CREEK GROUP - UMYMY 44-32.15 H 110-51.10

Mioun GeYsen

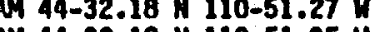

MIOWAY GEYSER BASIN - FAIRY CREEK GROUP - UNAMY 44-32.18 N $110-51.25$ Y
MIOWAY GEYSER BASIN - FLOOD GEYSER

MIDUAY GEYSER BASIN - FLOOD GEYSER

MIDKAY GEYSER BASIH - INDIGO SPRING

44-31. A 110-50.

44-31.2 110-50.0

$44-31.20 \mathrm{~N} 110-50.13 \mathrm{H}$

MIDWAY GEYSER BASIN - RABBIT CREEK GROUP - UMMA 44-30.58 N 110-49.18 H

MIDHAY GEYSER BASIM - RABBIT CREEK GROUP - UNMA 44-30.50 N 110-48.42

MIDWAY GEYSER BASIN - RABBIT CREEX GROUP - UMWA 44-30.49 H 110-48.42 Y

MIDWAY GEYSER BASIN - RABBIT CREEK GROUP - UNNA 44-30.50 H 110-48.42

HIDWAY GEYSER BASIN - UWMAMED CONE

MIDWAY GEYSER BASIN - INMAMMED SPRING

MORTH BUFFALO FORK SPRINGS - GROUP I

$44-30.57$ H $110-49.50$

44-31. H 110-50.

43-54.40 $110-11.70$ v 454111432

MORTH BUFFALO FORK SPRIUCS - GROUP

ORAMGE GROUP - UNHAMED SPRING

PIPEI INE CREEK SPRING

POTTS HOT SPRING BASIH - UWWMMED GEYSER

POTTS HOT SPRING BASIN - UNAMMED SPRIMG

SHOSHOHE GEYSER BASIN - DLUE GLASS SPRING

SHOSHONE GEYSER BASIN - MINUTE MAN GEYSER

SHOSHOHE GEYSER BASIN - TAURUS GEYSER

SHOSHONE GEYSER BASIN - UNION GEYSER

SHOSHONE GEYSER BASIH - UNNAMED SPRIME

TERRACE SPRING

TETOH YALLEY MAPM SPRINGS

UNMANED SPRING

UPPER GEYERS BASIN - UNMAMED SPRING

UPPER GEYERS BASIN - UNNAMEO SPRING

UPPER GEYSER BASIN - ARTEMESIA GEYSER

UPPER GEYSER BASIN - ARTEMESIA GEYSER

UPPER GEYSER BASIN - ARTEMISIA GEYSER

UPPER GEYSR BASIN - ARTEMISIA GEYSER

UPPER GEYSER BASIM - BEEHIVE GEYSER

UPPER GEYSR BASIN - BENCH SPRING

UPPER GEYSER BASIN - BOTTOMLSS PIT

UPPER GEYSER BASIN - CALTHIOS SPRING

UPPER GEYSER BASIH - CASTLE GEYSER

UPPER GEYSER BASIN - CHIMAMAH SPRING

$43-54.40$ N 110-11.70 W $45 N$ 111K

$43-54.40$
$44-27.77$
4
$4110-50.75$

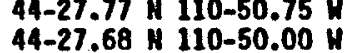

$\begin{array}{ccc}44-27.68 & 110-50.00 \\ 44-26.4 & 110-34.5 & W\end{array}$

$44-26.4$
$44-26.4$
$4110-34.5$

$44-26.4$ N $110-34.5$
$44-21.1$

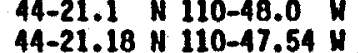

44-21.12 N 110-48.03 N

44-20.48 N $110-47.55$ W

44-21. N 110-48.

$44-39.00$ N $110-50.45$ N

43-37.43 N 110-36.37 N 42N 115K 110075077 18.0

44-31. $110-50$.

0020340

002034094.

$44-27.43$ N $110-50.00$

44-27.53 N $110-49.45$ N

$44-27.43$ N $110-50.00$ N

$44-27.43$ N $110-50.00$ H

$44-27.43$ N $110-50.00$ N

0020304

002025787.

TETON

TETON

IETON

UPPER GEYSER BASIN - CHINAMAH SPRING

44-27.73 N 110-51.35 H

002025

0020255

002032592.5

002032083.7

14-27.43 N 110-50.00

0020267

002033894.5

44-27.5 N 110-50.6

$44-27.43 \mathrm{~N} 110-50.00 \mathrm{~K}$

0020286

44-27.43 N 110-50.00 H

0020268

93.5

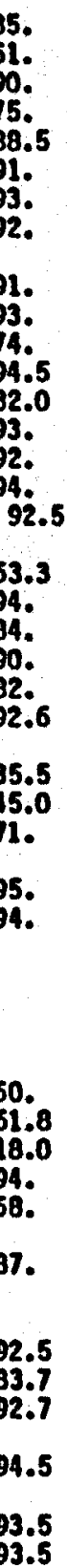


TETON

TEton

TETON

TETOA

TETON

TETON

TETON

TETON

TETON

TETON

TETON

TETON

TETON

TETON

IETON

TETOH

TETON

TETON

TETOH

IETON

TETON

reion

ETON

TETON

TETON

TETON

TETOA

TETOA

TETON

TETOA

TETO4

TETON

TETON

reTon

TETON

IETON

TETON

reton

reton

TETON

TETON

TETON

TETON

TETON

TETON

TETON

TETON

TETON

TETON

TETON

TETON

TEION

TETON

TETOH

TETON
UPPER GEYSER BASIN - CLIFF GEYSER

UPPER GEYSER BASIN - DAISY GEYSER

UPPER GEYSER BASIN - DAISY SPRING

UPPER GEYSER GASII - EAR SPRING

UPPER GEYSER BASIM - ECONOMIC GEYSER

UPPER GEYSER BASIN - ECONOAIC GEYSER

UPPER GEYSER BASIN - ENERALO POOL

UPPER GEYSER BASIN - GIAHTESS GEYSER

UPPER GEYSER BASIH - GRAND GEYSER

UPPER GEYSER BASIH - MILLSIDE NO. 1

UPPER GEYSER BASIN - IRON SPRING

UPPER GEYSER BASIN - JEMEL GEYSER

UPPER GEYSER BASIN - JEMEL GEYSER

UPPER GEYSER BASIN - LIONESS GEYSER

UPPER GEYSER BASIN - MORNING GLORY POOL

UPPER GEYSER BASIN - OBLONG GEYSER

OOPPER GEYSER BASIN - OLO FAITHFUL GEYSER A4-27.63 N 110-49.38 H .

UPPER GEYSR BASIN - OLDE SPRINGS GROUP - UMMMN 44-27.63 M II0-49.38 N

UPPER GEYSER BASIN - PUMCII BONL SPRING

UPPER GEYSER BASIN - PUNCHBOUL SPRING

UPPER GEYSER BASIN - RIVERSIDE GEYSER

UPPER GEYSER BASIN - SAPPHIRE SPRINO

UPPER GEYSER BASIN - SAMMILL GEYSER

UPPER GEYSER BASIH - SENTINEL SPRIHG

UPPER GEYSER BASIM - SOLITARY SPRING

UPPER GEYSER BASTI - SPASHOOIC GEYSER

UPPER GEYSER BASIN - SPLENDID GEYSER

UPPER GEYSER BASII - SPONGE GEYSER

UPPER GEYSER BASIH - SPOUTER SPRING

UPPER GEYSER BASIH - SULFIDE SPRING

UPPER GEYSER BASIN - TEAKETTLE SPRING

UPPER GEYSER BASIM - THREE SISTERS SPRING

UPPER GEYSER BASIH - THREE SISTERS SPRING

UPPER GEYSER BASIN - TOPAZ SPRING

UPPER GEYSER BASIH - TORTOISE SHELL SPRING

UPPER GEYSER BASIH - UMHAMED ORILL HOLE

UPPER GEYSER BASIN - UNNAMED SPRING

UPPER GEYSER BASIM - UNMAMED SPRING

UPPER GEYSER BASIN - UNWAMED SPRING

UPPER GEYSER BASIN - UNNAMED SPRING

UPPER GEYSER BASIN - UNNAMED SPRING

UPPER GEYSER BASIM - UMMAMED SPRING

UPPER GEYSER BASIK - UNNAMED SPRING

UPPER GEYSER BASIN - UWMAMED SPRING

UPPER GEYSER BASIM - UMMAMED SPRING

UPPER GEYSER BASII - UMYAYED SPRING

UPPER GEYSER BASIII - UMH

UPPER GEYSER BASIM - UNHAYED SPRING

UPPER GEYSER BASIM - UWMNED - UNTAMEO SPRING

UPPER GEYSER BASIH - UNHAYED SPRING

UPPER GEYSER BASIM - UNNAMEO SPRING

UPPER GEYSER BASIN - UMNAMED SPRING
$44-28.50$ N $110-50.00 \mathrm{H}$

$44-28.50$ N $110-50.00 \mathrm{~K}$

44-27.4 H 110-50.0

44-28.5 N 110-50.0

$44-28.5$
$44-27.28$

44-27.7 $N$ 110-49.5

44-28.20 / 110-51.52 W

$44-28.50 N 110-50.00$

44-28.5 H $110-50.0$ H

$44-28.05$ i $110-50.32$

44-27.63 * 110-49.38 *

$44-28.25$ II $110-51.95$

$44-28.25$ N $110-51.95$ N

44-28.48 A 110-51.15

44-27.7 110-51.3

$44-28.50$ H $110-50.00$

$44-27.43 N 110-50.00 M$

$44-27.43 N$

$44-28.5$ I1 $110-50.0 \mathrm{~N}$

44-28.5 N 110-50.0

44-27.43 N $110-50.00 \mathrm{~N}$

44-27.03 N 110-50.33

44-27.43 N 110-50.00 N

44-27.35 N 110-49.37 N

44-27.58 N 110-49.07 N

44-28.20 N 110-50.45

44-28.0 N 110-50.0 $N$

$44-27.35$ H $110-49.37$ H

44-28.0 110-50.0

44-27.50 N 110-50.00

44-28.50 N 110-50.00 K

$44-28.00$ N $110-50.00$ K

$44-28.20$ N $110-50.45$

$44-28.710-49.20$

$44-28.0$
$44-28.50$
44-27.43 N 110-50.00 N

$44-27.77 N 110-49.20$
002033789.6

002027789.

0020276

0020275183.6

002025193.

0020290 83.7

002029163.

002029770.

$\begin{array}{ll}0020324 & 94.3 \\ 0020249 & 94.1\end{array}$

002024994.1

0020264

002028090.8

002028493.7

002028588.

002032894.

002029871.

002033593.6

0020281

002031983

0020318 83.

002027483

$\begin{array}{ll}0020273 & 92.8 \\ 0020323 & 93.5\end{array}$

$0020258 \quad 94.5$

$0020330 \quad 89.6$

002033493.5

$0020321 \quad 92.5$

$0020329 \quad 93.5$

002033693.5

002032793.5

002029492.5

002028377 .

0020271

002027292.

002027890.5

0020279

$0020322 \quad 94.5$

0020282

002030055.

002029963.

002030581.

002030390.

002030181.

0020312

002030663.

002031071.

0020333 92.

0020292

0020309.80 .

0020302

002013

002031391.

002028991 . 

UPPER GEYSER BASIN - YELLON BELL SPRING NEST THUMB GEYSER BASIN - ABYSS POOL MEST THUMB GEYSER BASIM - BLACK POOL MEST THUMB GEYSER BASIN - EPHEDRA GEYSER MEST THUHB GEYSER BASIN - UNNAMED SPRING MEST THUMB GEYSER BASIN - UNNNHED SPRING VEST THUMB GEYSER BASIN - UNMAMED SPRING 44-27.53 N $110-49.46$ W 44-27.35 N 110-49.37 H 44-27.67 N $110-49.63$ H 44-27.53 N 110-49.45 W 44-28.4 $N$ 110-51.53 44-27.43 \& 110-50.00. 44-27.4 N 110-50.0 N 44-27.7 N 110-49.5 $44-28.44 N 110-51.52$

TETON

TETON

Teron

TETON

TETON

TETON YELLOWSTONE - BOILING SPRING

YELLONSTONE - EAR SPRING

YELLOUSTOHE - EXCELSIOR SPRING

YELONSTONE - FIREHOLE LAKE (LONER)

YELCOUSTOHE - HILLSIDE (UPPER)

YELONSTONE - LITIE MHIRLIGIG GEYSER

YELLONSTONE - AEAR CLEPSEDRA GEYSER

$44-28.43$ N $110-51.53$ K

44-25. N 110-34. 44-25. I 110-34. K 44-24. N 110-34. 44-25. II $110-34$.

44-25.5 N 110-34.2 N

TETON

TETOI

TETON

TETON

reron

TETON

TETON

TETOH

TETON YELLOUSTONE - OJO CALIENTE SPRING (RIVER GROUP 44-33.18 N 110-49.51 N

44-27.4 N 110-50.0

$44-31.23$ M $110-50.08$ K $44-32.30$ N $110-47.02$ $44-28.2$ i $110-51.5$ N YELLOWSTONE - RUSTIC GEYSER

VELLOXSTONE - SAPPHIRE SPRING

44-28.48 N 110-51.15

YLLLONSTONE - SNORT SPRINE

YELLOWSTORE - SPIKE GEYSER

YELLOUSTOHE - SPRING 192

YELLONSTONE - TERRACE

YELLOUSTONE - UPPER GEYSER BASTN - UWMAMED SPRI 44-39.00 A 110-50.45

YELLOUSTONE - PERPETUAL SPOUTER

002027584.

$\begin{array}{ll}0020317 & 64 \\ 0020316 & 89\end{array}$

002031689.

002031575.

002031482.

002026080.

002025384

0020252 89.

$0020250 \quad 62.5$

002026176.

0020259 80.

0020326

002048290.

002048378.5

002048589

0020484 84.1.

0020481

0020480

007508394.

907508394.

07509195.

co75089 76.

007508284.5

007508284.

007509a 91

0075086 92.

007508795 .

007508591.

007509095.

007508893

007509493.5

007508489.5

007508066

002030887.

007507889. 


\section{APPENDIX B}

Index to GEOTHERM sample file for the state of Wyoming sorted by county, township (TNS), range (RNG), and section (Sect.) Also given are the name of source, GEOTHERM record identifier (I.D.), and temperature (Temp. ${ }^{\circ} \mathrm{C}$ ). See Table 1 for explanation of alphabetic qualifiers proceeding temperature.

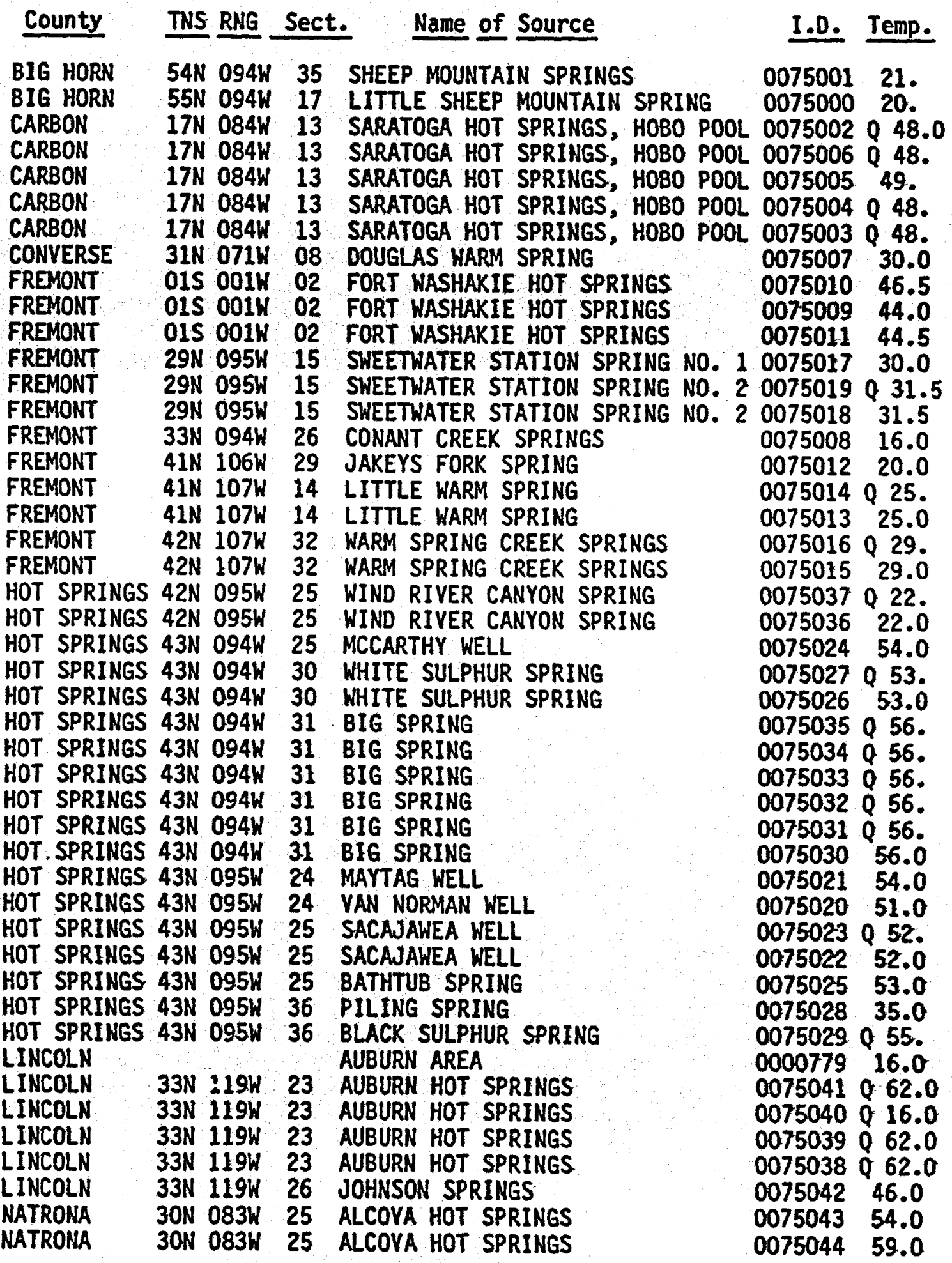




\begin{tabular}{|c|c|c|c|c|}
\hline $\begin{array}{l}\text { NATRONA } 32 N \quad 086 \mathrm{~W} \\
\text { PARK } \\
\text { PARK } \\
\text { PARK } \\
\text { PARK } \\
\text { PARK } \\
\text { PARK } \\
\text { PARK } \\
\text { PARK } \\
\text { PARK } \\
\text { PARK } \\
\text { PARK } \\
\text { PARK } \\
\text { PARK } \\
\text { PARK } \\
\text { PARK } \\
\text { PARK } \\
\text { PARK } \\
\text { PARK } \\
\text { PARK }\end{array}$ & $\begin{array}{c}35 \text { HORSE CREEK } \\
\text { YELLOWSTONE } \\
\text { YELLOWSTONE } \\
\text { YELLOWSTONE } \\
\text { MAMMOTH HOT } \\
\text { NORRIS GEYSE } \\
\text { NORRIS GEYSE } \\
\text { NORRIS GEYSE } \\
\text { NORRIS GEYSE } \\
\text { NORRIS GEYSE } \\
\text { NORRIS GEYSE } \\
\text { NORRIS GEYSE } \\
\text { NORRIS GEYSE } \\
\text { YELLOWSTONE } \\
\text { YELLOWSTONE } \\
\text { YELLOWSTONE } \\
\text { YELLOWSTONE } \\
\text { YELLOWSTONE } \\
\text { YELLOWSTONE } \\
\text { YELLOWSTONE } \\
\text { YELLOWSTONE } \\
\text { YELLOWSTONE } \\
\text { YELLOWSTONE } \\
\text { YELLOWSTONE } \\
\text { YELLOWSTONE } \\
\text { YELLOWSTONE } \\
\text { YELLOWSTONE } \\
\text { YELLOWSTONE } \\
\text { YELLOWSTONE } \\
\text { YELLOWSTONE } \\
\text { YELLOWSTONE } \\
\text { YELLOWSTONE } \\
\text { YELLOWSTONE } \\
\text { MAMMOTH HOT } \\
\text { MAMMOTH HOT } \\
\text { MAMMOTH HOT } \\
\text { MAMMOTH HOT } \\
\text { MAMMOTH HOT } \\
\text { MAMMOTH HOT } \\
\text { MAMMOTH HOT } \\
\text { MAMMOTH HOT } \\
\text { MAMMOTH HOT } \\
\text { MAMMOTH HOT } \\
\text { MAMMOTH HOT }\end{array}$ & 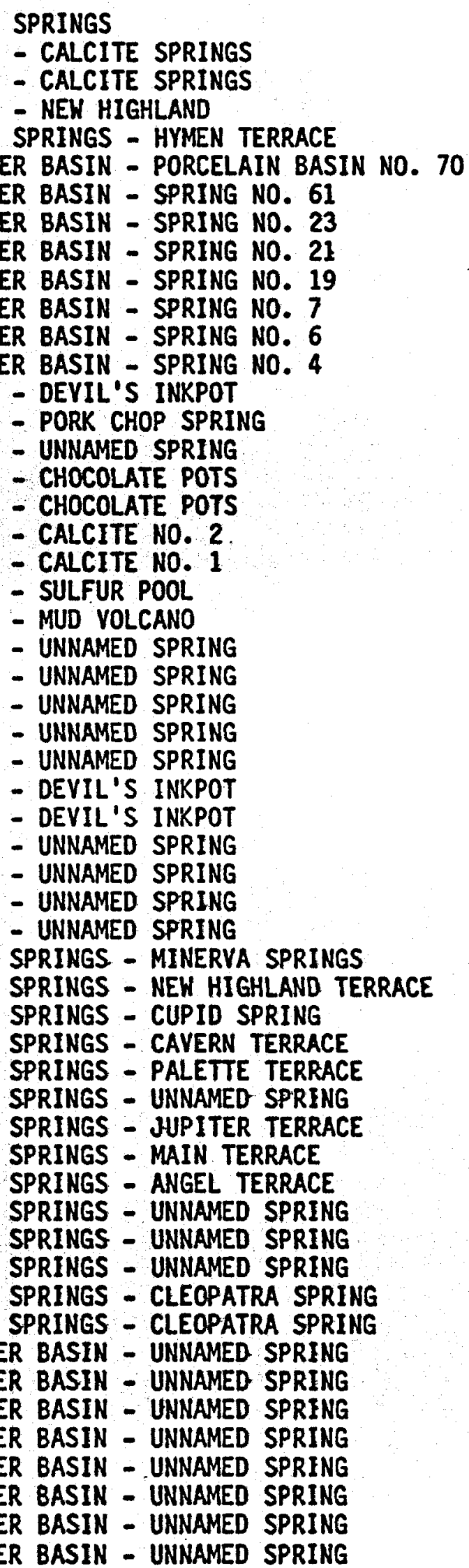 & $\begin{array}{l}0075045 \\
0020504 \\
0020502 \\
0075079 \\
0020465 \\
0020427 \\
0020417 \\
0020413 \\
0020412 \\
0020411 \\
0020407 \\
0020406 \\
0020404 \\
0020493 \\
0075093 \\
0020508 \\
0020507 \\
0020506 \\
0020505 \\
0020503 \\
0020501 \\
0020500 \\
0020499 \\
0020498 \\
0020497 \\
0020496 \\
0020495 \\
0020494 \\
0020492 \\
0020491 \\
0020490 \\
0020489 \\
0020488 \\
0020473 \\
0020472 \\
0020471 \\
0020470 \\
0020469 \\
0020468 \\
0020467 \\
0020466 \\
0020464 \\
0020463 \\
0020462 \\
0020461 \\
0020460 \\
0020459 \\
0020458 \\
0020457 \\
0020456 \\
0020455 \\
0020454 \\
0020453 \\
0020452 \\
0020451\end{array}$ & $\begin{array}{l}23.5 \\
73.5 \\
70.5 \\
\\
\\
\\
81.5 \\
71 . \\
52 . \\
51 . \\
89.4 \\
92.5\end{array}$ \\
\hline
\end{tabular}




\begin{tabular}{|c|c|c|c|c|c|}
\hline $\begin{array}{l}\text { PARK } \\
\text { PARK } \\
\text { PARK } \\
\text { PARK } \\
\text { PARK } \\
\text { PARK } \\
\text { PARK } \\
\text { PARK } \\
\text { PARK } \\
\text { PARK } \\
\text { PARK } \\
\text { PARK } \\
\text { PARK } \\
\text { PARK } \\
\text { PARK } \\
\text { PARK } \\
\text { PARK } \\
\text { PARK } \\
\text { PARK } \\
\text { PARK } \\
\text { PARK } \\
\text { PARK } \\
\text { PARK } \\
\text { PARK } \\
\text { PARK } \\
\text { PARK } \\
\text { PARK } \\
\text { PARK } \\
\text { PARK } \\
\text { PARK } \\
\text { PARK } \\
\text { PARK } \\
\text { PARK } \\
\text { PARK } \\
\text { PARK } \\
\text { PARK } \\
\text { PARK } \\
\text { PARK } \\
\text { PARK } \\
\text { PARK } \\
\text { PARK } \\
\text { PARK } \\
\text { PARK } \\
\text { PARK } \\
\text { PARK } \\
\text { PARK } \\
\text { PARK } \\
\text { PARK } \\
\text { PARK } \\
\text { PLATTE } \\
\text { PLATTE } \\
\text { SUBLETTE } \\
\text { SUBLETTE } \\
\text { SUBLETTE } \\
\text { SUBLETTE }\end{array}$ & $\begin{array}{ll}52 N & 102 W \\
52 N & 102 W \\
52 N & 102 W \\
52 N & 102 W \\
52 N & 102 W \\
52 N & 102 W \\
52 N & 102 W \\
52 N & 102 W \\
52 N & 103 W \\
26 N & 066 W \\
26 N & 066 W \\
28 N & 115 W \\
32 N & 107 W \\
32 N & 107 W \\
32 N & 107 W\end{array}$ & $\begin{array}{l}03 \\
03 \\
03 \\
03 \\
03 \\
03 \\
03 \\
03 \\
12, \\
04 \\
04 \\
20 \\
16 \\
16 \\
16\end{array}$ & 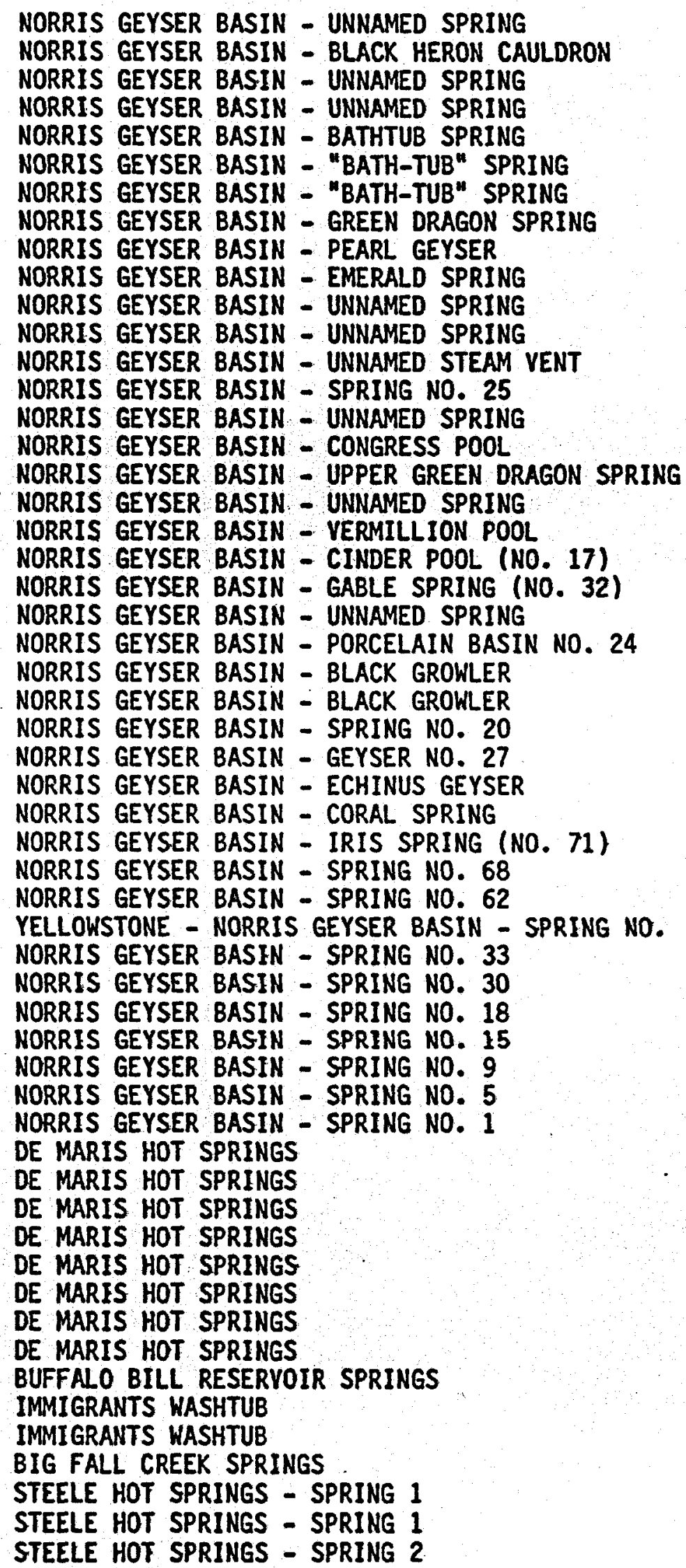 & $\begin{array}{l}0020450 \\
0020449 \\
0020448 \\
0020447 \\
0020446 \\
0020445 \\
0020444 \\
0020442 \\
0020441 \\
0020440 \\
0020439 \\
0020438 \\
002037 \\
002436 \\
0020435 \\
0020434 \\
0020443 \\
0020433 \\
0020432 \\
0020431 \\
0020430 \\
0020429 \\
0020428 \\
0020426 \\
0020425 \\
0020424 \\
0020423 \\
0020422 \\
002041 \\
002042 \\
0020419 \\
0020418 \\
0020416 \\
0020415 \\
0020414 \\
0020410 \\
0020409 \\
0020408 \\
0020405 \\
0020403 \\
0075047 \\
0075054 \\
0075050 \\
0075048 \\
0075049 \\
007553 \\
0075552 \\
0075051 \\
0075046 \\
0075056 \\
0075055 \\
0075062 \\
0075059 \\
0075060 \\
0075061\end{array}$ & 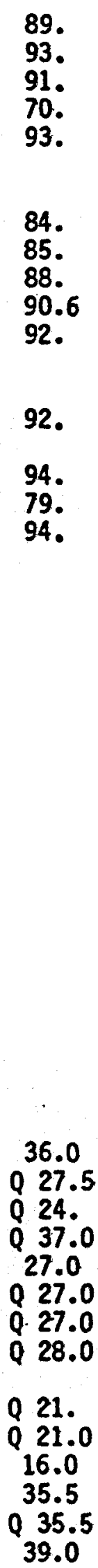 \\
\hline
\end{tabular}


SUBLETTE 38N 110W SUBLETTE 38N 110W TETON

TETON

TETON

TETON

TETON

TETON

TETON

TETON

TETON

TETON

TETON

TETON

TETON

TETON

TETON

TETON

TETON

TETON

TETON

TETON

TETON

TETON

TETON

TETON

TETON

TETON

TETON

TETON

TETON

TETON

TETON

TETON

TETON

TETON

TETON

TETON

TETON

TETON

TETON

TETON

TETON

TETON

TETON

TETON

TETON

TETON

TETON

TETON

TETON

TETON

TETON

TETON

TETON
02 KENDALL WARM SPRINGS

02 KENDALL WARM SPRINGS

MIDWAY GEYSER BASIN - FAIRY CREEK GROUP - UNNAM 0020351

MIDWAY GEYSER BASIN - FAIRY CREEK GROUP - UNNAM 0020353

MIOWAY GEYSER BASIN - FAIRY CREEK GROUP - UNNAM 0020354

MIDWAY GEYSER BASIN - FAIRY CREEK GROUP - UNNAM 0020355

LOWER GEYSER BASIN - UNNAMED STEAM VENT 0020357

LOWER GEYSER BASIN - IMPERIAL GEYSER

LOWER GEYSER BASIN - IMPERIAL GEYSER

LOWER GEYSER BASIN - UNNAMED MUD POT

LOWER GEYSER BASIN - UNNAMED SPRING

LOWER GEYSER BASIN - UNNAMED SPRING

YELLOWSTONE - TERRACE

SHOSHONE GEYSER BASIN - UNION GEYSER

UPPER GEYSER BASIN - UNNAMED SPRING

UPPER GEYSER BASIN - UNNAMED SPRING

UPPER GEYSER BASIN - UNNAMED SPRING

UPPER GEYSER BASIN - UNNAMED SPRING

UPPER GEYSER BASIN - EAR SPRING

UPPER GEYSER BASIN - GIANTESS GEYSER

UPPER GEYSER BASIN - THREE SISTERS SPRING

UPPER GEYSER BASIN - PUNCHBOWL SPRING

UPPER GEYSER BASIN - TOPAZ SPRING

UPPER GEYSER BASIN - CHINAMAN SPRING

UPPER GEYSER BASIN - BOTTOMLESS PIT

HILLSIDE GROUP - ASTA SPRING (NO. 3)

UPPER GEYSER BASIN - ARTEMISIA GEYSER

UPPER GEYSER BASIN - ARTEMISIA GEYSER

YELLOWSTONE - EXCELSIOR SPRING

TERRACE SPRING

LOWER GEYSER BASIN - IMPERIAL GEYSER

MIDWAY GEYSER BASIN - INDIGO SPRING

MIDWAY GEYSER BASIN - EXCELSIOR GEYSER

UPPER GEYSER BASIN - YELLOW BELL SPRING

UPPER GEYSER BASIN - SOLITARY SPRING

UPPER GEYSER BASIN - CASTLE GEYSER

YELLOWSTONE - SNORT SPRING

YELLOWSTONE - OJO CALIENTE SPRING (RIVER GROUP

YELLOWSTONE - NEAR CLEPSEDRA GEYSER

YELLOWSTONE - RUSTIC GEYSER

YELLOWSTONE - SPRING 192

YELLOWSTONE - BOILING SPRING

YELLOWSTONE - HILLSIDE (UPPER)

YELLOWSTONE - FIREHOLE LAKE (LOWER)

LOWER GEYSER BASIY - MORNING MIST SPR

LOWER GEYSER BASIN - MORNING MIST SPRINGS - UNN 0020383

SPRINGS - UNN 0020382

LOWER GEYSER BASIN - RIVER GROUP - UNNAMED SPRI 0020381

LOWER GEYSER BASIN - RIVER GROUP - UNNAMED SPRI 0020380

LOWER GEYSER BASIN - BATH SPRING 0020379

LOWER GEYSER BASIN - BATH SPRING 0020378

LOWER GEYSER BASIN - RIVER GROUP - 0J0 CALIENTE 0020377

LOWER GEYSER BASIN - RIVER GROUP - OJO CALIENTE 0020376

LOWER GEYSER BASIN - UNNAMED SPRING

LOWER GEYSER BASIN - UNNAMED SPRING

0020373

0020372

LOWER GEYSER BASIN - UNNAMED SPRING
0020371
29.5

Q 29.5

92.

93.

82.0

94.5

89.

87.

61.

88.5

75.

66.

82.

64.

89.

75.

93.

94.1

90.5

92.8

93.5

76.

61.8

91.

92.5

93.

95.

92.

91.

89.5

94.

84.5

93.

29.5

63.

64.

94.5

93.

92.

91. 
TETON

TETON

TETON

TETON

TETON

TETON

TETON

TETON

TETON

TETON

TETON

TETON

TETON

TETON

TETON

TETON

TETON

TETON

TETON

TETON

TETON

TETON

TETON

TETON

TETON

TETON

TETON

TETON

TETON

TETON

TETON

TETON

TETON

TETON

TETON

TETON

TETON

TETON

TETON

TETON

TETON

TETON

TETON

TETON

TETON

TETON

TETON

TETON

TETON

TETON

TETON

TETON

TETON

TETON

TETON
LOHER GEYSER BASIN - FIREHOLE POOL

0020370

LOWER GEYSER BASIN - FIREHOLE POOL

0020369

0020368

LOWER GEYSER BASIN - FIREHOLE POOL

LOWER GEYSER BASIN - FIREHOLE LAKE

0020367

LOWER GEYSER BASIN - KALEIDOSCOPE GROUP - UNNAM 0020366

LOWER GEYSER BASIN - FISSURE GROUP - EBONY SPRI 0020365

LOWER GEYSER BASIN - FOUNTAIN GROUP - FOUNTAIN 0020364

LOWER GEYSER BASIN - GREAT FOUNTAIN GROUP - DIA 0020363

LOWER GEYSER BASIN - GREAT FOUNTAIN GROUP - DIA 0020362

MIDWAY GEYSER BASIN - FAIRY CREEK GROUP - UNNAM 0020356

MIDWAY GEYSER BASIN - FAIRY CREEK GROUP - UNNAM 0020352

MIDWAY GEYSER BASIN - UNNAMED CONE

MIDWAY GEYSER BASIN - PRISMATIC LAKE

MIDWAY GEYSER BASIN - FLOOD GEYSER

MIDWAY GEYSER BASIN - UNNAMED SPRING

UPPER GEYSER BASIN - CALTHOS SPRING

UPPER GEYSER BASIN - CLIFF GEYSER

UPPER GEYSER BASIN - SPLENDID GEYSER

UPPER GEYSER BASIN - OBLONG GEYSER

UPPER GEYSER BASIN - SENTINEL SPRING

UPPER GEYSER BASIN - UNNAMED SPRING

UPPER GEYSER BASIN - GRAND GEYSER

UPPER GEYSER BASIN - WITCHES CAULDRON

UPPER GEYSER BASIN - SAWMILL GEYSER

UPPER GEYSER BASIN - SPASMODIC GEYSER

UPPER GEYSER BASIN - LIONESS GEYSER

UPPER GEYSER BASIN - SPONGE GEYSER

UPPER GEYSER BASIN - BEEHIVE GEYSER

UPPER GEYSER BASIN - GIANT GEYSER

UPPER GEYSER BASIN - RIVERSIDE GEYSER

UPPER GEYSER BASIN - TORTOISE SHELL SPRING

UPPER GEYSER BASIN - BENCH SPRING

UPPER GEYSER BASIN - UNNAMED SPRING

UPPER GEYSER BASIN - UNNAMED SPRING

YELLOWSTONE - UPPER GEYSER BASIN - UNNAMED SPRI

PIPELINE CREEK SPRING

UPPER GEYSER BASIN - UNNAMED SPRING

UPPER GEYSER BASIN - UNNAMED SPRING

UPPER GEYERS BASIN - UNNAMED SPRING

UPPER GEYSER BASIN - UNNAMED SPRING

UPPER GEYSER BASIN - UNNAMED SPRING

UPPER GEYSER BASIN - UNNAMED SPRING

UPPER GEYSER BASIN - UNNAMED SPRING

UPPER GEYSER BASIN - MORNING GLORY POOL

UPPER GEYSER BASIN - EMERALD POOL

BLACK SAND BASIN - UNNAMED SPRING

BLACK SAND BASIN - UNNAMED SPRING

0020346

0020345

0020341

0020339

0020338

0020337

0020336

0020335

0020334

0020333

0020332

0020331

0020330

0020329

0020328

0020327

0020325

0020324

0020323

0020322

0020320

0020312

0020310

0020308

0020307

0020306

0020305

0020304

0020303

0020301

0020300

0020299

0020298

0020297

0020296

0020295

UPPER GEYSER BASIN - SPOUTER SPRING

UPPER GEYSER BASIN - BLACK SAND POOL

UPPER GEYSER BASIN - ECONOMIC GEYSER

UPPER GEYSER BASIN - JEWEL GEYSER

UPPER GEYSER BASIN - UNNAMED DRILL HOLE

0020294

0020293

0020290

0020284

0020282

UPPER GEYSER BASIN - IRON SPRING

0020280

MIDWAY GEYSER BASIN - RABBIT CREEK GROUP - UNNA 0020350

UPPER GEYSER BASIN - UNNAMED SPRING
91.

90.5

84.0

93.

74.

92.6

63.3

R 92.5

94.5

89.6

93.5

93.6

93.5

92.5

79.

93.5

89.6

93.5

94.

93.5

92.5

94.3

93.5

94.5

83.7

71.

87.

63.

81.

90.

81.

55.

63.

77.

70.

88.

92.5

92.7

83.7

93.7

90.8

90. 
TETON

TETON

TETON

TETON

TETON

TETON

TETON

TETON

TETON

TETON

TETON

TETON

TETON

TETON

TETON

TETON

TETON

TETON

TETON

TETON

TETON

TETON

TETON

TETON

TETON

TETON

TETON

TETON

TETON

TETON

TETON

TETON

TETON

TETON

TETON

TETON

TETON

TETON

TETON

TETON

TETON

TETON

TETON

TETON

TETON

TETON

TETON

TETON

TETON

TETON

TETON

TETON

TETON

TETON

TETON
MIDWAY GEYSER BASIN - RABBIT CREEK GROUP - UNNA 0020347

MIOWAY GEYSER BASIN - FLOOD GEYSER

MIDWAY GEYSER BASIN - RABBIT CREEK GROUP - UNNA 0020348

UPPER GEYSER BASIN - UNNAMED SPRING

YELLOWSTONE - SPIKE GEYSER

UPPER GEYSER BASIN - HILLSIDE NO. 1

HILLSIDE GROUP - ASTA SPRING (NO. 3)

UPPER GEYSER BASIN - UNNAMED SPRING

YELLOWSTONE - EAR SPRING

UPPER GEYSER BASIN - UNNAMED SPRING

YELLOWSTONE - SAPPHIRE SPRING

SHOSHONE GEYSER BASIN - BLUE GLASS SPRING

JACKSON LAKE HOT SPRINGS

POTTS HOT SPRING BASIN - UNNAMED SPRING

POTTS HOT SPRING BASIN - UNNAMED GEYSER

WEST THUMB GEYSER BASIN - EPHEDRA GEYSER

WEST THUMB GEYSER BASIN - UNNAMED SPRING

WEST THUMB GEYSER BASIN - UNNAMED SPRING

WEST THUMB GEYSER BASIN - UNNAMED SPRING

HEART LAKE GEYSER BASIN - RUSTIC GROUP - UNNAME 0020479

SHOSHONE GEYSER BASIN - MINUTE MAN GEYSER

SHOSHONE GEYSER BASIN - TAURUS GEYSER

UNNAMED SPRING

UPPER GEYSER BASIN - PINE SPRINGS GROUP - UNNAM

UPPER GEYSER BASIN - UNNAMED SPRING

UPPER GEYSER BASIN - UNNAMED SPRING

HILLSIDE GROUP - UNNAMED SPRING

HILLSIDE GROUP - UNNAMED SPRING

UPPER GEYSER BASIN - UNNAMED SPRING

UPPER GEYSER BASIN - UNNAMED SPRING

UPPER GEYSER BASEN - WEST SPRING

UPPER GEYSER BASIN - ARTEMESIA GEYSER

UPPER GEYERS BASIN - UNNAMED SPRING

UPPER GEYSER BASIN - UNNAMED SPRING

UPPER GEYSER BASIN - UNNAMED SPRING

UPPER GEYSER BASIN - ECONOMIC GEYSER

TERRACE SPRING

LOWER GEYSER BASIN - MOUND SPRING

UPPER GEYSER BASIN - UNNAMED SPRING

UPPER GEYSER BASIN - UNNAMED SPRING

ORANGE GROUP - UNNAMED SPRING

UPPER GEYSER BASIN - JEWEL GEYSER

UPPER GEYSER BASIN - SULFIDE SPRING

UPPER GEYSER BASIN - THREE SISTERS SPRING

UPPER GEYSER BASIN - DAISY GEYSER

UPPER GEYSER BASIN - DAISY SPRING
0020342

0020309

0075094

0020349

0075092

0020258

0020281

0020271

0020264

0020263

0020318

0020311

0075091

0020313

0075090

0020474

0075073

0020487

0020486

0020485

0020484

0020483

0020482

0020481

0020480

0020478

0020476

0020475

0020340

94.

94.

84.

80 .

93.5

82.

91.

94.5

83.

75.

95.

91.

95.

72.0

94.

95.

89.

84.1

78.5

90 .

94.

83.

89.

82.5

65.

84 .

76.

80 .

80.

87.

68.

84.

0020253

0020292

0020291

0020402

0020400

0020289

0020288

0020287

0020285

0020283

0020279

0020277

0020276
63.

60.

94.

91.

87.

71 .

88.

77.

89.

88.6 
TETON

TETON

TETON

TETON

TETON

TETON

TETON

TETON

TETON

TETON

TETON

TETON

TETON

TETON

TETON

TETON

TETON

TETON

TETON

TETON

TETON

TETON

TETON

TETON

TETON

TETON

TETON

TETON

TETON

TETON

TETON

TETON

TETON

TETON

TETON (?)
UPPER GEYSER BASIN - UNNAMED SPRING

UPPER GEYSER BASIN - PUNCH BOWL SPRING

UPPER GEYSER BASIN - TEAKETTLE SPRING

UPPER GEYSER BASIN - CHINAMAN SPRING

LOWER GEYSER BASIN - SENTINEL GROUP - MOUND SPR 0020399

LOHER GEYSER BASIN - SENTINEL GROUP - UNNAMED S 0020398

LOWER GEYSER BASIN - SENTINEL GROUP - FLAT CONE 0020397

LOWER GEYSER BASIN - SENTINEL GROUP - ROSETTE G 0020396

LOWER GEYSER BASIN - SENTINEL GROUP - UNNAMED S 0020395

LOWER GEYSER BASIN - SENTINEL GROUP - QUEEN'S L 0020394

LOWER GEYSER BASIN - SENTINEL GROUP - QUEEN'S L 0020393

LOWER GEYSER BASIN - OUEEN'S LAUNDRY

0020392

LOWER GEYSER BASIN - SENTINEL GROUP - SENTINEL 0020391

LOWER GEYSER BASIN - SENTINEL GROUP - SENTINEL

LOWER GEYSER BASIN - SENTINEL CONE

LOWER GEYSER BASIN - UNNAMED SPRING

LOWER GEYSER BASIN - SURPRISE POOL

LOWER GEYSER BASIN - MORNING MIST SPRINGS - UNN OO20386

LOWER GEYSER BASIN - MORNING MIST SPRINGS - UNN 0020385

LOWER GEYSER BASIN - MORNING MIST SPRINGS - UNN 0020384

39N $113 \mathrm{~W} 06$

GRANITE FALLS HOT SPRINGS

39N 113W 06 GRANITE HOT SPRING

$39 \mathrm{~N} 113 \mathrm{~W} 06$

$39 \mathrm{~N} 116 \mathrm{~W} 32$

$41 \mathrm{~N} 116 \mathrm{~W} 02$

$41 \mathrm{~N} 117 \mathrm{~W} 36$

$42 \mathrm{~N} 115 \mathrm{~W} 02$

$42 \mathrm{~N} 115 \mathrm{~W} 11$

$45 \mathrm{~N} 111 \mathrm{~W} 32$

$45 \mathrm{~N} 111 \mathrm{~W} 32$

$48 \mathrm{~N} 115 \mathrm{~W} 20$

$48 \mathrm{~N} 115 \mathrm{~W} 20$

$48 \mathrm{~N} 115 \mathrm{~W} 20$

$48 \mathrm{~N} 115 \mathrm{~W}$
GRANITE HOT SPRING

ASTORIA SPRINGS

ABERCROMBIE WARM SPRINGS

BOYLES HILL SPRINGS

KELLY WARM SPRING

TETON VALLEY WARM SPRINGS

NORTH BUFFALO FORK SPRINGS - GROUP 2

NORTH BUFFALO FORK SPRINGS - GROUP 1

HUCKLEBERRY HOT SPRINGS

HUCKLEBERRY HOT SPRINGS

HUCXLEBERRY HOT SPRINGS

HUCKLEBERRY HOT SPRINGS

YELLOWSTONE - PERPETUAL SPOUTER

\section{6 . 45.0}

$0075066 \quad 45.0$

0075067 Q 41.0

$0075068 \quad 39.0$

$0075064 \quad 37.0$

$0075063 \quad 27.0$

007506530.0

$0075074 \quad 27.0$

$0075077 \quad 18.0$

$0075076 \quad 45.0$

$0075075 \quad 35.5$

0075069 Q 45.0

0075071 Q 61.0

0075070 Q 61.0

0075072071.0

007507889. 
APPEHDIX C

Index to GEOTHEPM sample flle for the state of Hyoming sorted into one-degree blocks by latitude and longltude. Records are sorted by name of source within each one-degree block. Adjacent one-degree blocks mich are published as a 1:250,000 map are combined under the approprlate map name. See Table 1 for explanatlon of alphabetic qualiffers proceeding temperature. I.D. - GEOTHERM record Identifler. Temp. - Temperature OC.

Latitude Longitude Name of Source

COORDIMATES NOT GIVEN BUT ALL RECORDS LIKELY TO BE IN ASHTOM 1:250,000

\begin{tabular}{|c|c|}
\hline $\begin{array}{l}\text { LOWER GEYSER } \\
\text { UPPER GEYSER } \\
\text { UPPER GEYSER } \\
\text { UPPER GEYSER } \\
\text { UPPER GEYSER } \\
\text { UPPER GEYSER } \\
\text { UPPER GEYSER } \\
\text { UPPER GEYSER } \\
\text { UPPER GEYSER } \\
\text { UPPER GEYSER } \\
\text { UPPER GEYSER } \\
\text { UPPER GEYSER } \\
\text { UPPER GEYSER } \\
\text { UPPER GEYSER } \\
\text { UPPER GEYSER } \\
\text { UPPER GEYSER } \\
\text { UPPER GEYSER } \\
\text { UPPER GEYSER } \\
\text { UPPER GEYSER } \\
\text { UPPER GEYSER } \\
\text { YELLOWSTONE - } \\
\text { YELLOWSTONE - } \\
\text { YELLOWSTONE - } \\
\text { YELLOWSTONE - } \\
\text { YELLOHSTONE - } \\
\text { YELLOWSTONE - } \\
\text { YELLOWSTONE - } \\
\text { YELLOWSTONE - } \\
\text { YELLOWSTONE - } \\
\text { YELLOWSTOHE - }\end{array}$ & 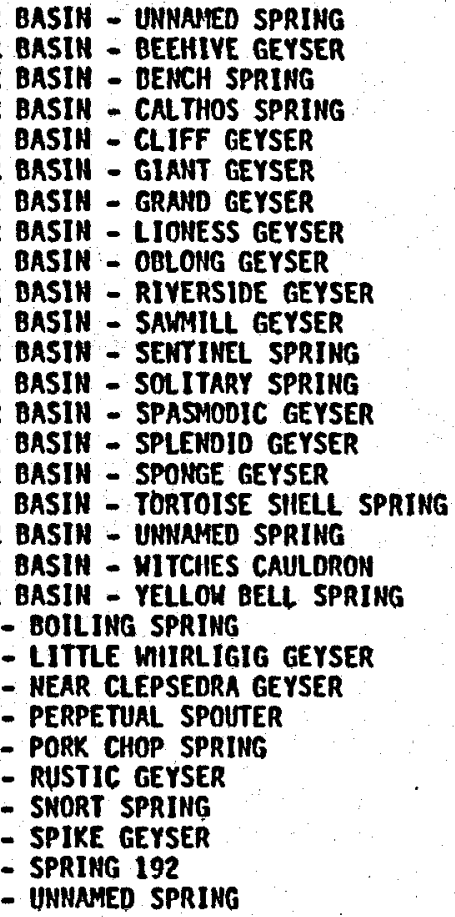 \\
\hline
\end{tabular}

RAMIINS 1:250,000

41-27.00 N 106-48.40 N SARATOGA HOT SPRINGS, HOBO POOL 41-27.00 N 106-48.40 $\mathrm{Y}$ SARATOGA IIOT SPRINGS, HOBO POOL 41-27.00 N 106-48.40 W SARATOGA IIOT SPRINGS, HOBO POOL 41-27.00 N 106-4B.40 W SARATOGA HOT SPRIHGS, HOBO POOL 11-27.00 N 106-48.40 W SARATOGA HOT SPRIHGS, HOBO POOL TORRIMGTON $1: 250,000$

42-14.97 N 104-46.C? N IMHIGRAMTS WASIITUB
County

1.D. Temp.

\begin{tabular}{ll:l} 
TETON & 0020388 & 90. \\
TETON & 0020325 & 92.5 \\
TETOH & 0020320 & 83.7 \\
TETOH & 0020338 & 94.5 \\
TETON & 0020337 & 89.6 \\
TETON & 0020324 & 94.3 \\
TETON & 0020332 & 79. \\
TETON & 0020328 & 94. \\
TETON & 0020335 & 93.6 \\
TETON & 0020323 & 93.5 \\
TETON & 0020330 & 89.6 \\
TETON & 0020334 & 93.5 \\
TETON & 0020321 & 92.5 \\
TETON & 0020329 & 93.5 \\
TETON & 0020336 & 93.5 \\
TETON & 0020327 & 93.5 \\
TETON & 0020322 & 94.5 \\
TETON & 0020333 & 92.5 \\
TETON & 0020331 & 93.5 \\
TETON & 0020326 & 94.5 \\
TETON & 0075083 & 94. \\
TETON & 0075092 & 91. \\
TETON & 0075086 & 92. \\
IETON (?) & 0075078 & 89. \\
PARK & 0075093 & 71. \\
TETON & 0075085 & 91. \\
TETON & 0075088 & 93. \\
IETOH & 0075094 & 93.5 \\
TETON & 0075084 & 89.5 \\
PARK & 0020508 & \\
\hline & &
\end{tabular}

CARBON

CARBOA

CARBON

CARBOH

PLATTE
0075002 Q 48.0

0075006948.

00750039 48.

007500549.

0075055021.0 
42-14.97 * 104-46.82 H IMMIGRANTS YASHTUB 42-39.88 W 105-23.62 H DOUGLAS HAPA SPRING CASPER 1:250,000

42-32.28 * 106-43.27 W ALCOYA HOT SPRIHGS 42-32.28 W 106-43.27 Y ALCOYA IOT SPRINGS 42-42.10 N 107-06.33 W HORSE CREEK SPRINGS

LANDER i:250,000

12-48.02 N 108-03.03 N CONANT CREEK SPRINGS

42-29.53 N 108-10.25 Y SWEETWATER STATION SPRING NO. I

12-29.53 N 108-10.25 N SWEETMATER STATION SPRING NO. 2

42-29.53 N 108-10.25 H SWEETWATER STATION SPRING MO. 2

42-44.98 N 109-36.97 W STEELE HOT SPRINGS - SPRING

42-44.98 N 109-36.97 H STEELE HOT SPRINGS - SPRING I

42-44.98 N 109-36.97 W STEELE HOT SPRIMGS - SPRING 2

PRESTON 1:250,000

42-50.42 N 110-59.50 K AUBURA HOT SPRINGS

42-50.42 N 110-59.50 $\mathrm{K}$ AUBURH HOT SPRINGS

42-50.42 N 110-59.50 Y AUBURH HOT SPRINGS

42-50.42 N 110-59.50 N AUBURN HOT SPRIHGS

42-23.92 N 110-30.68 N BIG FALL CREEX SPRINGS

42-49.08 N 110-59.50 N JOHNSON SPRINGS

42-49.50 N 111-00.00 W ALBURN AREA

THERMOPOL IS $1: 250,000$

\section{3-39.67 N 108-11.72 H BATHTUB SPRING}

43-39.27 N 108-11.62 W BIG SPRING

43-39.21 W 108-11.62 W BIG SPRING

43-39.27 N 108-11.62 N BIG SPRING

43-39.27 N 108-11.62 N BIG SPRING

43-39.27 N 108-11.62 W BIG SPRING

43-39.27 N 108-11.62 Y BIG SPRING

13-39.32 N 108-11.63 * BLACK SULPIUR SPRING

43-00.48 W 108-50.08 W FORT MASHAKIE HOT SPRIHGS

43-00.48 N 108-50.08 N FORT MASHAKIE HOT SPRINGS

43-00.48 N 108-50.08 * FORT WASHAKIE HOT SPRIMGS

43-40.47 N 108-12.23 Y MAYTAG WELL

43-39.90 N 108-11.67 H MCCARTIIY HELL

43-39.27 N 108-12.00 W PILING SPRING

43-40.30 N 108-12.23 W SACANAWEA HELL

43-40.30 N 108-12.23 H SACNAHEA MELL

43-40.47 N 108-12.50 W VAM MORMAN HELL

43-39.67 N 108-11.68 W VIIITE SIRPIUR SPRLNG

43-39.67 N 108-11.68 N WHITE SULPIHUR SPRING

43-34.92 N 108-13.73 W UIMD RIVER CANYOH SPRIMG

43-34.92 N 108-13.73 W HIND RIVER CAHYON SPRIHG

43-29.38 N 109-36.48 H JAKEYS FORK SPRING

43-31.22 N 109-40.00 N LITTLE HARM SPRING
PLATTE

COHYERSE

0075056 Q 21

$0075007 \quad 30.0$

MATROMA

NATROHA

MATRONA

$0075043 \quad 54.0$

007504459.0

FREMOHT

FREMONT

FREMONT

FREMONT

SUBLETIE

SUBLETTE

LIHCOLH

LINCOLN

LINCOLH

LINCOLA

SUBLETTE

Lincour

LINCOLN

HOT SPRIHGS

HOT SPRINGS

HOT SPRINGS

HOT SPRINGS

HOT SPRINGS

HOT SPRINGS

HOT SPRINGS

IIOT SPRINGS

FREMONT

FREMONT

IIOT SPRINGS

HOT SPRIMGS

HOT SPRINGS

HOT SPRIHGS

HOT SPRINGS

HOT SPRINGS

HOT SPRINGS

HOT SPRINGS

IIOT SPRINGS

HOT SPRINGS

HOT SPRINGS

FREMONT

FREMONT
007504523.5

$0075008 \quad 16.0$

007501730.0

0075019 Q 31.5

$0075018 \quad 31.5$

007505935.5

007506139.0

0075038 Q 62.0

0075041 Q 62.0

0075040 Q 16.0

0075039062.0

007505216

$0075042 \quad 16.0$

$0000779 \quad 16.0$

$0075025 \quad 53.0$

0075031 Q 56.

$0075030 \quad 56.0$

0075032 Q 56.

0075033 Q 56.

0075034 Q 56 .

0075035 Q 56 .

0075029 Q 55.

007501046.5

$0075009 \quad 44.0$

$0075011 \quad 44.5$

007502154

$0075024 \quad 54.0$

$0075028 \quad 35.0$

007502252.0

0075023 Q 52.

$0075020 \quad 51.0$

$0075026 \quad 53.0$

0075027 Q 53.

0075037 Q 22.

007503622.0

007501220.0

0075014 Q 25.
007502154.0 
43-31.22 N 109-40.00 W LITTLE WAPM SPRING

FREMONT 43-33.60 $109-43.08$ Y HAPY SPRING CREEK SPRINGS FREMONT $\begin{array}{ll}\text { 43-33.60 N 109-43.88 W WAPM SPRING CREEK SPRINGS } & \text { FREMONT } \\ 43-33.60 \mathrm{~N} \text { 109-43.88 } N \text { WARM SPRING CREEK SPRINGS } & \text { FREMONT }\end{array}$

007501325.0

43-32.75 N 110-44.37 * ABERCRONBIE WARM SPRINGS 43-18.00 N 110-46.48 W ASTORIA SPRINGS 43-28.30 N 110-50.08 H BOYLES HILL SPRINGS 43-21.90 N 110-26.57 W GRANITE FALLS HOT SPRINGS 43-22.20 N 110-26.68 H GRANITE HOT SPRING 43-22.20 N 110-26.68 Y GRANITE HOT SPRING 43-57.50 N 110-41.75 H JACKSON LAKE HOT SPRINGS 43-38.37 N 110-36.92 W KELLY HARM SPRING 43-16.93 N 110-01.13 W KENDALL HAPM SPRINGS 43-16.93 H 110-01.13 H KEMDALL HARH SPRIMGS

43-54.40 N 110-11.70 H NORTII BUFFALO FORX SPRIHGS - GROUP I 43-54.40 N 110-11.70 H NORTH BUFFALO FORK SPRINGS - GROUP 2

TETON

TETON

TETON

TETON

TETOH

TETON

TETON

TETON

SUBLETTE
SUBLETTE

SUBLETE

TETON 43-37.43 * 110-36.37 N TETON VALLEY UAPY SPRINGS

TETON

0075016 Q 29.

CODY $1: 250,000$

44-44.90 N 108-11.35 K LITILE SHEEP MOUNTAIN SPRING

44-36.45 N 108-09.13 H SHEEP HOUNTAIM SPRINGS

44-28.50 N 109-12.25 H BUFFALO BILL RESERYOIR SPRINGS

44-30.78 H 109-06.88 Y DE MARIS HOT SPRINGS

44-30.78 * 109-06.88 H DE HARIS HOT SPRINGS

44-30.78 " 109-06.88 Y DE MARIS HOT SPRIMGS

44-30.78 N 109-06.88 Y DE MARIS HOT SPRINGS

44-30.78 N 109-06.88 * DE MARIS HOT SPRIHGS

44-30.78 N 109-06.88 Y DE MARIS HOT SPRINGS

94-30.78 $\mathrm{N}$ 109-06.88 $\mathrm{K}$ DE MARIS HOT SPRINGS

BIG HORN

BIG HORN

PARK

PARK

PARK

PARK

PARK
PARK

PARK
PARK

PARK
PARK

PARK

ASHTON 1:250,000

PARK

007506327.0

$0075064 \quad 37.0$

$0075065 \quad 30.0$

$0075066 \quad 45.0$

0075067 Q 41.0

007506839.0

0075073072.0

0075074 Q 72.0

007507427.0

0075058 Q 29.5

007505729.5

0075075

$\begin{array}{ll}0075076 & 45.0 \\ 0075077 & 18.0\end{array}$

44-27.67 N 110-51.65 W BLACK SAND BASIN - UHNAMED SPRING : TETON

44-27.7 N 110-51.3 OLACK SANO BASIN - UNNAMED SPRIHG

44-16.55 11 110-30.20 H HEART LAKE GEYSER BASTH - PUSTIC GROUP - UMMAME TETOH

44-28.20 N 110-51.52 W HILLSIDE GROUP - ASTA SPRING (NO. 3) -

44-28.20 N 110-51.52 W HILSIDE GROUP - ASTA SPRING (NO. 3) TE TON

44-28.20 N 110-51.52 Y HILSIDE GROUP - UMHAMEO SPRING. 3) TETON

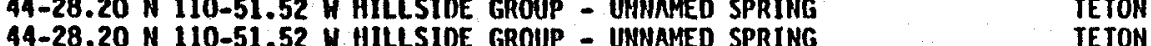

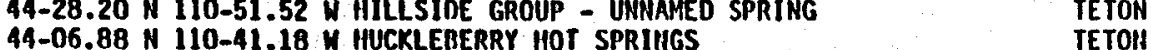

44-06.88 N 110-41.18 W HUCKLEBERRY HOT SPRIHIGS

44-06.8B N 110-41.18 W HUCKLEBERRY HOT SPRINGS

44-06.88 N $110-41.18$ W IUCKLEEERRY HOT SPRINGS
$44-06.88$ N $110-41.18$ N HUCKLEBERRY HOT SPRIHGS

44-31.47 N 110-52.32 Y LOMER GEYSER BASIN - IMPERINL GEYSER

44-32.2 N 110-50. $\mathrm{N}$ LONER GEYSER BASIN - BATH SPRING

44-33.2 N 110-50. W LOWER GEYSER BASIN - BATH SPRING

44-32.30 N 110-47.02 H LOWER GEYSER BASIN - FIREHOLE LAKE

44-32.30 N 110-46.9B Y LOWER GEYSER BASIN - FIREHOLE POOL

44-32.30 N 110-46.98 W LOWER GEYSER BASIN - FIREIIOLE POOL

TETON

TETON

TETON

TETON

TETON

TETON

14-32.30 N 110-46.90 W LOHER GEYSER BASIN - FIREHOLE POOL

TETOI.

TETOH

TETOH

TETOII

007500020.

007500121.

0075046

0075050 Q 24.

0075048 Q 37.0

0075048 Q 37.0

007504736.0

0075054027.5

007504927.0

0075053 Q 27.0

$\begin{array}{lll}0075052 & 9 & 27.0 \\ 0075051 & 0 & 28.0\end{array}$

14.

\author{
002029688. \\ 0020295 \\ 0020263 \\ 0020262 \\ 002026665 . \\ 002026584 . \\ 0075071961.0 \\ 0075070 Q 61.0 \\ 0075072 Q 71.0 \\ 0075069 Q 45.0 \\ 0020379 \\ 0020367 \\ 002036990.5 \\ 0020368 \\ 002037091.
}


14-31.4 N 110-47.7 W LONER GEYSER BASIN - FISSURE GROUP - EBOHY SPRI TETON 44-32.6 110-48.4 W LOHER GEYSER BASIN - FOUNTAIN GROUP - FOUNTAIN TETON 44-31.6 N 110-47.5 N LOWER GEYSER BASIN - GREAT FOUNTAIN GROUP - DIA TETON 44-31.6 N 110-47.5 W LONER GEYSER BASIN - GREAT FOUMTAIN GROUP - DIA TETON 44-31.47 N 110 52.32 W LONER GEYSER BASIN - IMPERIAL GEYSER - TETON 44-31.47 N 110-52.32 W LOWER GEYSER BASIN - IMPERIAL GEYSER TETON 44-31.5 N 110-4E.5 W LOWER GEYSER BASIN - KALEIDOSCOPE GROUP - UNMAM TETON 44-34.01 N 110-48.23 K LOWER GEYSER BASIN - MORNING MIST SPRINGS - UNN TETON 44-34.00 N 110-48.23 W LOWER GEYSER BASIN - NORNING MIST SPRINGS - UNM TETON 44-34.00 N 110-48.22 N LONER GEYSER BASIN - MORNING MIST SPRINGS - UNN TETON 14-34.00 N 110-48.25 N LOWER GEYSER BASIN - MORNING MIST SPRINGS - UHN TETOH 44-34.00 N 110-48.23 W LOWER GEYSER BASIN - MORNING MIST SPRINGS - UNN TETON 44-33.52 N 110-51.42 H LOWER GEYSER BASIN - MOUND SPRING 44-33.47 N 110-52.10 H LOHER GEYSER BASIN - QUEEN'S LAUNDRY TETON 44-33.47 N 110-52.10 W LOHER GEYSER BASIN - QUEEN S LAUNDRY 44-33. N 110-50. W LOWER GEYSER BASIN - RIVER GROUP - UNHAMED SPRI TETOH 44-33. N 110-50. W LOWER GEYSER BASIN - RIYER GROUP - UNNAMED SPRI TETON 44-33.18 N 110-49.51 W LOWER GEYSER BASIN - RIVER GROUP - OJO CALIENTE TETON 44-33.18 N 110-49.51 W LOWER GEYSER BASIN - RIVER GROUP - DJO CALIENTE TETON

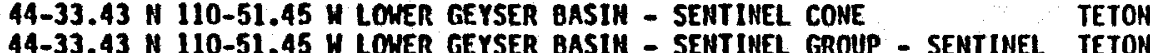
44-33.43 N 110-51.45 W LONER GEYSER BASIN - SENTINEL GROUP - SENTINEL TETON 44-33.43 N 110-51.45 W LOWER GEYSER BASIN - SENTINEL GROUP - SENTIMEL TETON 44-33.45 N 110-52.10 H LOUER GEYSER BASIN - SENTINEL GROUP - UMMAMED S TETON 44-33.47 11 110-52.10 H LOWER GEYSER DASIH - SENTIWEL GROUP - QUEEN'S L TETOH 44-33.52 M 110-52.10 H LOUER GEYSER BASIH - SENTIMEL GROUP - QUE 49-34.02 11 110-51.52 M LOUER GEYSER OASIM - SEMTIMEL GROUP - UNHAMED D. 94-34.05 N 110-51.48 W LOHER GEYSER BASIN - SENTINEL GROUP - FLAT CONE TETON ROSETTE G TETON 14-32.00 N 110-47.57 K LOWER GEYSER BASIN - SURPRISE POOL - ROSETTE G TETON 44-32. N 110-53. H LONER GEYSER BASIM - UMMAMED MUD POT TETON 44-32.29 H 110-46.57 H LOWER GEYSER BASIN - UNNAMED SPRING $\quad$ TETON 44-32. I 110-47. N LOWER GEYSER BASIN - UHMAMED SPRING TETON 44-32. N 110-47. H LOWER GEYSER BASIH - UWNAMED SPRING TETON 44-31.58 N 110-47.48 H LOWER GEYSER BASIN - UWHAMED SPRING TETON 14-32.2 N 110-47.30 W LOWER GEYSER BASIN - UMNAMED SPRING 44-31. N 110-52. LOWER GEYSER BASIN - UWHAMED STEAM YENT TETOM 44-58. N 110-42. H MANOTH HOT SPRINGS - ANGEL TERRACE PARK 44-58. N 110-42. N MANOTH HOT SPRINGS - CAVERN TERRACE PARK 44-58.5 N 110-42.6 H MMMOTII HOT SPRINGS - CLEOPATRA SPRING PARK 44-58.5 N 110-42.6 M MAMNOTH HOT SPRINGS - CLEOPATRA SPRING PARK 44-58. N 110-42. H MAPMOTH $110 T$ SPRINGS - CUPID SPRING

44-58. N 110-42. H MAMATH HOT SPRINGS - HYMEN TERRACE

44-58. N 110-42. N MAMNOTH HOT SPRINGS - JUPITER TERRACE

44-58. N 110-42. N MHMNTH $110 T$ SPRINGS - MAIN TERRACE

44-58. II 110-42. H MAMUTH HOT SPRINGS - MINERYA SPRINGS

Y MAMNDTH HOT SPRINGS - NEH HIGHLAND TER

N 110-42.

H MAYMOTH HOT SPRINGS - PALETTE TERRACE

N 110-42

W MAMOTII IIOT SPRIHGS - UNHAMED SPRING

44-58. N $110-42$.

W MAMAOTII IOT SPRINGS - UNMAATED SPRINIG

44-58. N $110-42$.
$44-31.23$

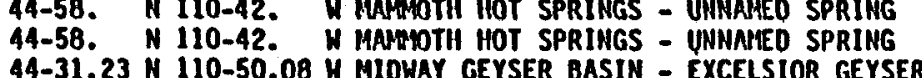

44-32.33 N $110-50.53$ H HLOMAY GEYSER BASIH - FAIRY CREEK GROUP - UMHAM TETON

44-32.18 N 110-51.25 U MIDUAY GEYSER BASII - FAIRY CREEK GROUP - UMHAM TEY

0020365

0020364

002036384.0

0020362

0020358

0020359 B.

0020366

0020383

002038229.5

0020386 75.

002038675.

002038429

002040094

0020392

002038163

002038064.

0020376

0020389

002039193.

$0020390 \quad 93.5$

002039389.5

$0020395 \quad 69.5$

0020394 86.

0020399

002039892 .

0020397

0020396

002038785.

002036161 .

002037191.

002037393.

002037292 .

002037488.5

002037575

0020357

0020464

002047066.4

0020460

0020459

002047171 .

$\begin{array}{ll}0020471 & 71 . \\ 0020465 & 70.5\end{array}$

00204657.

002046673 .

002047375 .

002047274 .

0020469

0020468

0020463

0020462

0020461

002034391.

002035693 .

002035274. 
44-32.18 N 110-51.27 W MIDWAY GEYSER BASIN - FAIRY CREEK GROUP - UNMAM TETON 44-32.15 N 110-51.10 W MIDWAY GEYSER BASIN - FAIRY CREEK GROUP - UNMAM TETON 44-32.18 N 110-51.25 W MIDHAY GEYSER BASIN - FAIRY CREEX GROUP - UWHAM TETON 44-32.33 N 110-50.53 N MIDHAY GEYSER BASIN - FAIRY CREEK GROUP - UHHAM TETON 44-31. N 110-50. W HIDWAY GEYSER BASIN - FLOOD GEYSER TETON 44-31. N 110-50. H MIDWAY GEYSER BASIN - FLOOD GEYSER TETON 44-31.2 110-50.0 W MIDHAY GEYSER BASIN - INDIGO SPRING TETON 44-31.20 N 110-50.13 H MIDWAY GEYSER BASIN - PRISMATIC LAKE TETON 44-30.50 N 110-48.42 H MIDWAY GEYSER BASIN - RABBIT CREEX GRDUP - UNNA TETON 44-30.50 N 110-48.42 H MIDWAY GEYSER BASIN - RABBIT CREEK GROUP - UNHA TETON 44-30.58 $110-49.18$ Y MIDUAY GEYSER BASIN - PABBIT CREEX GROUP - UNA TETON 44-30.49 N 110-48.42 H MIDUAY GEYSER BASIN - RABBIT CREEK GROUP - UMHA TETOH 14-30.57 $110-49.50$ H MIOUAY GEYSER BASIH - RABBIT CREEK GROUP - VNA TETON 44-31.5 N 110-49.50 N MTOWA GEYSER BASIN - ONMMED CONE 44-43. N 110-50. H MIDHAY GEYSER BASIN - UHNAMED SPRING 44-43. N 110-43. H NORRIS GEYSER BASIN - "BATH-TUB" SPRING PARK 44-43. N 110-43. H NORRIS GEYSER BASIN - "BATH-TUB" SPRIHG 44-43. N 110-43. H NORRIS GEYSER BASIN - BATTT-TUB SPRING 44-43. N 110-43. M NORRIS GEYSER BASIN - BLACK GROHLER 44-43. N 110-43. N NORRIS GEYSER BASIM - OLACK GROMER 44-43. N 110-42. W NORAIS GEYSER 110-43. H NORRIS GEYSER BASIN 110-42.00 W NORRIS GEYSER BASIN - CONGRES POOL PARK 44-43. N 110-42. H NORRIS GEYSER BASIH - CORAL 3 $110-42.42$ W NORRIS GEYSER BASIN - ECHINUS GEYSER PARK 44-43. N 110-43. H NORRIS GEYSER BASIN - EHERALD SPRING PARK - MAORIS GEYSER BASIH - GABLE SPRING (NO. 32) PARK 44-43.56 N 110-42.40 Y NOPRIS GEYSER BASIN - GEYSER NO. 27 . 44-43. H 110-43. Y NORRIS GEYSER BASIN - GREEN ORAGON SPRING PARK 44-44. N 110-42. H NORRIS GEYSER BASIN - IAIS SPRING (NO. 71) PARI 44-43. N 110-43. M NORRIS GEYSER BASIH - PEARL GEYSER

N NORRIS GEYSER gASIH - PORCELAIN BASIN NO. 24

$\checkmark$ MORRIS GEYSER BASIN - PORCELAIN BASIN NO. 70

44-43. N 110-42. W WORRIS GEYSER BASIN - SPRING NO. 15 44-43. N 110-42. W NORRIS GEYSER BASIN - SPRING NO. 18 44-43. N 110-42. N NORRIS GEYSER BASIN - SPRING NO. 19 44-43.75 N 110-42.47 H MORRIS GEYSER BASIN - SPRING NO. 20 44-43. N 110-42. W NORRIS GEYSER DASIN - SPRING NO. 21 44-43. N 110-42. Y MORRIS GEYSER BASIN - SPRING NO. 23 44-43.81 N $110-42.03$ H HORRIS GEYSER BASIN - SPRING ND. 25 44-43. 44-43. N 110-42. W HORRIS GEYSER BASIN - SPRING NO. 30 44-43. N 110-42. H NORRIS GEYSER BASIN - SPRING NO. 33 44-43. N 110-42. W NORRIS GEYSER BASIN - SPRING NO. 4 44-43. N 110-42. W WORRIS GEYSER BASIN - SPRING NO. 5 44-43. N 110-42. H NORRIS GEYSER BASIN - SPRING NO. 6 n Norris Geyser basin - SPRing ho. 61 n noRris geyser basin - spring no. 62 44-44. N 110-42. W NORRIS GEYSER BASIN - SPRING MO. 68 44-43. N 110-42. H NORRIS GEYSER BASIII - SPRING NO. 7 44-43. N 110-42. H NORAIS GEYSER BASIN - SPRING NO. 9 44-43.80 N 110-41.98 W NORRIS GEYSER BASIN - UHNAYEO SPRING 44-44. H 110-43. W NORRIS GEYSER BASIN - UNHAMEO SPRING 44-43. N 110-43. H MORAIS GEYSER BASIH - UWHAMED SPRING PARK PARK PARK PARK

0020353.93.

002035482.0

0020351 92:

002035594.5

0020342 . 94.

0020341 \& 92.5

0020344

002034563.3

002034982.

0020348 84.

002034794 .

0020350

002034692.6

0020339

0020445

0020444

002044693.

0020426

0020425

002044993

0020431

0020434

0020421

0020440 88.

0020430

84

0020442

0020420

002044185.

0020428 .

0020409

0020410

0020411

002042

0020424

0020412

0020413

0020436

0020414

0020415

0020404

0020405

0020406

0020417

0020418

0020419

0020407

0020408

002043592.

002043379.

002043892 . 
44-44. N 110-43. W NORRIS GEYSER BASIN - UANAMED SPRIHG 44-43.58 N 110-12.42 W HORRIS GEYSER BASIN - UNMAMED SPRING 41-43.5B N 110-42.42 H MORRIS GEYSER BASIN - UNMAMEO SPRING 44-43.8 $N$ 110-42.5 H NORRIS GEYSER BASIN - UNMAMEO SPRING 44-43.8 $110-42.5$ N HORRIS GEYSER BASIN - UMHAMED SPRING 44-43.7 N 110-42.6 H MORRIS GEYSER BASIN - UHMAPED SPRIMG 44-43.7 N 110-42.6 H NORRIS GEYSER BASIM - UNMAMEO SPRING 44-43.8 N 110-42.7 H NORRIS GEYSER BASIN - UMMAMED SPRING 44-43.8 N 110-42.7 H RORRIS GEYSER BASIN - UMMAMED SPRIHG 44-43.6 N 110-42.7 H MORRIS GEYSER BASIN - UMHAMED SPRING 44-43.8 N 110-42.7 W MORRIS GEYSER BASIH - UMHAMED SPRING 44-43.7 N 110-42.6 H NORRIS GEYSER BASIN - UMHAMEO SPRING 44-44. N 110-43. H MORRIS GEYSER BASIN - UMHAMED STEAM VEMT M 110-43. WORRIS GEYSER BASIN - UPPER GREEN DRAGON SPRING PARK 44-44. I 110-43. M MORRIS GEYSER BASIN - VERMILLION POOL PARK 44-27.77 N 110-50.75 N ORANGE GROUP - UHHAMED SPRING TETOH 44-27.68 N 110-50.00 Y PIPEL IHE CREEK SPRIMG

44-26.4 $110-34.5$ P POTTS IOT SPRIHG BASIN - UHHAMED GEYSER 44-26.4 N $110-34.5$ Y POTTS $10 T$ SPRING BASIN - WNMAMED SPRING TETON 44-21.1 N 110-48.0 SHOSHONE GEYSER BASIN - BLUE GLASS SPRING TETON 44-21.18 M 110-47.54 U SHOSHONE GEYSER BASIN - MINUTE MAN GEYSER TETON A4-21.18 N 110-47.54 $N$ SHOSHONE GEYSER BASTN - MINUTE MAN GEYSER TE 44-21.12 N I10-48.03 W SIOSHONE GEYSER BASIII - TAURUS GEYSER D TETON 44-21. N $110-48$. H SHOSHOHE GEYSER BASIN - UNNAMED SPRIMG TEION II0-48. 44-39.00 N 110-50.45 W TERRACE SPRING 44-31. N 110-50. H UNWAMEO SPRING 44-27.43 N 110-50.00 H UPPER GEYERS BASIN - UNNARED SPRING 49-27.43 14-27.43 M 110-50,00 M UPPER GEYSER BASIN - ARTEMISIA GEYSER 44-27.73 N 110-51.35 Y UPPER GEYSER BASIH - BLACK SAMD POOL . T 44-27.43 N 110-50.00 Y UPPER GEYSER BASIN - BOTTOMLESS PIT $44-27.5$ N $110-50.6$ H UPPER GEYSER BASIN - CASTLE GEYSER TER 44-27.43 N 110-50.00 Y UPPER GEYSER BASII - CHINAHAN SPRING TETON 44-27.43 N 110-50.00 H UPPER GEYSER BASIN - CHINAMAN SPRING 44-27.43 N 110-50.00 K UPPER GEYSER BASIN - CHINAMAN SPRING TETON 44-28.50 H 110-50.00 H UPPER GEYSER BASIN - DAISY SPRIMG 44-27,50 N 110-50.00 H UPPER GEYSER BASIN - DAISY SPRIMG 44-27.4 N 110-50.0 W UPPER GEYSER BASIH - EAR SPRING $44-28.5$
$44-28.5$ 110-50.0 H UPPER GEYSER BASIN - ECONONIC GEYSER $\quad$ TETON 44-28.5 N 110-50.0 H UPPER GEYSER BASIN - ECONOMIC GEYSER 44-27.7 N 110-49.5 H UPPER GEYSER BASIN - GIAITESS GEYSER 44-28.20 N 110-51.52 H UPPER GEYSER BASIM - HILLSIDE N0. 1 44-28.50 N 110-50.00 Y UPPER GEYSER BASIN - IRON SPRIMG TETON 44-28.5 $N$ 110-50.0 H UPPER GEYSER BASIN - JENEL. GEYSER TETON 44-28.5 N 110-50.0 H UPPER GEYSER BASIN - JEHEL GEYSER TETON 44-28.05 N 110-50.32 Y UPPER GEYSER BASIN - MORNING GLORY POOL TETON 44-27.63 N 110-49.38 N UPPER GEYSER BASIN - OLD FAITHFUL GEYSER. TETON 44-27.98 N 110-50.42 H UPPER GEYSER BASIN - PINE SPRINGS GROUP - UNMAM TETON 14-28.42 N 110-50.45 N UPPER GEYSER BASIN - PINE SPRINGS GROUP - UNNAM TETON 14-28.25 N 110-51.95 K UPPER GEYSER BASIM - PUICH BOHL SPRING - ONHAM TETON 44-28.25 N 110-51.95 W UPPER GEYSER BASIN - PUNCHBOHL SPRING

0020429

002044891

002044770 .

0020457

0020456

002045585.

002045473.

002045390 .

002045289.

002045190.

002045089.

002045880

0020437

002044394

002043294 .

002028771 .

0020307

002048695

002048794

0020474

0020478

0020475

0020477

0020476

002040260.

$0020401 \quad 61.8$

002034094.

0020304

002025468 .

002025787 .

0020256

$0020293 \quad 92.7$

0020267

020286

$0020268 \quad 93.5$

002026993.5

002027789.

002027688.6

002025193.

002029163.

002029083.7

002029770 .

002024994.1

0020264

002028090.8

002028493.7

002028588 .

002029877.

0020281

002031983.

002031983.

002031883.

002027392.8 
44-28.48 N 110-51.15 H UPPER GEYSER BASIN - SAPPHIRE SPRING 44-27.7 N 110-51.3 H UPPER GEYSER BASIN - SPOUTER SPRING 44-28.50 N 110-50,00 H UPPER GEYSER BASIN - SULFIDE SPRING 44-27.43 N $110-50.00$ H UPPER GEYSER BASIN - TEAKETTLE SPRING $44-27.43$
$44-28.5$ $\begin{aligned} & 44-28.5 \\ & 44-28.5\end{aligned} 110-50.0$ W UPPER GEYSER BASIN - THREE SISTERS SPRING $44-28.5$ N $110-50.0$ W UPPER GEYSER BASIN - THREE SISTERS
$44-27.43$ N $110-50.00$ W UPPER GEYSER BASIN - TOPAZ SPRING $44-27.43$ N 110-50.00 W UPPER GEYSER BASIN - TOPAZ SPRING
$44-27.03$ N 110-50.33 Y UPPER GEYSER BASIN - UNHAMEO DRILL HOLE 14-27.43 N 110-50.00 W UPPER GEYSER BASIN - UNMAMEO SPRING 44-27.43 N 110-50.00 W UPPER GEYSER BASIN - UNHAMEO SPRING 44-27.35 N 110-49.37 N UPPER GEYSER BASIN - UMHAMED SPRING 44-27.58 N 110-49.07 $\mathrm{W}$ UPPER GEYSER BASIN - UNMAMED SPRING 44-28.20 N 110-50.45 W UPPER GEYSER BASIN - UMMAMED SPRIMG 44-27.35 N 110-49.37 U UPPER GEYSER BASIN - UMMAMEO SPRIMG 44-28.0 II 110-50.0 $Y$ UPPER GEYSER DASIN - UMUAMED SPRIMG 44-28.0 $N$ IIO-50.37 $W$ UPPER GEYSER BASIN - UNMRED SPRING 44-28.0 N $110-50.0$ W UPPER GEYSER BASIN - UMHAMED SPRIHG 44-27.7 N 110-59.5 H UPPER GEYSER BASIN - UNMAYED SPRING 44-27.4 N 110-50.0 H UPPER GEVSER BASIN - UNHAMED SPRING 44-28.5O N 110-50.00 W UPPER GEYSER BASIN - UNNAHEO SPRING 44-27.43 N 110-50.00 $N$ UPPER GEYSER BASIN - UNNAMED SPRING 44-28.20 N 110-50.45 Y UPPER GEYSER BASIN - UNNAMED SPRING 44-28.50 N 110-50.00 Y UPPER GEYSER BASIN - UNNAMED SPRING 44-28.25 N 110-51.95 W UPPER GEYSER BASIN - UNMAMED SPRING 44-28.4 N 110-51.53 W UPPER GEYSER BASIN - UMNAMED SPRING 44-28.44 N 110-51.52 H UPPER GEYSER BASIN - UNNAMED SPRING 44-27.77 N 110-49.20 Y UPPER GEYSER BASIN - UWNAMED SPRING 44-28.0 N 110-50.0 W UPPER GEYSER BASIN - UMNAMED SPRIHG 44-28.00 N 110-50.00 W UPPER GEYSER BASIN - UMNAMED SPRING 44-27.53 N 110-49.46 W UPPER GEYSER BASIN - UMHAMEO SPRIMG 44-27.35 H 110-49.37 H UPPER GEYSER BASIN - UMHAMED SPRIHG 44-27.67 N 110-49.63 Y UPPER GEYSER BASIN - UIHIMED SPRING 14-27.53 N 110-49.45 U UPPER GEYSER BASIM - UMHIUED SPRIMG 14-27.53 $N$ 110-49.45 $Y$ UPPER GEYSER BASIN - UMNMED SPRING 44-27.50 N 110-50.00 4 UPPER GEYSER BASIM - UNMAMED SPRIM 44-25. $M$ IIO-34.53 H UPPER GEYSER BASIN - NEST SPRING 44-25. N 10-34. H WEST INUND GEYSER BASIN - ABYSS POOL 44-25. N 110-34. W NEST TINM GEVSER BASIN - BLACK POOL 44-24. N 110-34. Y YEST TIMMB TEYSER BASIN - EPHEDRA GEYSER 44-24. N 110-34. W WEST TILMB GEYSER BASIH - UNHAMED SPRING 44-25. N 110-34. N WEST THUNB GEYSER BASIN - UNMAMED SPRING 44-25.5 N 110-34.2 N WEST THUHB GEYSER BASIN - UNHAMED SPRING 44-54.65 N 110-23.78 Y YELLOWSTONE - CALCITE NO. 1 44-54.65 N 110-23.78 W YELLONSTONE - CALCITE NO. 2 44-54.65 N 110-23.78 N YELLOMSTOHE - CALCITE SPRINGS 44-54.65 N 110-23.78 N YELLOWSTONE - CALCITE SPRIMGS 44-42.78 N 110-44.67 W YELLOYSTONE - CHOCOLATE POTS 44-42.77 N 110-44.67 N VELLOHSTONE - CHOCOLATE POTS 14-45.53 N 110-25.85 N YELLOYSTOHE - DEYIL'S INKPOT 44-45.53 N 110-25.85 W YELLOHSTONE - DEYIL'S IMKPOT 44-45.53 N 110-25.85 Y YELLOWSTONE - DEVIL'S IMKPOT 44-27.4 N 110-50.0 H YELLOHSTONE - EAR SPRING 44-31.23 N 110-50.08 Y YELLOWSTOME - EXCELSIOR SPRING 44-32.30 N 110-47.02 Y YELLOHSTONE - FIREHOLE LAKE (LOWER) 44-28.2 N 110-51.5 W YELLOWSTONE - IIILLSIDE (IIPPER)
TETOH

TETOH

TETOH

TETON

IETOH

TETOH

TETON

TETON

TETON

TETON

TETON

TETON

TETON

IETON

TETON

TETON

TETON

TETOH

TETON

TETON

TETON

IETON

IETON

TETON

IETON

IETON

TETON

IETON

TETON

TETON

TETON

TETOA

IETON

TETON

TETON

TETON

TETON

TETON

TETON

IETON

TETOH

PARK

PARK

PARK

PARK

TETON

IE TON

TETON
IETON
$0020258 \quad 94.5$

002029492.

002028377

0020271

$\begin{array}{ll}0020272 & 92 . \\ 0020278 & 90.5\end{array}$

0020279

0020270

0020282

002030055.

002029963.

002030390 .

002030190 .

0020306 81.

002030663.

002031071

0020312

$0020250 \quad 82.5$

002025289.

0020289 91.

002025384.

0020302

0020292

002027584.

002026080 .

002026176.

002031391.

002031175 .

002030980 .

002031764 .

002031689.

002031575

0020314 .

02034 82.

002028887.

002025980.

002048378.5

0020485 78.5

0020484 B4.

0020481 .

0020480

002050392.5

002050589.4

0020502

0020504

002050651.

0020507

0020492

002049381.5

007509195 .

007509195.

007508976.

007508284.5 
44-37.28 N 110-25.58 $M$ VELLONSTONE - SULPUR POOL

44-39.00 N 110-50.45 $W$ YELLOWSTONE - TERRACE

44-32.38 N 110-15.22 Y YELLONSTONE - UNNAMEO SPRING

TETON

44-32.48 N 110-15.35 W YELLOWSTOME - UNWAHED SPRING

44-46.00 N 110-25.80 H YELLOWSTONE - UNHAMED SPRING

TETON

44-46.00 N 110-25.80 K VELLOWSTONE - UNMAMED SPRING

44-46.00 N 110-25.80 $N$ YELLOWSTONE - UMNAMED SPRING

14-39.53 N 110-28.75 K YELLOUSTONE - INNAMED SPRING

44-39.50 N 110-29.00 K YELLLWSTONE - UMMAMED SPRING

44-39.50 N 110-28.93 W YELLOUSTOHE - UNHAMED SPRING

44-39.50 N 110-28.93 W YELLOUSTONE - UNMANED SPRING

PARK

PARK

PARK

PARK

PARK

PARK

PARK

PARK

PARK

44-28.5 N 110-50,0 H YELLOWSTONE - UPPER GEYSER BASIN - UNMAMED SPRI TETOH

002050085.5

007507973.5

0020416

007508795

0075090 95.

0020501

007508066.

002049986.

002049888.

002049788.

0020496

0020495

002049187.

002048985.

002048879.8

0020308 87.

8


APPENDIX D

Sources for the records in the GEOTHERM sample file for Wyoming. Each reference is preceded by its abbreviated form (called CODE) used in the sample file (Table 1). Entries in this computer-generated appendix are sorted by CODE.

CODE = ALLEN AND DAY, 1935

ALLEN, E. T., AND DAY, A. L., 1935, HOT SPRINGS OF THE YELLOWSTONE NATIONAL PARK: CARNEGIE INSTITUTION OF WASHINGTON PUBLICATION NO. $466,525 \mathrm{P}$.

CODE = BRECKENRIDGE AND HINCKLEY, 1978

BRECKENRIDGE, R. M. AND HINCKLEY, B. S., 1978, THERMAL SPRINGS OF WYOMING: WYOMING GEOLOGICAL SURVEY BULLETIN 60, $104 \mathrm{P}$.

CODE = GOOCH AND WHITFIELD, 1888

GOOCH, F. A., AND WHITFIELD, J. E., 1988, ANALYSES OF WATERS OF YELLOWSTONE NATIONAL PARK, WITH AN ACCOUNT OF THE METHODS OF ANALYSIS EMPLOYED: U.S. GEOLOGICAL SURVEY BULLETIN $47,84 \mathrm{P}$.

CODE $=$ GUNTER, 1973

GUNTER, B. D., 1973, AQUEOUS PHASE-GASES PHASE MATERIAL BALANCE STUDIES OF ARGON AND NITROGEN IN HYDROTHERMAL FEATURES AT YELLOWSTONE NATIONAL PARK: GEOCHIMICA ET COSMOCHIMICA ACTA, V. 37 , NO. $3, P$. 495-513.

CODE = GUNTER AND MUSGRAVE, 1966

GUNTER, B. D., AND MUSGRAVE, B. C., 1966, GAS CHROMATOGRAPHIC MEASUREMENTS OF GEOTHERMAL EMANATIONS AT YELLOWSTONE NATIONAL PARK: GEOCHIMICA ET COSMOCHIMICA ACTA, V. 30, NO. 11, P. 1175-118.

CODE = MAZOR AND WASSERBURG, 1965.

MAZOR, E., AND WASSERBURG, G. J., 1965, HELIUM, NEON, ARGON, KRYPTON AND XENON IN GAS EMANATIONS FROM YELLOWSTONE AND LASSEN VOLCANIC NATIONAL PARKS: GEOCHIMICA ET COSMOCHIMICA ACTA, Y. 29, NO. $5, P$. 443-454.

CODE = TRUESDALE AND FOURNIER, 1976

TRUESDALE, A. H. AND FOURNIER, R. 0., 1976, CONDITIONS IN THE DEEPER PARTS OF THE HOT SPRING SYSTEMS OF YELLOWSTONE NATIONAL PARK, WYOMING: U. S. GEOLOGICAL SURVEY OPEN-FILE REPORT 76-428, 29 P. 
CODE = WHITE AND WILLIAMS, 1975

WHITE, D. E. AND WILLIAMS, D. L., 1975, ASSESSMENT OF GEOTHERMAL RESOURCES OF THE UNITED STATES-1975: U. S. GEOLOGICAL SURVEY CIRCULAR 726, 155 P. 